\title{
TEMPERATURE OF AIRCRAFT CARGO FLAME EXPOSURE DURING ACCIDENTS INVOLVING FUEL SPILLS
}

by

Joseph A. Mansfield

JM Technical

$$
\begin{array}{r}
\text { FiECEIVED } \\
\text { JANUARY } 1993 \text { AllF } 31993 \\
\text { USTI }
\end{array}
$$

prepared for

\section{LAWRENCE LIVERMORE NATIONAL LABORATORY LIVERMORE, CA 94550}




\section{DISCLAIMER}

Work performed under the auspices of the U.S. Department of Energy by Lawrence Livermore National Laboratory under contract number W-7405-ENG-48.

This document was prepared as an account of work sponsored by an agency of the United States Government. Neither the United States Government nor the University of Culifornia nor any of their employees, makes any warranty. express or implied, or assumes any legal liability or responsibility for the accuracy, completeness, or usefulness of any information, apparatus, product, or process disclosed, or represents that its use would not infringe privately owned rights. Reference herein to any specific commercial products, process, or service by trade name, trademark, manufacturer, or otherwise, does not necessarily constitute or imply its endorsement, recommendation, or favoring by the United States Government or the University of California. The views and opinions of authors expressed herein do not necessarily state or reflect those of the United States Government or the University of California, and shall not be used for advertising or product endorsement purposes. 


\section{TEMPERATURE OF AIRCRAFT \\ CARGO FLAME EXPOSURE DURING ACCIDENTS INVOLVING FUEL SPILLS}

by

Joseph A. Mansfield

JM Technical

JANUARY 1993

\section{prepared for
LAWRENCE LIVERMORE NATIONAL LABORATORY LIVERMORE, CA 94550}




\section{FOREWARD}

This work was carried out in support of nuclear weapons risk assessments at the Lawrence Livermore National Laboratory. Probabilistic temperature distributions were derived for accidents involving hydrocarbon fuel fires for certain aircraft locations. Effects of fuel spil rate, location and number of spills, wind conditions (direction and speed), and aircraft orientation were considered to derive the distributions, using previously published fire-test data.

The examples selected for analysis were for strategic bombers (B-52 and B-1) on strip alert at several former SAC bases; both engine fires and collision with other aircraft were studied. The results are specific for these cases, but the methodology is believed to be applicable to other abnormal environments involving fuel fires.

The work was performed under LLNL contracts B108499 and B199139. 


\section{CONTENTS}

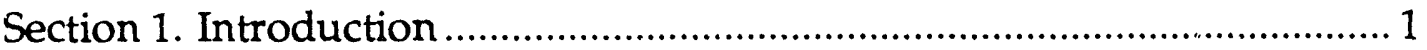

Section 2. Evaluation of Flame Temperature Exposure:

Data Base, Procedures, Assumptions ............................................ 3

Section 3. Predicted Temperatures of Flame Exposure .............................. 49

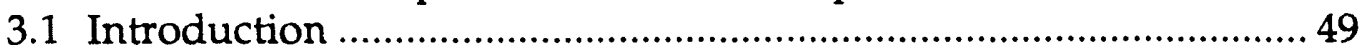

3.2 Engine Start Accident Results: B-52 .............................................. 51

3.3 Engine Start Accident Results: B-1B …………...............................69

3.4 Aircraft Collision Results: B-52/B-1B ……..................................... 89

3.5 Uncertainty Analysis................................................................. 121

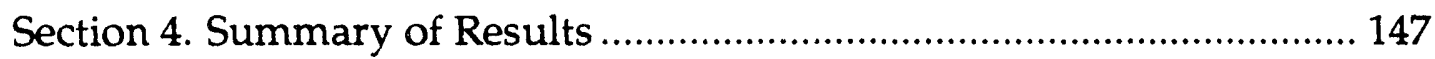

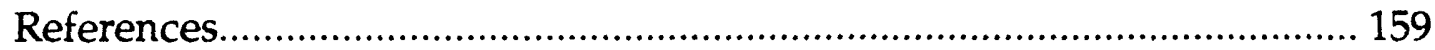

Appendices:

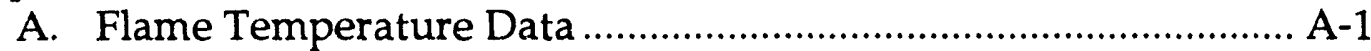

B. Aircraft/Air Base Orientation ................................................ B-1

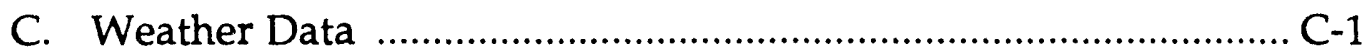

D. Fuel Spill Location/Rate Data …………….............................. D-1

E. Computer Program: Listing, Explanation, User Instruction, Example Output ............................................ E-1

F. Supplementary Results .............................................................. F-1 


\section{1 INTRODUCTION}

This report describes an evaluation of flame exposure temperatures of weapons contained in alert (parked) bombers due to accidents that involve aircraft fuel fires. The evaluation includes two types of accident, collisions into an alert aircraft by an aircraft that is on landing or take-off, and engine start accidents. Both the B-1B and B-52 alert aircraft are included in the evaluation.

The temperature of weapon exposure depends on a number of factors that are inherently statistical, for example, on the wind speed and direction at the time of an accident, and for collisions, on the detailed nature of the collision (angle of collision, speed, etc). The evaluation results are then also statistical in nature; most results are given in terms of the probability that the weapon exposure will exceed a given temperature for a situation of interest, for example, for the B-1B aircraft at a particular air base or appropriately averaged over all B-1B bases.

The evaluation of exposure temperatures is based on several types of data, including fire test data, weather data, and "fuel spill location and rate" data. For fuel spill related data, data was developed of necessity by an estimative procedure, and the estimates contain a sizeable uncertainty. The data base and the nature or source of its uncertainties are described in Section 2 of the report, along with various assumptions made in the evaluation. Numerical estimates of the data base uncertainties, and of the consequent uncertainty in exposure temperature probabilities, are given in subsection 3.5. (These uncertainty values are given in terms of plus or minus one standard deviation of error.) Although subject to these uncertainties, the evaluation is thought to provide a useful and "reasonable" estimate of the probability that weapons would experience given 
exposure temperatures. The evaluation is useful further in that it identifies the relative importance of the factors that govern weapon exposure in such accidents. It also indicates the relative magnitude of uncertainties and in turn then indicates the information required for improved predictions.

Section 4 provides a brief summary of the evaluated probabilities that weapon exposure will exceed given temperatures, and includes uncertainties of these results. The more detailed presentation of results is given in subsections 3.2 through 3.4. Section 4 is intended for readers interested in general results who wish to avoid the details given in Sections 2 and 3.

Details of the data base are given in Appendices A through D. The computer program which was developed for the evaluation of exposure temperatures is described in Appendix E, including a listing and detailed explanation of its use. 


\section{2}

\section{EVALUATION OF FLAME TEMPERATURE EXPOSURE: DATA BASE, PROCEDURES, ASSUMPTIONS}

The purpose of Section 2 is to (1), show the data base upon which weapon exposure estimates were made, thereby also providing an indication of the sources of uncertainty, and (2), describe the calculational procedure for evaluating weapon exposure. The report is written so that the reader interested primarily in weapon exposure predictions rather than the basis of the predictions can omit Section 2.

There was a policy in evaluating weapon exposure that should be noted. To the extent that it was possible, the best estimate of weapon exposure probability was sought, with the idea that an uncertainty analysis would reveal the potential inaccuracies of the best estimate; in other words, it was not the policy to seek conservative or pessimistic values of the probability of exposure to given temperatures. However, circumstances did arise where the conservative or pessimistic approach was unavoidable. These cases are noted in Section 2.

It will be seen in the discussion below that the probability of exposure to given temperatures depends on:

(1) the manner in which the flame temperature varies with location within the fire, and the dependence of this variation on wind speed and fuel spill rate,

(2) the direction (or directions) that the alert aircraft are pointed at a given air base

(3) the statistical character of the wind speed and wind direction at a given base, 
(4) for the aircraft collision accidents, the dependence of aircraft traffic (number of landings and take-offs) on time of day, and

(5) the likelihood that the fuel spills will occur at given locations with respect to the alert aircraft and at given spill rates.

All of these factors are discussed in Section 2, starting with a brief description of the flame temperature characteristics of fuel spill fires.

The flame temperature data used is from a series of six fire tests, where the fuel was "spilled" at a constant rate onto a level surface, as illustrated on Figure 2.1 (Refs. 1 and 2). This test is believed to provide the only fire data (1), in which temperature measurements were made at sufficient spatial resolution throughout a sufficient fraction of the flame volume to permit an evaluation of expected exposure temperatures, (2), that was conducted under the condition of fuel "spilling" onto a flat surface (the condition of primary interest for the accidents considered in this report), ${ }^{*}$ and (3), in which there was a systematic exploration of the effect of the wind speed and spill rate on flame characteristics.

\section{SIMULATED FLIGHT DECK \\ $(100 \times 100 \mathrm{ft})$}

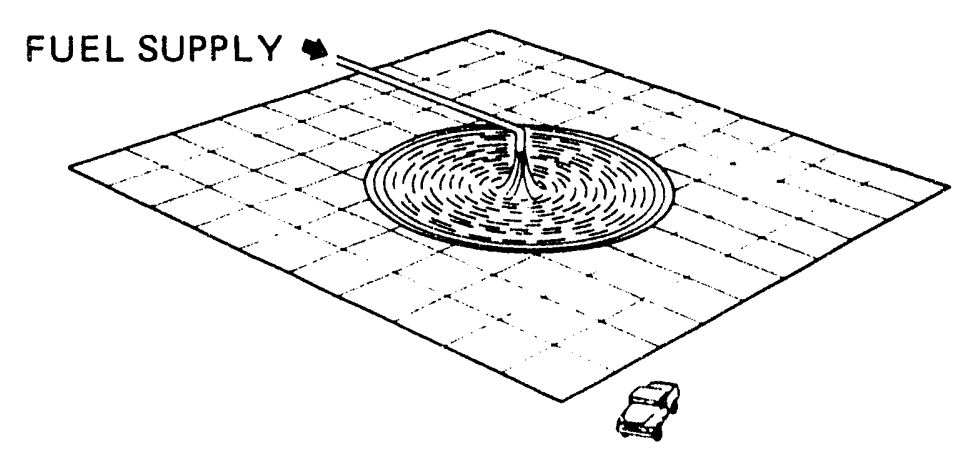

Figure 2.1 Fire Test Configuration

\footnotetext{
* Most large fuel fire tests are conducted for reasons of experimental simplicity under a condition known as a "pool fire", where there is a pool of fuel on the "ground surface" that is contained along it's periphery by a physical barrier. There are technical reasons to believe that flame temperatures or temperature patterns would differ for "spill" and "pool" fires of comparable fuel diameter. It is unclear if the flame differences are or are not significant, and at the present time, there are no equivalent tests from which a comparison can be made.
} 
The wind speed and spill rate conditions of the tests are given in Table 2-1 below. It can be seen that two tests were conducted for the medium wind speed at a spill rate of $300 \mathrm{gpm}$; the difference in flame temperature from the two tests is used as a measure of test to test reproducibility, and the effect of this difference on the probability of exposure to given temperatures is shown in subsection 3.5.

TABLE 2-1 Fire Test Condition Organization

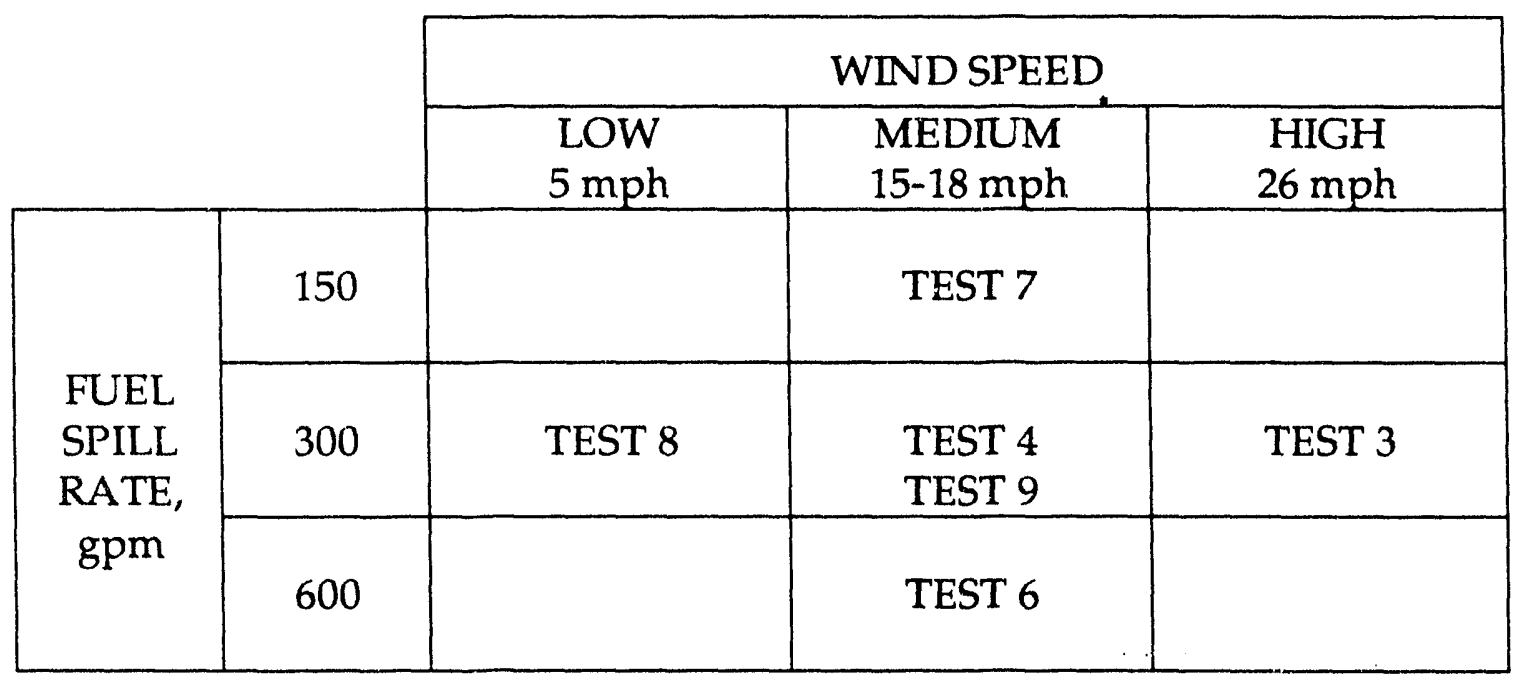

An illustration of the spatial patterns in flame temperature is given in Figure 2.2 for the wind of $5 \mathrm{mph}$ and the fuel spill rate of $300 \mathrm{gpm}$. (Contours for all test data used are given in a somewhat different format in Appendix A.) Figure 2.2 shows horizontal contours of the time-average temperature at the four heights of 1, 4,7 and 10 feet; the horizontal coordinate axis is parallel to the wind direction, this coordinate corresponding to the downwind distance from the fuel spill point, and the vertical coordinate is the crosswind distance from the fuel spill point. It can be seen that the time-average temperature contours are more or less circular, that the centers of the contours are downwind of the fuel spill point, that the highest temperature region is increasingly downwind with increasing height, and that the highest temperature and the dimensional area of the higher temperature regions increases with increasing height. It can also be seen that there are small regions at the 7 and 10 foot height which exceed $2150^{\circ} \mathrm{F}$; temperatures above $2150^{\circ} \mathrm{F}$ are found only for the $5 \mathrm{mph}$ wind speed. Examination of data for higher wind speeds shows that the magnitude of the highest temperature tends to decrease with increasing wind speed; there is also the trend that the height of the highest temperature region decreases with increasing wind speed. 


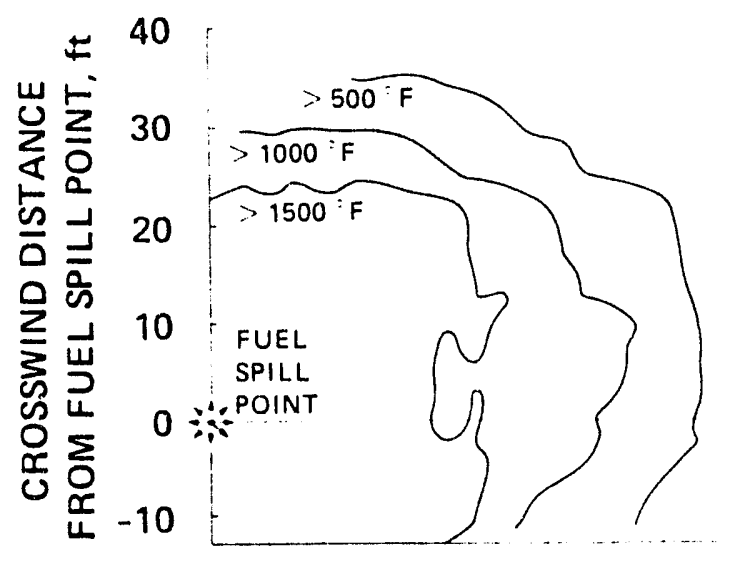

(a) 1-ft HEIGHT

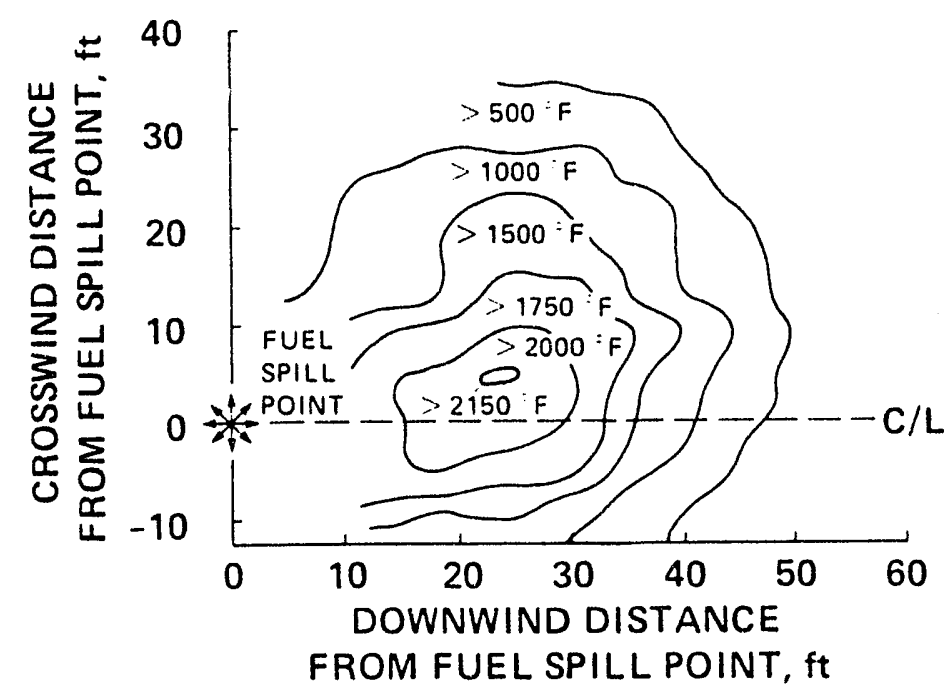

(c) 7-ft HEIGHT

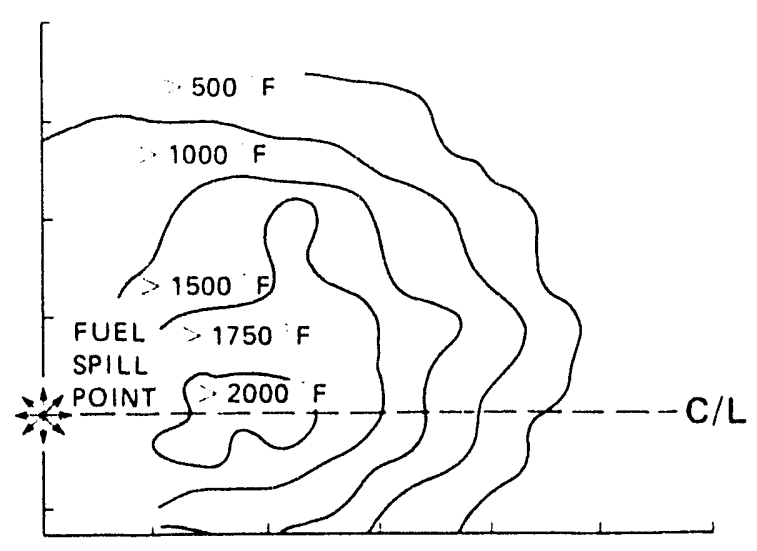

(b) 4-ft HEIGHT

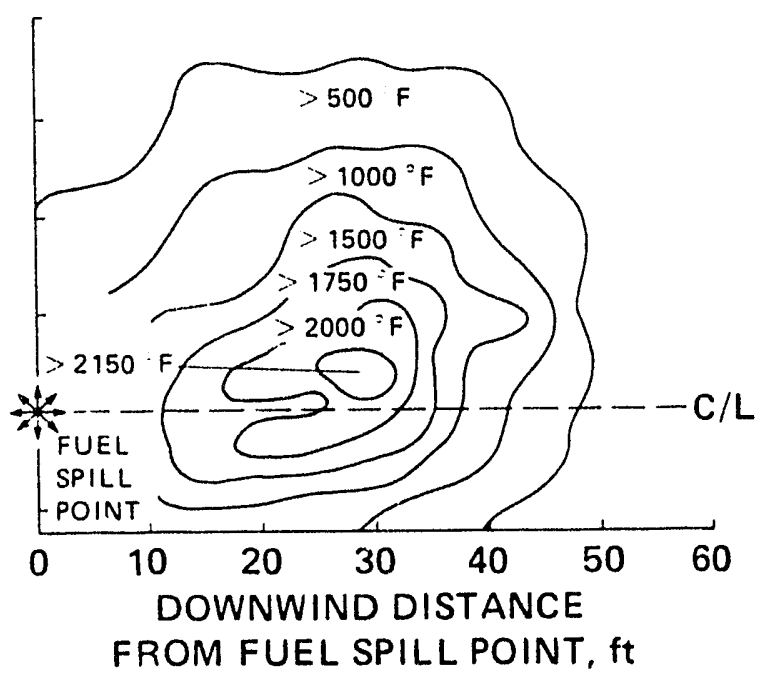

(d) 10-ft HEIGHT

\section{Figure 2.2 Temperature Contours for $5 \mathrm{mph}$ Wind Speed}

An example of the temperature contour patterns for a spill rate again of $300 \mathrm{gpm}$ but at the wind speed of $15 \mathrm{mph}$ is given on Figure 2.3. In contrast to the circular contours for the $5 \mathrm{mph}$ speed, the contours for $15 \mathrm{mph}$ are elongated along the wind direction axis.* For the $15 \mathrm{mph}$ speed, the flame footprint areas are comparatively large (although the spill rates and fuel layer diameters are the same as for $5 \mathrm{mph}$ ), and the "centers" of the contours are significantly further downwind. Figure 2.4 shows, for example, the relative downwind location of the center of the highest temperature regions for the 5 and $15 \mathrm{mph}$ wind speeds.

* The anomalous narrow, vertical contour in the upper right of the contour map for the 7 foot height is thought to be due to the "splash" of liquid fuel onto insulation near the temperature sensors. 


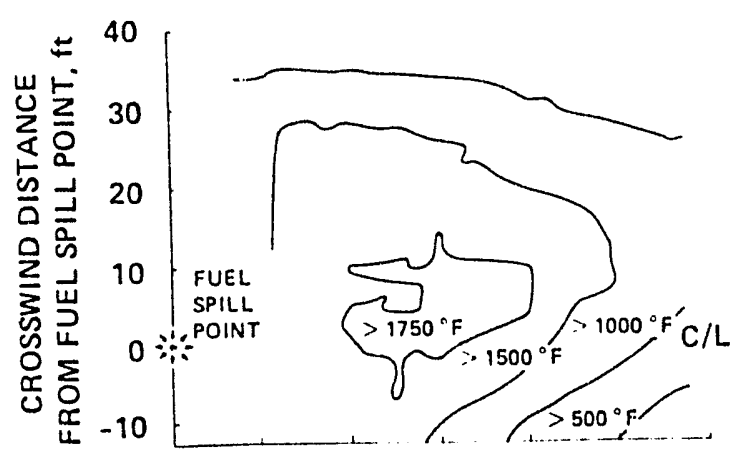

(a) 1.ft HEIGHT

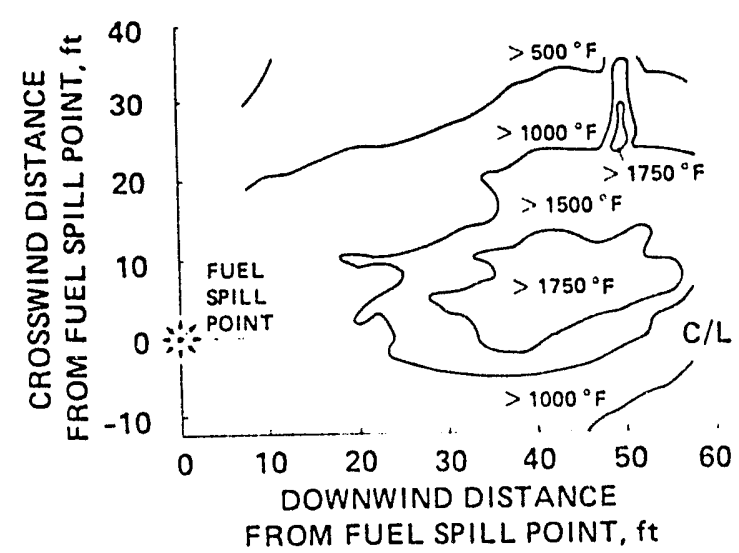

(c) $7 . \mathrm{ft} \mathrm{HEIGHT}$

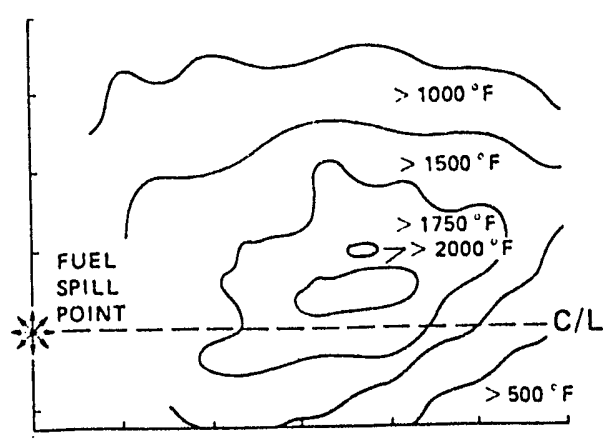

(b) 4.ft HEIGHT

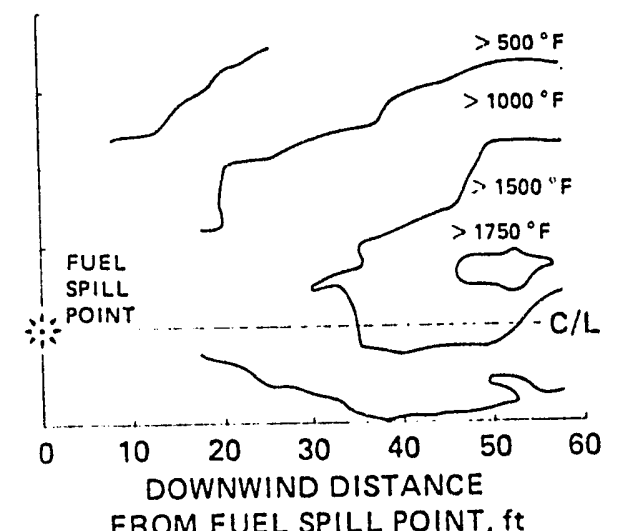

(d) 10-ft HEIGHT

Figure 2.3 Temperature Contours for $15 \mathrm{mph}$ Wind Speed

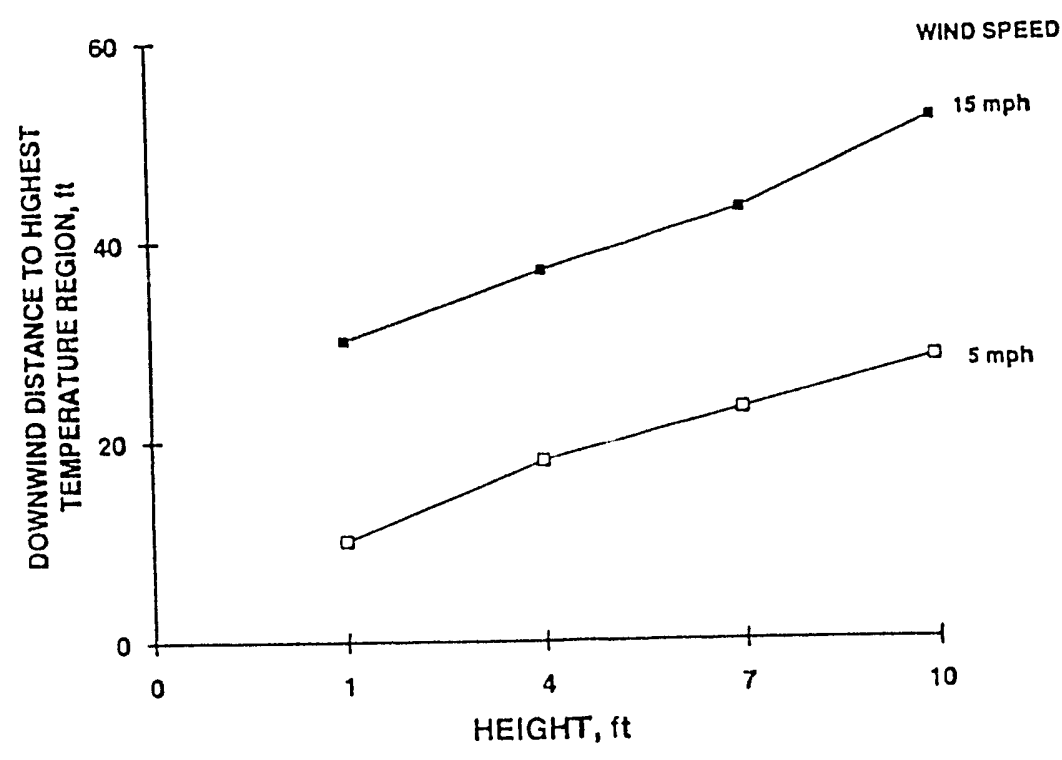

Figure 2.4 High Temperature Region Location for Different Wind Speeds 
This illustrates that the temperature of exposure depends a great deal on wind speed. Exposure level also depends on the fuel spill rate, but the impact of spill rate is considerably less than wind speed. Fuel spill rate influences the fire "size" in the sense that the diameter of the liquid fuel layer depends on the spill rate. The fuel layer diameter increases from the initiation of the fuel spill until it reaches a maximum and thereafter constant diameter. Analytic and experimental data show that this maximum fuel diameter is related to the spill rate approximately as $D=3.5(S)^{1 / 2}$, where $D$ is the diameter in feet for a spill rate $\mathrm{S}$ in $\mathrm{gpm}$. It would be expected that as the fires get larger, the probability of weapon exposure would increase. While this is true, it will be seen in Section 3 that the effect is not particularly significant.

Temperature was measured in the six tests referred to above at locations over four horizontal planes (at heights of 1, 4, 7 and 10 feet), and the distance interval between measurement locations in each horizontal plane was 2.5 feet in both horizontal directions. For purposes of computation, the time-average temperature at each location for each test is stored on magnetic disc; the data is arranged so that when a given fire location is input, the temperature at that location is output.*

Temperature measurements for the six fire tests are "free field measurements" in the sense that there were no significant objects or structures near to or immersed in the flame regior. The presence of an aircraft will modify the temperature and temperature patterns, in most cases locally, but in certain cases the entire flame region will be affected. It is important to discuss the unavoidable consequence of using free field measurements. In this regard, it is useful to discuss various characteristics of the flame region for fires of this type, and it is convenient to start with fires under conditions of no wind. The discussions will also serve to indicate the nature of heat transfer to objects immersed in fires of this type.

Regarding the nature of this type of fire, fuel is vaporized along the surface of the liquid fuel due primarily to radiant heating from the flame region. The fuel vapor rises into the flame region, starting at "very low speed" near the liquid surface and increasing in speed with height. Example calculated and measured values of the vertical drift velocity along the axis of a 50 foot diameter pool fire are given in Figure 2.5; it can be seen that speeds of approximately $60 \mathrm{ft} / \mathrm{sec}$ are achieved at a height of 20 feet for fires of this size (Ref 3). As discussed below, there is a rotational or spin velocity superimposed on the above drift velocity.

Part of the fuel vapor will pyrolyze as it progresses from the fuel surface up through the flame, and soot formation is significant. Example measurement

* This temperature data is available from: JM Technical, 531 NW Canyon Dr., Redmond, OR 97756, (503)-923-2107 (Attn: Joe Mansfield); Lawrence Livermore National Laboratory, P.O. Box 808, Livermore, CA 94550 (Attn: Douglas Stephens). 
results given on Figure 2.6 for the 50 foot diameter fire show an increase in soot mass fraction with height to a maximum of about 3 percent at the 37 foot height; the lower mass fraction at the 70 foot height is primarily a result of "excessive" air entrainment at that height (Ref 3$)$. Soot formation is enhanced in the lower region of the fire, particularly the lower central region, by the absence of free oxygen. For the 50 foot fire, again, Figure 2.7 shows very small mass fractions of oxygen in the central region even at heights of 9.4 and 13.8 feet. Air is drawn into the flame region from outside the fire, and significant penetration to the lower central region does not occur in the absence of wind.

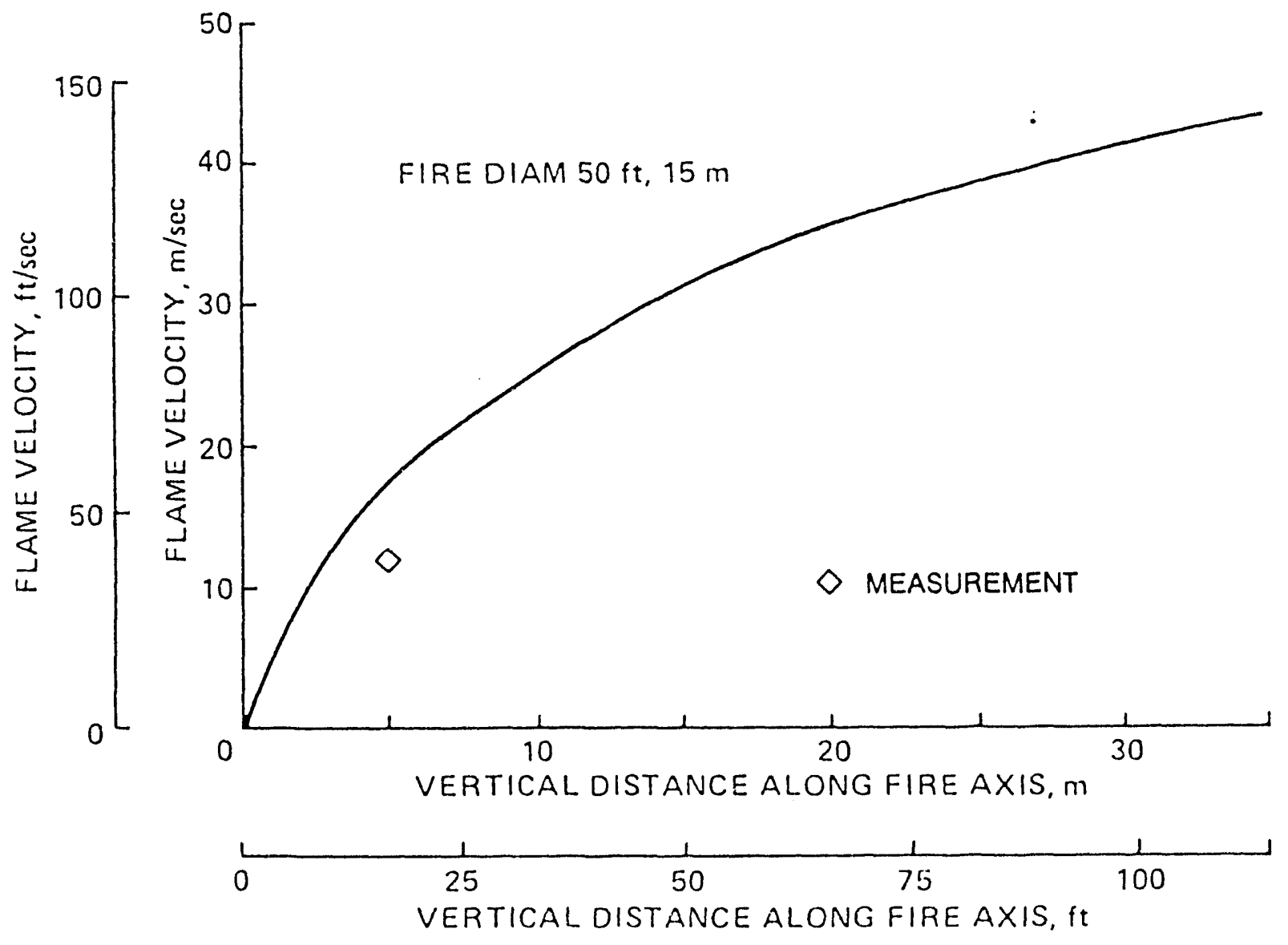

Figure 2.5 Vertical Drift Velocity in 50 foot Diameter Pool Fire 


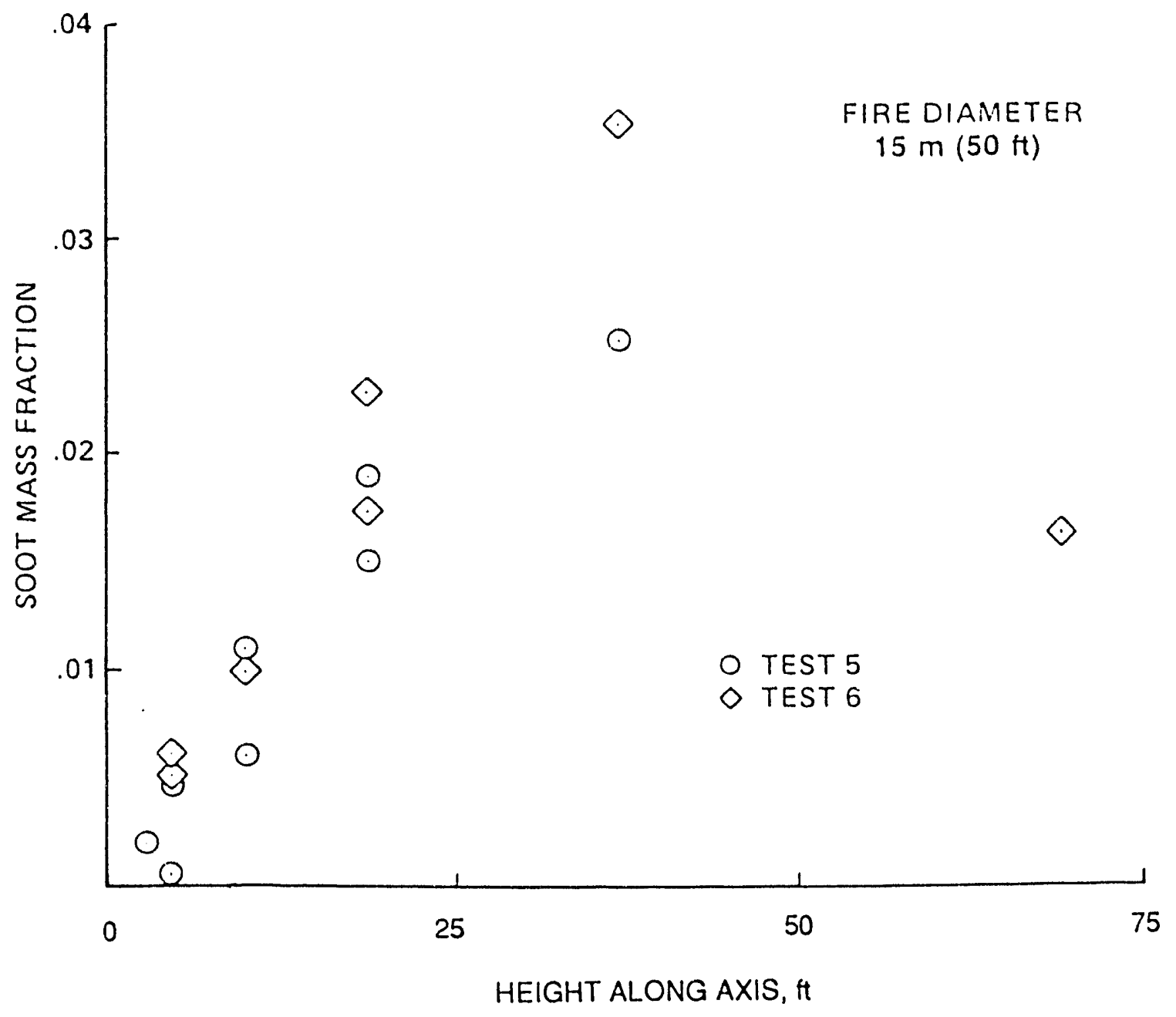

Figure 2.6 Soot Mass Fraction with Height in 50 foot Diameter Pool Fire

The mechanism of air entrainment in the absence of wind relates to buoyancy. Given that there is a "well-mixed" fuel/air quantity at some location in the fire, the mixture will combust, rise in temperature, expand and buoyantly rise. The vertical buoyant motion will in turn cause air to be drawn into the region from outside the fire, thereby providing additionai oxygen and the means for continuing the mixing/combustion/buoyant rise/air entrainment sequence. This sequence is pulsating or cyclic in nature; combustion will occur in a local region, the combustion product mass (and inerts) will rise from the region, and the region subsequently experiences a time period (e.g., a fraction of a second to perhaps 2 or 3 seconds) of relative chemical inactivity followed by reinitiation of combustion. There is a spin motion of the rising combustion product mass, forming a so-called "turbule." There is a sizeable variation in the dimension of turbules, but they appear to be typically of the order of 10 feet or more in 


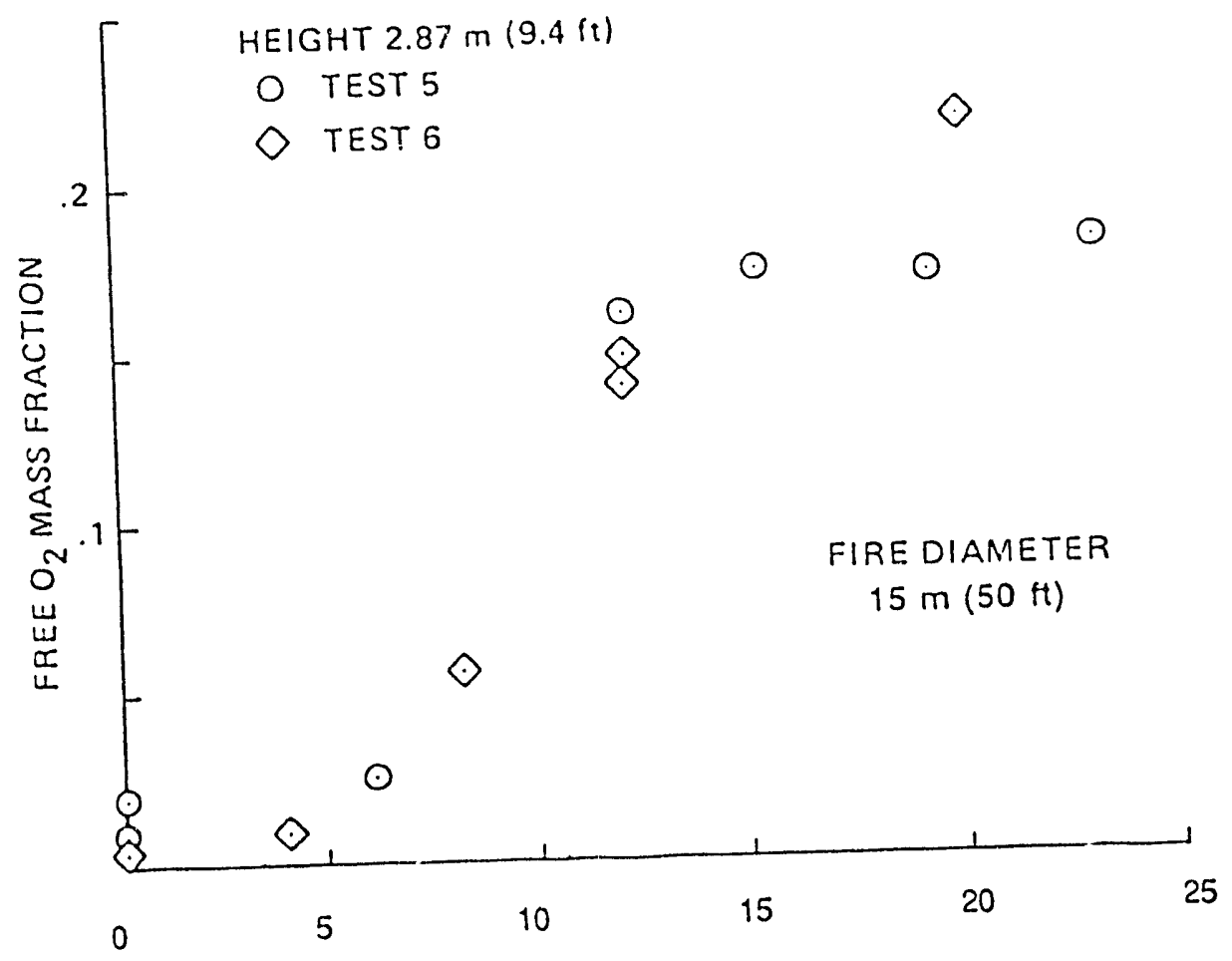

(a) RADIAL DISTANCE, $\mathrm{ft}$

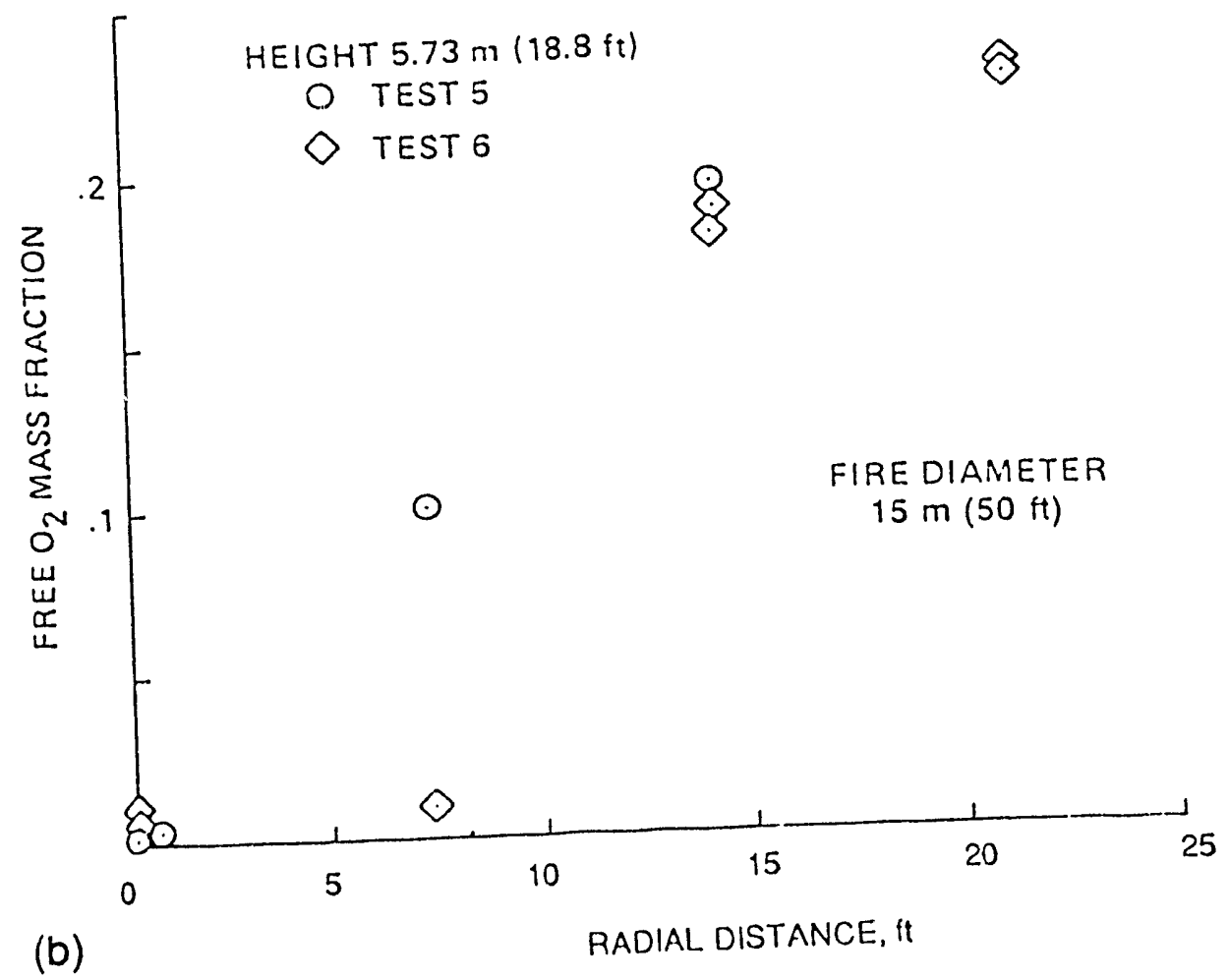

Figure 2.7 Free Oxygen Mass Fraction in 50 foot Diameter Pool Fire 
diameter. For fires with no wind, the spin of the turbules is oriented as illustrated on Figure 2.8, that is, the spin velocity is upward on the turbule side nearest the fire axis. In order for combustion to occur, fuel and oxygen must of course mix at a "molecular level", and the physical process of mixing is an important determinant of the fire character and specifically of the maximum temperatures that are achieved. The turbulence associated with the turbules is unquestionably related to the rate of fuel/air mixing. Figure 2.9 shows the mass fraction of raw fuel with height along the axis of the 50 foot diameter fire (Ref 3 ). Although substantial oxygen is available in the upper regions of the fire, it can be seen that measurable quantities of fuel occur up to the 70 foot height. There are also measurable quantities of free hydrogen in the upper regions of the fire (Ref 4).

With the addition of wind, the horizontal air flow interacts with the upflowing fire constituents, and there is some air penetration and mixing of the air with the flame constituents (in addition to that caused by buoyancy). On the basis of the fuel spill tests (Ref 1), temperatures at very low heights (e.g., 1 foot) tend to increase with increasing wind speed; apparently, wind causes increased combustion in the lower regions, probably due to greater air entrainment in the region, perhaps also due to enhanced fuel/air mixing. As noted earlier, however, there is a decrease in the maximum flame temperature and the height of the maximum temperature with increasing wind speed. The decrease in maximum temperature may be due to the cooling effect of "excessive" air entrainment, although there are no confirming data on the distribution of chemical composition for fires with wind. It might be anticipated that the entrainment from a "steady" wind would reduce the turbulence compared to the no wind fire. Purely on the basis of visual test observation, this does not appear to be the case; the pulsating nature of air entrainment, turbule formation, and the highly turbulent rise of the turbules appears no less intense and is a prominent feature of the fires with or without wind.

If any step in the combustion/buoyant rise/air entrainment/fuel vaporization sequence described above is by some means reduced, the entire sequence will be curtailed. The placement of objects of sufficient surface area in regions of the fire near or below what would ordinarily be the primary region of combustion can substantially change the fire, tending to reduce the overall rates of combustion, air entrainment, fuel vaporization, and so on. For certain fuel spill locations and fire sizes, the surface area of the wing/fuselage of an alert aircraft is sufficient to alter the overall fire. The most extreme case appears to be for a fire centered 


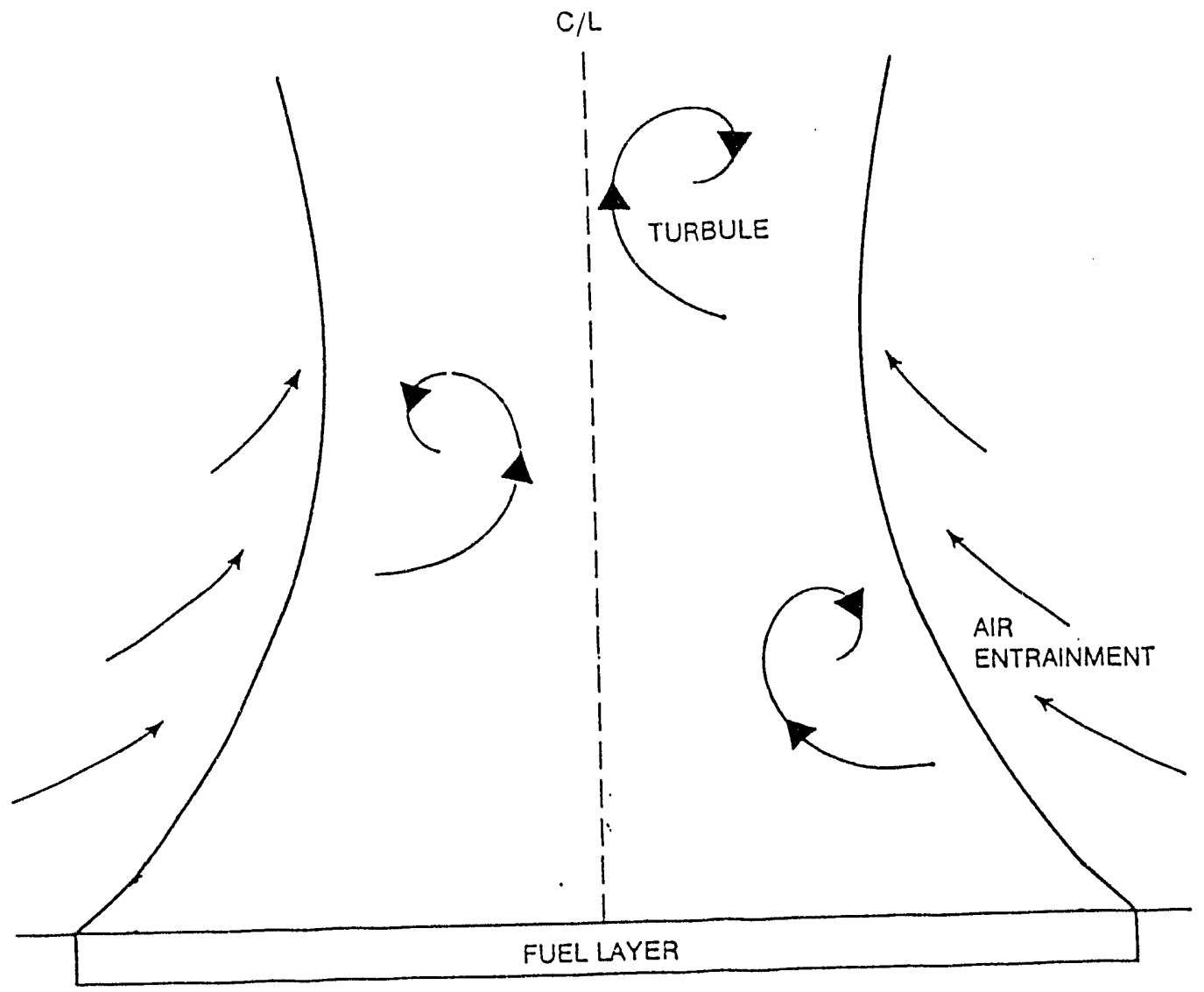

Figure 2.8 Turbule Orientation for Fire with No Wind

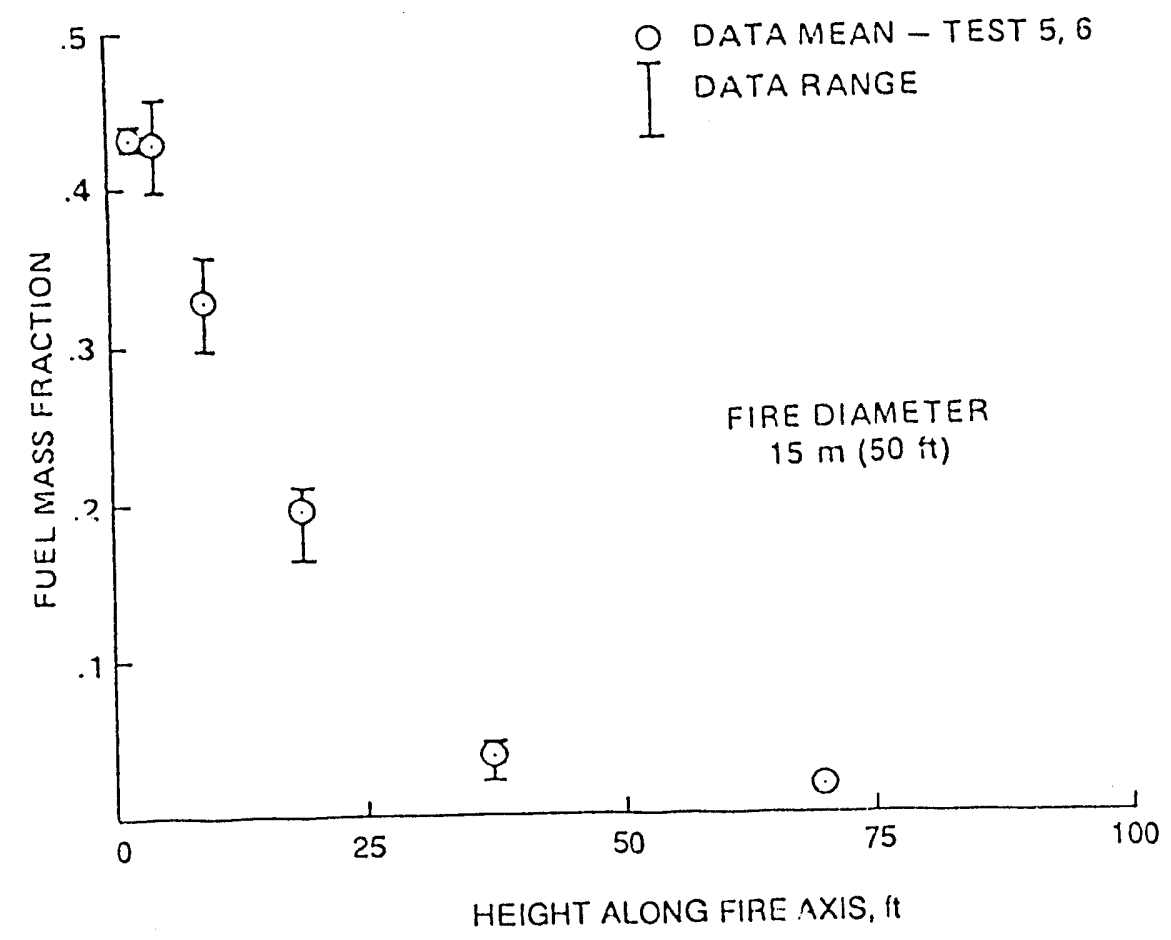

Figure 2.9 Raw Fuel with Height for 50 foot Diameter Pool Fire 
below the engine/central fuselage region of the $B-1 B$; this is illustrated for a 70 foot diameter fuel layer on Figure 2.10. For this fire diameter under conditions of "low" wind, the primary region of combustion in the absence of the B-1B would be at and above the fuselage height of the B-1B, and it is thought that the total rates of combustion, fuel vaporization and air entrainment will be reduced, and that the temperature averaged over the flame

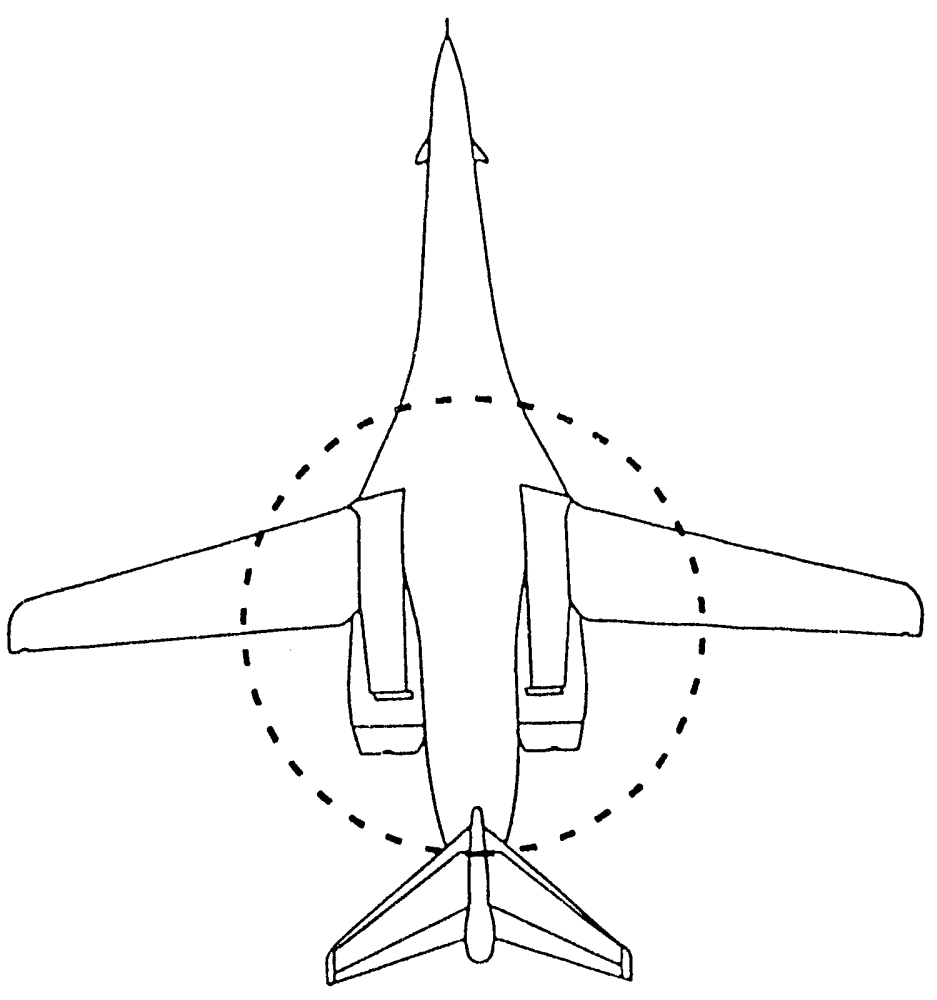

Figure 2.10 Configuration of High Aircraft Influence on Fire

volume will be reduced. This does not mean that the temperature at every location will be reduced, and due to flow $r$ 'tern modification, certain locations where low to moderate temperatures occur is. te absence of the alert aircraft can 
actually be higher in temperature with the presence of the alert aircraft; an increase in the maximum temperature due to the presence of the aircraft is not anticipated. Thus, for a specific set of conditions (e.g., fuel spill location, wind speed, spill rate, etc.), the predicted temperature using free field measurement dati: can be lower than would occur; however, it is thought that more often than not, the prediction would be higher than would occur. From a statistical point of view (i.e., when all conditions are appropriately weighted), it is thought that predicted probabilities of exceeding a given exposure temperature using free field measurements will tend to be higher than would occur with the aircraft present. The above statement is judgmental and an appropriate series of fire tests with simulated aircraft immersed in the fire would improve the reliability of prediction.

It is more common that a structure immersed in the fire will influence the fire primarily in the local region of the structure, rather than changing the dynamics of the overall fire as described above. For many accidents of interest, the weapon itself will be temporarily protected from direct exposure to flame by the fuselage. Regarding protection by the fuselage, analysis of accident reports has shown for landing accidents, for example, that on the average 50 percent of the aircraft structure is consumed by fire, although these studies and reports do not provide a clear indication of the rate of consumption (Ref 5 ). On the basis of work conducted by the FAA, FAA personnel indicate "that fuselage burn-through (aluminum) typically commences in the time period of 40 to 60 seconds, and that while the rate of fuselage consumption depends on the type and amount of fuselage liner, the time to progress to extensive flame exposure of cargo is typically of the order of 3 to 4 minutes" (Ref 6). Fuselage burn-through, then, appears to occur in small time periods compared to "medium or long" duration fires. Because burn-through time is apparently comparatively small, and because there is a sizeable uncertainty in time to burn-through, the conservative policy of assuming immediate exposure appeared reasonable and was adopted.

Once fuselage burn-through is sufficient to result in direct flame exposure, the flame will tend to be modified locally by the presence of remaining fuselage structure. In the statistical sense, it is thought that temperature predictions based on free-field measurements would be once again more severe than the actual temperatures. However, the presence of fuselage fuel tanks in the vicinity of the weapons, both for the B-52 and B-1B, is a factor that should be considered. Tests and calculations indicate that flame exposure of aluminum fuel tanks will not cause tank wall melting in the fuel-wetted region of the tank (Refs. 7, 8), although melting will result in the ullage or vapor region. Exposure will cause boil-off of liquid fuel at estimated rates of about $0.1 \mathrm{gpm}$ per square foot of tank area exposed to the flame. Thus, there appears to be the potential of a reasonably significant source of fuel vapor in the region of the weapons, 
probably at a height near or above the location of the highest weapon. It is not anticipated that the fuel source would create an abnormally severe weapon exposure, but the effect of an elevated source is far too complicated for reliable judgement; reliable assessment would require analysis or testing.

Regarding heat transfer to objects in fires of this type, the factors that primarily dictate the mode of heat transfer are the "high" soot concentration and "intense" turbulence characteristic of such fires. It was shown in Figure 2.6 above that soot mass fractions of approximately 0.03 were achieved. For this mass fraction, and for soot particle diameters and photon wavelengths typical of these flames, the radiation absorption coefficient is of the order of $40 / \mathrm{ft}$ (see for example Ref 9 ).* Thus, 90 percent of the photons of the photon beam (IR beam) are absorbed in about $3 / 4$ inch (and the emissivity of a soot cloud layer somewhat thicker than $3 / 4$ inch approaches 1.0). Radiant heat transfer to an immersed object, then, originates from and depends on the temperature within the very thin layer that surrounds the object. This layer is thicker of course in regions of the fire where the soot concentration is smaller.

As a "hot" gas flows around an immersed object, a boundary layer is developed around the object, and the boundary layer will be laminar or turbulent depending on the circumstances. The boundary layer tends to be cooler than the rest of the flow due to energy loss to the object. For fires of interest here, the temperature of the layer surrounding the object will be increased intermittently by the passing of the high temperature turbules described above, and this temperature influence on the layer will occur in two fundamentally different ways. When the object is in the path of the rising turbule, the high velocities of the turbule (spin and drift) essentially result in a momentary replacement of the layer constituents with the constituents of the turbule, that is, the original boundary layer will be virtually but momentarily eliminated. During this period, then, there is direct radiant transfer to the object from the high temperature soot that exists in the turbule. When the turbule passes by the object at some distance from the object and does not directly "interact" with the object, there is no direct radiation from the turbule to the object. However, as the turbule progresses past the object, the cool layer around the object can be influenced by the turbule in the following way. As the turbule progresses, the thin layer immediately surrounding the turbule (about $3 / 4$ inch thick) is first heated by the turbule by direct radiation from the turbule, that layer in turn radiates to the next layer (and also back to the turbule), and so on to the successive layers. However, in such a "radiation propagation", the

* For purposes of estimate, a soot particle diameter of 1.5 micron and photon wavelength of 2 microns (the peak wavelength from a blackbody at about $1850^{\circ} \mathrm{F}$ ) was assumed. Also, total crossection (absorption and scattering) was used. 
temperature of successive layers decreases, and it also takes time for the propagation to occur, that is, there is some average propagation speed. (This is somewhat analogous to the speed of thermal conduction in a solid.) To our knowledge, the magnitude of temperature influence for given distances from a high temperature turbule has not been calculated nor measured; purely from intuition, we suspect that for turbules of 10 foot diameter, the influence will be negligible for distances larger than about 5 to 10 feet.

The discussion turns now from temperature data to the orientation of the alert aircraft and the wind characteristics. Figure 2.11 shows a sketch of the runway and alert aircraft arrangements for Wurtsmith and McConnell AF Bases as example aircraft/airport configurations. (Comparable configurations for all twelve air bases considered in this report are given in Appendix B.) A common arrangement of alert aircraft is that a number of aircraft are pointed in each of two directions; for example, for Wurtsmith AF Base on Figure 2.11 it can be seen that there are five aircraft positions where the aircraft are pointed to approximately 140 degrees and four where the aircraft are pointed to approximately 230 degrees; for McConnell AF Base, Figure 2.11 shows five aircraft at each of the angles 10 and 190 degrees.*

\footnotetext{
* It has been indicated (private communication, Park Winter, LLNL) that parking arrangements can vary in the sense (1), that the number parked in a given direction will change from time to time, (2), that for some bases there is occasionally one aircraft pointed in a third direction, and (3), that in some cases the parking direction can only be specified within an angular range (e.g., \pm 20 degrees). For purposes of this report, a baseline arrangement for each base was selected on the basis of the information provided. The pointing directions and number of aircraft at each direction for the baseline arrangement for each base is given in Appendix B.
} 

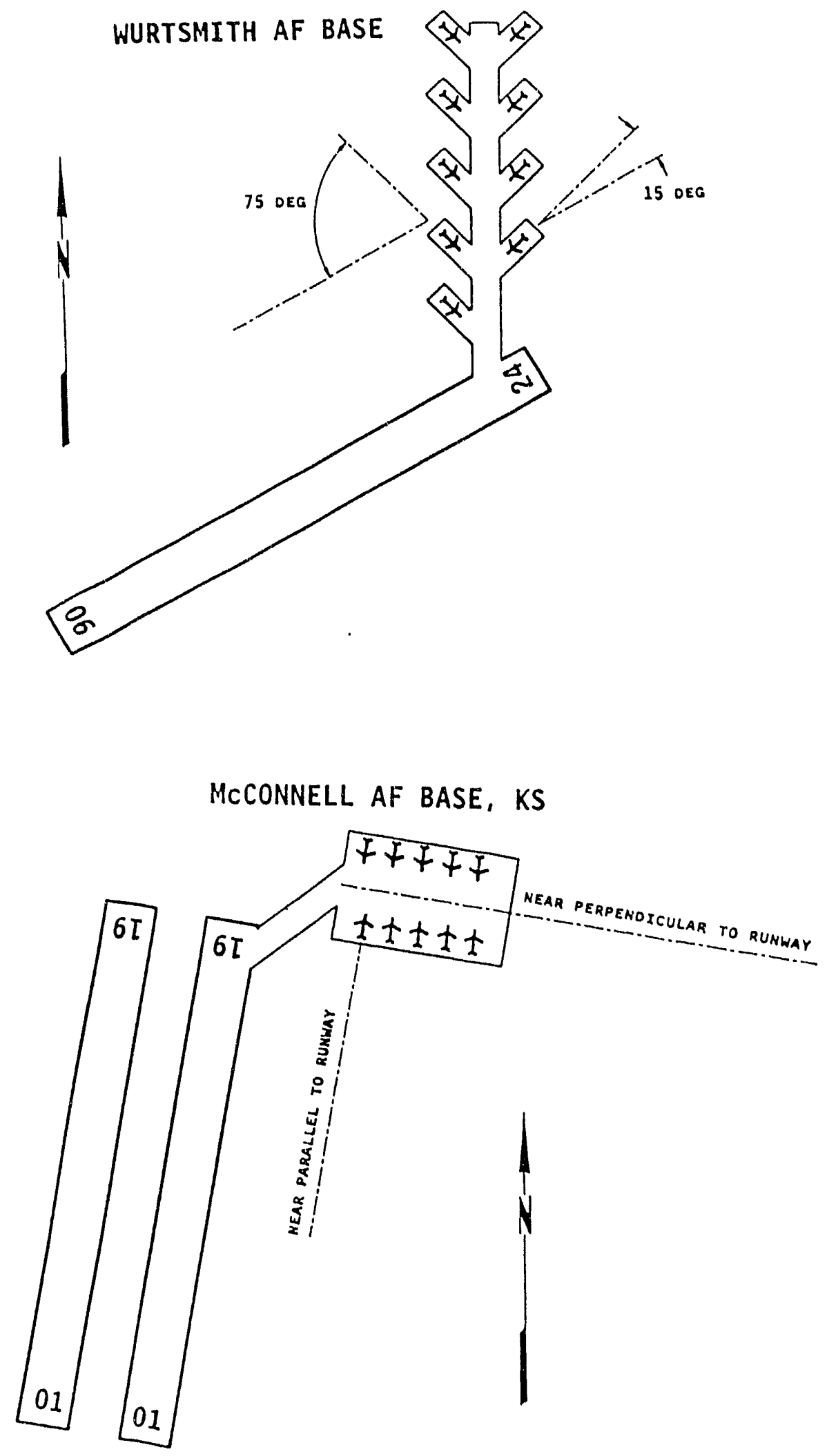

Figure 2.11 Aircraft/Runway Configuration at Wurtsmith \& McConnell AF Bases 
Regarding the weather, Figure 2.12 shows the probability that the wind is from a given direction for Wurtsmith and McConnell AF Bases. (Wind direction plots and other weather data for the twelve bases considered in this report are given in Appendix C.) There is a tendency for the wind to be predominant for two direction ranges which are separated by about 180 degrees. From Figure 2.12 this tendency is evident for McConnell AF Base, where there is a predominant wind direction range centered at about 180 degrees and a range of somewhat less prominence centered at about 10 degrees. For Wurtsmith AF Base, the tendency for the two predominant ranges is not as clear; there is clearly a predominance whose peak is at 200 degrees, and some suggestion of a second predominance at about 40 degrees. Examination of Figures 2.11 and 2.12 shows that the alignment of the runways tends to coincide with the predominant wind directions, and wind direction probabilities favor take-offs from or landings on the alert pad end of the runway, that is, for take-offs or landings that have a component of wind flow opposing aircraft motion, take-offs from or landings on the alert pad end of the runway are statistically more probable. Calculation shows this probability is $69 \%$ and $57 \%$ for Wurtsmith and McConnell, respectively; these probabilities range from about $50 \%$ to the most extreme case at Castle AF Base of $82 \%$.

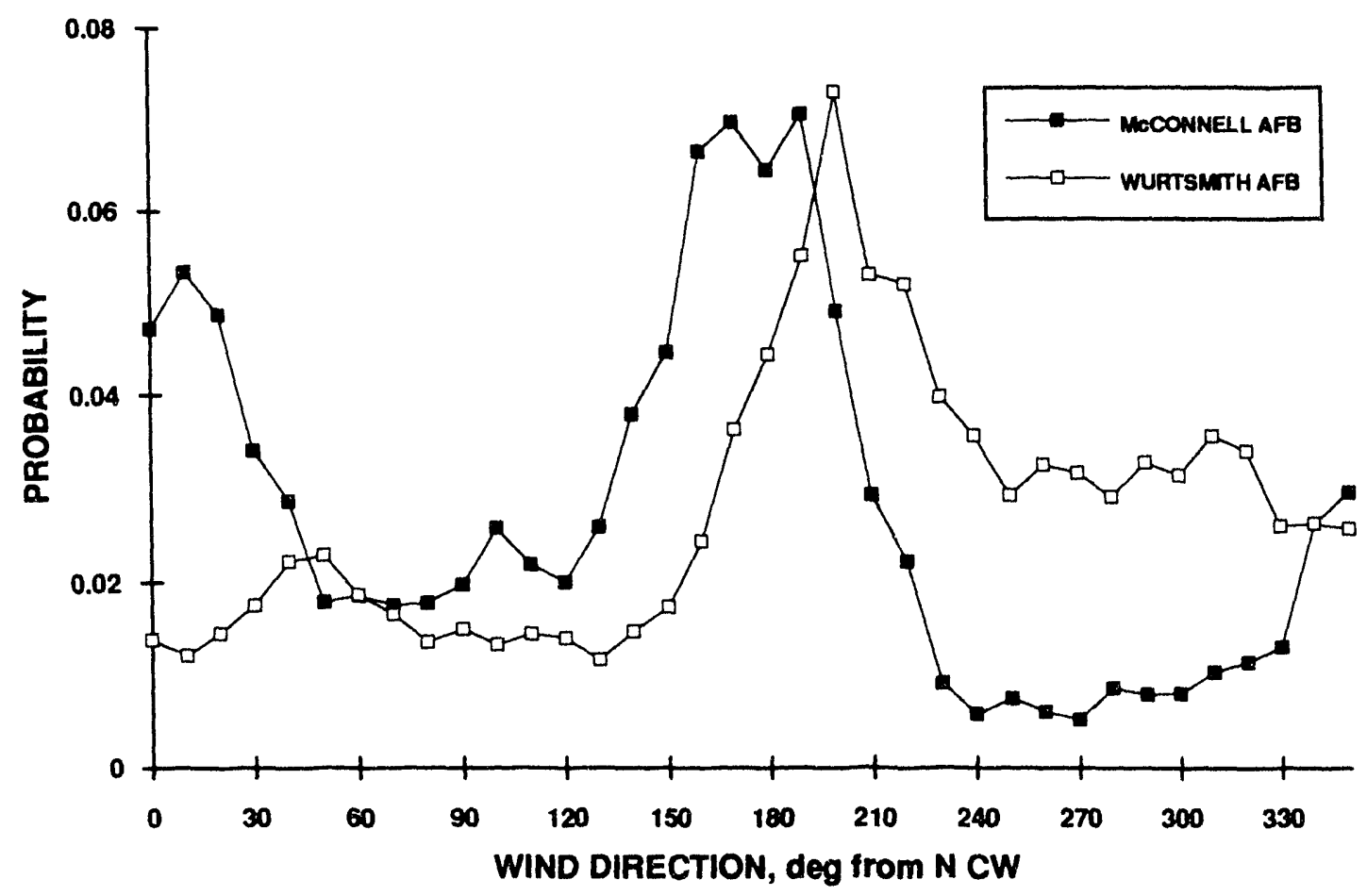

Figure 2.12 Wind Direction Distribution at Wurtsmith \& McConnell AF Bases 
The probability that a given wind speed will occur is illustrated on Figure 2.13 for McConnell, Wurtsmith, Grand Forks and Castle AF Bases, where the probabilities for Grand Forks and Castle AF Bases have respectively the highest and lowest average speeds of any base considered. The probability that the wind speed will lie within the ranges 0 to 10,10 to 20 , and above $20 \mathrm{mph}$ is given in Table 2-2, where these speed ranges correspond to those selected above to represent the low, medium and high speed ranges associated with the temperature data. Because the low wind speed is the most probable wind, the character of the low wind speed temperatures will tend to have the greatest influence on the probability of exposure to various temperatures, and the rarity of the high speeds will cause the high speed temperature data to have little influence on the results. Table 2-2 also lists the average wind speed for each of the four bases, and the average is listed for all twelve bases in Appendix $C$. The wind speed averaged over all twelve bases is $7.9 \mathrm{mph}$.

Consider next that the wind conditions will vary somewhat with time of day at a given base. Consider also that airport traffic (the rate of landings and takeoffs) varies with the time of day. It is expected that the number of accidents would increase in proportion to the number of landings and take-offs; thus,

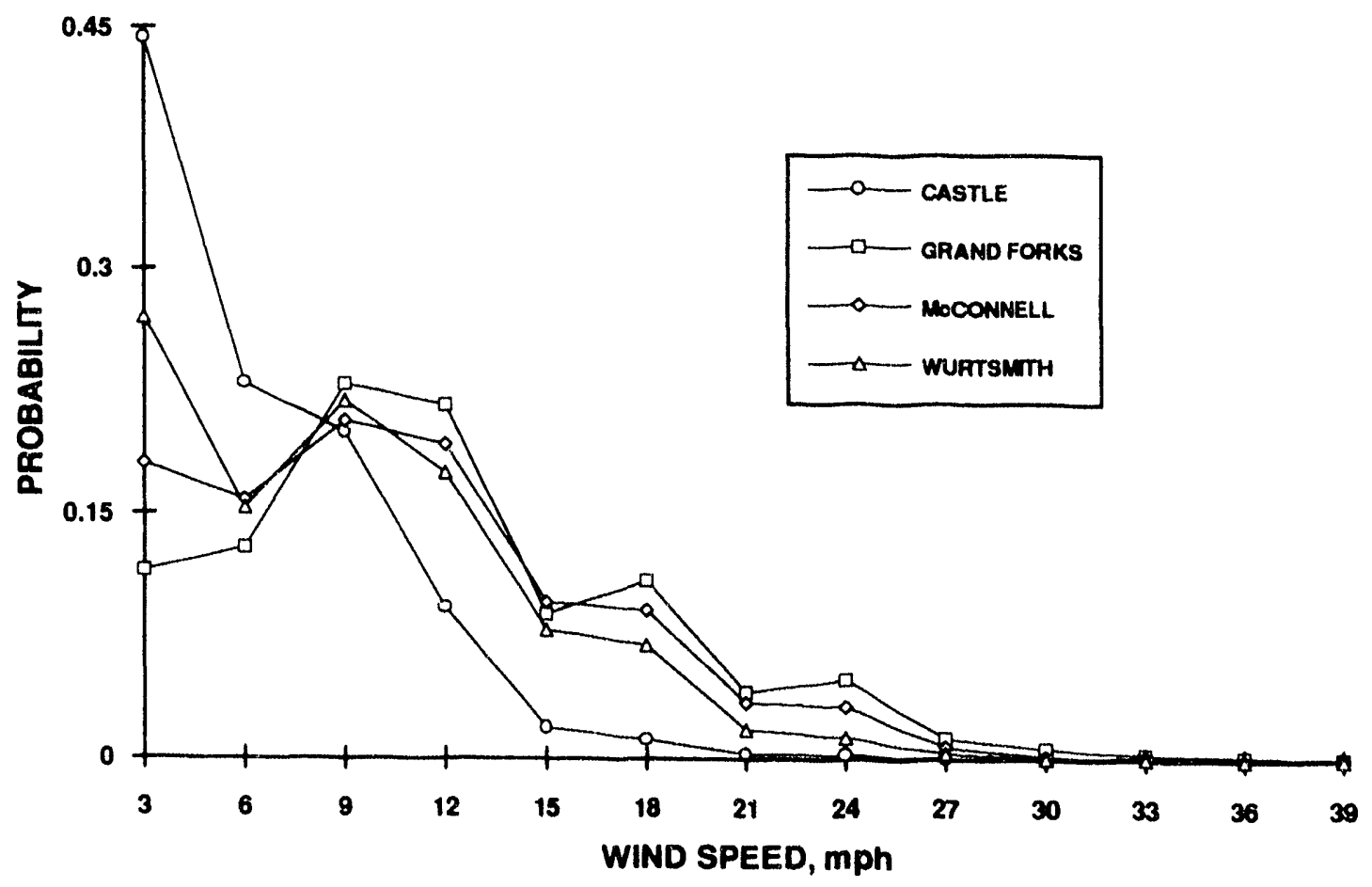

Figure 2.13 Wind Speed Probability Distributions 
TABLE 2-2 Probability Wind Speed Will Be Within Given Ranges

\begin{tabular}{|c|c|c|c|}
\hline \multirow{2}{*}{} & \multicolumn{3}{|c|}{ WIND SPEED RANGES, mph } \\
\cline { 2 - 4 } & $0-10$ & $10-20$ & $>20$ \\
\hline $\begin{array}{c}\text { MCCONNELL } \\
\text { (Avg. 8.6 mph) }\end{array}$ & .59 & .37 & .04 \\
$\begin{array}{c}\text { WURTSMITH } \\
\text { (Avg. 7.1 mph) }\end{array}$ & .67 & .31 & .02 \\
$\begin{array}{c}\text { GRAND FORKS } \\
\text { (Avg. 10.4 mph) }\end{array}$ & .53 & .39 & .08 \\
$\begin{array}{c}\text { CASTLE } \\
\text { (Avg. 5.0 mph) }\end{array}$ & .91 & .09 & $<.005$ \\
\hline
\end{tabular}

there would be a greater probability for an accident to occur under the wind conditions at peak traffic times than at others. The effect of traffic and wind conditions vs time of day was included in the evaluation of exposure temperatures.*

Available traffic data consisted of the relative traffic for four each six hour periods starting at midnight. Typical traffic data for these time periods is given in Table 2-3; traffic for other bases is given in Appendix $C$. There is not a great deal of base to base variation in relative traffic vs time of day, and it can be seen from Table 2-3 that about one-half of landings and take-offs are during the "afternoon" hours (1200-1800 hrs).

* There will be some dependence of wind conditions with time of year, and there may be some variation of traffic with time of year. This effect was not included in exposure evaluation.

Also, the likelihood of landing/take-off accidents is comparatively high under certain severe weather conditions. Exposure evaluation does not account for this weather dependency. 
TABLE 2-3 Fractions of Landings or Take-offs at Various Times of Day

\begin{tabular}{|c|c|c|c|c|}
\hline & \multicolumn{4}{|c|}{ TIME OF DAY (GMT) } \\
\cline { 2 - 5 } & $0000-0600$ & $0600-1200$ & $1200-1800$ & $1800-2400$ \\
\hline DYESS & .06 & .22 & .51 & .21 \\
GRAND FORKS & .06 & .28 & .52 & .14 \\
SAWYER & .06 & .24 & .53 & .17 \\
\hline
\end{tabular}

The effect of the time variation of traffic was incorporated into the evaluation of exposure temperatures through an appropriate data processing of weather data. In this regard, weather data was first processed to determine the relative probability of occurrence of any wind direction/wind speed/time of day combination: in terms of an example combination, the probability is determined that the wind direction will be within the range $60 \pm 5$ degrees within the wind speed range of 10 to $20 \mathrm{mph}$ at a time between 0600 and 1200 hours. Since there are 36 wind direction ranges, 3 wind speed ranges and 4 time-of-day intervals, une number of combinations is $36 \times 3 \times 4=432$. For the second step, the probability value for each combination is adjus $\Leftrightarrow$ d in proportion to the traffic for that combination. Specifically for this step, the 108 probability values for the time interval 0000-0600 hours are multiplied by the traffic fraction for that time of day (. .06 for Dyess on Table 2-3), the 108 probability values for the interval 0600-1200 hours are multiplied by the traffic fraction for that time interval $(0.22$ for Dyess on Table 2-3), and so on; the resulting probability set is then normalized so that the sum of 432 probability values is unity. The adjusted probability values each represent the relative probability that an accident will occur for a given wind speed/wind direction combination. This probability set, along with other probability sets discussed below, is then used in the evaluation of exposure temperatures. Weather/traffic probability sets have been developed for all twelve bases, and an example set is given in Appendix $C$. 
It is mentioned here that there are more accidents during landing than take-off, and this is a factor that must be accounted for in the exposure evaluation. A study of commercial carrier accidents that occurred over a period of eleven years shows that for aircraft crashes that resulted in fuel fires, the ratio of landing to take-off accidents was 3.0; this study comprised aircraft ranging in size from the Boeing 737 to the DC-10 (Ref 5). For military bases, the ratio of landing to take-off accidents is approximately 2.0 for "high performance" aircraft and 3.8 for "air carriers" (Ref 10); however, the ratios for military bases were compiled without distinction between accidents that did or did not result in fires. While the ratios for commercial and military base accidents are mutually supporting, the ratio for military base accidents that result in fire is unknown. Thus, a value of 3.0 is used as a baseline ratio, and the effect of the uncertainty in the ratio is given in subsection 3.5 .

The next input considered pertains to fuel spills. Exposure temperature evaluation requires knowledge of the relative probability that fuel spills will occur at various locations and at various volumetric spill rates. While this is a necessary input for aircraft collision accidents, there is essentially no data base indicating locations or rates, nor information from which they may be derived. Consequently, a procedure was developed to estimate the required probabilities for various types of aircraft collision. While the procedure is systematic, it inherently involves technical judgement and is subject to corresponding error. It was initially anticipated that while there is a sizeable uncertainty in this input, resulting exposure temperature results would not be particularly sensitive to errors in this input; while the results did prove to be reasonably insensitive, fuel spill probabilities represent the primary source of uncertainty in temperature evaluation. Subsection 3.5 includes estimates of uncertainties caused by uncertainties in the fuel spill input.

Regarding the fuel spill input for the aircraft collision, consider first the location of fuel tanks on the alert aircraft. Sketches illustrating fuel location for the B-1B and B-52 are given on Figure 2.14. For purposes of calculation, a set of discrete locations must be selected which represent potential fuel spill locations. The selected spill locations are illustrated in Figure 2.15; numerical specification of these locations is given in Appendix D.

The labeling of potential spill points on Figure 2.15 should be noted. Fuselage tank spill points have been designated F1 through F6 for the B-1B, and F1 through F5 for the B-52. Left wing spill points are designated L1 through L5, and right wind points $\mathrm{R} 1$ through $\mathrm{R} 5$. These designations will be reft. 1 ed to below in the discussion of the procedure for evaluating spill probabilities. 


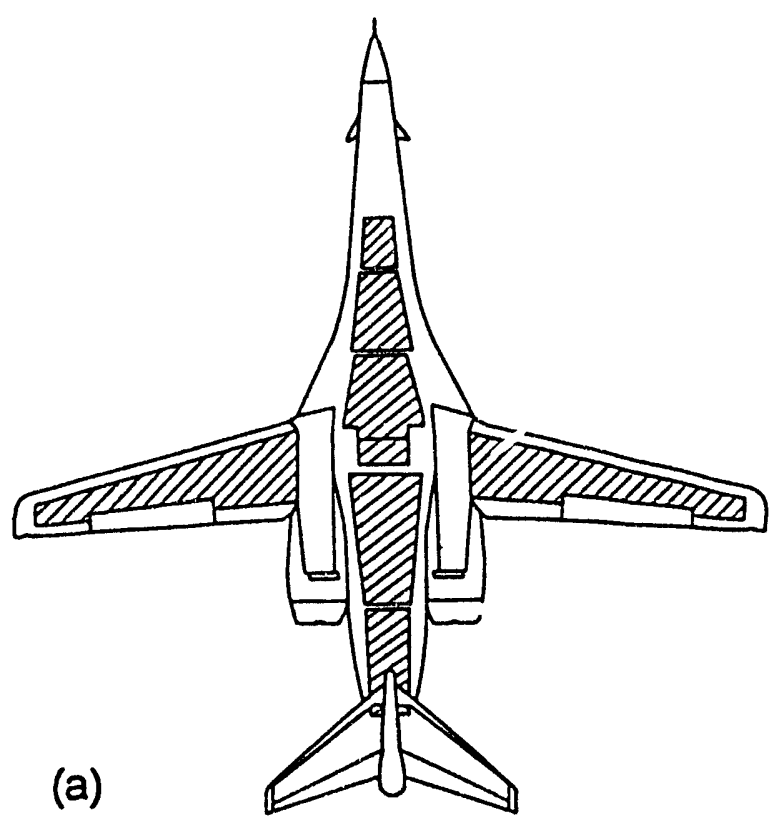

FUEL
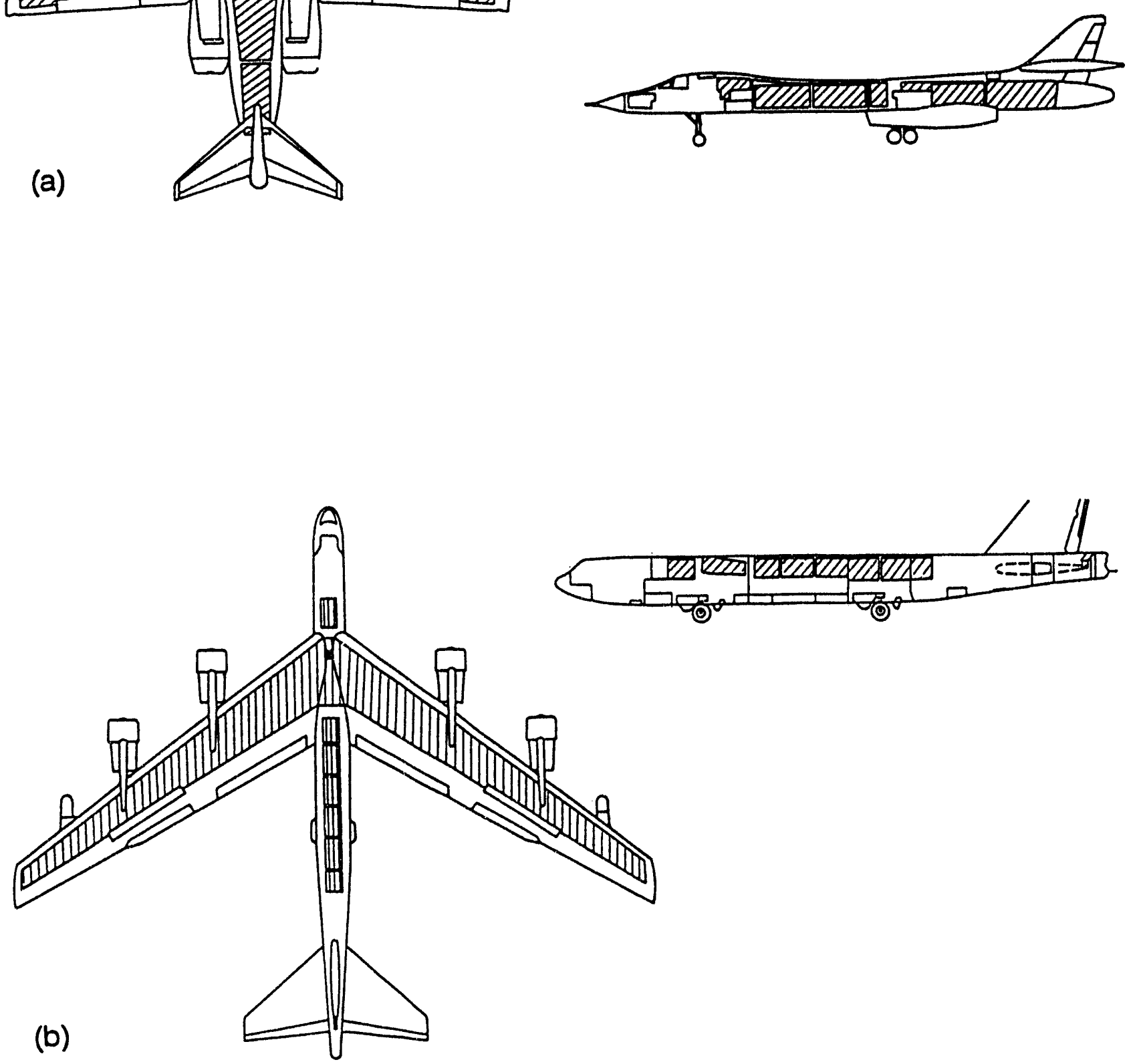

Figure 2.14 Alert Aircraft Fuel Locations 


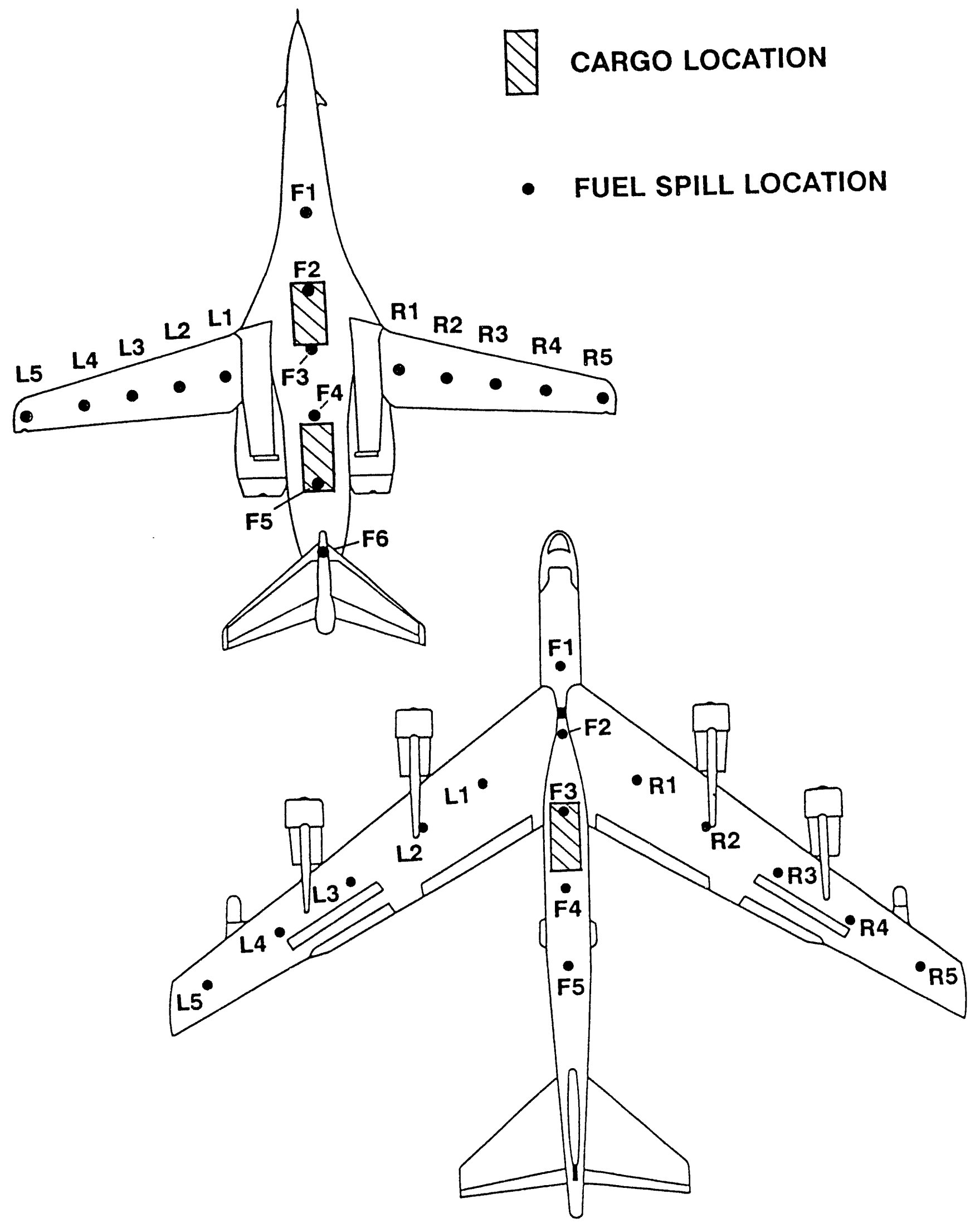

Figure 2.15 Fuel Spill Locations and Designations 
In addition to the alert aircraft, there will be fuel spills from the colliding aircraft, and fires from spills at the colliding aircraft (when it comes to rest) can contribute to weapon exposure in the alert aircraft. It is necessary then to locate fuel spills from the colliding aircraft with respect to the alert aircraft. The manner in which these locations are designated is illustrated in Figure 2.16. Figure 2.16 shows a grid of potential spill locations designated $C n_{1} n_{2}$, where $n_{1}$ and $n_{2}$ are numbers which indicate the lateral position and vertical position, respectively. The spacing between grid lines is 25 feet.

Regarding the procedure for estimating probabilities of spills at various locations and rates, for purposes of illustration consider the broadside collision of an aircraft into a B-52. The sketch on Figure 2.17 illustrates first of all a subcategory of this type of collision, where the left wing tip of the colliding aircraft impacts the fuselage nose of the B-52; specifically the sketch is intended to represent a collision in which the left wing tip guide line shown in Figure 2.17 lies between the upper two dashed lines, or in the region labeled "1". It can be seen that the broadside collision has been separated into 12 collision regions, and as described below, each region represents a subcategory of the broadside collision where the events of the collision for each subcategory (and the locations and types of damage) are distinct. Since it is as likely for the colliding aircraft to be in one position as another, the relative probability for a collision associated with a given region is proportional to distance between the dashed curves for that region. For the wing tip/nose impact on Figure 2.17, the region 1 collision, the impact is to the "crew stations" section of the B-52, and the impact is judged to be remote enough from the forward fuel tanks (F1 spill location) to not cause a spill from the B-52 tanks. However, the left wing tip region of the colliding aircraft will sustain tank damage. The extent of damage and the eventual location of the fuel spill will depend on the speed of the colliding aircraft at impact. The speed at impact can vary from near zero (where the aircraft comes in ground contact well away from the alert aircraft and skids and slows before impact) to above landing/take-off speed (where ground contact is coincident or at near coincidence with aircraft collision). It is as likely that the colliding aircraft will make ground contact at one distance from the alert aircraft as any other distance, and one collision speed is thereby as probable as any other in this speed range. For purposes of analysis, the speeds at collision have been divided into three ranges referred to as low, medium and high speed, where the low speed range corresponds to the range from zero to $1 / 4$ of maximum possible speed, medium from $1 / 4$ to $1 / 2$ of maximum, and high from $1 / 2$ maximum to maximum; the relative probability that the impact speed will be in a given range is thereby 0.25 for the low speed range, 0.25 for medium and 0.50 for high. 


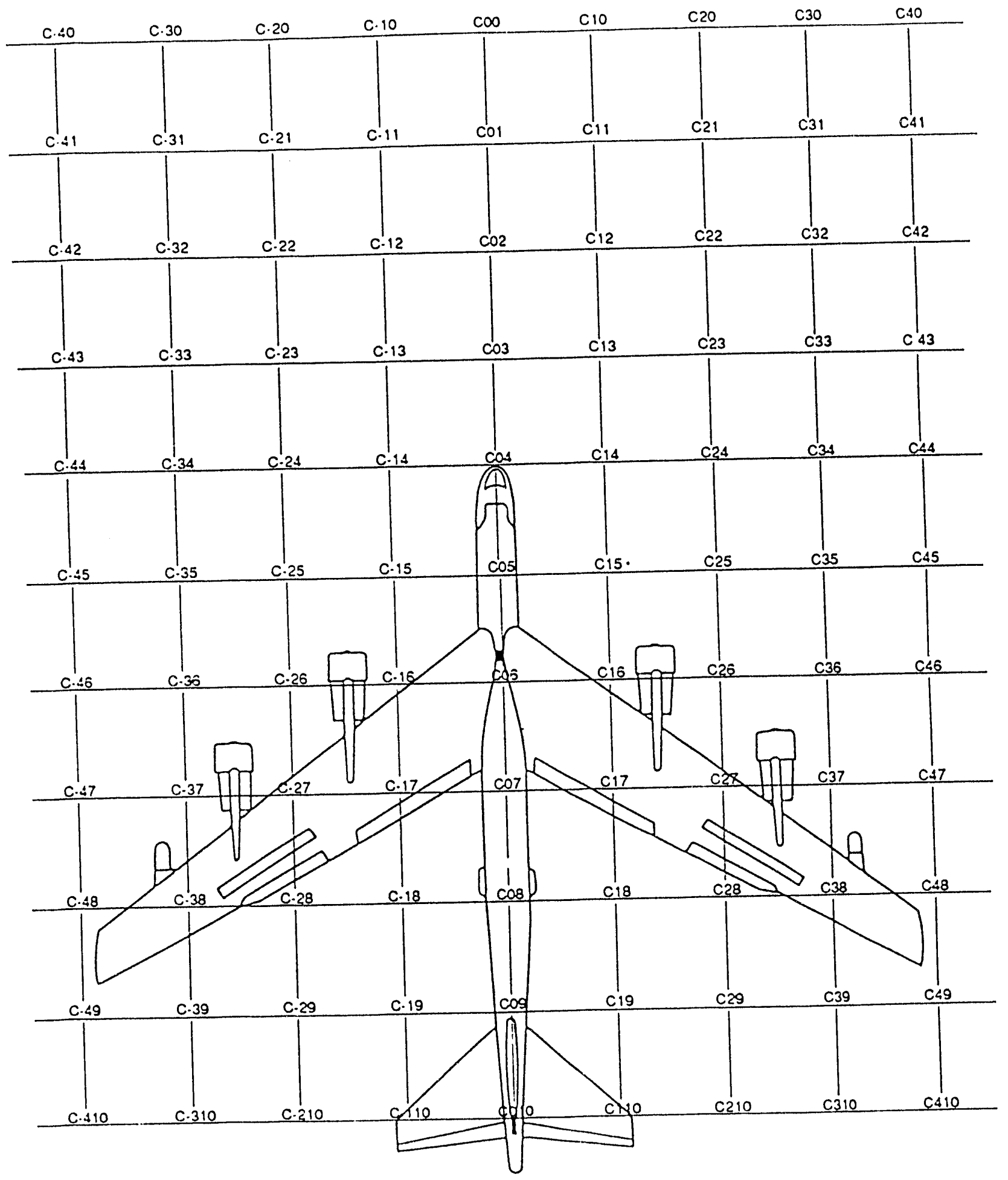

Figure 2.16 Fuel Spill Location Designation for Spills from Colliding Aircraft 


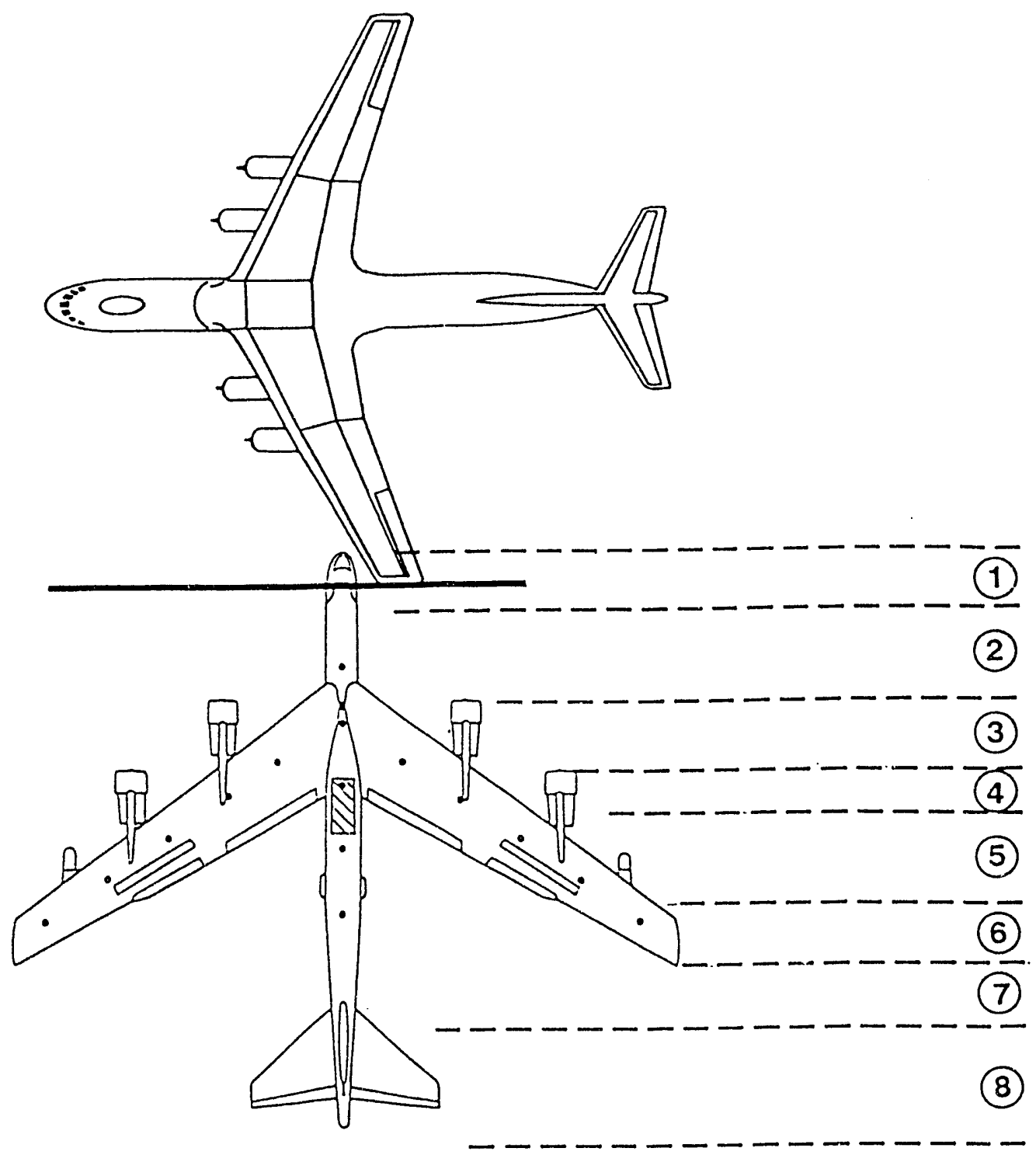

(9)

- - - - - - - - - - - -

(10)

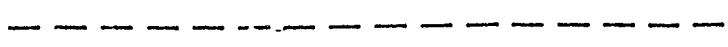

(11)

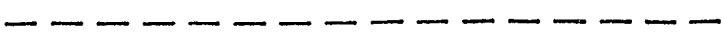

(12)

Figure 2.17 Region 1 Broadside Collision into B-52 
On the basis of these relative probabilities for a collision to occur within a given speed range, and from an evaluation of the relative probabilities of the collision occurring in the various regions shown in Figure 2.17, the relative probabilities for a collision occurring in a given region within a given speed range can be evaluated. These 36 probability values (12 regions $\times 3$ speed ranges) for the B-52 broadside collision are given in Table 2-4 (columns 3,4 and 5). The corresponding probabilities for collisions into the $\mathrm{B}-1 \mathrm{~B}$ and the $\mathrm{B}-52$ at all collision angles considered are given in Appendix D.

TABLE 2-4 Probabilities For Various Types of Broadside Collisiın into B-52 at Various Collision Speeds

\begin{tabular}{|c|c|c|c|c|}
\hline \multirow[t]{2}{*}{$\begin{array}{l}\text { COLLISION } \\
\text { REGION }\end{array}$} & \multirow{2}{*}{$\begin{array}{c}\text { RELATIVE } \\
\text { PROBABILITY FOR } \\
\text { COLLISION IN } \\
\text { GIVEN REGION }\end{array}$} & \multicolumn{3}{|c|}{$\begin{array}{l}\text { RELATIVE PROBABILITY FOR } \\
\text { COLLISION IN GIVEN REGION AT } \\
\text { GIVEN COLLISION SPEED RANGE }\end{array}$} \\
\hline & & $\begin{array}{c}\text { LOW } \\
0-40 \mathrm{mph} \\
\end{array}$ & $\begin{array}{c}\text { MED } \\
40-80 \mathrm{mph}\end{array}$ & $\begin{array}{c}\text { HIGH } \\
80-160 \mathrm{mph}\end{array}$ \\
\hline 1 & .05156 & .01289 & .01289 & .02579 \\
\hline 2 & .07736 & .01934 & .01934 & .03868 \\
\hline 3 & .06160 & .01540 & .01540 & .03080 \\
\hline 4 & .04012 & .01003 & .01003 & .02006 \\
\hline 5 & .07736 & .01934 & .01934 & .03868 \\
\hline 6 & .05444 & .01361 & .01361 & .02722 \\
\hline 7 & .06016 & .01504 & .01504 & .03009 \\
\hline 8 & .10316 & .02579 & .02579 & .05158 \\
\hline 9 & .11176 & .02794 & .02794 & .05587 \\
\hline 10 & .10888 & .02722 & .02722 & .05444 \\
\hline 11 & .11892 & .02973 & .02973 & .05946 \\
\hline 12 & .13468 & .03367 & .03367 & .06733 \\
\hline TOTAL & 1.0000 & .25000 & .25000 & .50000 \\
\hline
\end{tabular}


The next task is to estimate the location of fuel spills for collisions at each region for each speed range. There are a number of factors to consider in making this estimate, and the following discussion illustrates the procedure of estimate. For purposes of demonstration, consider the region 2 broadside collision into the B-52 which is illustrated in Figure 2.18. It is first of all judged that fuel tank ruptures will occur (1), on the left wing of the colliding aircraft at a position about $1 / 3$ wing length inward from the wing tip, and (2), at the F1 (forwardmost) fuselage spill point of the B-52. For the low speed range collision, there is some question whether damage would be sufficient to rupture tanks in the $\mathrm{F} 1$ region since the initial fuselage/wing contact at impact is somewhat forward of tankage in the F1 region; when it becomes difficult to judge whether a rupture will or will not occur at a location, but there appears to be a reasonable probability for rupture, it is the procedure policy to assume the rupture. For this collision, impact will cause the colliding aircraft to rotate CCW (about a vertical axis), and there can be some tilting of the wing of the colliding aircraft in either direction (rotation about the fuselage axis). Regarding the wing tilting, the sketch on Figure 2.19 shows the relationship of the left wing of the colliding aircraft and the B-52 nose under their normal parking state. Under this idealized state the wing tip impact would be about center height of the fuselage nose. There is probably a tendency for the colliding aircraft fuselage to be slightly lower than at normal parking due to wheel retraction in some cases and wheel system damage at ground impact in others. For the case where the wings of the colliding aircraft are reasonably level at collision, there is probably a slight tendency for the left wing tip to be driven down and perhaps under the B-52 nose. When the left wing tip is lower than the right wing tip at collision, it appears much more likely that the left wing will be driven down and under the B-52 nose. For these cases, the left wing tip may or may not make contact with the ground surface Contact with a "tarmac" surface (probably momentarily) would appear to contribute only slightly to the CCW rotation of the colliding aircraft (compared to the contribution of impact with the B-52 nose). When the left wing tip is higher than the right at collision, the left wing would tend to be driven upward and perhaps over the B-52 nose. The right wing may contact the ground surface, and with contact, the frictional forces would provide some counteraction of the CCW rotation of the colliding aircraft. It is judged that the speed reduction of the colliding aircraft due to ground contact of either wing tip would be insignificant. It is judged that the momentum or speed loss of the colliding aircraft due to the impact between the wing and nose is of the order 1020 percent, so that the skid distance of the colliding aircraft would be of the order of 20-35 percent less than the distance for an equivalent but unobstructed skid. (100 feet has been assumed as the unobstructed skid distance for a speed that is $1 / 4$ of maximum speed). It is judged that forces lateral to the direction of motion acting on the colliding aircraft by the B-52 fuselage during the impact process are insufficient to significantly change the direction of motion of the center of mass of the colliding aircraft. Likewise, lateral forces on the colliding 


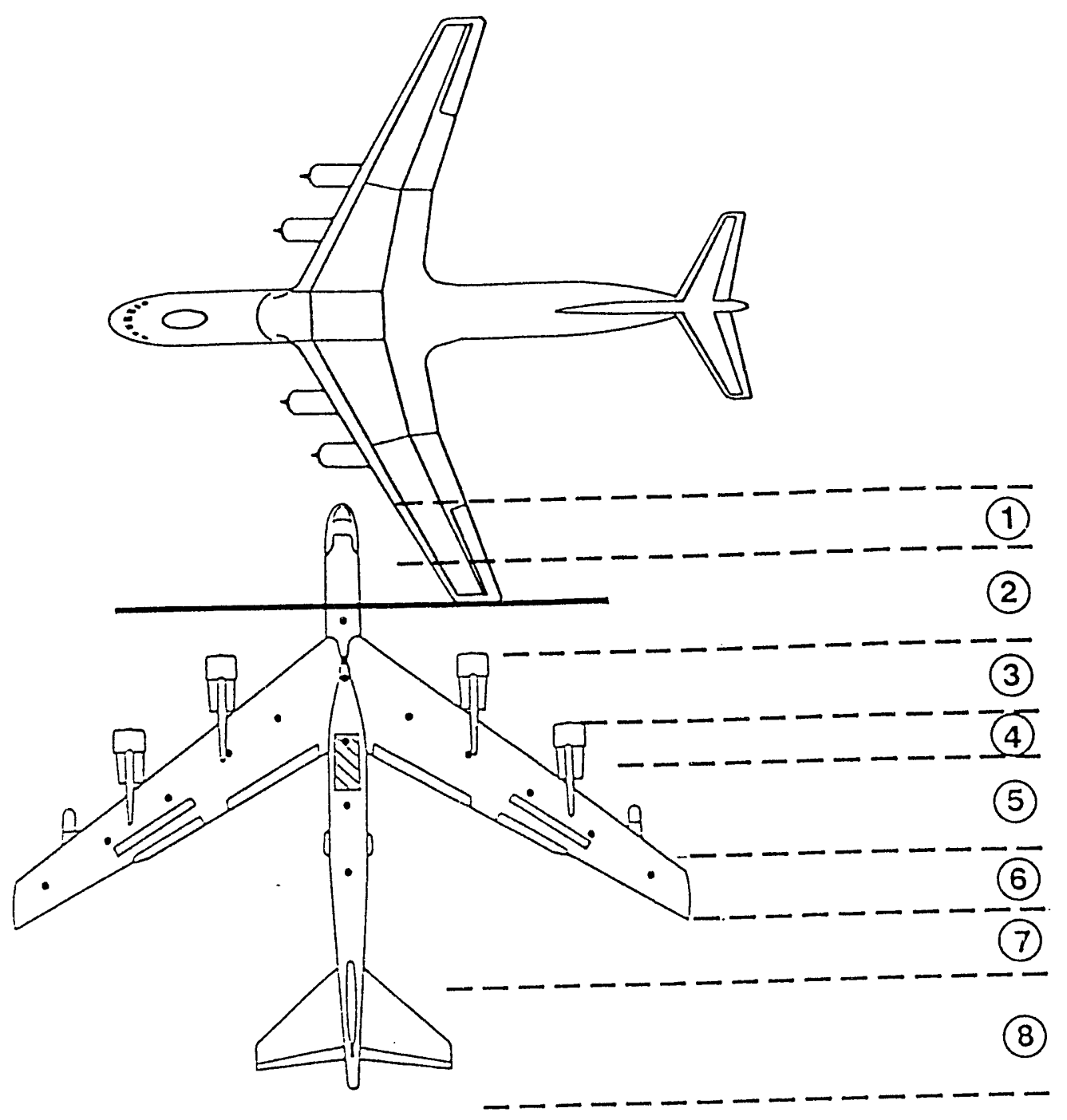

(9)

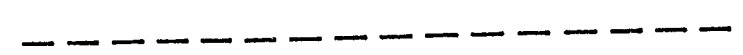

(10)

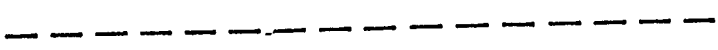

(11)

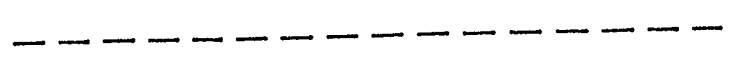

(12)

Figure 2.18 Region 2 Broadside Collision into B-52 


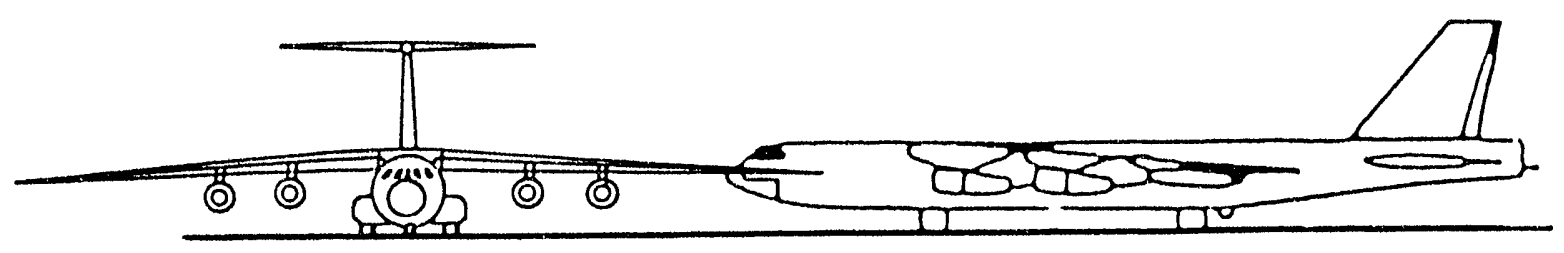

\section{Figure 2.19 Example Wing/Fuselage Collision Position}

aircraft by the ground are judged to be insufficient to significantly change the motion, and the CCW rotation of the colliding aircraft is judged to be in the range of 10 to 30 degrees. Rotation of the B-52 is judged to be insignificant.

On the basis of factors in the discussion above, the rupture in the left wing of the colliding aircraft when at rest is judged to be at any of the following locations for the low speed impact: C-13, C-14, C-23, C-24, C-25; the general arrangement with respect to the B-52 of these five locations, along with the $\mathrm{F} 1$ location, is illustrated in Figure 2.20. The reason that there are five locations listed for the colliding aircraft is a consequence of the uncertainty of determining this spill location, and there are several sources of this uncertainty. First, there is the uncertainty in the rotation of the colliding aircraft. Second, this case is for the low speed impact range, which includes impact speeds from near zero to $1 / 4$ of maximum speed, and the range of skid distances appropriate to the velocity range must be included. Third, even for a known impact velocity, there is an uncertainty in the skid distance. Fourth, there is an uncertainty regarding the speed loss due to impact. Finally, there is an uncertainty in the position of the rupture on the left wing.

With these uncertainties, the location cannot be determined to a greater accuracy than is represented by an area that encloses the five points, and we cannot determine that a spill at any one of the five locations is more likely than spills at any of the other four. The policy then is to treat each of the five locations as equally probable spill sites, and the probability of a spill at each is thereby $1 / 5$ of the probability of a region 2 collision at low speed (i.e., from Table $2-4,1 / 5 \times 0.01934=0.00387)$. Thus in the exposure evaluation, weapon exposure from fires for spills at all five locations is determined, but the probability for each of the fires is 0.00387 . It is important to recognize however 


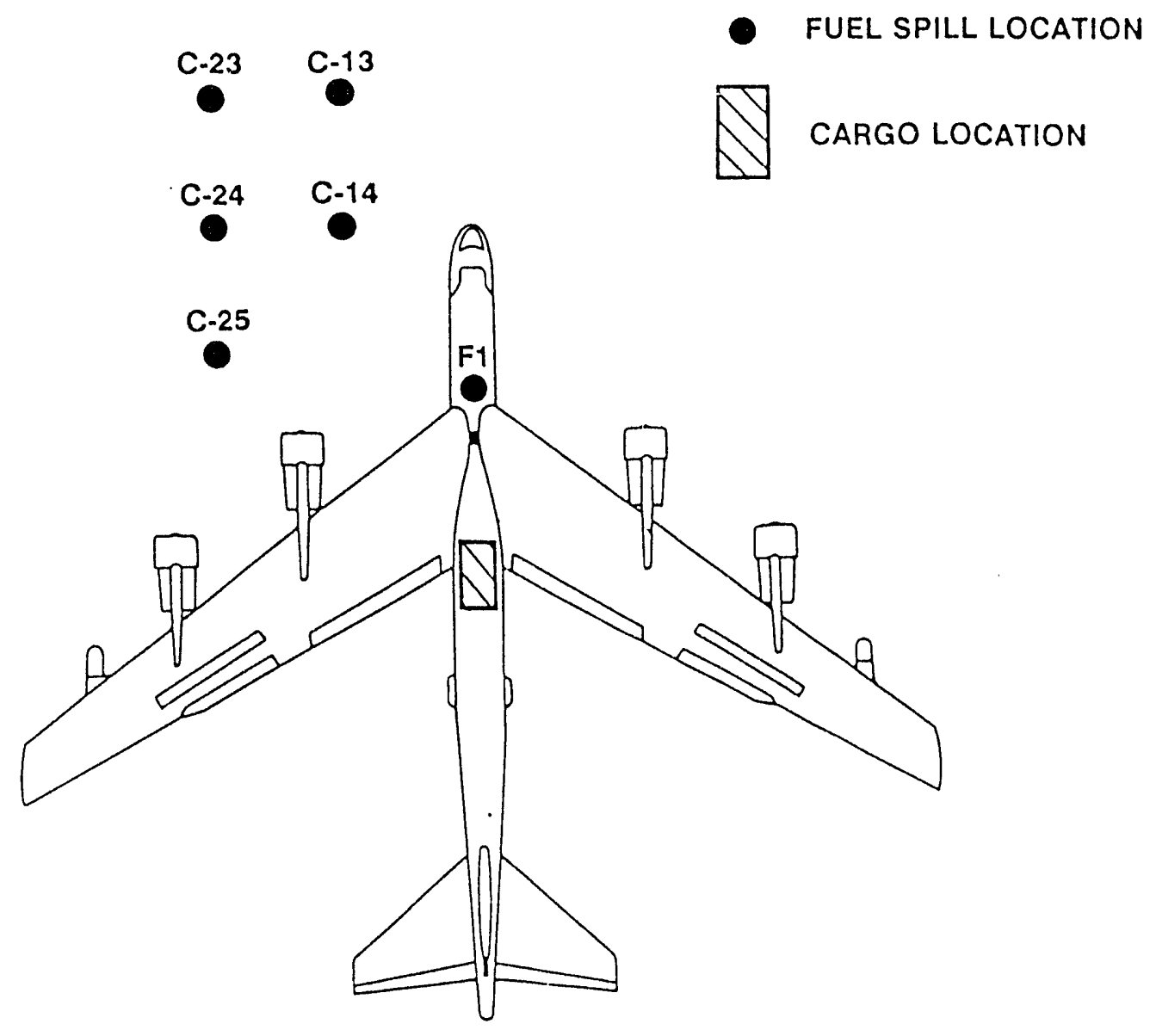

Figure 2.20 Example Double Spill Locations

that there is also a concurrent rupture at the F1 location, that is, the region 2 collision results in simultaneous spills at F1 and at a wing location. There are then the following five "double" rupture cases to consider:

$\mathrm{F} 1$ and $\mathrm{C}-13$

$\mathrm{F} 1$ and $\mathrm{C}-14$

$\mathrm{F} 1$ and $\mathrm{C}-23$

$\mathrm{F} 1$ and $\mathrm{C}-24$

$\mathrm{F} 1$ and $\mathrm{C}-25$,

and the probability of each combination is 0.00387 .

When there are two (or more) ruptures, the interest is solely with the fire causing weapon exposure (or the most severe exposure) for the wind conditions then under consideration. Thus, the weapon exposure temperatures for both fires are determined, and only temperature values from the fire which results in the highest temperature at the weapon are retained for evaluation of the probability that weapons will be exposed to various temperatures. 
Multiple concurrent ruptures are common for collisions in the various regions. In applying the procedure for estimating spill locations, it was found that as many as four concurrent ruptures occur, and triple ruptures were found to be by far the most common case. A region 4 collision at high speed illustrated in Figure 2.21 is an example which led to a triple rupture, with one rupture at the F1 or F2 location, a second rupture in the right wing at R2, R3 or R4, and a wing rupture in the colliding aircraft located at C15, C04, C-13, C-14, C-23, C-24, $\mathrm{C}-33$ or C-34. For this case then, there are $2 \times 3 \times 8=48$ equally probable fire combinations (from Table 2-4, the probability of each combination is $0.02006 / 48=0.00418$ ), and the weapon exposure temperature is evaluated for $48 \times 3=144$ fires.

The existence of concurrent fires has a significant bearing on the predicted probabilities that weapon exposures will exceed given temperatures. For a single fire, there tends to be a sizeable range of wind directions at which the flame is directed away from the weapon. With two or more concurrent fires, wind directions that are unfavorable to exposure from one fire are often favorable to exposure from another. Recognition that the existence of multiple fires would be common and would affect exposure probabilities is largely why a significant effort was devoted to estimating fire locations and their probabilities. It is not so important that the multiple fires be precisely located, but it is important that their presence be included in the analysis.

There are certain instances where multiple fires can contribute an uncertainty to exposure prediction. The sketch on Figure 2.22 illustrates the situation where the wind direction is along the line between two spill locations. For spill locations that are sufficiently close together, the presence of each of these fires will influence the other. The upwind fire would probably cause a reduction of air penetration into the downwind fire, and temperatures in the downwind fire would tend to be abnormally low. It is thought that the presence of the downwind fire may cause mass flow in the upwind fire to be directed somewhat more vertically (a slight shifting of temperature regions), but that the general temperature values would not be appreciably affected. Since temperature data from multiple fires is not available, data from the two single-spill fires is used. It will be seen later that the downwind fire for these wind directions will rarely expose the weapon, and the error in exposure probability is believed to be insignificant.

For certain collision regions, it was found that the colliding and alert aircraft remain coupled (or in close proximity) after the collision. Coupling tends to occur when the fuselage of the colliding aircraft impacts at or near the midsection of the alert aircraft. For the broadside collision into the B-52, for example, coupling was judged to occur for collision regions 7,8 and 9 . 


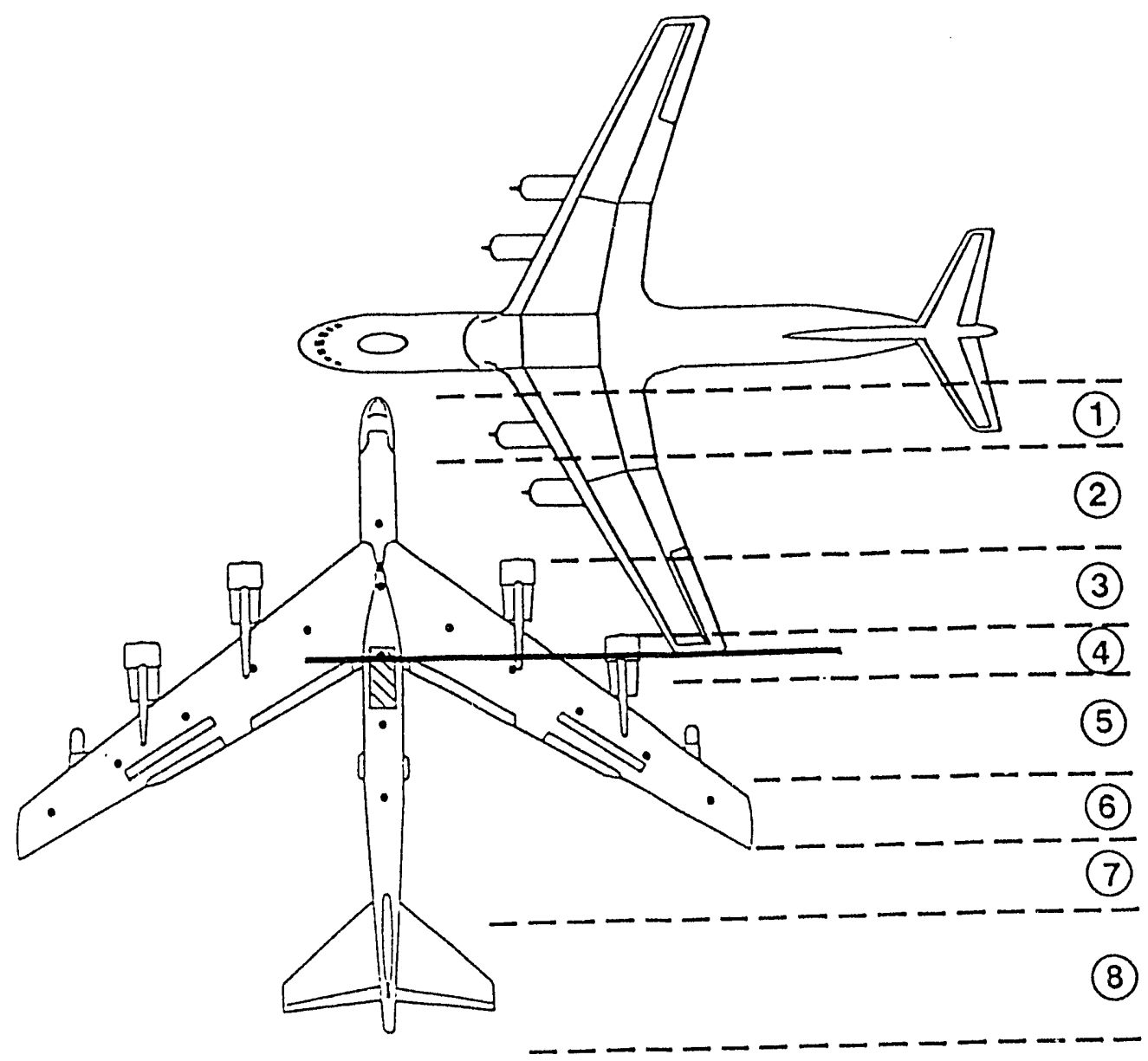

(9)

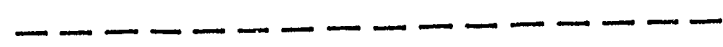

(10)

- - - - - - - - - - - -

(11)

- - - - - - - - - - -

(12)

Figure 2.21 Region 4 Broadside Collision into B-52 


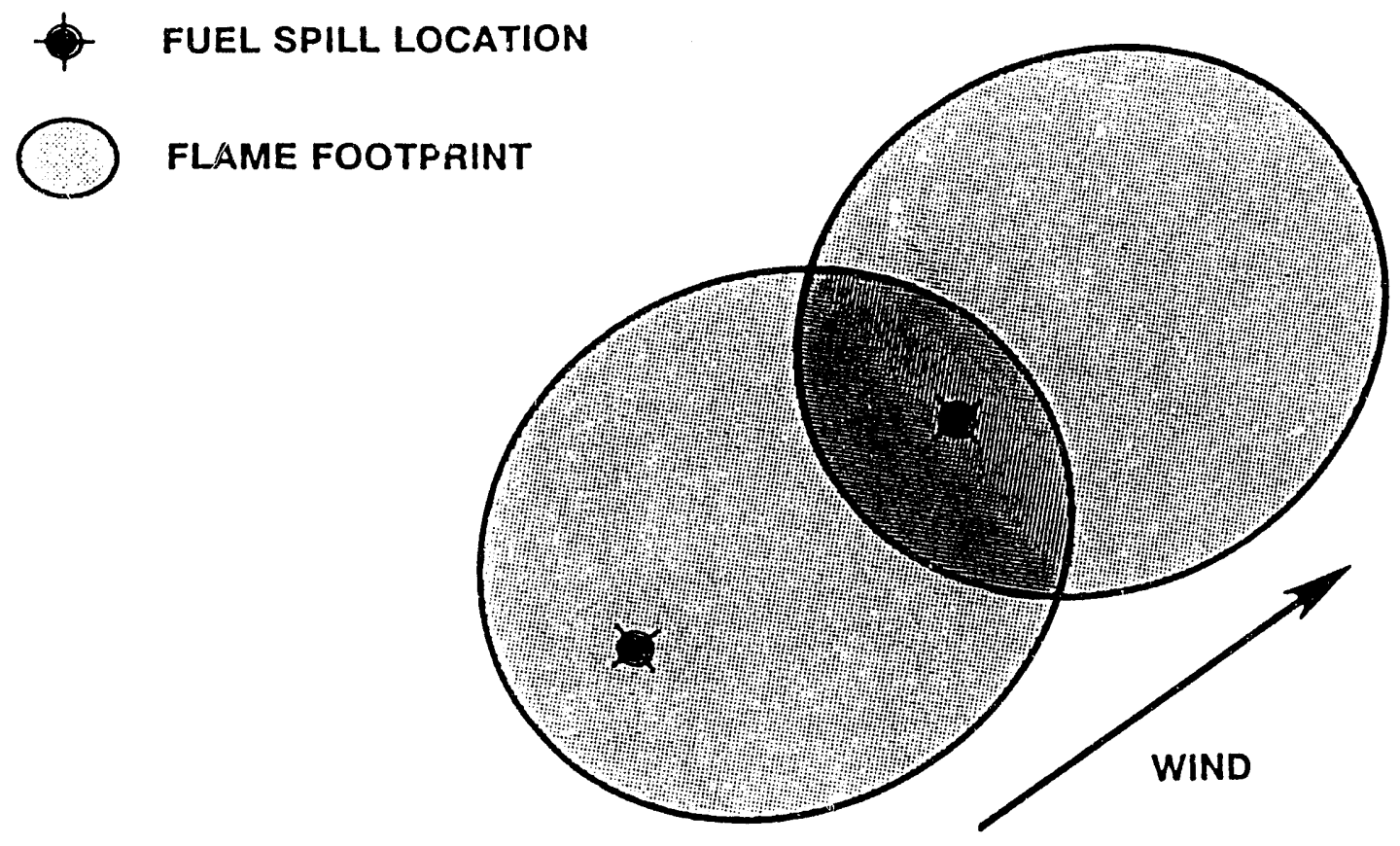

Figure 2.22 Aligned Fires

For collisions that result in aircraft coupling, fuel tank damage points from both aircraft will often be in close proximity. For instance, for the region 8 collision, a fuel spill from the left wing of the colliding aircraft is judged to be near a spill from the mid to aft fuselage section of the B-52 (F2, F3 or F4). In cases where two fuel spills of given rates ar? judged to be in close proximity, it is the policy in the procedure to treat the spills as a single spill whose spill rate is the sum of the two individual rates. Coupling was judged to occur for 28 percent of broadside collisions into the B-52, and for the various coilision angles into both the B-52 and $\mathrm{B}-1 \mathrm{~B}$, the percentage of coupled collisions ranged from about 20 to 35 percent.

Turning now to volumetric spill rates, estimates of the rate must be made for ev sry rupture that occurs. Rate estimates are unquestionably the most uncertain input, but it will be shown in Subsection 3.5 that the probability of exposure to various temperatures is particularly insensitive to errors in spill rate; it will be seen, for example, that the reduction of all spill rate category inputs by one 
category ( 3 to 2 and 2 to 1) reduces the average probability of exceeding a given temperature (averaged over the temperature range) by approximately 8.7 percent.

Some of the general trends in the rate estimates are given in Table 2-5, where the rate category estimates are listed for collision impacts between various aircraft components, e.g., the fuselage nose of the colliding aircraft impacting the engine/engine mount region of the alert aircraft. The category estimates in Table 2-5 are for the first impact of a given collision. Most collisions will involve more than one impact between various aircraft components; for example, the first impact of a collision may be the impact of the colliding aircraft wing with the wing of the alert aircraft, and continued motion may subsequently result in the impact of the colliding aircraft fuselage with the fuselage of the alert aircraft. For some types of collision, there can be significant slowing of the colliding aircraft before a second or third impact occurs, and consequently the rate estimate for second or third impacts is occasionally smaller than listed in Table 2-5 for a given type of impact. Detailed examination of spill rate tables for all coilisions considered (Appendix D) will show occasional departures from the trends or values given in Table 2-5 even for first impact of aircraft components; the reason for the departure; is a consequence of some particular circumstance of the impact, for example, on the angle of impact. The pur rose $_{f}$ Table $2-5$ is to illustrate the general magnitude of spill rates that have been estimated, and to show their dependence on type of impact and speed of impact. The difficulty of assessing spill rates and the factors used in their assessment is illustrated in the following paragraphs.

Consider first those collisions where "hn colliding aircraft wing region that is outboard of it's engine impacts the aleri ircraft wing region outboard of it's engines. One example of this type of collision is where the trailing edge of the alert aircraft wing is impacted by the leading eage of the colliding wing. The wing fuel tanks of the alert aircraft are well forward of the actual trailing edge of the wing (forward of flaps, spoilers or ailerons), and it appears that the trailing edge section provides some protection to the fuel tanks. For the B-1B, for example, the trailing edge section is of composite and fiberglass construction, but with steel "rod-like" pieces lying in the chord direction spaced at intervals along the span. There is first of all some possibility that the steel rods will be driven to puncture the wing tanks of the alert aircraft. It is assumed that this type of puncture will occur for the medium and high speed impacts and will not occur for low speed impacts. On the basis of the crossectional dimensions of the rods, effective puncture sizes are estimated to be about 10 to 20 square inches. For purpnses of relating spill rate to rupture dimensions, Table 2-6 lists approximate volume spill rates through circular tank openings for a range of opening diameters or areas and a range of heights from opening to upper fuel surface; actual rates will depend somewhat on the shape of the opening, and the 
TABLE 2-5 Estimates of Fuel Spill Rates for Impacts Between Various Aircraft Components

\begin{tabular}{|c|c|c|c|}
\hline TYPE OF COMPONENT & IMPACT & \multicolumn{2}{|c|}{ SPILL RATE RANGE CATEGORY* } \\
\hline $\begin{array}{l}\text { Wing outboard of engine } \\
\text { impacting wing outboard of } \\
\text { engines }\end{array}$ & $\begin{array}{l}\text { HIGH } \\
\text { MED } \\
\text { LOW }\end{array}$ & $\begin{array}{c}\text { FOR ALERT } \\
\text { AIRCRAFT WING } \\
2,1^{* *} \\
2,1 \\
1\end{array}$ & $\begin{array}{c}\text { FOR COLLIDING } \\
\text { AIRCRAFT WING } \\
2, \\
2, \\
1\end{array}$ \\
\hline $\begin{array}{l}\text { Impact between engine/ } \\
\text { engine mount region of either } \\
\text { alert or colliding aircraft with } \\
\text { wing outboard of engines of } \\
\text { other aircraft }\end{array}$ & $\begin{array}{l}\text { HIGH } \\
\text { MED } \\
\text { LOW }\end{array}$ & $\begin{array}{c}\text { FOR ALERT } \\
\text { AIRCRAFT WING } \\
(\text { B-52)* } \\
2,3^{* *} \\
2 \\
2,1\end{array}$ & $\begin{array}{c}\text { FOR COLLIDING } \\
\text { AIRCRAFT WING } \\
2,3 \\
2 \\
2,1\end{array}$ \\
\hline $\begin{array}{l}\text { Impact of engine/engine } \\
\text { mount region of colliding } \\
\text { aircraft impacting engine/ } \\
\text { engine mount region of alert } \\
\text { aircraft (B-52) }\end{array}$ & $\begin{array}{l}\text { HIGH } \\
\text { MED } \\
\text { LOW }\end{array}$ & $\begin{array}{c}\text { FOR ALERT } \\
\text { AIRCRAFT WING } \\
\text { (B-52)* } \\
2 \\
2 \\
2\end{array}$ & $\begin{array}{l}\text { FOR COLLIDING } \\
\text { AIRCRAFT WING }\end{array}$ \\
\hline $\begin{array}{l}\text { Fuselage "nose" of colliding } \\
\text { aircraft impacting fuel tank } \\
\text { region of fuselage of alert } \\
\text { aircraft }\end{array}$ & $\begin{array}{l}\text { HIGH } \\
\text { MED } \\
\text { LOW }\end{array}$ & \multicolumn{2}{|c|}{$\begin{array}{c}\text { FOR FUSELAGE TANKS OF ALERT } \\
\text { AIRCRAFT } \\
3 \\
3 \\
2\end{array}$} \\
\hline $\begin{array}{l}\text { Fuselage "nose" of colliding } \\
\text { aircraft impacting "crew } \\
\text { compartment region" of alert } \\
\text { aircraft }\end{array}$ & $\begin{array}{l}\text { HIGH } \\
\text { MED } \\
\text { LOW }\end{array}$ & \multicolumn{2}{|c|}{$\begin{array}{c}\text { FOR FUSELAGE TANKS OF ALERT } \\
\text { AIRCRAFT } \\
2,1 \\
2,1 \\
1\end{array}$} \\
\hline $\begin{array}{l}\text { Fuselage "nose" of colliding } \\
\text { aircraft impacting wing } \\
\text { outboard of engines of alert } \\
\text { aircraft (B-52) }\end{array}$ & $\begin{array}{l}\text { HIGH } \\
\text { MED } \\
\text { LOW }\end{array}$ & \multicolumn{2}{|c|}{$\begin{array}{l}\text { FOR ALERT AIRCRAFT WING (B-52) } \\
\qquad \begin{array}{c}3,2 \\
2 \\
2\end{array}\end{array}$} \\
\hline $\begin{array}{l}\text { Fuselage "nose" of colliding } \\
\text { aircraft impacting engine/ } \\
\text { engine mount region or alert } \\
\text { aircraft (B-52) }\end{array}$ & $\begin{array}{l}\text { HIGH } \\
\text { MED } \\
\text { LOW }\end{array}$ & \multicolumn{2}{|c|}{$\begin{array}{c}\text { FOR ALERT AIRCRAFT WING (B-52) } \\
3 \\
3,2 \\
2\end{array}$} \\
\hline $\begin{array}{l}\text { Engine region of colliding } \\
\text { aircraft impacting fuselage of } \\
\text { alert aircraft }\end{array}$ & $\begin{array}{l}\text { HIGH } \\
\text { MED } \\
\text { LOW }\end{array}$ & $\begin{array}{c}\text { FOR ALERT } \\
\text { AIRCRAFT } \\
\text { FUSELAGE } \\
3 \\
2 \\
2 \\
\end{array}$ & $\begin{array}{c}\text { FOR COLLIDING } \\
\text { AIRCRAFT WING } \\
2 \\
2 \\
1\end{array}$ \\
\hline
\end{tabular}

- Spill rate range categories 1, 2 and 3 correspond to volume spill rates of less than 225 gpm, 225 to $450 \mathrm{gpm}$ and greater than $450 \mathrm{gpm}$ respectively.

* Different rates were sometimes evaluated for the same general type of collision. 
TABLE 2-6 Spill Rates (in gpm) vs Rupture Size and Fuel Pressure Head

\begin{tabular}{|cc|rrrrrr|}
\hline $\begin{array}{c}\text { HOLE } \\
\text { DIAM } \\
\text { inch }\end{array}$ & $\begin{array}{c}\text { HOLE } \\
\text { AREA } \\
\text { sq in }\end{array}$ & \multicolumn{7}{|c|}{1} & \multicolumn{6}{|c|}{ FUEL PRESSURE HEAD IN FEET } \\
\hline 12 & 113 & 1750 & 2470 & 3040 & 3495 & 3910 & 4280 \\
8 & 50 & 775 & 1095 & 1345 & 1550 & 1735 & 1900 \\
6 & 28 & 435 & 615 & 760 & 870 & 975 & 1070 \\
5 & 20 & 310 & 435 & 535 & 620 & 690 & 755 \\
4 & 13 & 195 & 275 & 335 & 390 & 435 & 475 \\
3.5 & 10 & 155 & 220 & 270 & 310 & 345 & 380 \\
3 & 7 & 110 & 155 & 190 & 215 & 245 & 265 \\
2 & 3 & 48 & 68 & 84 & 96 & 108 & 118 \\
1 & .8 & 12 & 17 & 21 & 24 & 27 & 30 \\
0.5 & .2 & 3.0 & 4.3 & 5.3 & 6.1 & 6.8 & 7.4 \\
\hline
\end{tabular}

listed rates apply when there is a path for gas flow to the tank ullage. (In many cases, the rate of fuel vaporization due to heating from the fire is sufficient to maintain normal ullage pressure.) Pressure heads will tend to decrease during the course of the fire, and average values of 1 and 3 feet are assumed for wing ruptures and fuselage tank ruptures, respectively.* From Table 2-6, the wing rupture of 10 to 20 square inches corresponds to spill rates of about 155 to 310 $\mathrm{gpm}$; in terms of spill rate category these rates correspond to approximately the middle of spill rate category 1 (less than $225 \mathrm{gpm}$ ) to near the middle of spill rate category 2 (225 to $450 \mathrm{gpm}$ ).

The steel rods mentioned above may also puncture the wing tank of the colliding aircraft. The puncture dimension range and corresponding spill rate range is judged to be the same as those given above for the alert aircraft. thickness. 
In addition to the steel rod penetration of the wing tank, structural elements of the colliding aircraft wing, particularly the comparatively rigid structures such as stiffeners, may penetrate the tank wall of the alert aircraft. Moreover, during this penetration there will be a relative motion of the two wings that is lateral to the initial motion of the colliding aircraft; forces acting on the colliding aircraft during impact would cause it to rotate about a vertical axis and, concurrently, the impacting wing of the colliding aircraft would tend to be forced upward due to the swept-back angle of the leading edge of the colliding aircraft wing and of the trailing edge of the alert aircraft wing. With this relative motion, structures that have or have not penetrated the alert aircraft wing may "cut" or "tear" the aft and upper surface of the alert aircraft wing. It appears that various tank opening dimensions may occur. For the high and medium impact speeds, it is judged that the openings may range from narrow but relatively long "cuts" (e.g., 0.1 inch $\times 36$ inch $=3.6$ square inch) to wider but shorter openings (e.g., 2 inch $\times 12$ inch $=24$ square inch). From Table 2-6, spill rates from these openings range from about 50 to $370 \mathrm{gpm}$, corresponding once again to spill rate categories 1 and 2. For the low speed impact, openings are judged to be on the low end of the size range above (and perhaps substantially smaller), corresponding to the spill category 1 . Wing damage to the colliding aircraft is judged to be similar to those given above for the alert aircraft.

Another type of impact between aircraft components where spill rate assessment is particularly difficult is the case of the fuselage nose of the colliding aircraft impacting the fuel tank region of the fuselage of the alert aircraft (the fourth type of impact listed in Table 2-5). The spill rate categories listed in Table 2-5 for this type of impact are 3 for the high and medium speed impact and 2 for the low speed impact, the highest overall rates of any of the component impacts.

It is thought that the most likely mode of initial tank rupture for this collision is the penetration of a comparatively "sharp" structural element (or elements) into the tank. However, for purposes of discussion it is useful to compare the tank response from two types of high-force, impulsive loading on the tank wall, one where the force is concentrated at one or more discrete points (as with the penetration of sharp structural elements mentioned above), and the second relatively unlikely case where the force is "smoothly" distributed along the surface of the tank wall. Alert aircraft tanks are nearly full, and for the smooth distribution, the incompressibility of the liquid would result in a pressure rise in the liquid that would resist the buckling of the impacted wall. Sizeable tank failures such as seam failures from the pressure wave in the liquid appear to be likely in this case. Also, forces on the tank wall may act to dislodge the tank from its attachment or mounting points, and tank failure may occur from this structural failure. In contrast, penetration of the "thin" tank wall by sharp structural elements requires a comparatively small force. With the structural break-up of the colliding aircraft nose region during the early stages of collision, 
it is thought that there would likely be an abundance of structural elements capable of tank penetration. Once openings have been initiated, there are paths for liquid escape, and continued loading of the wall would act to expand the openings. With the sizeable momentum of the colliding aircraft, it is thought that certain medium and high speed collisions may result in openings at or in excess of 200 square inches (e.g., 36 inch by 6 inch $=215$ square inches). Rupture areas of this size raises an issue with regard to temperature data. The highest spill rate at which temperature data are available is for $600 \mathrm{gpm}$, a rate that corresponds to an opening area of about 22 square inches. This is nearly an order of magnitude less than the estimates mentioned above, and it is desirable to have temperature data at spill rates substantially larger than $600 \mathrm{gpm}$. However, openings of this magnitude first of all represent a small fraction of fuel spills. Second, fire durations for these large spill rates tend to be comparatively small (to an approximation, duration varies inversely with spill rate). Consequently, it is thought that the absence of this data will not seriously impact the probability of weapon exposure to various temperatures.

The above paragraphs illustrate the difficulty and judgmental nature of the spill rate assessment. For several reasons, it is not expected that detailed studies of accident reports would provide an adequate data base for spill rates; burn damage, for example, will often destroy evidence from which spill rates may be deduced. At this time, a single reasonably well established spill rate is available. This data is from a Navy aircraft carrier collision of an EA-6B at landing speed into a parked F-14 (Ref 11), and data recorded indicate a single spill from the F-14 at an approximate rate of $415 \mathrm{gpm}$. A reasonably detailed structural analysis or simulated testing between aircraft components would undoubtedly improve the uncertainty of the spill rate input.

Regarding the engine start accident, it will be seen that fuel spill distribution also represents the most uncertain input to weapon exposure evaluation for this type of accident. It is noted first that this report addresses one special case of the engine start accident, as explained below. In the event of an "engine fire" during an engine start procedure, fuel flow to the engine can and would normally be stopped by returning the throttle to the closed position or by other control switches (Ref. 12). Assuming correct operation of the fuel control/valve system and "normal" response of the operator, burning residual fuel should be confined or essentially confined to the engine compartment. This type of limited fire is not addressed in this report. In the comparatively unlikely event of fuel control system failure or operational failure, fuel would eventually spill from the engine compartment to the ground surface; estimates show that a small fraction of the fuel supplied can be combusted in the engine compartment in the absence of air flow through the engine for typical fuel supply rates. This case where a continued fuel supply spills to the ground is the case evaluated for this report. 
Regarding spill locations for the engine start accident, first of all right-board or left-board accidents have essentially (but not exactly) the same likelihood of occurring, and an equal probability for the two sides has been assumed. The main difficulty is in determining the axial position of the spill or spills from the engine compartment. The path of liquid fuel flow is difficult to establish primarily because of the difficulty of assessing where and when burn-through (melting) of aluminum parts of the structure that affect the path will occur. For purposes of baseline calculations, it has been assumed that spills will occur with equal likelihood at either the exhaust or inlet of the engine (but not both concurrently). The baseline fuel spill location distribution then comprises non-concurrent spills of equal probability at any of the following four locations: the left or right inboard engine exhaust or intake. Because of the sizeable uncertainty of this distribution, the effect of substantially different distributions is shown in the uncertainty portion, subsection 3.5.

In the discussion above, data used in predicting weapon exposure has been briefly described, including data on flame temperatures, airport/aircraft configurations, wind speeds and directions, and fuel spill locations and rates. The discussion now turns to the procedure for evaluating weapon exposure.

A first step in the procedure is to establish the appropriate fuel spill location/rate distribution. Suppose, for example, that the accident of interest is a collision on landing at the alert pad end of the runway at Eaker AF Base, and further that the direction of motion of the landing aircraft at collision is parallel to the runway. Referring to the airport/aircraft arrangement sketch for Eaker AF Base on Figure 2.23, a collision into an aircraft in the immediate row of alert aircraft would be a rear-end collision, and the fuel spill distribution file for a rear-end collision into a B-52 would be selected. A collision into the second, less accessible row of alert aircraft can occur, and this second type of collision (broadside in this case) could readily be included in the evaluation; collisions into the second row have been excluded in the evaluation on the basis that the likelihood of this collision is small compared to collision into the immediate row.

In the next stage of the procedure, the weapon exposure temperature is first determined for a particular set of the following conditions: wind speed, wind direction, time of day, fuel spill location and fuel spill rate. As illustrated in Figure 2.24, the exposure temperature is determined in effect by appropriately superimposing the flame temperature data onto the weapon. Thus, flame temperature data is selected on the basis of wind speed and fuel spill rate, and the data is located and oriented on the basis of the fuel spill location and wind direction. The particular conditions illustrated on Figure 2.24 are for a spill at the rear most location of the fuselage, the wind flow direction is 50 degrees 


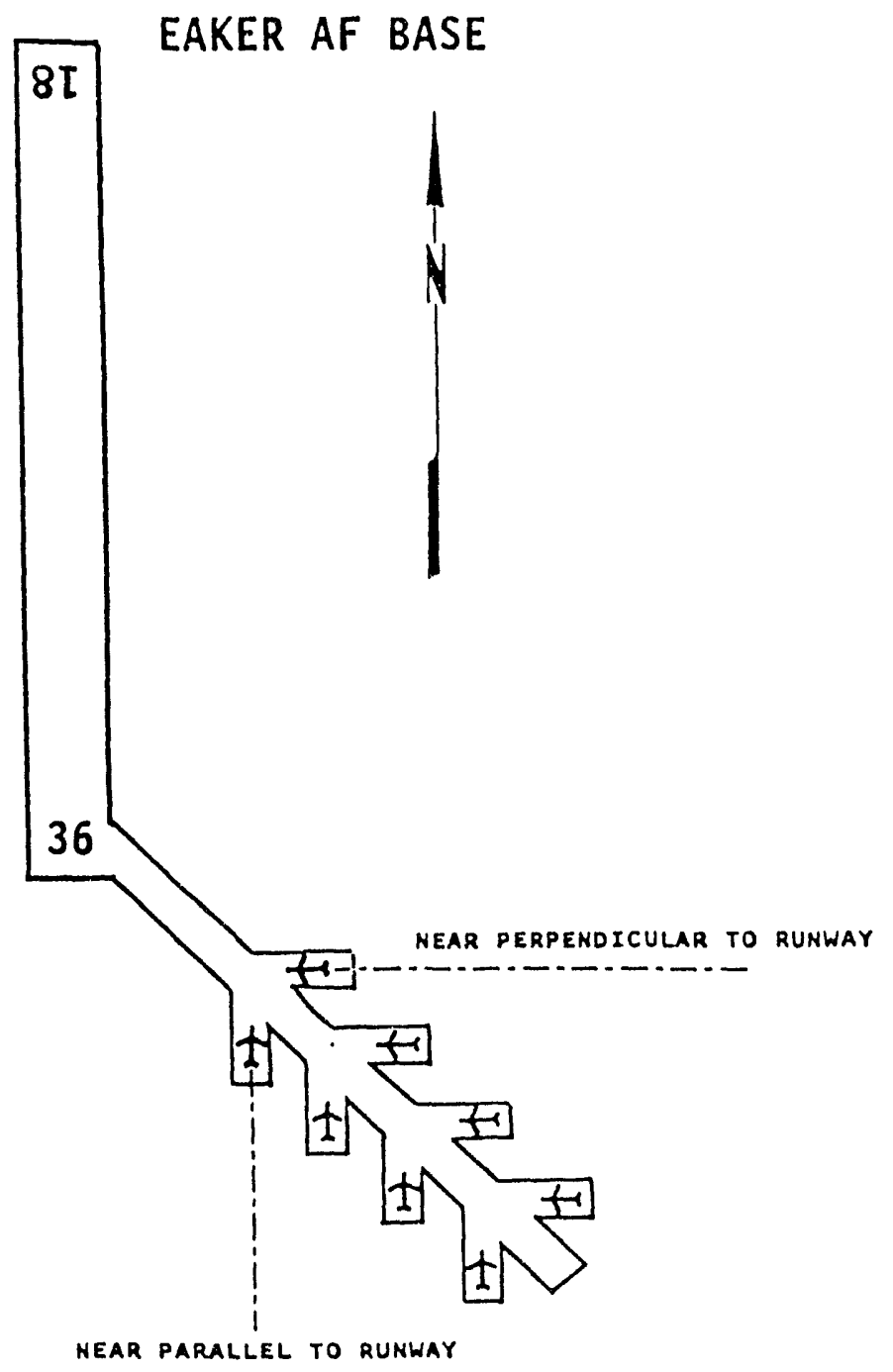

Figure 2.23 Aircraft/Airport Arrangement at Eaker AF Base

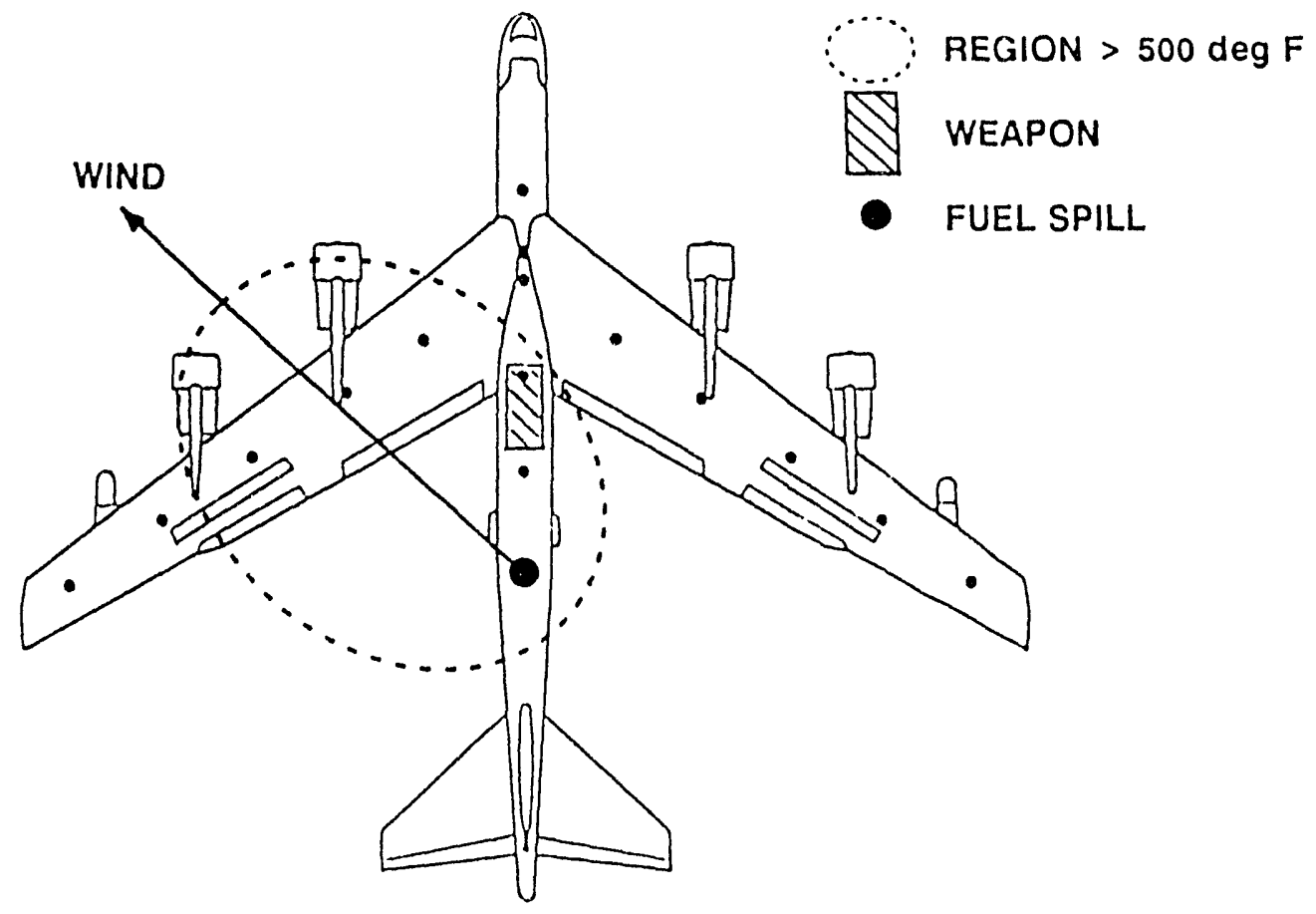

Figure 2.24 Example Flame "Footprint" for a Particular Set of Conditions 
counter clockwise of the pointing direction of the B-52, and the flame boundary location with respect to the spill point correspond approximately with a spill rate of $300 \mathrm{gpm}$ and wind speed of $15 \mathrm{mph}$. It can be seen that the weapon volume is located near the edge of the fire in a relatively cool region, and the temperature over the weapon volume will vary from about $500^{\circ} \mathrm{F}$ where the weapon is near the fire edge to perhaps $1000^{\circ} \mathrm{F}$ where the weapon is closer to the fire center. It is the calculational policy in evaluating weapon exposure to use the highest temperature that exists over the weapon volume, on the basis that the highest temperature will first affect a weapon response.

Each set of conditions has a certain probability of occurring. In the earlier discussion of the data files, there were probabilities associated with given wind conditions and with given fuel spill locations and rates. Specifically, the relative probability was established that an accident would occur at each wind speed/ wind direction/ time of day combination, which will be designated here as the probability PW, and the relative probability was established (for each collision type) that a fuel spill would occur at a given location and rate, which will be designated here as PFS. Then the relative probability, $\mathrm{P}$, that an accident will occur with a given fuel spill location and rate at a given wind speed, wind direction and time of day is $\mathrm{P}=\mathrm{PW} * \mathrm{PFS}$. Thus, there is a probability associated with the exposure temperature evaluated for the first set of conditions, and this connected pair - the temperature value and the probability value - will be retained as a connected pair for later use in evaluating the probability of weapon exposure, as explained below.

The next step in the procedure is to repeat the temperature evaluation for the next set of conditions, thus obtaining a second connected pair. This temperature evaluation is repeated for all sets of conditions involved. There are 432 different conditions for a weather/time file, ( 36 wind direction $\times 3$ wind speeds $\times 4$ times of day $=432$ ). The number of fuel spill location/rate combinations depends on the type of collision. The maximum number of fuel spill combinations is 2998 for the rear-end collision into the B-1B, and the minimum is 829 for the collision into the B-52 45 degrees from the front. The maximum number of sets of conditions (and temperatures evaluated) for the collision accident is then $432 x$ $2998=1,295,136$, and the minimum 358,128 . (The maximum number for the engine start accident is $432 \times 4=1728$ ).

Once the temperature/probability pairs have been established for all sets of conditions, the overall probability of weapon exposure to given temperatures is evaluated (for example, for the landing collisions at Eaker). This is accomplished by first separating all the temperature/probability pairs into temperature level categories, where these categories are: $\angle 475^{\circ} \mathrm{F}, 475$ to $525^{\circ} \mathrm{F}$, 525 to $575^{\circ} \mathrm{F}, 575$ to $625^{\circ} \mathrm{F}, \ldots \ldots . ., 2475$ to $2525^{\circ} \mathrm{F}$. Then for each temperature level category, the connected probabilities associated with that temperature category are added to establish the probability that the exposure temperature is 
within that temperature category. The probability that the weapon will be exposed to a temperature from 975 to $1025^{\circ} \mathrm{F}$ for example is the sum of the probabilities for each condition that resulted in an exposure between 975 and $1025^{\circ} \mathrm{F}$. A graph of these sums plotted against temperature as illustrated on Figure 2.25 represents the probability of weapon exposure to given temperatures for a given accident case (for example, for the landing collision at Eaker mentioned above).

The next step is to convert the probability of exposure to given temperatures to the probability that the exposure will exceed given temperatures, the latter probability usually being more useful in engineering applications. This is accomplished by adding the probabilities as shown on Figure 2.25 from the temperature of interest to the maximum temperature. The results of this addition for all temperatures is plotted on Figure 2.26, and represents the probability that weapon exposure will exceed a given temperature.

The discussion of procedure above was for a landing collision accident. To determine the overall probability for collisions at a given air base, the possible collisions into an alert aircraft by an aircraft taking off must also be included, and the probabilities for landing collisions and take-off collisions must be appropriately combined. For collision accidents, it was mentioned above that a weighted mean probability for an air base is obtained by weighting the landing collision probabilities and take-off collision probabilities in proportion to the relative number of landing to take-off accidents that have historically resulted in fuel fires. In subsection 3.4, examples of landing, take-off and weighted mean probabilities for various bases are given. Also given in subsection 3.4 is an overall weighted mean for all B-1B bases, where the base to base weighting is in proportion to the relative number of landing/take-off operations at each base. For the engine start accident, the probability of exceeding given temperatures depends on the direction the alert aircraft is pointed. Since the aircraft at a given base are usually pointed in more than one direction, it is necessary to conduct the procedure of evaluating the probability for each pointing direction, and appropriately weight the probabilities to determine the probability for a base. The weighting in this case is in proportion to the relative number of aircraft pointed in the various directions. In subsections 3.2 and 3.3, examples for various bases of the probabilities for the various directions and the weighted mean for that base are given. Weighted means over all B-1B bases and all B-52 bases are also given. 


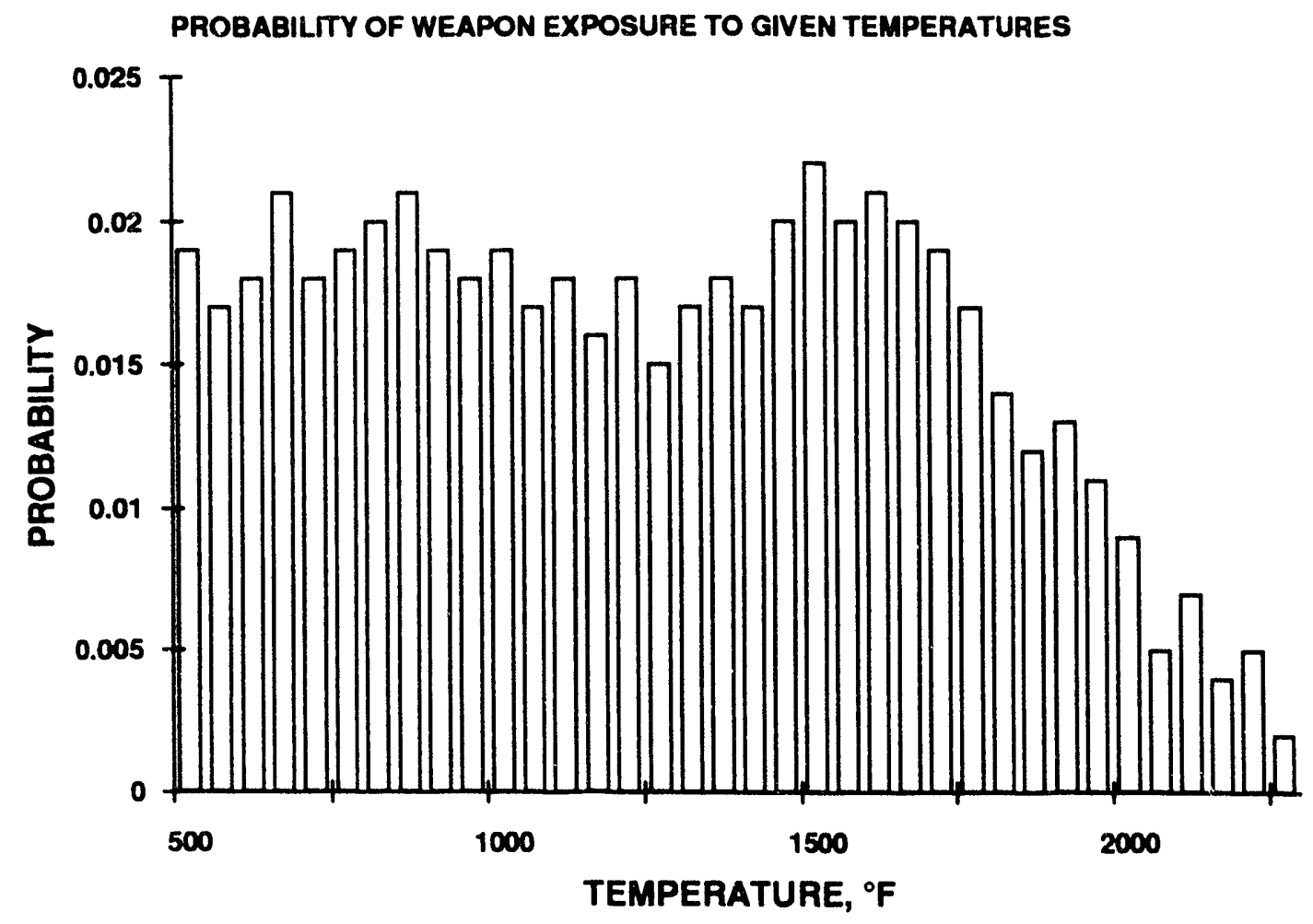

Figure 2.25 Illustration of Probability of Weapon Exposure to Given Temperatures

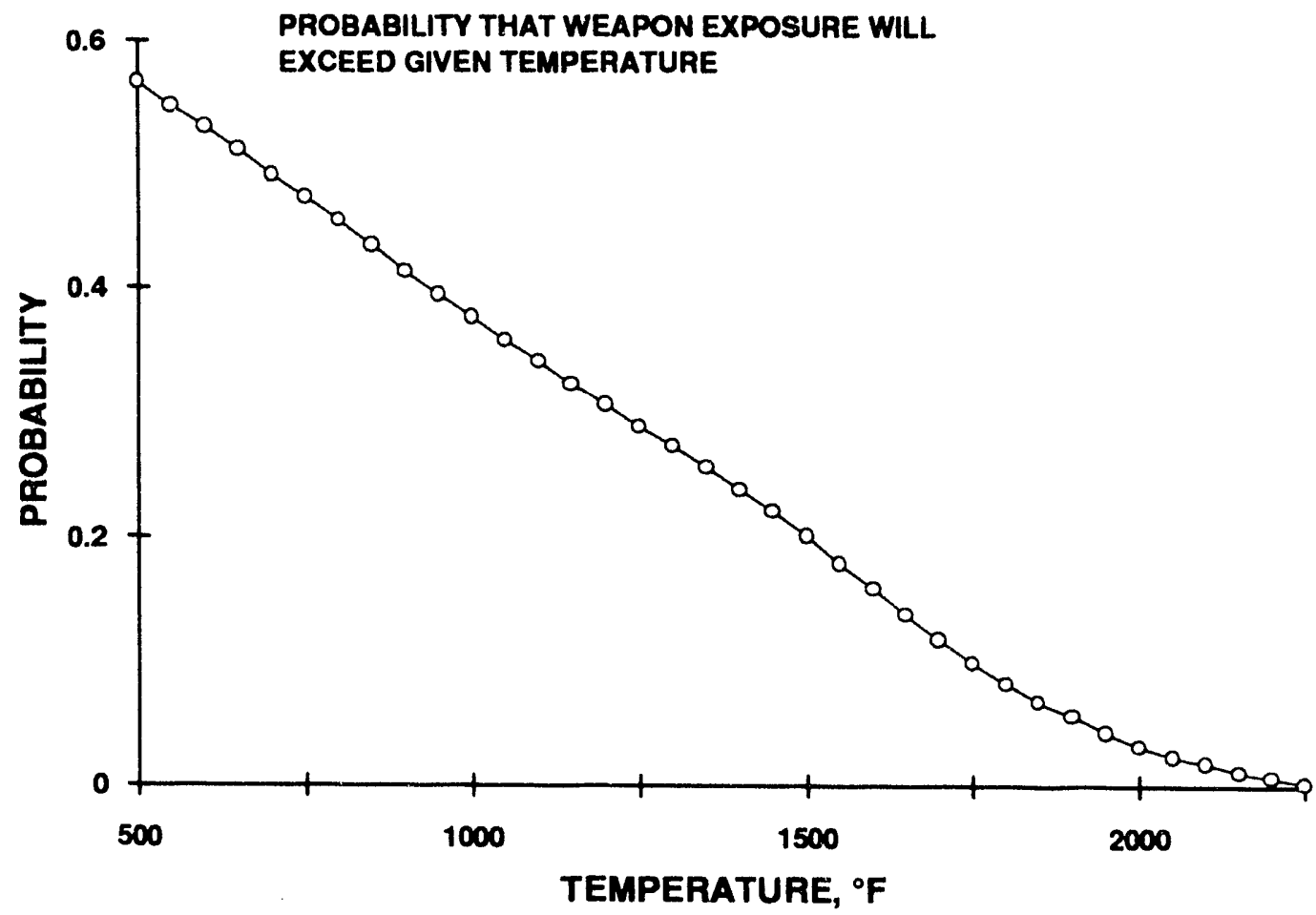

Figure 2.26 Illustration of Probability that Weapon Exposure will Exceed a Given Temperature 
In the description earlier of the development of the fuel spill location distributions for the collision accidents, it was mentioned that there is most often more than one concurrent fuel spill location and fire in each particular collision situation. An illustration of triple concurrent fires is given on Figure 2.27, showing fuel spills at the rear most fuselage location, at a right outboard wing location of the alert aircraft, and at a left outboard location of the colliding aircraft. It can be seen in the illustration that only the fire from the fuselage spill has resulted in weapon exposure for the particular condition of wind direction. The interest is only with the fire that is causing the exposure (or the most severe exposure) of the weapon. For multiple concurrent fires, it is the calculational policy to determine the maximum temperature of exposure (if any) from all concurrent fires, and to retain only the temperature/probability pair from the fire resulting in the highest temperature value for the evaluation of the probability of exposure.

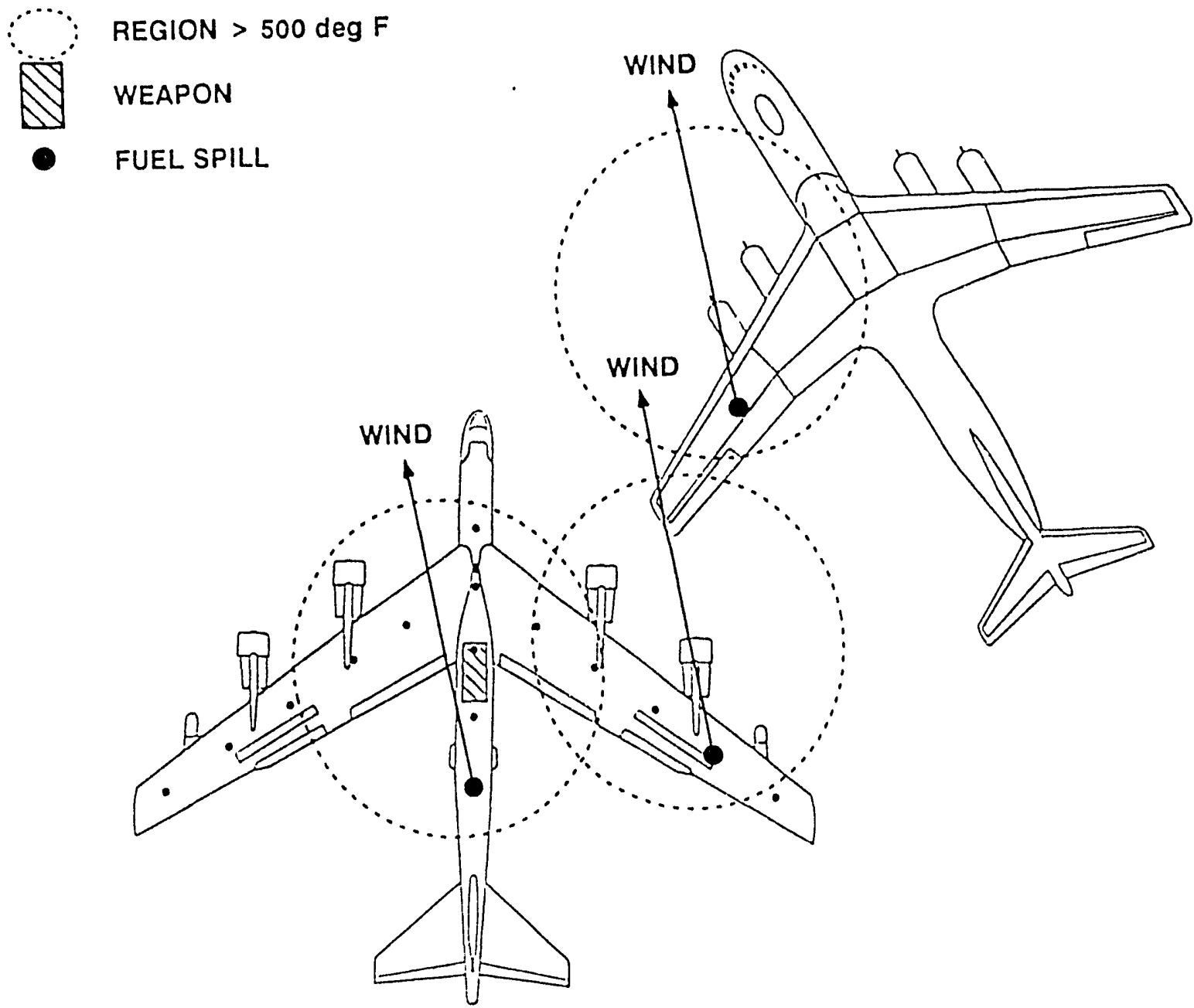

Figure 2.27 Example Weapon Exposure for Triple Concurrent Fuel Spill 
48 


\section{PREDICTED TEMPERATURE OF FLAME EXPOSURE}

\subsection{INTRODUCTION}

The purpose of Section 3 is (1), to present all results which indicate the probability of weapon exposure to given temperatures, (2), to reveal the manner and extent that the various controlling factors influence the results, and (3), to provide information that reveals the credibility, limitations and uncertainties of the results. Thus, Section 3 extends beyond a summary of results; a concise summary is given in Section 4 .

Regarding the organization of Section 3, the discussion starts with those cases which are most easily explained and proceeds to cases which tend to involve an increasing number of variables; the relative importance of a case, then, has no bearing on its position within the discussion. The discussion starts with the engine start accident, first for the B-52 and then for the B-1B. Considered next is the collision of an aircraft landing or taking off into an alert aircraft, and finally a discussion of uncertainty of results is given.

Results are primarily presented in terms of plots of the probability of exceeding a given exposure temperature vs temperature. Section 3 also contains a number of sketches, many of which serve to illustrate to scale the relative locations of the fuel spill points, weapon (cargo), and regions of the flame at or above a given temperature, that is, the flame "footprints". These sketches are often used as a convenient means of illustrating how the controlling factors affect the result. The flame footprints used in the sketches are "elliptical" approximations to the actual footprints, and while the approximation is close enough to correctly illustrate various points, it should be viewed as a convenient means of illustration only. Footprints are shown for various heights for the 0 to $10 \mathrm{mph}$ and the 10 to $20 \mathrm{mph}$ wind speed ranges, and the discussion will refer to 
these ranges as the low and mediurn speeds, respectively. Wind speed exceeding $20 \mathrm{rnph}$ (the high wind speed range) have little impact on overall results due to their statistical rarity, and few sketches with high wind speed footprints are included.

The term "baseline" conditions appears periodically in Section 3, and represents the set of conditions that is most common. For example, the baseline weapon heights are 7 and 10 feet for the B-52 and B-1B, respectively, since these heights are the approximate weapon heights under "normal" conditions or under conditions that would be most often encountered. However, while the majority of results presented is for the baseline conditions, results for other conditions are also given. The eifect of weapun height is examined, for example. Also, results are presented for the normal $\mathrm{cr}$ baseline parking orientations of the alert aircraft (as lirted for each airfield in Appendix B), but the effect of aircraft orientation on flame exposure is shown as well.

Many results not included in Section 3 are given in Appendix F. Details of the conditions, assumptions and procedures for the evaluation of probability vs temperature are given in Section 2. 


\subsection{ENGINE START ACCIDENT RESULTS: B-52}

An example rosult from an engine start accident is shown in Figure 3.1. The example is for Minot AF Base with the weapon (cargo) at the B-52 baseline height of 7 feet. Figure 3.1 gives the probability that the cargo exposure will exceed a given temperature. The two curves labeled ' 330 deg' and ' 240 deg' on Figure 3.1 are the individual results for the alert aircraft pointed in the 330 and 240 degree directions, as illustrated on Figure 3.2. The individual results are combined to produce a "weighted mean", where the mean is generated by statistically weighting the individual results in proportion to the number of aircraft pointed in each direction.

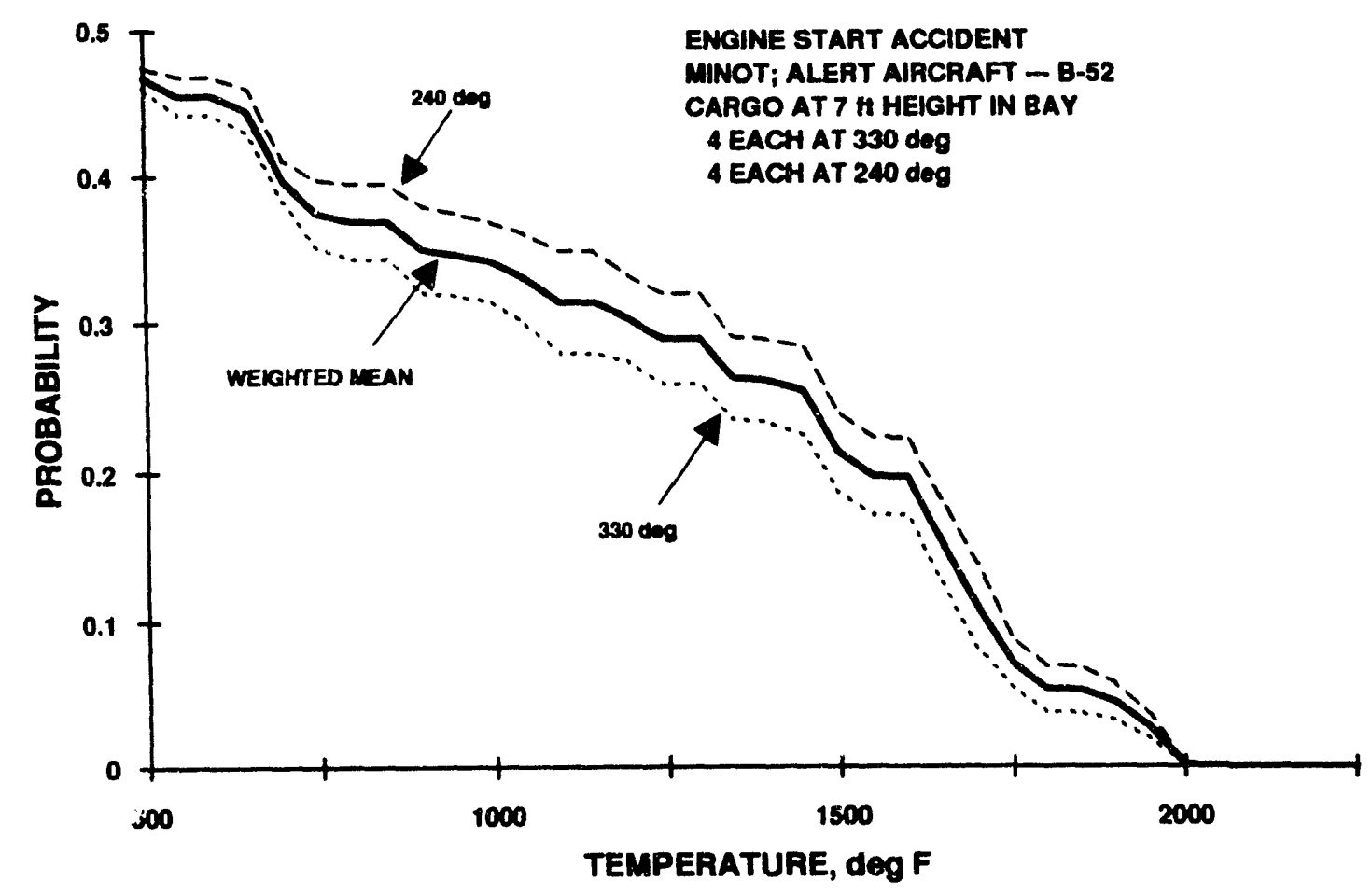

Figure 3.1 Results of Engine Start Accident at Minot AF Base

The general magnitude and character of the mean curve on Figure 3.1 is typical for B-52 engine start accidents at all bases, and various base to base comparisons are given later. The difference in magnitude of the two individual results for Minot AF Base is also reasonably typical. The largest overall difference between results for the two angles occurs for Eaker AF Base, and these results are given in Figure 3.3. The cause of the variation in magnitude for different aircraft orientations is related to wind characteristics, and the nature of this relationship is discussed in the next paragraphs. 
MINOT AF BASE, ND

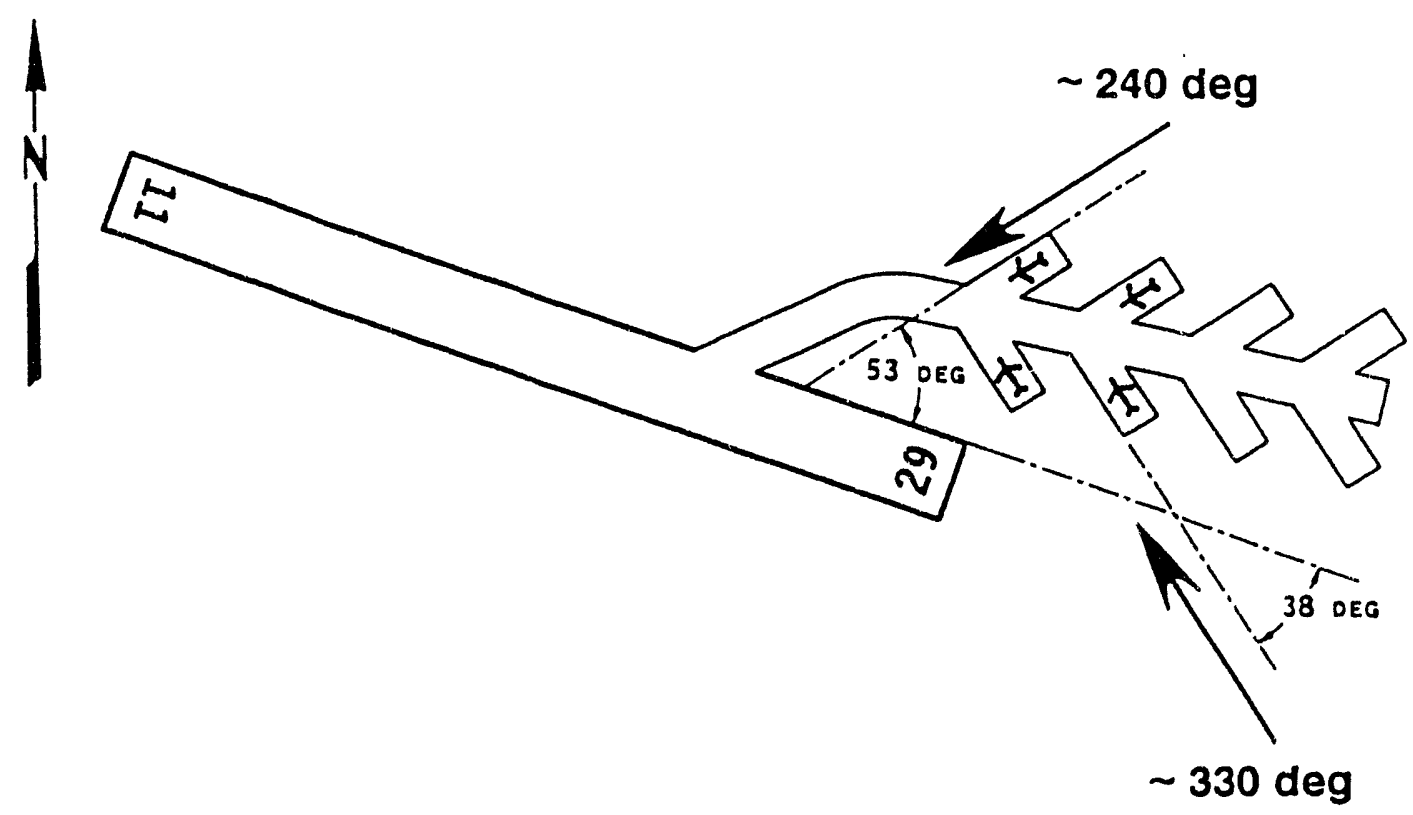

\section{Figure 3.2 Aircraft Orientation for Minot AF Base}

Regarding this relationship, consider first the wind direction probability curve for Eaker AF Base shown on Figure 3.4. From Figure 3.4, it can be seen that winds ranging from about 150 to 210 degrees from the north are quite predominant, with a predominance of lesser magnitude occurring over the comparatively small range between zero and 30 degrees. Consider next the sketch on Figure 3.5, which illustrates the locations of the cargo and the four fuel spill points and four associated flame footprints when the alert aircraft is pointed to zero degrees. On Figure 3.5a, the footprints are for a wind direction of 180 degrees, where 180 degrees is the median angle of the most predominant wind range.* It can be seen that the cargo is not exposed to flame for any of the four cases, nor clearly would there be exposure for wind angles throughout the predominant range around 180 degrees ( 150 to $210 \mathrm{deg}$ ). Figure $3.5 \mathrm{~b}$ shows the footprints for the wind direction of 15 degrees, the median for the other predominant range of zero to 30 degrees. For this wind direction, there is exposure for a single spill location, although there would be exposure from a second spill point near the 30 degree extremity of the predominant wind range. Thus, for the zero degree aircraft orientation, it appears that wind directions of highest probability tend to result in a 'small' likelihood of cargo exposure.

* The flame footprints on Figure 3.5 are representative of the mid-speed wind range. The point could have been equally well illustrated with low wind speed footprints. 


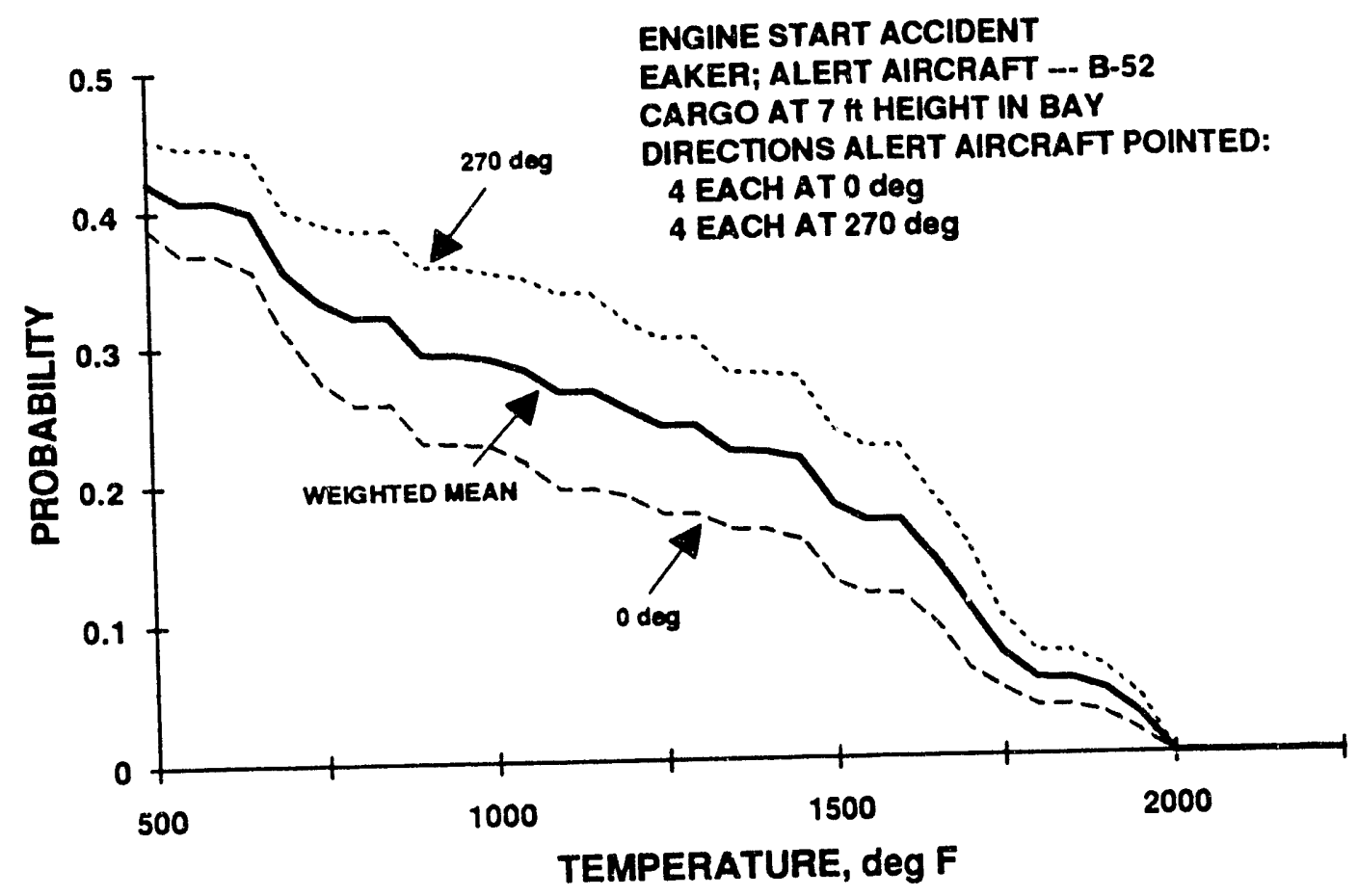

Figure 3.3 Results for Engine Start Accident at Eaker AF Base

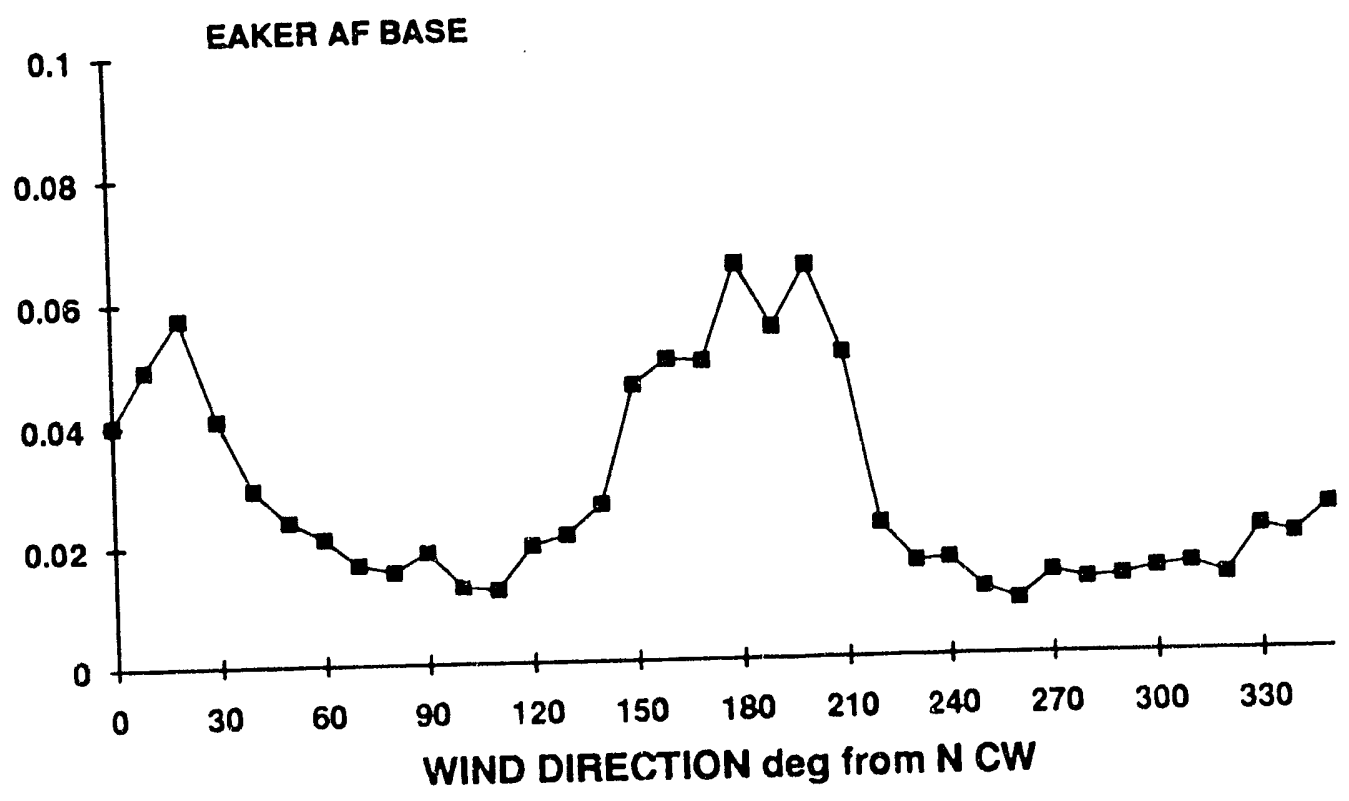

Figure 3.4 Frequency of Wind Direction for Eaker AF Base 


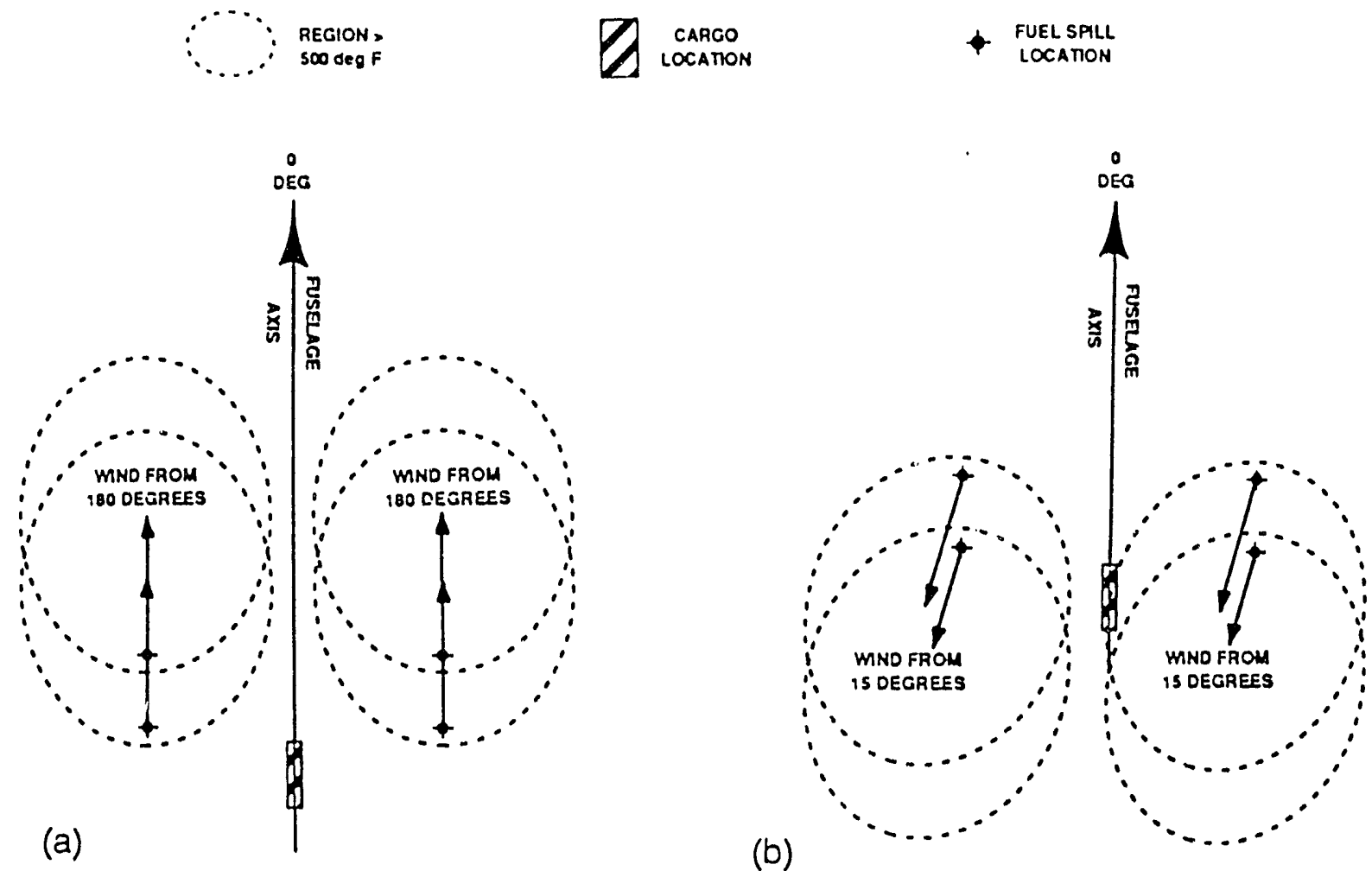

Figure 3.5 Flame Footprints for Predominant Winds at Eaker AF Base for Zero Degree Parking Angle

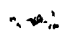
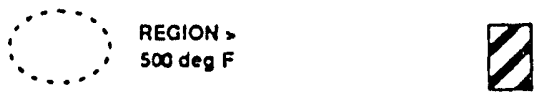

CARGO

LOCATION

FUEL SPILL
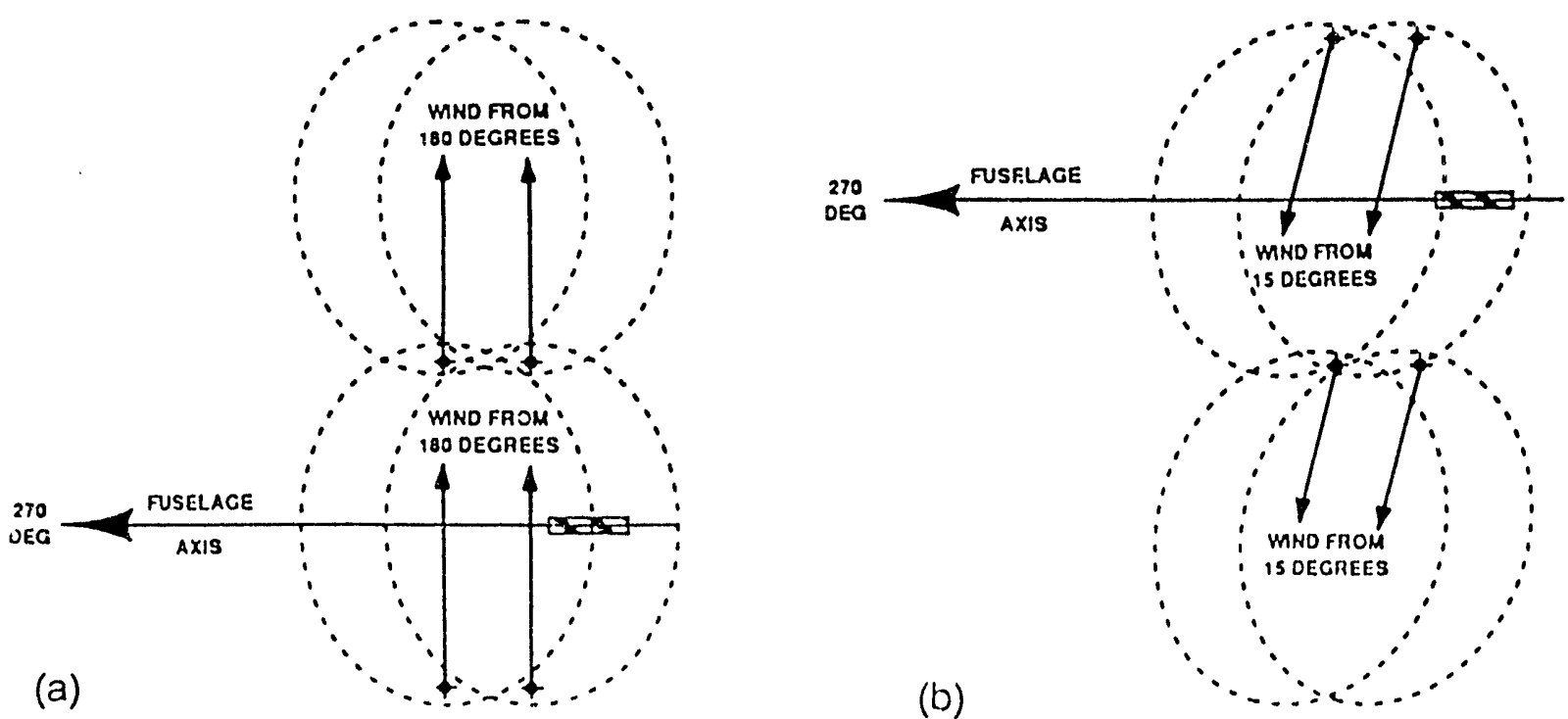

Figure 3.6 Flame Footprints for Predominant Winds at Eaker AF Base for 270 Degree Parking Angle 
Figure 3.6 shows the analogous arrangement for the aircraft pointed at 270 degrees. From Figure 3.6a it can be seen that there is cargo exposure for two spill locations for the wind direction 180 degrees; a more detailed examination shows that there is exposure for one spill location over the entire predominant wind range of 150 to $210 \mathrm{deg}$, and exposure over most of the range for a second spill location. Similarly, for the 15 degree wind direction on Figure $3.6 \mathrm{~b}$ there is exposure for one spill location, and further examination shows exposure throughout the wind range of 0 to 30 degrees for one spill location and throughout about one-half the range from a second location.

These examples have demonstrated the reason that there is a greater probability of flame exposure for the 270 degree parking angle than the zero degree parking angle. It also becomes evident that there will be a general dependence of probability on parking angle. This dependence has been examined, and Figure 3.7 contains plots of the probability that the exposure will exceed various temperatures vs aircraft angle for Eaker AF Base.

For Eaker AF Base, and for most bases, there are two parking angles that tend to maximize the exposure or severity of exposure, and these will occur when the aircraft fuselage axis is "nearly" perpendicular to a predominant wind direction; that is, these will occur when the cargo is downwind of one or more spill points for a predominant wind. Curves such as those on Figure 3.7 can then be expected to vary somewhat from base to base in a manner that corresponds to weather differences from base to base. An illustration of the base to base variation is given on Figure 3.8, which shows the dependence of the probability of exceeding $500^{\circ} \mathrm{F}$ (the probability of flame exposure) for four bases. It can be seen that the maximum probabilities and angle of maximum probabilities do vary with base. For Castle AF Base there is a single maximum, and the magnitude of the variation with angle is clearly more extreme than for other bases. The results for Castle were particularly included on Figure 3.8 because of the unique wind characteristics at this base. At most bases, there are two predominant wind direction ranges which tend to be separated by about 180 degrees (see Appendix B). The wind direction distribution at Castle AF Base, as shown in Figure 3.9, is unique in that it comprises a single predominant range, and it is unusually predominant. 


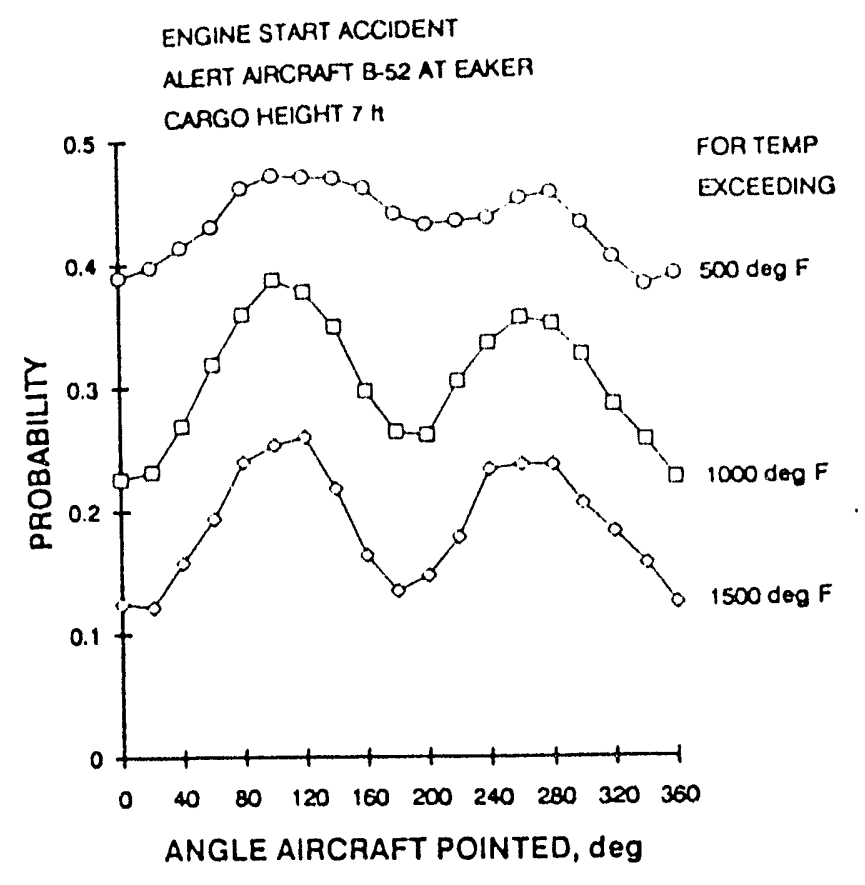

\section{Figure 3.7 Probability of Exceeding Various Temperatures vs Aircraft Orientation for Eaker AF Base}

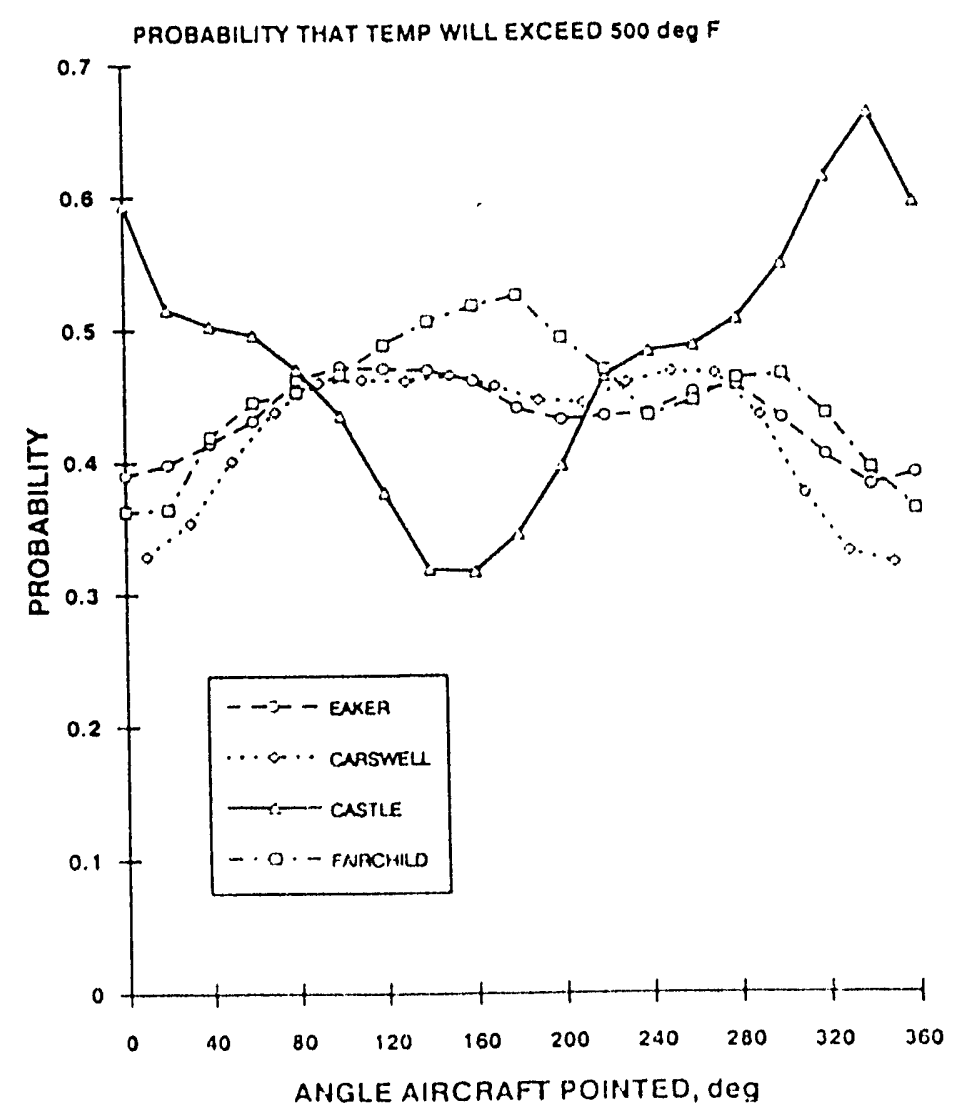

Figure 3.8 Probability of Exceeding $500^{\circ} \mathrm{F}$ vs Aircraft Orientation for various B-52 Bases 
The cause of the comparatively high probability for parking angles in the vicinity of 330 degrees for Castle AF Base is apparent from Figure 3.10a.* At the parking angle of 330 degrees, there is cargo exposure for four spill points when the wind is from the highly predominant direction of 330 degrees. It is also clear that there would be exposure from two spill points for wind directions throughout the predominant wind range of 320 to 350 degrees. The sketch on Figure $3.10 \mathrm{~b}$ also illustrates why the minimum probability for Castle AF Base on Figure 3.8 is for parking angles in the vicinity of 150 degrees. It can be seen from Figure $3.10 \mathrm{~b}$ that exposure would not occur for any spill point for wind directions throughout the predominant wind range 320 to 350 degrees.

Plots of the effect of aircraft pointing direction on the probability of exceeding temperature levels of 1000 and $1500^{\circ} \mathrm{F}$ are given on Figure 3.11. It is noted that the curves for Castle AF Base have two maximum and minimum values, whereas there was a single maximum and minimum for the corresponding curve for $500^{\circ} \mathrm{F}$ on Figure 3.8. Further, the minima on right side of Figure $3.11 \mathrm{a} \& \mathrm{~b}$ are at parking angles in the vicinity of 330 degrees, while there was a maximum at this parking angle on Figure 3.8. The sketch on 3.12 helps to illustrate how this minimum and maximum can occur at the same parking angle for different temperature levels. It can be seen on Figure 3.12 that the region above $1500^{\circ} \mathrm{F}$ is "too narrow" for cargo exposure at these angles, whereas there is exposure above $500^{\circ} \mathrm{F}$ for all four spill points. Further examination of various wind angles for the parking angle of 330 degrees has shown that exposure to temperatures above $1500^{\circ} \mathrm{F}$ occurs only at wind angles less than about 305 degrees (out of the predominant wind range) for the left-board spill points, and occurs only at wind angles larger than about 355 degrees (also out of the predominant wind range) for right-board spill points. In other words, there is a very small angular range within the predominant wind range at which there is exposure to temperatures in excess of $1500^{\circ} \mathrm{F}$ for a parking angle of 330 degrees. There is exposure over a greater fraction of the predominant wind range for temperature exposure exceeding $1000^{\circ} \mathrm{F}$.

Wind speeds at Castle AF Base are unusually small compared to other locations; the ratio of probabilities of speeds in the 0 to $10 \mathrm{mph}$ range to the 10 to $20 \mathrm{mph}$ range is 10 for Castle AF Base. Consequently, the ellipses used in Figure 3.10 are representative for low wind speed range. 


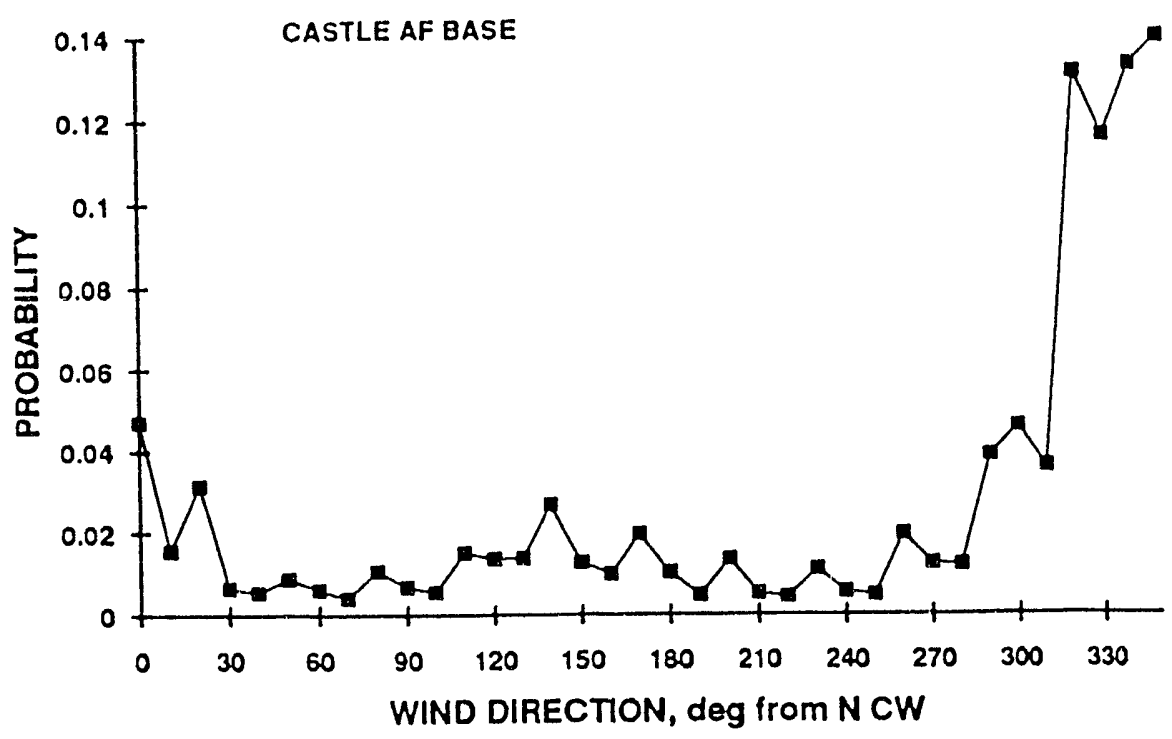

Figure 3.9 Wind Direction Distribution for Castle AF Base
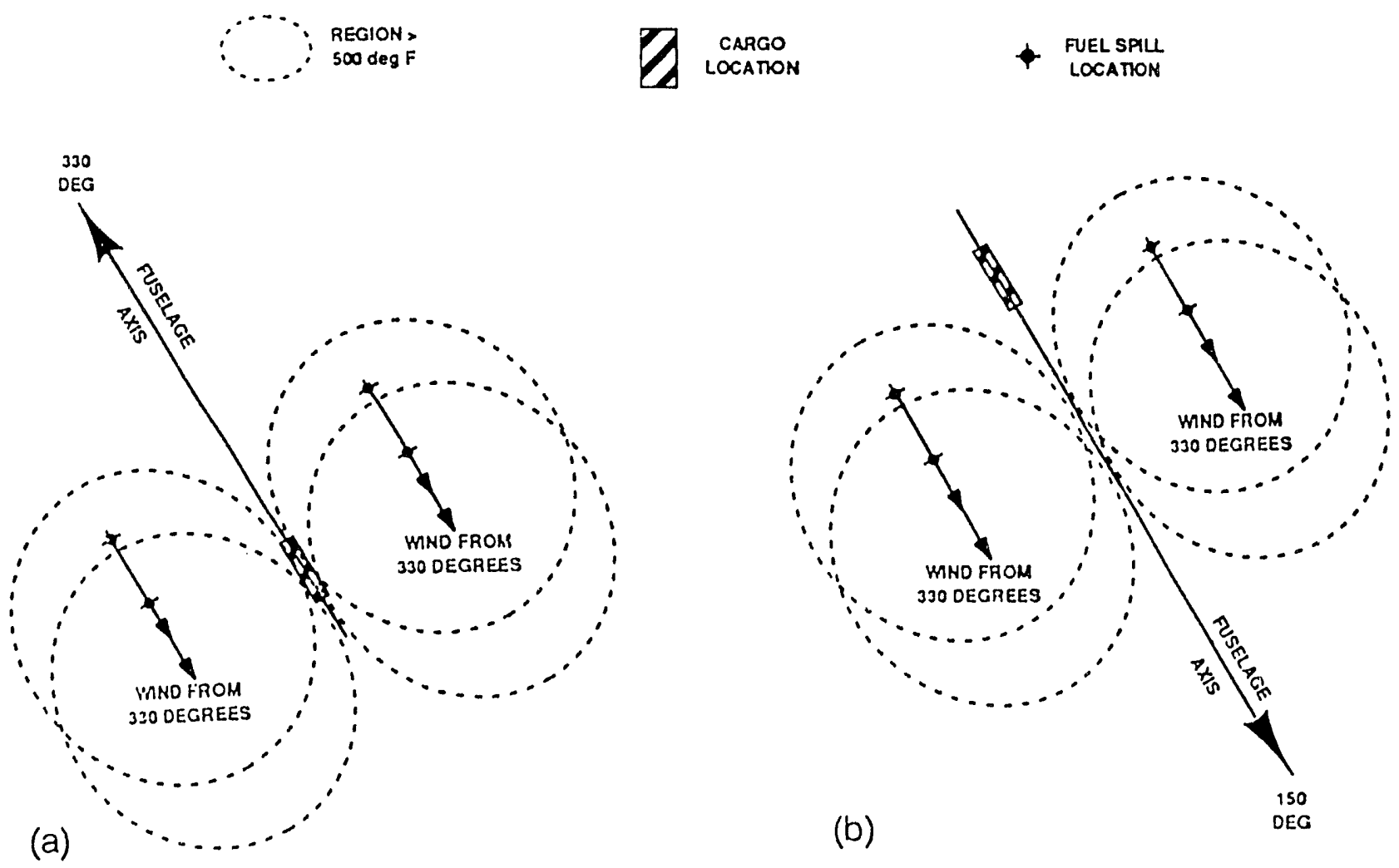

Figure 3.10 Flame Footprints for Predominant Winds at Castle AF Base 


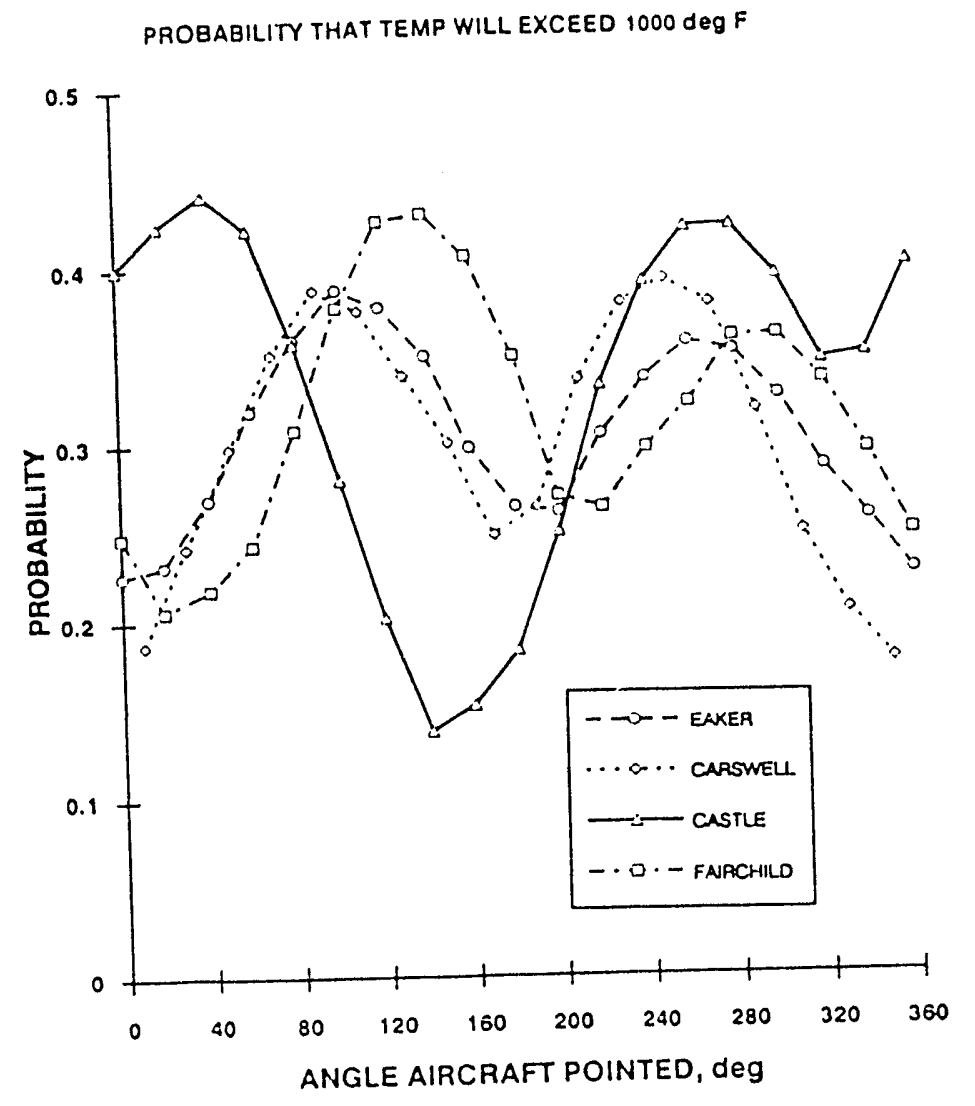

(a)

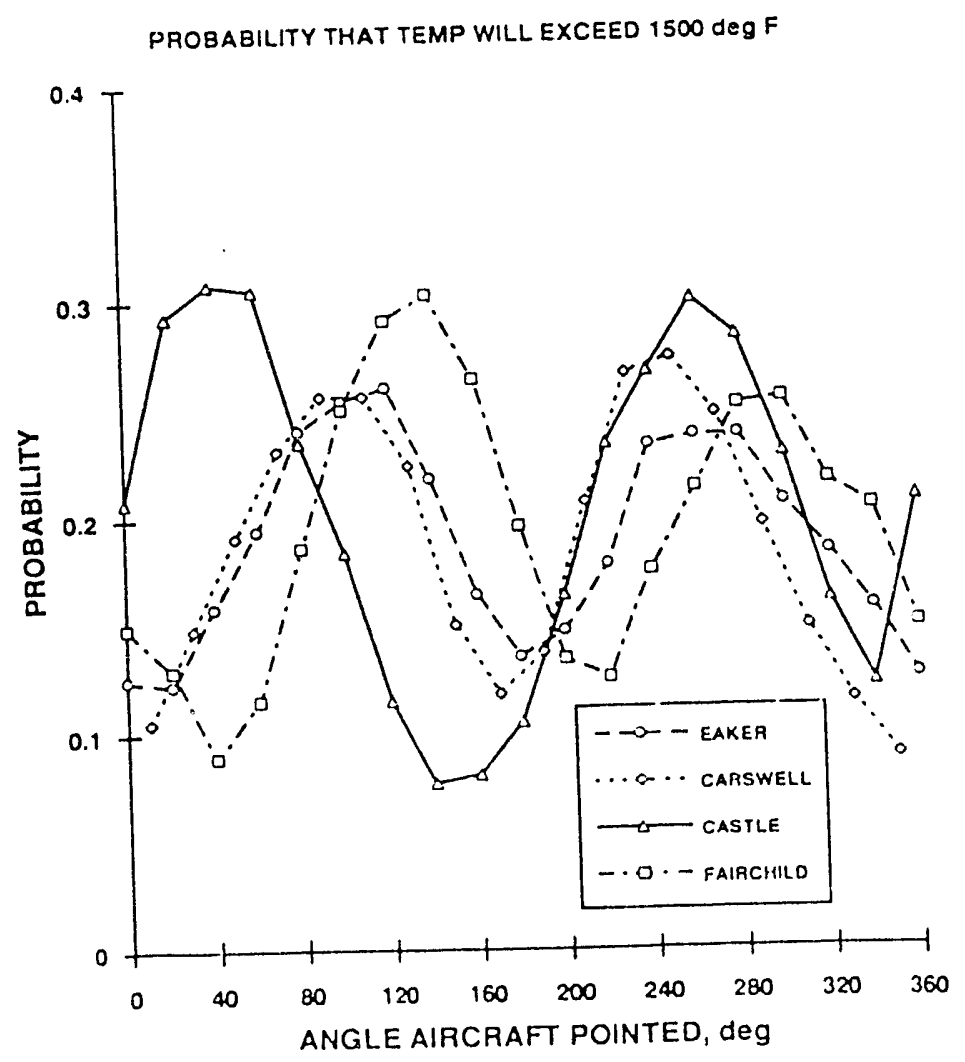

(b)

Figure 3.11 Probability of Exceeding 1000 and $1500^{\circ} \mathrm{F}$ vs Parking Angle for Various B-52 Bases 

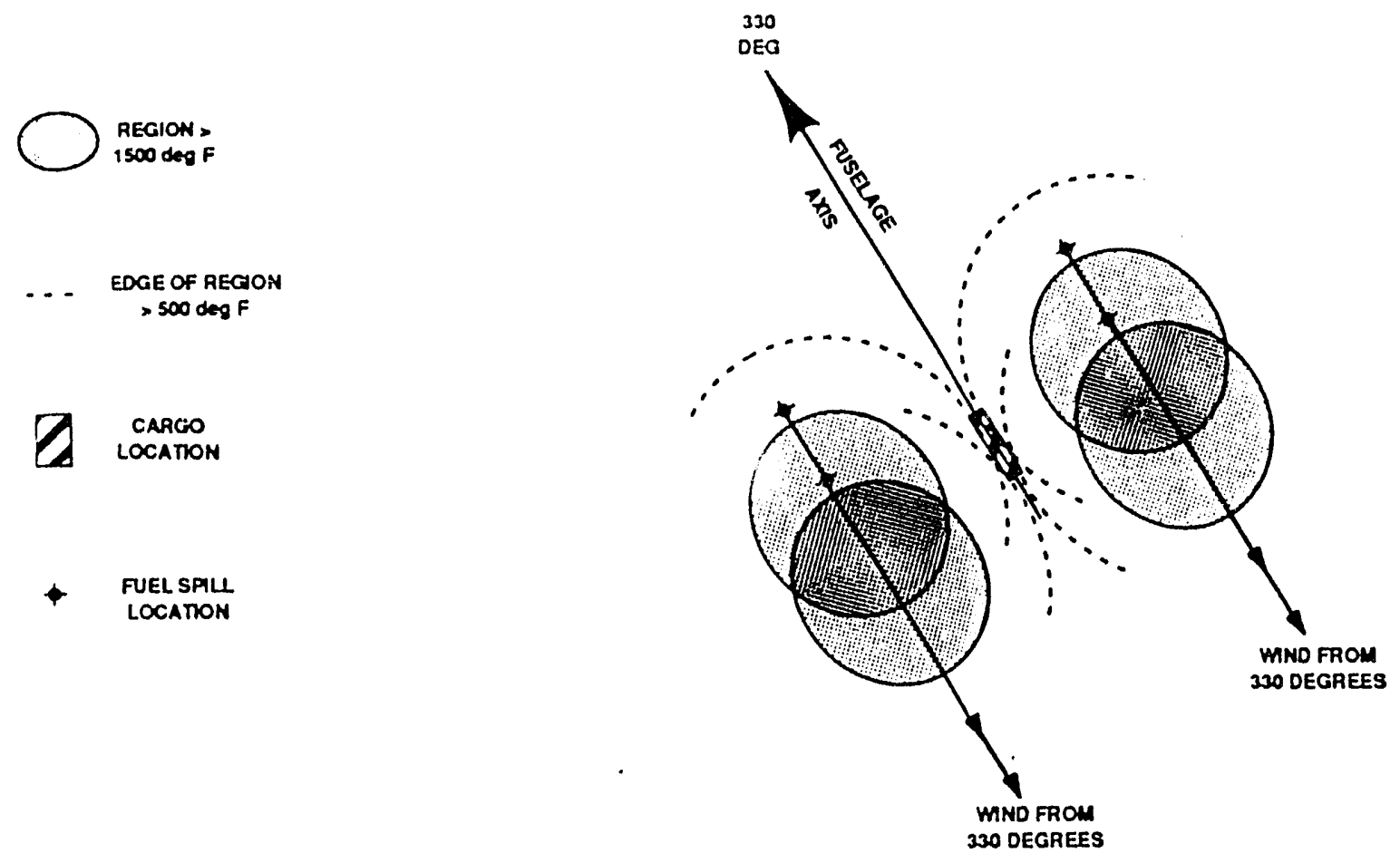

Figure 3.12 $1500^{\circ}$ F Footprints for Predominant Winds at Castle AF Base

The weighted mean probability vs temperature curve was introduced earlier in this section, where the mean represented the statistical weighting of the individual probabilities for each actual parking angle. Returning to these mean values, Figure 3.13 shows the probability of exceeding a given temperature for all eight B-52 bases considered. In view of the variation of parking angles and weather among bases, the range of probability results among bases appears remarkably small. 


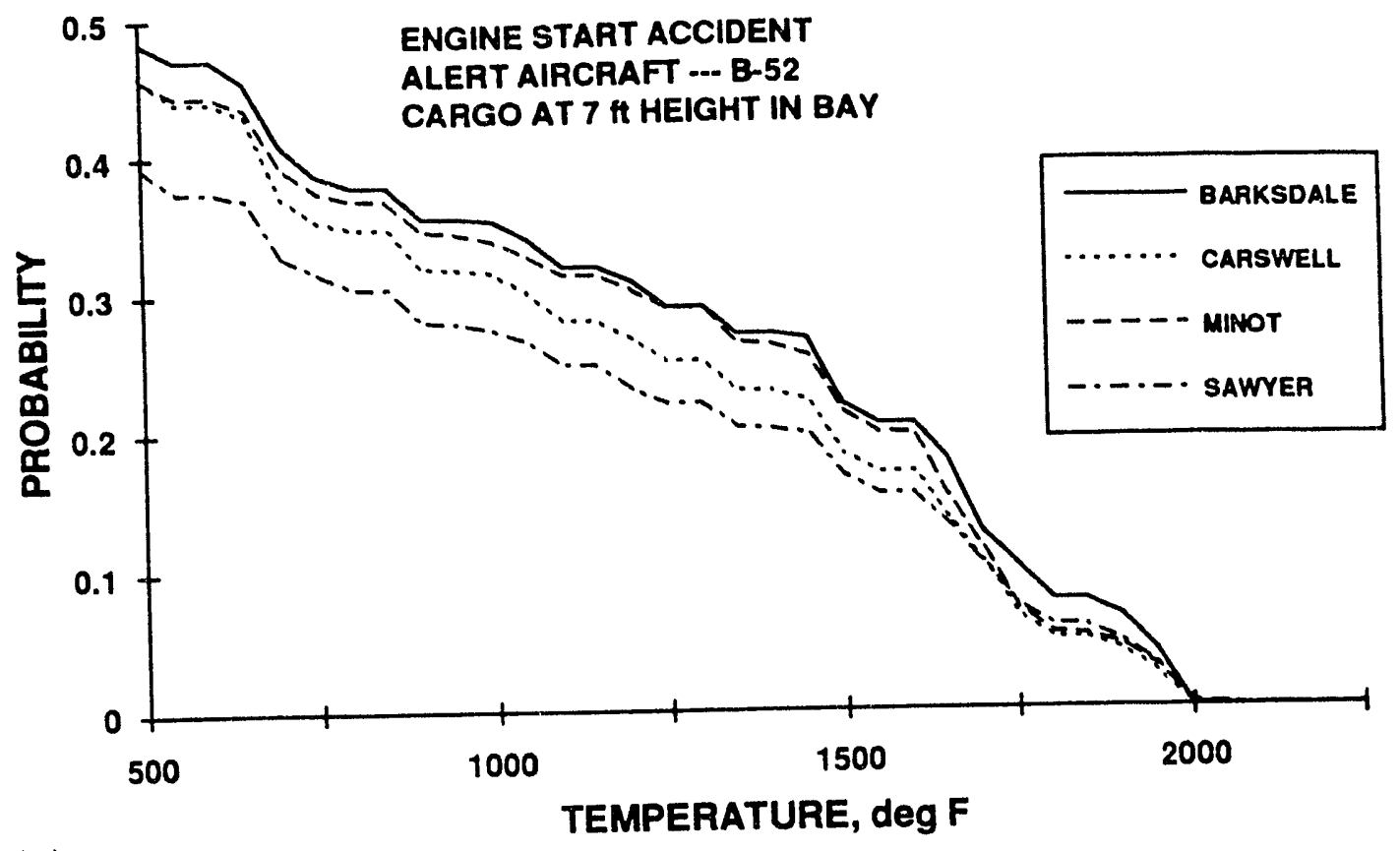

(a)

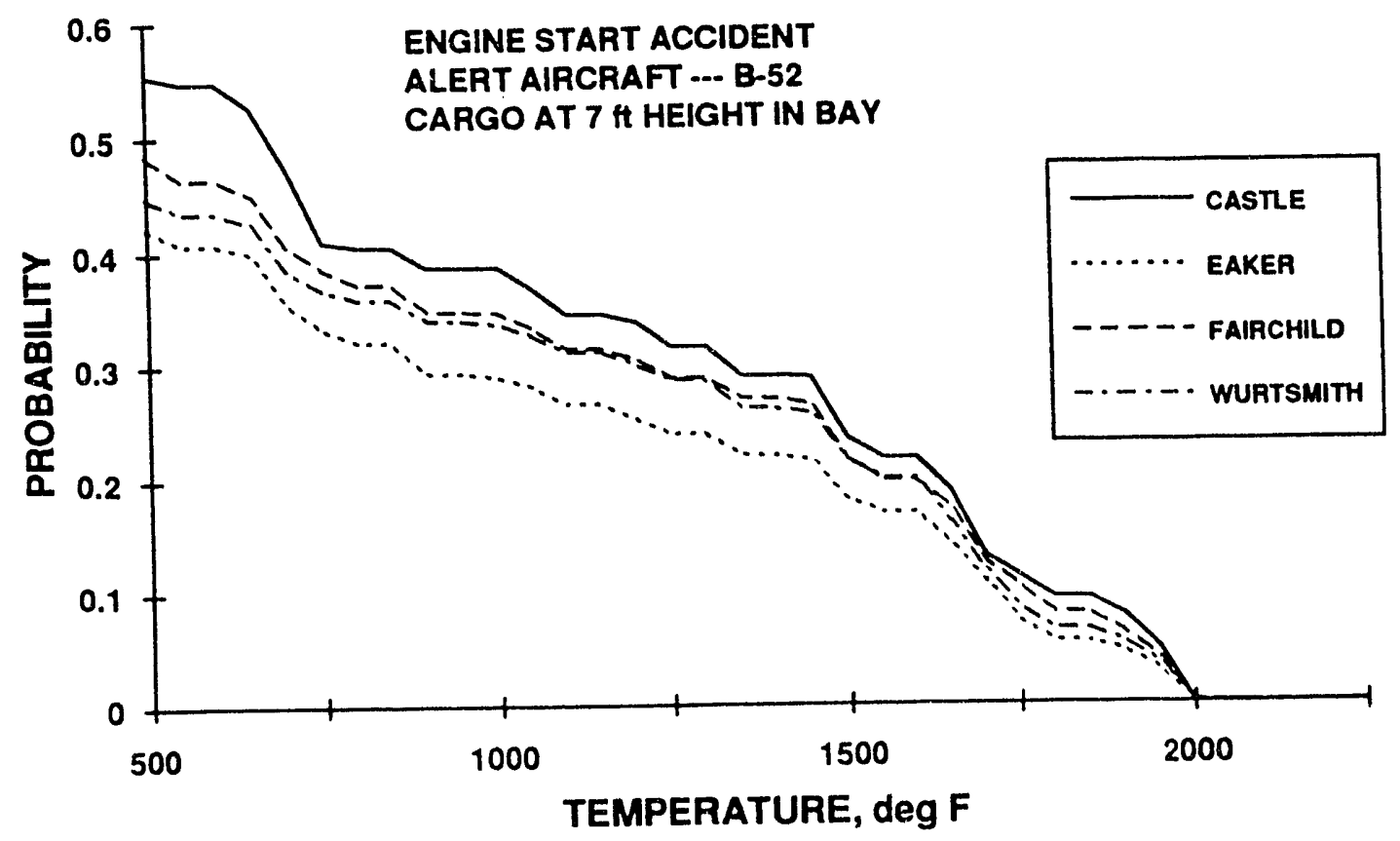

(b)

Figure 3.13 Base to Base Variation of Results for B-52 Engine Start Accidents 
Figure 3.14 shows the overall mean probability vs temperature averaged over the eight B- 52 bases. This mean was obtained by weighting in proportion to the number of aircraft parked at each direction at each base. Figure 3.14 also shows the lowest and highest mean probability obtained for any base, thus, the curves on Figure 3.14 represent for the normal or baseline parking angles the mean probability over all B-52 bases and the range in probability for all B-52 bases.

It is noted that probability values above $2000^{\circ} \mathrm{F}$ on Figure 3.13 and 3.14 are consistently negligible, and the reason for the absence of significant values above $2000^{\circ} \mathrm{F}$ deserves some discussion. Regarding the reasons, first the temperature does not reach $2000^{\circ} \mathrm{F}$ at the 7 foot height for the mid- and high-wind speeds for the low spill rates used in the engine start accidents. Second, while there is a significant region above $2000^{\circ} \mathrm{F}$ for the low wind speed, the cargo is too distant from the forward spill points to intersect the $2000^{\circ} \mathrm{F}$ region, and the downwind edge of the $2000^{\circ} \mathrm{F}$ region 'barely intersects a corner' of the cargo for the rear spill points. Thus, in the calculation, values at or greater than $2000^{\circ} \mathrm{F}$ are rarely encountered. However, it would be misleading here to imply

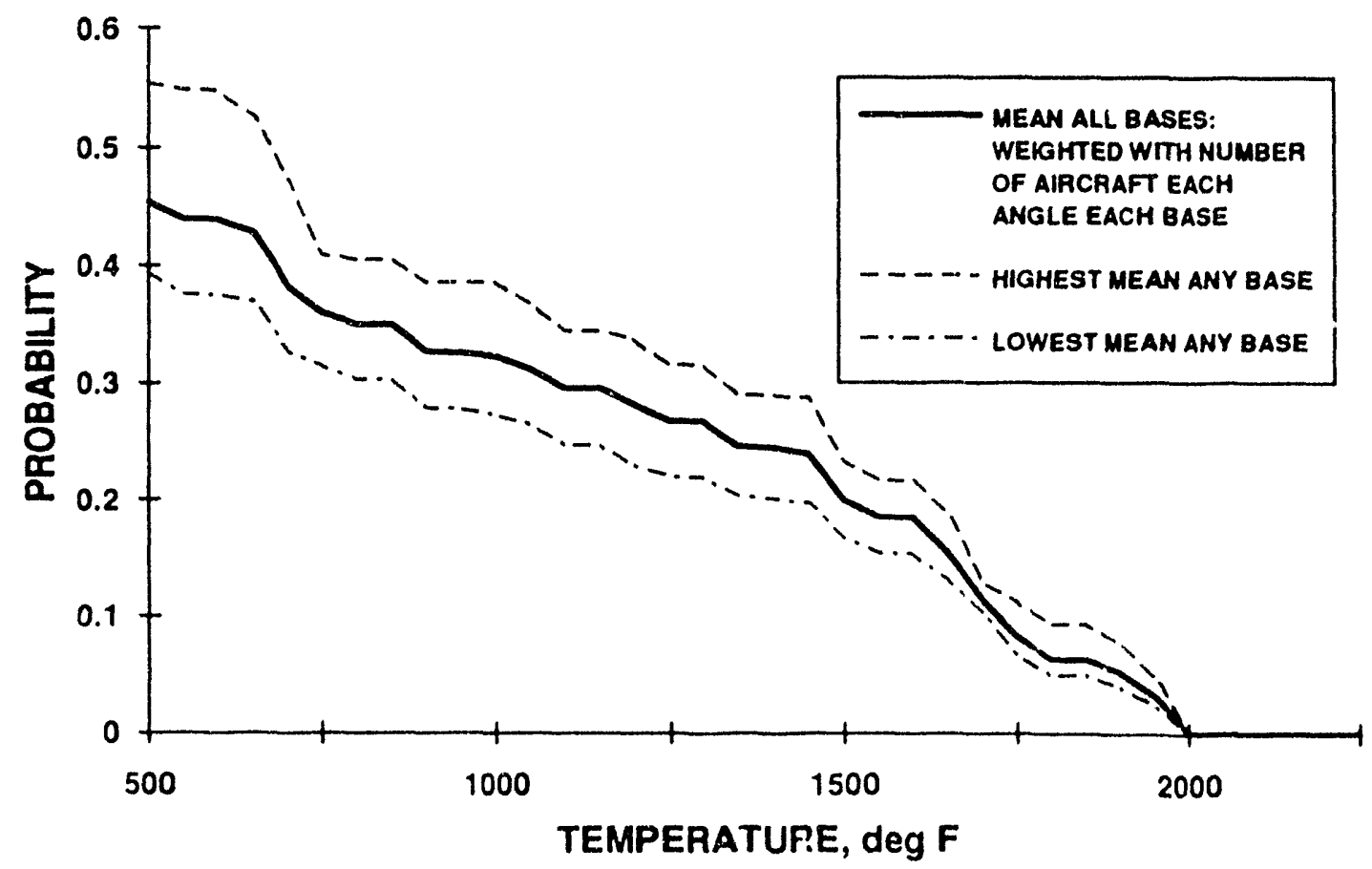

Figure 3.14 Mean Results Over Eight B-52 Bases for Engine Start Accidents 
that probabilities of some significance for temperatures at or above $2000^{\circ} \mathrm{F}$ cannot occur. The region above $2000^{\circ} \mathrm{F}$ just misses the cargo under some conditions, and in view of the uncertainty of location and size of a given temperature region (see Appendix A) and the location of the spill points, credible probabilities may exist for temperatures at or above $2000^{\circ} \mathrm{F}$. In addition, cargo location is often in the forward section of the bay and at one or the other sides of the bay, whereas for the chosen "baseline" location, the cargo center is located midway in the bay along the fuselage axis. In order to illustrate the magnitude of the effect of these distance variations, calculations were conducted for (1), all spill points located 2 feet closer to the fuselage, and (2), cargo located in the forward part of the bay (7 feet forward of the "baseline" location). The results of these calculations can be compared to the "baseline" results for Fairchild AF Base on Figure 3.15.

It can be seen that the spill point adjustment resulted in a significant probability at $2000^{\circ} \mathrm{F}$ (6.4 percent), although the probability remained negligible at $2050^{\circ} \mathrm{F}$. For the cargo adjustment, the probability remained negligible even at $2000^{\circ} \mathrm{F}$. For both adjustments, the probability level between 500 and $2000^{\circ} \mathrm{F}$ has been elevated.

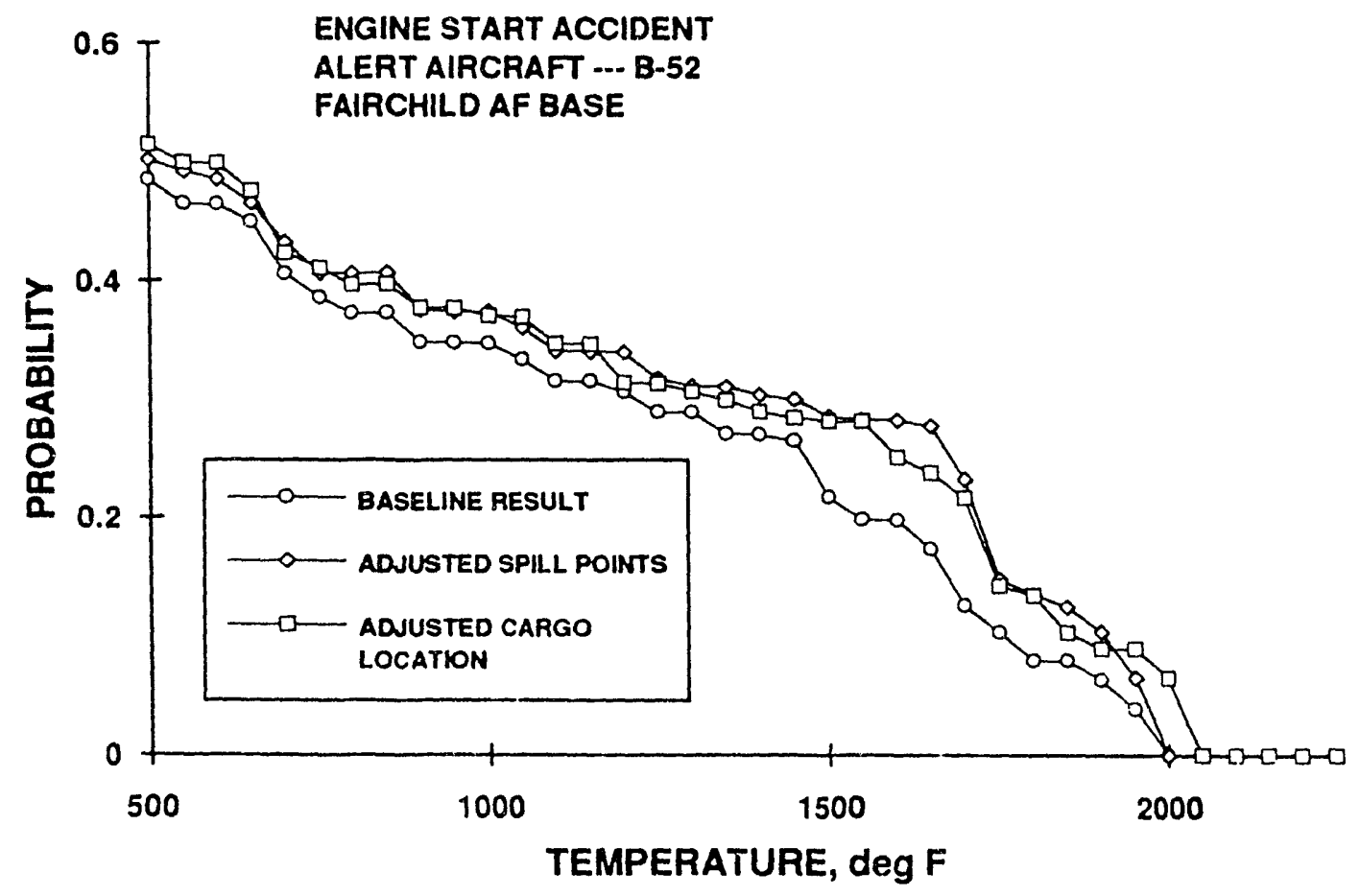

Figure 3.15 Effect of Adjusted Spill/Cargo Location 
The results thus far presented have been limited to the baseline cargo height for the B-52 of 7 feet. Other cargo locations can occur due, for example, to flame induced failure of the cargo mounting structure, landing gear failure, etc. Probability vs temperature plots for the heights of $1,4,7$ and 10 feet are given on Figure 3.16 for various bases. There are a number of general trends which the pir on Figure 3.16 serve to illustrate. First of all, the probability of exceeding $500^{\circ} \mathrm{F}$ tends to increase with decreasing height. This particular trend is illustrated on Figure 3.17 for several bases. The reason for this trend is to a large extent related to the fact that the upwind edge of the footprint is increasingly upwind with decreasing height. The effect of this on exposure is illustrated in Figure 3.18. Figure 3.18a and $3.18 \mathrm{~b}$ each show two footprints for the medium wind speed due to spills from the left rear spill point, and the heights for Figure $3.18 \mathrm{a}$ and $3.18 \mathrm{~b}$ are for 7 and 1 foot, respectively. The two footprints in each case are for wind directions such that the footprint edge just intersects the edge of the cargo. Thus, cargo exposure will occur for any wind direction within the angular range labeled " $\alpha$ ". " can be seen that the range $\alpha$ is substantially larger for the 1 foot than for the 7 toot height; this proves to be a trend with height, and the trend also applies to other wind speeds. 


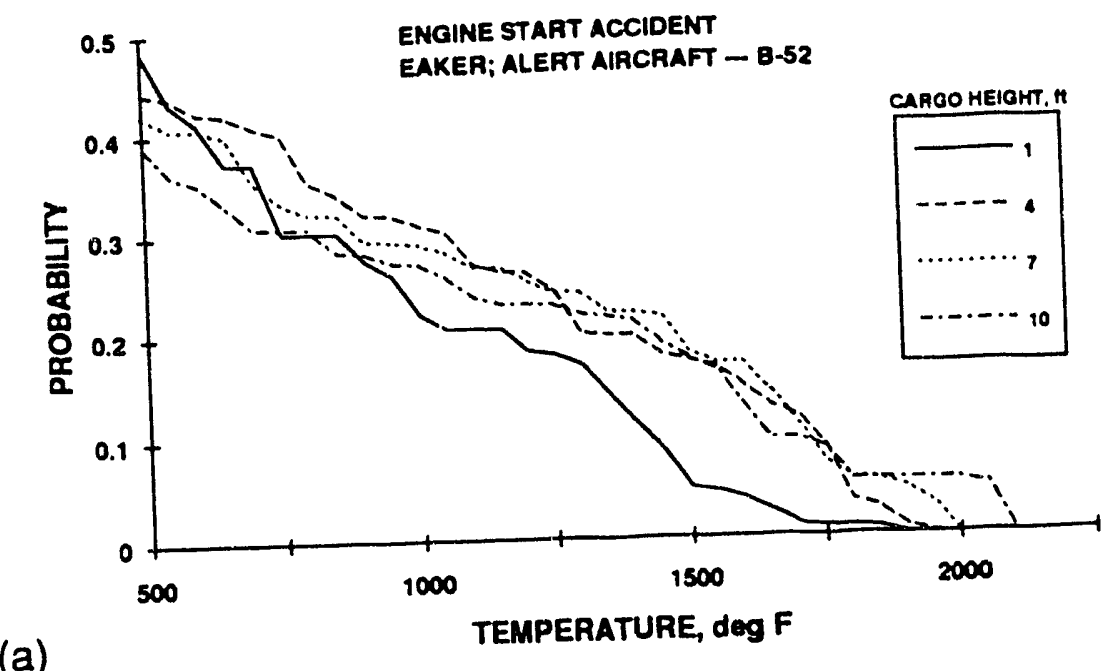

(a)

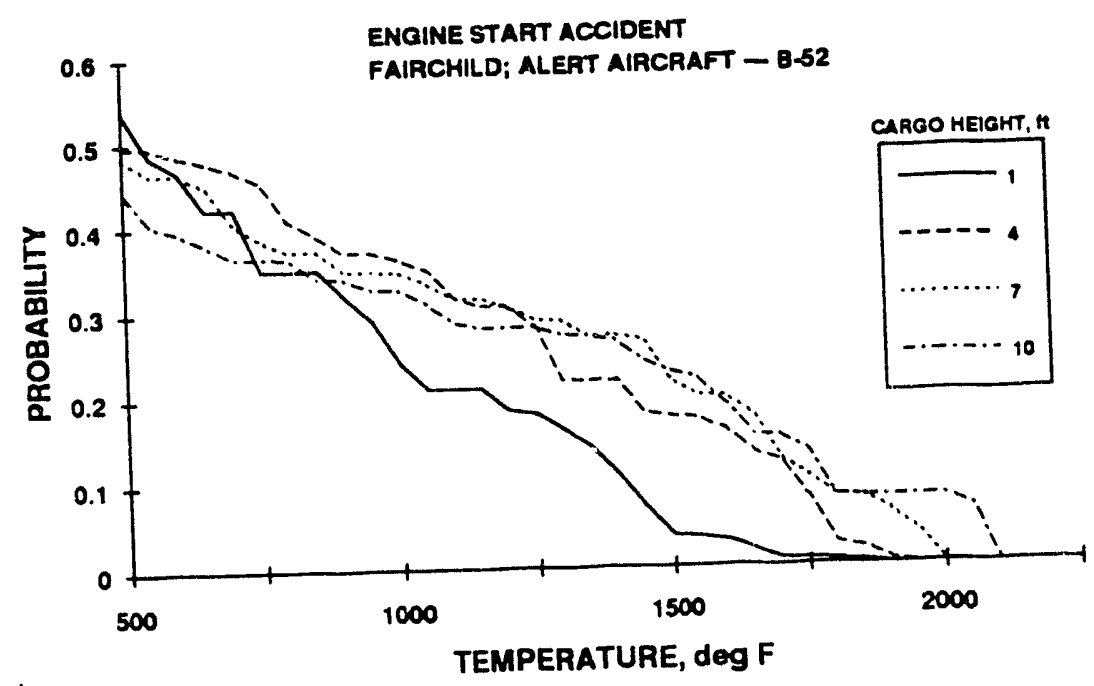

(b)

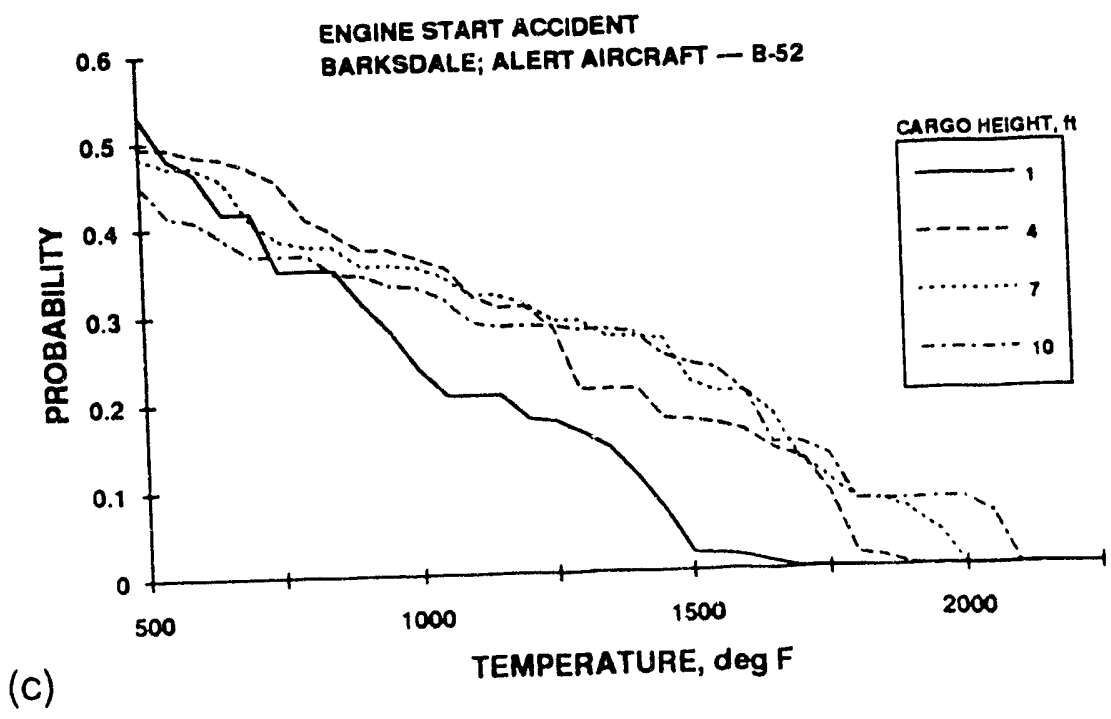

Figure 3.16 Engine Start Results for Various Cargo Heights 


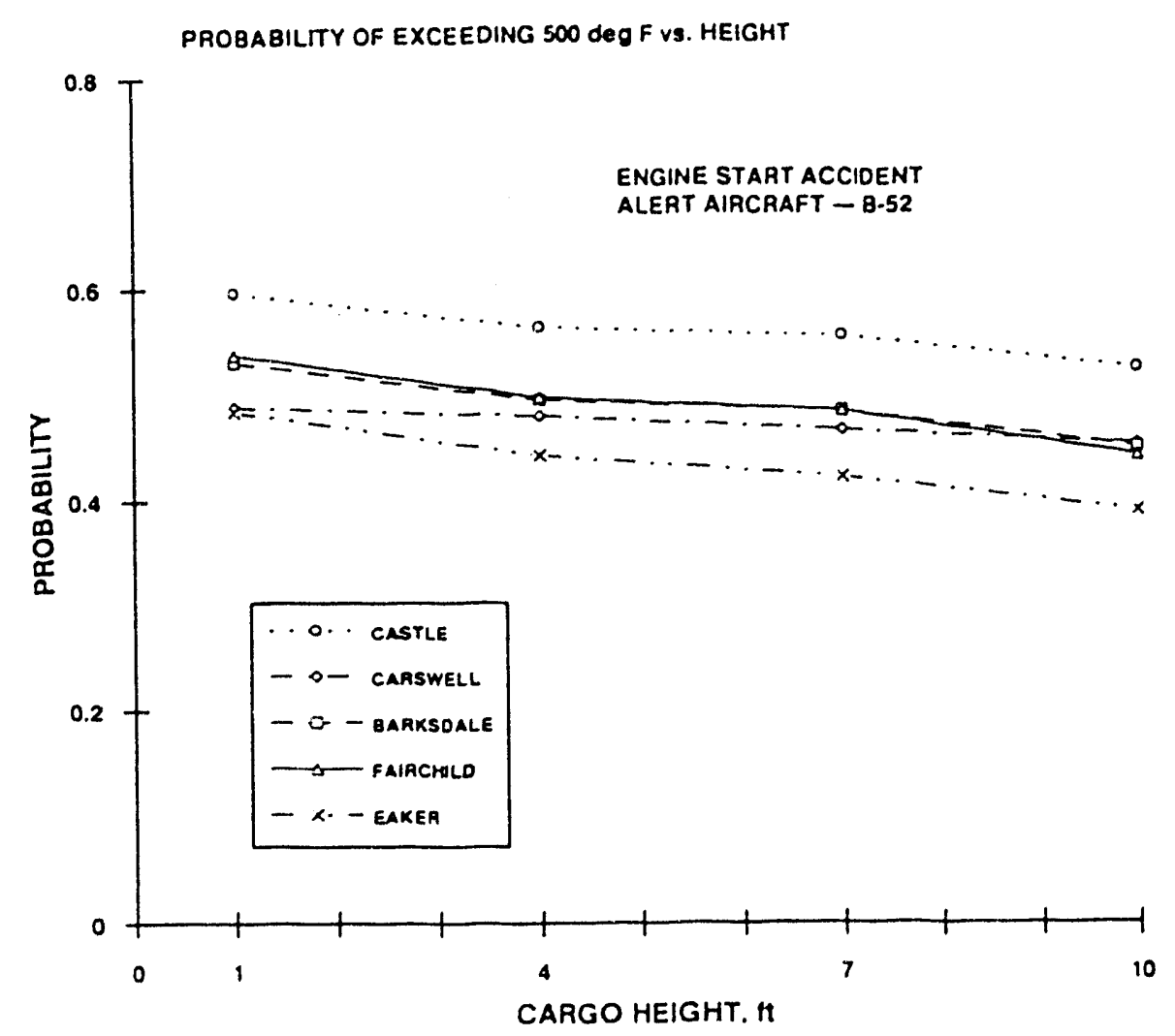

Figure 3.17 Probability of Exceeding $500^{\circ} \mathrm{F}$ for Various Heights at Various B-52 Bases; Engine Start Accident
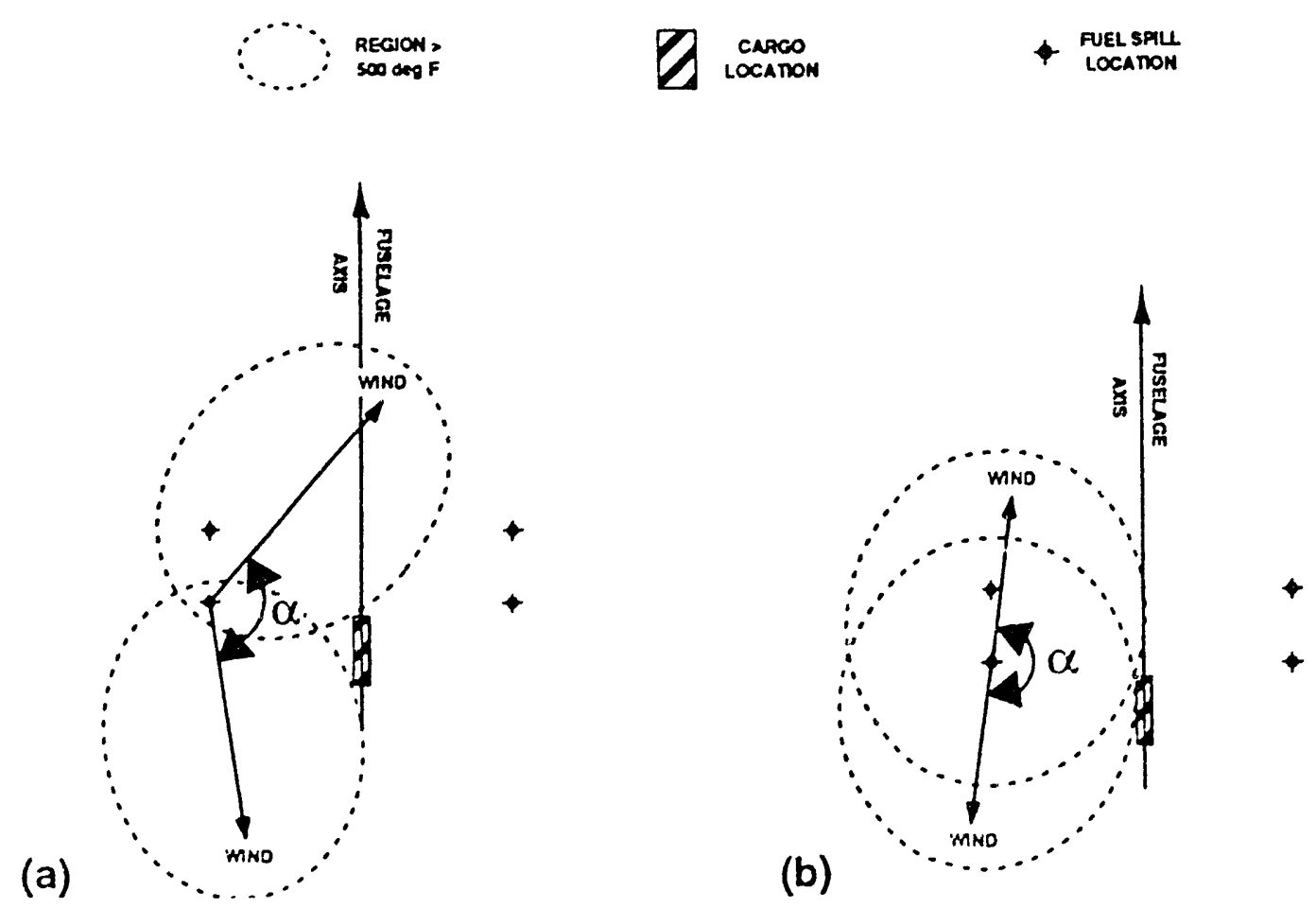

Figure 3.18 Wind Direction Range Resulting in Cargo Exposure for Two Heights 
Consider next on Figure 3.16 the probabilities at temperatures above $500^{\circ} \mathrm{F}$. It can be seen that the probability for the 1 foot height becomes and remains less than the probability for other heights for temperatures greater than about 700 to $900^{\circ} \mathrm{F}$. The reason for this reversal is illustrated on Figure 3.19. For the cargo located downwind of the left forward spill point, Figure 3.19a and 3.19b show shows the approximate location for the medium wind speed of the region above $1500^{\circ} \mathrm{F}$ for the 7 and 1 foot heights, respectively. For the 1 foot height, it can be seen that the region above $1500^{\circ} \mathrm{F}$ does not extend far enough downwind to intersect the cargo, whereas there is significant exposure above $1500^{\circ} \mathrm{F}$ at the 7 foot height. Further examination shows that the result is the same for the low wind speed for either the forward or rear spill point, although there is some exposure above $1500^{\circ} \mathrm{F}$ for the medium wind speed for the rear spill point.
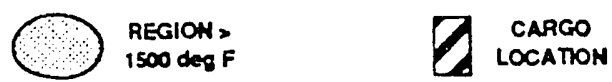

+ FUEL SPIL

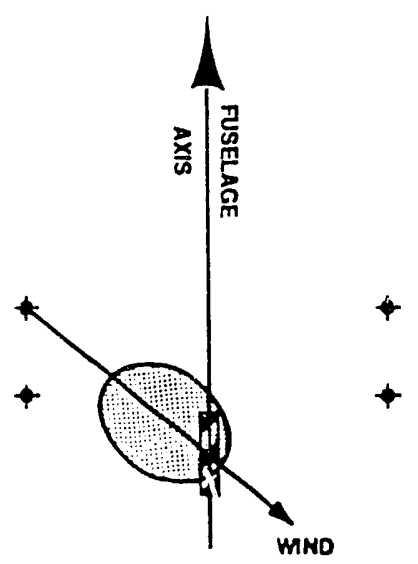

(a) 7 Foot Height

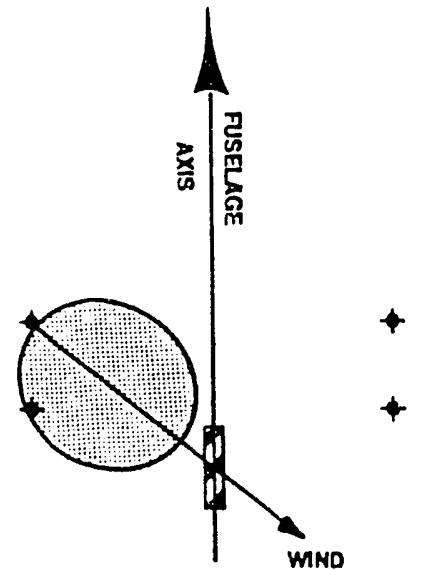

(b) 1 Foot Height

Figure $3.191500^{\circ} \mathrm{F}$ Footprints at 7 and 1 Foot Heights

Consider next on Figure 3.16 the temperature region at or above $2000^{\circ} \mathrm{F}$. It can be seen that a significant probability is reached at the 10 foot height for temperatures above $2000^{\circ} \mathrm{F}$, whereas for lower heights the probability is negligible at or above $2000^{\circ} \mathrm{F}$. Temperature regions above $2000^{\circ} \mathrm{F}$ occur for the low wind speed (and only for the low wind speed) at heights of 4, 7 and 10 feet. For the 4 foot height, the region does not reach far enough downwind to intersect the cargo for any of the spill locations, and for 7 foot the region "barely intersects a corner of the cargo" for the two rear (nearest) spill points. For the 10 foot height, the region extends well into the cargo for the rear spill points. This exposure at the 10 foot height results in a negligible probability at $2100^{\circ} \mathrm{F}$, but the probabilities at $2050^{\circ} \mathrm{F}$ are significant (the mean probability at $2050^{\circ} \mathrm{F}$ averaged over 5 bases, for example, is .064). 


\subsection{ENGINE START ACCIDENT RESULTS: B-1B}

The presentation in subsection 3.3 for the B-1B is similar in organization to that in subsection 3.2 for the B-52, with certain differences noted below. A primary difference for the B-1B is that weapons can be contained in either the middle bay or the aft bay, or both.* A weapon in the middle bay only or the aft bay only simply adds an additional variable to consider, but weapons concurrently in both bays complicates the analysis somewhat in that it becomes appropriate to evaluate the probability that the exposure to one or the other weapon exceeds a given temperature. The case of a weapon only at 10 foot height in the middle bay is treated as the baseline case, but the presentation of results is extensive for the cases of aft bay only and both bays.

Example results for weapons in the middle bay are given in Figure 3.20, which shows (as in the previous subsection) the probability for McConnell AF Base and Ellsworth AF Base vs temperature for each of the two parking angles and the weighted mean probability. The general character of these results is similar to those given for the B-52, and those differences that do exist are periodically noted below.

The largest overall difference in probability at the two parking angles is for McConnell AF Base, as shown on Figure 3.20. An example of the dependence of probability on parking angle is given for Ellsworth AF Base on Figure 3.21. It can be seen that the dependence on parking angle is similar to that shown above for the B-52.

* It has been indicated that weapons are rarely, if ever,
and no results pertaining to the forward bay have been included. 


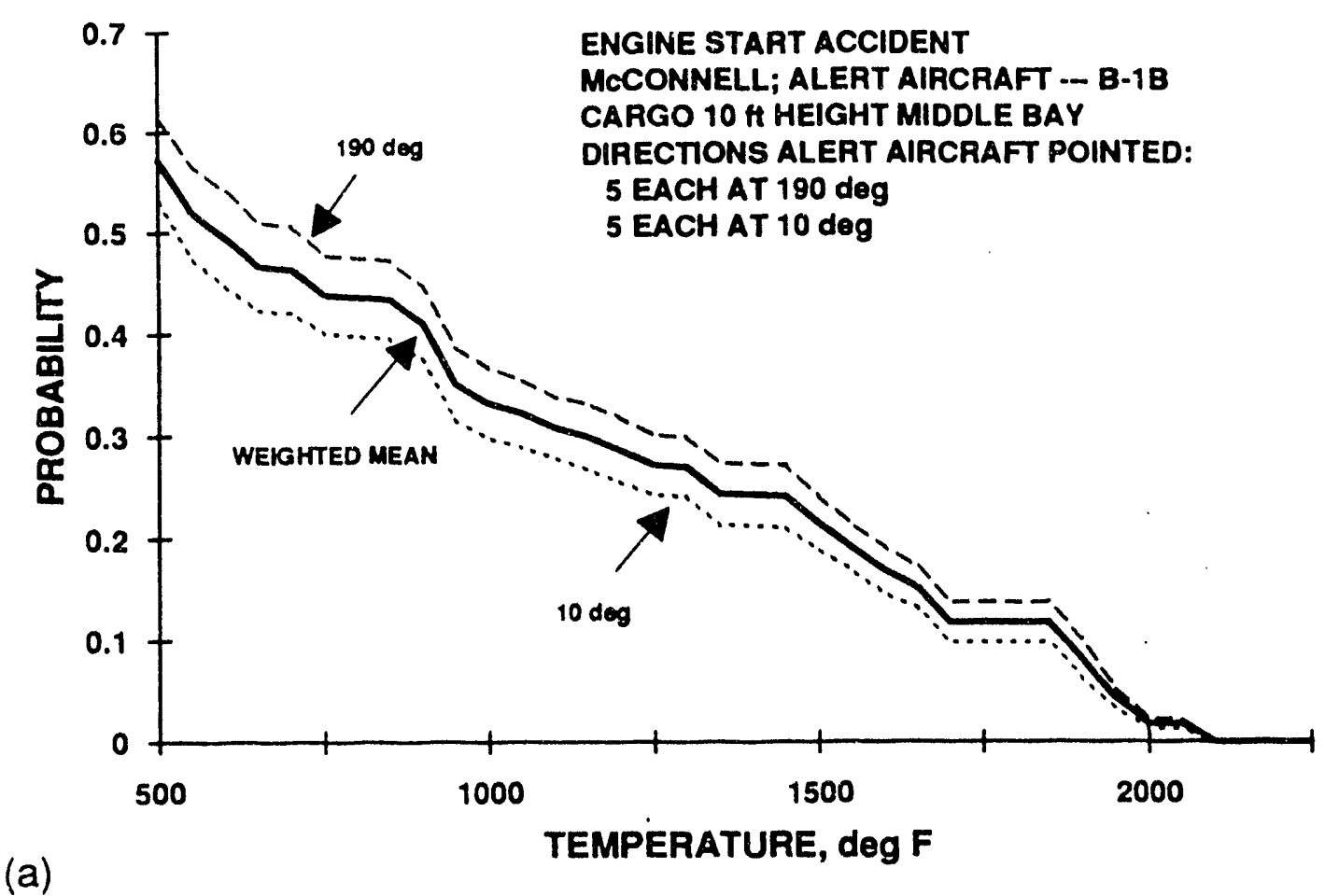

(a)

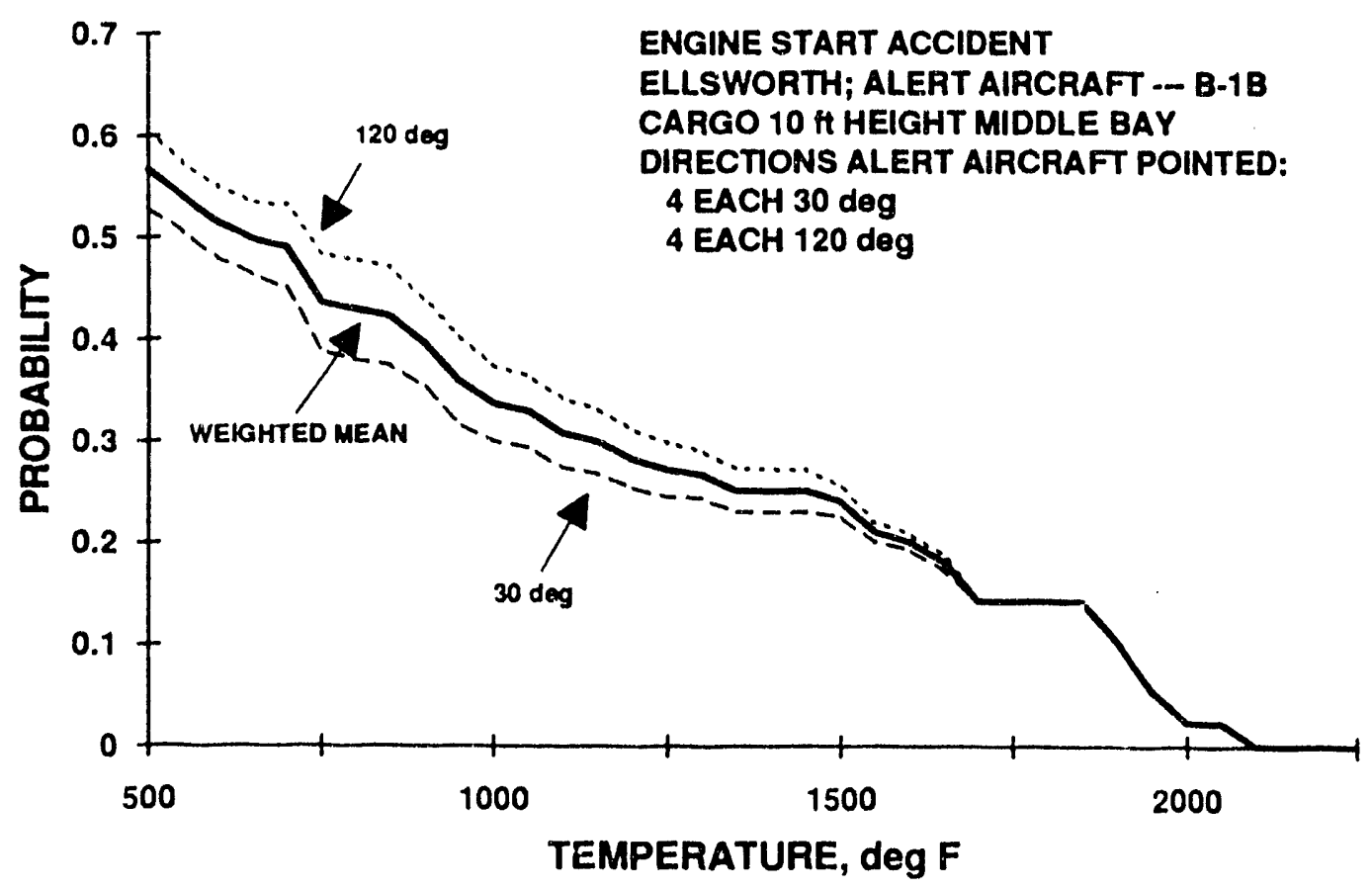

(b)

Figure 3.20 Example Results for B-1B Engine Start Accident for Cargo in Middle Bay 


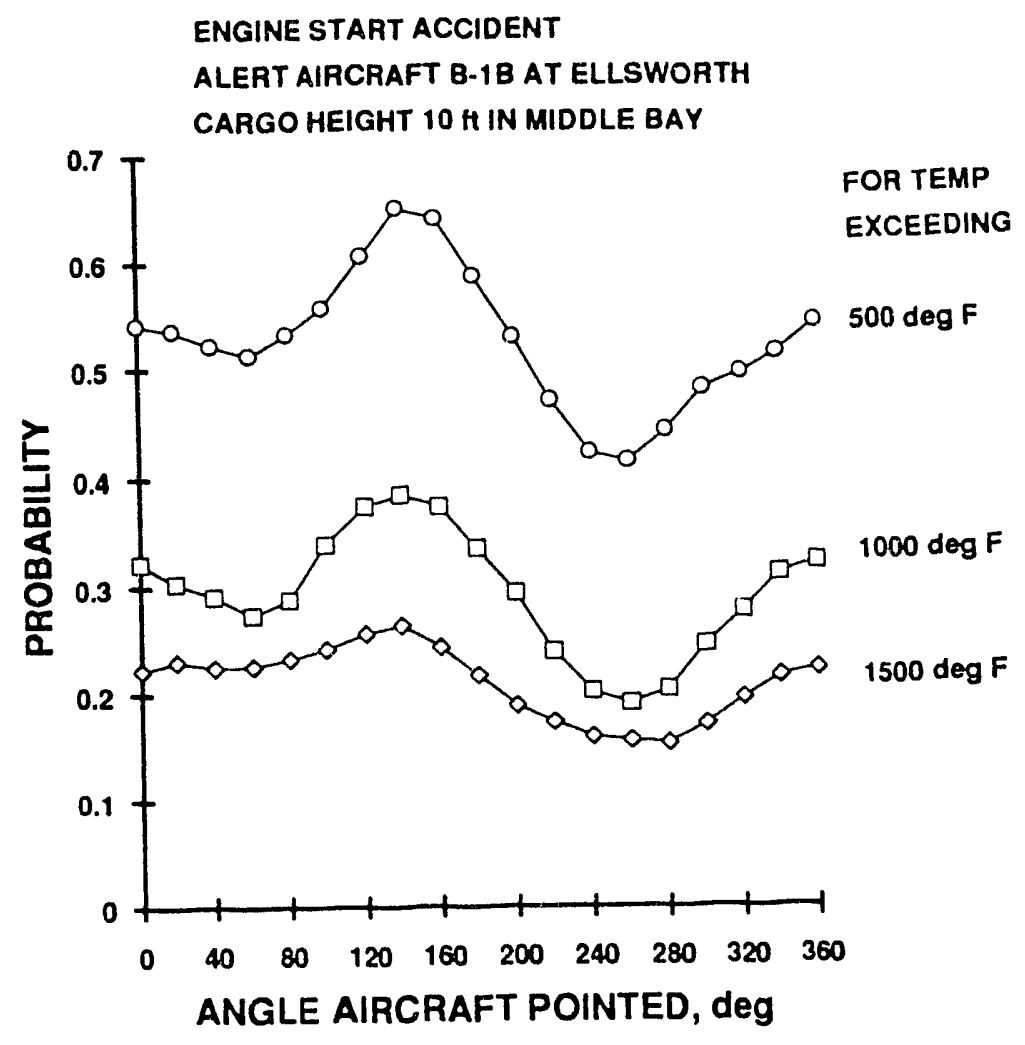

Figure 3.21 Probability vs Aircraft Orientation at Ellsworth AF Base

The weighted mean for each of the four B-1B bases for cargo in the middle bay is given on Figure 3.22a, and Figure 3.22b shows the mean weighted over all bases along with the highest and lowest mean obtained for any base. The corresponding curves for the aft bay are given in Figure 3.23a and 3.23b. Finally, so that the results for the middle and aft bays may be compared, Figure 3.24 shows the mean over all bases for cargo in the middle or aft bay.

Referring to Figure 3.24, it can be seen that the results for the middle and aft bay are quite similar, with the most significant difference occurring over the temperature range of 1950 to $2150^{\circ} \mathrm{F}$. The cause of the difference in result over the high temperature range is illustrated on the sketches of Figure 3.25. Figure 3.25a shows the approximate locations of the regions above 2000 and $2150^{\circ} \mathrm{F}$ (for low wind speed) when the middle bay cargo is downwind of the left rear spill point. It can be seen that neither high temperature region extends far enough downwind to expose the middle bay cargo. Figure $3.25 \mathrm{~b}$ shows these temperature region locations when the aft cargo is downwind of the left forward spill point, and in this case there is significant exposure of the aft cargo to both temperature regions. Additional examination has shown that while the middle bay cargo barely intersects the region above $2000^{\circ} \mathrm{F}$ for the forward spill points, there is no case of extensive exposure to the middle bay cargo such as that shown on Figure $3.25 \mathrm{~b}$ for the aft cargo. Thus, the comparatively high probability for the 1950 to $2150^{\circ} \mathrm{F}$ range for the aft bay on Figure 3.24 is a happenstance of the distance between cargo and spill points. 


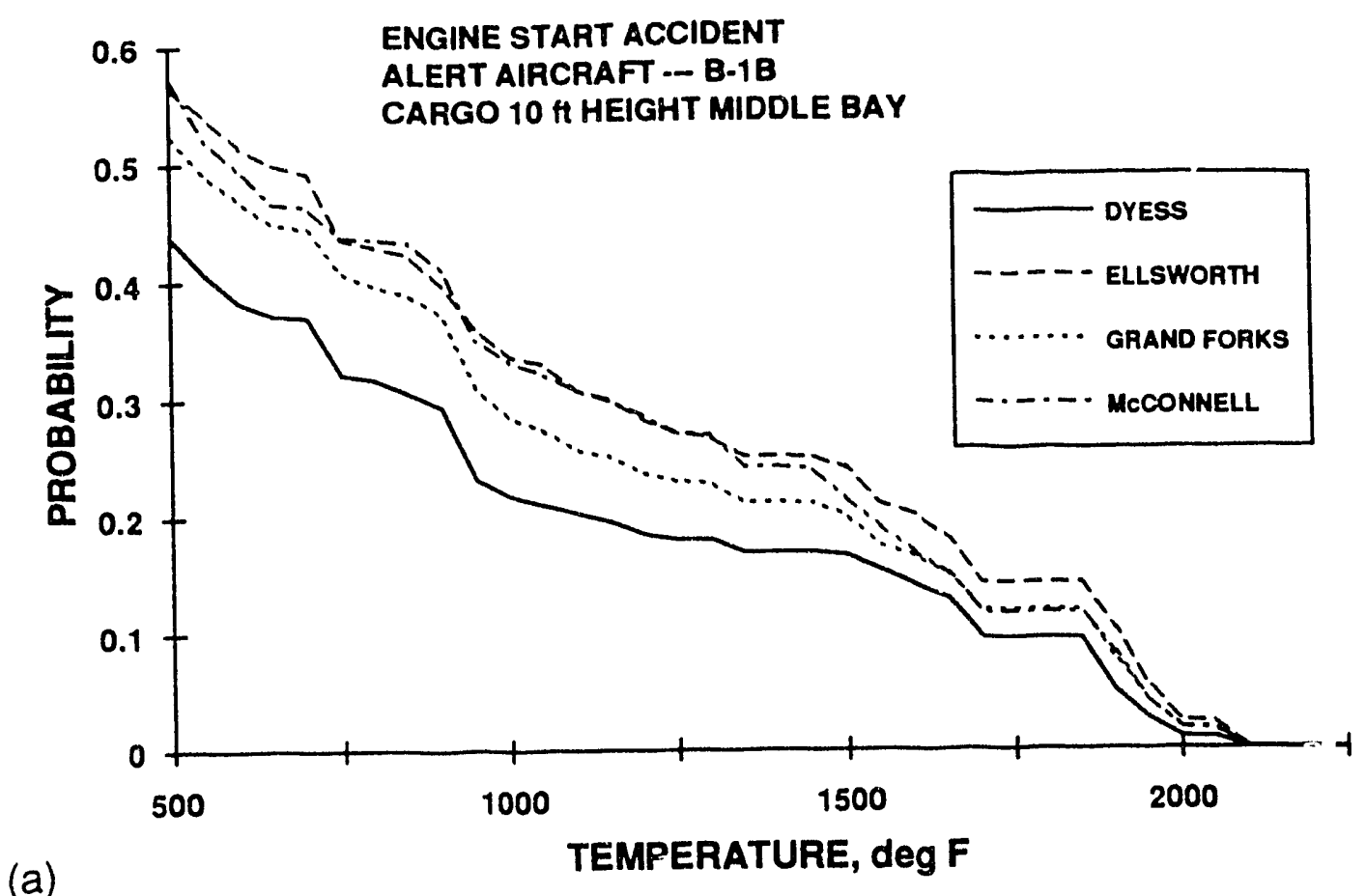

(a)

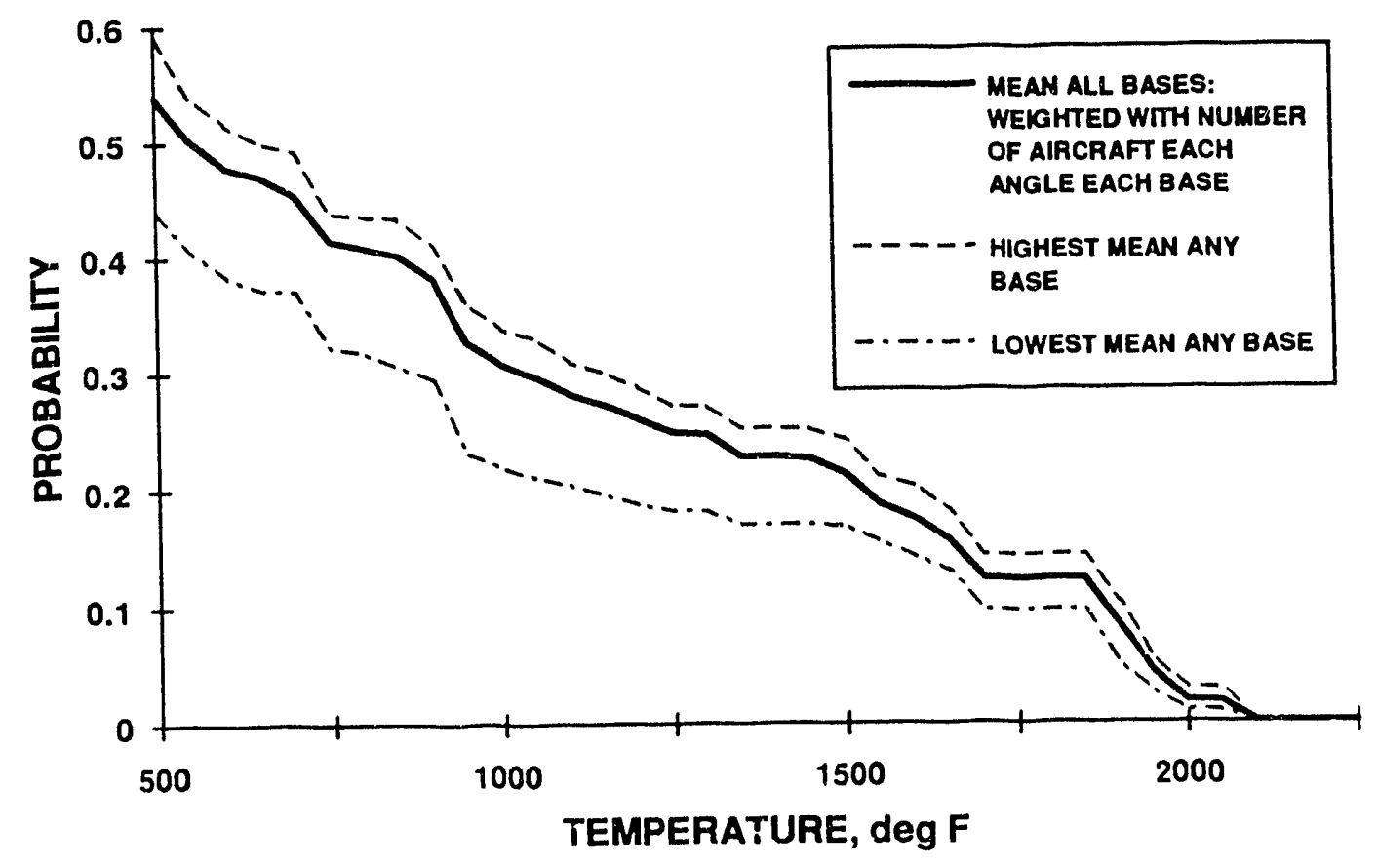

(b)

Figure 3.22 Results of B-1B Engine Start Accident for Cargo in the Middle Bay Only 


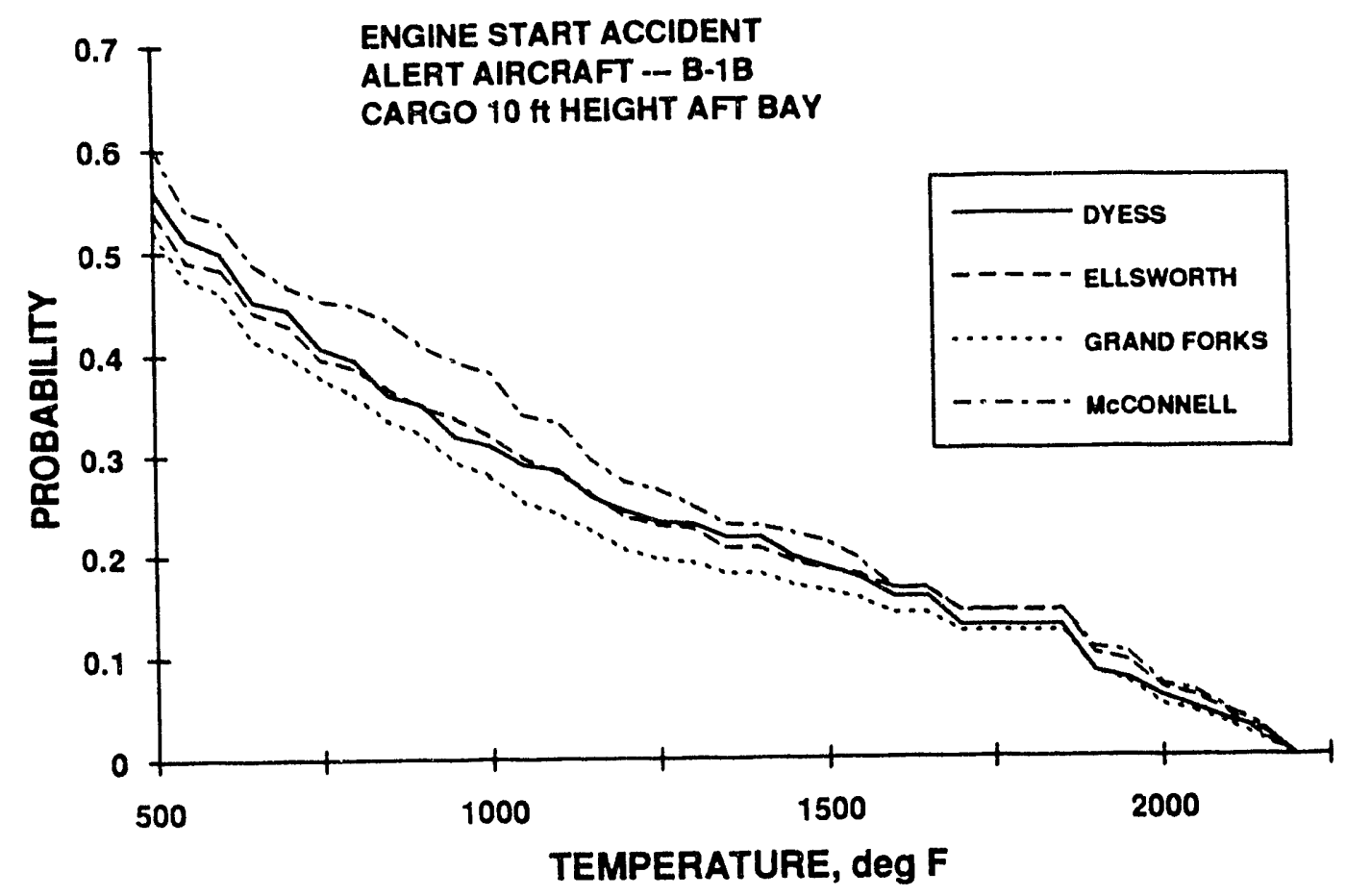

(a)

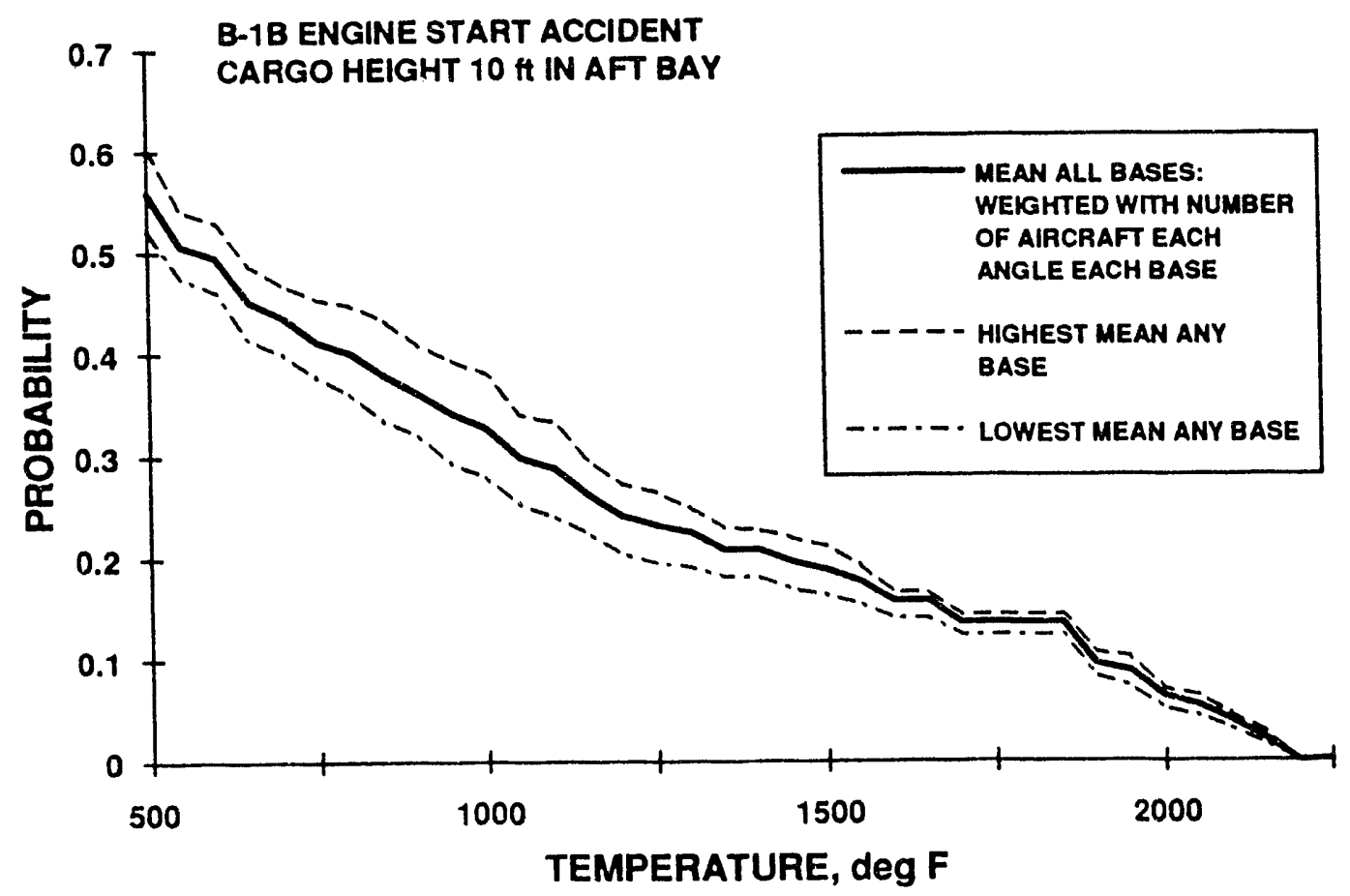

(b)

Figure 3.23 Results of B-1B Engine Start Accident for Cargo in the Aft Bay Only 


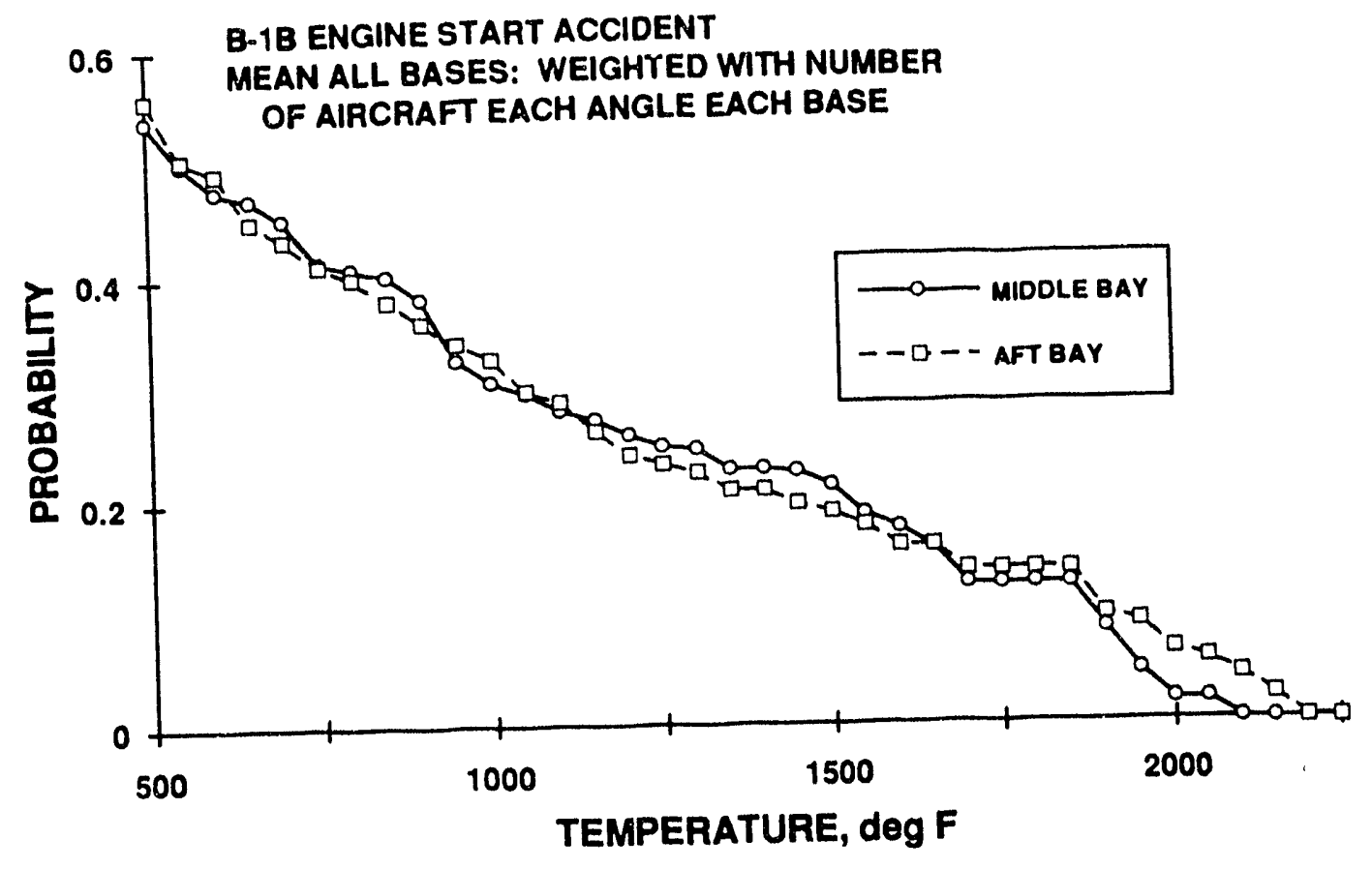

Figure 3.24 Engine Start Accident B-1B Base Mean Results for Middle and Aft Bavs

$\int_{2000 \operatorname{dog} F}^{\text {RECNON } F}$

REGION:

2150 dog F F LOCATIOH

- FUEL SPILL
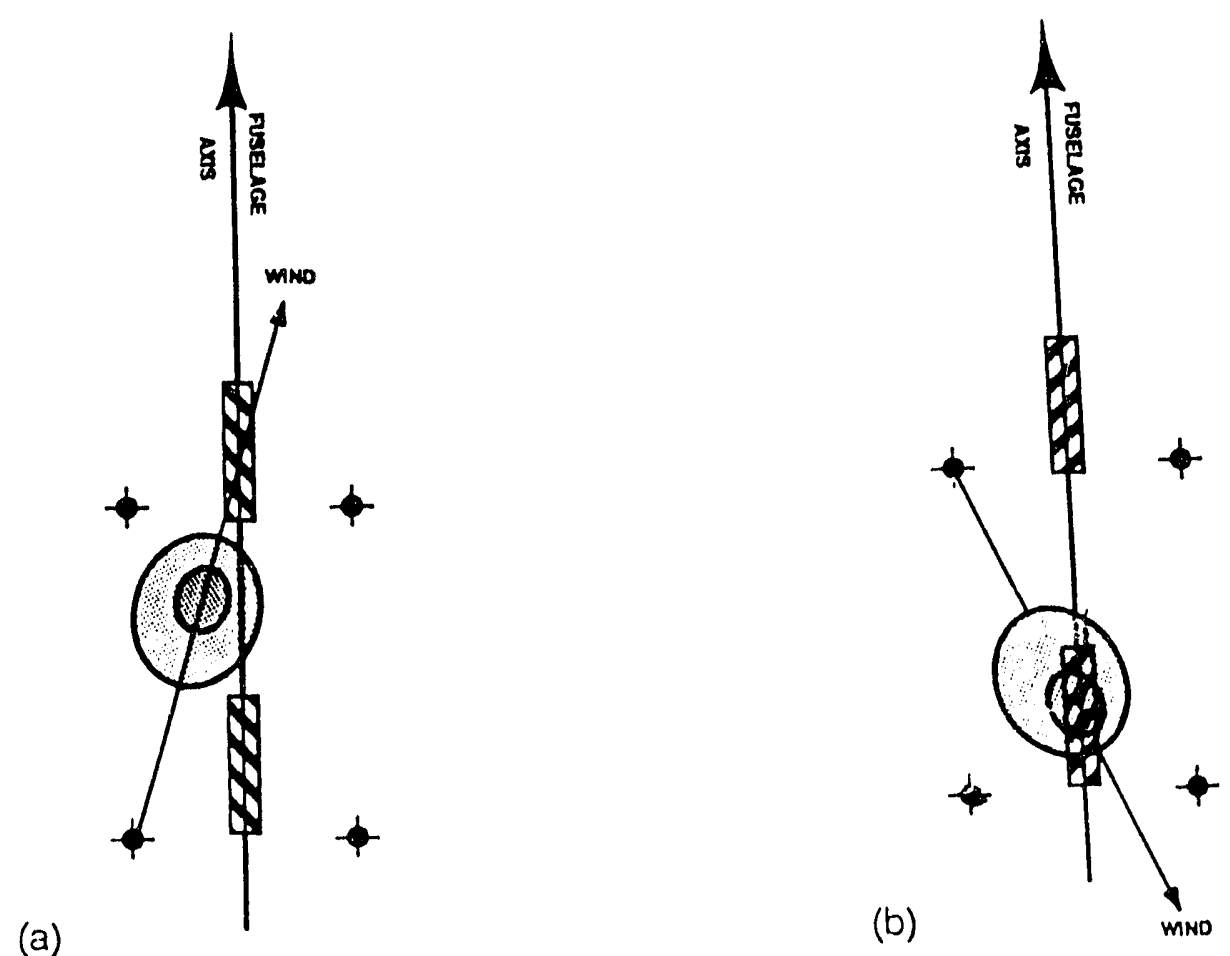

Figure 3.25 High Temperature Exposure for B-1B Engine Start Accident 
Regarding the case where weapons are simultaneously contained in the middle and aft bay, Figure 3.26 shows the mean probability vs temperature for each of the four B-1B bases; for purposes of comparison, Figure 3.26 also includes the mean probabilities for weapons contained in the middle bay only and the aft bay only.

It can be seen on Figure 3.26 that, except for the very high temperatures, the probability for weapons concurrently in both bays is somewhat higher than the higher of the probabilities for the middle bay only or aft bay only. It is instructive to examine the magnitude of the probability for the double weapon case compared to the single weapon cases. In this regard, consider first the sketches on Figure 3.27. Figure 3.27a shows two footprints which correspond to regions which exceed $1500^{\circ} \mathrm{F}$ for the low wind speed at 10 foot height due to spillage at the left rear spill point. The two footprints are for wind directions such that the footprint edge just intersects the edge of the weapon in the middle bay, and middle bay weapon exposure exceeding $1500^{\circ} \mathrm{F}$ will thus occur for any wind direction within the angular range labeled " $\alpha_{M}$ ". Figure $3.27 \mathrm{~b}$ shows the analogous angular range of exposure for the aft bay weapon, " $\alpha_{A}$ ". On figure $3.27 \mathrm{c}$, the various angles of Figure 3.27a and $\mathrm{b}$ are superimposed. Figure 3.27 shows the angular range labeled " $\beta$ " over which the two exposure ranges $\alpha_{M}$ and $\alpha_{A}$ overlap, that is, for the double weapon case, there is concurrent exposure exceeding $1500^{\circ} \mathrm{F}$ of both weapons. For conditions where this overlap occurs, the double weapon case is, in effect, evaluated by determining the highest exposure temperature of each weapon for each set of conditions (wind direction, spill location, parking angle, etc.) and using only the higher of these two temperatures in the analysis to determine probability vs temperature. Probabilities for the double weapon case, then, will not exceed the sum of the probabilities of the two single weapon cases, and the double weapon probability will always exceed either single weapon probability. Since the size of a region that exceeds a given temperature decreases with increasing temperature, the overlap angle $(\beta)$ will tend to decrease with increasing temperature, and there can be temperatures above which there is no overlap. At temperatures above 


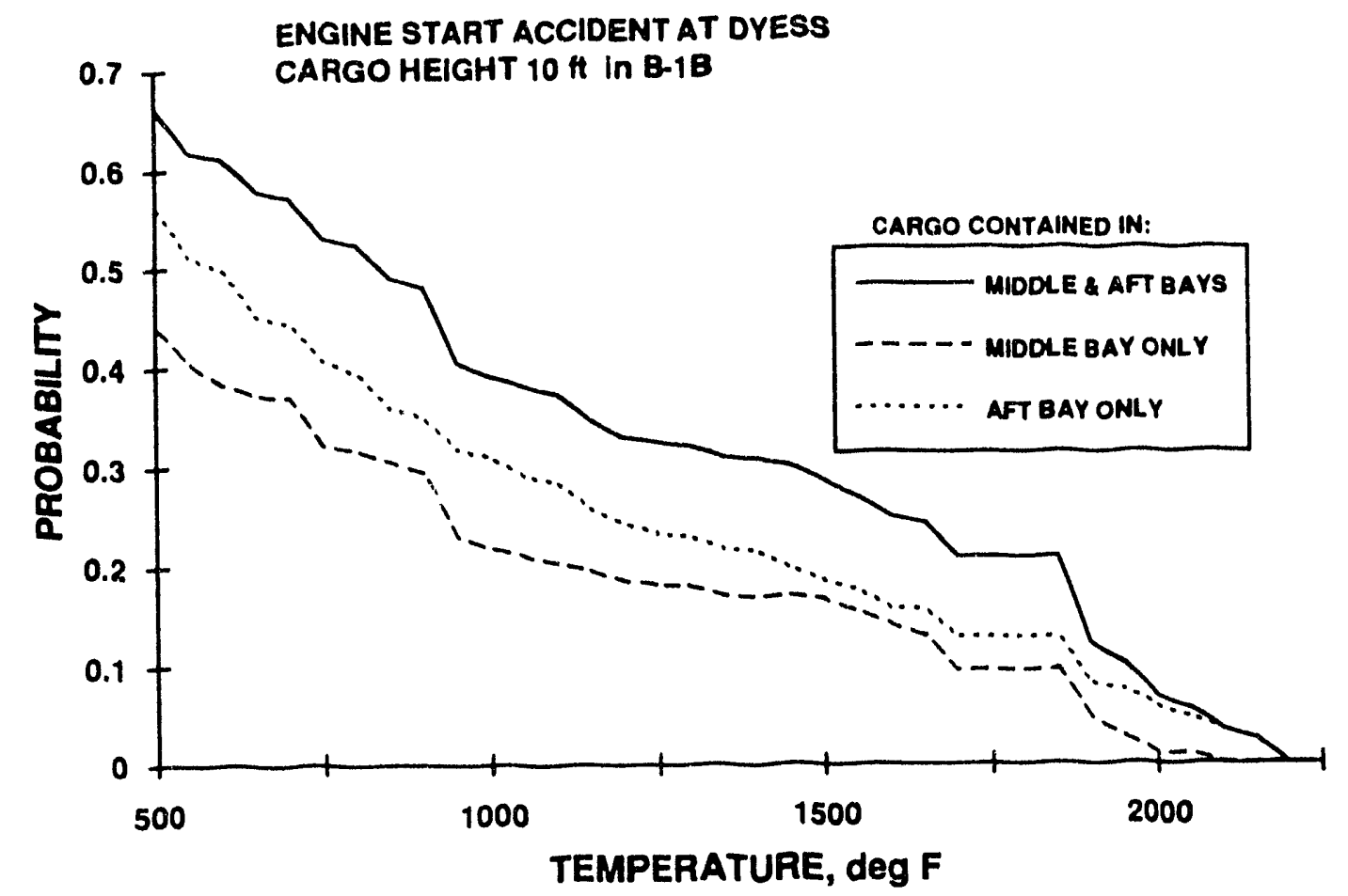

(a)

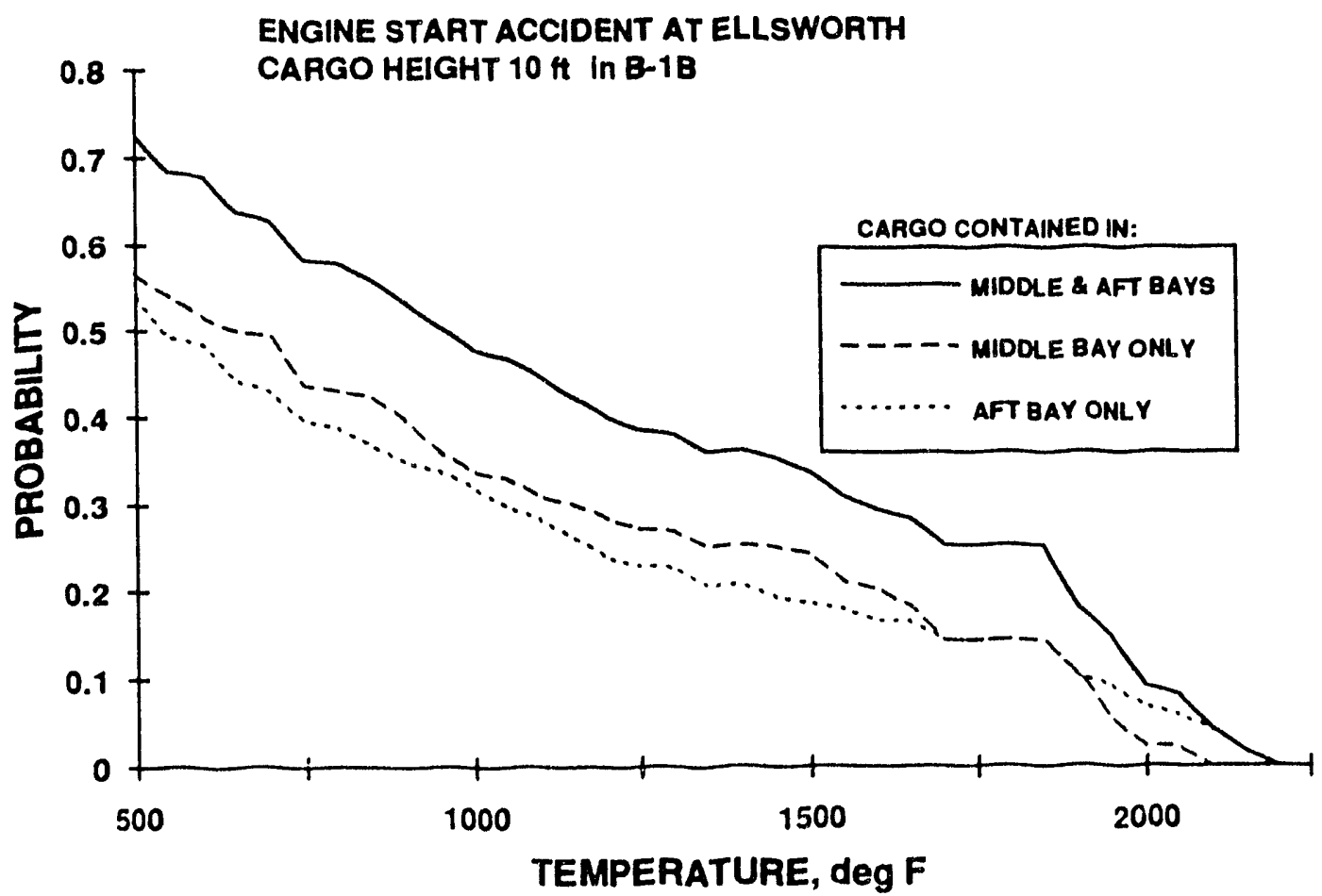

(b)

Figure 3.26 a \& b Engine Start Accident Results for Double and Single Weapon Cases at 10 Foot Height 


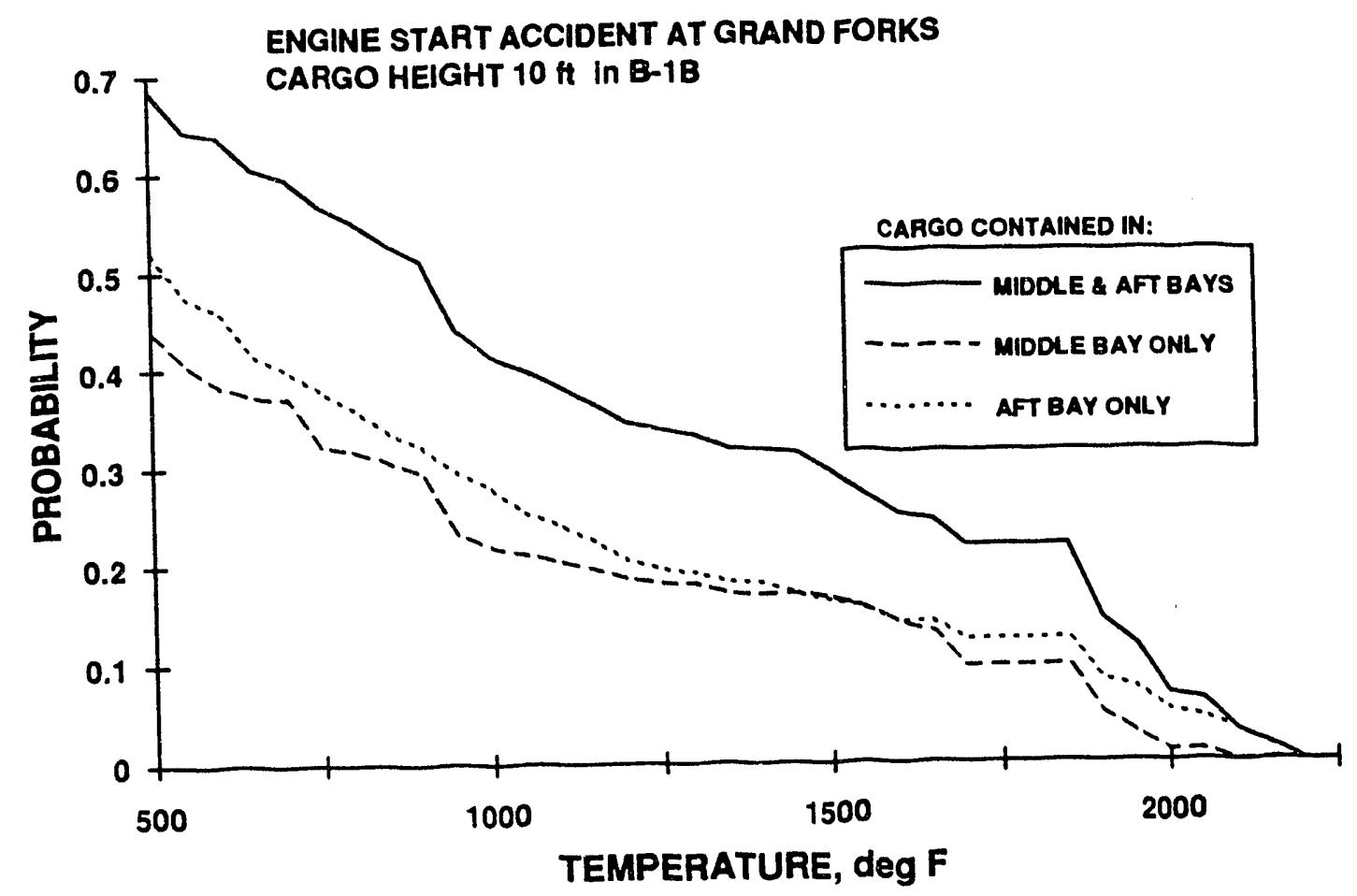

(c)

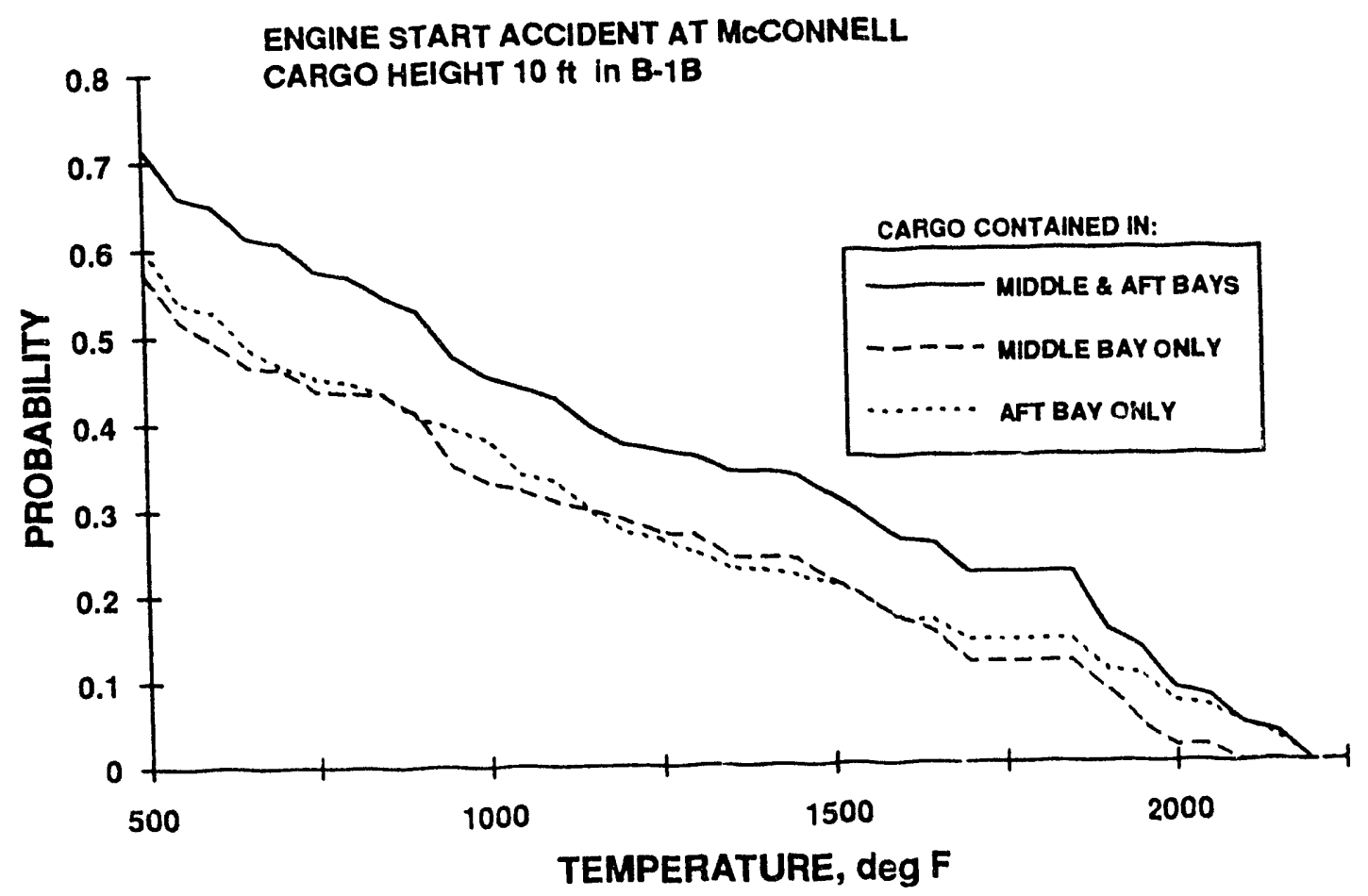

(d)

Figure $3.26 \mathrm{c} \& \mathrm{~d}$ Engine Start Accident Results for Double and Single Weapon Cases at 10 Foot Height 


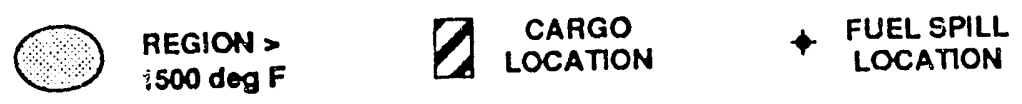

(a)
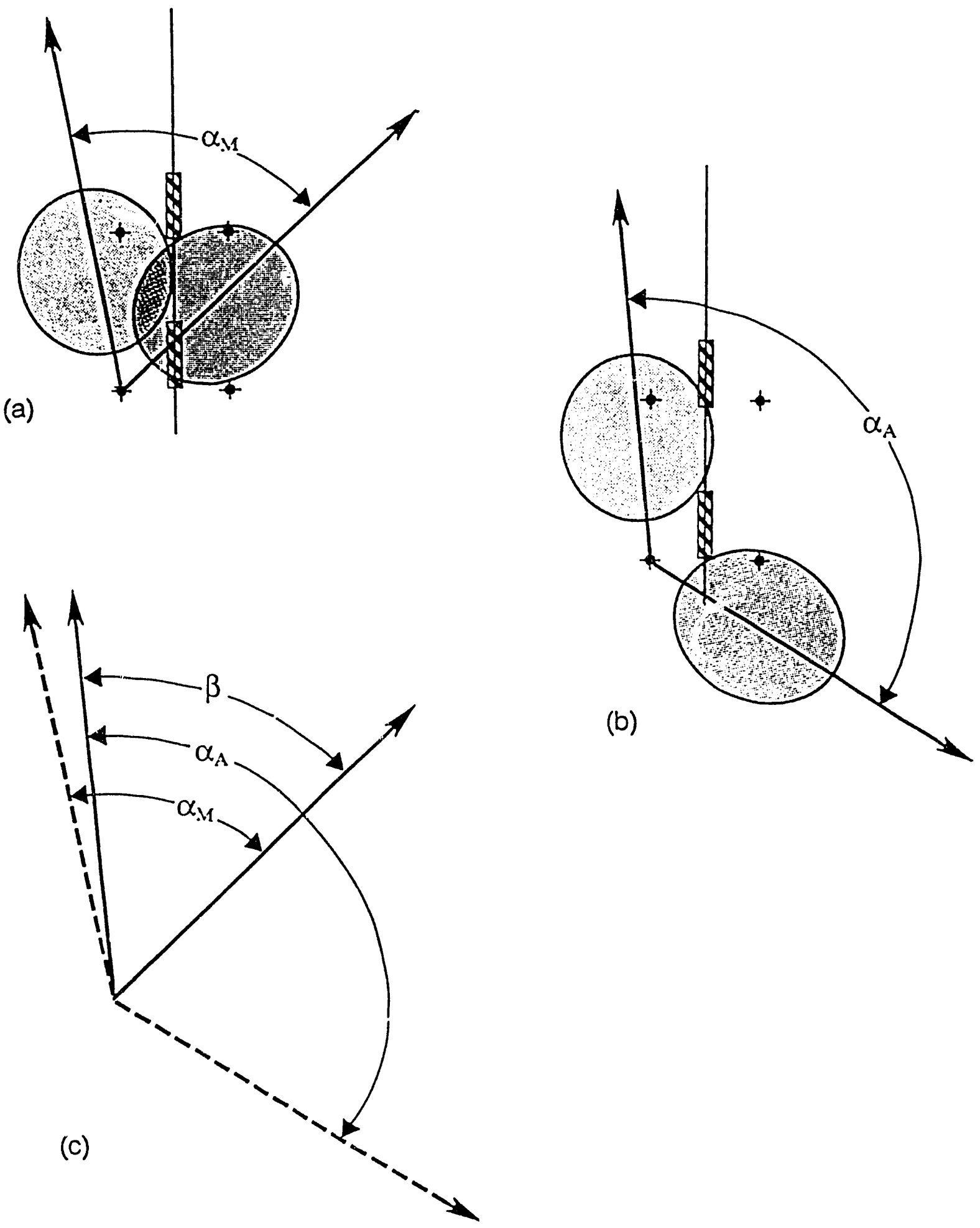

$\equiv$

Figure 3.27 Configuration for Exposure Overlap of Middle/Aft Bay Cargo 
this point, the double weapon probability becomes the sum of the single weapon probabilities. The graph on Figure 3.28 shows the dependence on temperature of the ratio of double weapon probability to the sum of the single weapon probabilities. It can be seen that this ratio increases with temperature, approaching 1.0 at about $2000^{\circ} \mathrm{F}$.

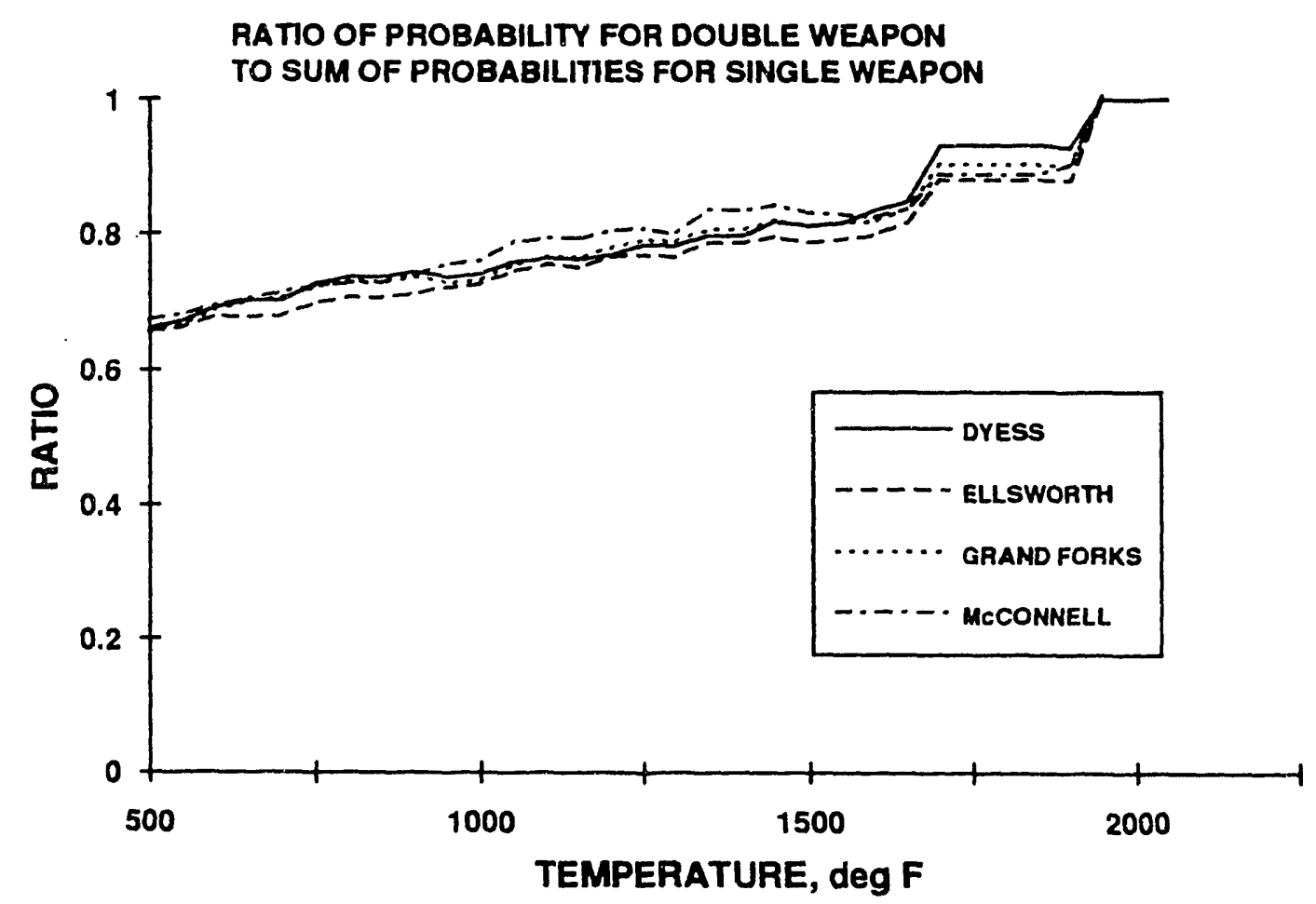

Figure 3.28 Ratio of Probability for Double Weapon to Sum of Probabilities for Single Weapon 
For purposes of base to base comparison, the double weapon probability vs temperature curves for all four B-1B bases are given in Figure 3.29.

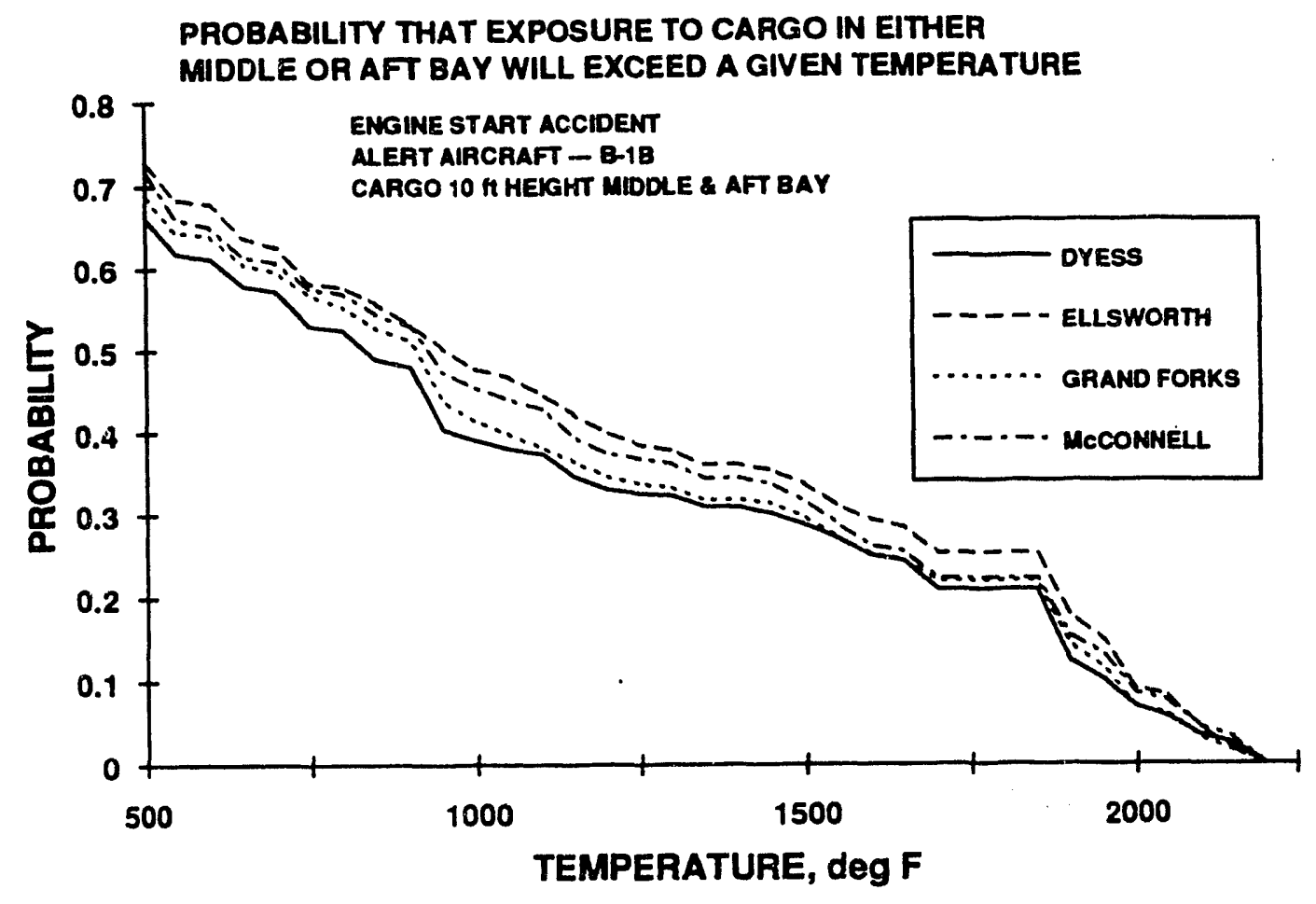

Figure 3.29 Double Weapon Results for Engine Start Accidents at All B-1B Bases

Regarding the effect of cargo height, probability vs temperature curves for the middle bay position for the heights of $1,4,7$ and 10 feet are given on Figure 3.30 for the four B-1B bases. The corresponding curves for the aft bay are given on Figure 3.31 for two bases.

Several features of these curves deserve some discussion. The B-1B results differ in many respects from those for the B-52, and it is revealing to discuss the B-1B result features in terms of comparisons to those for the B-52. The primary cause of these differences is the difference in distance betwron fuel spill points and cargo. 


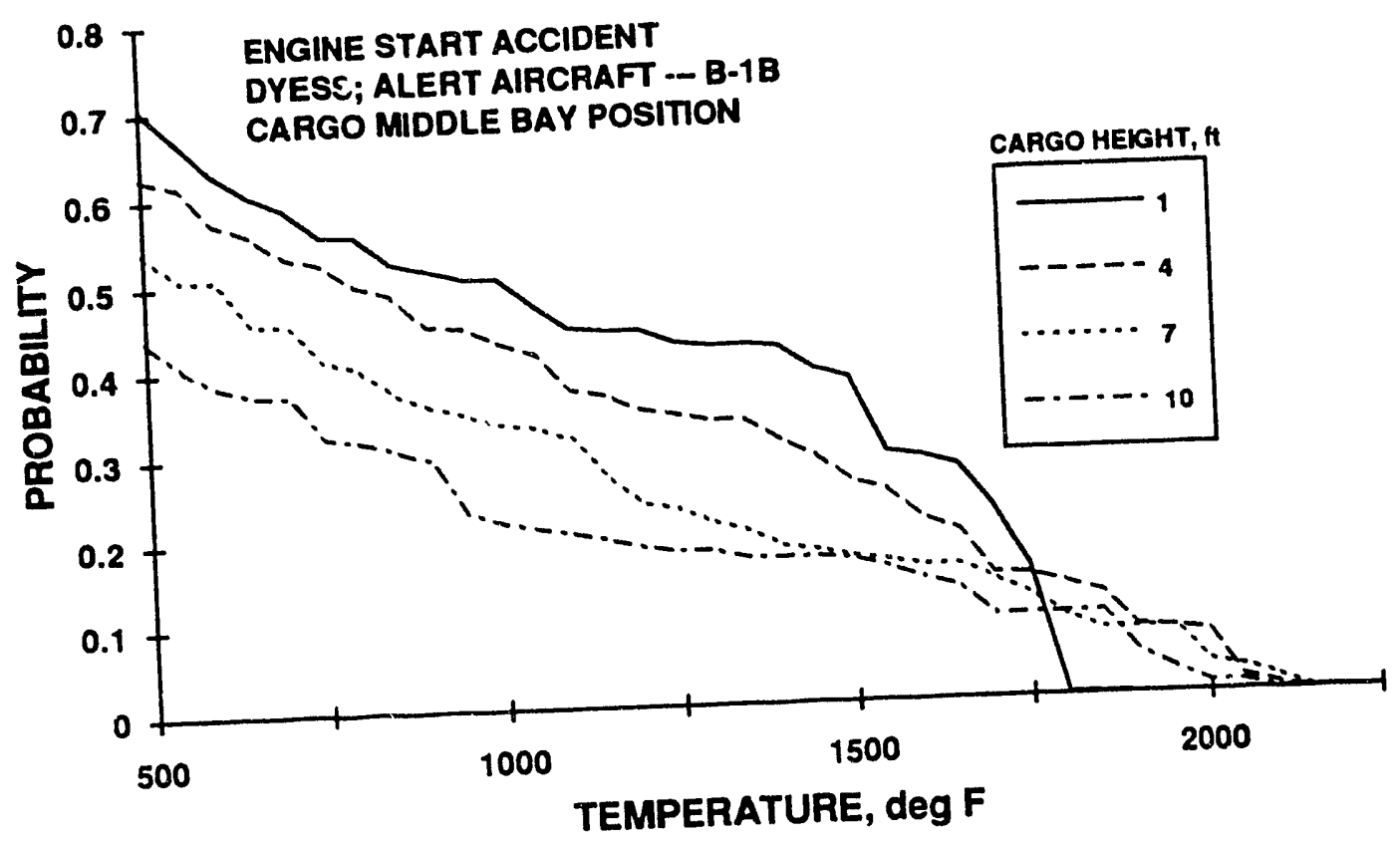

(a)

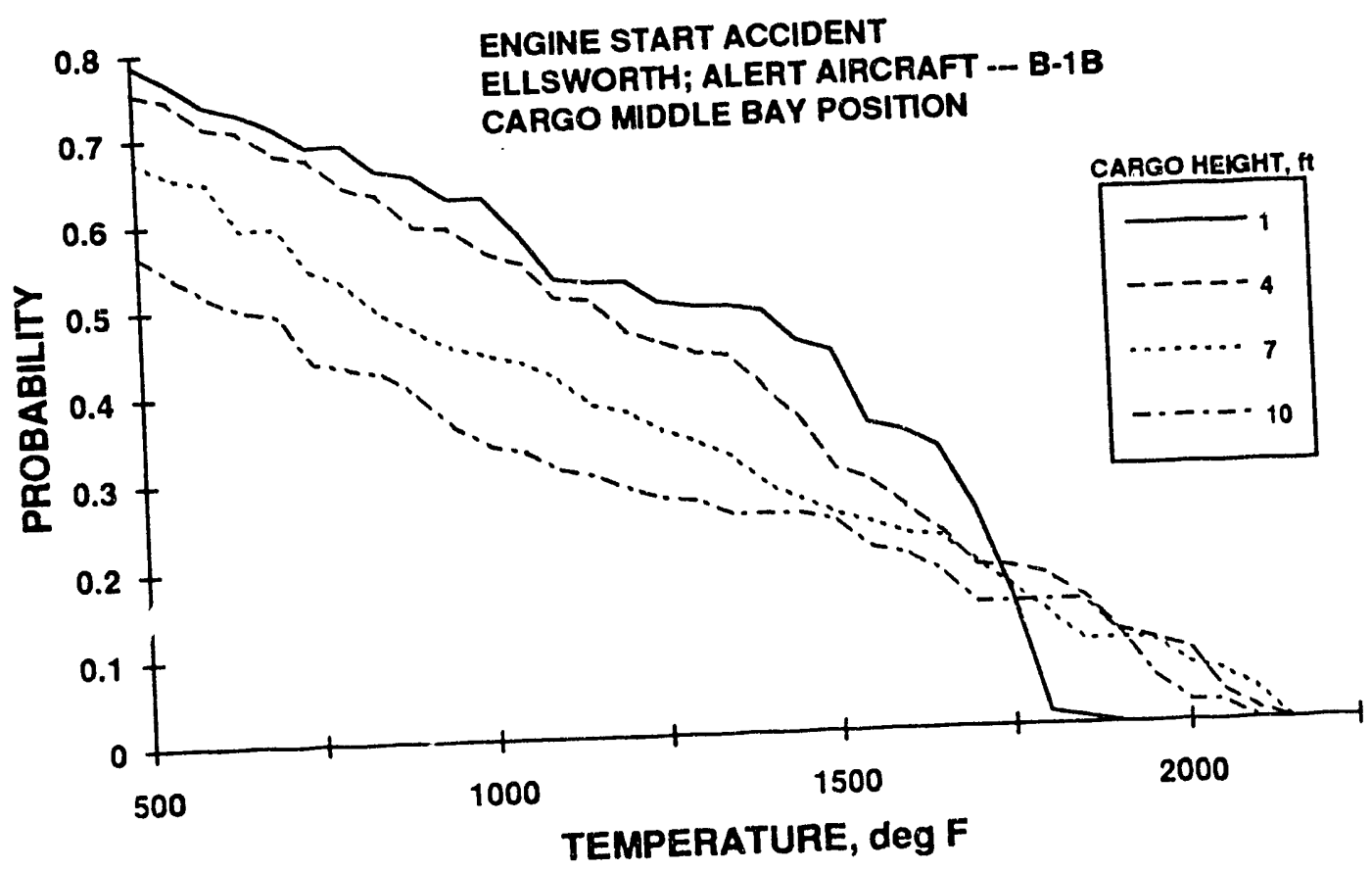

(b)

Figure 3.30 a \& b B-1B Engine Start Results for Various Cargo Heights for Middle Bay 


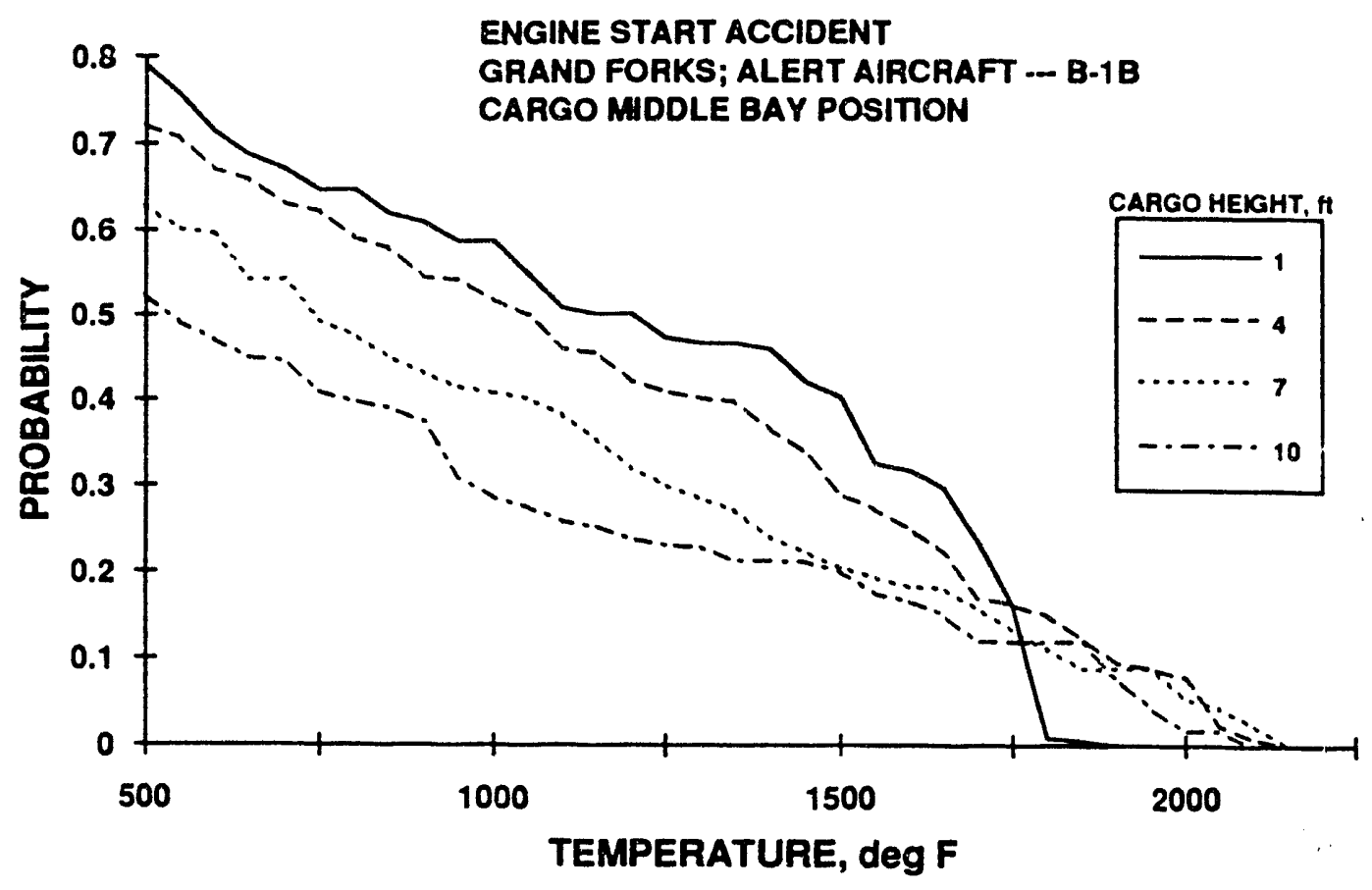

(c)

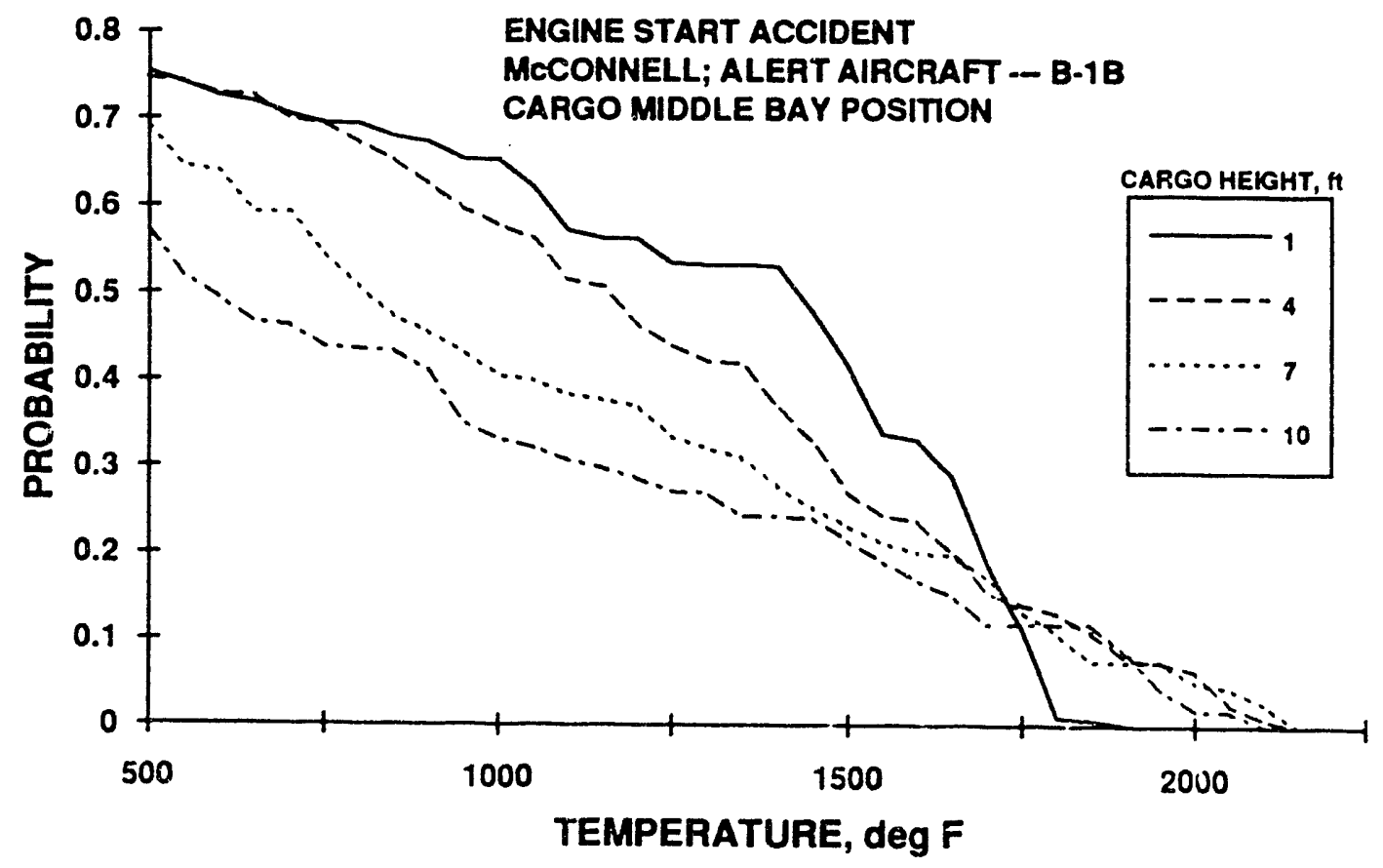

(d)

Figure 3.30 c \& d B-1B Engine Start Results for Various Cargo Heights for Middle Bay 


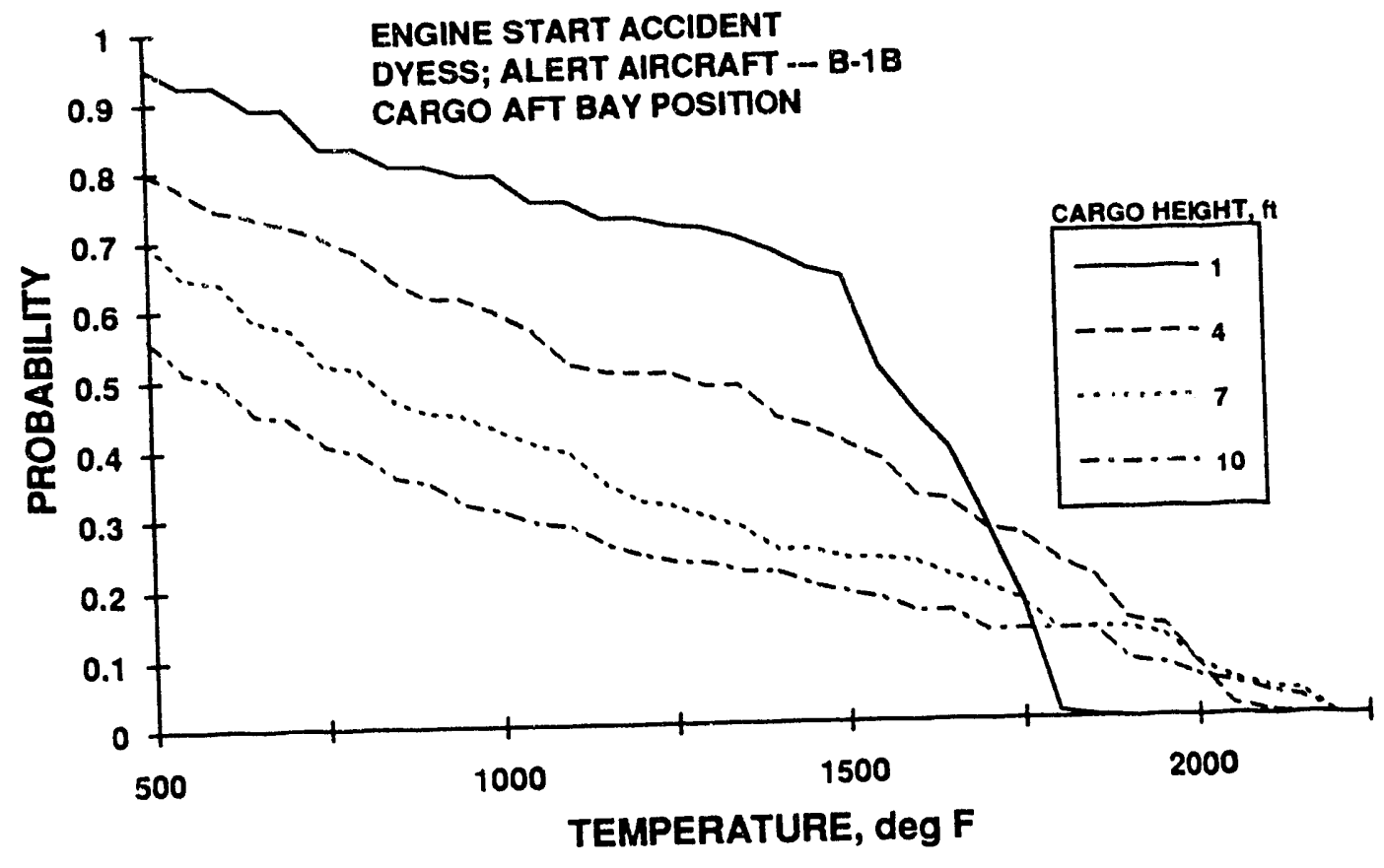

(a)

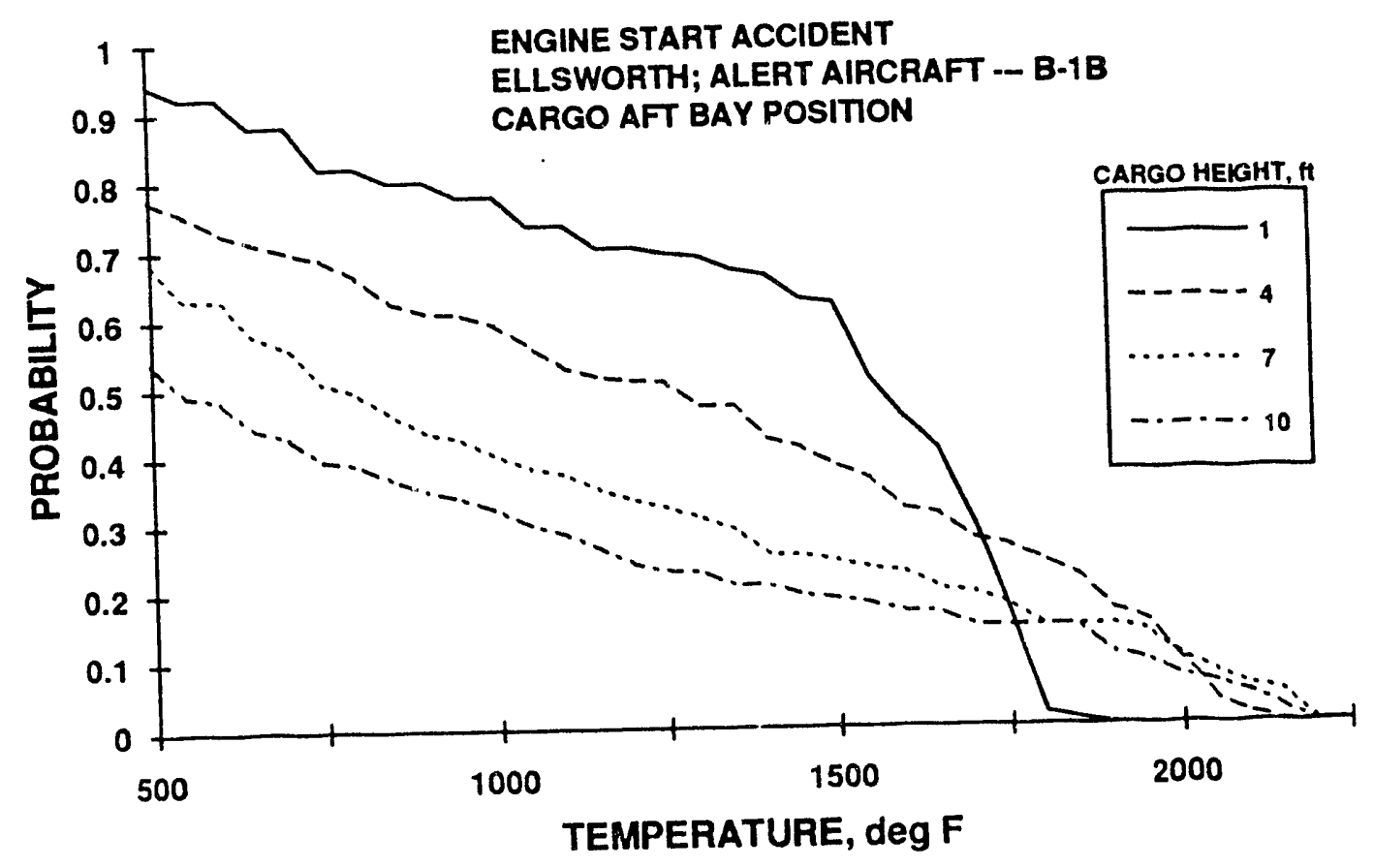

(b)

Figure 3.31 B-1B Engine Start Results at Various Cargo Heights for Aft Bay 
Consider first the exposure probability of $500^{\circ} \mathrm{F}$ at the 1 foot height. For the $\mathrm{B}-1 \mathrm{~B}$, these values are in the range 0.7 to 0.8 for the middle bay and above 0.9 for the aft bay; for the B-52, the probability range is about 0.5 to 0.6 (ref. Figures 3.16 and 3.17). The footprints of regions at or above $500^{\circ} \mathrm{F}$ for the low wind speed given on Figure 3.32 illustrate why these three probability ranges differ. It can be seen on Figure 3.32a for the B-1B that both the middle and aft weapons are exposed even when the weapons are upwind of the forward spill point (both weapons are exposed for any wind direction for the low wind speed). From Figure $3.32 \mathrm{~b}$, it can be seen that spills of the rear spill points result in exposure of the aft weapon for any wind direction, but exposure of the middle bay weapon would occur only over a limited range of wind directions (the actual range is approximately $135^{\circ}$ ). Thus, the probability of exposures of $500^{\circ} \mathrm{F}$ or above are somewhat smaller for the middle bay weapon than the aft weapon. On the basis of low wind speed alone, the probability of aft weapon exposure would be 1.0; it is the character of the medium wind speed footprints (and to a much lesser extent the high wind speed footprints) that prevents this.

Regarding the B-52, it can be seen from Figure 3.32c that the weapon is not exposed when the wind is directed away from the weapon for spills at either the forward or rear spill point. (The actual angular range of exposure is approximately 230 and 130 degrees for the rear and forward spill point, respectively.) Thus, the probability for the B-52 is significantly less than for the B-1B at either weapon location.

Regarding the probability vs temperature curves at temperatures above $500^{\circ} \mathrm{F}$, it can be seen on Figures 3.30 and 3.31 that the curve for the 1 foot height is above the curves for other heights throughout a large fraction of the entire temperature range, but that the 1 foot curve decreases abruptly with temperature, falling to negligible values at about $1800^{\circ} \mathrm{F}$. This behavior is qualitatively similar to that for the B-52, although the 1 foot curves fall below the other curves at about $1750^{\circ} \mathrm{F}$ compared to about $800^{\circ} \mathrm{F}$ for the B-52 (ref. Figure 3.16). 

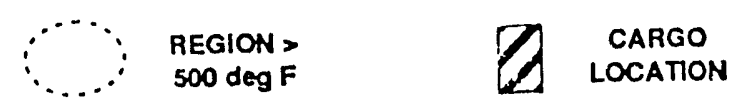

FUEL SPILl
LOCATION

(a)
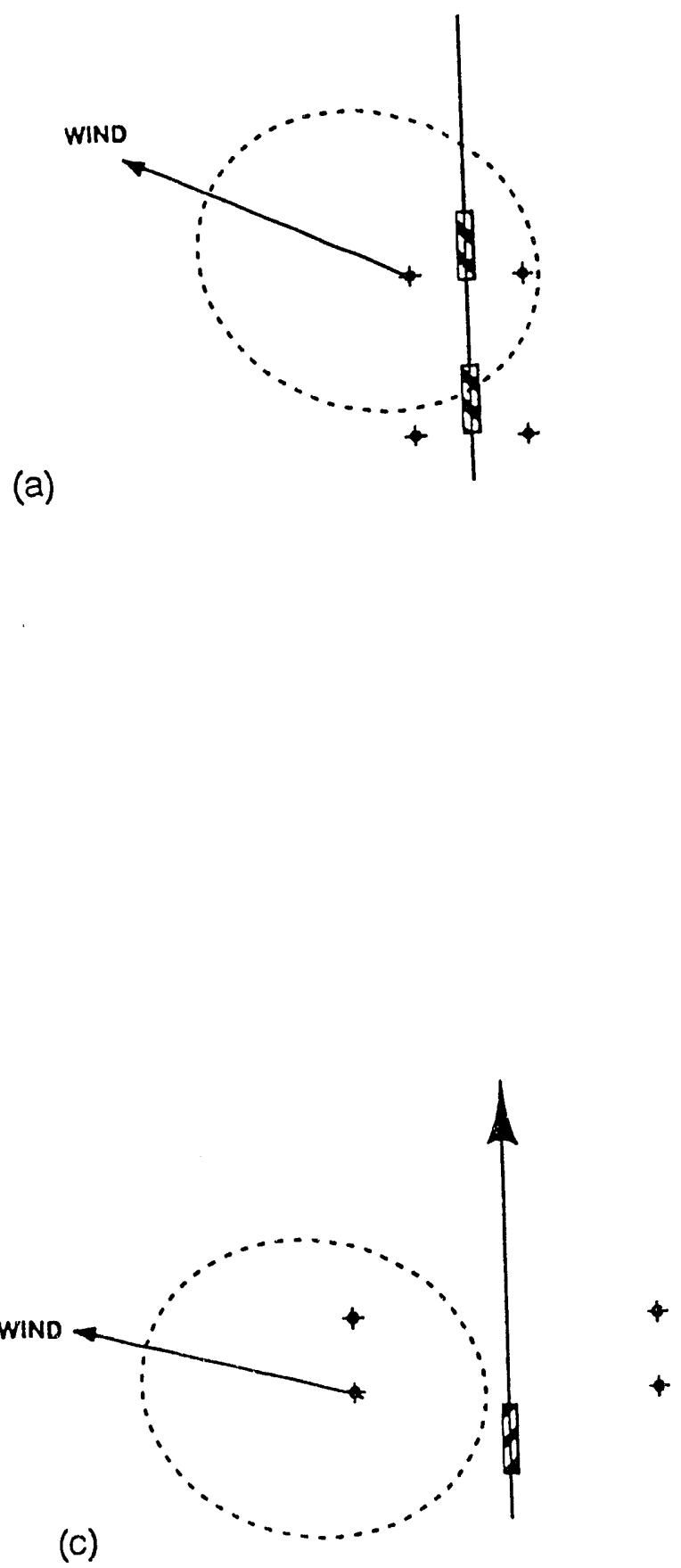

(b) W!ND

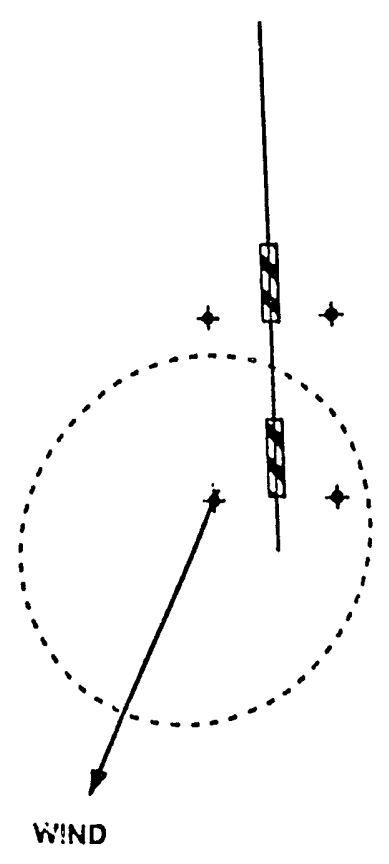

Figure 3.32 B-52/B-1B Exposure Illustration for 1 Foot Height 
Plots of the probability of exceeding $500^{\circ} \mathrm{F}$ vs height are given on Figure 3.33a for the middle bay position and Figure 3.33b for the aft bay position. The dependence of probability on height is more significant for the aft position than the middle position, and for both positions the dependence is more significant than for the B-52 (ref. Figure 3.17).

Plots of the probability vs temperature at the 1 foot height for weapons concurrently at the middle and aft bay positions are given on Figure 3.34 for two bases; for purposes of comparison, curves for weapons at the middle bay position only and the aft bay position only are included. For both bases, the double weapon probability is 1.0 over the temperature range 500 to $600^{\circ} \mathrm{F}$, that is, the probability is 1.0 that the exposure of at least one of the weapons will exceed $600^{\circ} \mathrm{F}$. 


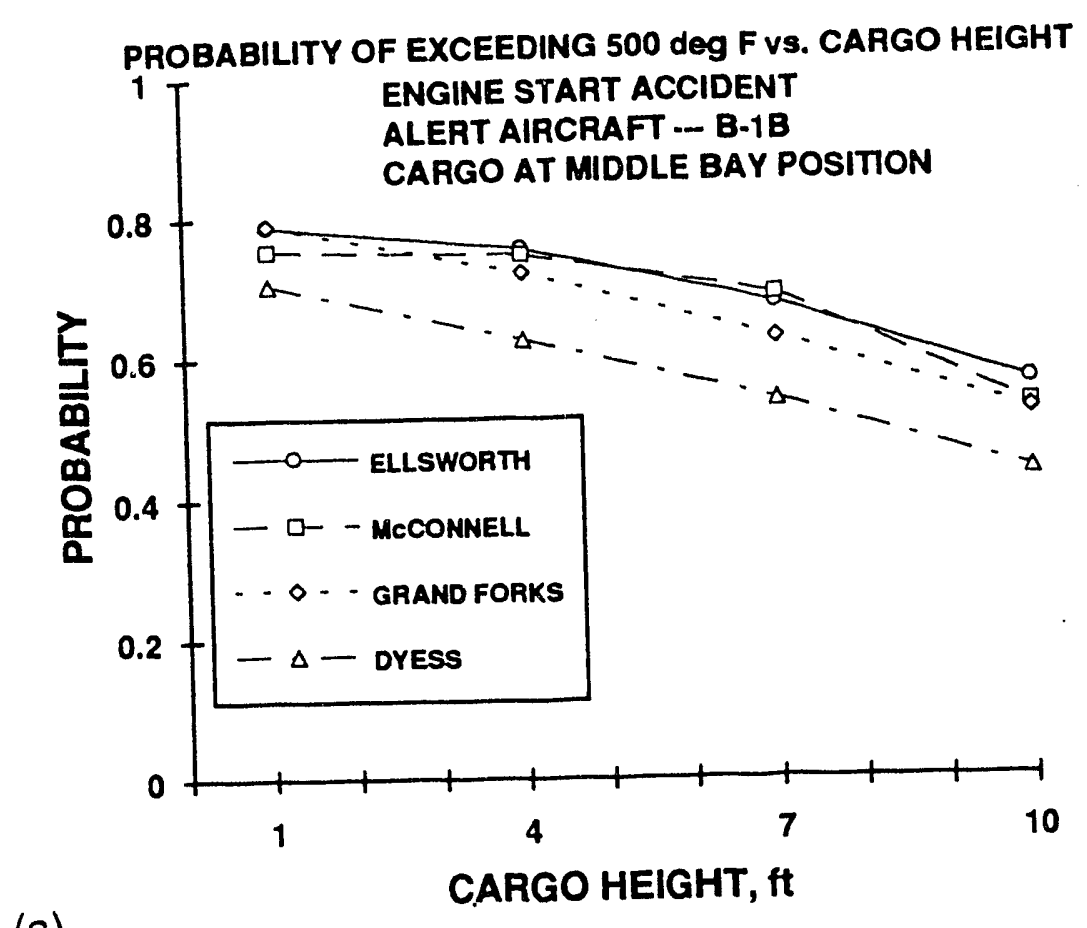

(a)

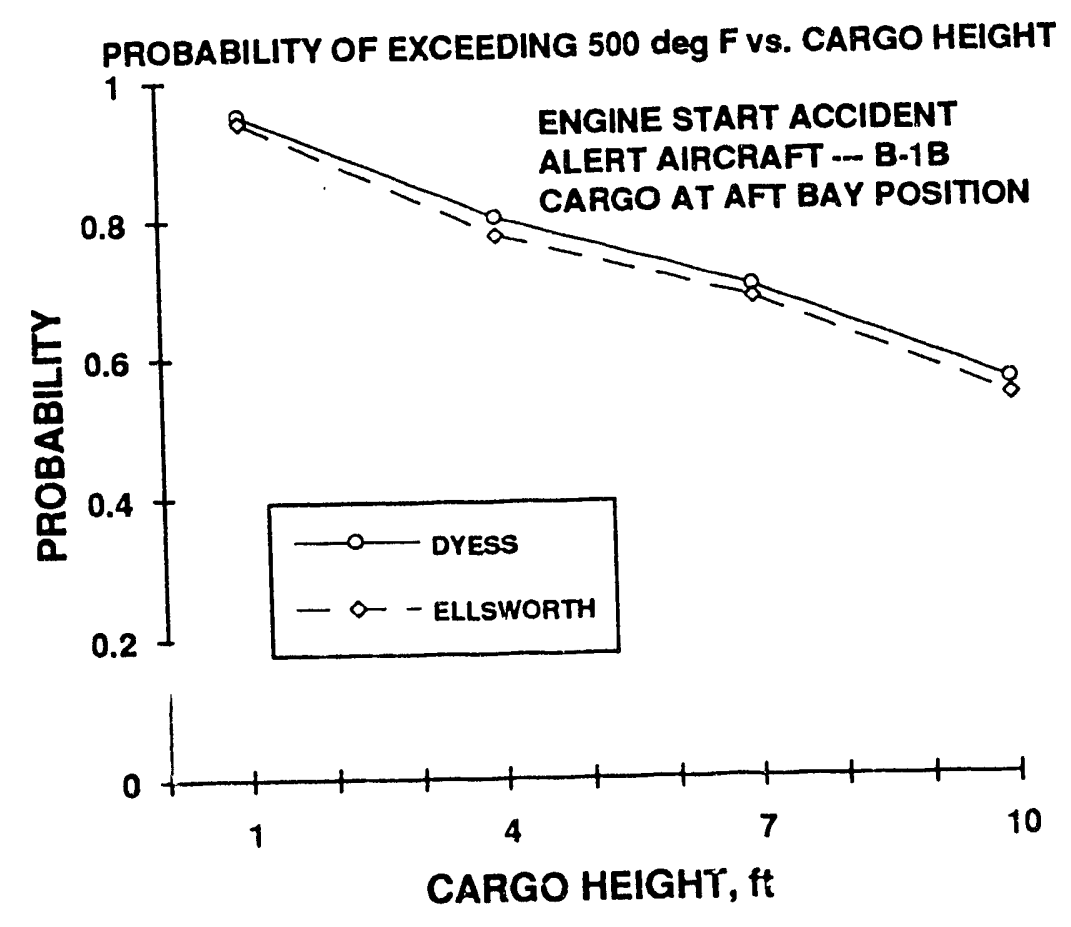

(b)

Figure 3.33 Probability vs Cargo Height for Engine Start Accident for Middle and Aft Bays 


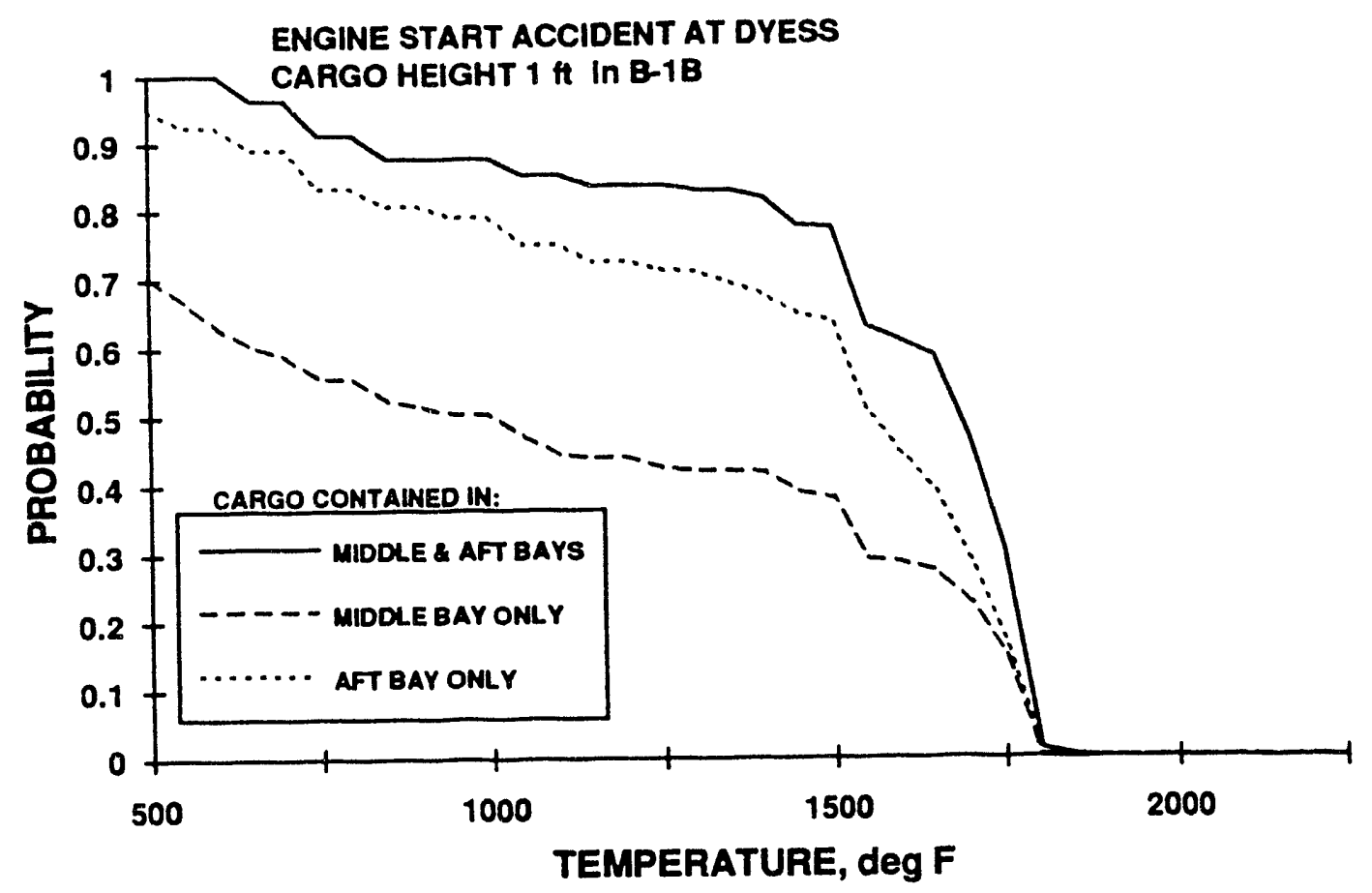

(a)

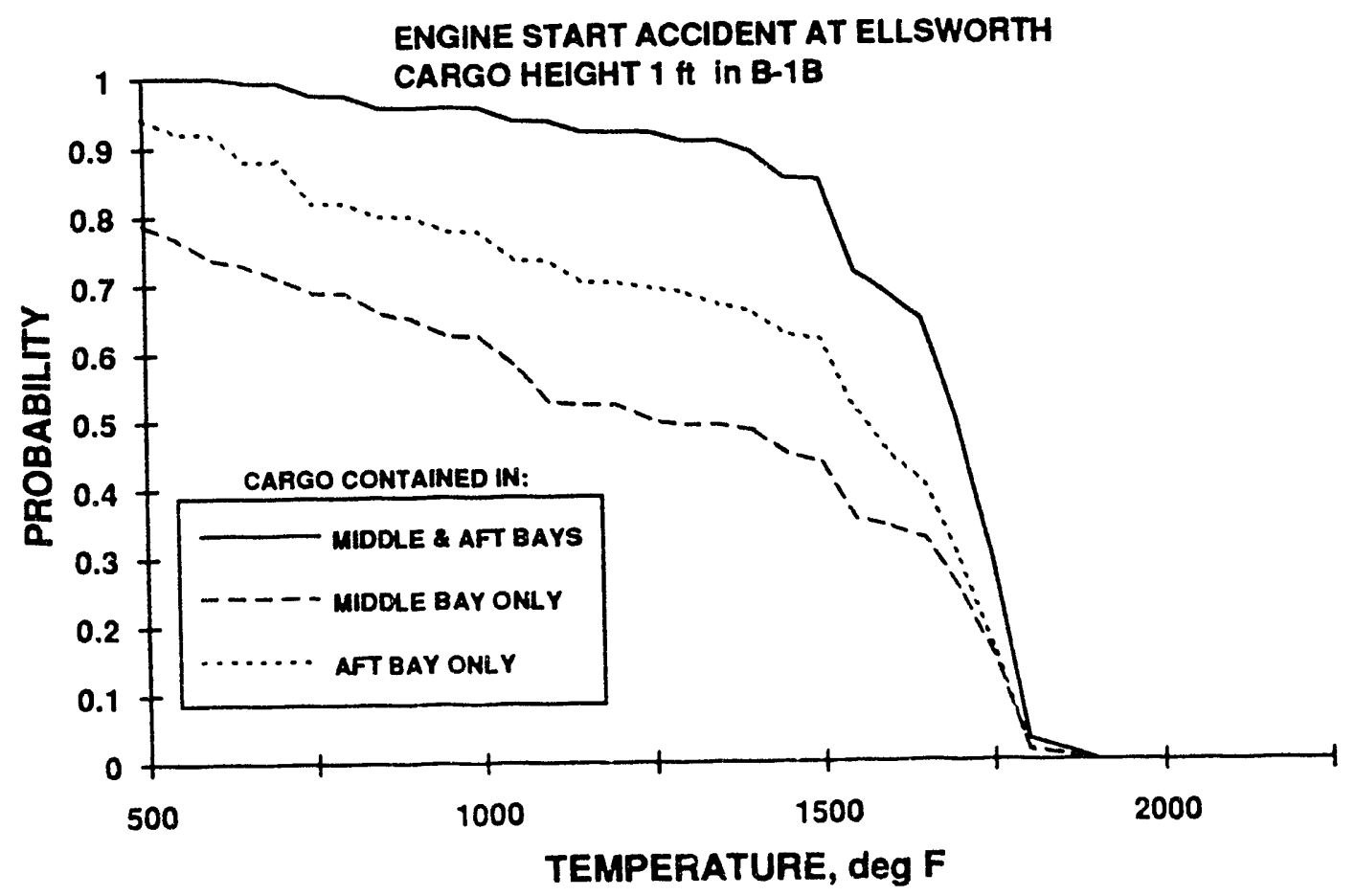

(b)

Figure 3.34 Engine Start Accident Results for Double and Single Weapon Cases at 1 Foot Height 


\subsection{AIRCRAFT COLLISION RESULTS: B-52/B-1B}

Results presented in subsection 3.4 are for the exposure of weapons in an alert aircraft due to a collision into the alert aircraft of an aircraft that is landing or taking off. As described in detail in Section 2, the results depend on the direction of motion of the colliding aircraft with respect to the alert aircraft, that is, whether the collision is a broadside, rear-end, head-on, or from some intermediate angle. The evaluation of a given case then requires input of the parking direction of the alert aircraft and the motion direction of the colliding aircraft at collision. It is believed that a direction of motion of the colliding aircraft which is parallel or "nearly" parallel to the runway would be by far the most likely case, and this direction of motion is considered the "baseline" case. The earlier results given in this section are for baseline conditions, and results for other motion directions are given later in the section.

Another aspect of the baseline conditions relates to restrictions imposed on wind direction range for landing or take-off collisions; these restrictions and the reasons for them are as follows. Figure 3.35 provides an illustration of the four take-off and landing configurations that can occur, Figure $3.35 \mathrm{a}$ and $3.35 \mathrm{~b}$ showing take-off from the alert pad end of the runway and the end opposite the alert pad, respectively, and Figure $3.35 \mathrm{c}$ and $3.35 \mathrm{~d}$ showing landing on the alert pad end and on the end opposite the alert pad, respectively. Take-offs from the alert pad end, as in Figure 3.35a, will not result in take-off collision into an alert aircraft. Collision can occur for take-offs from the opposite end, but assuming that take-offs are into the wind, take-off collisions would only occur for wind flows having a component opposing the aircraft taking off. This 180 degree wind direction range, ranging \pm 90 degrees from the runway direction is illustrated on Figure 3.35b. Regarding landing, collision can occur for landing on the alert pad end, but only for the 180 degree wind direction range shown in Figure 3.35c. Collision potential is not as clear cut for the case of landing on the end opposite the alert pad (Figure 3.35d). However, it is thought that the probability of collision into an alert aircraft is comparatively slight, and for baseline purposes, collisions for this configuration are excluded. Note that the take-off part of a "touch and go" would be considered a take-off. Thus, the configurations represented by Figures $3.35 \mathrm{~b}$ and $3.35 \mathrm{c}$ only are included in baseline probability evaluation. (Note that wind restrictions do not arise in engine start accidents.)

The assumption that take-off and landing is into the wind has an impact on the probability of temperature exposure. While a collision can result in fuel spills at any fuel tank location of the alert aircraft (and of the colliding aircraft), there is a tendency to have more damage and thus more spills on the impact side (or end) of the alert aircraft. With take-offs and landings into the wind, there is 
TAKE-OFF

FROM ALERT PAD END

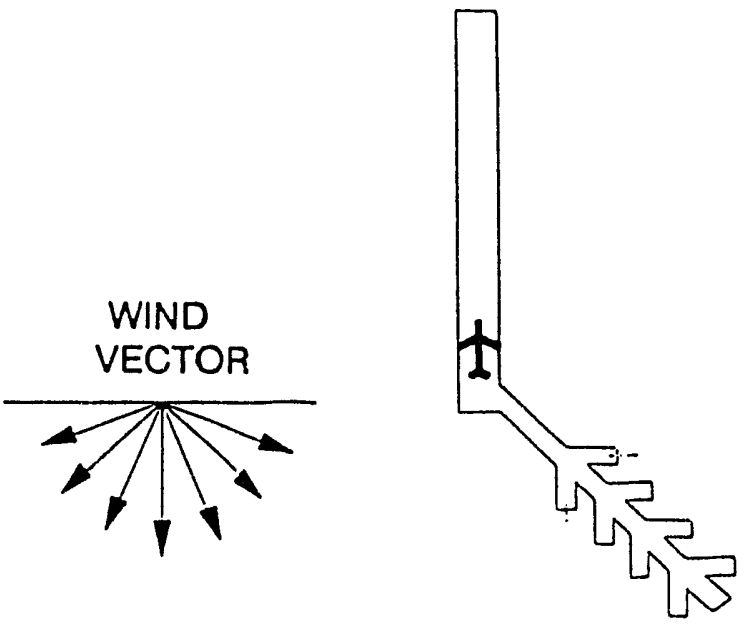

NO COLLISION

(a)
TAKE-OFF

FROM END OPPOSITE ALERT PAD
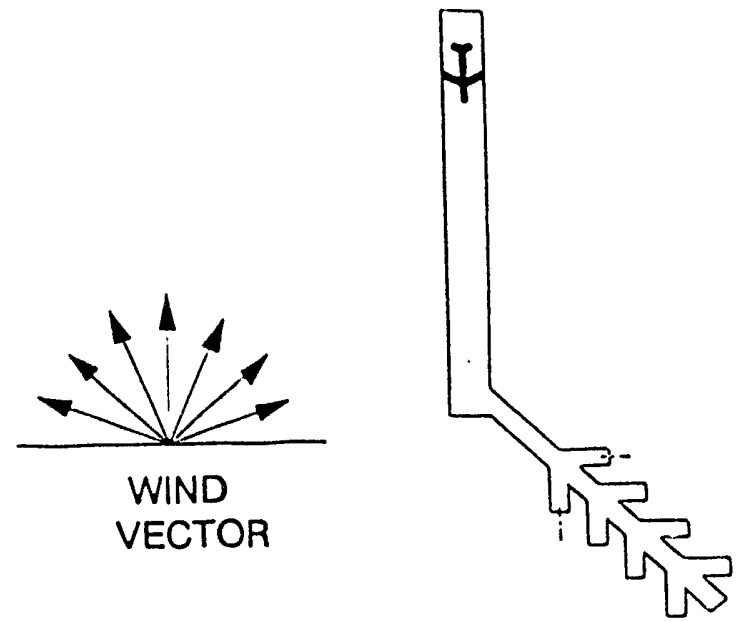

POTENTIAL COLLISION (b)

\section{LANDING}

TOUCHDOWN ON ALERT PAD END

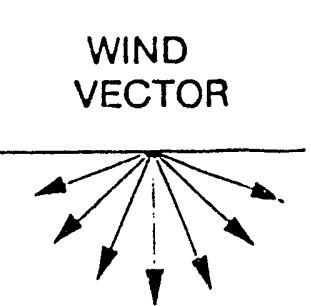

$\pm$

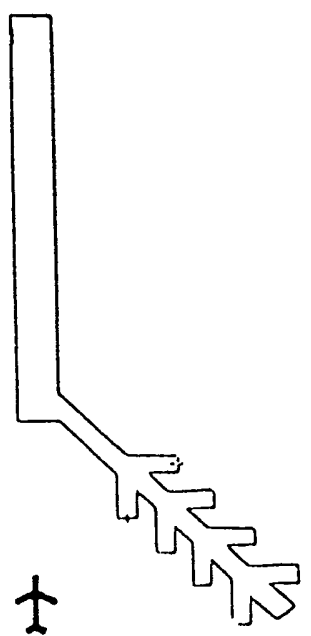

(c)

$$
\text { POTENTIAL. COLLISION }
$$

\section{LANDING}

TOUCHDOWN ON END OPPOSITE ALERT PAD

$\Varangle$
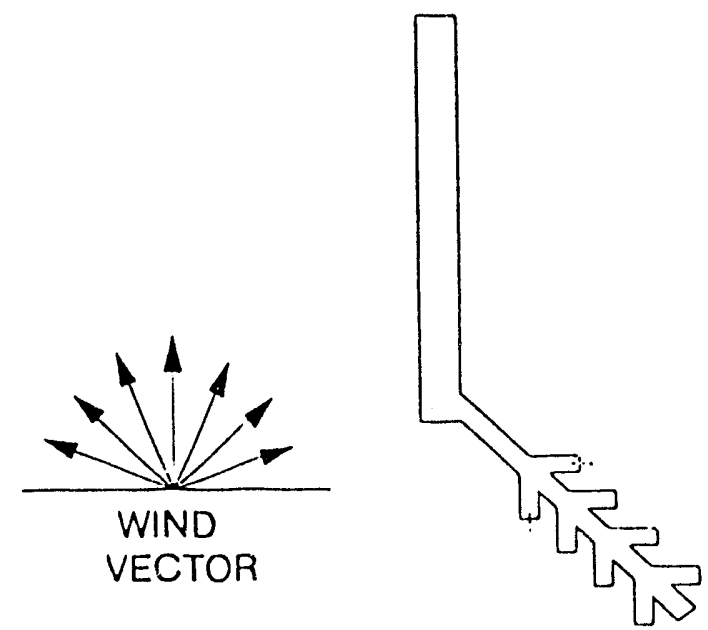

Figure 3.35 Landing and Take-off Configurations 
then a preponderance of spills on the downwind side (or end), a situation which minimizes the chance of cargo flame exposure.

The collision of a moving aircraft into an alert aircraft is quite different from the engine start accident in that (1), fuel spills can occur at any point over a comparatively extensive region, and (2), the range of fuel spill rates is comparatively large. To provide a perspective of the collision case, the sketches on Figure 3.36 illustrate to scale flame footprints, under various wind conditions from various spill locations at various rates.

Figure 3.36a shows flame footprints for the low, medium and high wind speeds at the 10 foot height for a medium rate spill (225 to $450 \mathrm{gpm}$ ) at the most remote wing location of the $\mathrm{B}-1 \mathrm{~B}$; the wind vector in this case bisects the line between the middle and aft weapon bays. It can be seen that exposure at both bays occurs for the high wind speed (the "longest" footprint) and medium speed. For the low wind speed (by far the most probable wind), the footprint does not reach either bay, and the spill point would have to be about mid-wing for exposure at low wind speed to occur. For wind directions favorable to exposure, low speed footprints for the rearmost fuselage spill point would reach the aft bay but not the middle bay. Similarly, for the forwardmost fuselage spill point, the middle but not the aft bay can be exposed for low speed winds.

Figure 3.36b shows a comparable arrangement for the B-52, where in this case the footprints and footprint locations correspond to the 7 foot height. With the longer wing of the B-52, bay exposure occurs only for the relatively infrequent high wind speed. Exposure can occur at medium speed for all but the most outboard wing spill points, and the low speed footprint would barely reach the bay for a mid-wing spill. For winds favorable to exposure, exposure can occur for any fuselage spill location for any wind speed.

The footprint with the largest crosssectional area (which does not have the greatest length) is illustrated on Figure 3.36c. This footprint is for the 1 foot height from a $600 \mathrm{gpm}$ spill at medium wind speed. It can be seen that there is exposure for the second wing spill point from the fuselage even when the cargo is upwind of the spill point. 

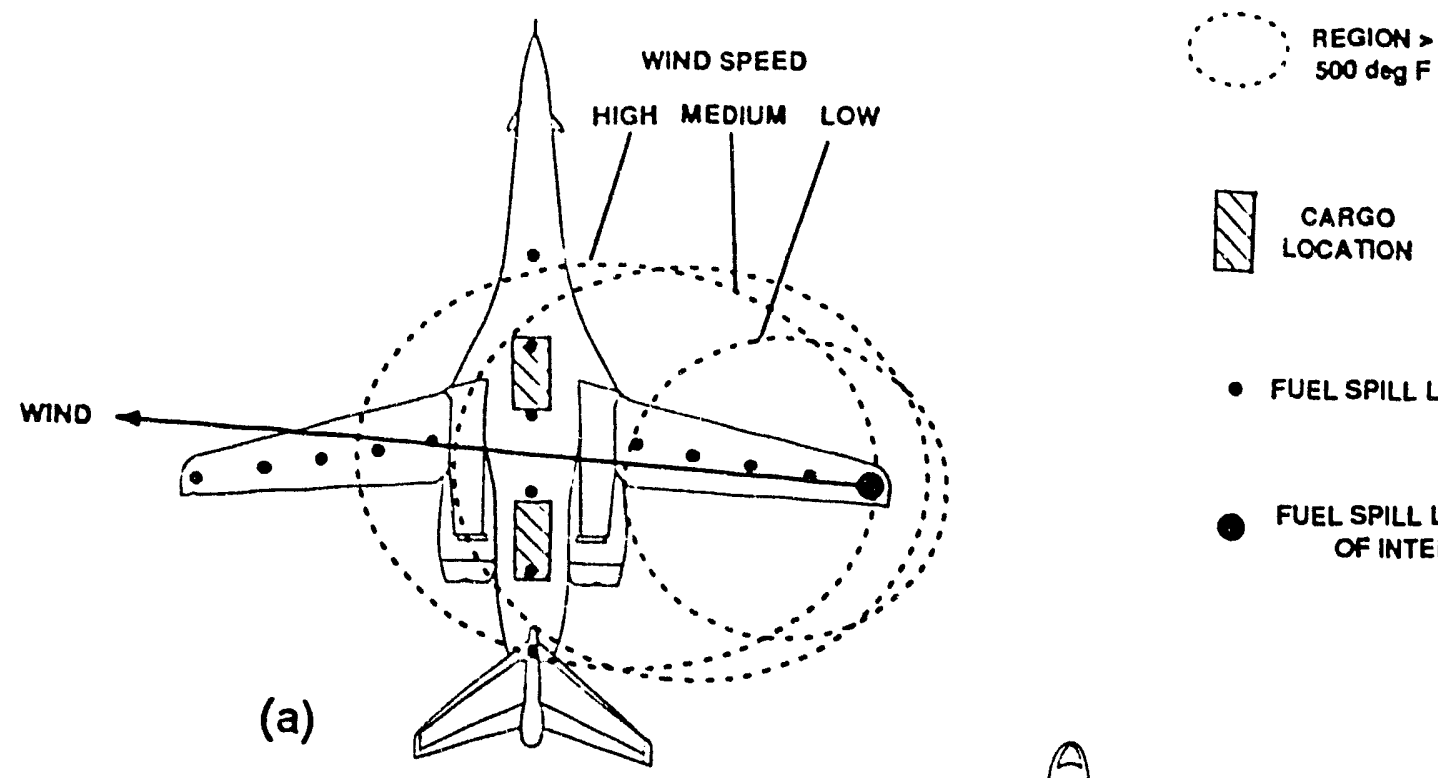

D. CARGO

- fuel spill location

FUEL SPILL LOCATION OF INTEREST

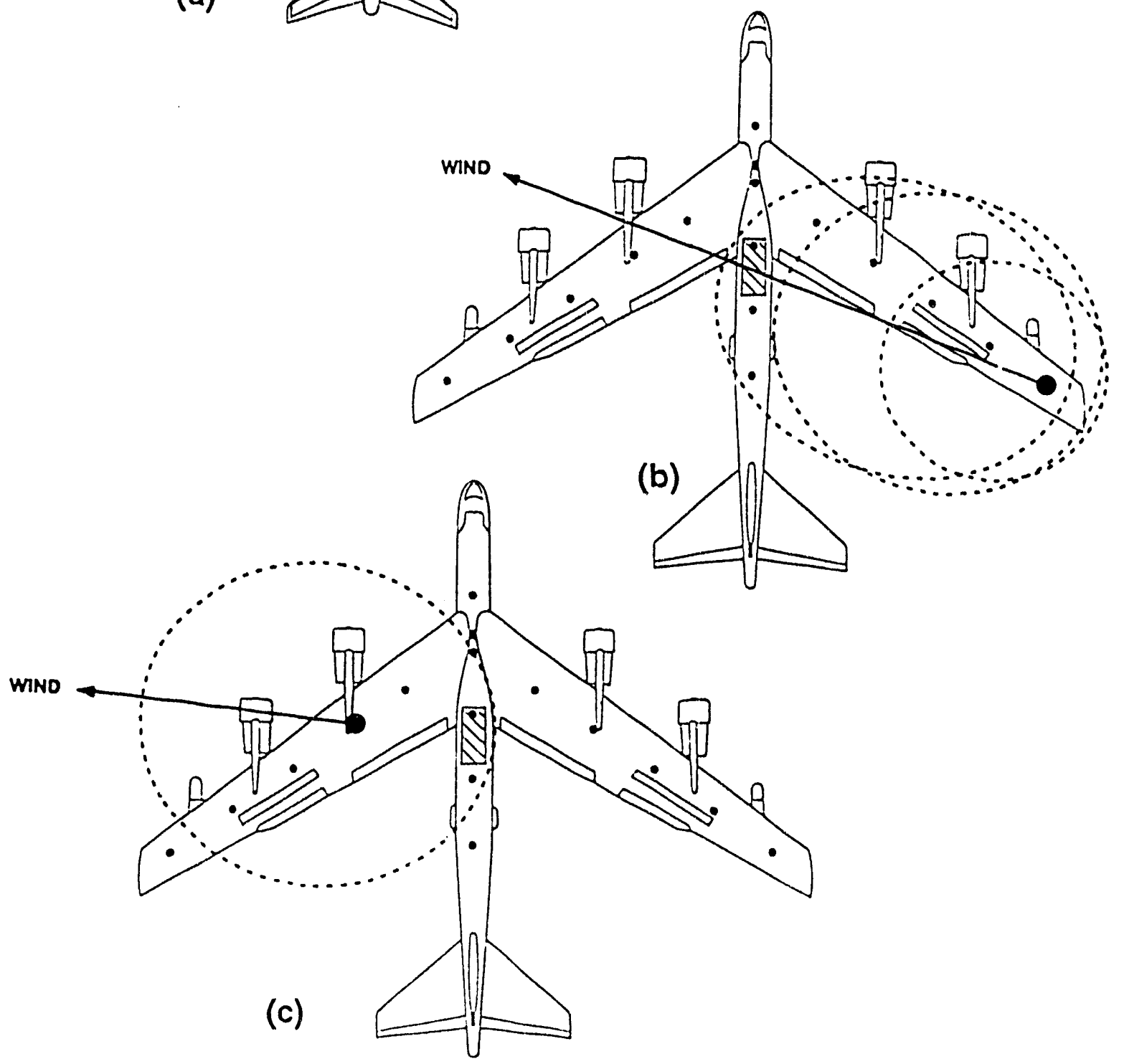

Figure 3.36 Example Flame Footprints for Collisions 
The collision of two aircraft can, of course, result in more than one fuel spill location. Indeed, on the basis of the fuel spill location distributions developed for collisions at various angles, by far the most common number of spills for all collision distributions is three. Fairly often, a triple spill case is comprised, as illustrated on Figure 3.37a, of a wing location and fuselage location on the alert aircraft, and a wing location on the colliding aircraft. In evaluating probabilities of temperature exposure for multiple spills, the temperature chosen as the exposure temperature for a given set of conditions is the highest temperature the cargo experiences from any of the flames that exist. Moreover, since it is often only possible to determine the location of a given spill to within a location range, the analysis is conducted for more than one location for that given spill. Thus, for a triple spill, for example, there are several possible locations for each of the three spills, and the analysis includes the evaluation of exposure temperature for all combinations of where the three spills can be located; there is then an appropriate statistical weighting for these combinations.

Even though the triple spill is common, there is a sizeable number of conditions (wind directions, wind speeds, etc.) for which the cargo is not exposed to flame. Figure $3.37 \mathrm{~b}$ is an illustration of such conditions, and it is easy to visualize that a sensible fraction of conditions will not result in cargo
exposure. 


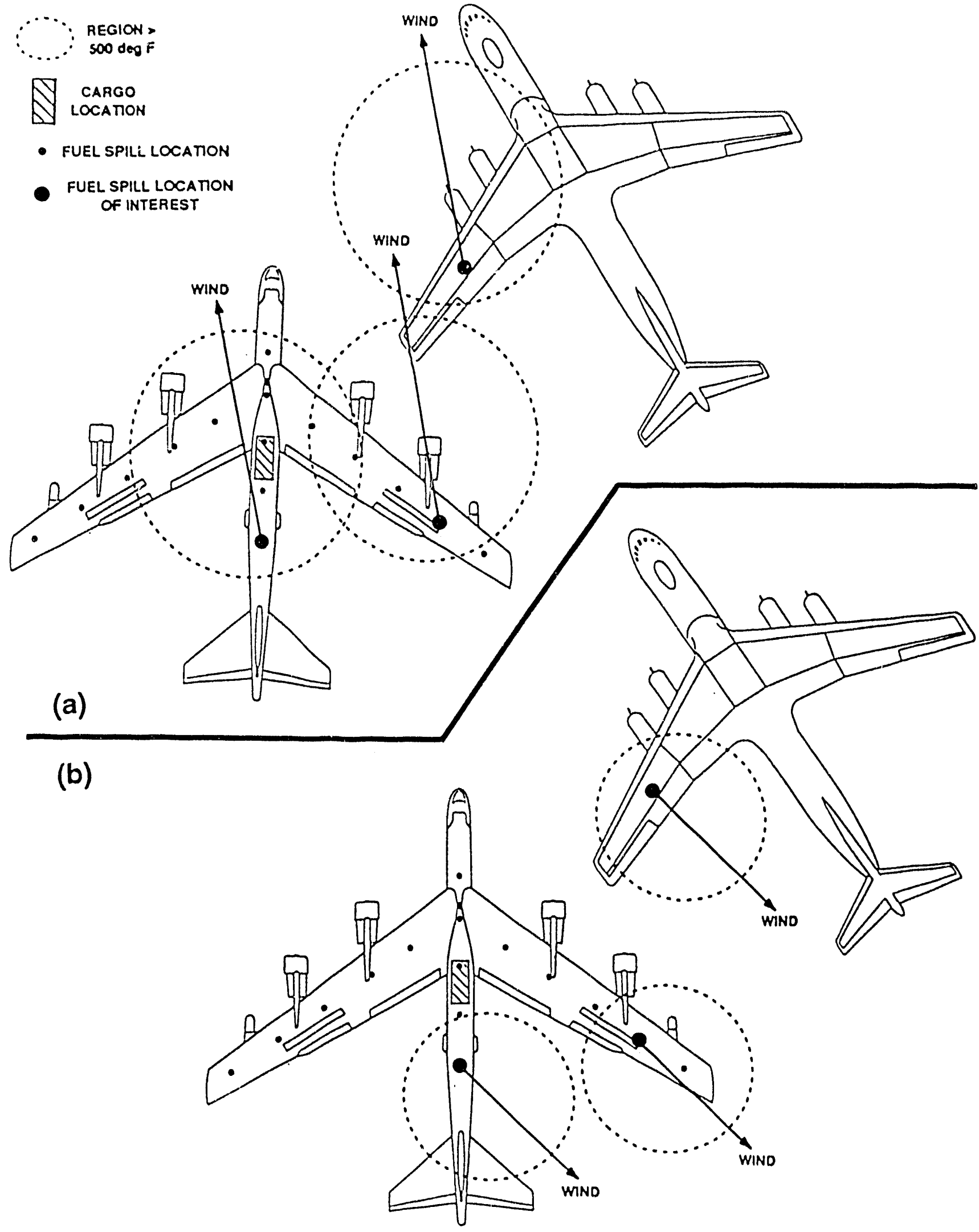

Figure 3.37 Example Flame Footprints for Triple Fuel Spill 
Example results for the B-52 alert aircraft are given on Figure 3.38. Each graph on Figure 3.38 includes three probability vs temperature curves, one for the case where the colliding aircraft is on landing, a second where the colliding aircraft is on take-off, and the third representing the weighted mean probability for collisions on landing and take-off, where the mean is statistically weighted according to the relative number of landing and take-off "crashes" which result in fuel fires. Based on references 5 and 10, a baseline value of 3.0 is used for the ratio of landing to take-off accidents involving fires; the effect of a variation in this ratio is given in subsection 3.5 .

The results for Barksdale and Eaker AF Base on Figure 3.38a and 3.38b are typical for the B-52, with regard to both the general magnitude of the probabilities with temperature and the difference between the probabilities for landing and take-off. The magnitude of the take-off probability for Castle AF Base on Figure 3.38c, however, is unusually low. The cause of this low probability relates primarily to the unusual wind characteristics at Castle AF Base. As shown in subsection 3.2, winds at Castle AF Base are characterized as having a single highly predominant angular range from about 320 to 350 degrees. As illustrated in Figure 3.39, take-offs starting from the end of the runway opposite the alert pad (take-offs that can result in collision) occur only for wind directions with an air flow velocity component which opposes the motion of the aircraft taking off, that is, for winds over the 180 degree direction range from 50 to 230 degrees. The predominant wind range is then outside of the 50 to 230 degree range, and the probability for winds in the 50 to 230 degree range is unusually small. Take-offs that result in collisions with an alert aircraft thus represent an unusually small fraction of the total number of take-offs (from both ends of the runway), and the probability vs temperature values for take-offs at Castle AF Base are consequently comparatively small.

A summary of the mean probability vs temperature for all B- 52 bases is given on Figure 3.40. The overall mean probability averaged over all bases has not been evaluated; the overall mean depends on the relative number of operations (landings and take-offs) from base to base, and traffic data was not available for all B-52 bases. 


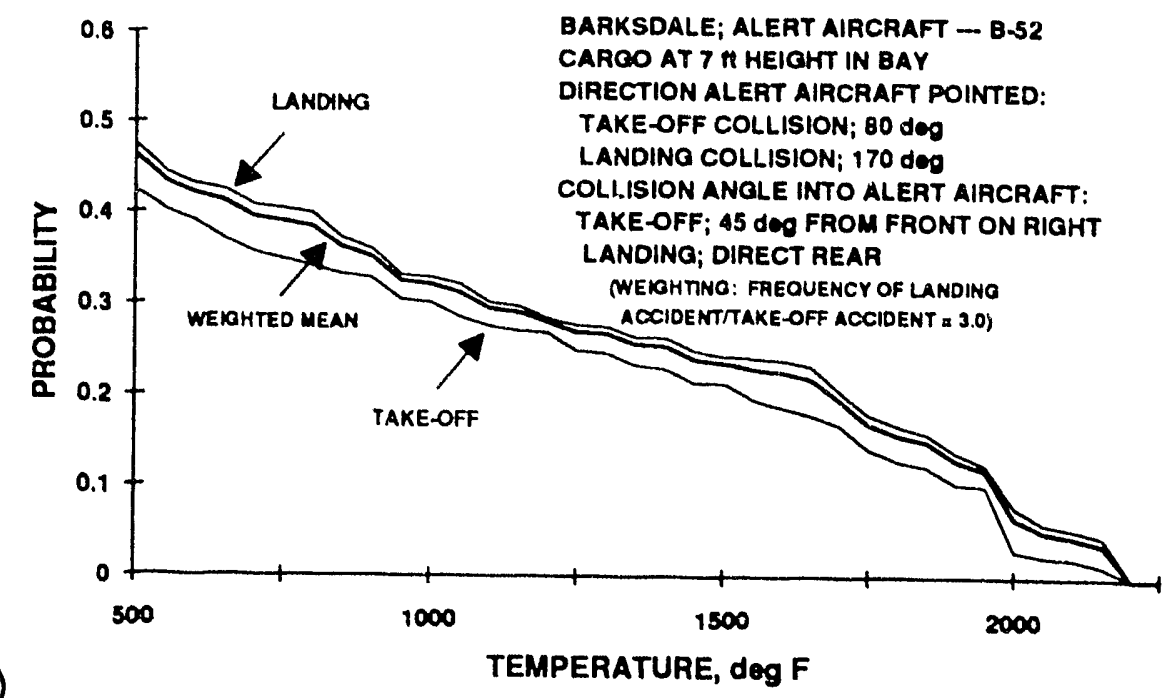

(a)

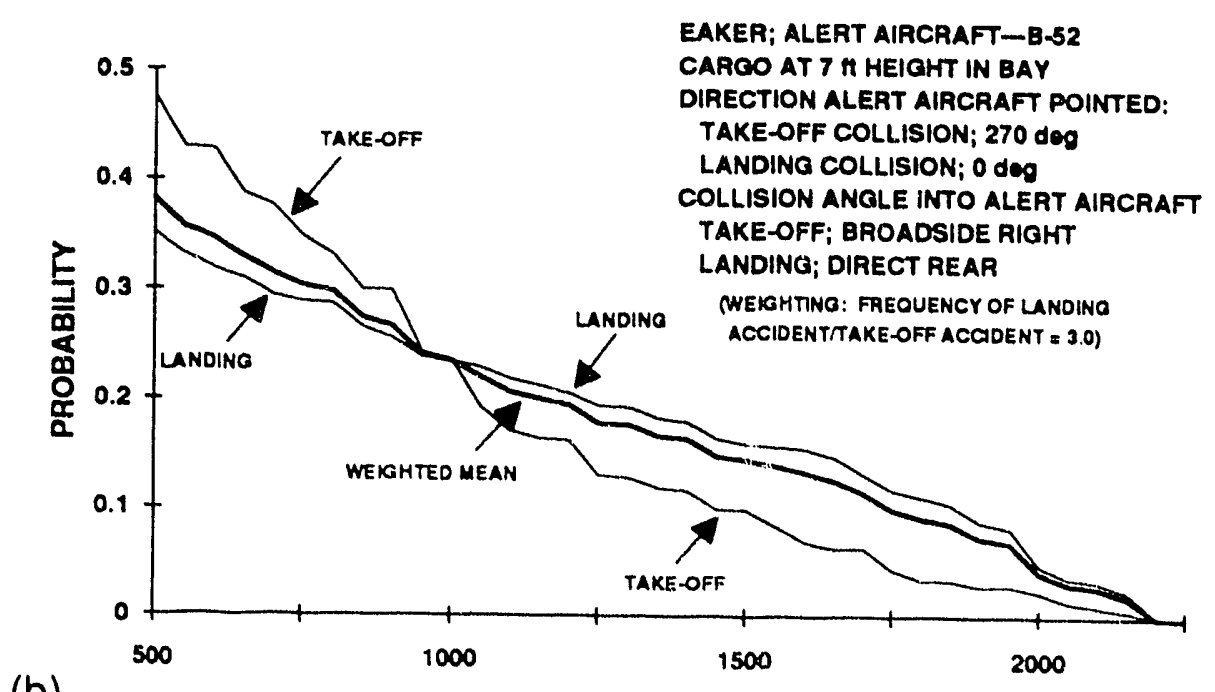

(b)

TEMPERATURE, $\operatorname{deg} F$

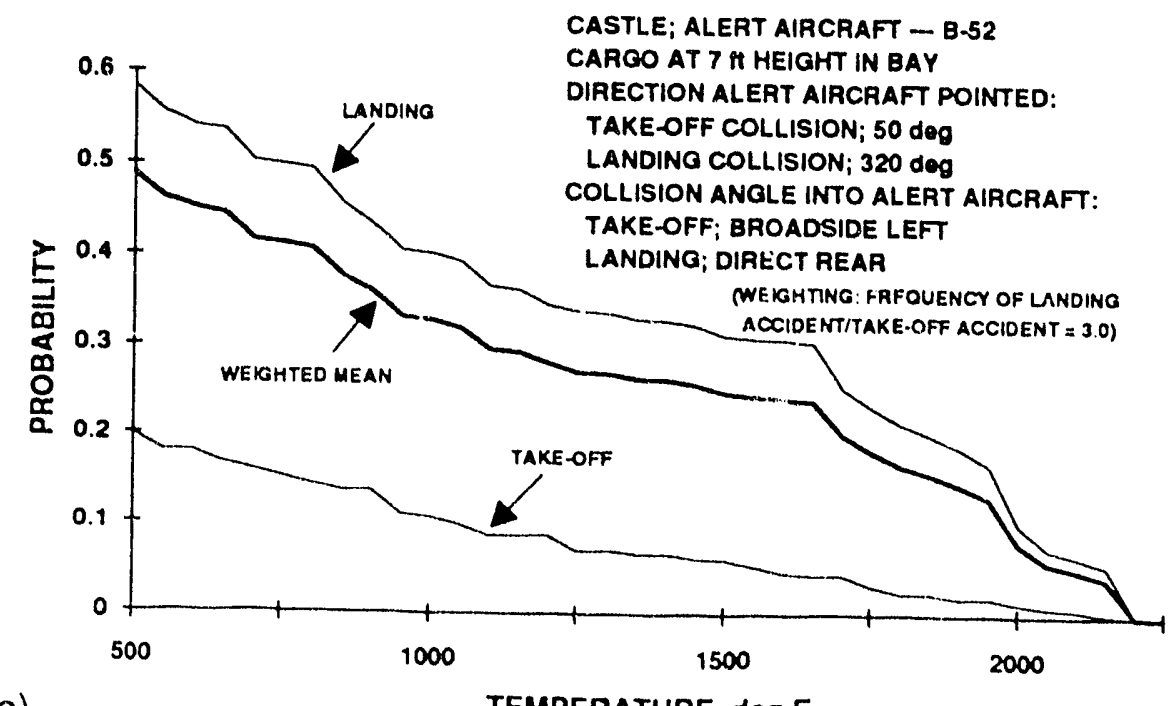

(c)

TEMPERATURE, deg $F$

Figure 3.38 Example Landing/Take-off Collision Baseline Results for B-52 


\section{CASTLE AF BASE, CA}

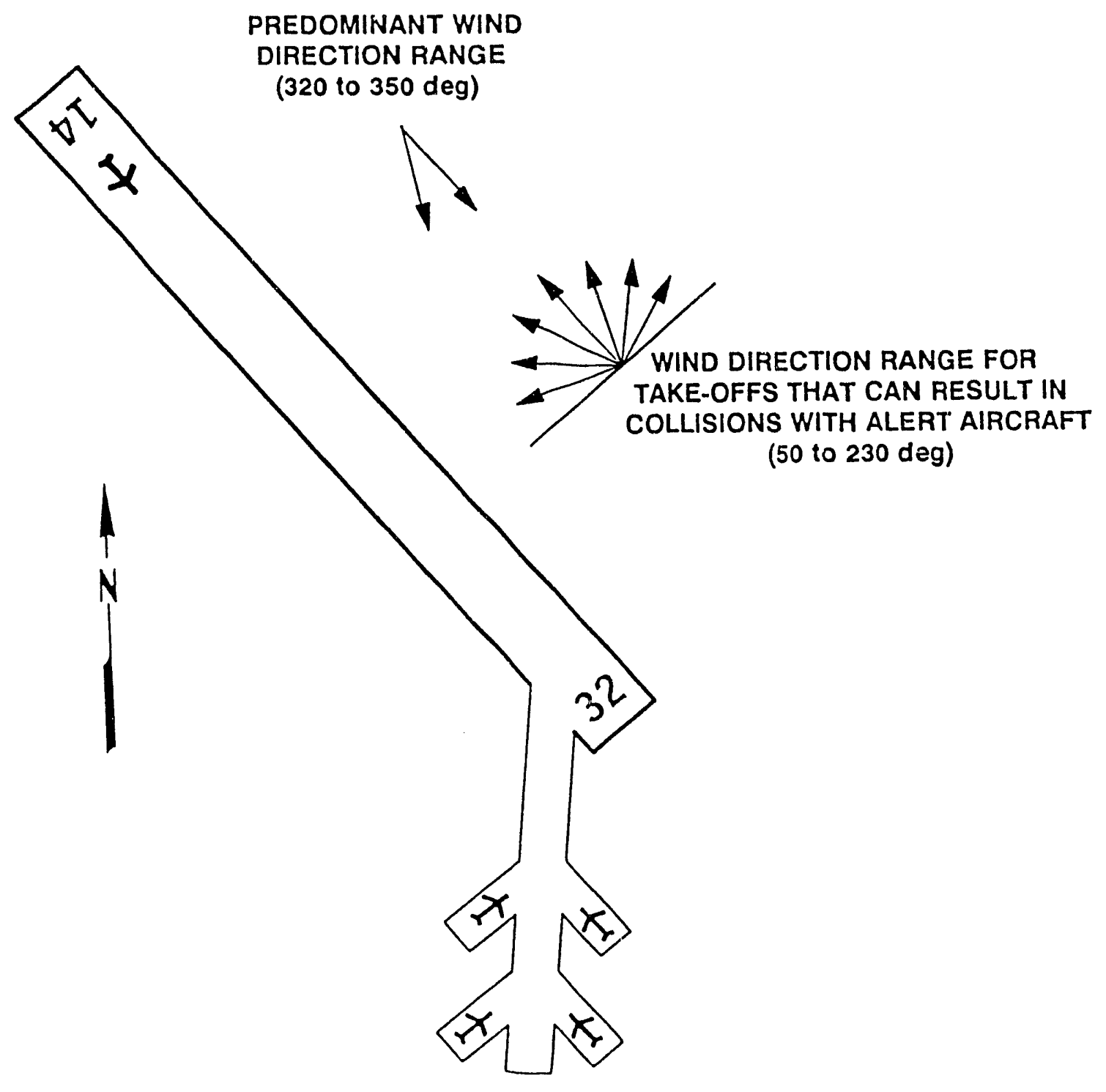

Figure 3.39 Collision Configuration at Castle AF Base 

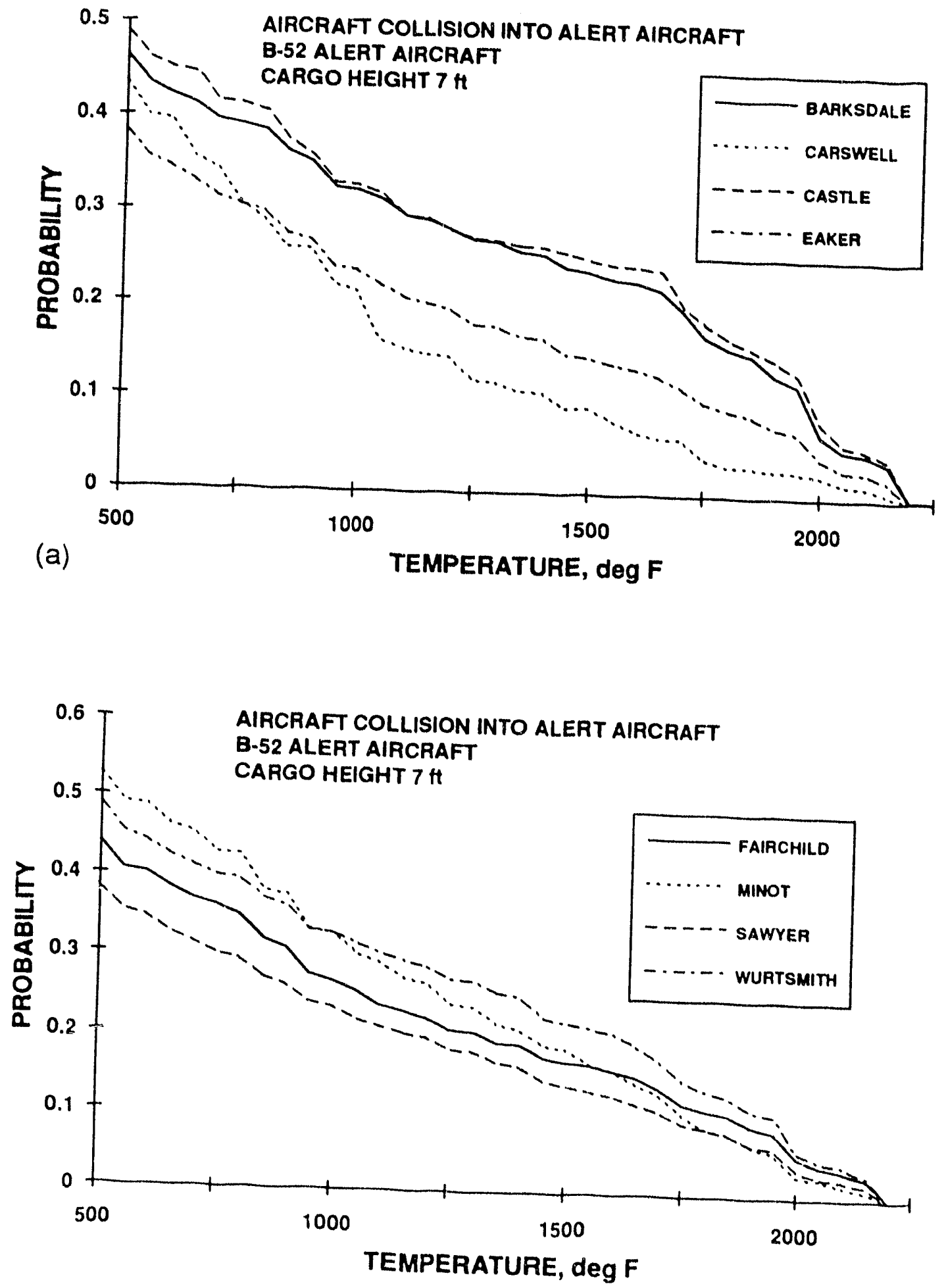

(b)

Figure 3.40 Summary of Baseline Results for Collision into Alert B-52 
For the B-1B with weapons in the middle bay, plots of the probability for landing, take-off and the weighted mean are given for all four bases on Figure 3.41. A summary of the weighted mean probability for each of the bases is given on Figure 3.42 .

It can be seen from Figure 3.41 that the probability for take-off at Ellsworth AF Base (Figure 3.41b) is comparatively low, and that the probability for both landing and take-off is comparatively low for McConnell AF Base (Figure 3.41d). Rather than weather, the primary cause of these low values is related to collision angle and the relative location of cargo and fuel spills. Regarding the cause, it is noted first that the collision angle into the alert aircraft in these cases (and no other) is "direct rear". Consider next the wing and fuselage fuel spill locations for the B-1B on Figure 3.43a. The wing spill points are all aft of, and therefore downwind of, cargo in the middle bay. In addition, for a direct rear collision, the engine and wing structures provide considerable "protection" to the forward fuselage tanks, particularly those that are near and forward of the middle bay. In this regard, fuel spill data along the fuselage has been extracted from the fuel spill probability distribution that was developed for a direct rear collision into a B-1B. This extracted data is plotted on Figure 3.44 in terms of probability of a fuselage fuel spill vs distance from the center of cargo in the middle bay. It can be seen that the probability for fuel spills forward or upwind of the cargo is comparatively small.

For the B-52, it may be recalled from Figure 3.38 that direct rear collisions did not result in unusually low probabilities with temperature. In this regard, it can be seen from Figure $3.43 \mathrm{~b}$ that, in contrast to the B-1B, there are wing spill points forward or upwind of the cargo. In addition, there is less "protection" of fuselage tanks near or forward of the cargo compared to the B-1B. A data extraction from the fuel spill probability distribution developed for a direct rear collision into the B-52 shows on Figure 3.44 that the probability of fuselage spills forward or near the cargo is significantly higher for the B-52 than for the B-1B. 


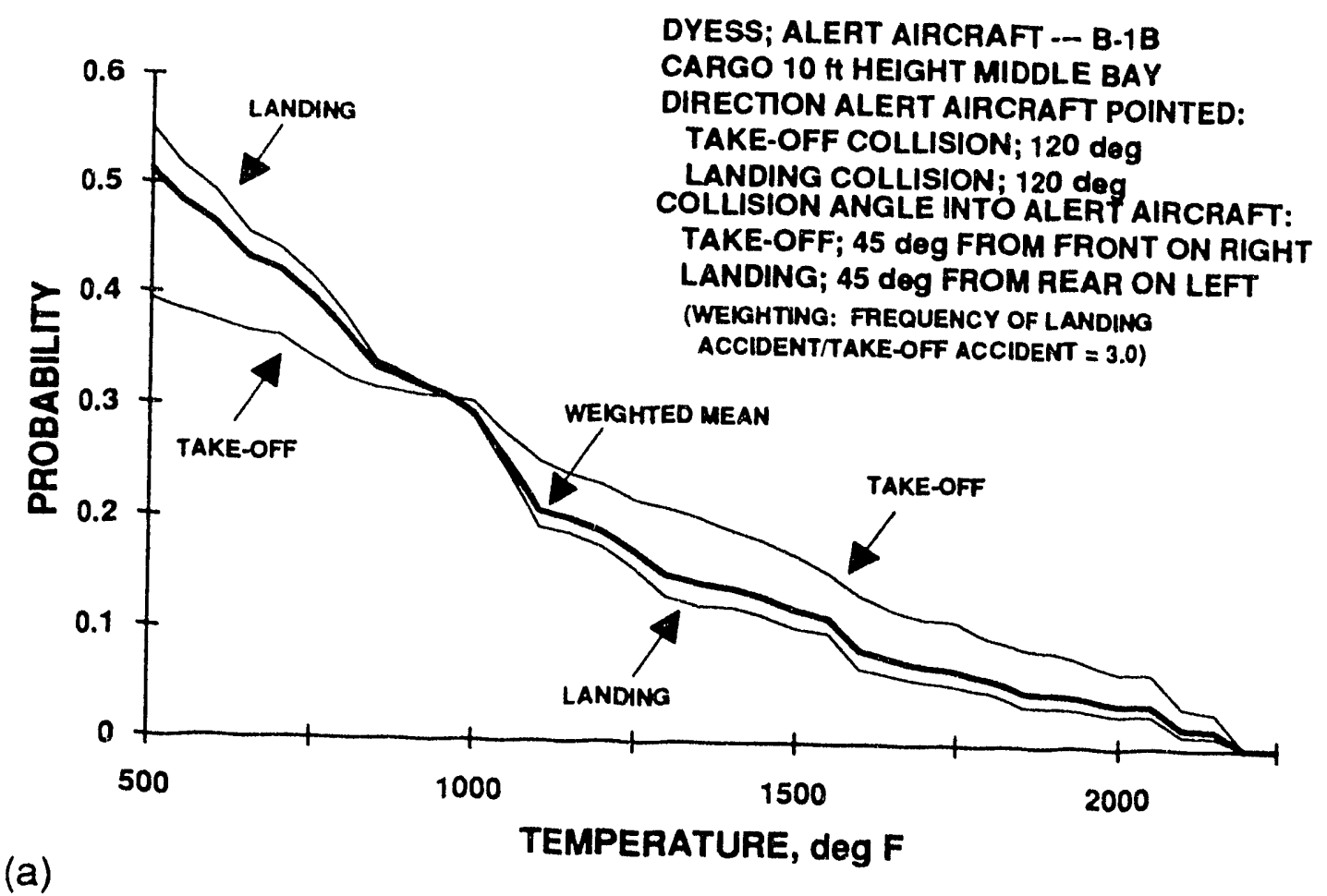

(a)

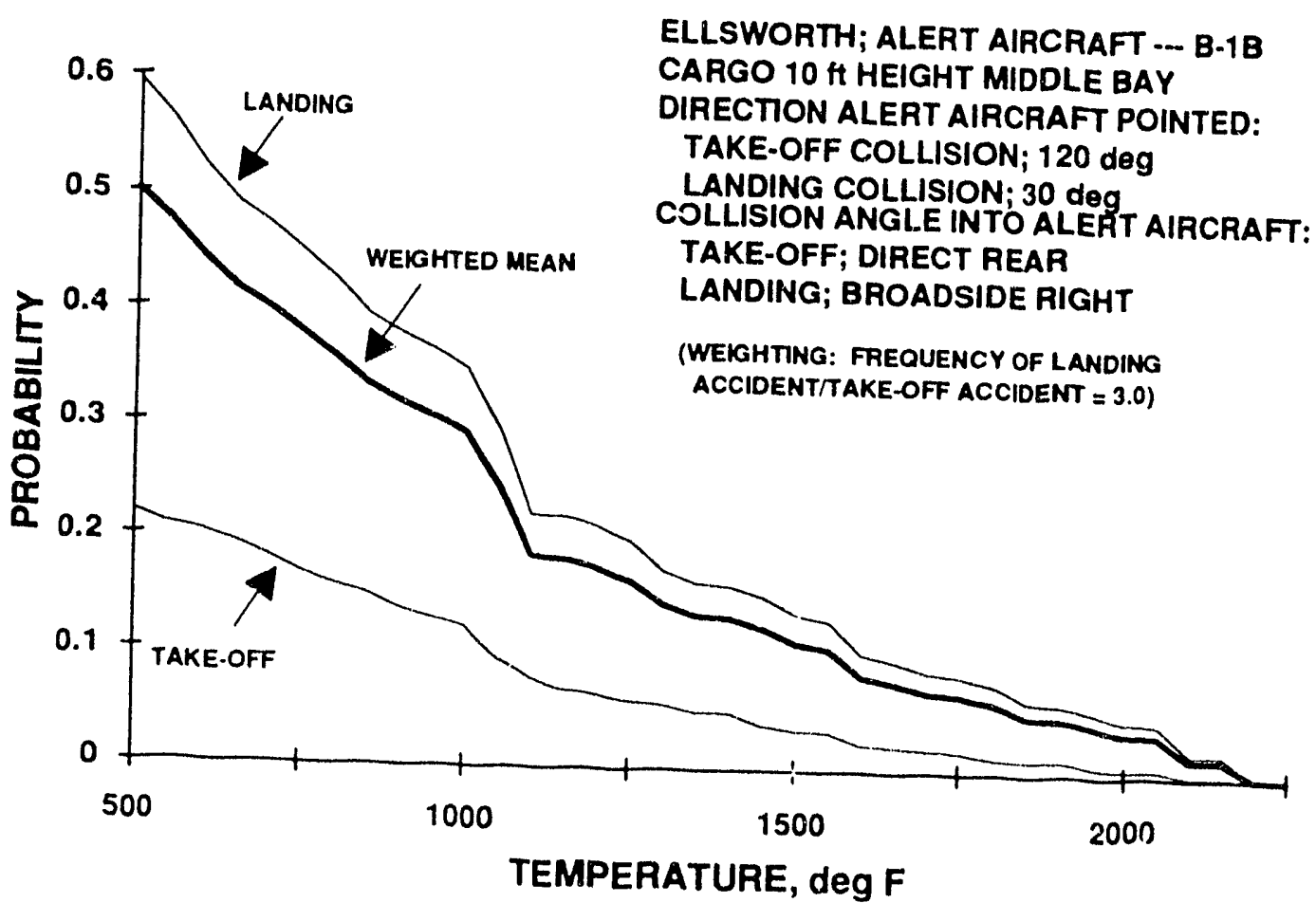

Figure 3.41 a \& b Landing/Take-off Collision Baseline Results for B-1B at Middle Bay 


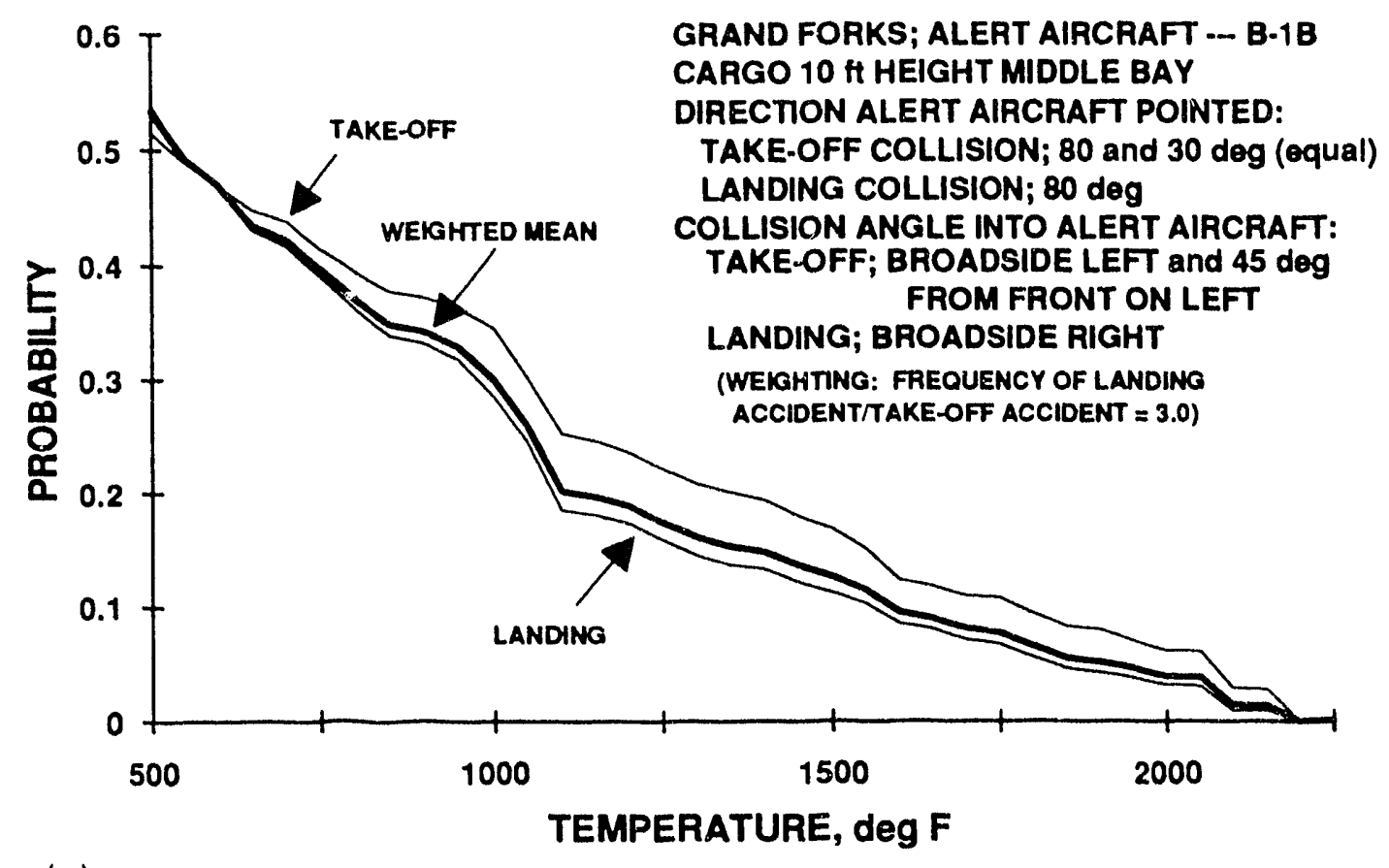

(a)

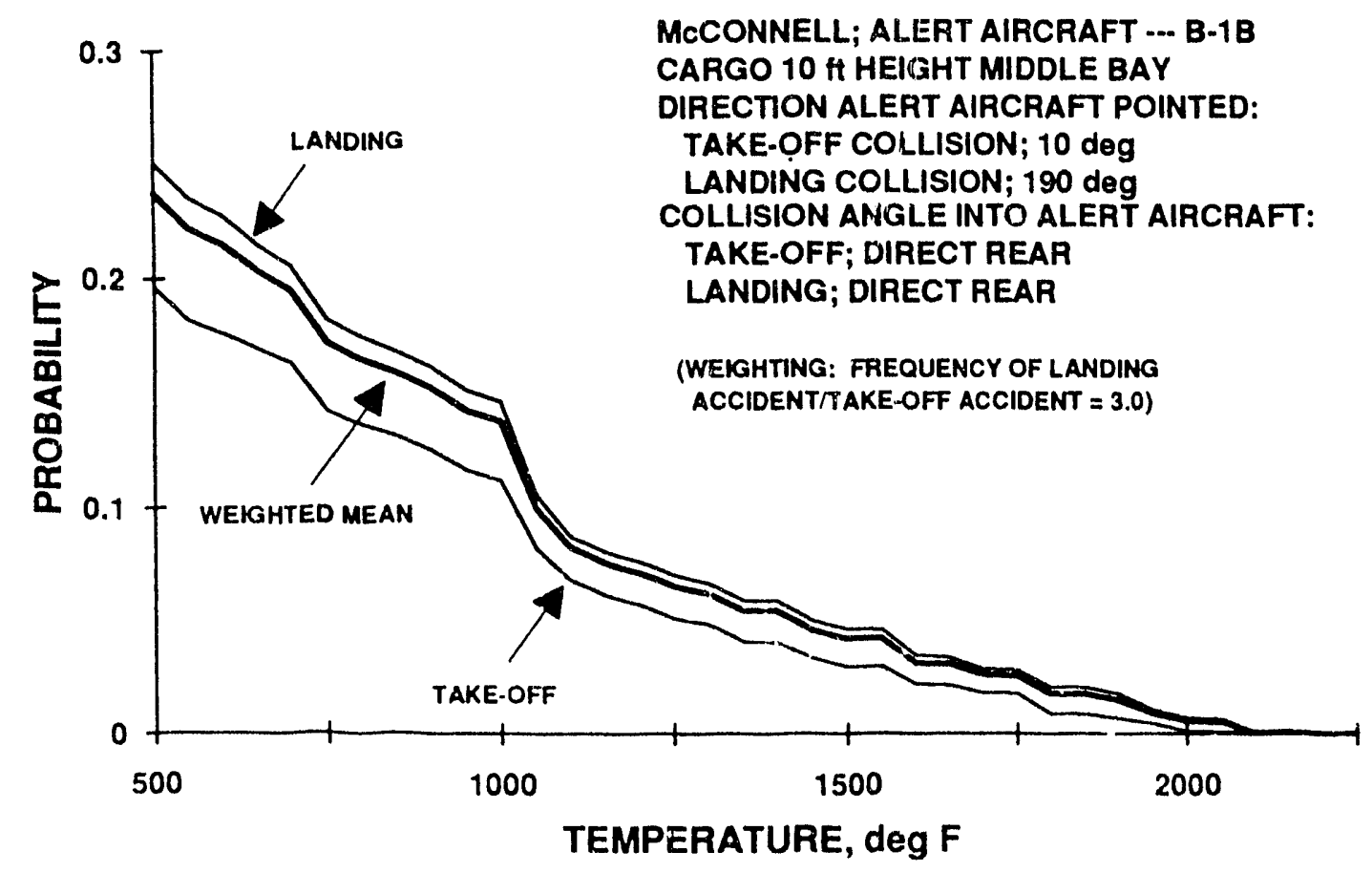

(b)

Figure 3.41 c \& d Landing/Take-off Collision Baseline Results for B-1B at Middle Bay 


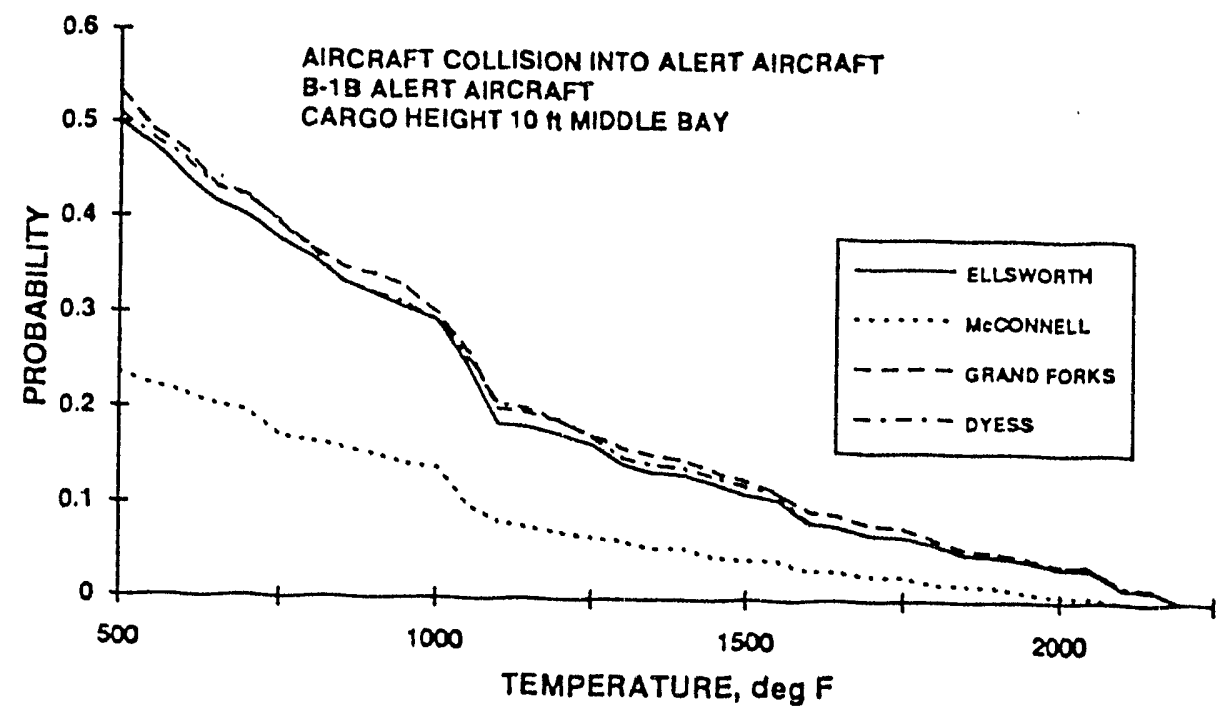

Figure 3.42 Summary of Collision Baseline Results for B-1B at Middle Bay

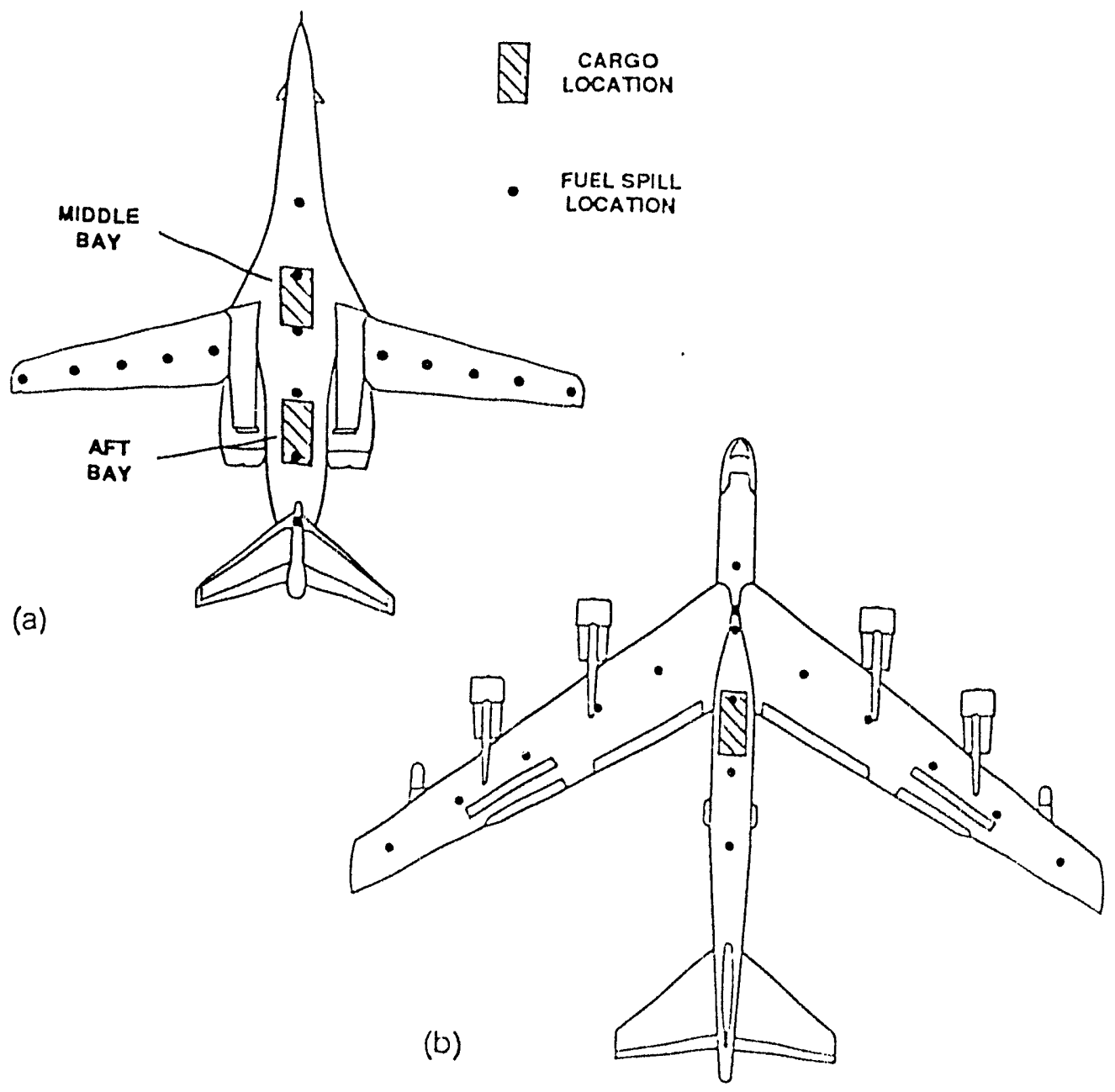

Figure 3.43 Fuel Spill/Cargo Location Relationship for Collisions 
FUEL SPILL PROBABILITY DISTRIBUTION ALONG FUSELAGE

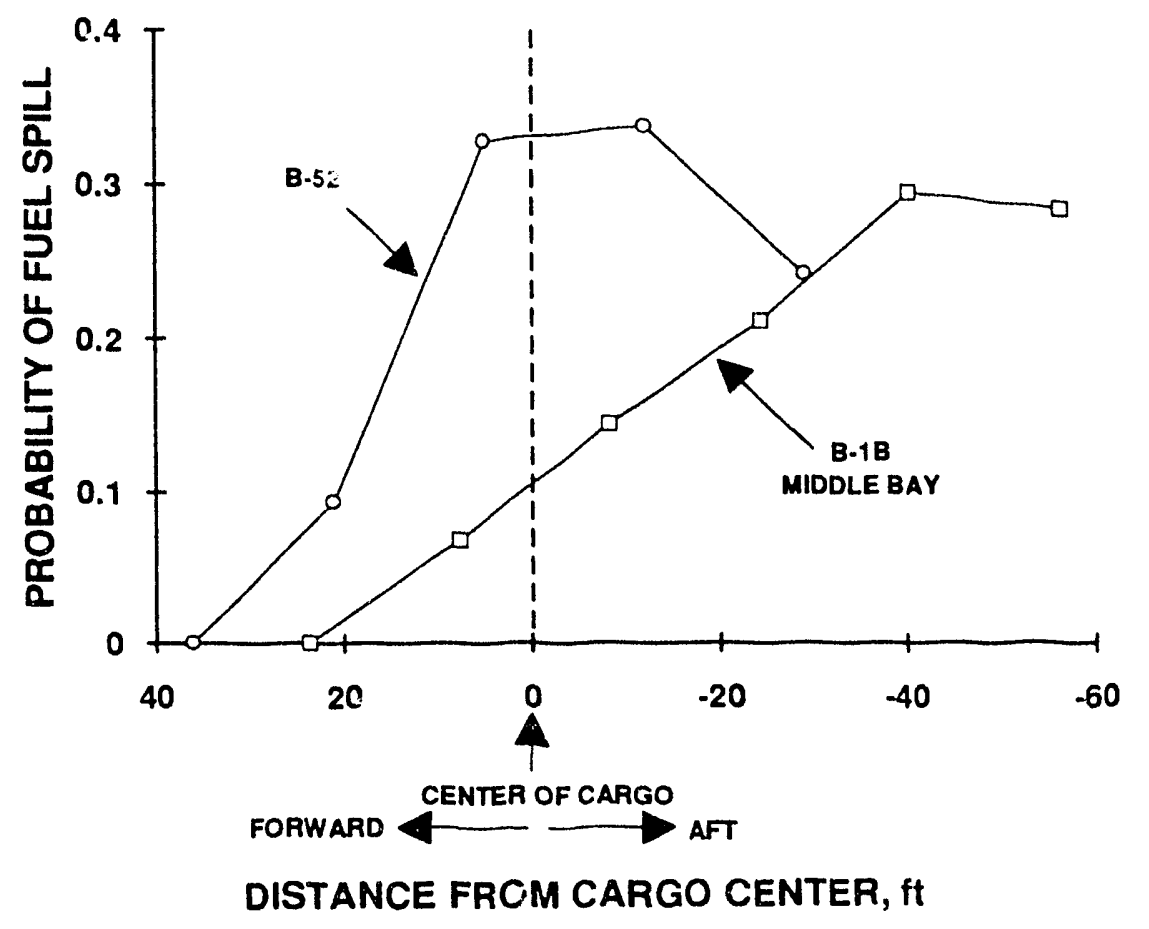

Figure 3.44 Fuselage Fuel Spill Distributions

For the case of cargo in the aft bay of the B-1B, the likelihood of spills forward or upwind of the cargo is substantially higher than for the middle bay. Wing spills are now forward of the cargo, and the "protection" of fuselage tanks near or forward of the bay is reduced. The landing, take-off and mean probability with temperature for aft bay cargo at McConnell AF Base is given in Figure 3.45. It can be seen that the probatility of exposure for direct rear collisions is significantly higher at the aft bay than the middle bay (ref. Figure $3.41 d)$.

The mean probabilities for the aft bay for each of the B-1B bases, weighted by the relative frequency of landing and take-off crashes, is given on Figure 3.46 . 


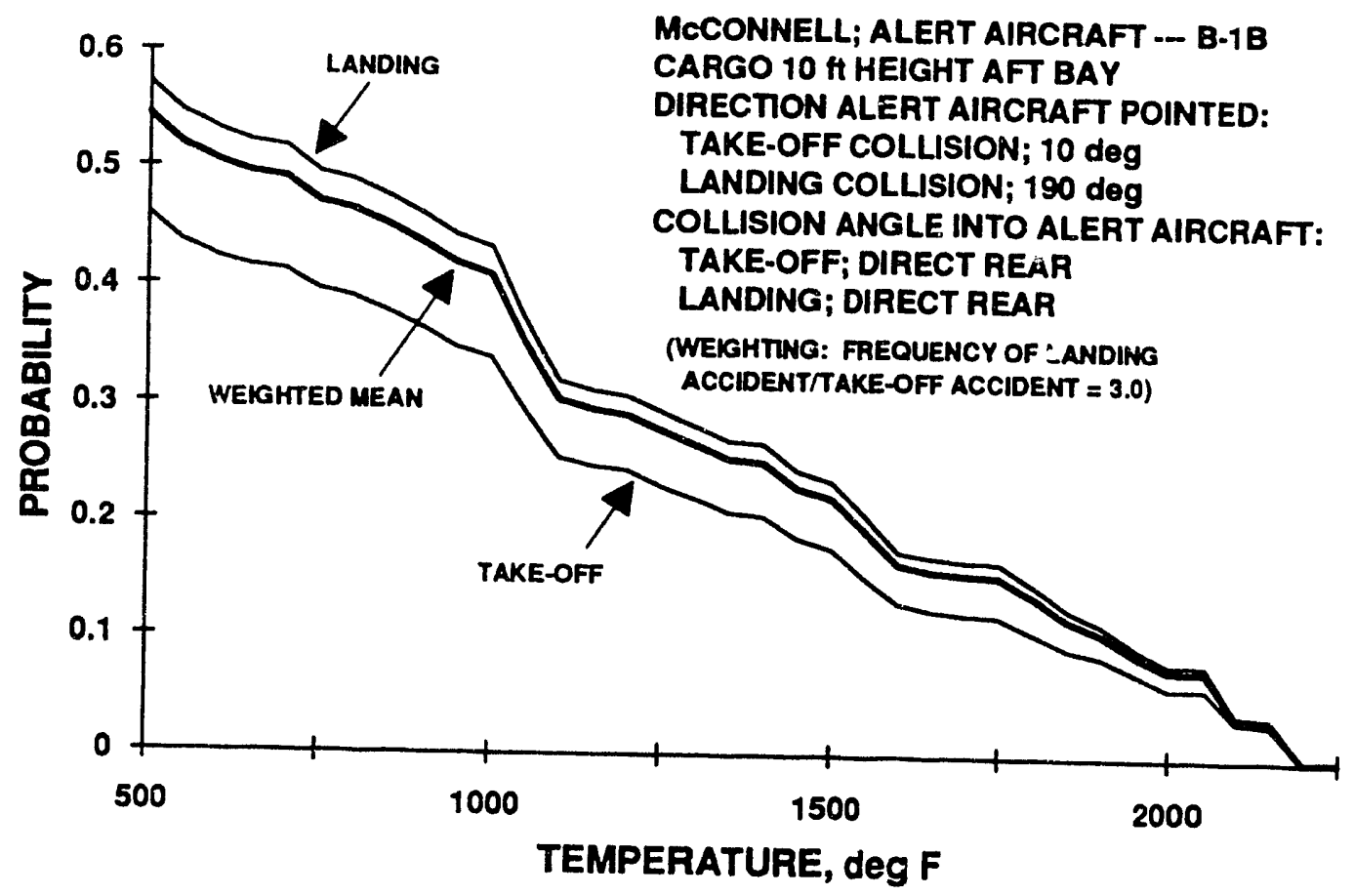

Figure 3.45 Landag/Take-off Results for B-1B at Aft Bay

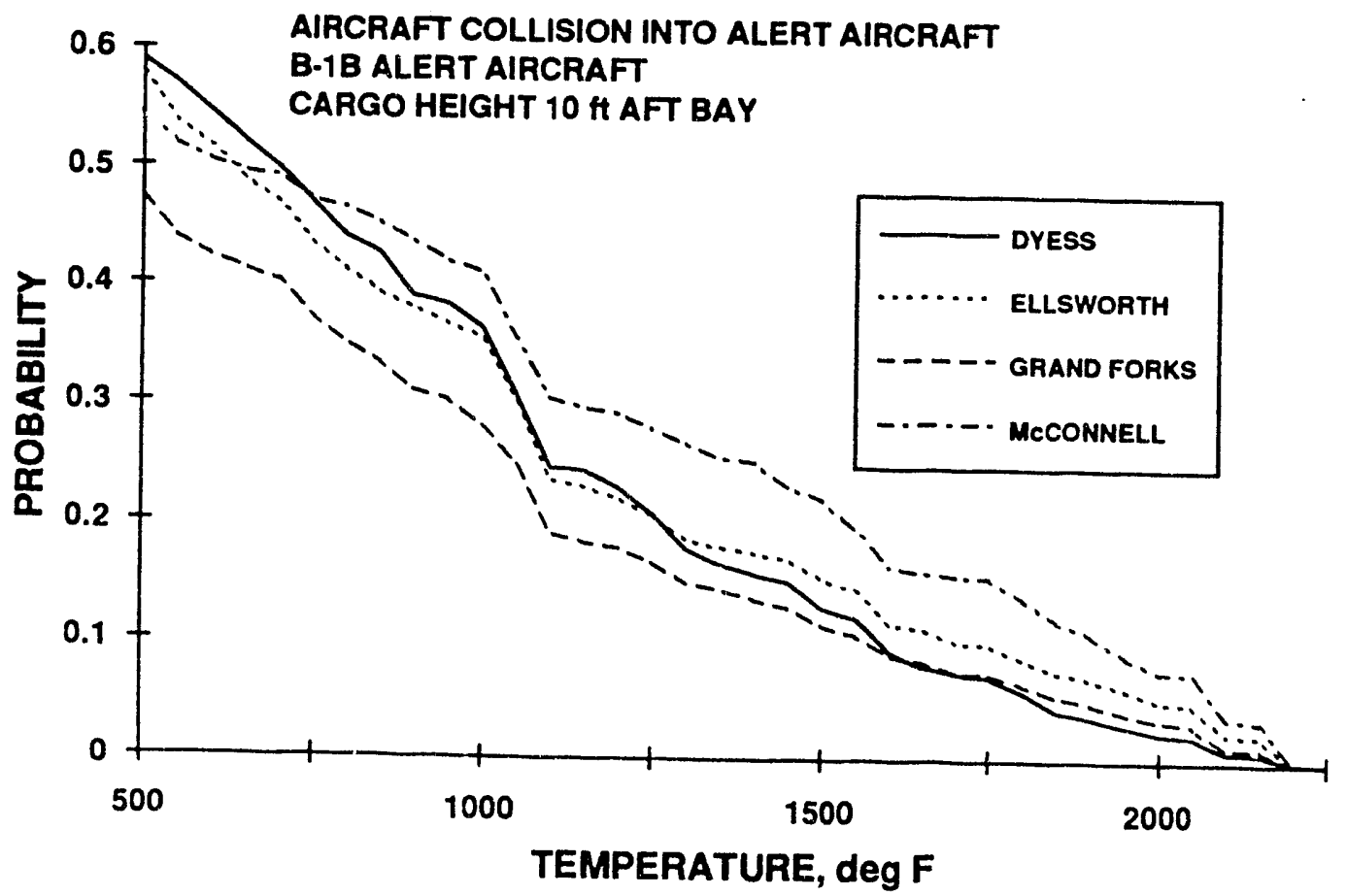

Figure 3.46 Summary of Results for B-1B at Aft Bay 
Data on the number of landing or take-off operations for all four B-1B bases is available in reference 13 . Consequently, the mean B-1B base probability can be evaluated, where the probability for each base is weighted in proportion to the number of landing/take-off operations (per unit time) at each base. Based on reference 13, the ratio of the number of operations at each base to the total number of operations at B-1B bases is:

\begin{tabular}{ll} 
DYESS & 0.27 \\
ELLSWORTH & 0.21 \\
GRAND FORKS & 0.15 \\
MCCONNELL & 0.37 \\
\hline TOTAL & 1.00
\end{tabular}

The mean B-1B base probability vs temperature, based on these weighting factors, is given on Figure 3.47 for the middle and aft bays for the 10 foot height. For these mean probabilities, the values for the middle bay are significantly lower than for the aft bay. The comparatively low probability for the middle bay is due largely to the low probability and high fraction of total operations at McConnell AF Base.

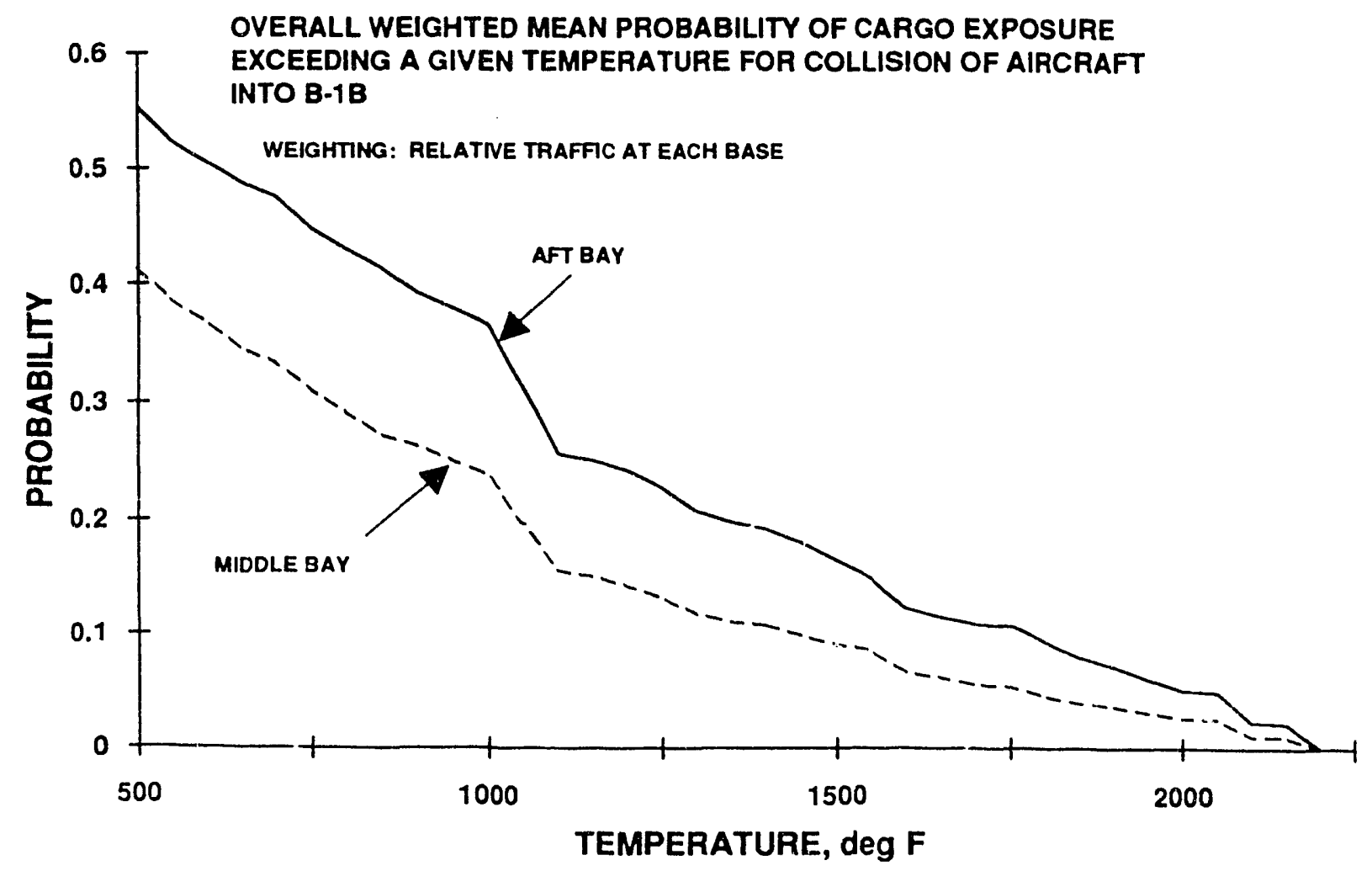

Figure 3.47 Mean B-1B Collision Results 
Results presented thus far have been for the condition that the direction of motion of the colliding aircraft at collision is parallel or nearly parallel to the runway, whereas collisions for other directions of motion can occur. "Off-angle" collision results provide a great deal of insight into the nature of aircraft collision flame exposure, and a number of results are presented and discussed below.

The sketch for Eaker AF Base on Figure 3.48 helps to illustrate the general circumstance of off-angle collisions. For Eaker AF Base, the normal landing direction is the same as the pointing direction of the alert aircraft, so that a baseline landing collision is a direct rear collision, and this would occur only for wind flow directions in the range 90 to 270 degrees, as illustrated in Figure $3.48 \mathrm{a}$. If the direction of motion of the landing aircraft at impact has become 45 degrees clockwise of the normal landing direction, the collision into the alert aircraft will be on the left of the alert aircraft from the direction 45 degrees from the rear of the alert aircraft, and the fuel spill probability distribution for a 45 degree from rear on left collision will be used to evaluate the temperature probabilities; however, the wind range involved in evaluating this landing collision remains 90 to 270 degrees, since otherwise this landing attempt on the alert pad end of the runway would not have occurred.

Similarly, for collisions on take-off at Eaker AF Base, the collision would be broadside right for motion directions parallel to the runway, as illustrated in Figure $3.48 \mathrm{~b}$, and the wind flow direction range would be limited to 270 (through 0 ) to 90 degrees. For a direction of motion that has become 45 degrees clockwise from the normal take-off direction, the collision will be on the right of the alert aircraft from the direction 45 degrees from the rear of the alert aircraft, and the fuel spill probability for a 45 degree right rear collision will be used for temperature probability evaluation. 


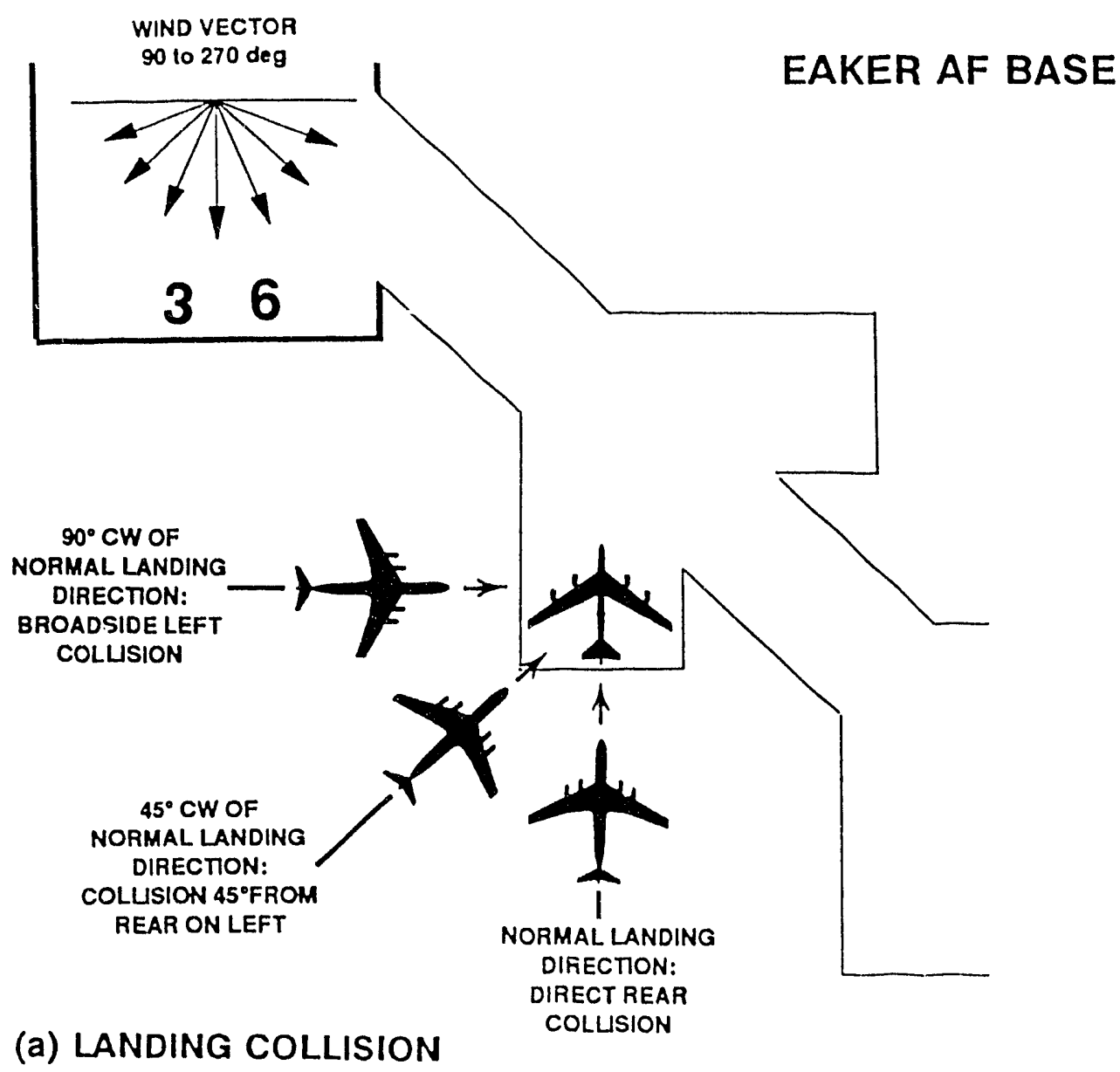

(a) LANDING COLLISION

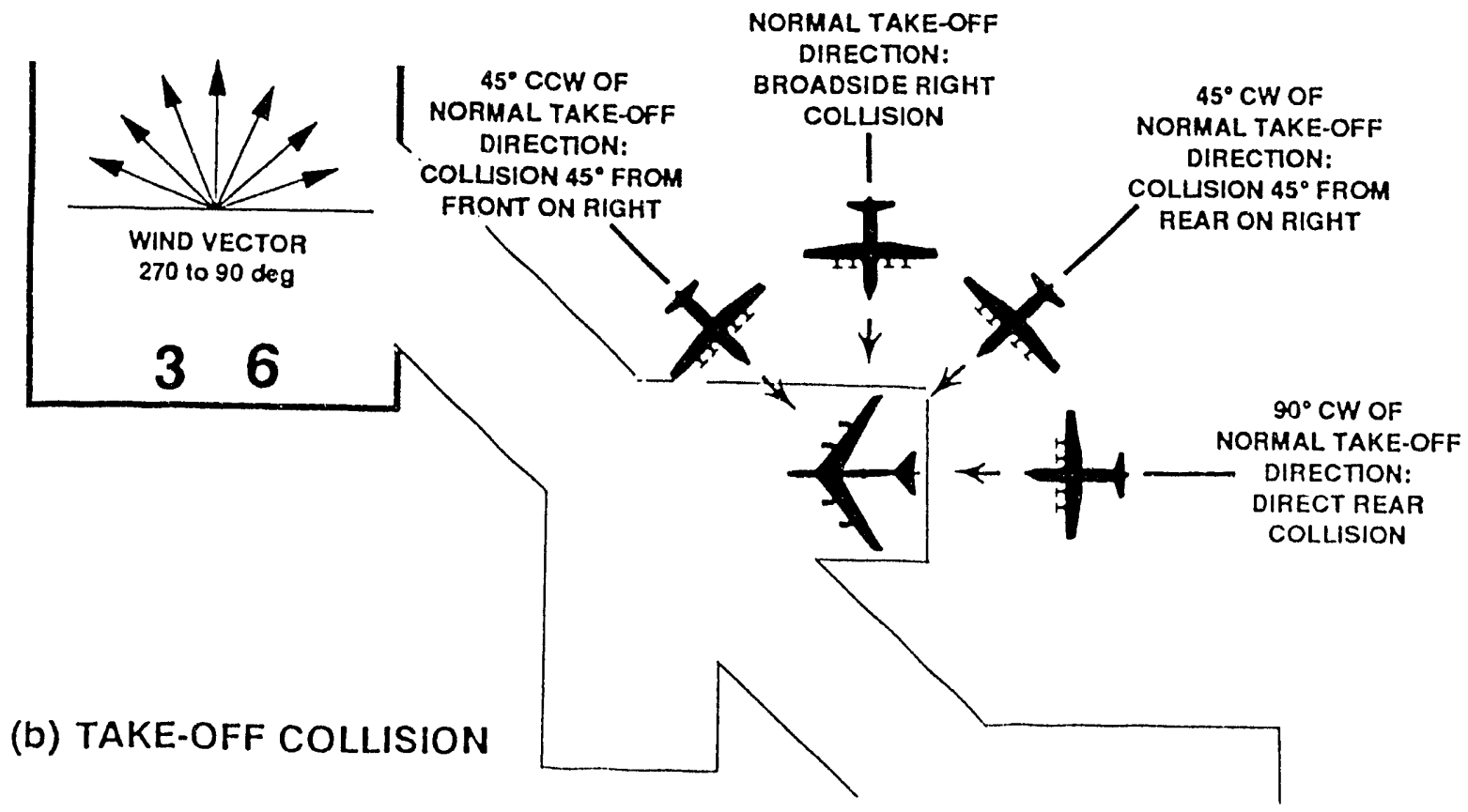

Figure 3.48 Off-Angle Collision Configuration for Eaker AF Base 
Example results for various motion angles at collision are given on Figure 3.49 for a landing collision at McConnell AF Base. It can be seen that the probabilities are significantly dependent on motion angle, and that probability appears to increase with motion angle from zero, where zero angle in the case of a landing collision at McConnell represents a direct rear collision. It is more revealing to present these results in terms of the probabilities vs motion angle for various temperature levels. Such plots are given for landing and take-off

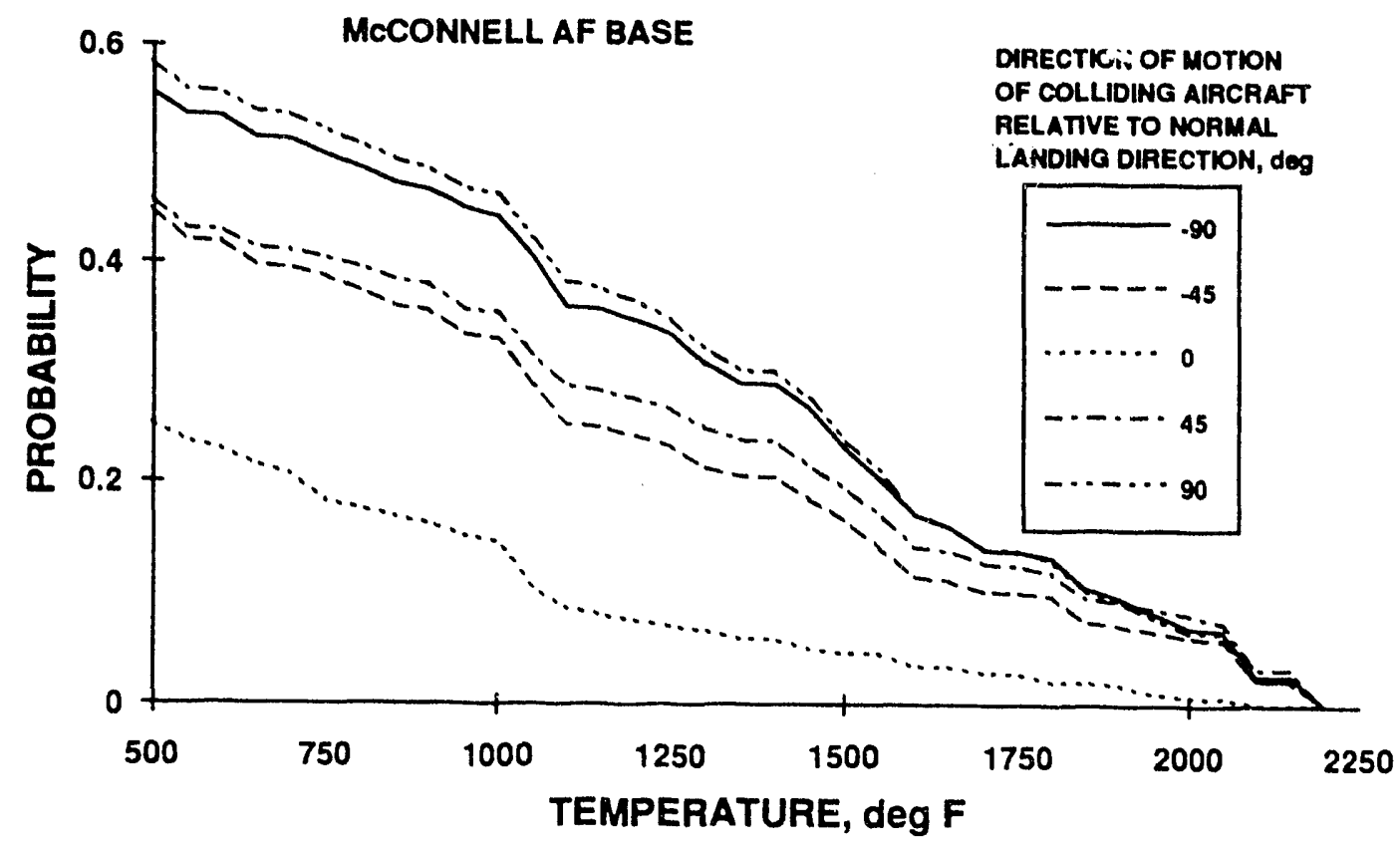

Figure 3.49 Probability vs Temperature for Off-Angle Collisions at Mcronnell AF Base

collisions at McConnell on Figures 3.50a and 3.50b, respectively, and for landing collisions at Dyess and Ellsworth on Figures $3.50 \mathrm{c}$ and $3.50 \mathrm{~d}$, respectively. On each of these graphs, the motion angle corresponding to a direct rear collision is indicated by an "arrow". It can be seen on each graph that the probability is mirimum for the angle corresponding to a direct rear collision, and that the probability increases with angle from the direct rear collision angle. Thus, the probabilities appear to be chiefly dependent on the type of collision (e.g., direct rear, broadside, etc.), and it is particularly revealing then to plot the probability vs type of collision. For three B-1B bases, plots of this type are given for a probability of exceeding $500^{\circ} \mathrm{F}$ for landing and take-off on Figures $3.51 \mathrm{a}$ and $3.51 \mathrm{~b}$, respectively, and for a probability of exceeding $1000^{\circ} \mathrm{F}$ for landing and take-off on Figures 3.51c and 3.51d, respectively. 

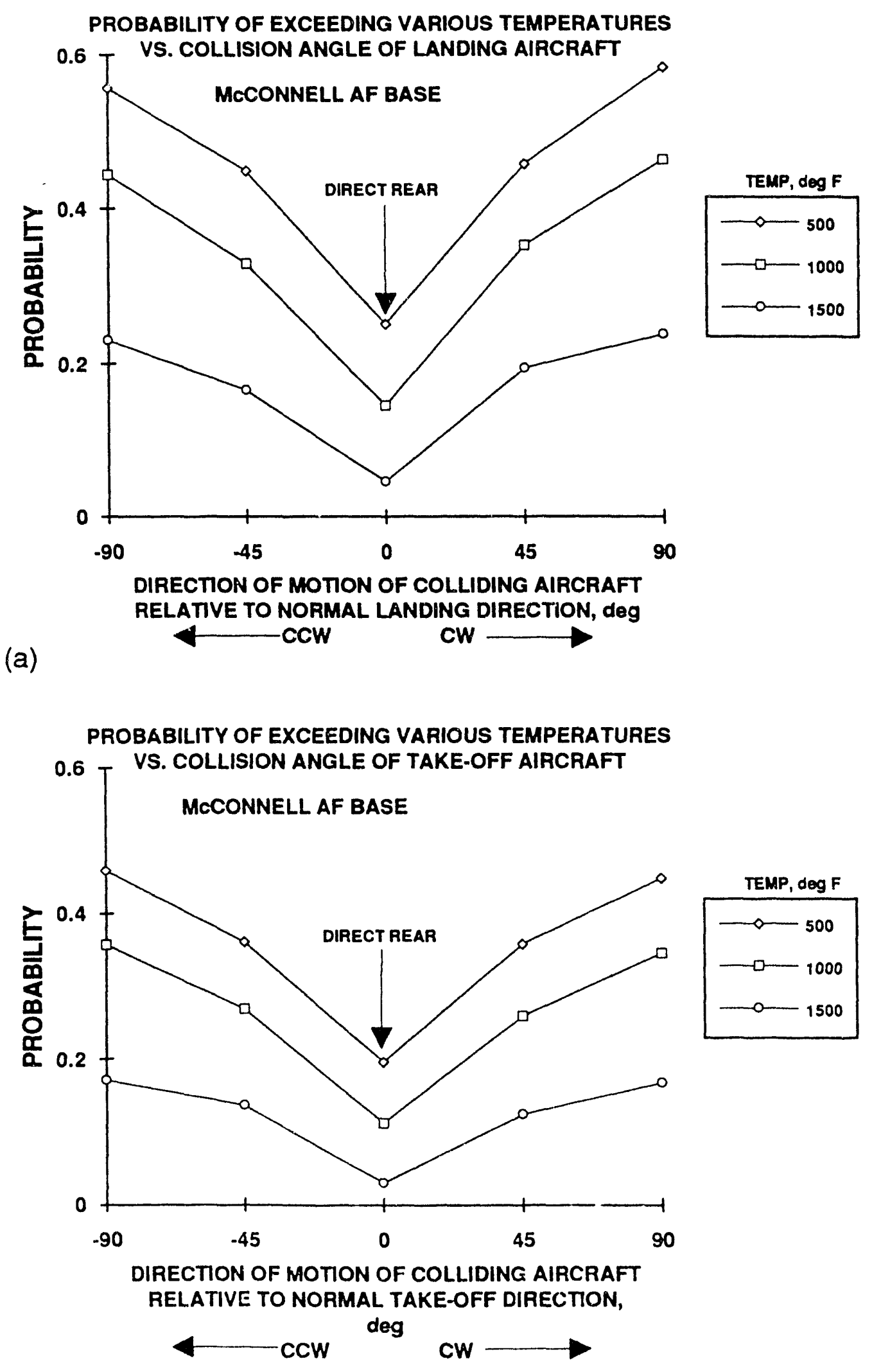

(b)

Figure $3.50 \mathrm{a} \& \mathrm{~b} \quad$ Probability vs Collision Angle for Various B-1B Bases 
PROBABILITY OF EXCEEDING VARIOUS TEMPERATURES

VS. COLLISION ANGLE OF LANDING AIRCRAFT

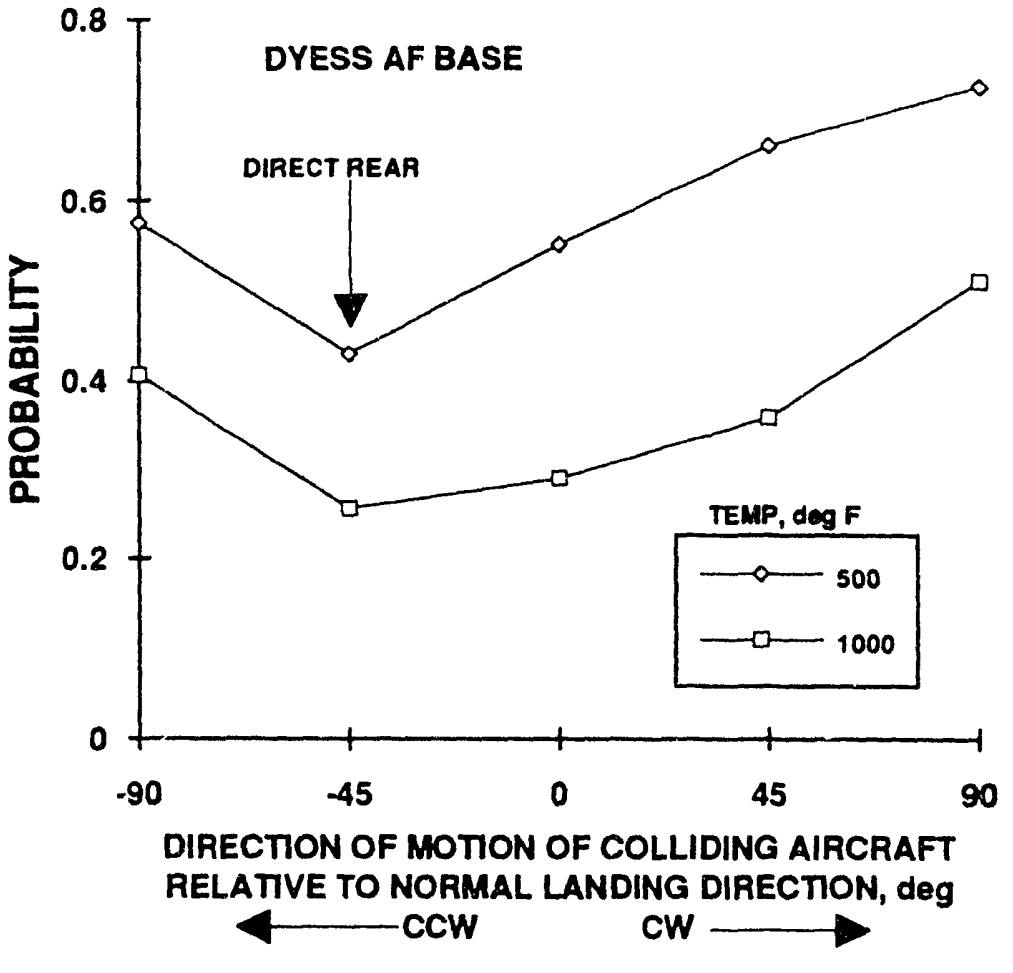

(c)

PROBABILITY OF EXCEEDING VARIOUS TEMPERATURES

VS. COLLISION ANGLE OF LANDING AIRCRAFT

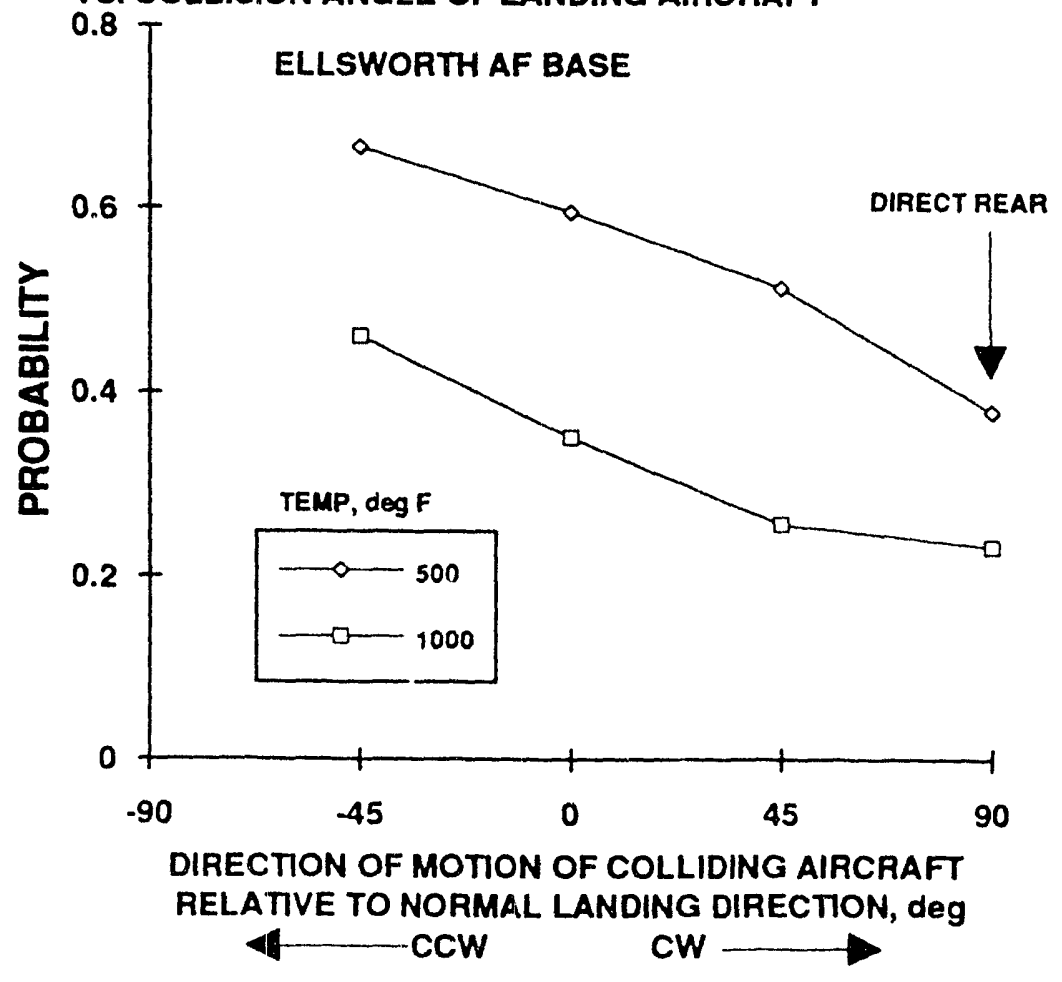

(d)

Figure 3.50 c \& d Probability vs Collision Angle for Various B-1B Bases 

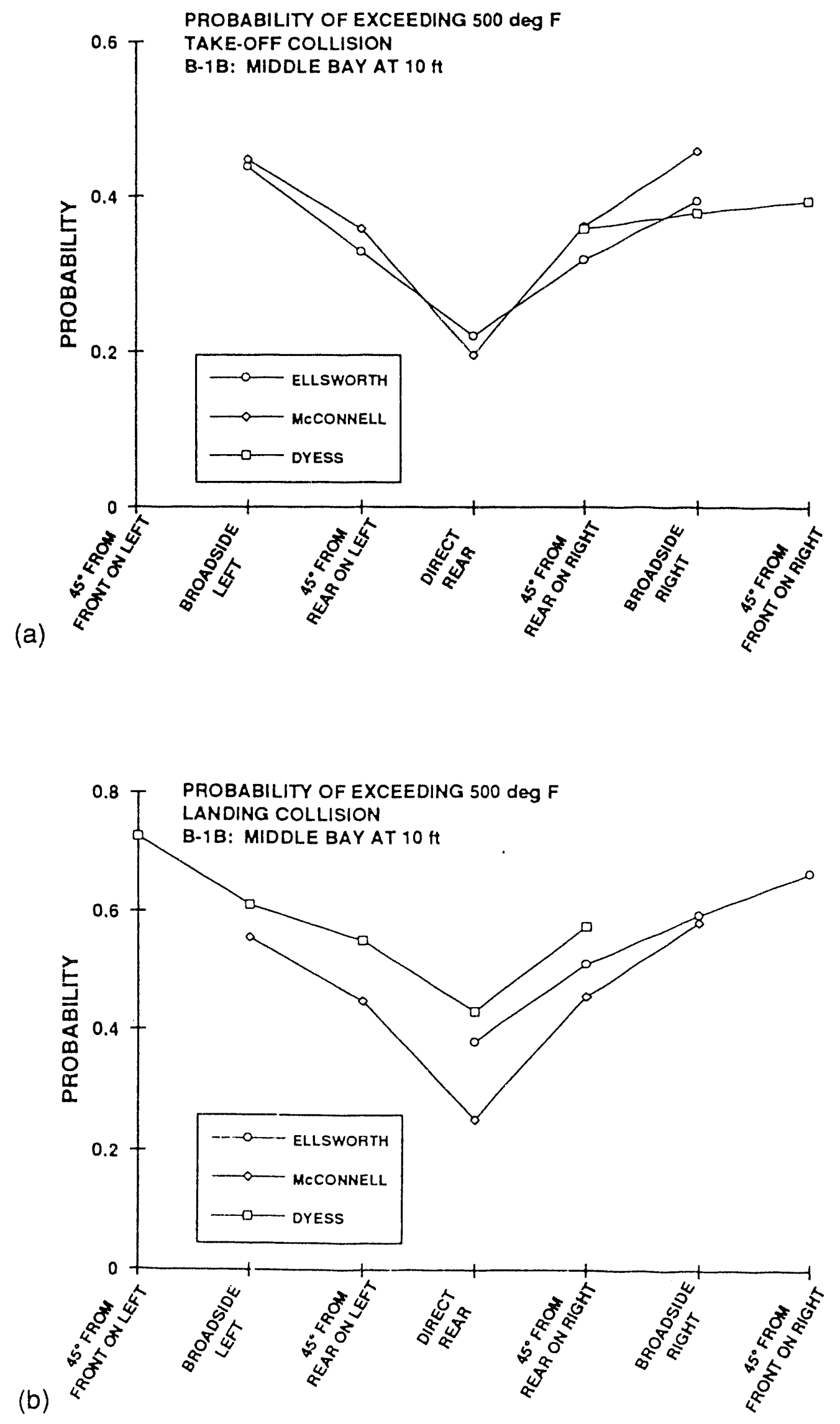

Figure 3.51 a \& $b \quad$ Probability vs Type of Collision for B-1B 

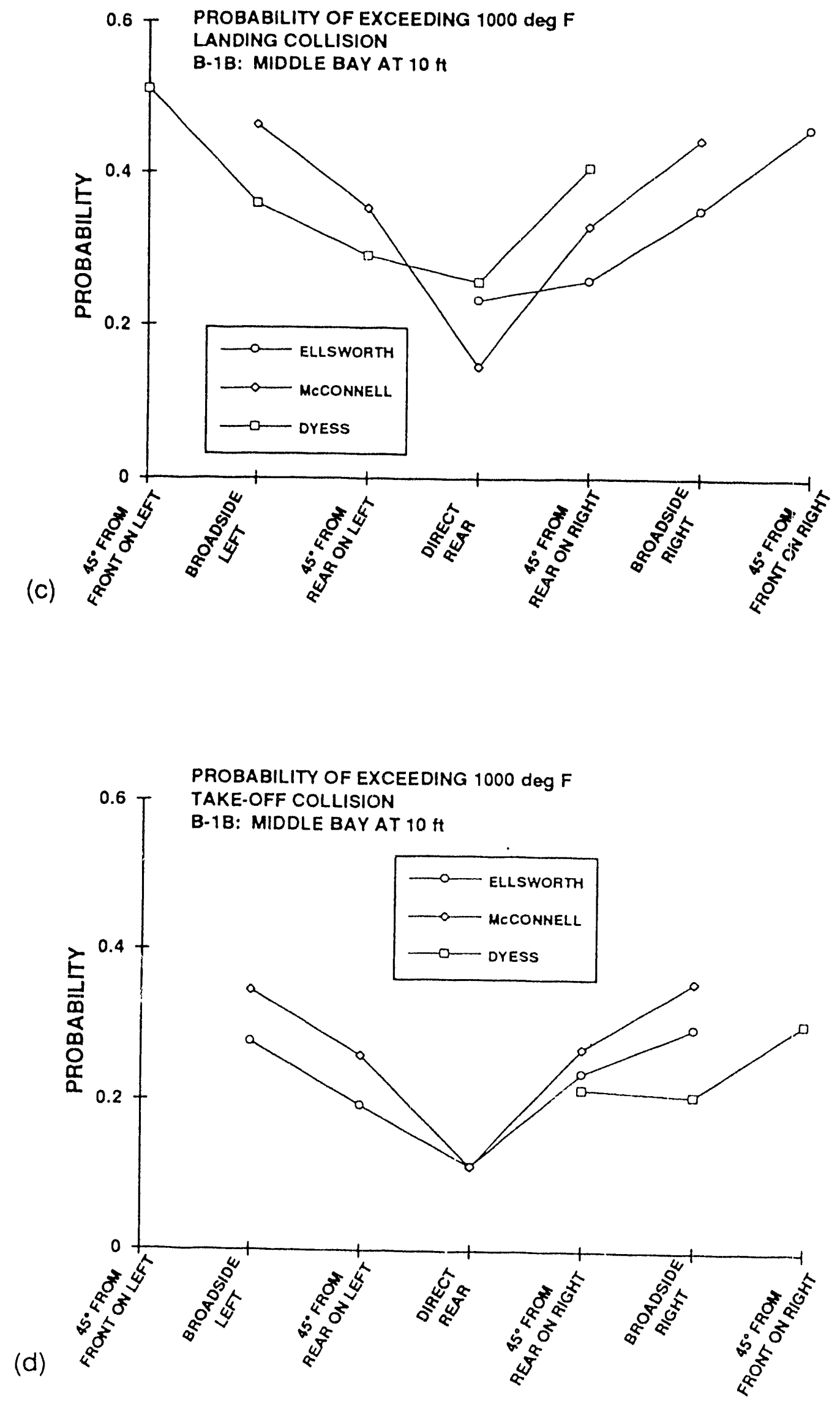

Figure $3.51 \mathrm{c} \& \mathrm{~d}$ Probability vs Type of Collision for B-1B 
In the format of Figure 3.51, the data trend mentioned above is quite apparent. These results indicate that as the collision progresses from a rear end collision to a front collision, there is an increasing opportunity for fuel spills that are favorable to cargo exposure, or more specifically, that there is an increasing occurrence of fuel spills that tend either to be upwind of the cargo or are near enough to the cargo for cargo exposure to occur.

Close inspection of Figure 3.51 reveals that the probability values for landings tend to be larger than for take-offs. This would follow from the tendency mentioned above that there are fewer take-offs from the end of the runway that can result in collisions than there are landings on the end of the runway that can result in collisions.

Base to base differences in probability can only be due to differences in (1), weather and (2), parking angles of the alert aircraft. The relative contribution of these two has not been determined.

An example of the effect of cargo location (aft vs middle bay) on the probability vs collision type is given on Figure 3.52. The example is for landing collisions at McConnell AF Base. On the basis that there are simply many more spill point locations "upwind" of the cargo for the aft bay, it would be expected that the aft bay probabilities would be larger than for the middle bay for any of the collision types shown. While that is seen to be true on Figure 3.52, there is a comparatively large aft to middle bay difference for direct rear collisions. This is consistent with the idea mentioned above of greater "protection" provided to fuel tanks near and forward of the B-1B middle bay cargo for direct rear collisions.

For collisions into an alert B-52, plots of probability vs type of collision for three bases are given for landing and take-off collisions on Figures 3.53a and $3.53 \mathrm{~b}$, respectively. It can be seen that the trend of increasing probability with increasing collision angle from a direct rear collision also applies to the B-52, although the dependence is clearly less pronounced for the B-52. From a comparison of Figures 3.53a and 3.52a, a great deal of similarity can be seen between the probability curves for the B-52 and for the aft bay of the B-1B.

Referring again to Figure 3.53 only, the results for Castle AF Base have been included because of the unusual weather conditions. When the wind so highly favors landings on and take-offs from the alert pad end of the runway, it will cause the probabilities to be abnormally high for landing collisions and low for take-off collisions. This result can be seen to occur from Figure 3.53, although the shape of the probability curve for Castle AF Base is similar to those for other bases. 

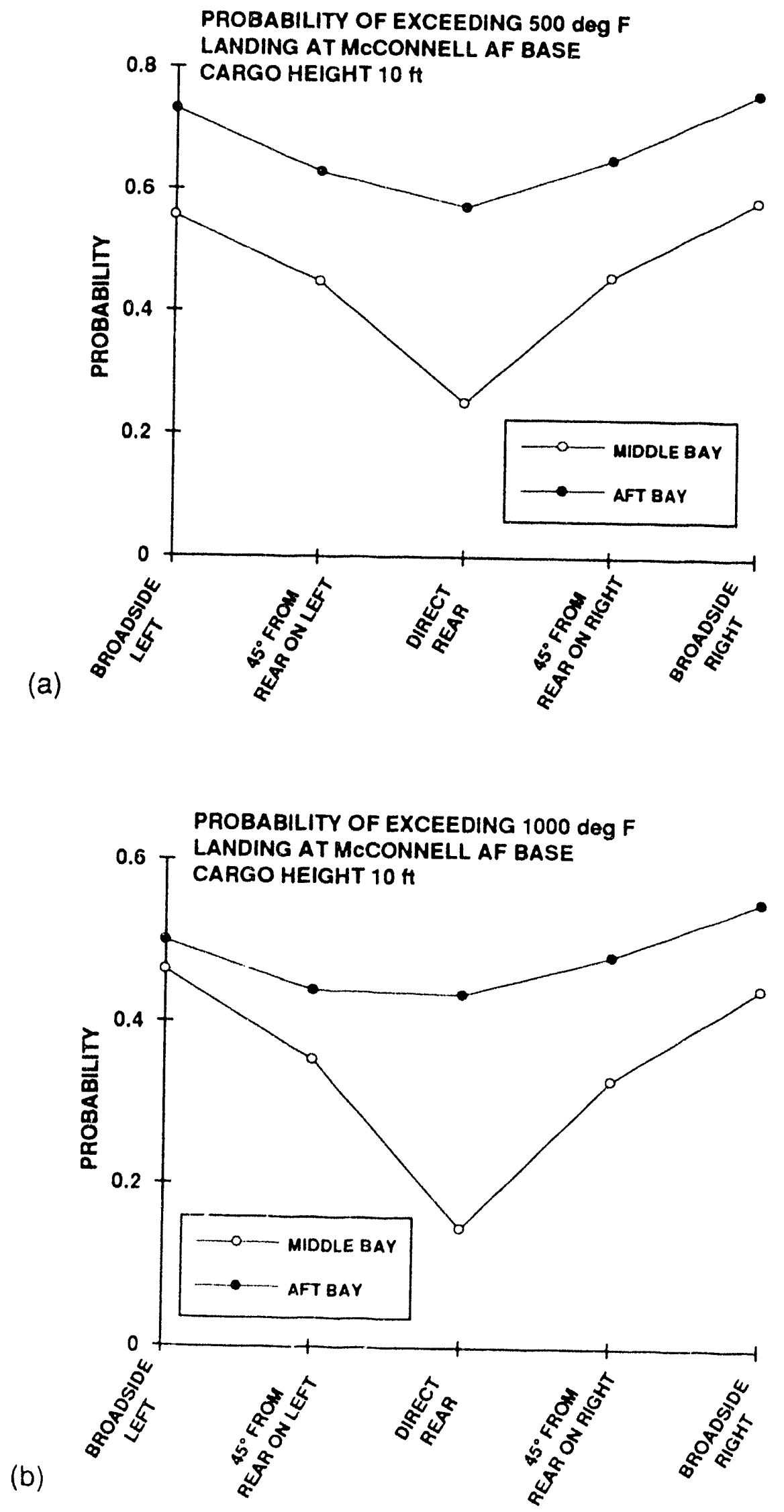

Figure 3.52 Probability vs Type of Collision for Middle and Aft Bays 

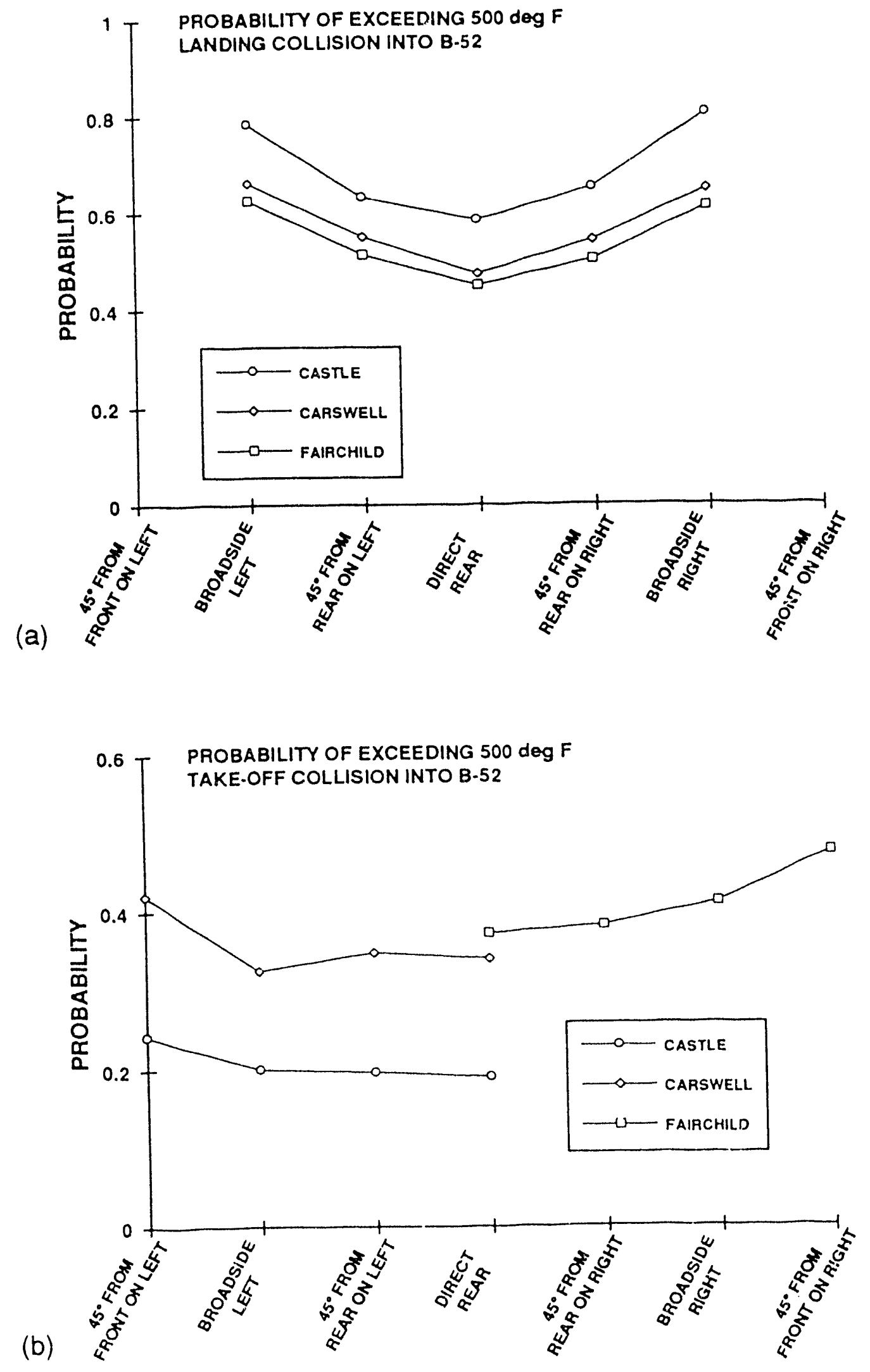

Figure 3.53 Probability vs Type of Collision for B-52 
Regarding the effect of cargo height on the probability vs temperature, examples for collisions into the B-1B are given on Figure 3.54; the examples are for cargo in the middle bay at Dyess AF Base on Figure 3.54a, and for cargo in the middle bay and aft bay at Ellsworth AF Base on Figures 3.54b and 3.54c, respectively. Similarly, cargo height effects for three B-52 bases are given in Figure 3.55 .

On the basis of Figures 3.54 and 3.55, the general character of the probability curves appears to be reasonably independent of the type of alert aircraft and, in the case of the B-1B, of which bay is involved. In all cases the probability at the one foot height is larger than for other heights throughout most of the temperature range, but then drops sharply, starting at about $1500^{\circ} \mathrm{F}$, to zero at about 1800 to $1850^{\circ} \mathrm{F}$. The probability for the 4,7 and 10 foot heights persists to temperatures above $2000^{\circ} \mathrm{F}$, with the probability reaching zero for the 4 foot height at about $2150^{\circ} \mathrm{F}$ and for the 7 and 10 foot heights at about $2200^{\circ} \mathrm{F}$.

Plots of the probability of exceeding $500^{\circ} \mathrm{F}$ vs cargo height are given on Figure 3.56 for the B-1B with cargo in the middle or aft bay and for the B-52. The effect of height on the probability of flame exposure is not particularly pronounced nor particularly different for any of the three conditions shown. The probability magnitude for cargo in the middle bay at McConnell AF Base is comparatively low (as would be expected from results presented earlier), and this large difference does not occur for the aft bay at McConnell (also expected from previous results). 


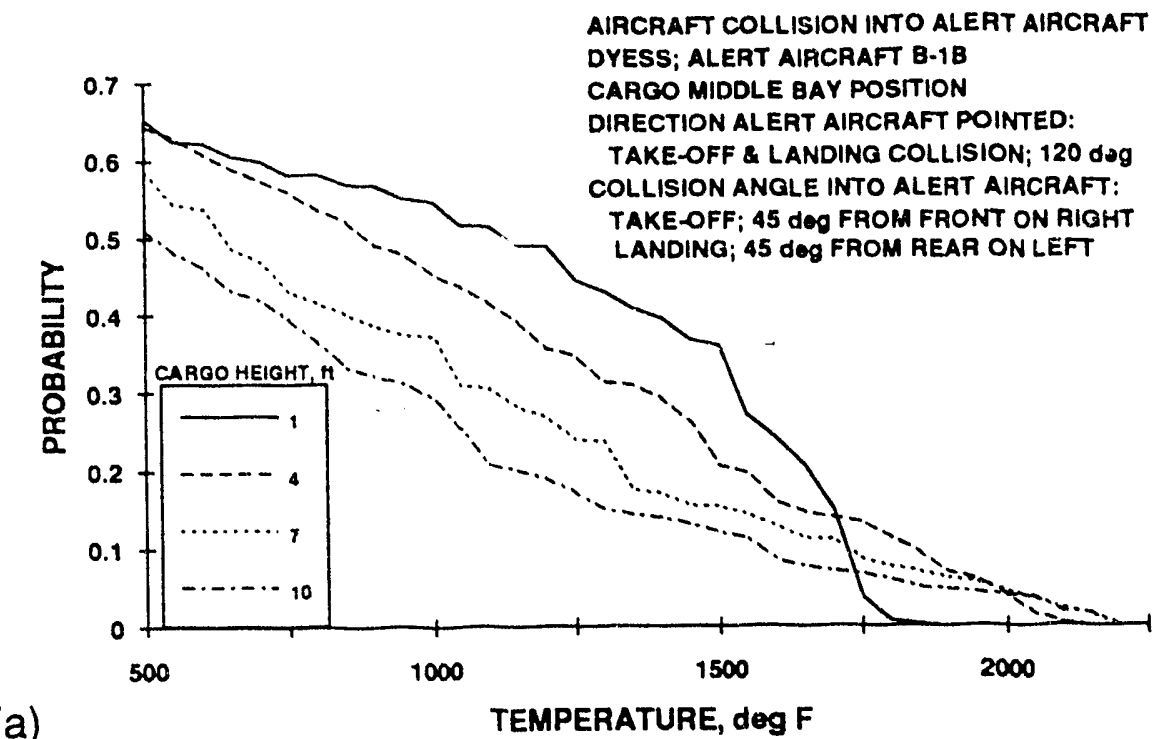

(a)

\section{TEMPERATURE, dog F}
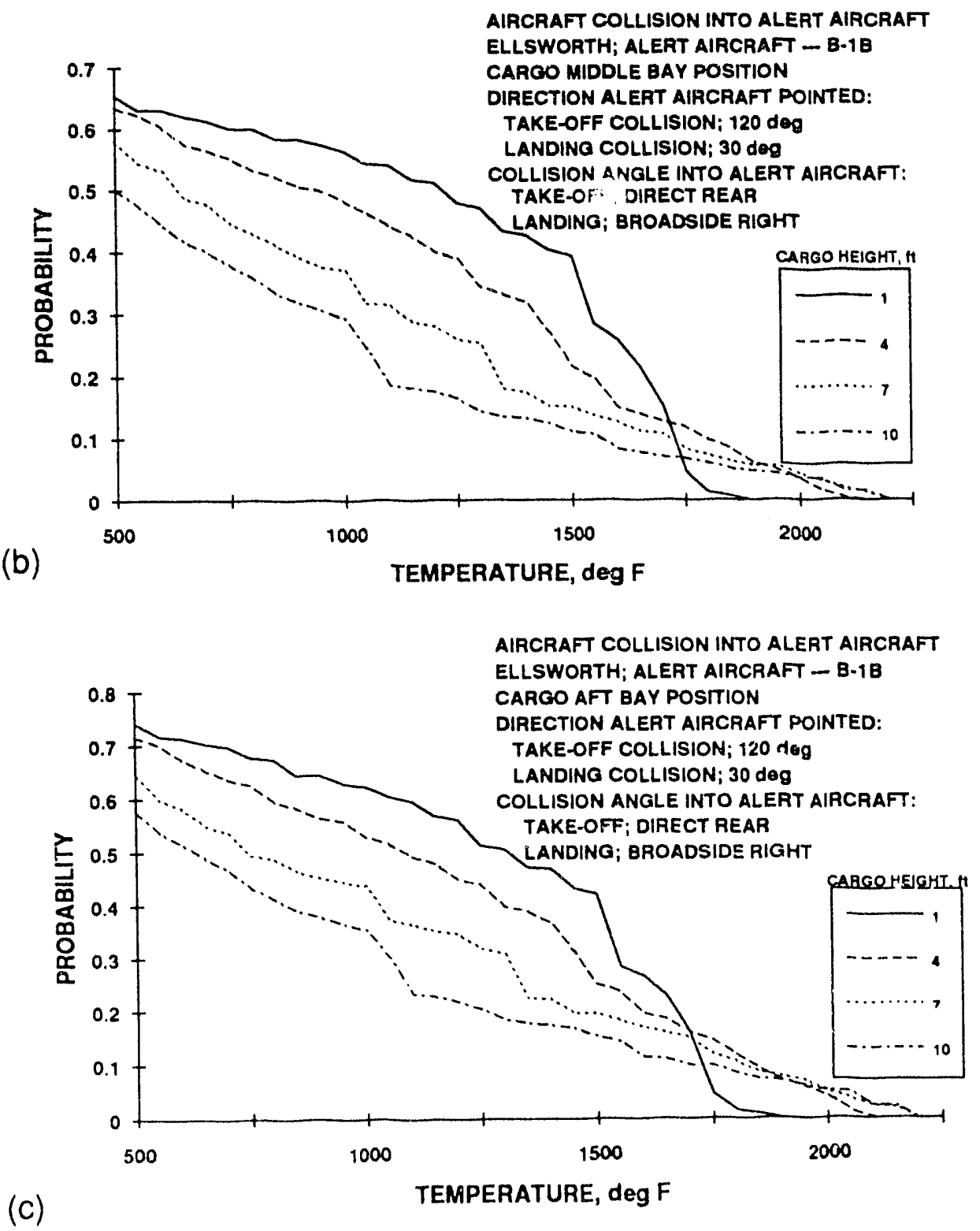

Figure 3.54 Effect of Cargo Height for B-1B Collision Accidents 

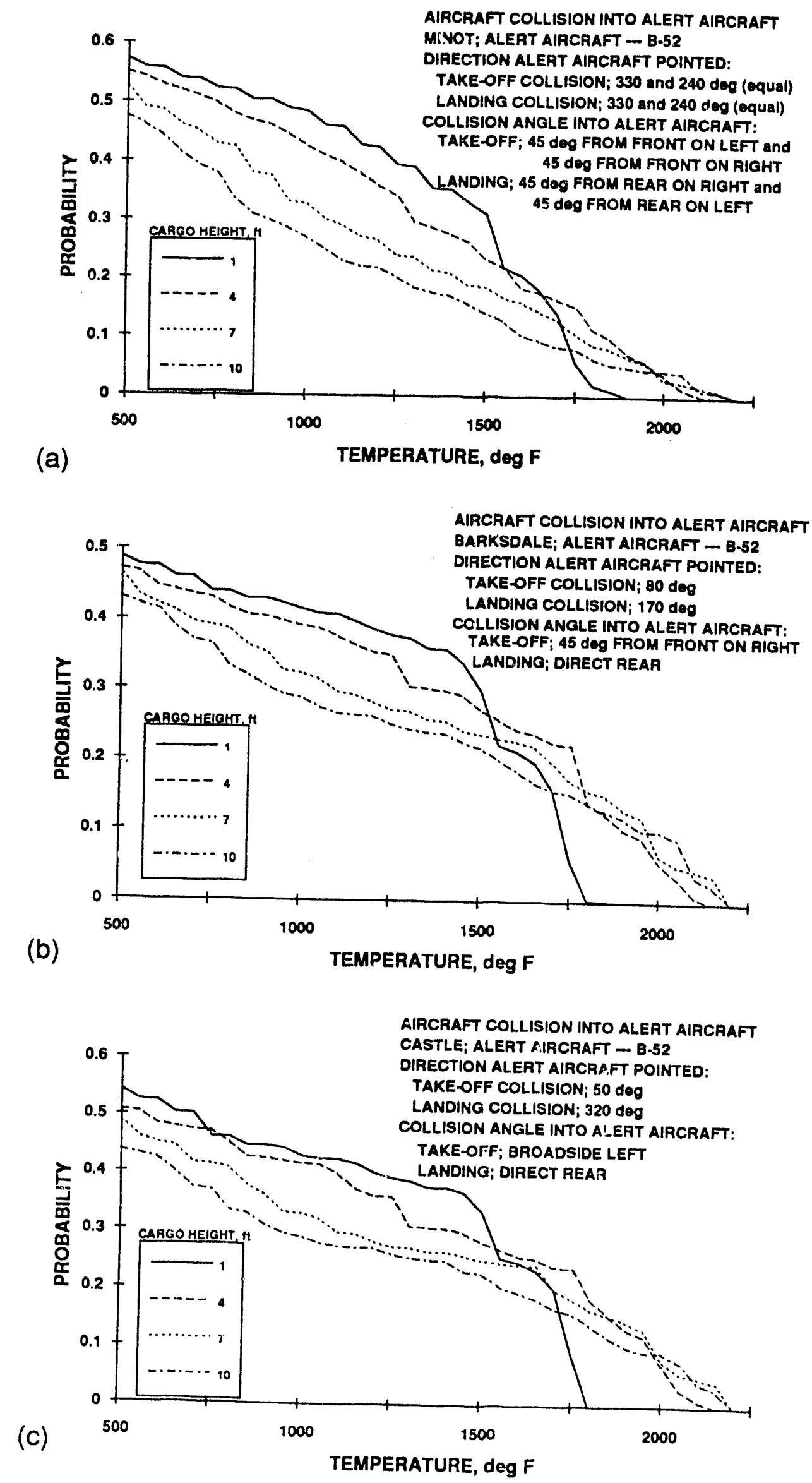

Figure 3.55 Effect of Cargo Height for B-52 Collision Accidents 


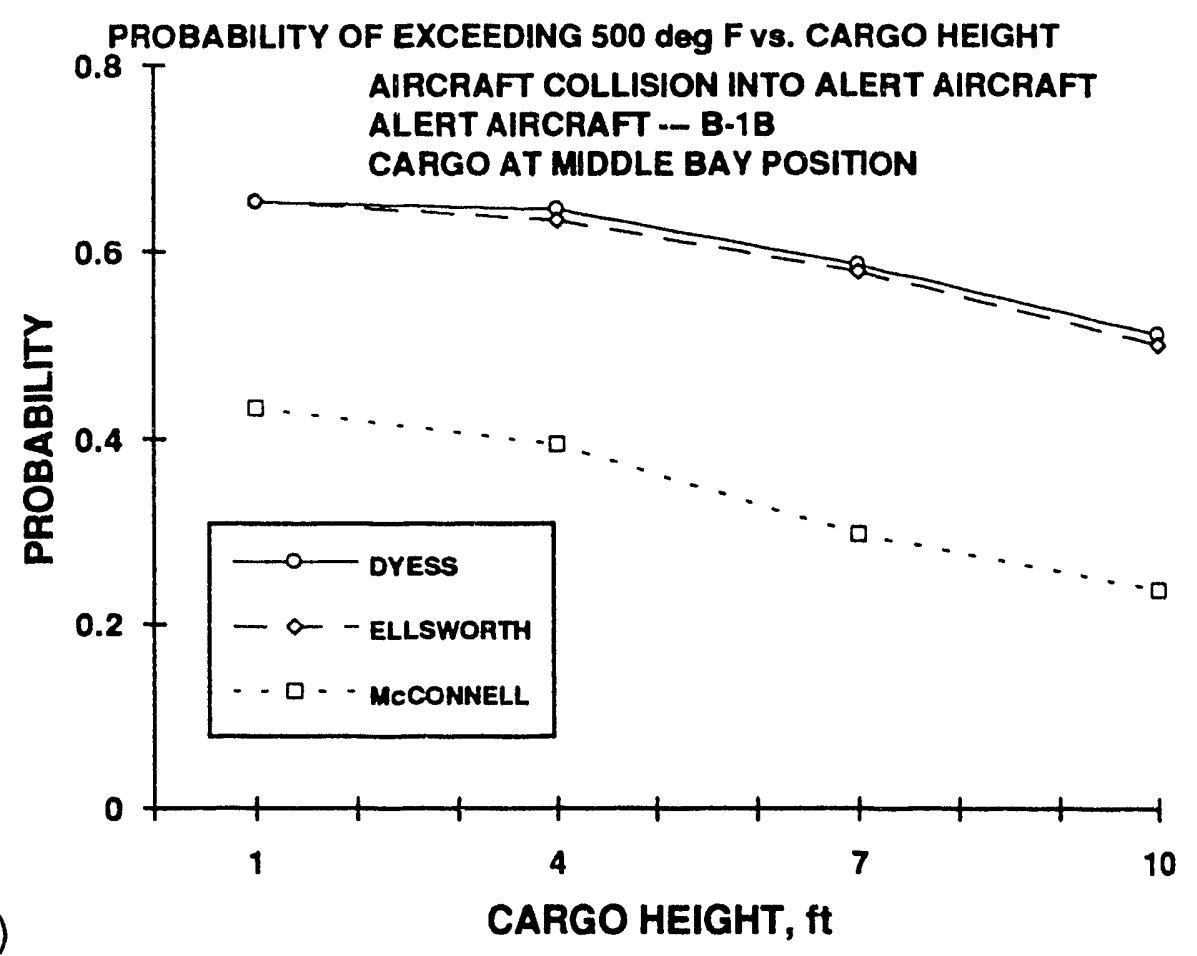

(a)

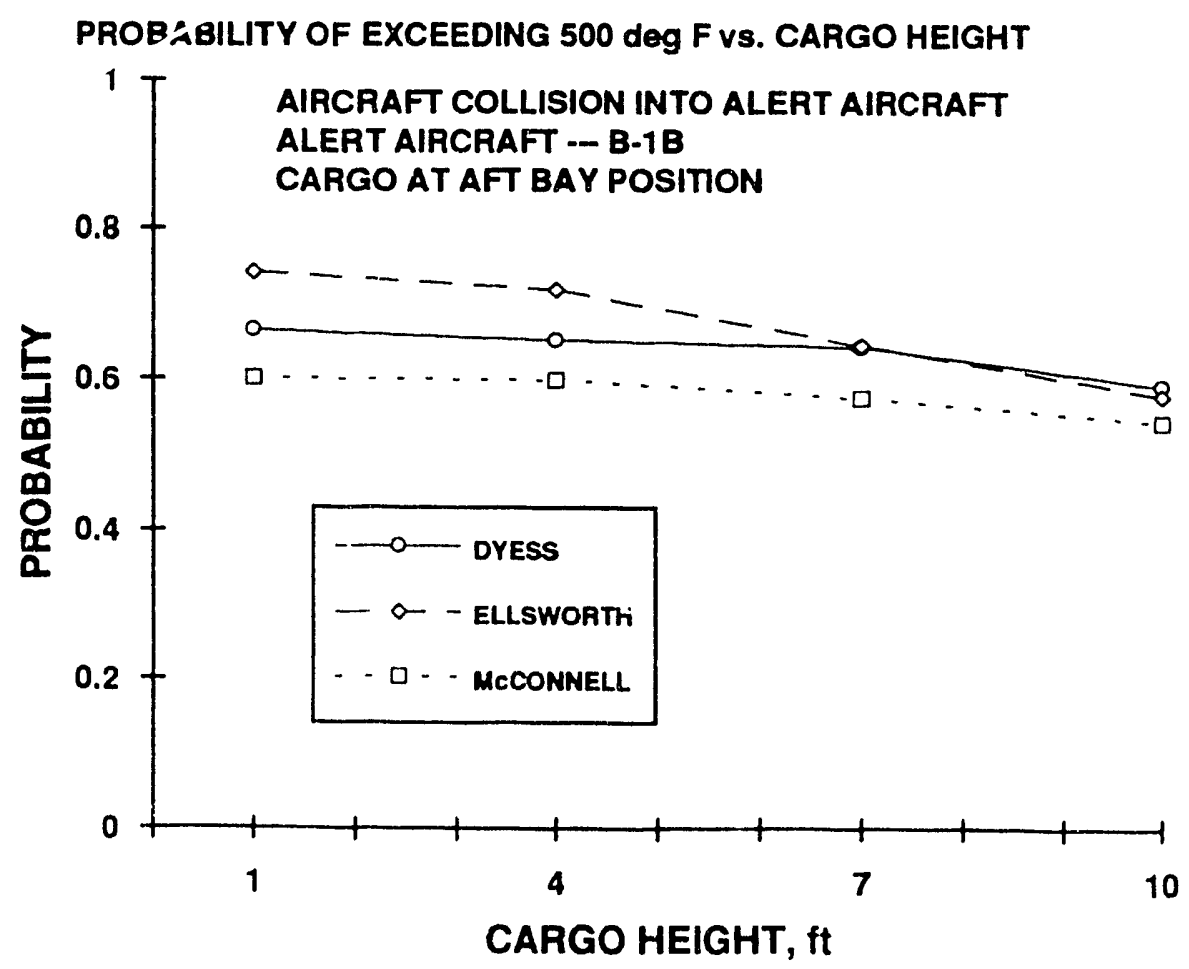

Figure $3.56 \mathrm{a} \& \mathrm{~b}$ Probability of Exposure vs Cargo Height for Collision Accidents 


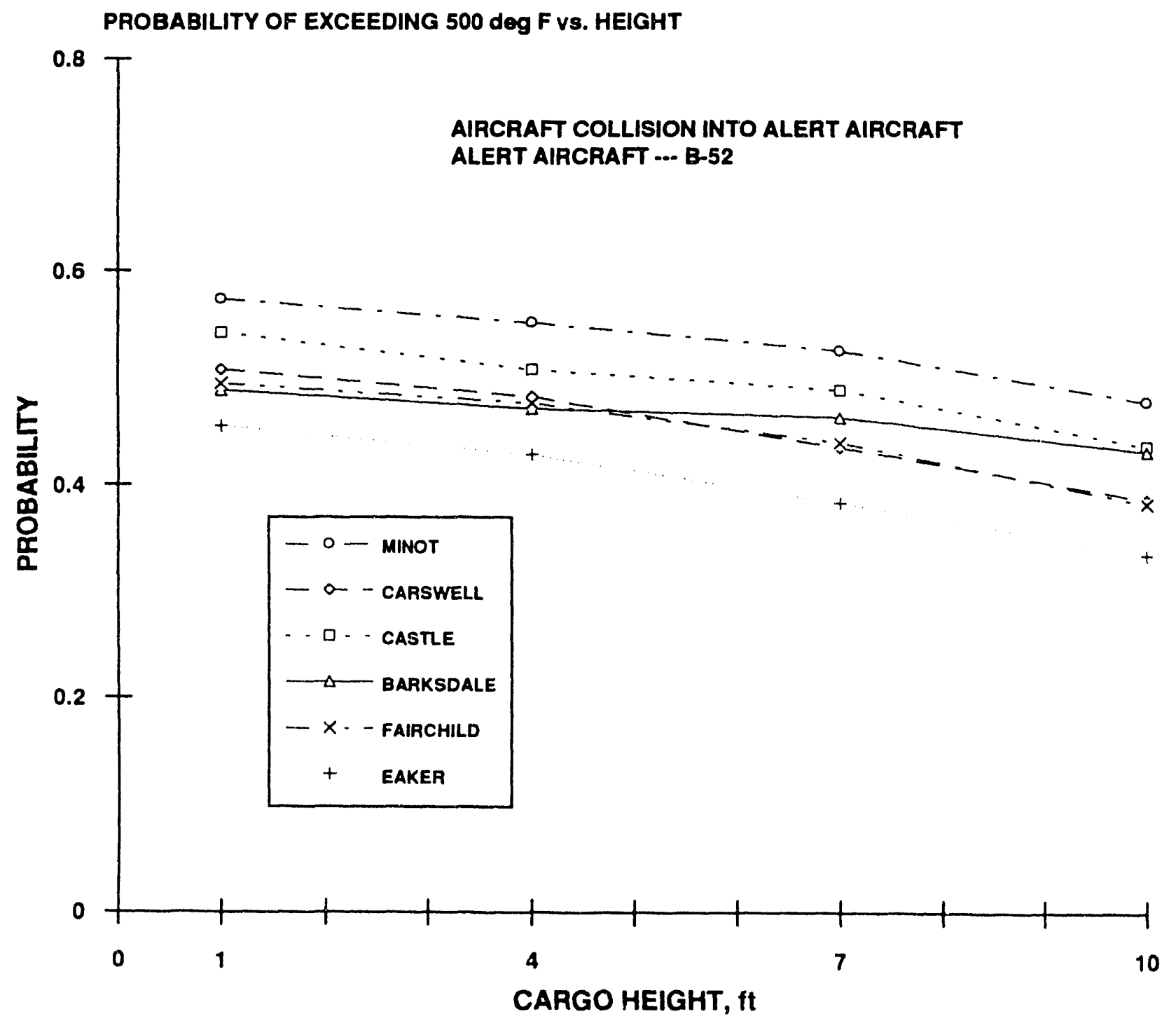

(c)

Figure 3.56c Probability of Exposure vs Cargo Height for Collision Accidents 


\subsection{UNCERTAINTY ANALYSIS}

The purpose of this subsection is to indicate sources of error and their effect on the predicted probabilities of exceeding given temperatures. Uncertainties for the collision accident are considered first, followed by those for the engine start accident. It is noted that the uncertainty values presented in this report are intended to represent one standard deviation of error $( \pm 1 \sigma)$.

There are several sources of error with regard to flame temperature. First of all, the temperature values used are based on a series of fire tests, and the reproducibility of the test results is thereby an issue. Two of the tests were conducted under "nearly" the same conditions of wind speed and fuel spill rate (designated Test 4 and Test 9 in Appendix A), and these results serve as a measure of reproducibility. The effect of test to test differences in temperature field has been evaluated by using the data from each of the two tests separately to produce the probability of exceeding given temperatures. Example differences in probability for the two tests are shown on Figure 3.57 for aircraft collisions at Dyess and Ellsworth AF Bases. It can be seen from Figure 3.57 that the impact of the test data change on the overall result is not highly significant. It is noted, however, that the probability calculation uses data from five tests, four of which did not contain any data variation. Including data variation from all five tests, and assuming the variations for all tests are equivalent, there would be an increase in the impact over that shown in Figure 3.57 by approximately $\sqrt{5}=2.24$. $^{*}$

It is noted that the effect of test reproducibility appears to be absent on Figure 3.57 for temperatures above about $1750^{\circ} \mathrm{F}$. This is due to the general lack of temperatures above $1750^{\circ} \mathrm{F}$ for Tests 4 and 9 (at heights of interest). The plots on Figure 3.57 are misleading in that test to test differences would extend to temperatures above $1750^{\circ} \mathrm{F}$; if replicate test data would have been available for the $5 \mathrm{mph}$ wind, where the higher temperatures at the height of interest occur, the effect of test reproducibility would have been seen at the higher temperatures. To offset this deficiency, the average difference in probability between 500 and $1500^{\circ} \mathrm{F}$ was evaluated and will be used as a measure of this uncertainty for temperatures from 500 to $2250^{\circ} \mathrm{F}$. The average probability difference is approximately 0.0056 , and the estimated uncertainty in probability is $\Delta \mathrm{P}_{\text {rep }}=0.0056 * \sqrt{5}=0.013$.

* A given error in data for the low wind speed would tend to have a larger impact on the overall result than for the medium speed, since the low wind speed is a more probable speed. However, test to test variation tends to be significantly smaller for the low wind speed tests, more or less balancing the effect of higher probability of low wind speed. 

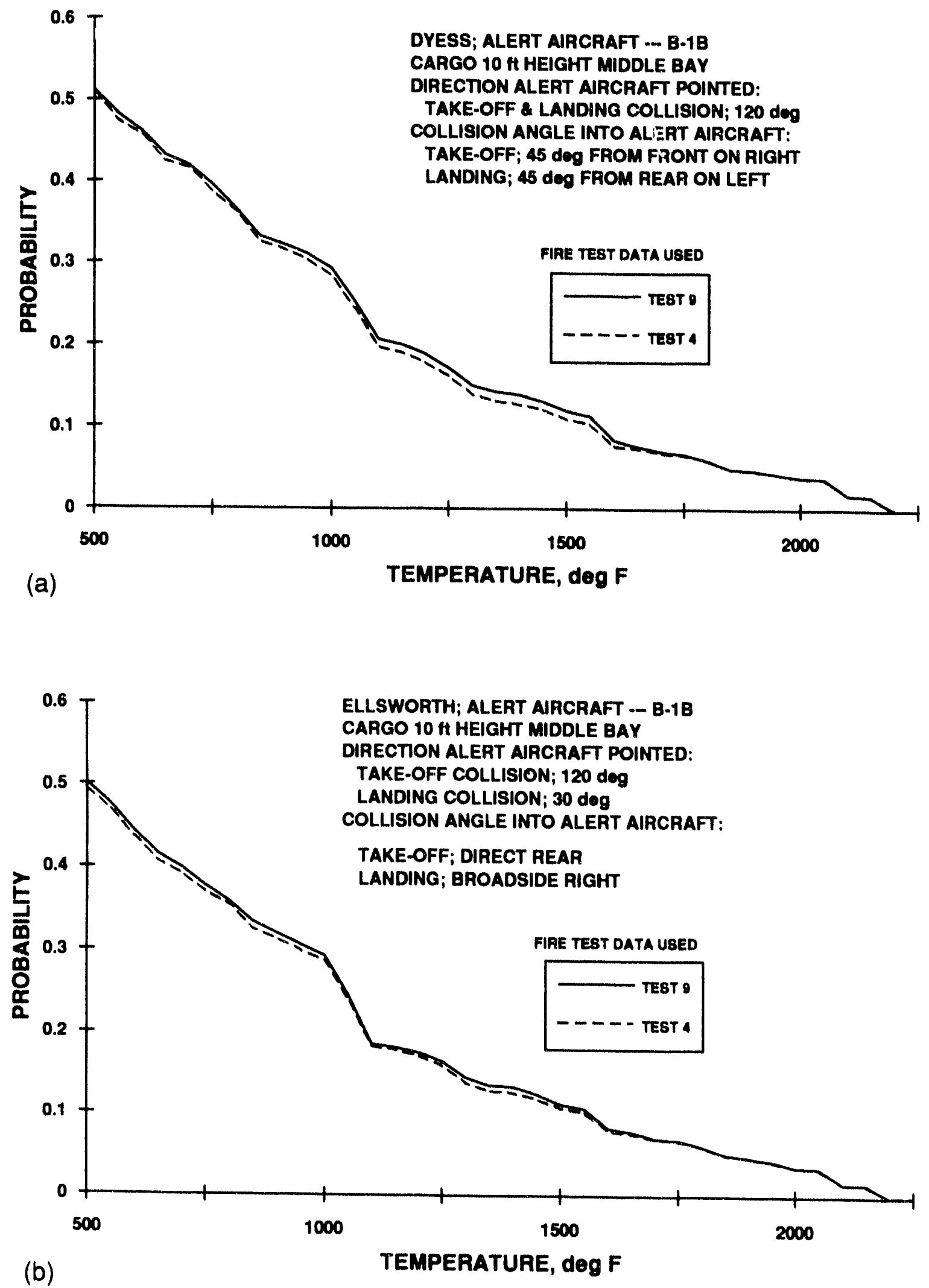

Figure 3.57 The Effect of Fire Test Data Reproducibility 
Another source of temperature error relates to extrapolation of temperatures over the low temperature regions of the fire. For the fire test series used in the analysis, temperature measurements were made over $50 \times 47.5$ foot horizontal areas which were centered as near to the highest flame temperature region as possible. Temperatures outside of the measurement area were evaluated by extrapolation of measured temperatures to the edge of the flame, where the edge location was estimated from film coverage and knowledge of the location of the periphery of the liquid fuel layer. The flame edge and extrapolated temperatures can be subject to significant error, and calculations to reveal the related uncertainty in probability have been conducted. For these calculations, the extrapolated temperature values were modified to reflect errors that were probable; for example, temperature values in the extrapolated portion of the baseline temperature data file for Test 8 , the TAX8 file, were changed to create a modified file called TAZ8. These modified temperature distributions were then used to evaluate the uncertainty in the probability of exposure to given temperatures.

It is mentioned that for low wind speed, the measurement area nearly covered the entire flame region, and little extrapolation is required. As the wind speed increases, a decreasing fraction of the flame is within the measurement area. Thus, the temperature field uncertainty increases with wind speed, but this tends to be negated by the pronounced decrease in the probability of experiencing a given speed with increasing wind speed.

Figure 3.58 shows the effect of extrapolation for a modification of the temperature field for the medium wind speed at $300 \mathrm{gpm}$ spill rate. Clearly this modification has had little impact; the small impact is at least in part due to the small likelihood of the medium wind speed occurring, and in part because this temperature distribution represents only one fuel spill category (225 to 450 gpm). In any case, extrapolation appears to have a small impact on probability at the "lower" temperatures, and has no effect at the "higher" temperatures.

Example differences in the probability of exceeding a given temperature for modifications of the low wind speed temperature field are given on Figure 3.59. It can be seen on Figure 3.59 that the modified temperature field resulted in a small increase in probability at and near $500^{\circ} \mathrm{F}$; this was a necessary result because in effect the modification increased the size of the fire. There are also small increases in the 1000 to $1250^{\circ} \mathrm{F}$ region, where there was initially an abrupt drop. There can, of course, be no changes at the "higher" temperatures since temperature extrapclation was required only for the lower temperatures. 


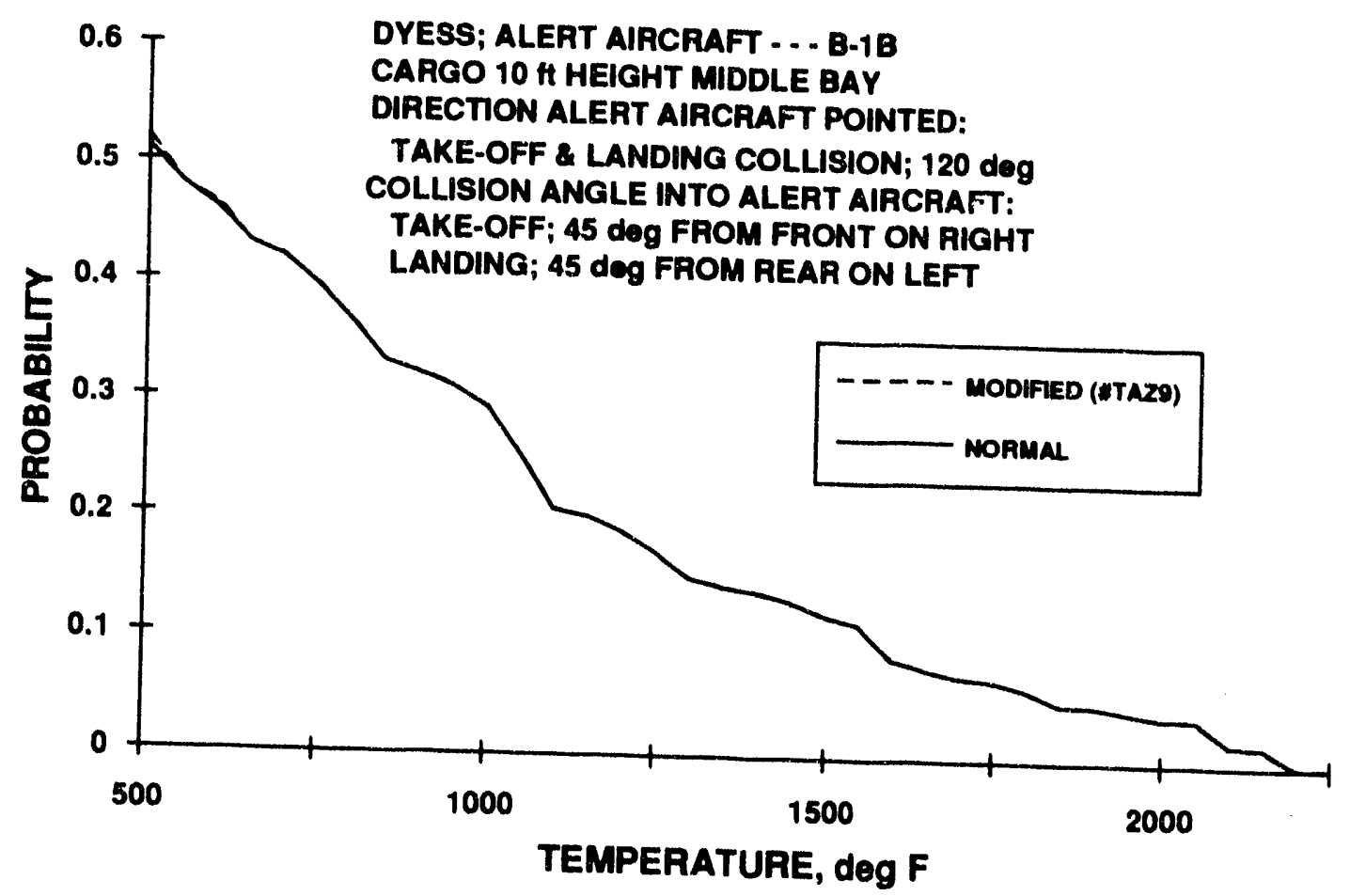

\section{Figure 3.58 Effect of Temperature Field Extrapolation for Medium Wind Speed Data}

The difference between the probability of exceeding given temperatures for the modified and standard temperature fields was evaluated for several accidents for the low wind speed case. From an averaging of these results, the probability difference exhibits a general decrease with increasing temperature, becoming zero at approximately $1650^{\circ} \mathrm{F}$. A curve fit (linear) of this difference in probability with temperature, used as a measure of the uncertainty caused by temperature field extrapolation, is given by $\Delta P_{\text {ext }}=-0.0000783(T-500)+0.09$, where $T$ is the temperature in deg $F$. 


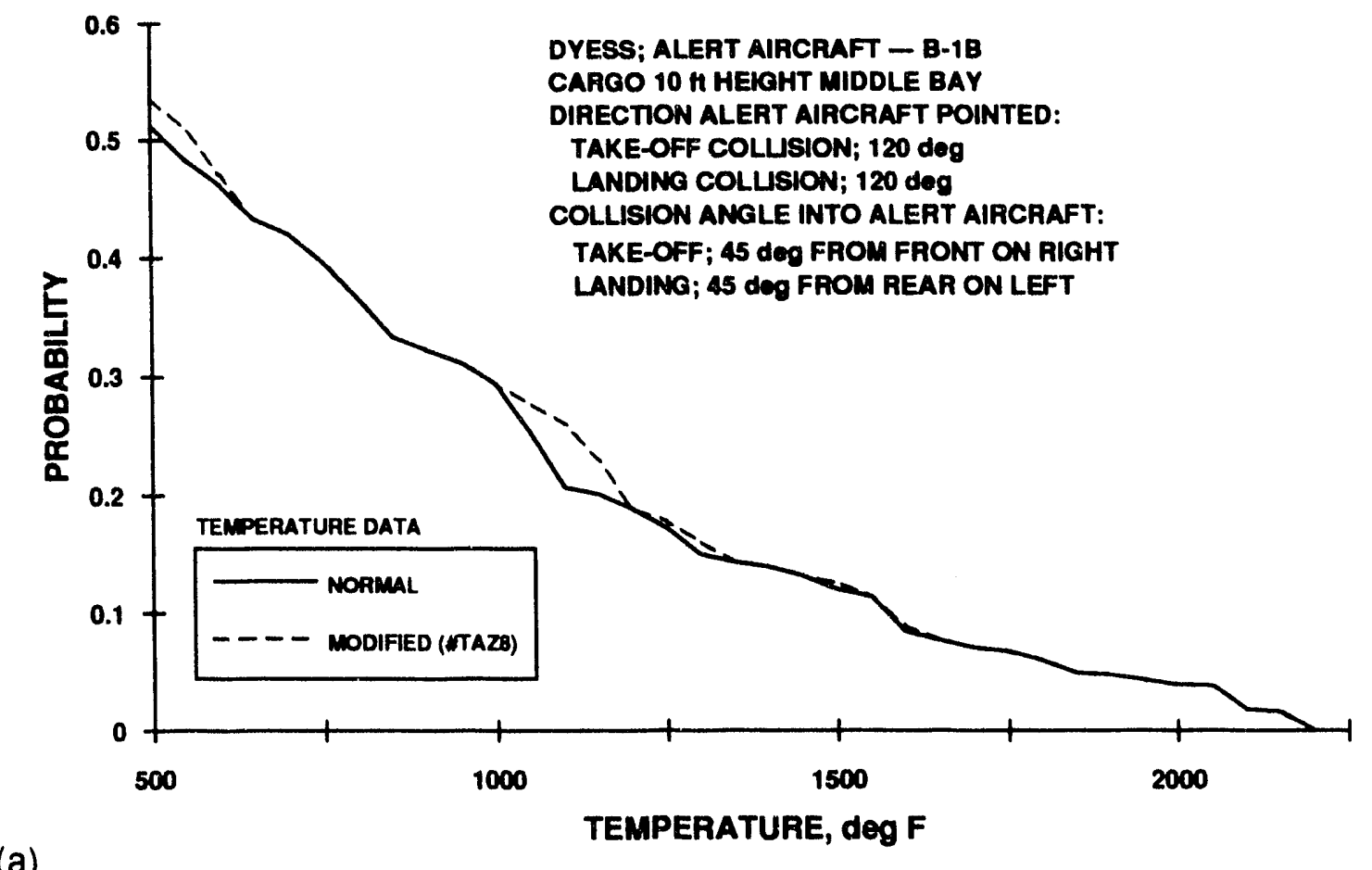

(a)

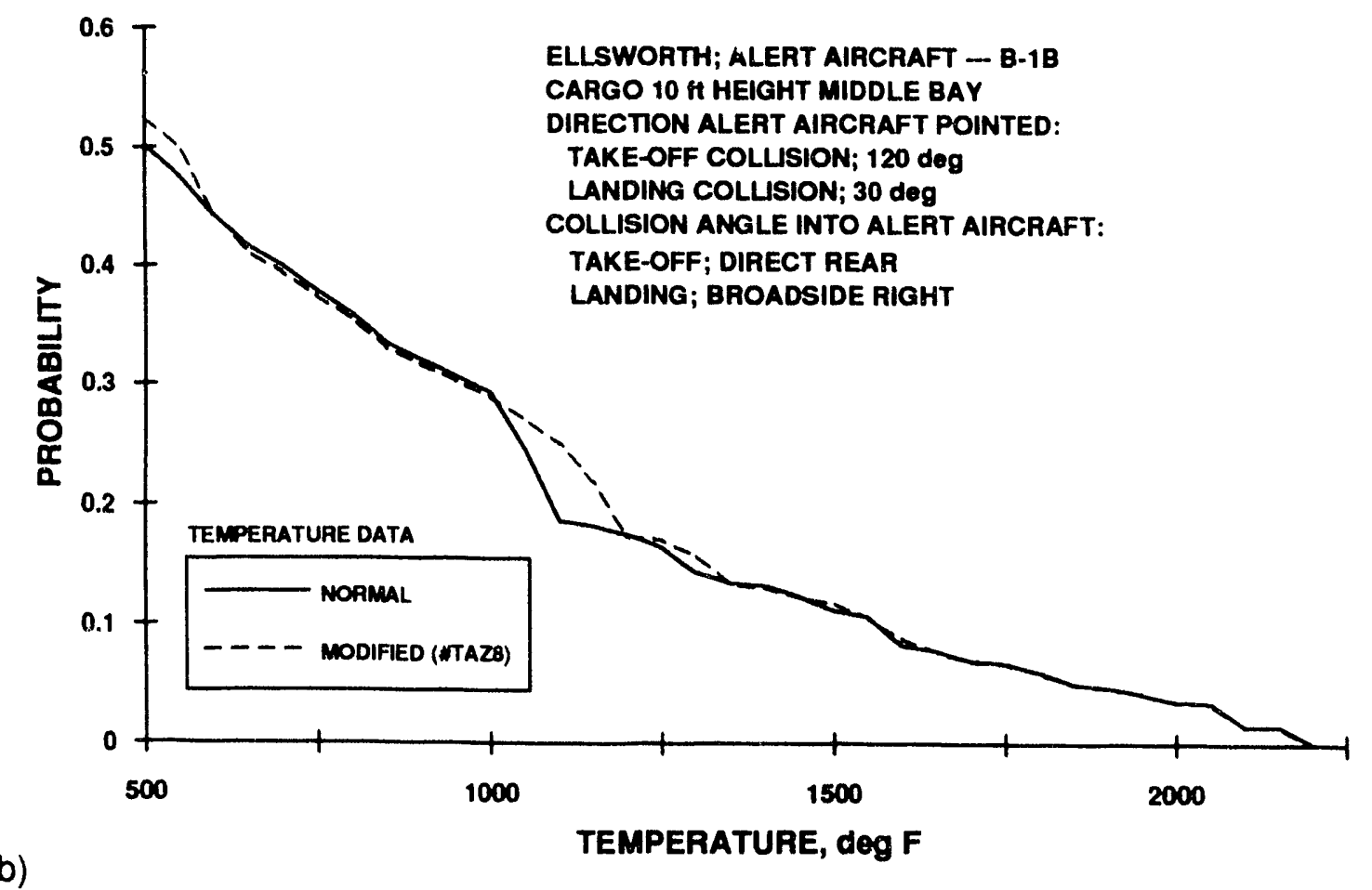

Figure 3.59 Effect of Temperature Field Extrapolation for Low Wind Speed Data 
Errors in evaluating the probability of exposure to given temperatures can occur when the measurement duration of the temperature data used in the evaluation is much shorter than the duration of interest.* Since the evaluation in this report uses data based on measurement durations of about two minutes, and some of the durations of interest are much longer than two minutes, there is a potential for this type of error. For this circumstance, the predicted probabilities of exposure based on two-minute measurements will be larger than would tend to occur for longer durations for temperatures above a particular temperature and the predicted probabilities will be smaller than would tend to occur for longer durations for temperatures below this particular temperature. An estimate of this error has been obtained.

The procedure of evaluating this error estimate comprises a two step analysis. In the first step, the statistics of flame temperatures from long duration fire tests $\left(1 / 2\right.$ hour) were examined (by Lawrence Livermore Lab personnel $\left.{ }^{* *}\right)$. Specifically, the scatter about the mean temperature (i.e., time-average temperature over the test duration of time-average temperatures averaged over two minutes, and also averaged over ten minutes, was analyzed. It was determined from this analysis that the standard deviation of the scatter about the mean was $106^{\circ} \mathrm{F}$ for the two-minute averages and $38^{\circ} \mathrm{F}$ for the ten-minute averages. In the second step, a mathematical relationship between flame temperature and location in the fire was first developed; this relationship, while fictitious, was representative of the temperature fields obtained from the six fuel spill tests. This temperature field was then applied to an evaluation of the probability that weapon exposure would exceed given temperatures for a particular type of accident (for example, for an aircraft crash at Eaker AF Base). Next, several temperature fields were developed whose temperatures at given fire locations scattered about the above fictitious temperature field by magnitudes that are associated with a standard deviation of $106^{\circ} \mathrm{F}$ (assuming a normal distribution). Each of these "scattering" temperature fields was then also applied to the evaluation of the probability that exposure would exceed given temperatures for the same accidents as for the fictitious field. Likewise, several fields were developed whose temperature scattered about the fictitious field by magnitudes associated with a standard deviation of $38^{\circ} \mathrm{F}$, and these fields were also applied to an evaluation of the probability of exceeding given temperatures. A summary comparison of results from this analysis can be seen on Figure 3.60.

It can be expected that the curve for the $106^{\circ} \mathrm{F}$ standard deviation case will tend to be higher in probability than for the $38^{\circ} \mathrm{F}$ s.d. curve and the fictitious curve, and the $38^{\circ} \mathrm{F}$ s.d. curve would be higher than the fictitious curve, for temperatures larger than some particular value, and that these curves would

* The origin of this error is explained in detail in Ref. 1.

* Reference 14, memo by John Creighton dated 12/30/91. 


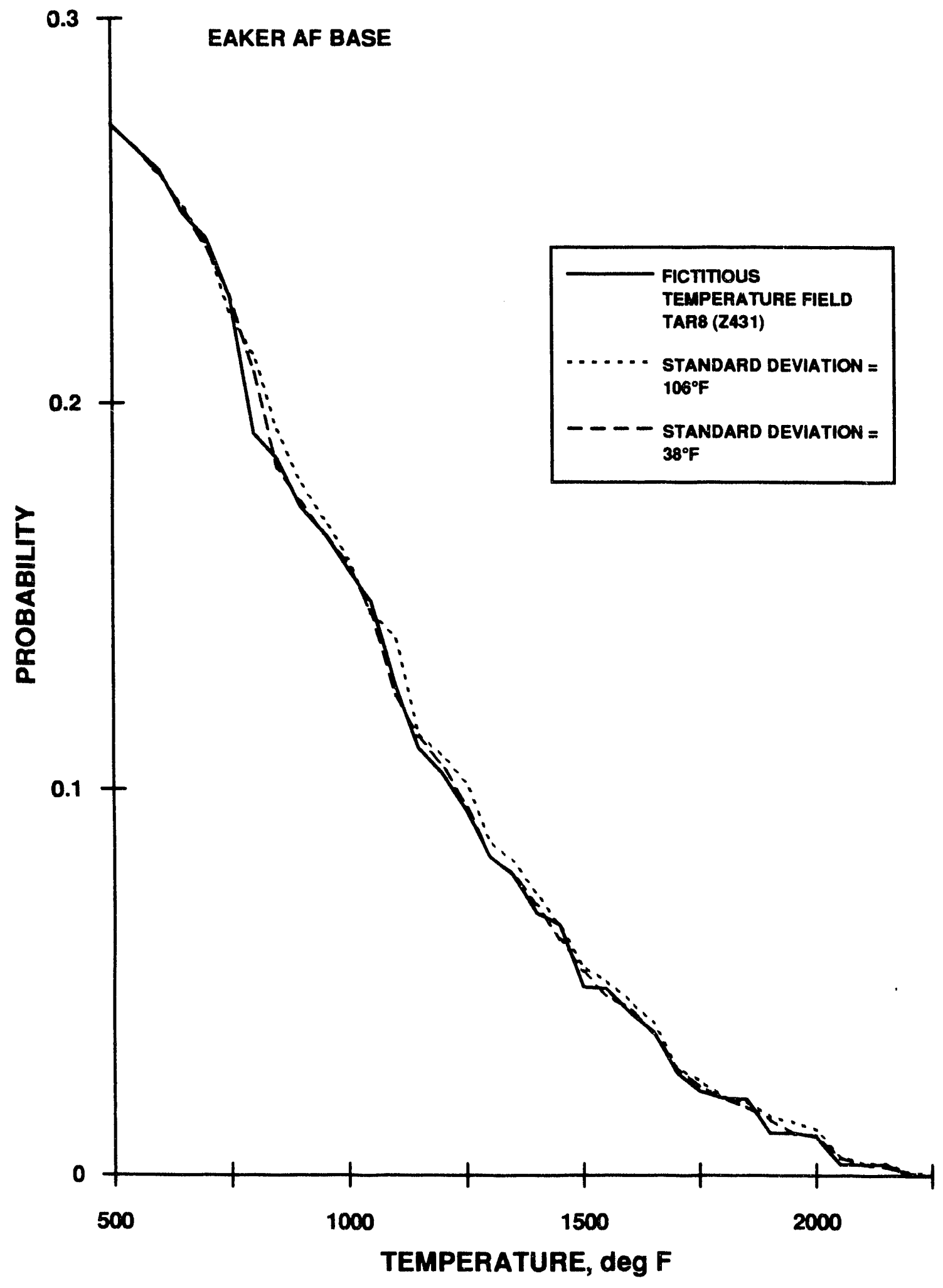

Figure 3.60 Effect of Temperature Data Duration on Probability of Exceeding Given Temperatures 
reverse their order for temperatures below the particular value. These trends do occur, although due to the statistical nature of the analysis, the rule is violated at a few temperature values (had a large enough number of curves been developed, the point violations would not be expected). Also due to the statistical nature of the analysis, it is difficult to identify a precise temperature where the probabilities crossover from above to below the curve based on the fictitious temperature field, but it appears to be in the 700 to $800^{\circ} \mathrm{F}$ region.

The major goal of the above analysis was to determine if predicted probabilities based on two-minute duration data would be significantly in error for longer durations (e.g., 10 minutes). On the basis of the small curve to curve departure seen on Figure 3.60, duration of measurement is simply not a significant error factor for the circumstances involved in this report and the issue beyond the above analysis is ignored. (It is noted that there are special situations where duration is a significant issue.)

There is an additional temperature related error that should be discussed. Consider, by way of example, that appropriate fire test data is not available for fuel spill rates less than $150 \mathrm{gpm}$. Experimental data (ref 1) and analytical treatments (ref 15) show that the highest temperature region of a fire decreases in height with decreasing fire size (fire diameter or fuel spill rate). It can happen then that a $75 \mathrm{gpm}$ spill could result in a maximum exposure temperature for some specific instance, whereas the analysis for lack of data would indicate temperatures below maximum. There would also be instances where the predicted temperature would be higher than the actual. On the basis of experience with this analysis and the nature of fires, it is my judgement that the absence of temperature data does not have a serious impact on the overall results, but there may be cases of significant error in a given temperature region. However, it is also my judgement that this type of fire will not lead to exposure temperatures that are higher than the maximum indicated in this report (about $2200^{\circ} \mathrm{F}$ ), and that probabilities in the $2000^{\circ} \mathrm{F}$ and above range based on a more extensive set of temperature data may be lower than, but not significantly higher than, those given in this report.

There is some dependence of weapon exposure on the direction that the alert aircraft is pointed for a fixed angle of collision (broadside, rear-end, etc). An illustration of this dependence is given on Figure 3.61, which shows the probability of exceeding given temperatures for various pointing directions for landing accidents. For some collisions, alert aircraft direction will change during the collision process, that is, there will be some rotation of the aircraft about a vertical axis. In the calculations of the probability of exceeding given temperatures, the initial directions of the parked aircraft are input, and there is no accounting for the effect of rotation for those collisions that involve rotation. Since it is not included, the uncertainty of excluding rotation should be examined, and this examination is considered next. 


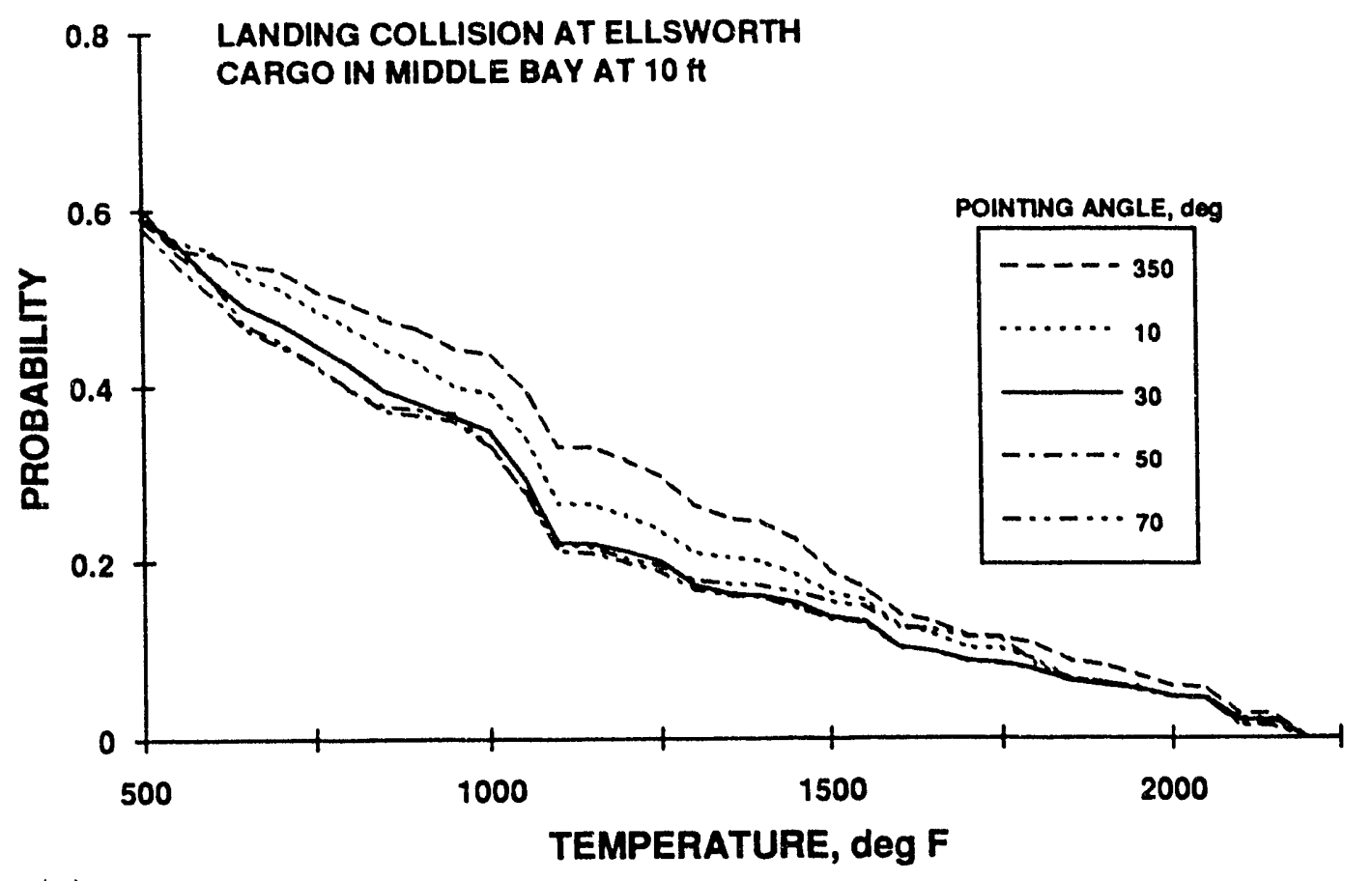

(a)

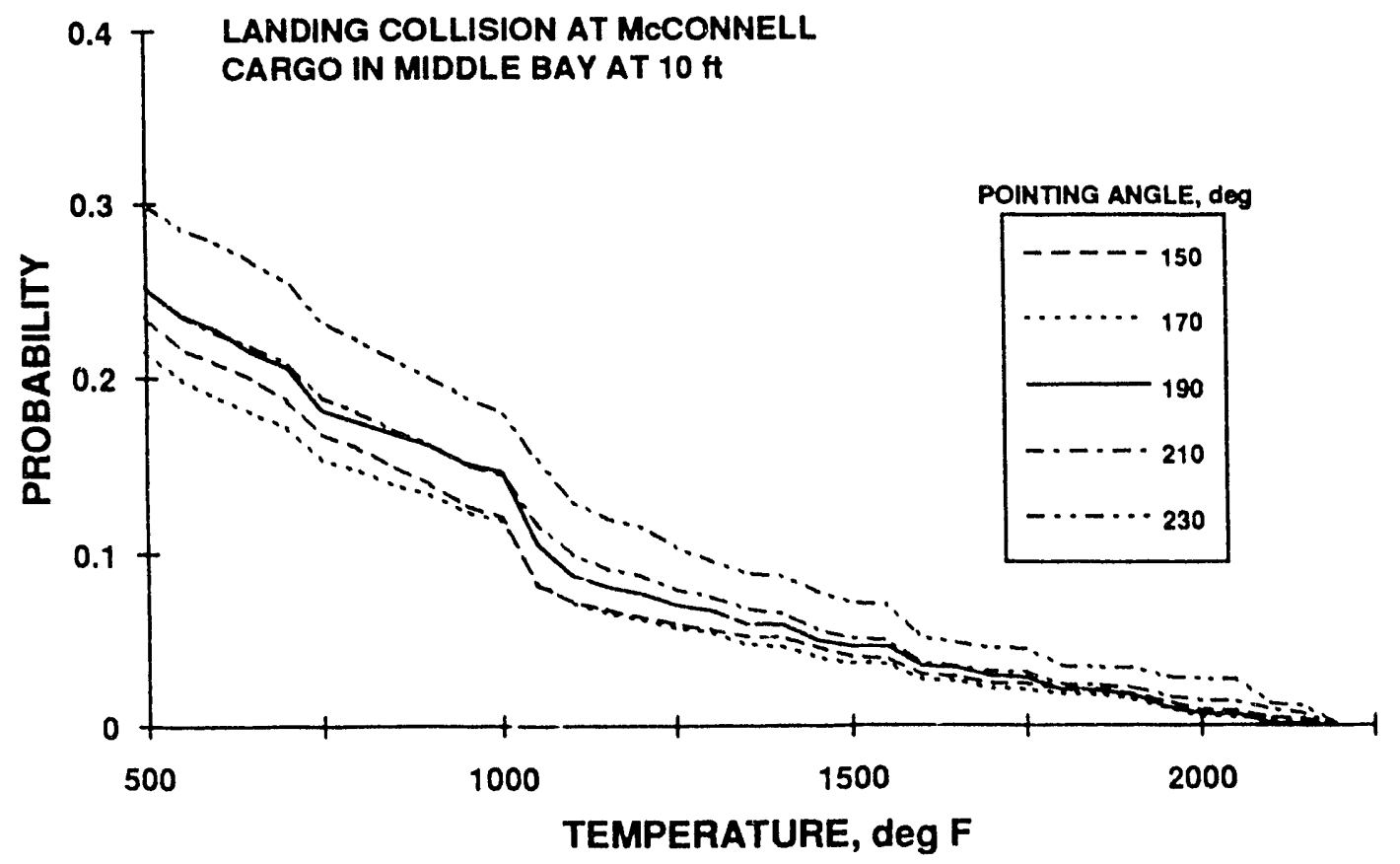

(b)

Figure 3.61 The Effect of Alert Aircraft Redirection During Collision 
Calculations of the effect of rotation on the probability of exceeding given temperatures have been made for landing and take-off collisions at several bases, and standard deviations of the variation in probability for 20 and 40 degree rotations have been evaluated. These deviation results are given in Table 3-1 for various temperature ranges. The fractions of accidents that fall into various collision categories of lateral position and impact speed for all collision angles into the B-1B and B-52 were next examined. It was concluded with reasonable confidence that parked aircraft would not rotate for over one-third of collisions, and while highly judgmental, it was estimated that the rotations would be 20 degrees or less for an additional one-third. On this basis, the deviations for 20 degree rotations (Table 3-1) have been chosen as a measure of uncertainty due to rotation. An illustration of the magnitude of this uncertainty is given in Figure 3.62 .

TABLE 3-1 Standard Deviation of Probability From Aircraft Rotation During Collision

\begin{tabular}{|c|c|c|c|}
\cline { 3 - 4 } \multicolumn{1}{c|}{} & \multicolumn{2}{|c|}{ Standard Deviation of Probability } \\
\cline { 3 - 4 } & $500-1000$ & $20^{\circ}$ rotation & $40^{\circ}$ rotation \\
\hline $\begin{array}{c}\text { Temp } \\
\text { Range, }\end{array}{ }^{\circ}$ F & $1000-1500$ & .0210 & .0336 \\
& $1500-2000$ & .0226 & .0418 \\
& $2000-2250$ & .0121 & .0296 \\
\hline
\end{tabular}

The most uncertain input (but an essential input) in the evaluation of probability vs temperature for collision accidents is the probability distribution of fuel spill locations. It was anticipated that the results would be "reasonably" insensitive to spill distribution error, and significant effort was devoted to the development of spill distributions for various types of collision (described in Section 2 and Appendix D). The explanation of uncertainty due to fuel spill distribution uncertainties is considered next. 


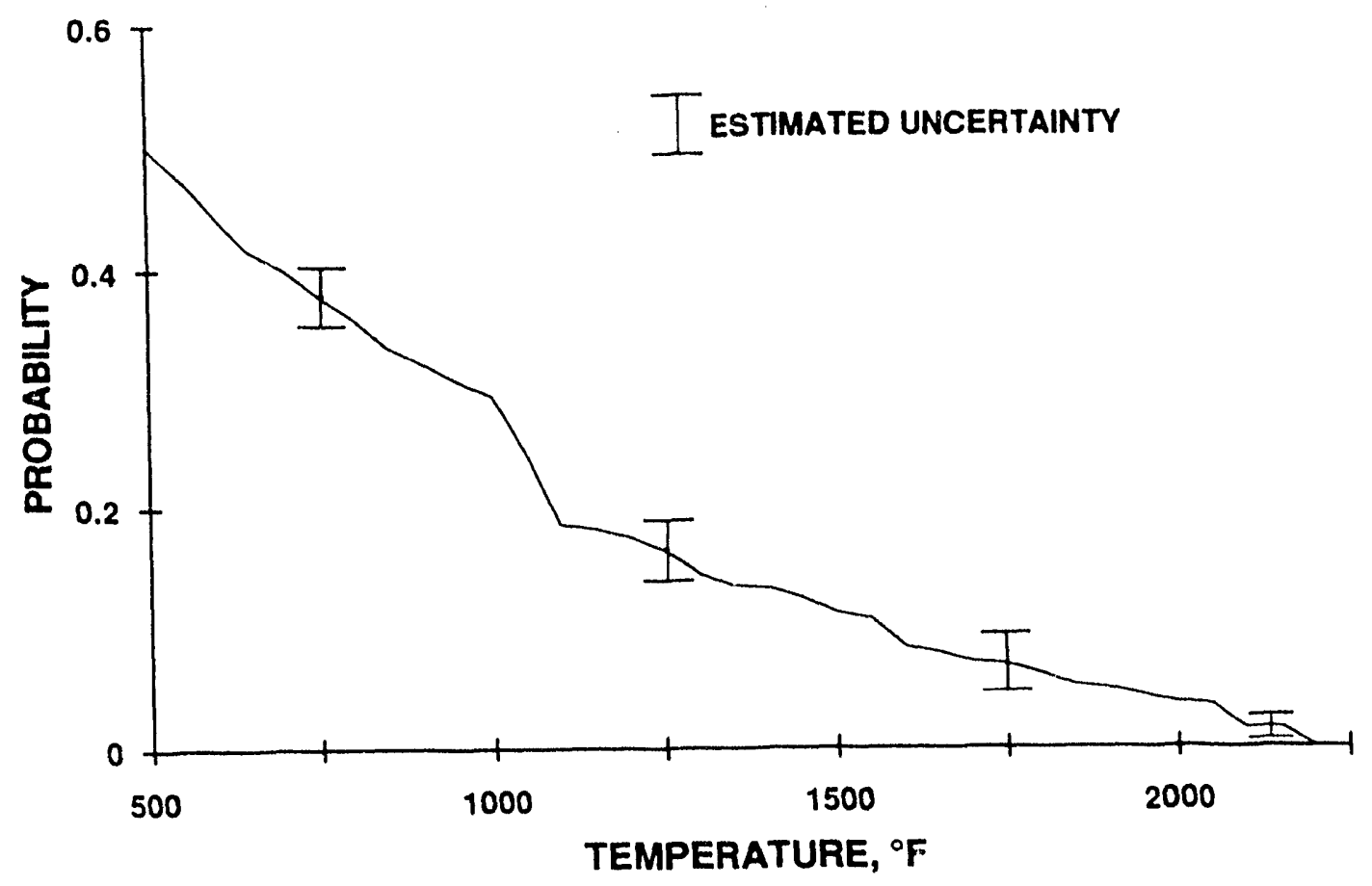

Figure 3.62 Uncertainty Due to Rotation During Collision

In this regard, consider a broadside collision into the B-1B. For this collision, there tends to be comparatively more wing spill points on the impact side of the aircraft, and the impact side also tends to be the downwind side. The initial spill distribution developed for the broadside collision reflects this tendency. There is a greater probability for spills on the "downwind" wing of the alert aircraft, as illustrated in Figure 3.63a. It is possible that there are more spills on the upwind wing than was incorporated in the initial distribution as illustrated in Figure $3.63 \mathrm{~b}$, and spills on the upwind wing would tend to have a maximum impact on the likelihood of cargo exposure. (We have not envisioned an error that could cause a greater impact.) In order to evaluate the effect of upwind wing spills, two modified versions of the standard broadside fuel spill distributions were developed. For one version, upwind spills were added at the outboard wing fuel spill location, which is 65.5 feet from the fuselage axis (this 


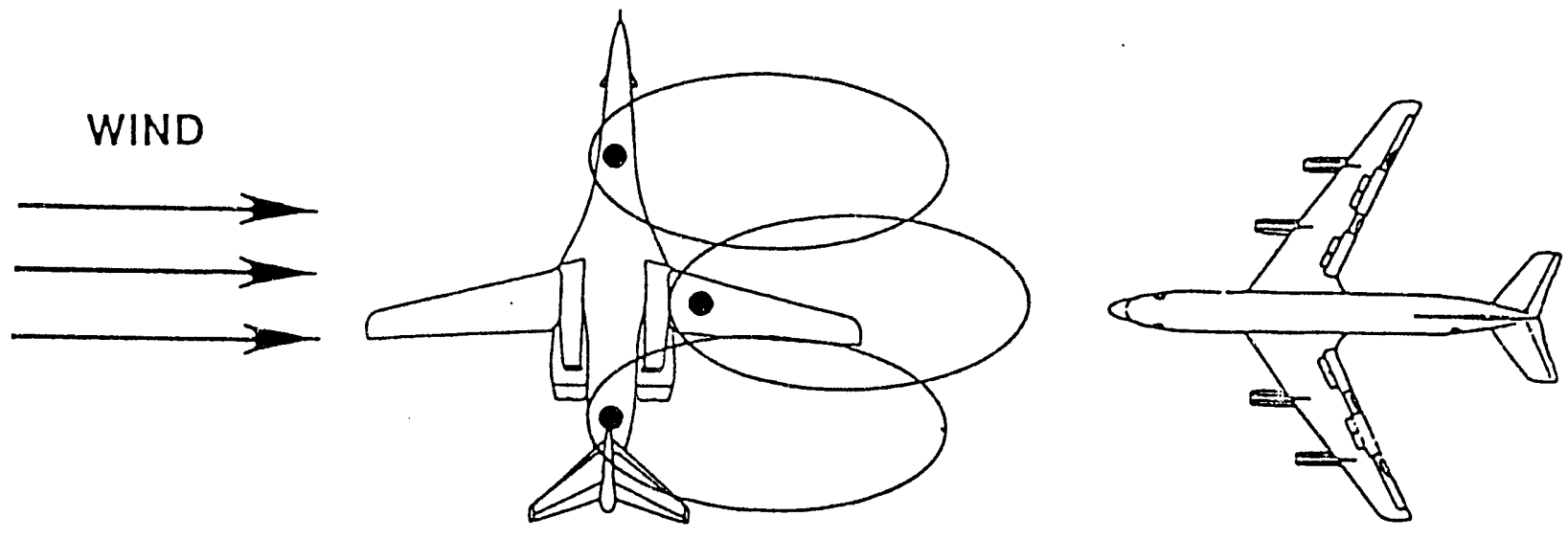

(a)

MODIFIED FUEL SPILL DISTRIBUTION (\# XCZ5)
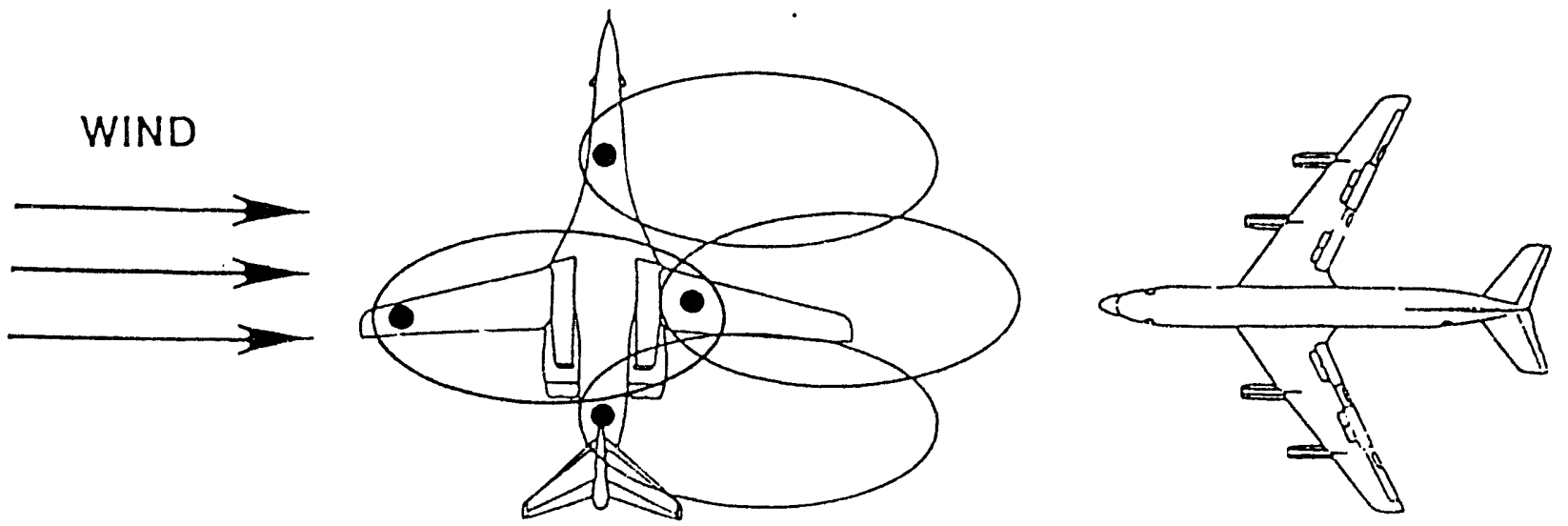

ADDITION.AL UPWIND SPILL CASES

(b)

Figure 3.63 Illustration of Modified Fuel Spill Distribution 
distribution set is referred to as the XCZ5 distribution). For the other version, upwind spills were added to a mid-wing fuel spill location, which is 30.2 feet from the fuselage axis (this distribution set is referred to as the XCW5 distribution). For the standard broadside spill distribution, there are 446 different fire "arrangements," each differing in spill locations and/or rates. For each of the new distributions, fuel was spilled from the new upwind wing location for 160 of the 446 arrangements. The spills at the new locations included all three spill rate categories. There are probabilities of occurrence of each arrangement, and for the new distributions the relative probability that a given arrangement would include a spill at the new upwind location was just over 0.21 . On the basis of the above description, it can be seen that the quantity of added upwind spills is significant. Because of this, and because the added spills are strategically located to have a maximum impact on weapon exposure, a sizeable effect on the probability of exceeding given temperatures would be expected.

The effect of the new XCZ5 distribution and XCW5 distribution can be seen on Figures 3.64 and 3.65 for collisions at Ellsworth and Grand Forks. Comparison of the probability results from the XCZ5 and XCW5 distributions with those from the standard broadside collision distributions can be made on Figures 3.64 and 3.65, respectively. in the overall sense, it can be seen that the effect of the upwind spills is significant. On Figure 3.64, it can be seen that there is no difference in probability for temperatures above about $1800^{\circ} \mathrm{F}$. The reason that there is no high temperature effect for the XCZ5 distribution is that the spill location is remote enough from the fuselage ( 65.5 feet) that the high temperature regions of the low wind fires do not extend to the weapon bay. For the XCW5 distribution, it can be seen from Figure 3.65 that the probability differences extend to the maximum temperatures since, in this case, the spill to fuselage distance of 30.2 feet results in exposure to the high temperatures of low wind speed fires under various wind direction conditions. It is thought that spills 


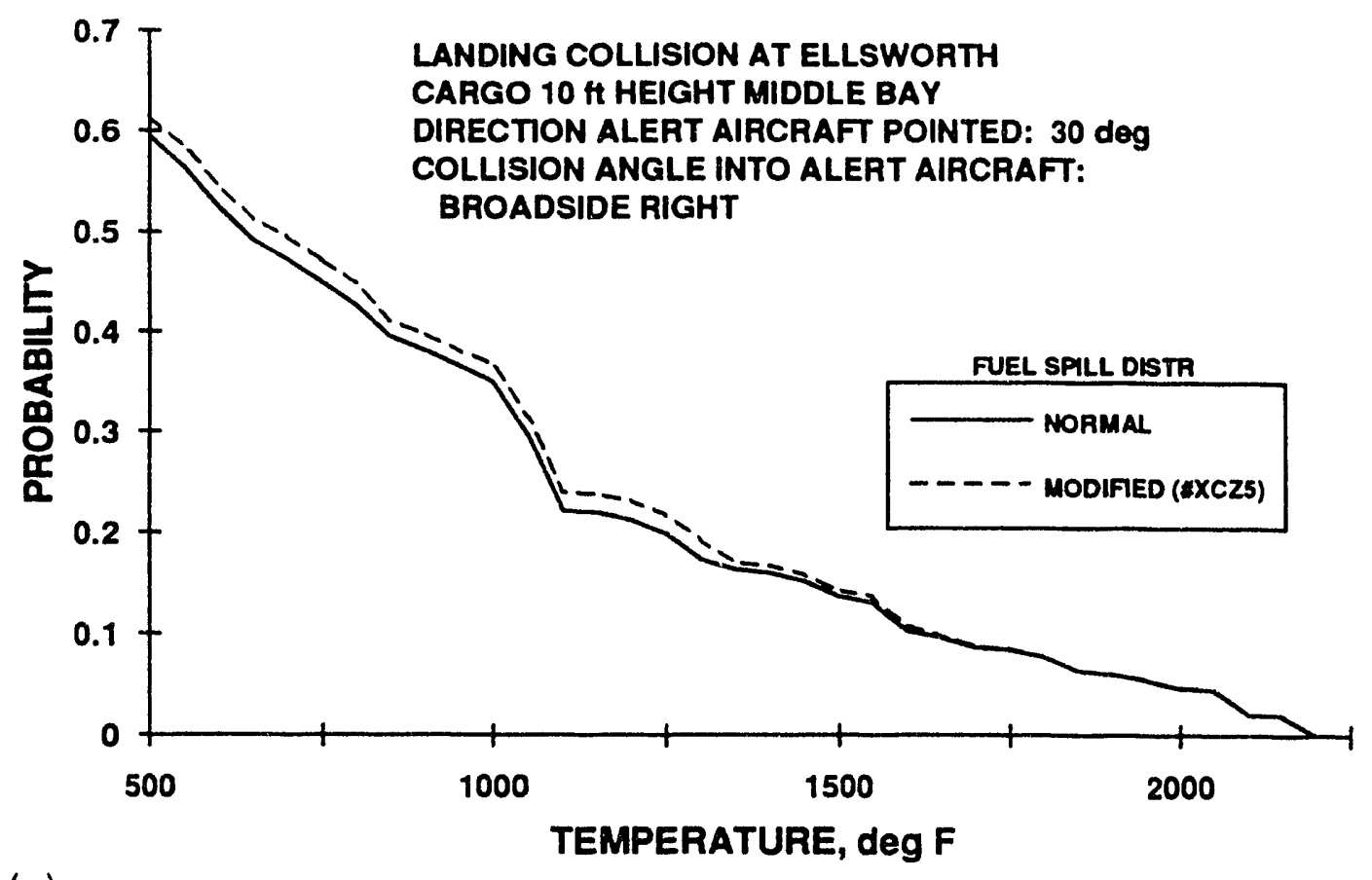

(a)

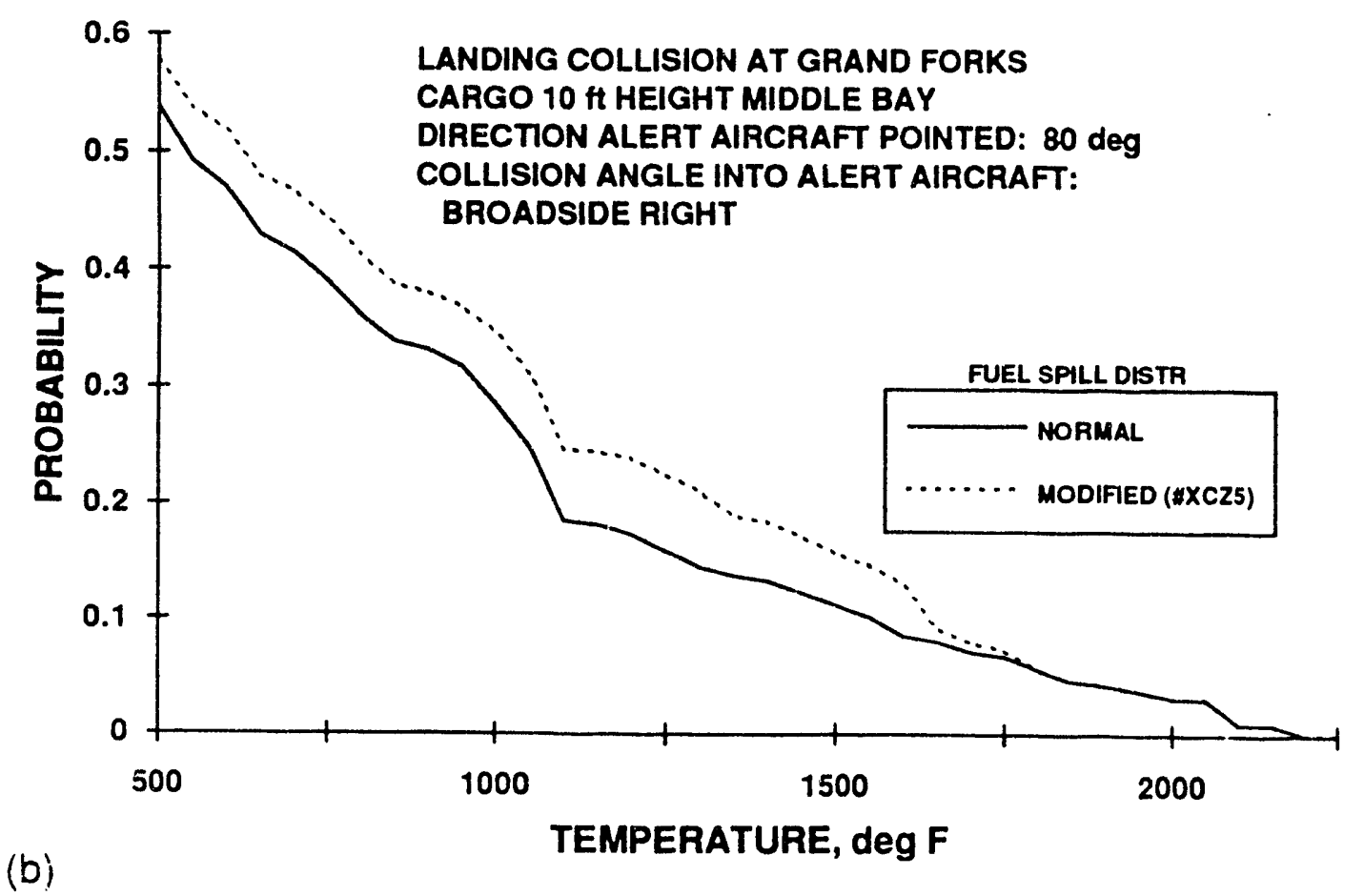

Figure 3.64 The Effect of the XCZ5 Fuel Spill Distribution Errors 

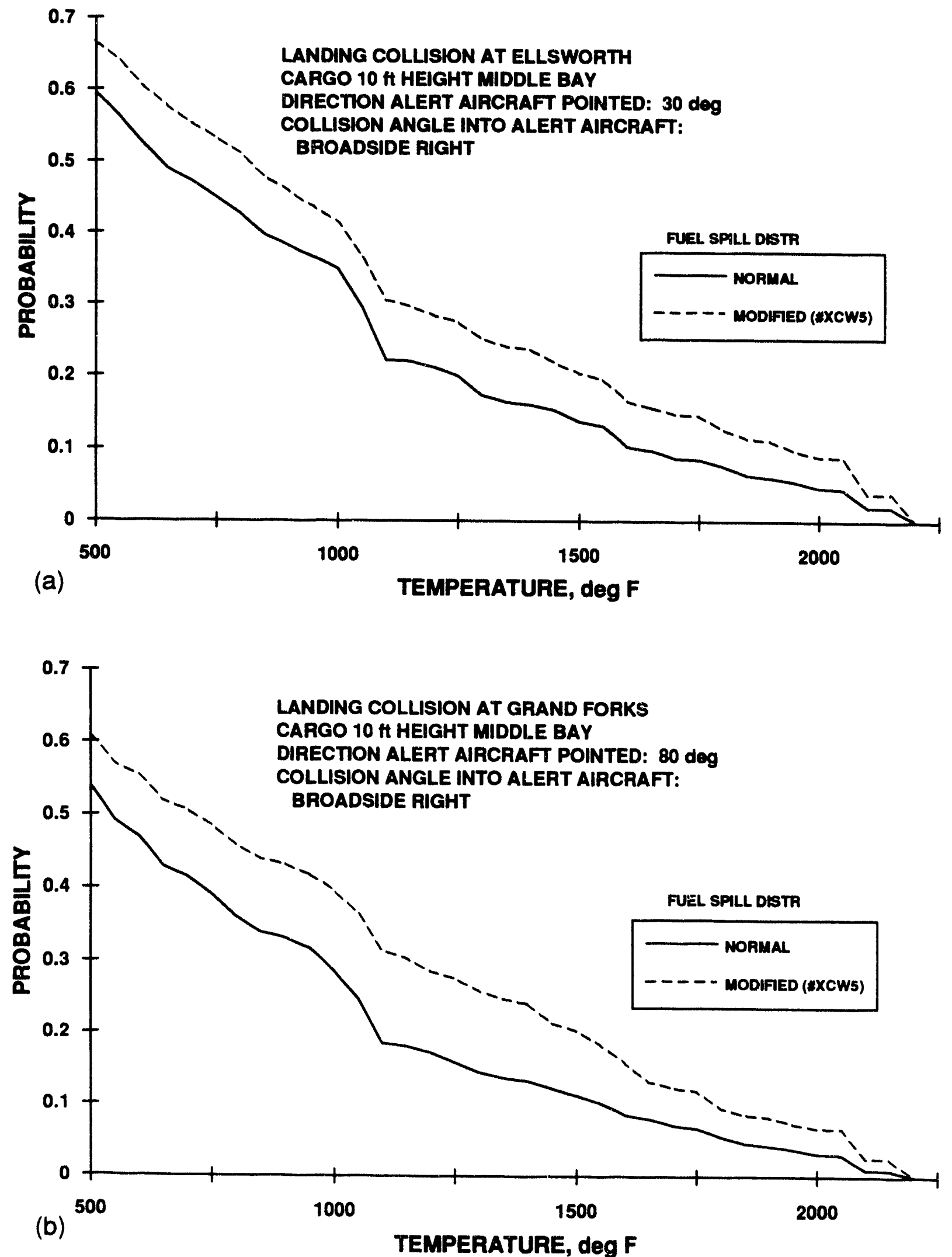

Figure 3.65 The Effect of the XCW5 Fuel Spill Location Distribution Errors 
from both upwind wing locations are possible. It is thought that tank damage near the wing tip can occur, for example, for high impact speed collisions which cause a rotation of the alert aircraft, where the wing tip region can interact with the ground or ground objects. It is thought that mid-wing tank damage can occur during high impact speed collision through penetration of tankage by structures associated with the wheel/ wheel strut mechanism. It is noted that the distributions for the standard (baseline) broadside collision include spills at these upwind wing locations, and that for the XCZ5 and XCW5 distributions the number and probability of spills from these locations was simply expanded significantly.

Regarding estimates of uncertainty, the addition of this abundant number of upwind spills at these strategic locations is viewed as an "extreme case." However, the development of the fuel spill distributions was highly judgmental, and we have thereby chosen to use the effect of the XCZ5 and XCW5 distributions (combined) as an estimate of uncertainty. The corresponding standard deviations are given in Table 3-2, and the deviations are illustrated in Figure 3.66. In using these deviation numbers, it is assumed that the deviation for take-off collisions is equivalent to those for landing collisions.

\section{TABLE 3-2 Estimated Uncertainty Due to Fuel Spill Distribution Input}

\begin{tabular}{|c|c|}
\hline $\begin{array}{c}\text { Temperature } \\
\text { Range, }{ }^{\circ} \mathrm{F}\end{array}$ & $\begin{array}{c}\text { Standard Deviation } \\
\text { of Probability }\end{array}$ \\
\hline $500-1000$ & 0.068 \\
$1000-1500$ & 0.074 \\
$1500-2000$ & 0.039 \\
$2000-2250$ & 0.016 \\
\hline
\end{tabular}

Fuel spill distributions are also uncertain in the fuel spill rates selected. While rate selection is a particularly uncertain input, it was anticipated that the probability would be particularly insensitive to rate. Calculations were made which illustrate the insensitivity of fuel spill rate, and example results are shown on Figure 3.67. For the dashed-line probability curve shown on Figure 3.67, the 


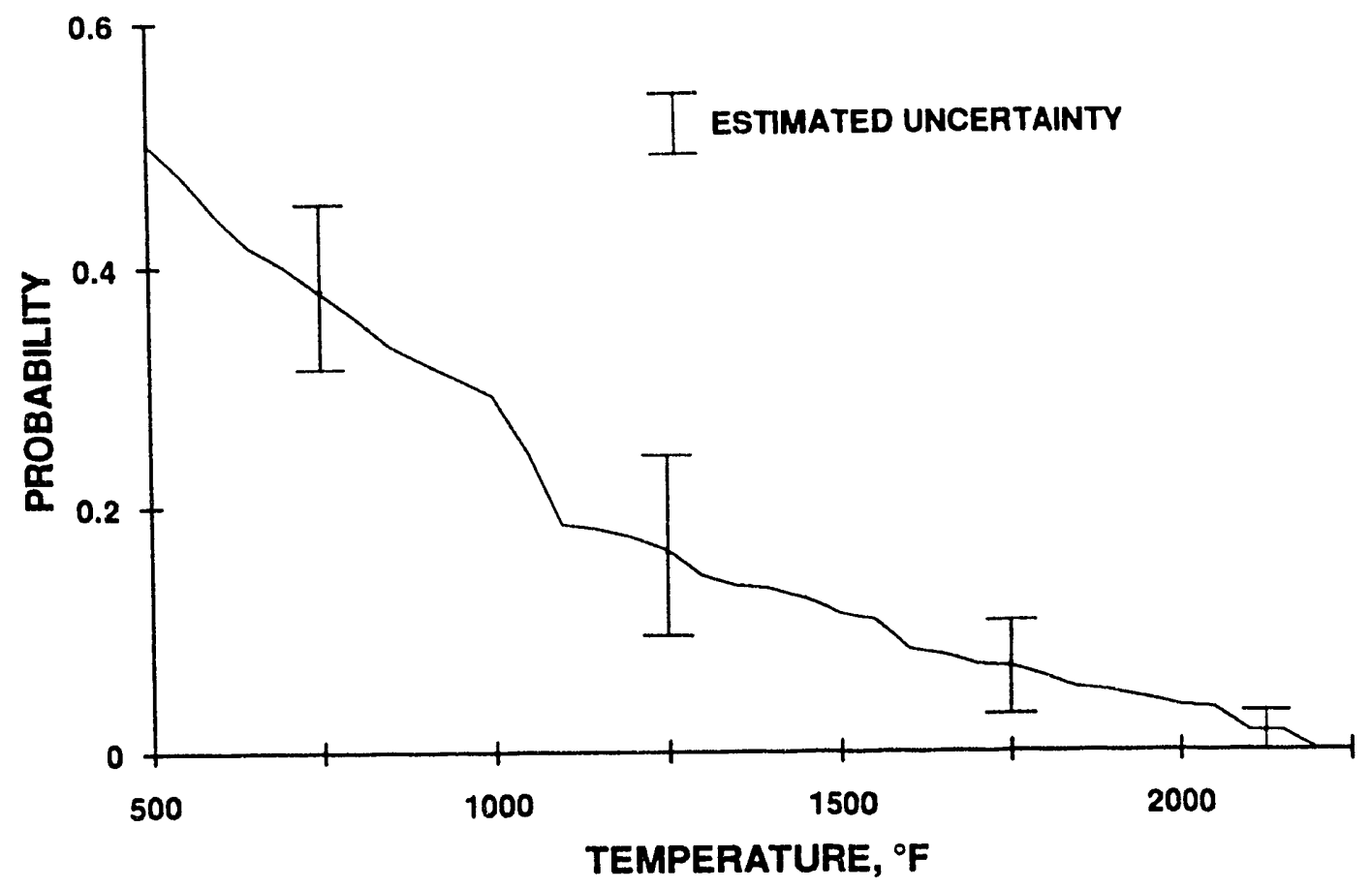

Figure 3.66 Illustration of Uncertainty Due to Fuel Spill Distribution

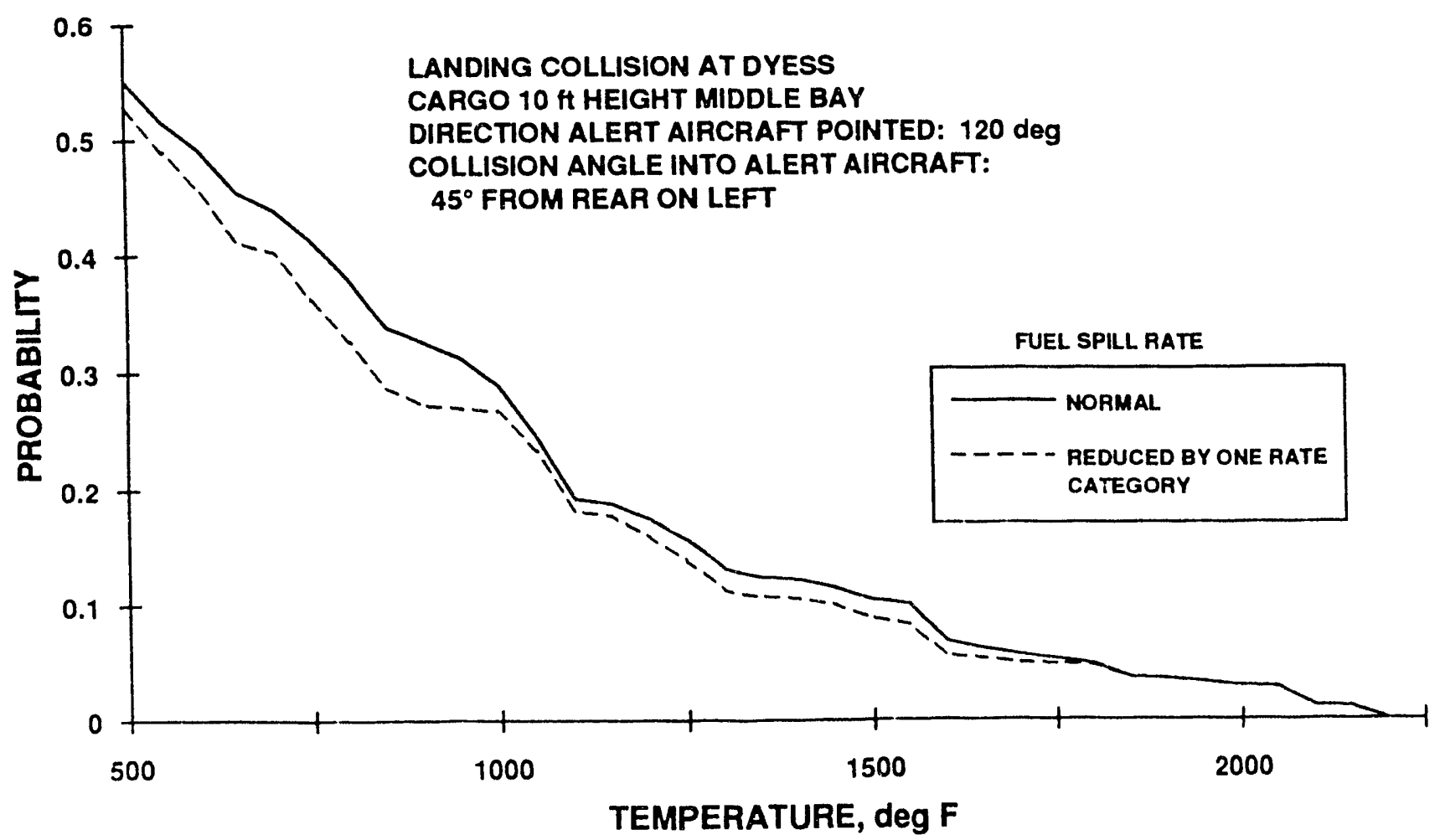

Figure 3.67 Illustrated Effect of Fuel Spill Rate on the Probability of Exposure 
fuel spill rate category in the entire fuel distribution rate file was reduced by one number (all category 3 to category 2, all category 2 to category 1 ). It can be seen that this significant change in rate has caused a small reduction in the probability with temperature. (A corresponding increase in rate causes a corresponding increase in probability.) We have chosen to ignore rate error effects on the basis of these calculations.

It is mentioned in Section 2 that certain types of collision appear to result in fuel spill rates that are substantially larger than the maximum rate of $600 \mathrm{gpm}$ for which temperature data are unavailable. It is thought that these comparatively high rates are statistically infrequent, and would not significantly alter the general results that have been given. (It is noted, also, that these high spill rates result in comparatively small fire durations). If results for larger spill rates (and larger fires) could be included, two effects on the results would be anticipated. First, the probabilities over the "medium" temperature range may increase somewhat, simply on the geometric basis that the fires are larger in diameter and exposure is correspondingly more likely to occur. Second, it is anticipated that the probabilities over the "high" temperature range would decrease. This decrease is anticipated because the "highest temperature region" in fires tends to increase its height with increasing fire diameter, and would rise above the weapon region for fires somewhat larger than the $600 \mathrm{gpm}$ fire.

A discussion in Section 2 indicated that the probability of exposure to given temperatures depends to some extent on the ratio of the number of landing vs take-off accidents that result in fires. For commercial transports, the ratio was identified as 3.0. From the available military accident data, the ratio of landing to take-off accidents ranged from about 2 to 4 depending on the type of aircraft, but the data did not distinguish between accidents that did or did not result in fires. There is some uncertainty, then, with regard to the fire related ratio for military application, and the effect of this uncertainty is considered next.

Figure 3.68 shows the probability of exceeding given temperatures for various values of the ratio for Barksdale and Ellsworth AF Bases. The almost negligible effect of the ratio shown on Figure 3.68.a for Barksdale is typical. The effect shown on Figure 3.68b for Ellsworth AF Base is one of the two extreme cases in which the dependence on the ratio was far more substantial than that for other bases. On the basis of these results, the effect of the uncertainty in this ratio will be ignored.

The uncertainties that have been examined are independent in the sense that the value of one does not systematically depend on the value of another. The overall uncertainty for collision accidents, $\Delta \mathrm{P}_{\text {COLL }}$ may then be determined by 

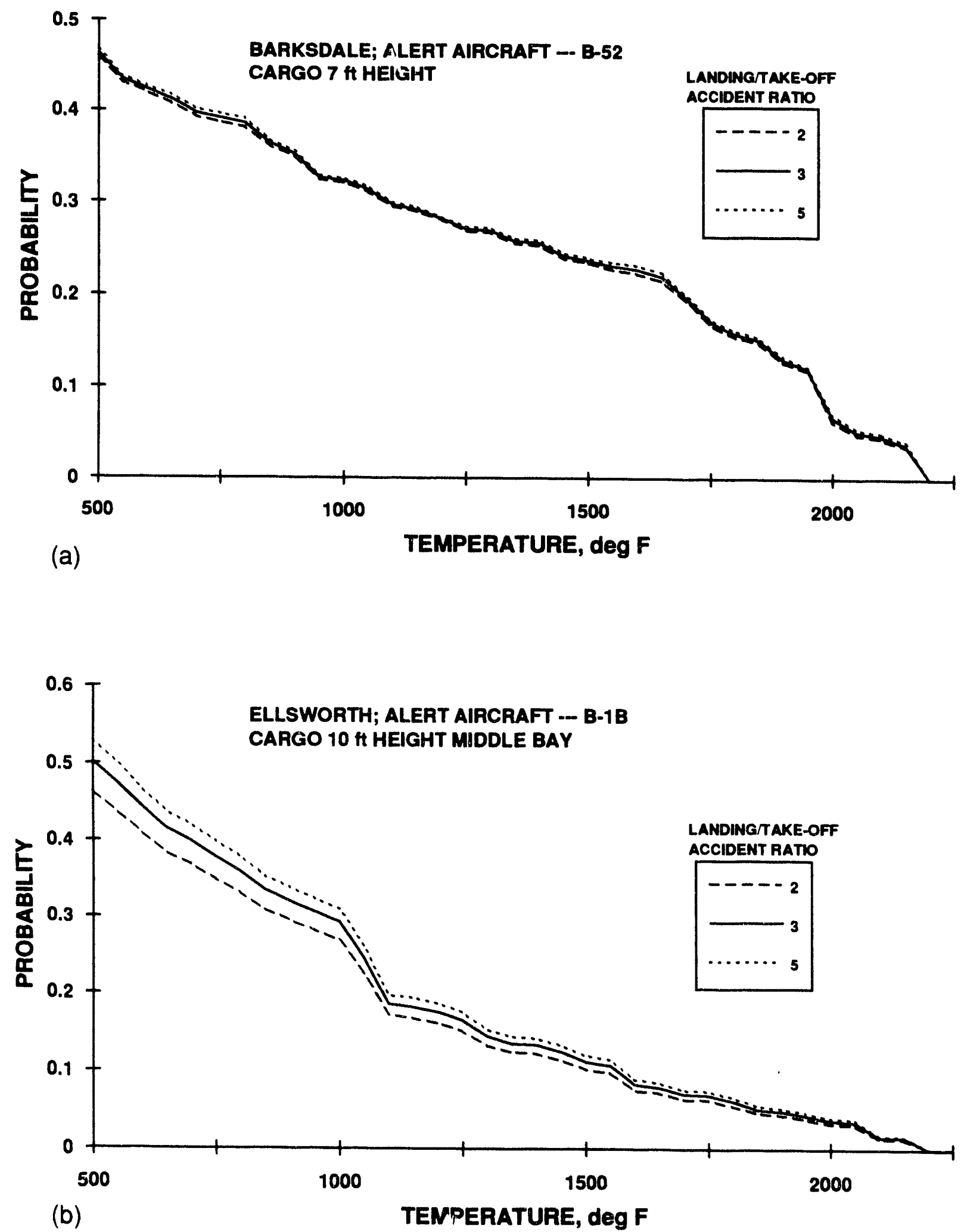

Figure 3.68 The Effect of the Ratio of Landing to Take-off Accidents 
combining the individual uncertainties from the various sources, $\Delta \mathrm{P}_{i}$, using the rule $\Delta \mathrm{P}_{\mathrm{COLL}}=\left(\sum_{i}\left(\Delta \mathrm{P}_{i}\right)^{2}\right)^{1 / 2}$. A summary of the individual and total uncertainties for collision accidents is given on Table 3-3. An illustration of the magnitude of the total uncertainty is given on Figure 3.69. (Extension of the uncertainty to a negative value at $2125^{\circ} \mathrm{F}$ on Figure 3.69 is a consequence of the method of uncertainty evaluation, and of course has no meaning.)

TABLE 3-3 Summary of Uncertainties for Collision Accident

\begin{tabular}{|c|c|c|c|c|c|}
\hline & \multicolumn{4}{|c|}{ TEMPERATURE RANGE, ${ }^{\circ} \mathrm{F}$} \\
\hline & & $500-1000$ & $1000-1500$ & $1500-2000$ & $2000-2250$ \\
\hline \multirow{4}{*}{$\begin{array}{l}\text { UNCERTAINTY } \\
\text { SOURCE }\end{array}$} & $\begin{array}{l}\text { TEMPERATURE FIELD } \\
\text { REPRODUCIBILITY }\end{array}$ & 0.013 & 0.013 & 0.013 & 0.013 \\
\hline & $\begin{array}{l}\text { TEMPERATURE FIELD } \\
\text { EXTRAPOLATION }\end{array}$ & 0.070 & 0.031 & $\cdots$ & $\cdots$ \\
\hline & $\begin{array}{c}\text { FUEL SPILL } \\
\text { DISTRIBUTION }\end{array}$ & 0.068 & 0.074 & 0.039 & 0.016 \\
\hline & $\begin{array}{l}\text { ALERT AIRCRAFT } \\
\text { ROTATION DURING } \\
\text { COLLISION }\end{array}$ & 0.021 & 0.023 & 0.019 & 0.012 \\
\hline \multicolumn{2}{|c|}{ TOTAL UNCERTAINTY } & 0.100 & 0.084 & 0.045 & 0.024 \\
\hline
\end{tabular}

The discussion now turns to the uncertainties for the engine start accidents, which are considerably different in nature than for the collision accident. Uncertainties caused by extrapolating the flame temperature field beyond the measurement region depend greatly on the geometric relationship between the four spill points and the weapon volume. In many cases, for all wind directions that result in weapon exposure, weapon location is entirely within that part of the temperature field that was measured; in these cases, then, there is no uncertainty due to extrapolation of the temperature field. Calculations to obtain the probability of exposure to given temperatures for engine start accidents use test data from tests 3,7 and 8 only. For Test 8 for the B-52, and for Test 7 for both the B-1B and the B-52, there is no weapon exposure to the extrapolated part of the temperature field for any wind direction. For Test 8 for the B-1B, there is exposure to the extrapolated temperature field only in the 500 to $750^{\circ} \mathrm{F}$ range for some wind directions. For this case, the difference in the probability of exceeding given temperatures for the baseline and modified temperature files (TAX8 and TAZ8) is given in Figure 3.70 for all B-1B bases. These values are significant in the $500-700^{\circ} \mathrm{F}$ range, but the difference is zero for temperatures of $750^{\circ} \mathrm{F}$ and above. Examination of the geometry of exposure for Test 3 shows that there is weapon exposure in the extrapolated region for some wind directions only in the $500-750^{\circ} \mathrm{F}$ temperature range for the $\mathrm{B}-1 \mathrm{~B}$, and only in the $500-800^{\circ} \mathrm{F}$ 


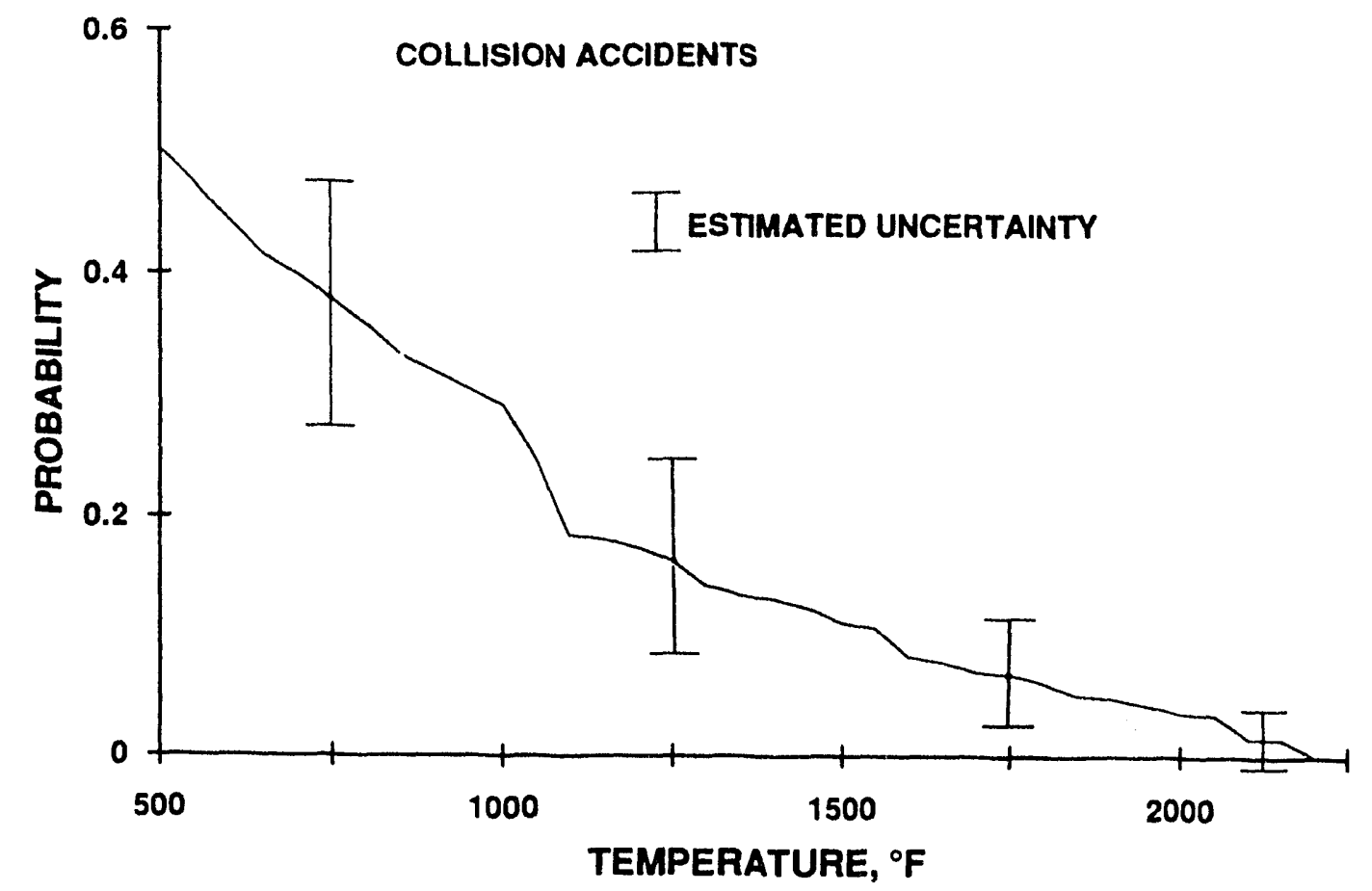

Figure 3.69 Illustration of Total Uncertainty for Collision Accidents

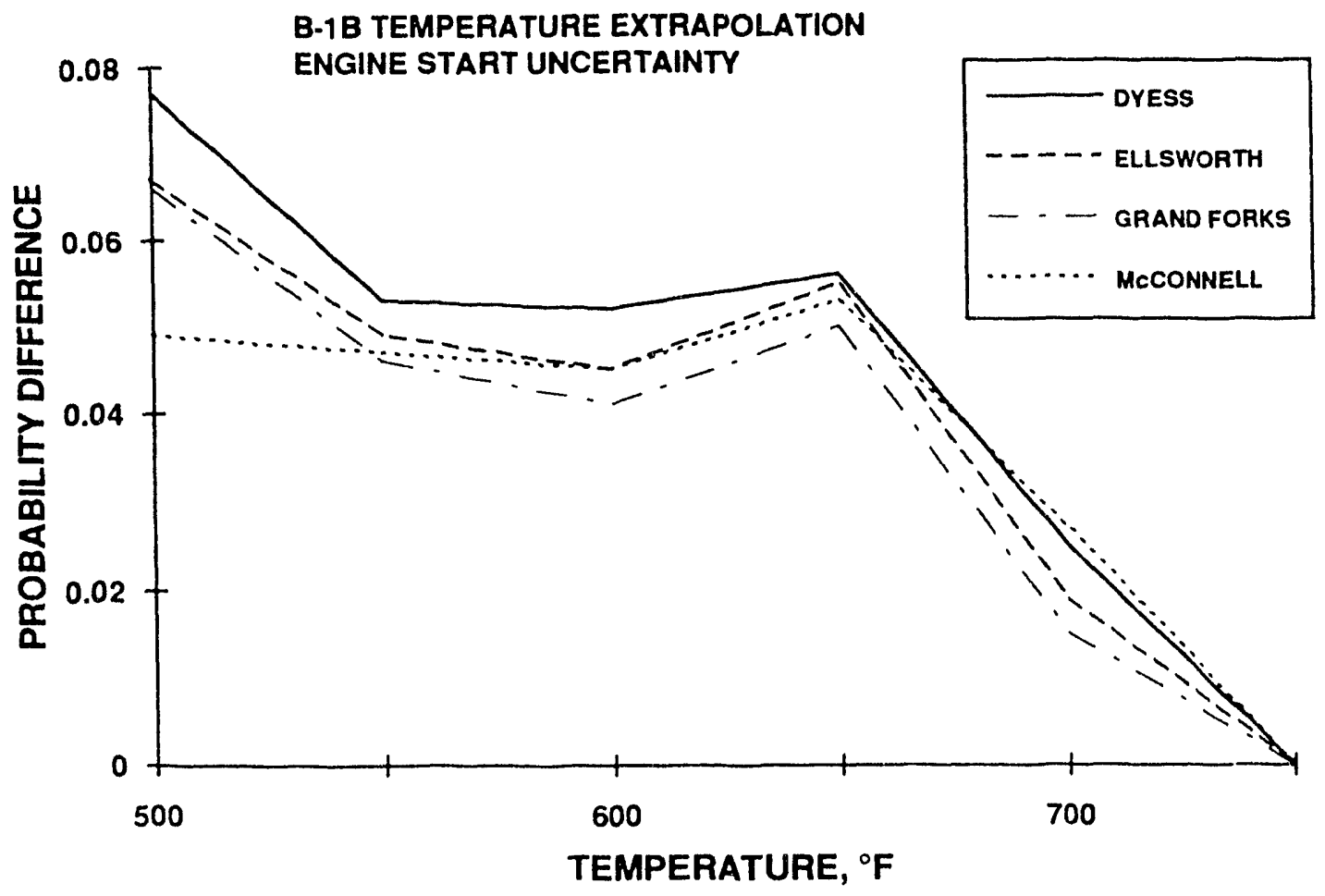

Figure 3.70 Uncertainty Due to Temperature Extrapolation for B-1B Engine Start Accident 
range for the B-52, similar in extent to B-1B for Test 8 above. The probability of wind speeds in excess of $20 \mathrm{mph}$ (corresponding to Test 3 ) is 0.035 (averaged over all twelve bases), compared to 0.67 for wind speeds in the $0-10 \mathrm{mph}$ range (corresponding to Test 8 ). It is anticipated then that the probability differences for Test 3 would be approximately $5 \%(0.035 / 0.67)$ of those shown for Test 8 on Figure 3.70, and we have chosen to ignore the perturbation caused by Test 3 .

Regarding fire test reproducibility, as mentioned above, calculations to obtain the probability of exposure to given temperatures for engine start accidents use test data from tests 3,7 and 8 only. The replicate tests, tests 4 and 9 , cannot then be directly applied to evaluate the uncertainty as was done with the collision accidents. The assumption that the effect for engine start accidents is comparable to that for collision accidents is reasonable provided the assumption is limited to average effects over fairly broad temperature ranges, e.g., 1000 to $1500^{\circ} \mathrm{F}$, etc.; it is anticipated that if data from a replicate for Test 7 was available and applied, the uncertainty effect may vary with temperature to a greater extent than for the collision accident. We have chosen to use the uncertainty evaluated for the collision accidents as the measure of uncertainty for engine start accidents, with the limitation mentioned above. The uncertainty in probability for all temperature ranges is then $0.0056 \times \sqrt{3}=0.01$, where the value " 3 " (rather than 5) enters in the engine start case since the data from three tests are used.

The origin and difficulties of the fuel spill distributions for the engine start accidents were discussed in Section 2. For the baseline distributions, it was assumed that there would not be multiple concurrent spills and that fuel spills were equally likely at one of the following four locations: the left or right inboard engine exhaust or intake; this is the basis of all results given in subsections 3.2 and 3.3. The only alternate distribution that appears sensible is the distribution where it is equally likely to have spills at the right inboard exhaust and intake concurrently, or at the left inboard exhaust and intake concurrently. The alternate is probably less likely, and since there are always two fires with the alternate and thus a greater chance of exposure, the probability resulting from the alternate will always be substantially higher than those from the baseline distribution. Example results showing the probability vs temperature for the baseline and alternate distributions are given on Figure 3.71. It can be seen that the probability difference from the two types of distribution is significant, particularly for the B-1B at McConnell. The probability difference patterns for the B-1B and the B-52 on Figures 3.71 $a$ and $b$, respectively, are very typical. The difference is much larger for the B-1B than the B-52 over most of the temperature range, and for the $B-52$, the difference tends to be largest in the 1500 to $1800^{\circ} \mathrm{F}$ range. These characteristics depend on the geometric relationship between spill points and weapon volume, not on the differences in airbase or weather. 


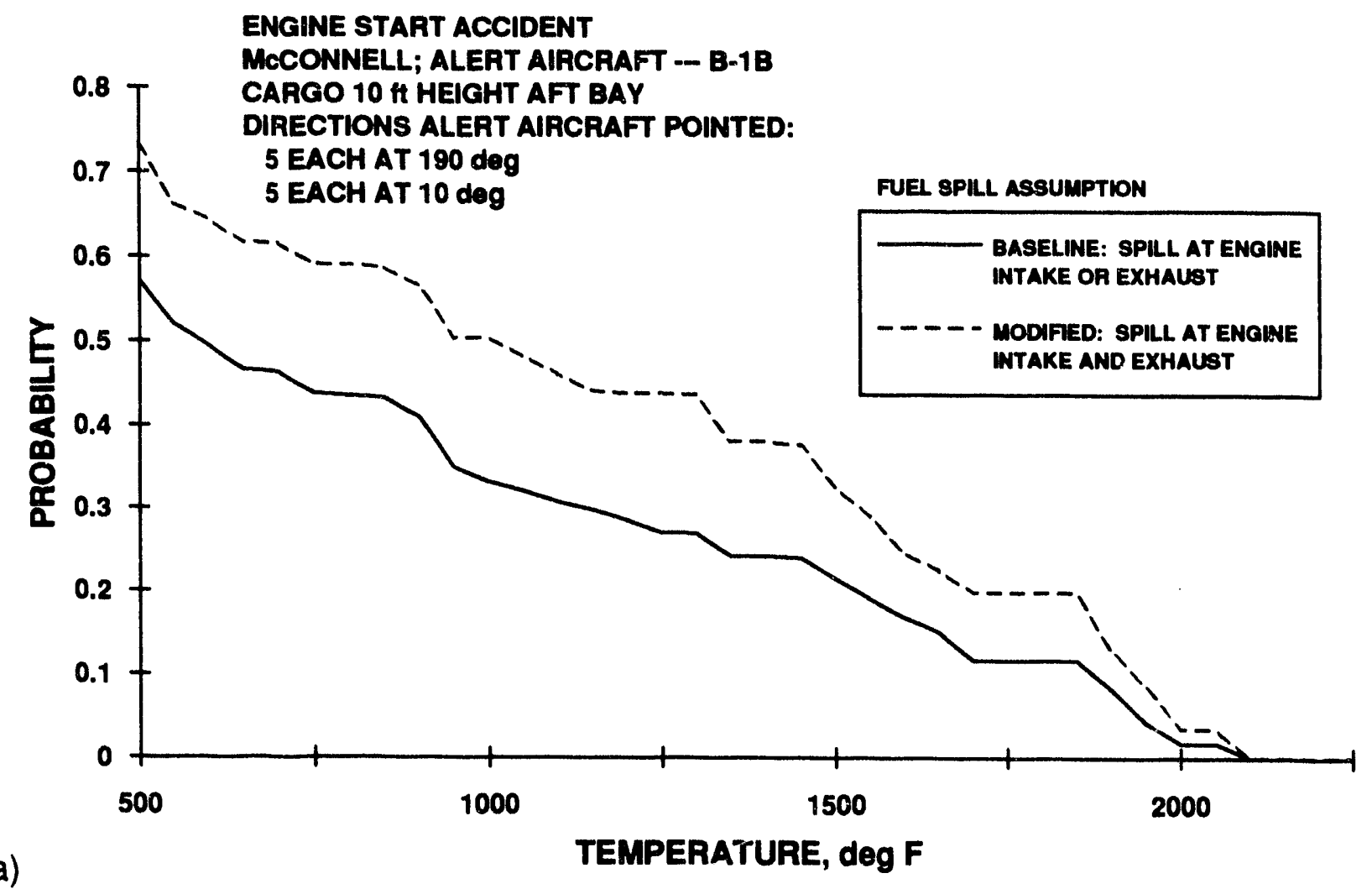

(a)

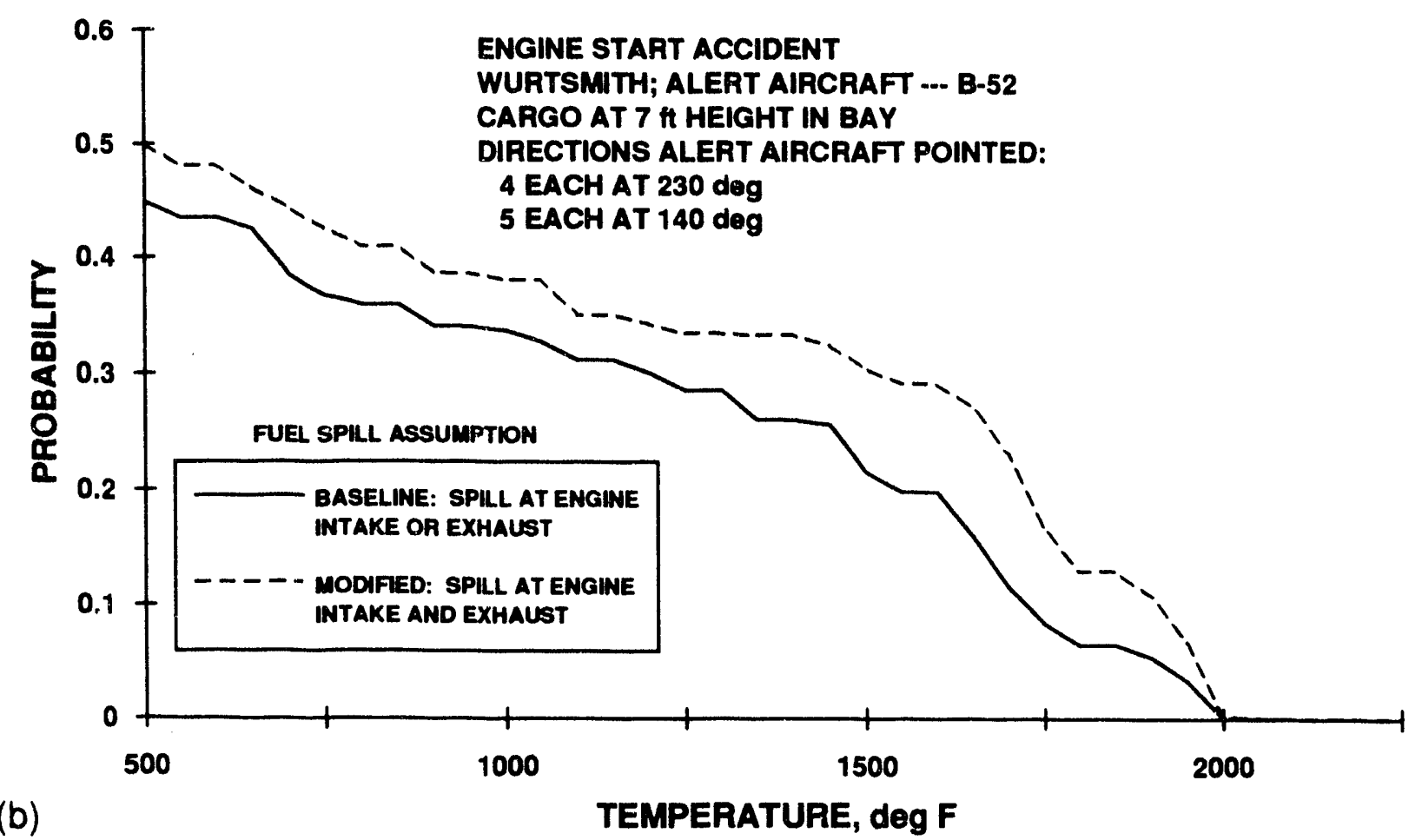

Figure 3.71 The Effect of Alternate Fuel Spill Distributions for Engine Start Accidents 
It is difficult in the engine start case to establish a sound basis for estimating the uncertainty due to fuel spill uncertainties. We have chosen the difference in probability resulting from the two types of distribution as a "reasonable" measure of the uncertainty. Standard deviations based on the results from nine of the twelve bases are given in Table 3-4.

TABLE 3-4 Standard Deviations of Probability for Engine Start Accidents

\begin{tabular}{|c|cccc|}
\cline { 2 - 5 } \multicolumn{1}{c|}{} & \multicolumn{4}{c|}{ TEMPERATURE RANGE, $^{\circ} \mathrm{F}$} \\
\cline { 2 - 5 } \multicolumn{1}{c|}{} & $500-1000$ & $1000-1500$ & $1500-2000$ & $2000-2100$ \\
\hline B-1B & 0.149 & 0.130 & 0.076 & 0.019 \\
& & & 0.083 & 0 \\
B-52 & 0.051 & 0.063 & 0.083 \\
\hline
\end{tabular}

A summary of the uncertainties and the total uncertainty for the engine start accidents is given in Table 3-5. The total uncertainty for engine start accidents is illustrated in Figure 3.72. (Extension of the uncertainty to a negative value at $2050^{\circ} \mathrm{F}$ on Figure 3.72 is a consequence of the method of uncertainty evaluation, and of course has no meaning.)

A brief summary of the results given in Section 3 is given in Section 4.

TABLE 3-5 Summary of Engine Start Accident Uncertainties

\begin{tabular}{|c|c|c|c|c|c|}
\hline & \multicolumn{4}{|c|}{ Temperature Range, ${ }^{\circ} \mathrm{F}$} \\
\hline & & $500-1000$ & $1000-1500$ & $1500-2000$ & $2000-2100$ \\
\hline \multirow{4}{*}{ B-1B } & $\begin{array}{l}\text { Temperature } \\
\text { Extrapolation }\end{array}$ & $.047^{*}$ & $-\cdots$ & -- & $-\cdots$ \\
\hline & $\begin{array}{c}\text { Fire Test } \\
\text { Reproducibility }\end{array}$ & .010 & .010 & .010 & .010 \\
\hline & $\begin{array}{c}\text { Fuel Spill } \\
\text { Distribution }\end{array}$ & .149 & .130 & .076 & 019 \\
\hline & TOTAL & .157 & .130 & .077 & .021 \\
\hline \multirow{3}{*}{ B-52 } & $\begin{array}{c}\text { Fire Test } \\
\text { Reproducibility }\end{array}$ & .010 & .010 & .010 & .010 \\
\hline & $\begin{array}{c}\text { Fuel Spill } \\
\text { Distribution }\end{array}$ & .051 & .063 & .083 & 0 \\
\hline & TOTAL & .052 & .064 & .084 & .010 \\
\hline
\end{tabular}

* The average for the four bases over the temperature range 500 to $700^{\circ} \mathrm{F}$ is treated as the uncertainty for the range 500 to $1000^{\circ} \mathrm{F}$. 

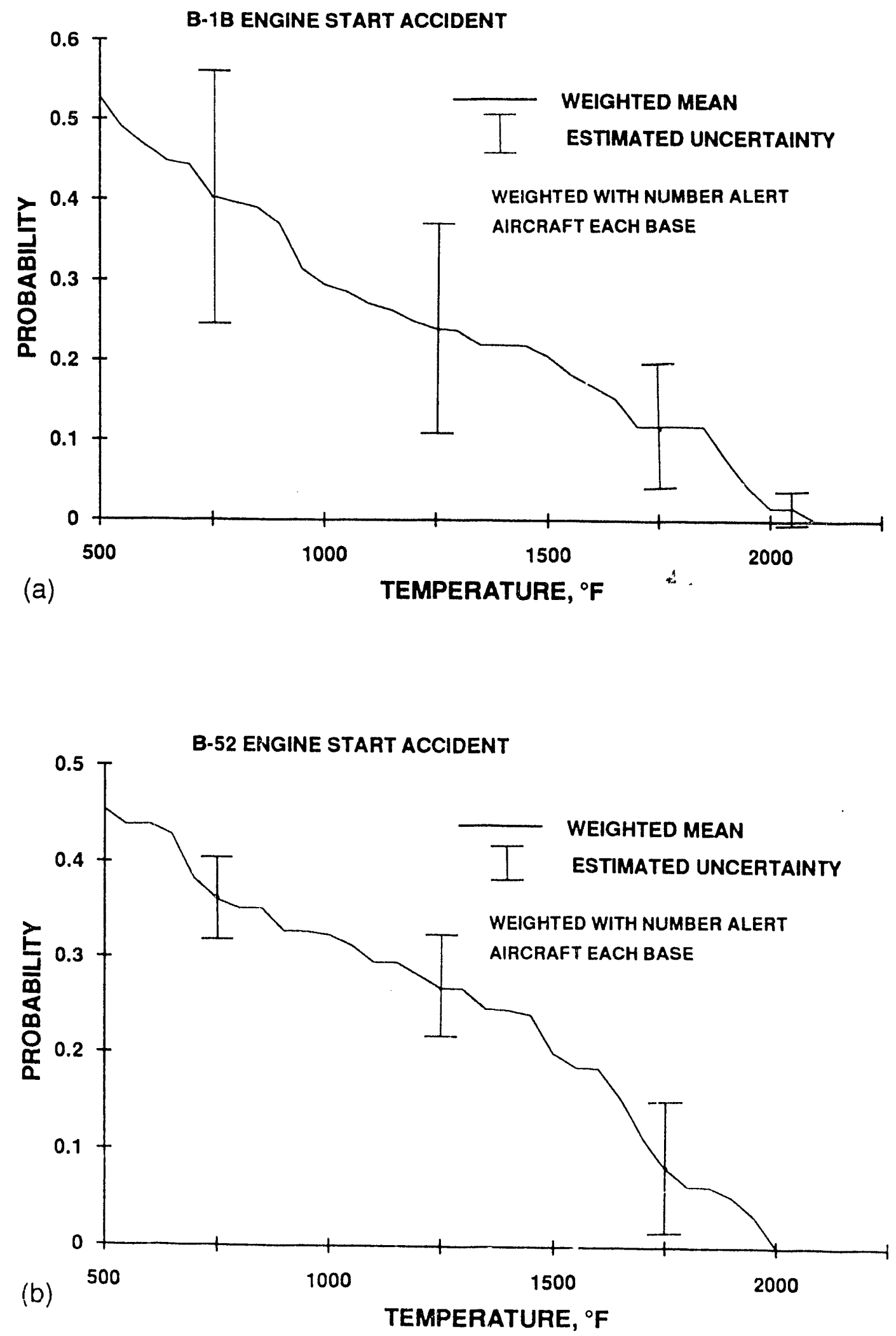

Figure 3.72 Weighted Mean Probabilities and Uncertainties for Engine Start Accidents 


\section{4 SUMMARY OF RESULTS}

The intent of Section 4 is to provide a summary of the predicted probabilities that weapon exposure will exceed given temperatures. Estimated uncertainties of these predictions are also given; uncertainty values presented in this report are intended to represent one standard deviation of error $( \pm 1 \sigma)$.

It is noted that the terms "weapon" and "cargo" are synonymous.

Figure 4.1 shows the weighted mean probability of exceeding given temperatures for the collision of aircraft into the B-1B, where the weighting is in proportion to the relative number of operations (landing/take-off) at each of the four bases (27\% for Dyess, 21\% for Ellsworth, 15\% for Grand Forks, and 37\% for McConnell); the weighted mean probabilities on Figure 4.1 for collision into the B-1B (as well as for other types of accident) are given in tabular form in Table 4-3 at the end of Section 4. Figure 4.1 also indicates the estimated uncertainty of the predicted probabilities for the collision accident. The probability curve given on Figure 4.1 is for the common or "baseline" condition where the weapon is located at the ten foot height in the middle weapon bay, and where the motion of the colliding aircraft is parallel or nearly parallel with the runway; the results for other conditions are illustrated below.

Numerical values of the estimated uncertainty shown in Figure 4.1 are given in Table 4-1. Those are "total" uncertainties, resulting from the combined effect of several sources of uncertainty; these sources include uncertainties in: flame temperature data, the rotation of the parked/alert aircraft during collision, the relative number of collisions that occur during landing or take-off, and fuel spill locations and fuel spill rates that occur under various conditions of collision. The uncertainty of fuel spill location is the major contributor to the total uncertainty (see Subsection 3.5).

TABLE 4-1 Uncertainty in the Probability of Exceeding Given Temperatures for Collision Accidents

\begin{tabular}{|c|cccc|}
\cline { 2 - 5 } \multicolumn{1}{c|}{} & \multicolumn{4}{c|}{ TEMPERATURE RANGE, ${ }^{\circ} \mathrm{F}$} \\
\cline { 2 - 5 } & $500-1000$ & $1000-1500$ & $1500-2000$ & $2000-2250$ \\
\hline $\begin{array}{c}\text { UNCERTAINTY IN } \\
\text { PROBABILITY }\end{array}$ & 0.100 & 0.084 & 0.045 & 0.024 \\
\hline
\end{tabular}

The mean probability of exceeding given temperatures for weapons in the aft bay of the B-1B is given in Figure 4.2, where the weighting is the same as described above; tabular values of the weighted mean are given in Table 4-3. The mean probability for the middle bay (as given in Figure 4.1) is included in Figure 4.2 for convenience of comparison. 


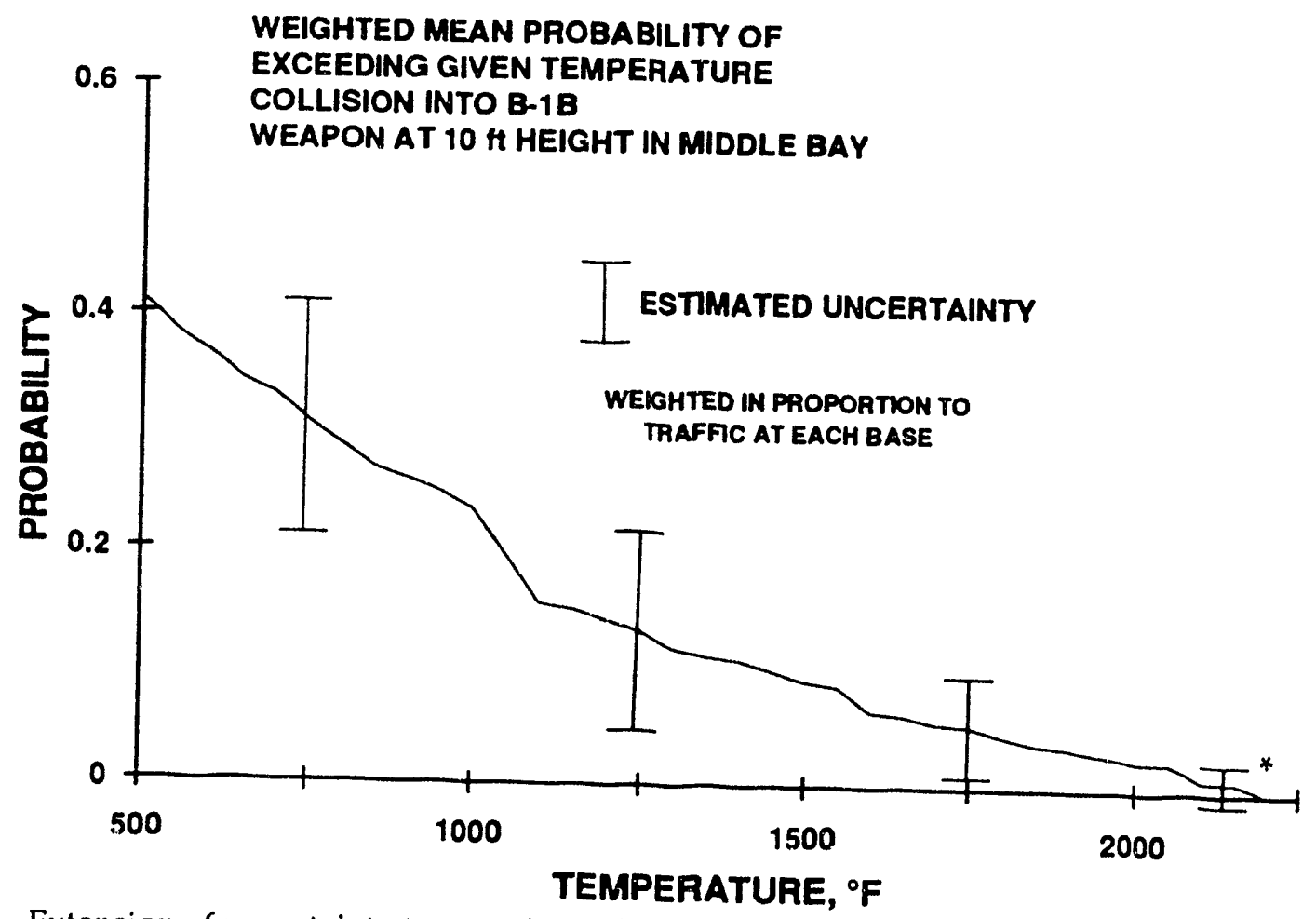
* Extension of uncertainty to negative value is a consequence of the method of uncertainty
evaluation, and has no meaning.

Figure 4.1 Weighted Mean Probability of Exceeding Given Temperature for Collision into B-1B with Weapon in Middle Bay

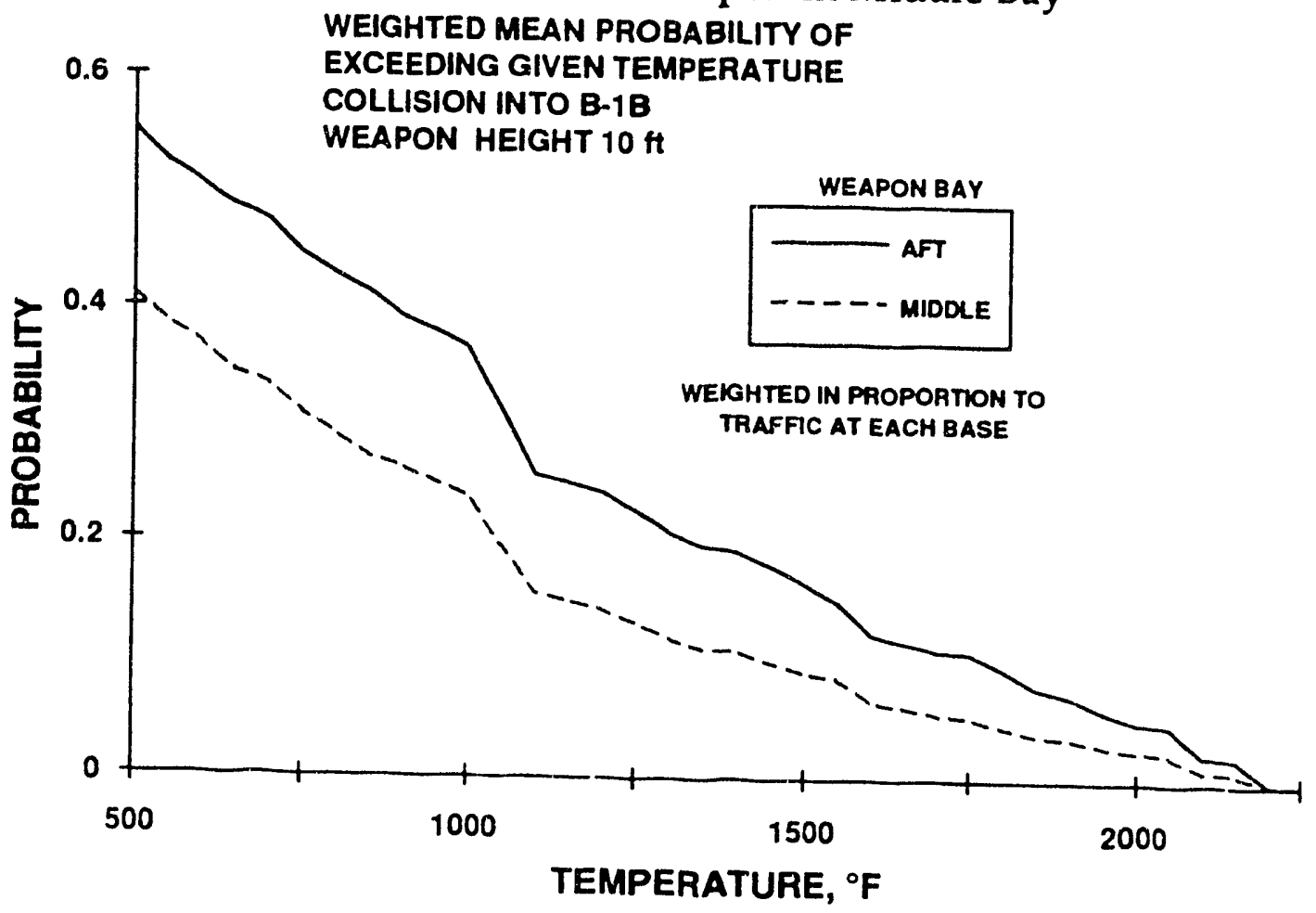

Figure 4.2 Weighted Mean Probability of Exceeding Given Temperature for Collision Accident with Weapon in Middle or Aft Bay of B-1B 
The probabilities of exceeding given temperatures for aircraft collisions into the B-52 are given in Figure 4.3 for the baseline weapon height of 7 feet. In this case, the probability curves are given for individual bases; mean probabilities weighted over all bases (as given in Figure 4.1 for the B-1B) are not given for the B-52 because data on the base to base variation in the number of landing/take-off operations was not available. The estimated uncertainties in Table 4.1 apply to collisions into the B-52.

The results given in Figure 4.1, 4.2 and 4.3 are for the baseline weapon heights of 10 feet for the B-1B and 7 feet for the B-52. Different weapon heights can occur due, for example, to wheel strut collapse and to fire induced weakening of the weapon support structure. The probability of exceeding given temperatures has some dependence on weapon height because there is some variation in flame temperature patterns and levels with height and because the location of the flame area with respect to the spill point will vary with height. Typical examples of exceeding given temperatures are given in Figure 4.4 for both the B-1B and B-52.

The results in this section have been for the condition that the aircraft that collides on landing or take-off into the alert (parked) aircraft is moving parallel or nearly parallel to the runway, that is, the aircraft landing or taking off did not significantly alter its flight direction prior to collision. Collisions can of course occur where the motion is not parallel to the runway, and these collisions, designated here as "off-angle" collisions have been examined. Example results are given on Figure 4.5, which shows the probability of exceeding various temperatures for a range of motion directions both clockwise and counter clockwise about the normal landing direction. It can be seen that there is a sizeable effect on the probabilities, and that there is no consistent increasing or decreasing pattern in the probability for given changes in motion direction from the normal landing direction, that is, counter clockwise changes from the normal landing direction, for example, may lead to an increase or decrease in probability. Further examination of the effect has shown that consistent patterns do emerge when the probabilities are plotted against the direction of the moving aircraft with respect to the pointing direction of the alert aircraft that is, against whether the accident is a broadside collision, rear-end collision, etc. 

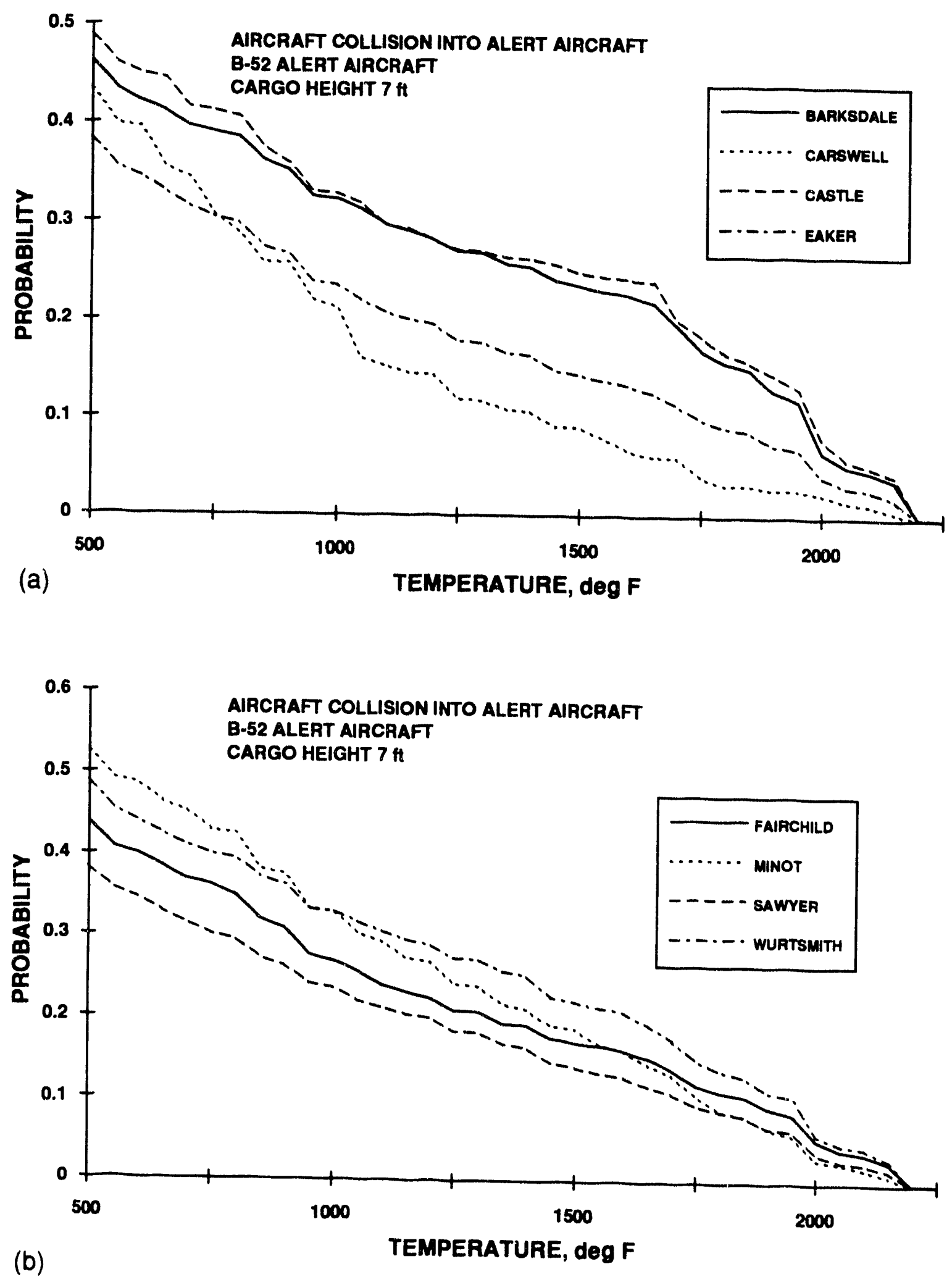

Figure 4.3 Probability of Exceeding Given Temperatures for Eight B-52 Bases 

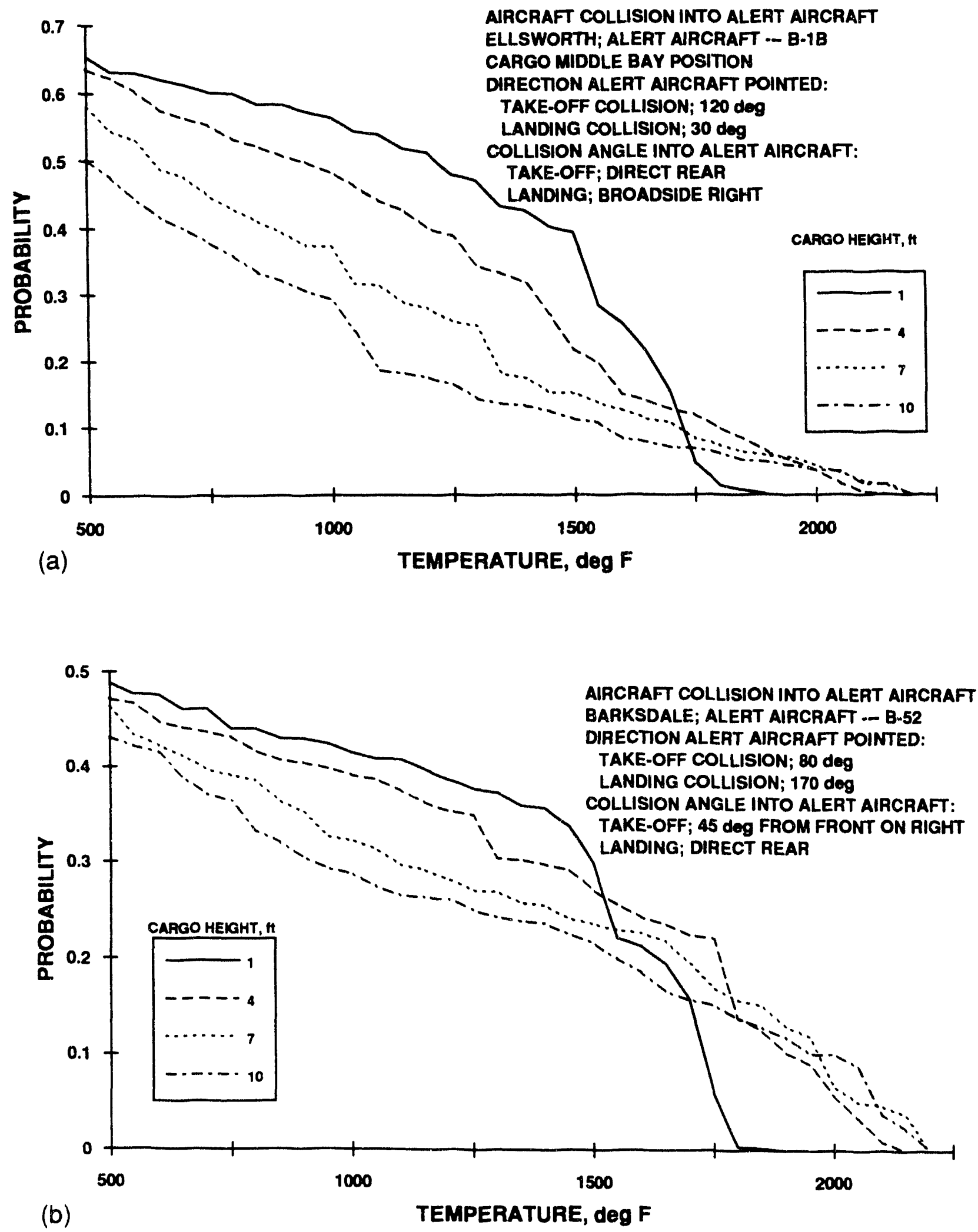

Figure 4.4 Examples of the Effect of Weapon Height on the Probability of Exceeding Given Temperatures for Collision Accidents 

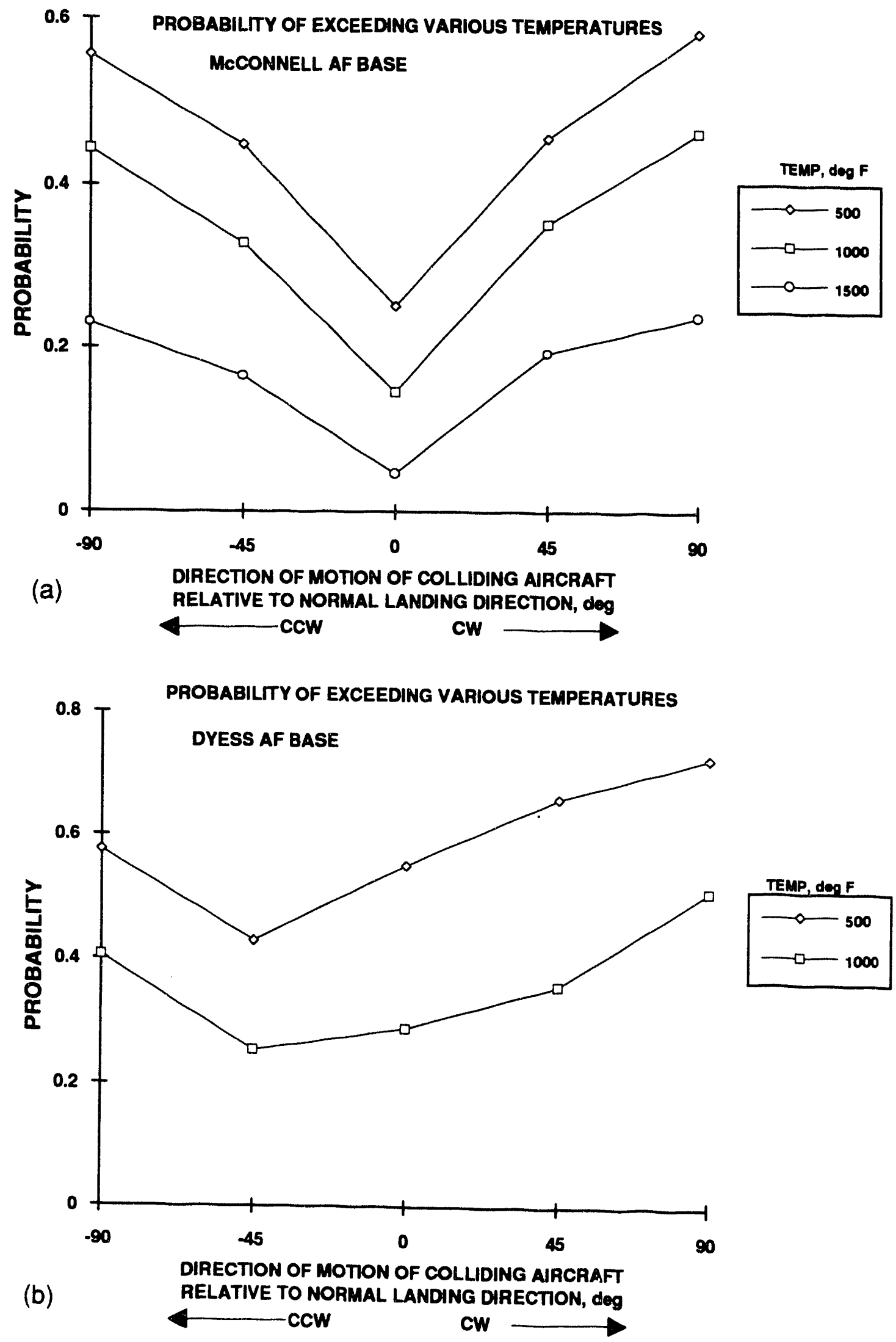

Figure 4.5 Probability of Exceeding Various Temperatures for Off-Angle Collisions 
Example results in these terms are given on Figure 4.6, which shows the probability of exceeding $500^{\circ} \mathrm{F}$ for a range of "collision angles" into both the B-1B and B-52. It can be seen on Figure 4.6 that the probability increases as the collision angle changes from the rear end collision. These plots suggest, and detailed analysis confirms, that collision angle is a major governing factor of the probability of exposure.

(a)

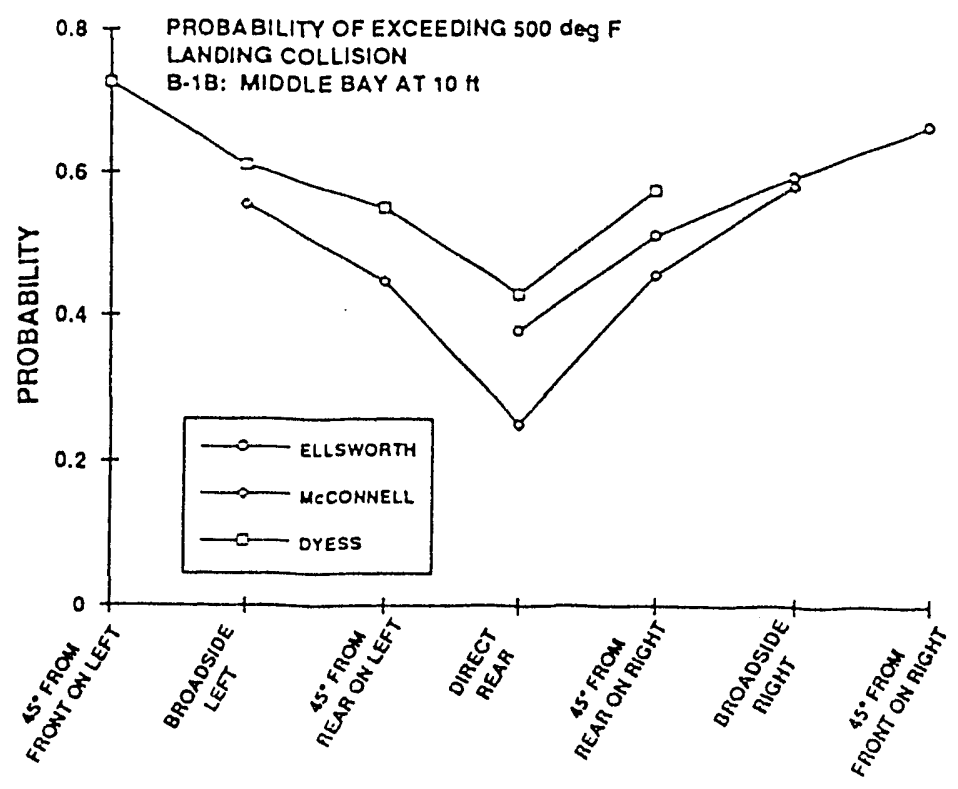

(b)

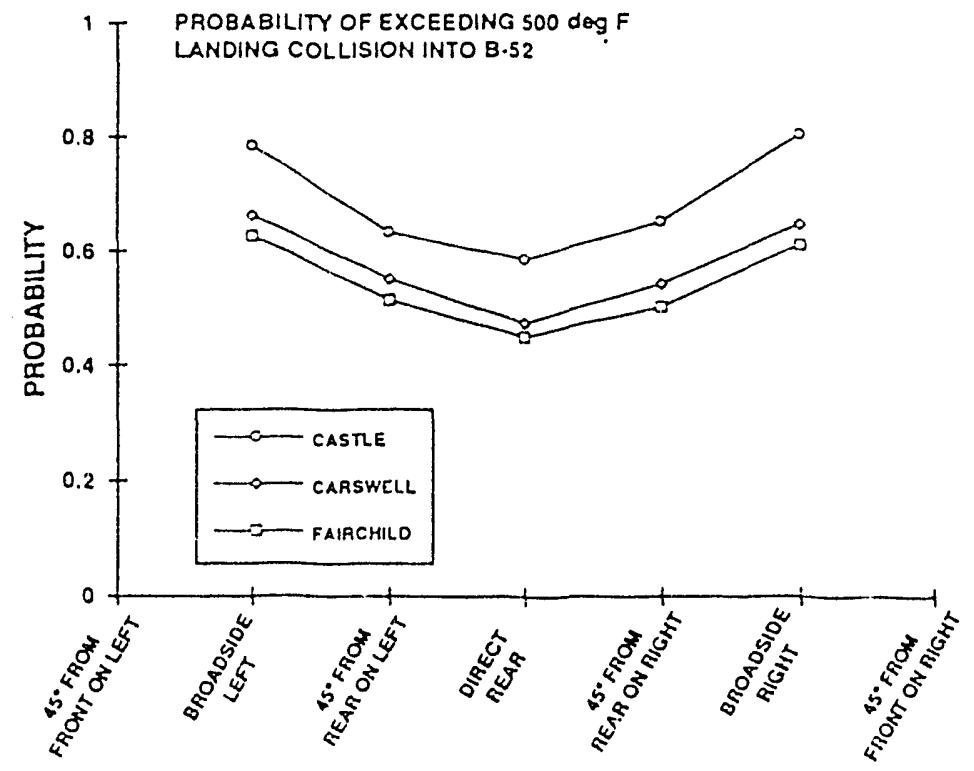

Figure 4.6 Probability of Exceeding Given Temperatures vs Collision Angle for Various Bases 
The plot on Figure 4.7 illustrates the difference in probability when the weapon is in the aft vs middle bay. For the middle bay, there appears to be a comparatively abrupt drop for the rear end collision, and this has been found to be consistent for all B-1B bases. Weapons in the middle bay are first of all located forward of most of the fuel tanks. Second, fuel spill location distributions that were developed for this project show abnormally few spills that are near or forward of the middle bay for the rear end collision, reflecting that some protection is provided to the forward fuselage tanks by the engine/engine mount structure.

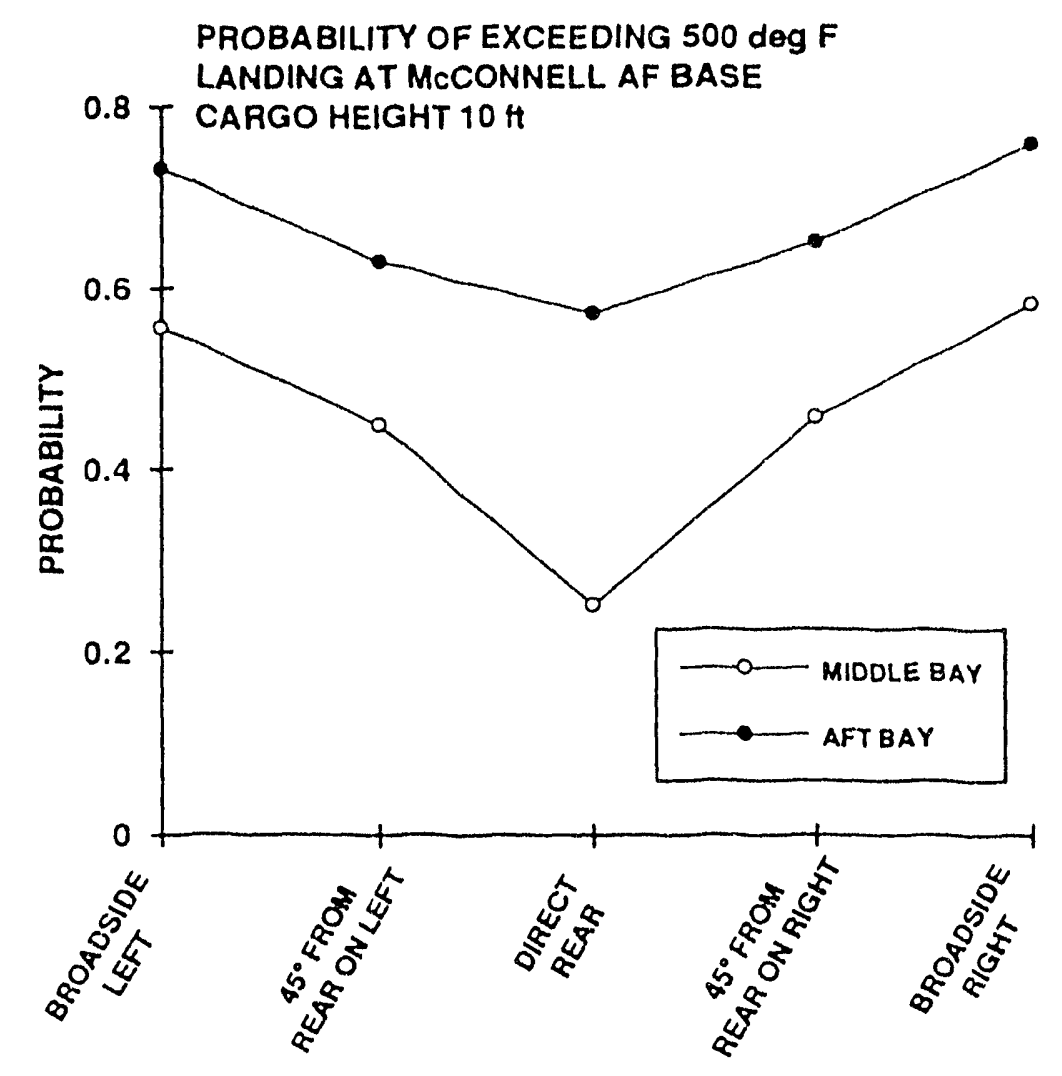

Figure 4.7 Example Probability of Exposure vs Collision Angle for Weapons in Middle or Aft Bay 
Shifting the discussion now to the engine start accident, the weighted mean probability of exceeding given exposure temperatures is given on Figures 4.8 and 4.9 for the B-1B and B-52, respectively, and tabulated values of these weighted mean curves are given in Table 4-3 at the end of Section 4. Weighting for the engine start accident is in proportion to the number of alert aircraft parked in the various directions at the various bases. These plots are for the selected baseline weapon heights of 10 and 7 feet for the B-1B and B-52, respectively, and regarding the $\mathrm{B}-1 \mathrm{~B}$, for weapons contained in the middle bay. The plots also provide an indication of the predictive uncertainty. For engine start accidents, the uncertainties given for the B-1B and B-52 are different; numerical values of the uncertainty for various temperature ranges are given in Table 4-2. As with the collision accident, the predominant contribution to the total uncertainty for engine start accidents is from the uncertainty in the fuel spill distribution.

TABLE 4-2 Engine Start Accident Uncertainties

\begin{tabular}{|c|c|c|c|c|}
\cline { 2 - 5 } \multicolumn{1}{c|}{} & \multicolumn{4}{c|}{ TEMPERATURE RANGE, $^{\circ} \mathrm{F}$} \\
\cline { 2 - 5 } \multicolumn{1}{c|}{} & $500-1000$ & $1000-1500$ & $1500-2000$ & $2000-2100$ \\
\hline B-1B & .157 & .130 & .077 & .021 \\
B-52 & .052 & .064 & .084 & .010 \\
\hline
\end{tabular}

Example probability curves for weapons in the aft bay or the middle bay of the B-1B are given on Figure 4.10. Figure 4.10 also shows the probability of exceeding given temperatures when weapons are concurrently in the middle and aft bays. The results given on Figure 4.10 are typical of those obtained for other bases.

The effect of height on the probability of exceeding given temperatures is illustrated on Figure 4.11 for the B-1B (middle or aft bay) and B-52. The height effects on Figure 4.11 are typical of results obtained for other bases. 


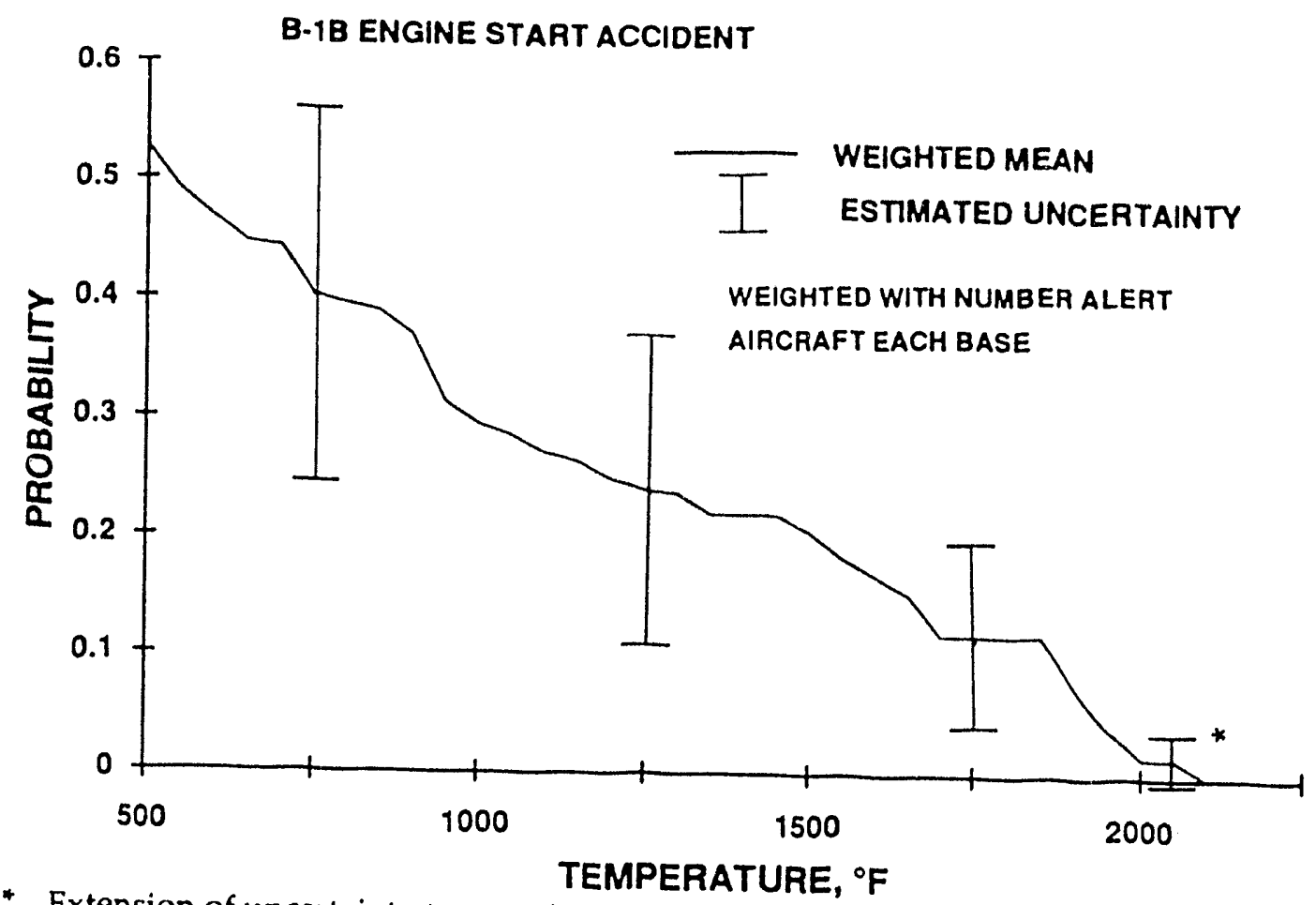

*xtension of uncertainty to negative value is a consequence of the method of uncertainty
evaluation, and has no meaning.

Figure 4.8 Weighted Mean Probability of Exceeding Given Temperatures for B-1B Engine Start Accident

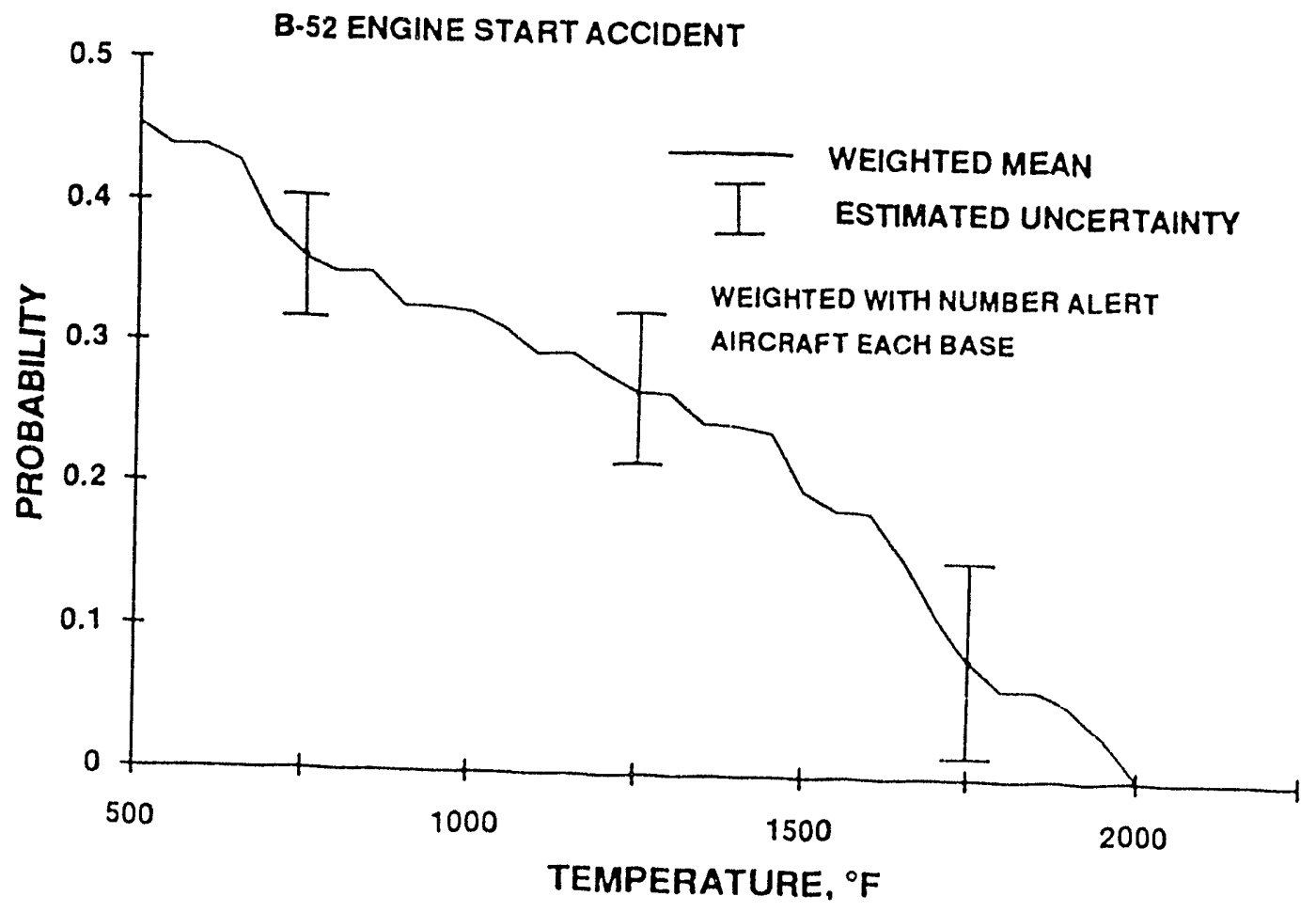

Figure 4.9 Weighted Mean Probability of Exceeding Given Temperatures for B-52 Engine Start Accident 

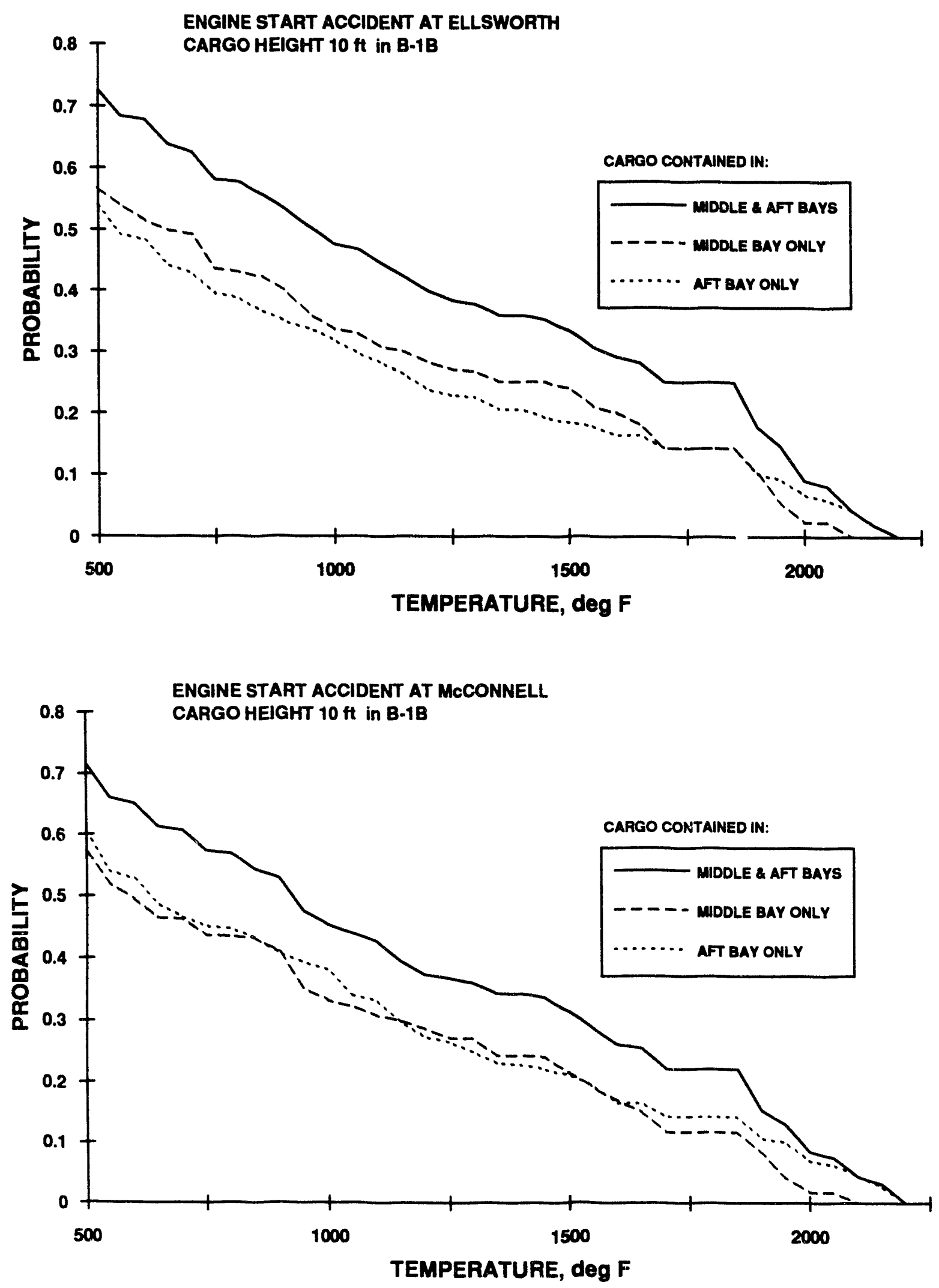

Figure 4.10 Results for Engine Start Accidents for Weapons in Middle, Aft or Both Bays 

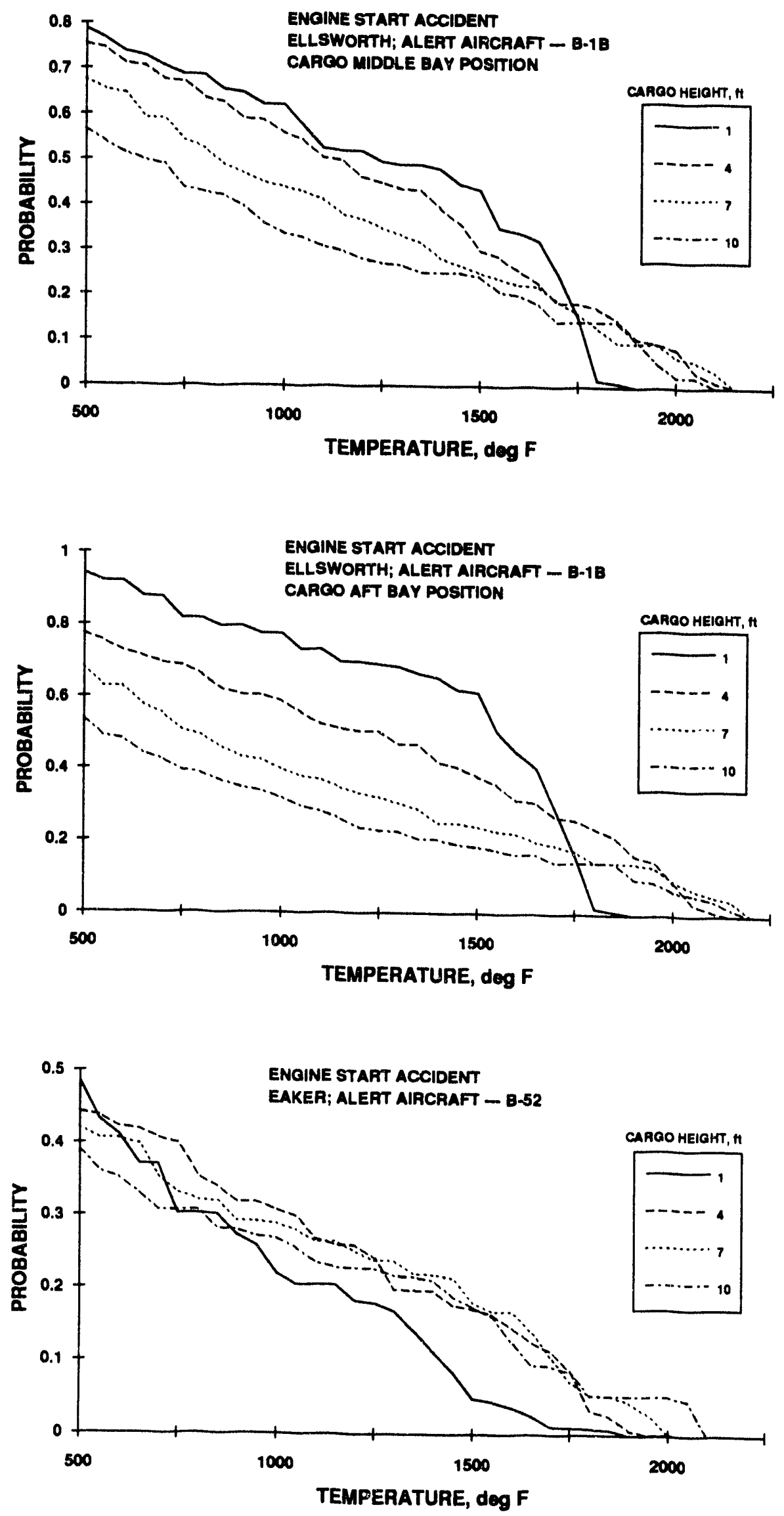

Figure 4.11 The Effect of Height for Engine Start Accidents 
TABLE 4-3. Weighted Mean Probability Over All Air Bases that Weapon Exposure will Exceed a Given Temperature

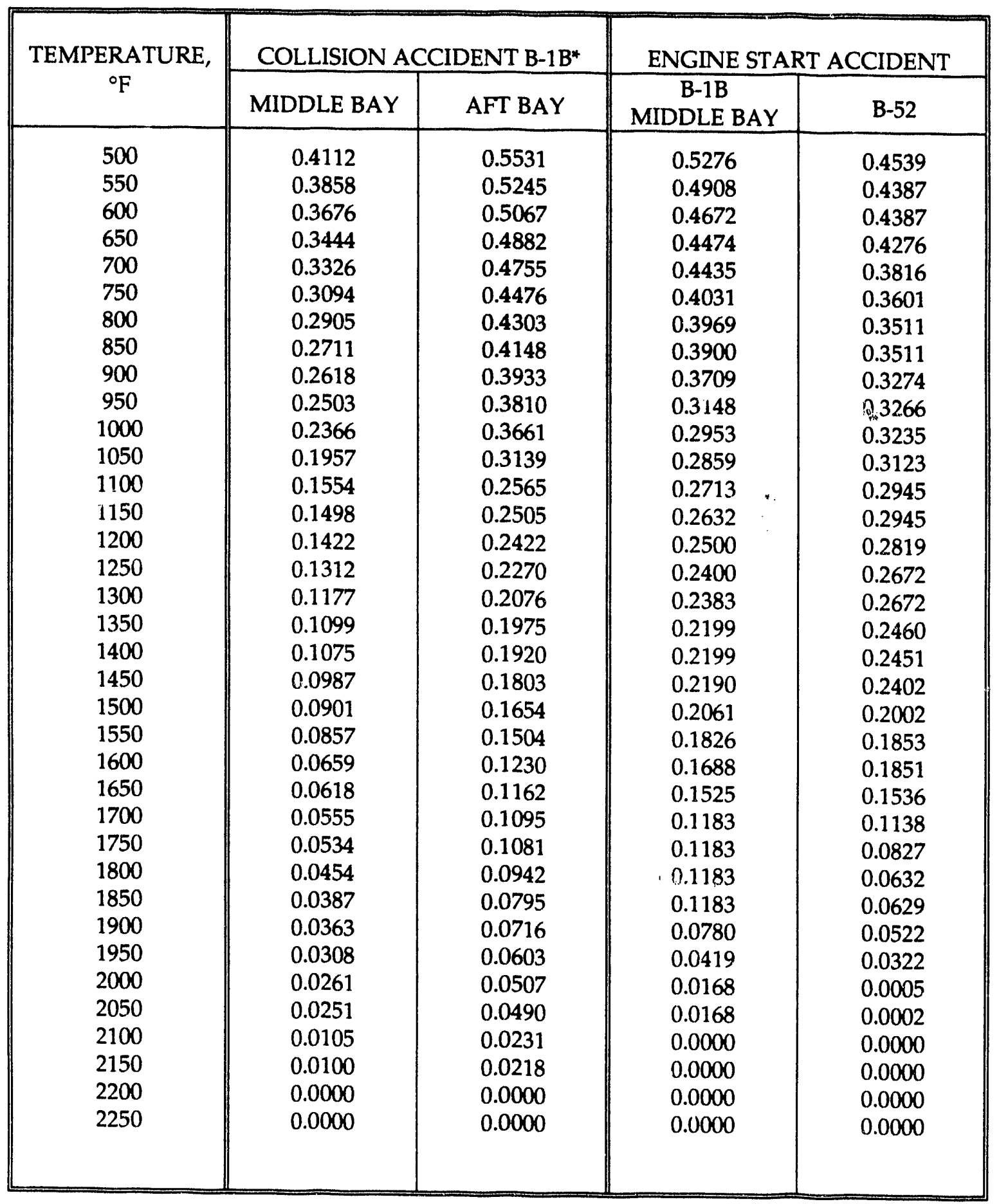

* There is no equivalent weighted mean data for the B-52 collision accident due to the lack of data on the relative base to base traffic. 


\section{REFERENCES}

1. Measurement and Statistical Analysis of Flame Temperatures From Large Fuel Spill Fires, J.A. Mansfield and L.J. Linley, Final Report NWC TP 7061, Naval Weapon Center, China Lake, CA 93555, January 1991.

2. A Statistical Analysis of Temperature Measurements in Large Fuel Spill Fires, J.A. Mansfield and L.J. Linley, presented at the 1990 JANNAF Propulsion Systems Hazard Meeting, John Hopkins University, Laurel, MD, April 3-6, 1990.

3. Large Pool Fires: Experiment and Analysis, P.T. Harsha, W.N. Bragg, R.B. Edelman, and J.A. Mansfield, Combustion Institute 83-2, Pasadena, CA,11 April 1983.

4. Measurement of the Spatial Dependence of Temperature and Gas and Soot Concentrations Within Large Open Hydrocarbon Fuel Fires, H.T. Johnson, L.J. Linley and J.A. Mansfield, NASA TM 58230, March 1982.

5. Advanced Risk Assessment of the Effects of Graphite Fibers on Electronic and Electric Equipment: Final Phase 1 Report, L.S. Pocinki, L.D. Kaplan, M.E. Cornell, and R. Greenstone, NASA Contract Report 159027, ORI, Inc., Silver Spring, MD, May 1979.

6. Private Communication, Thor Eklund, FAA Technical Center, Atlantic City, N.J.

7. Graphite-Epoxy Composite Material Response to Carrier Deck Fire, J.S. Fontenot, Naval Weapon Center TM 3351, China Lake, CA, November 1979.

8. A Model for Fuel Fire Duration and Application to the B-1B Bomber, D.E. Magnoli, Report UCRL-ID-112576, Lawrence Livermore National Laboratory, December 15, 1992.

9. Emissivity of Dispersed Carbon Particles, V.R. Stull and G.N. Plass, Journal of the Optical Soc. of America, Vol 50, Number 2, February 1960.

10. Private Communication, Mr. Park Winter, Lawrence Livermore National Laboratory. 
11. Navy Report CCG4: PEJ 5830, Naval Weapon Center, China Lake, CA, 30 June 1981.

12. Boeing T.O. 1B-52H-1, 15 July 1967.

13. U.S. Air Force SAC Bomber Base Usage Report, P.S. Winter, UCRL-ID 107231, Lawrence Livermore National Laboratory, 1 February 1991.

14. Internal Lawrence Livermore Lab Memo from Dr. J. Creighton on standard deviation of SANDIA temperature data, dated 12/30/91.

15. A Mathematical Model of a Large Open Fire, P.T. Harsha, W.N. Bragg, and R.B. Edelman, SAI-81-026-CP, Science Applications, Inc., Canoga Park, CA, prepared under NASA Contract No. NAS2-10675, April 1981. 


\section{Appendix A \\ FLAME TEMPERATURE DATA}

The flame temperature data used in weapon exposure predictions for this report were obtained from a series of six fire tests. This data is presented in Appendix A in terms of horizontal contours of time-average temperature at the heights of $1,4,7$ and 10 feet.

For the test series, fuel (JP-5) was "spilled" at a constant rate onto a level surface, with deliberate fuel ignition occurring at the instant the fuel first impacted the surface. The liquid fuel flow along the surface radially outward from the point of impact was unrestricted. Under this circumstance, the diameter of the fuel layer on the surface will increase until it reaches a maximum and thereafter constant value. This maximum diameter is given approximately as $D=3.5(S)^{1 / 2}$, where $D$ is the diameter in feet for a spill rates in gpm. Tests were conducted under three wind speed and three fuel spill rate conditions. The matrix of test conditions for the six tests is given in Table A-1.

TABLE A-1. Test Condition Matrix

\begin{tabular}{|c|c|c|c|c|}
\cline { 2 - 4 } \multicolumn{2}{c|}{} & \multicolumn{3}{c|}{ WIND SPEED } \\
\cline { 2 - 5 } \multicolumn{2}{c|}{} & $\begin{array}{c}\text { LOW } \\
5 \mathrm{mph}\end{array}$ & $\begin{array}{c}\text { MEDIUM } \\
15-18 \mathrm{mph}\end{array}$ & $\begin{array}{c}\text { HIGH } \\
26 \mathrm{mph}\end{array}$ \\
\hline & 150 & & TEST 7 & \\
\cline { 2 - 4 } $\begin{array}{c}\text { FUEL } \\
\text { SPILL } \\
\text { RATE, } \\
\text { gPm }\end{array}$ & 300 & TEST 8 & $\begin{array}{c}\text { TEST } 4 \\
\text { TEST } 9\end{array}$ & TEST 3 \\
\cline { 2 - 5 } & 450 & & TEST 6 & \\
\hline
\end{tabular}


The temperature contours mentioned above are given in Figures A-1 through A-6. The contours are based on temperature measurements over each of the horizontal planes, where the distance between measurement locations over each horizontal plane is 2.5 feet in both horizontal directions. On the figures, the "dotted enclosure" indicates the area over which temperature measurements were made. The temperature contours outside of this enclosure are estimates based on film coverage and knowledge of the location of the edge of the fuel layer. The film coverage was used to identify the position of the edge of the fire at each height relative to the position of the edge at other heights. The edge of the fire at ground level was identified from knowledge of the position of the edge of the fuel layer relative to the spill point.

In fires of this sort, there is an initial time period of "flame build-up" after the fuel supply is started and ignited. The build-up period, typically of the order of one minute, is due in part to the time for the liquid fuel to spread to the maximum diameter. There is then a second period during which the flame has "stabilized," where stabilized means that while there are significant local fluctuations in temperature, temperature averages over one minute periods at a given location would be essentially constant with time. There is then a third period of "flame decay" after the fuel supply has been stopped. The timeaverage temperatures given on Figures A-1 through A-6 are based only on data obtained during the stable period.

Details of the tests and test results are given in Reference 1, and a summary is given in Reference 2. The test facility design, fire test series and initial data analysis was sponsored by the Navy Department, Naval Weapons Center, China Lake, California (contact, John Fontenot). The tests were conducted at NASA, White Sands Test Facility, Las Cruces, NM (contact, Larry Linley). The initial time-dependent raw temperature data is availabie from Mr. Linley at the White Sands Facility; this includes data over the duration of fire growth, the duration over which the time-average temperature patterns are steady, and for the time after the fuel supply was stopped. The temperature data over the "steady" duration of the fire (either time-dependent or time-averaged) is available from Douglas Stephens, Lawrence Livermore National Laboratory, Livermore, California, or Joe Mansfield, JM Technical, Redmond, Oregon. 


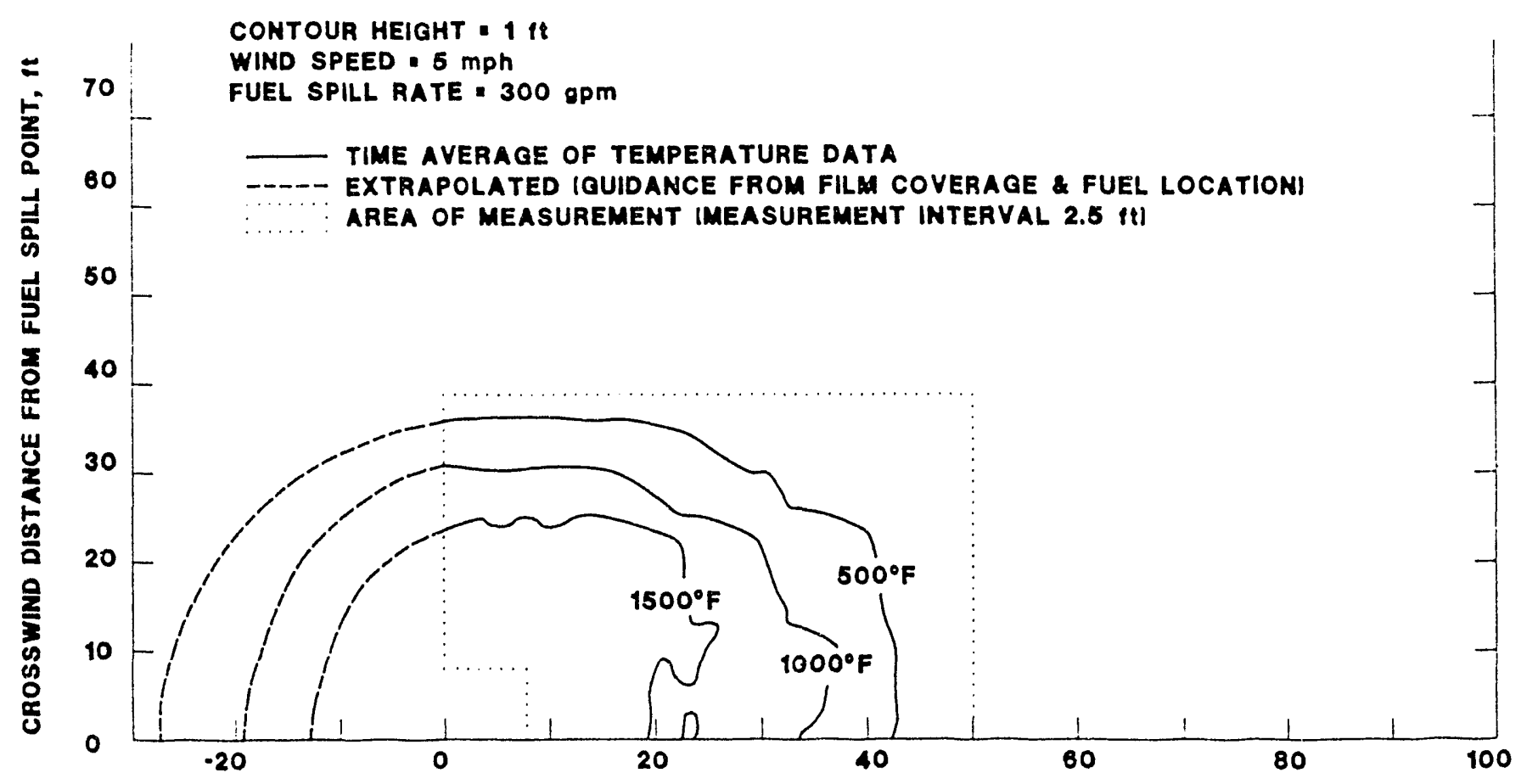

DOWNWIND DISTANCE FROM FUEL SPILL POINT, it

Figure A-1.1 Time-Average Temperature Contours at 1 foot height for $5 \mathrm{mph}$ Wind Speed and $300 \mathrm{gpm}$ Fuel Spill Rate (Test \#8)

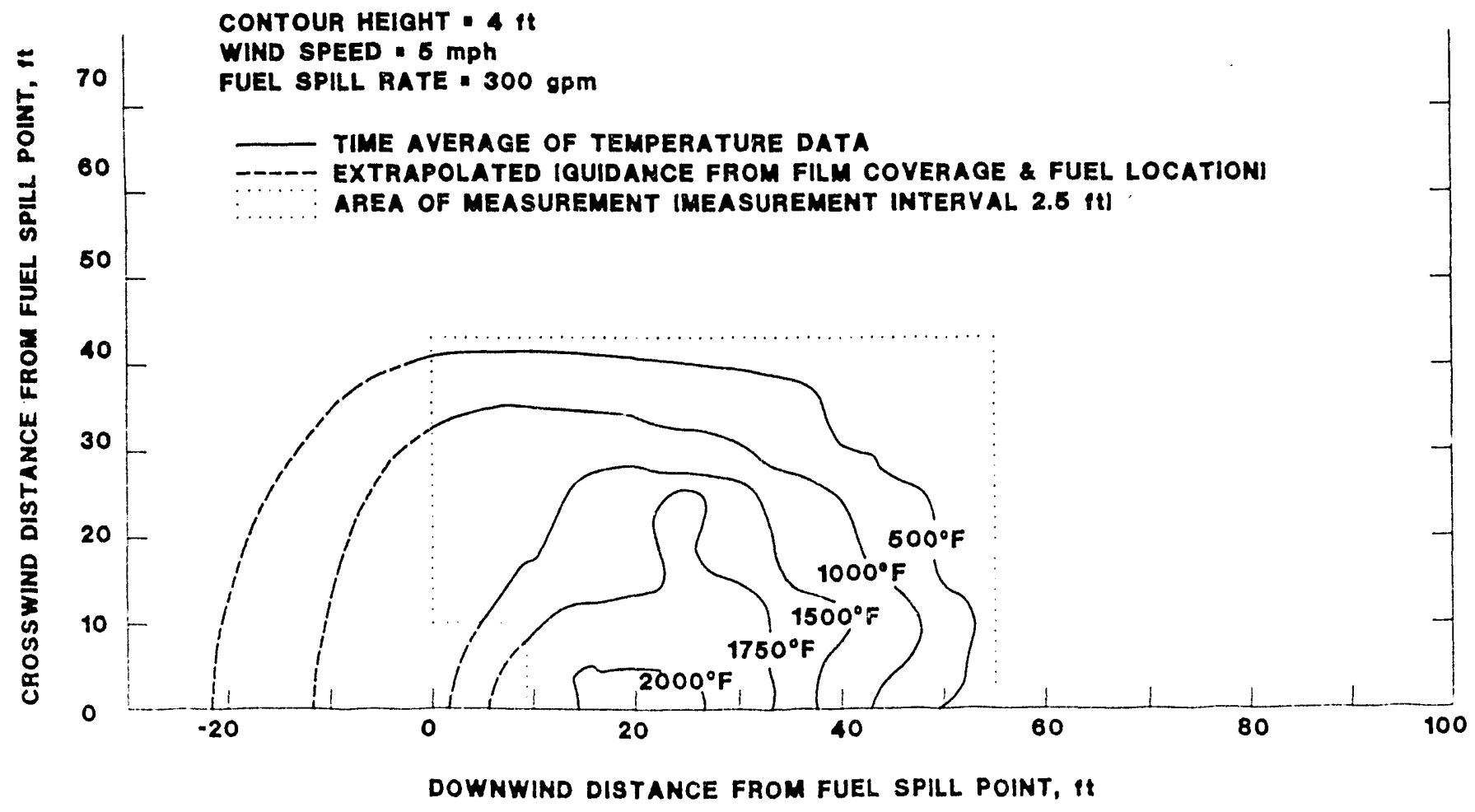

Figure A-1.2 Time-Average Temperature Contours at 4 foot height for $5 \mathrm{mph}$ Wind Speed and $300 \mathrm{gpm}$ Fuel Spill Rate (Test \#8) 


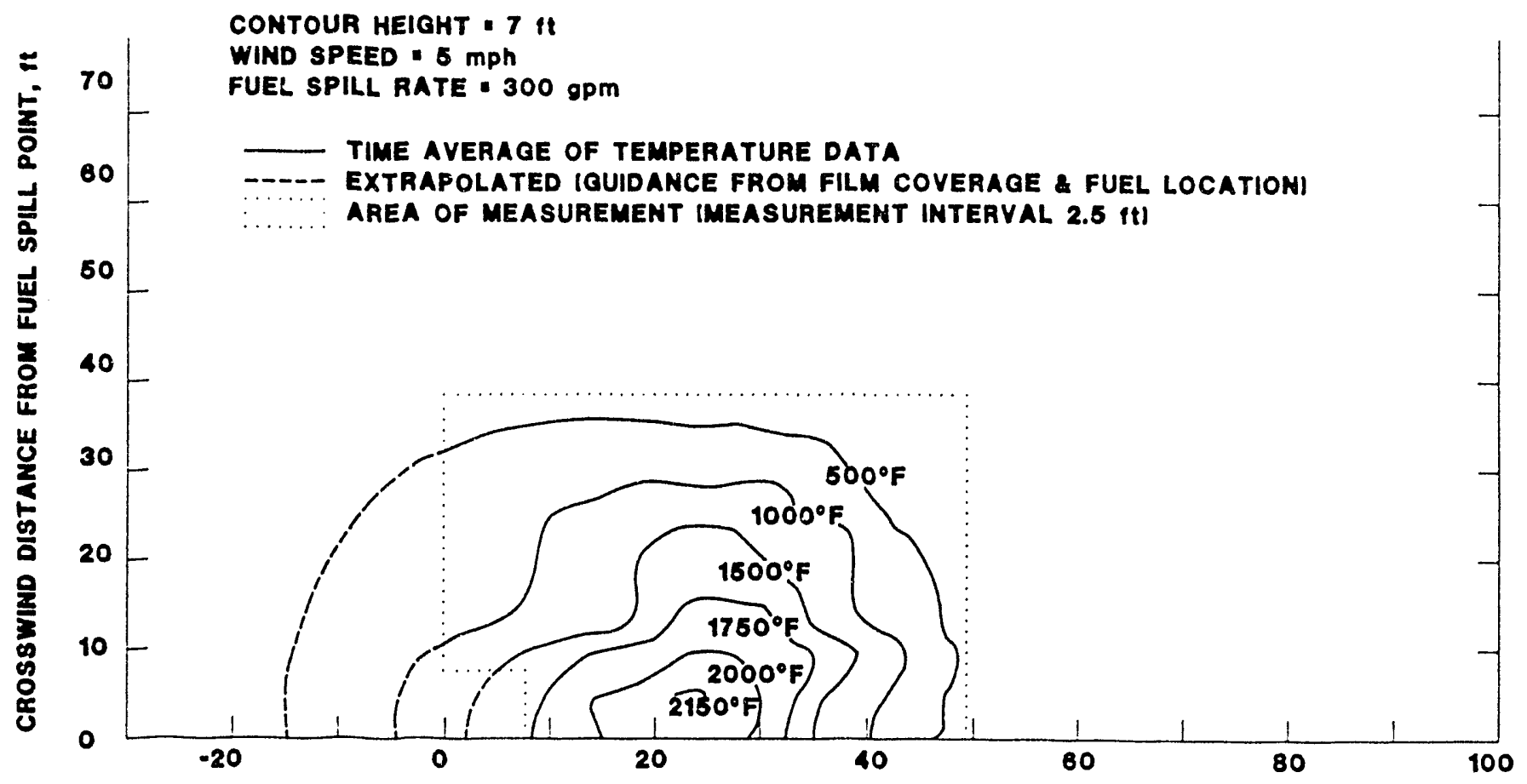

DOWNWIND DISTANCE FROM FUEL SPILL POINT, it

Figure A-1.3 Time-Average Temperature Contours at 7 foot height for $5 \mathrm{mph}$ Wind Speed and 300 gpm Fuel Spill Rate (Test \#8)

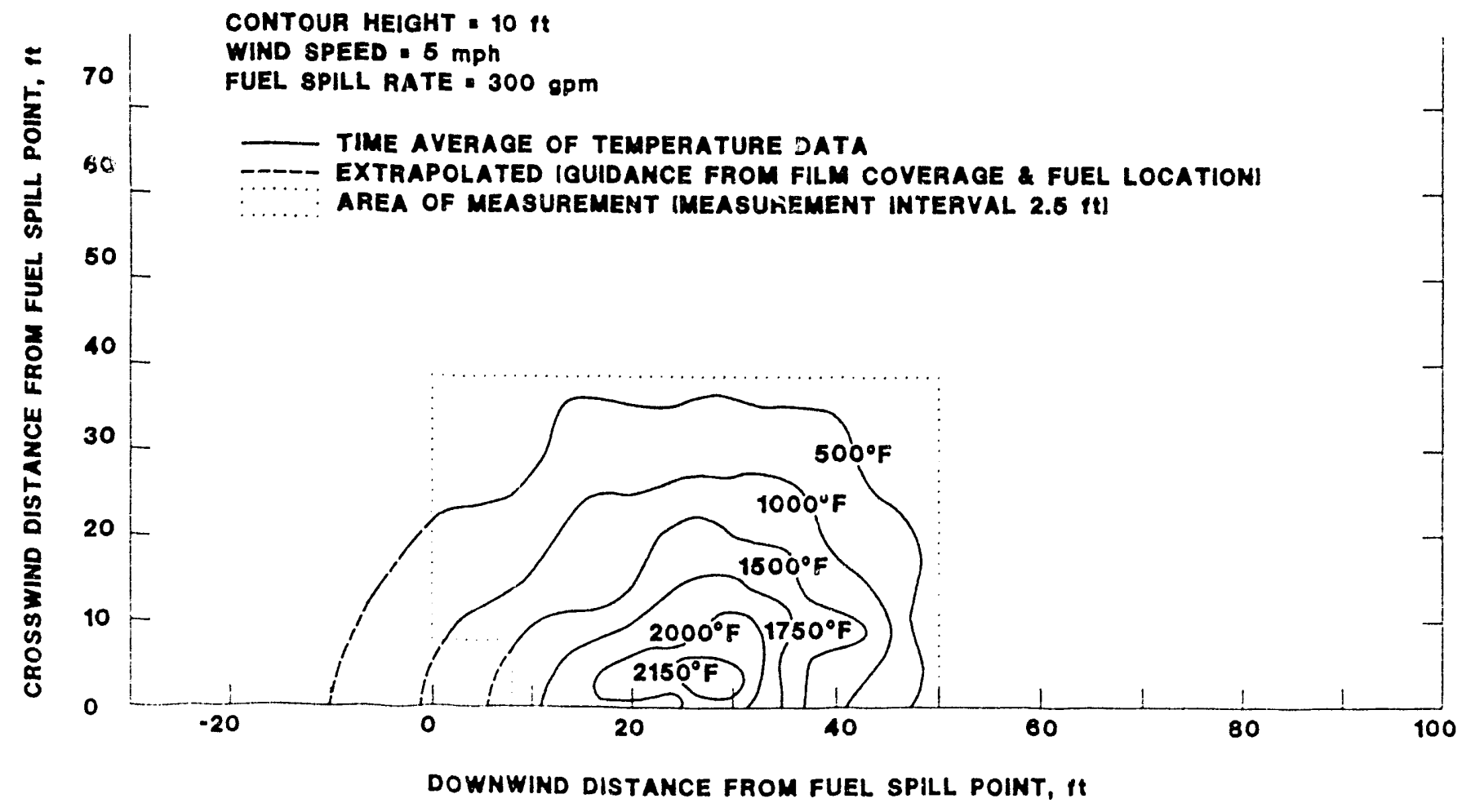

Figure A-1.4 Time-Average Temperature Contours at 10 foot height for $5 \mathrm{mph}$ Wind Speed and $300 \mathrm{gpm}$ Fuel Spill Rate (Test \#8) 


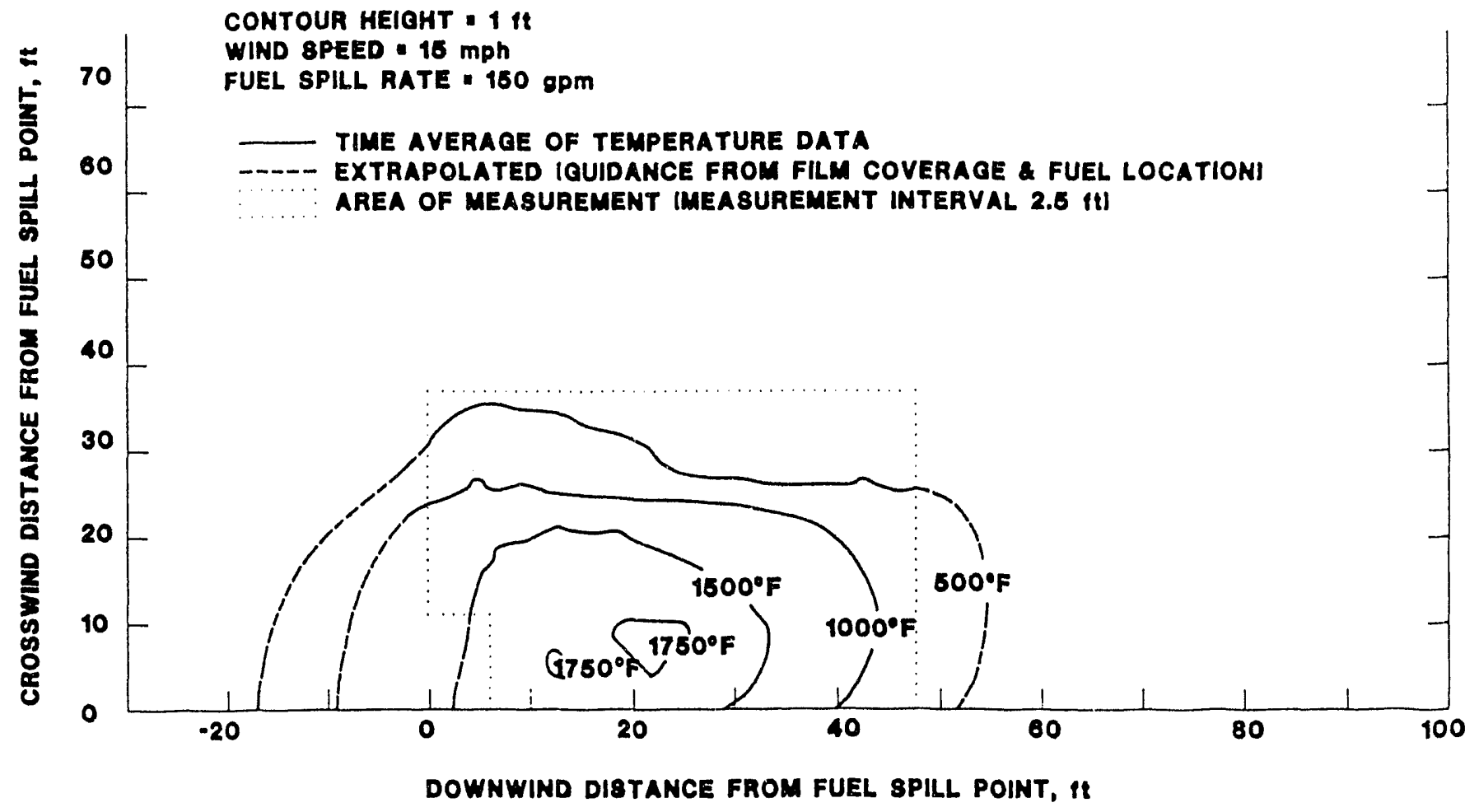

Figure A-2.1 Time-Average Temperature Contours at 1 foot height for $15 \mathrm{mph}$ Wind Speed and $150 \mathrm{gpm}$ Fuel Spill Rate (Test \#7)

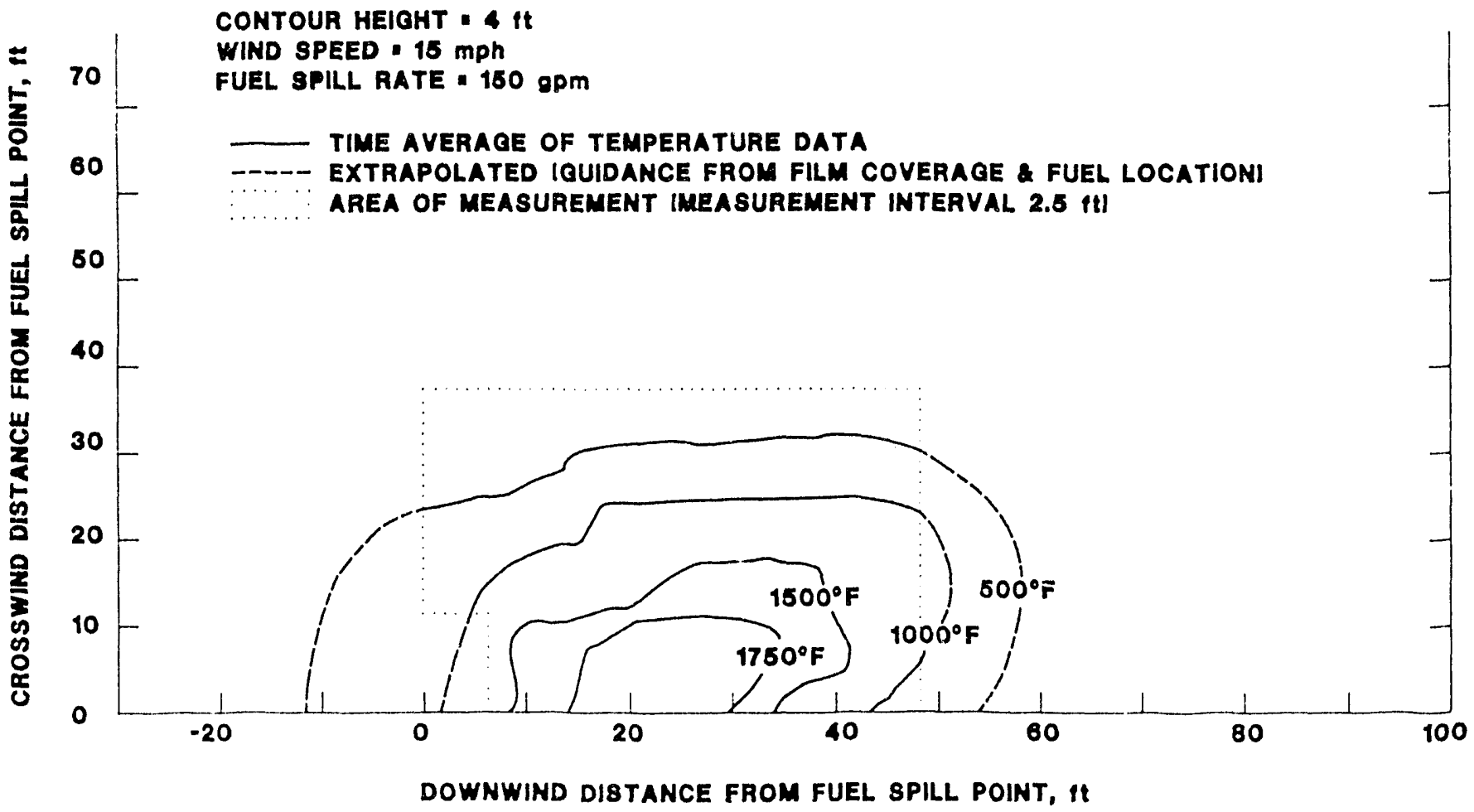

Figure A-2.2 Time-Average Temperature Contours at 4 foot height for $15 \mathrm{mph}$ Wind Speed and $150 \mathrm{gpm}$ Fuel Spill Rate (Test \#7) 


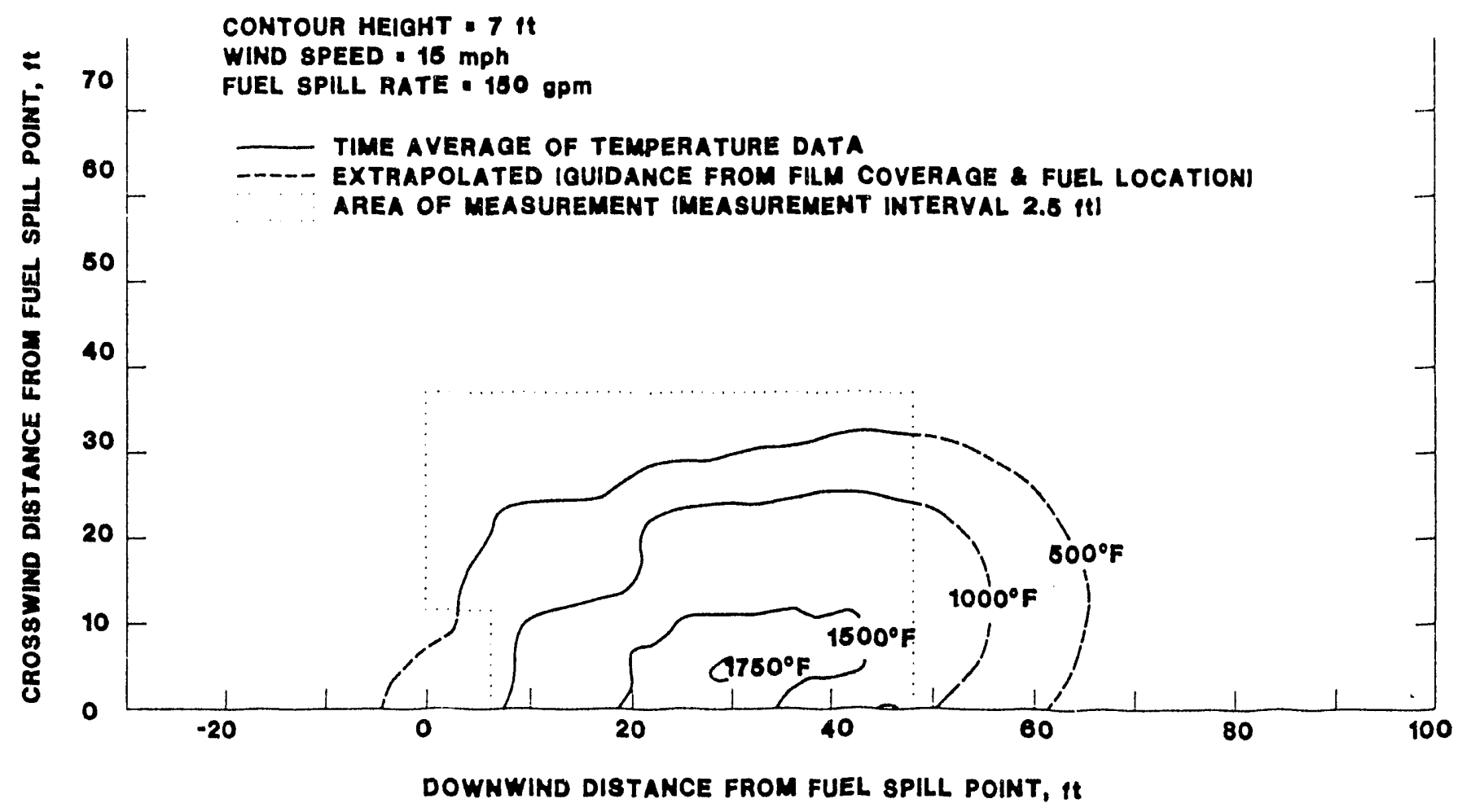

Figure A-2.3 Time-Average Temperature Contours at 7 foot height for $15 \mathrm{mph}$ Wind Speed and $150 \mathrm{gpm}$ Fuel Spill Rate (Test \#7)

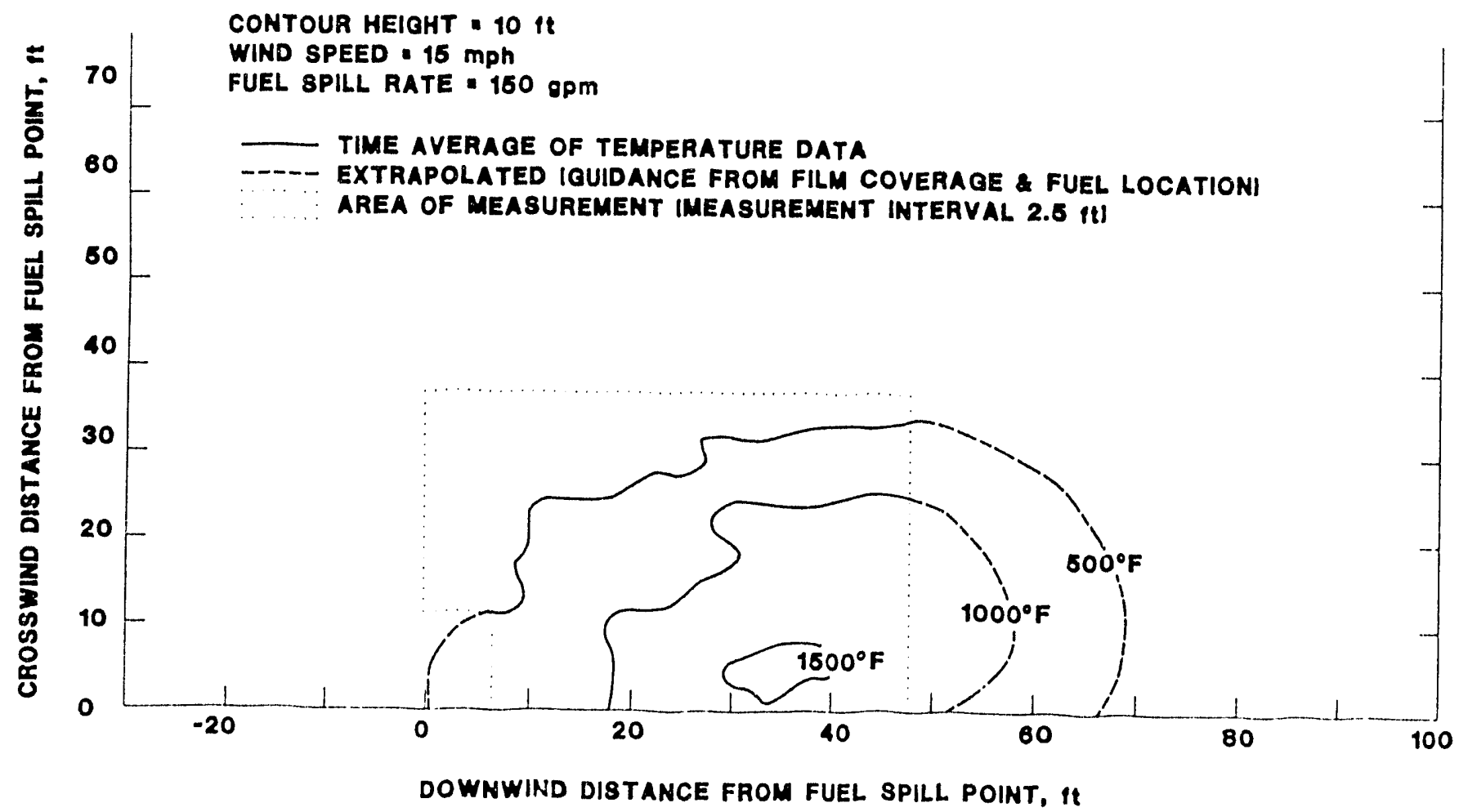

Figure A-2.4 Time-Average Temperature Contours at 10 foot height for $15 \mathrm{mph}$ Wind Speed and $150 \mathrm{gpm}$ Fuel Spill Rate (Test \#7) 


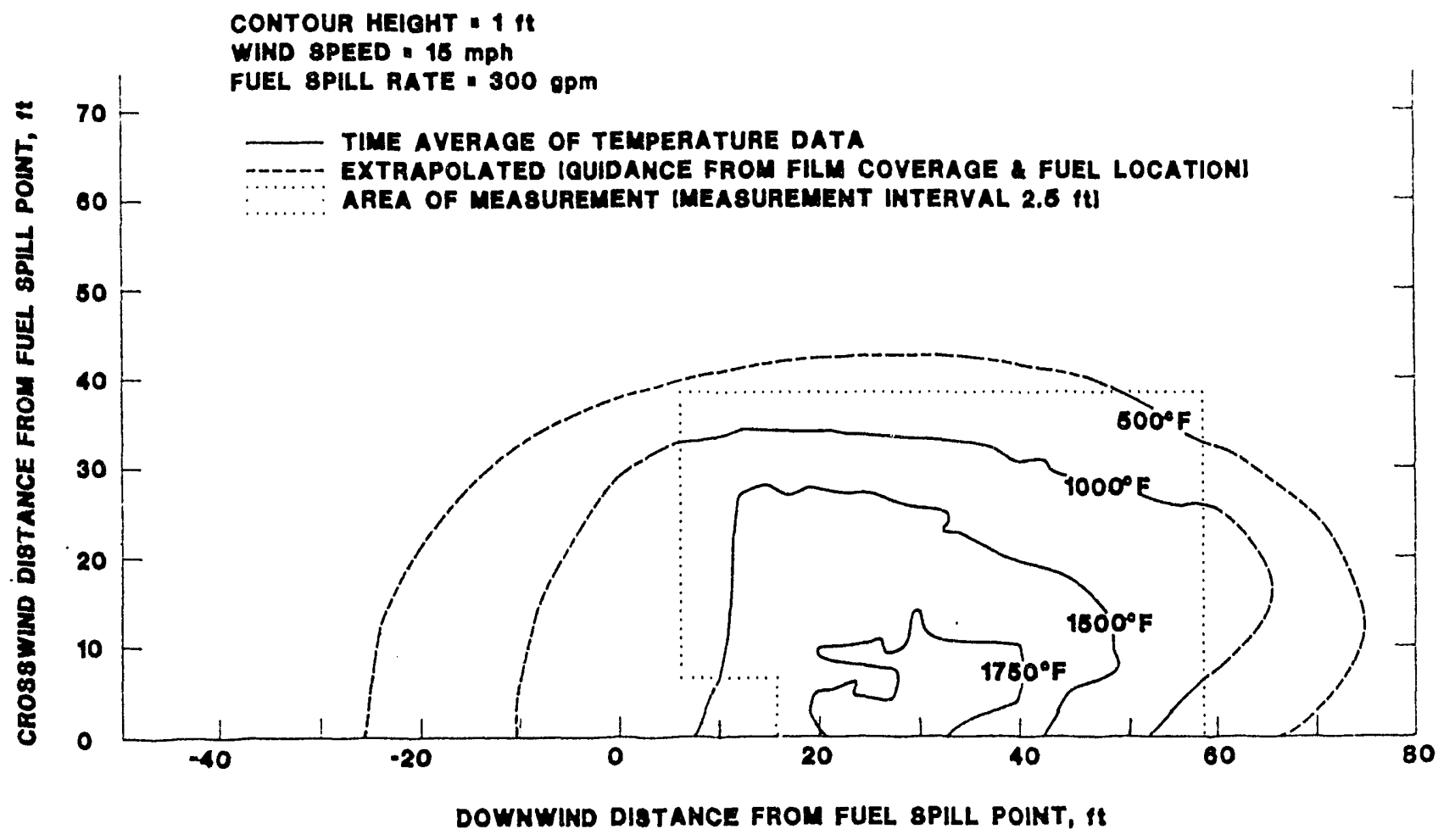

Figure A-3.1 Time-Average Temperature Contours at 1 foot height for $15 \mathrm{mph}$ Wind Speed and $300 \mathrm{gpm}$ Fuel Spill Rate (Test \#9)

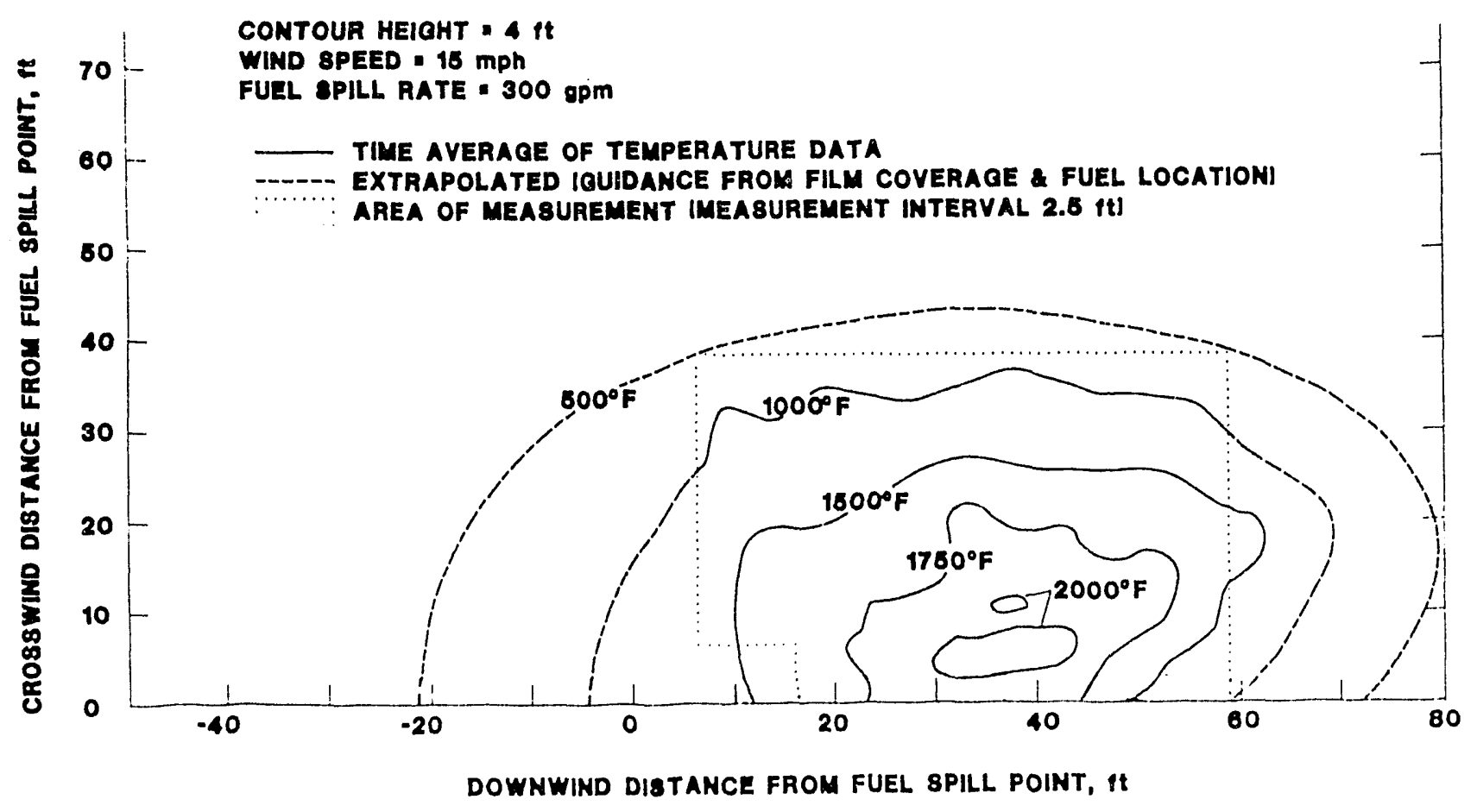

Figure A-3.2 Time-Average Temperature Contours at 4 foot height for $15 \mathrm{mph}$ Wind Speed and $300 \mathrm{gpm}$ Fuel Spill Rate (Test \#9) 


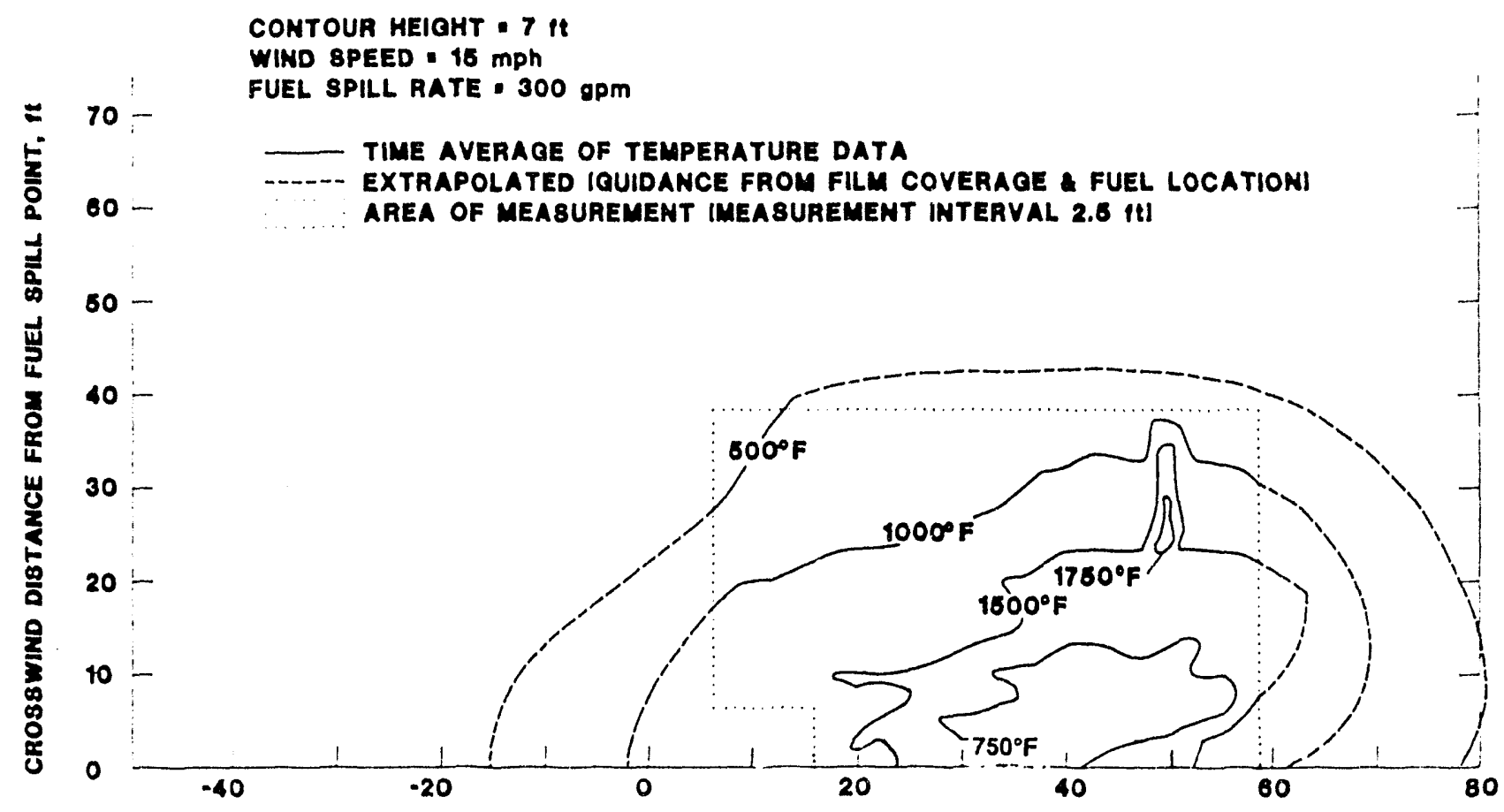

DOWNWIND DISTANCE FROM FUEL SPILL POINT, it

Figure A-3.3 Time-Average Temperature Contours at 7 foot height for $15 \mathrm{mph}$ Wind Speed and 300 gpm Fuel Spill Rate (Test \#9)

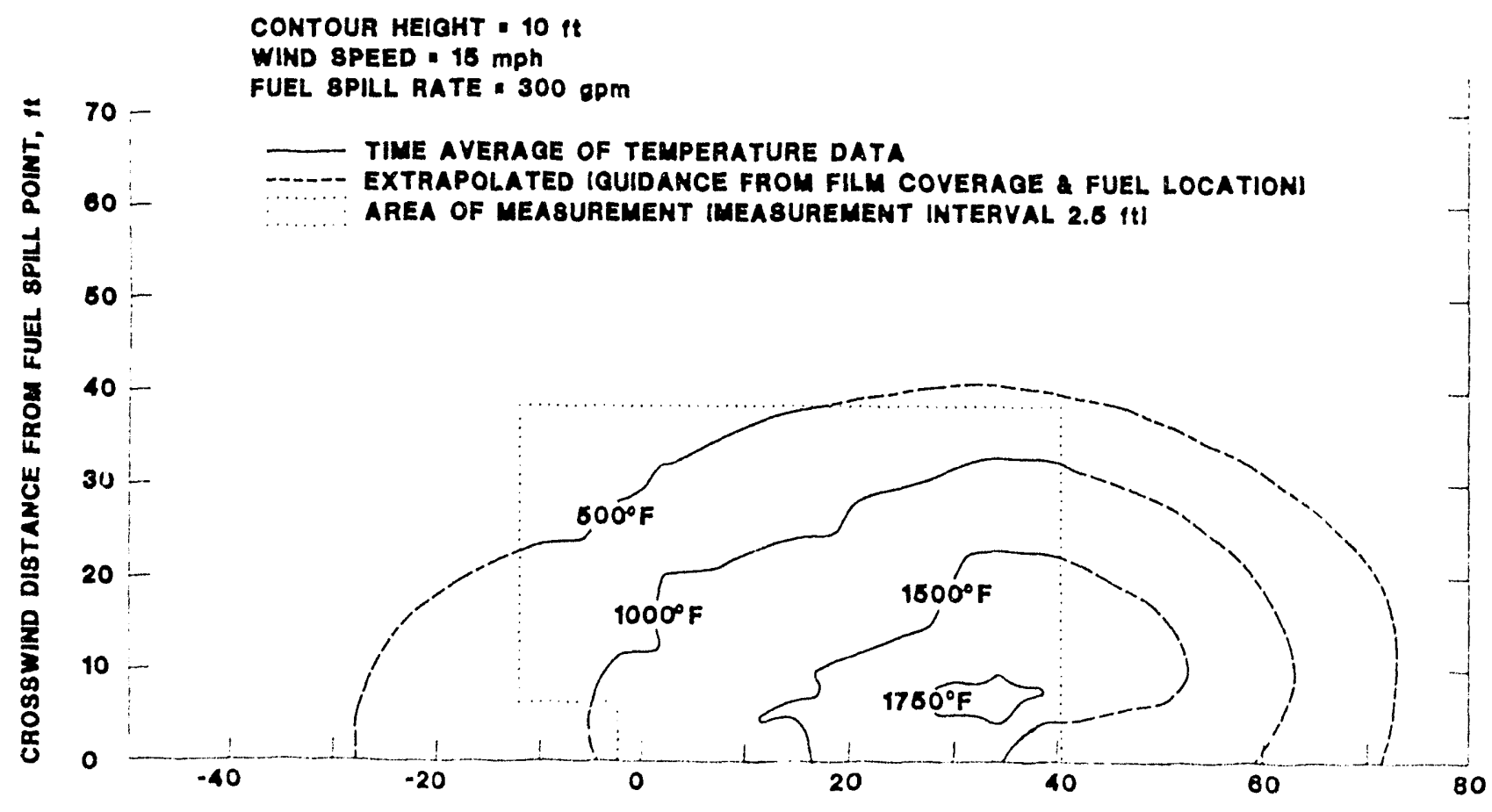

DOWNWIND DISTANCE FROM FUEL SPILL POINT, it

Figure A-3.4 Time-Average Temperature Contours at 10 foot height for

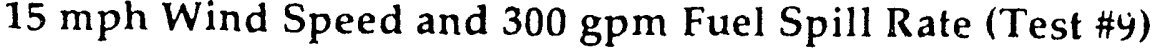




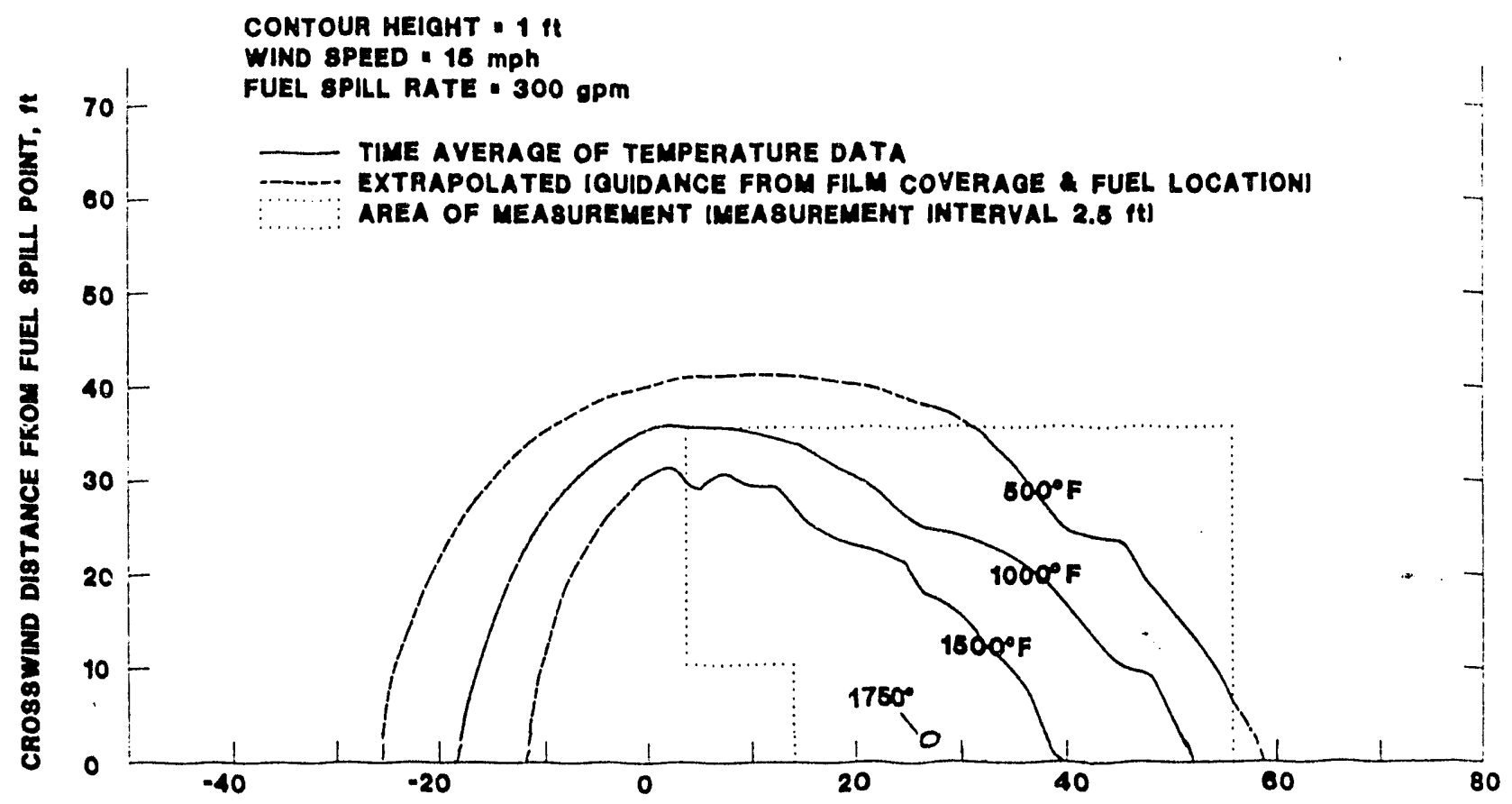

DOWNWIND DISTANCE FROM FUEL 8PILL POINT, it

Figure A-4.1 Time-Average Temperature Contours at 1 foot height for $15 \mathrm{mph}$ Wind Speed and $300 \mathrm{gpm}$ Fuel Spill Rate (Replicate Test - Test \#4)

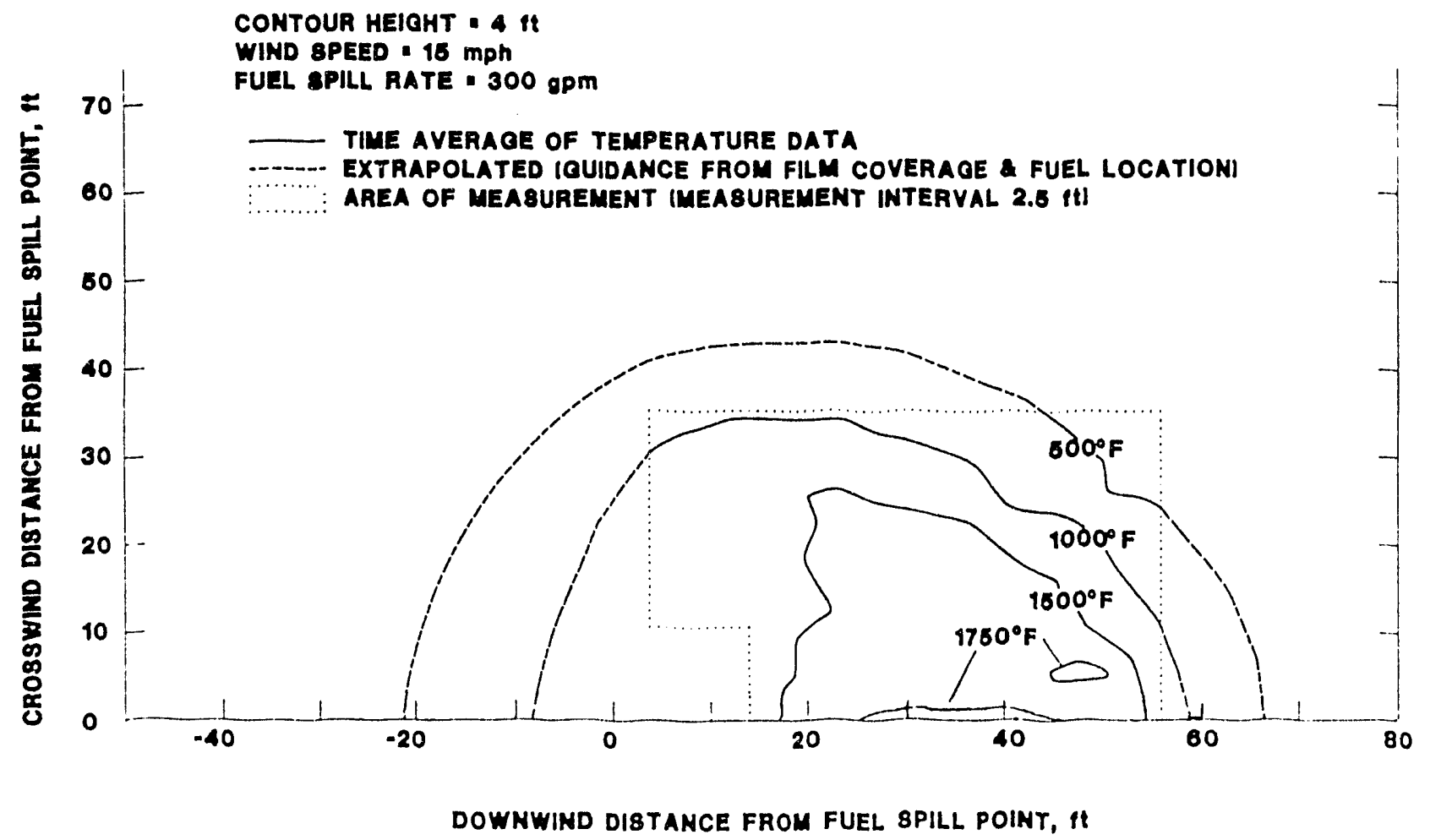

Figure A-4.2 Time-Average Temperature Contours at 4 foot height for $15 \mathrm{mph}$ Wind Speed and $300 \mathrm{gpm}$ Fuel Spill Rate (Replicate Test - Test \#4) 


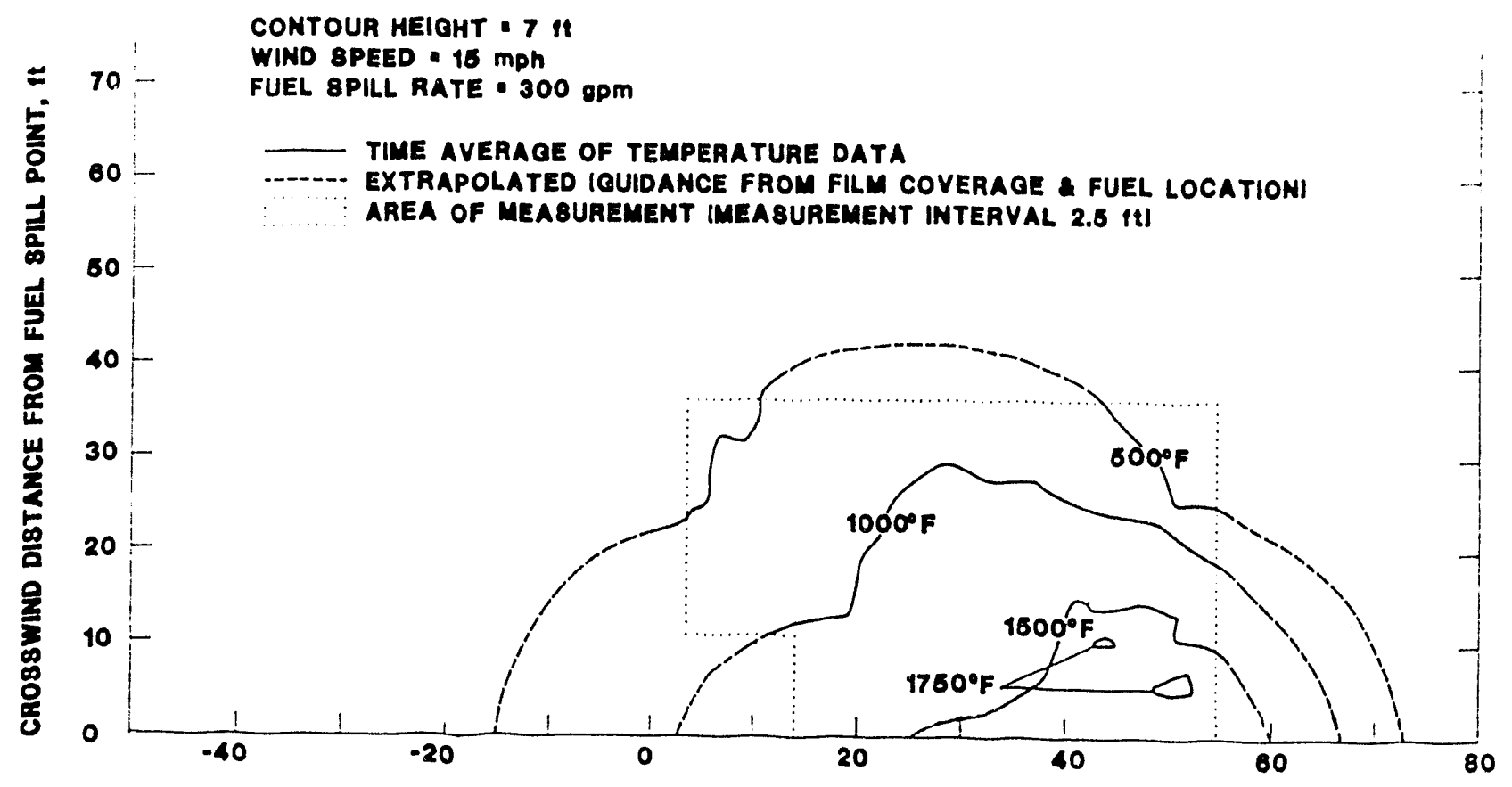

DOWNWIND DISTANCE FROM FUEL BPILL POINT, it

Figure A-4.3 Time-Average Temperature Contours at 7 foot height for $15 \mathrm{mph}$ Wind Speed and $\mathbf{3 0 0} \mathrm{gpm}$ Fuel Spill Rate (Replicate Test - Test \#4)

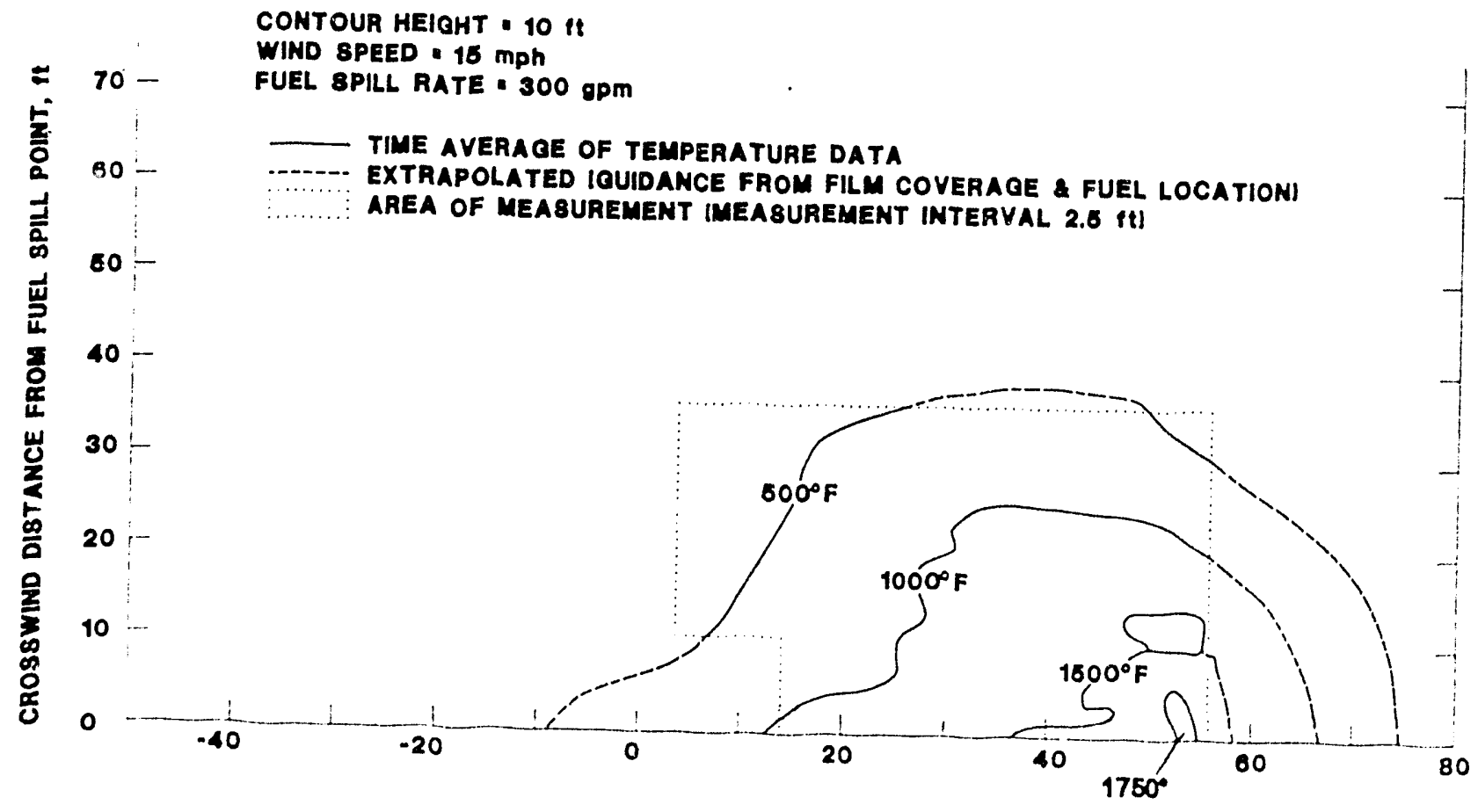

DOWNWIND DI8TANCE FROM FUEL BPILL POINT, it

Figure A-4.4 Time-Average Temperature Contours at 10 foot height for $15 \mathrm{mph}$ Wind Speed and $300 \mathrm{gpm}$ Fuel Spill Rate (Replicate Test - Test \#4) 


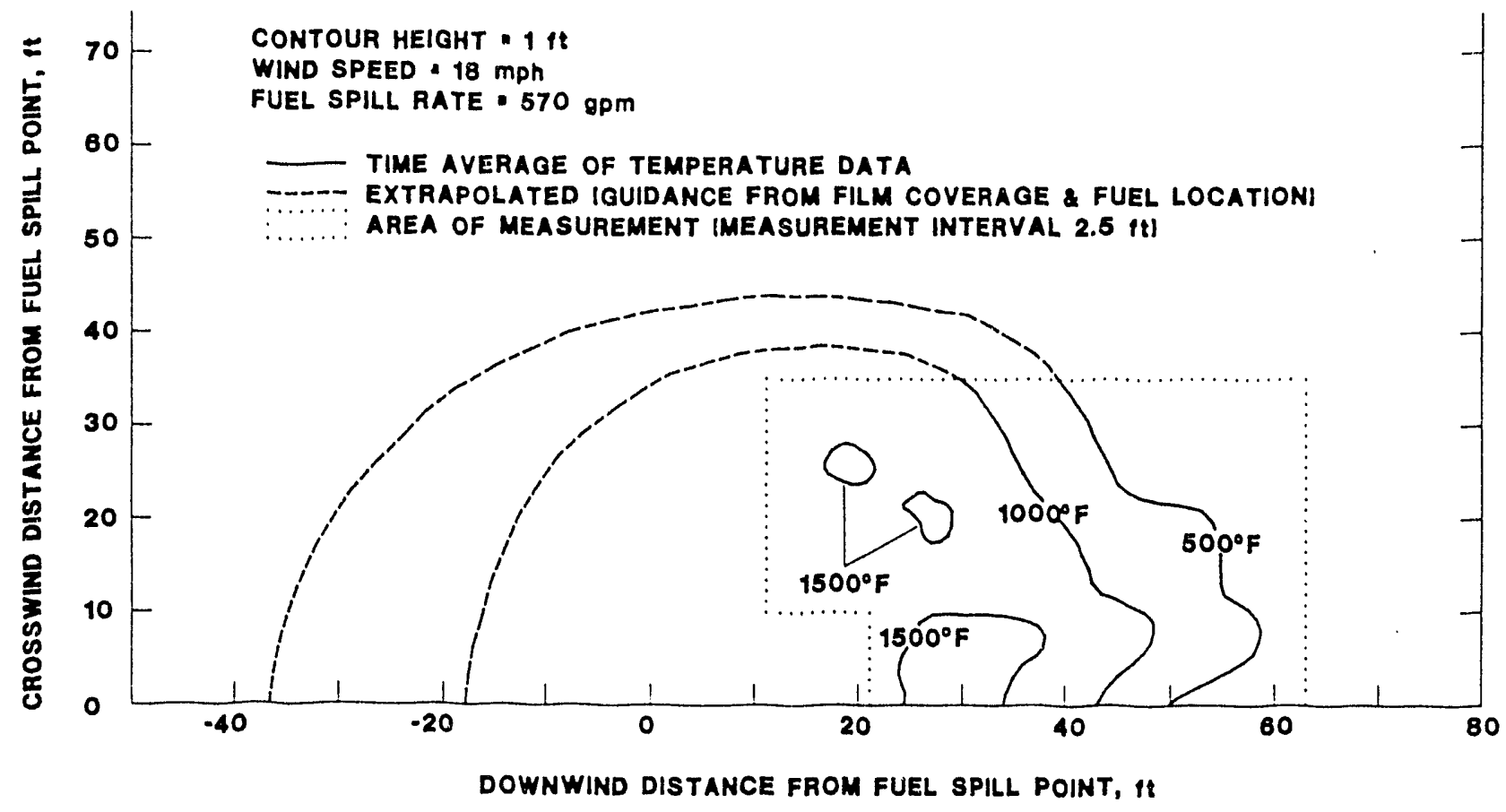

Figure A-5.1 Time-Average Temperature Contours at 1 foot height for $15 \mathrm{mph}$ Wind Speed and $600 \mathrm{gpm}$ Fuel Spill Rate (Test \#6)

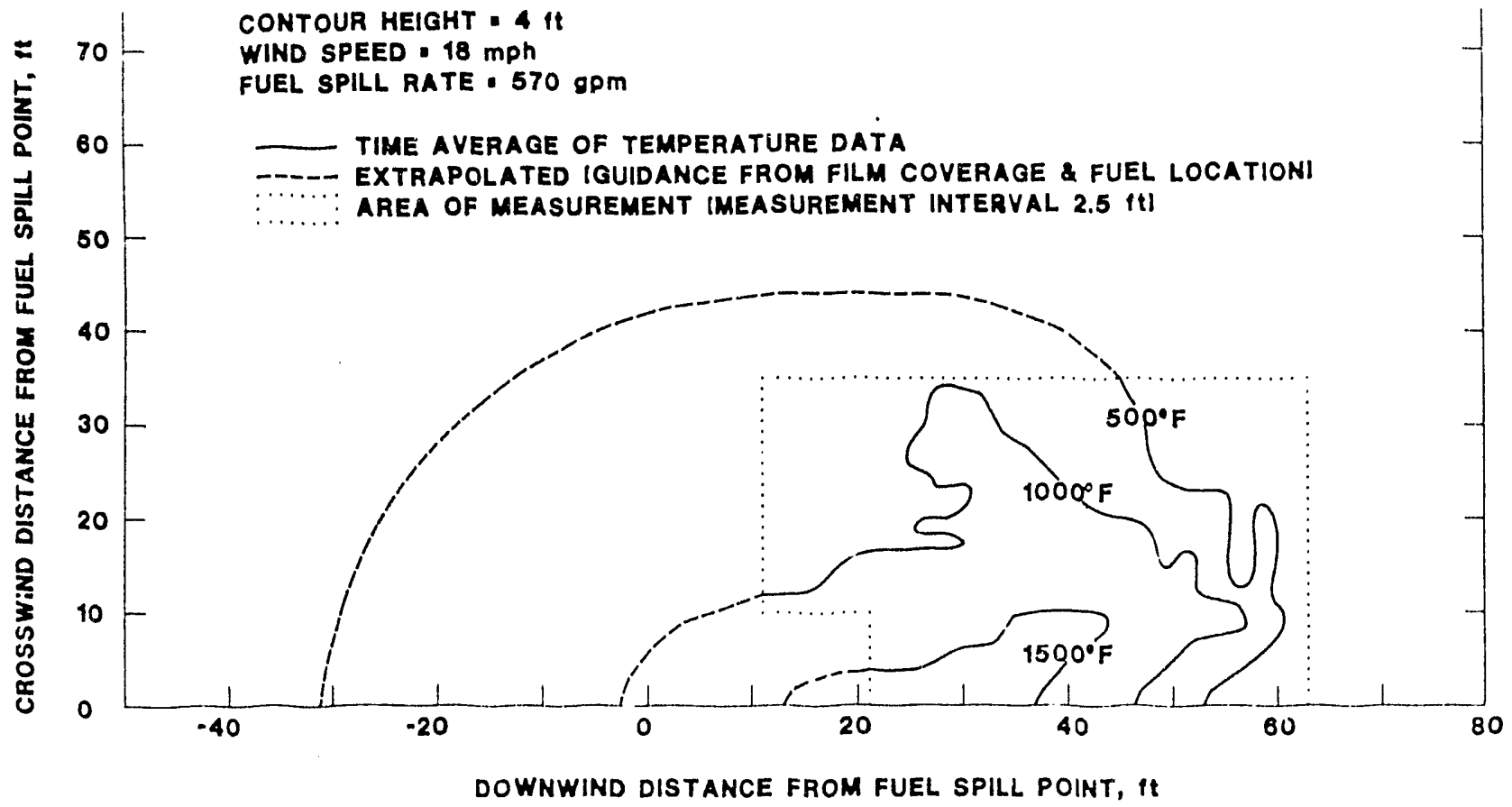

Figure A-5.2 Time-Average Temperature Contours at 4 foot height for $15 \mathrm{mph}$ Wind Speed and $600 \mathrm{gpm}$ Fuel Spill Rate (Test \#6) 


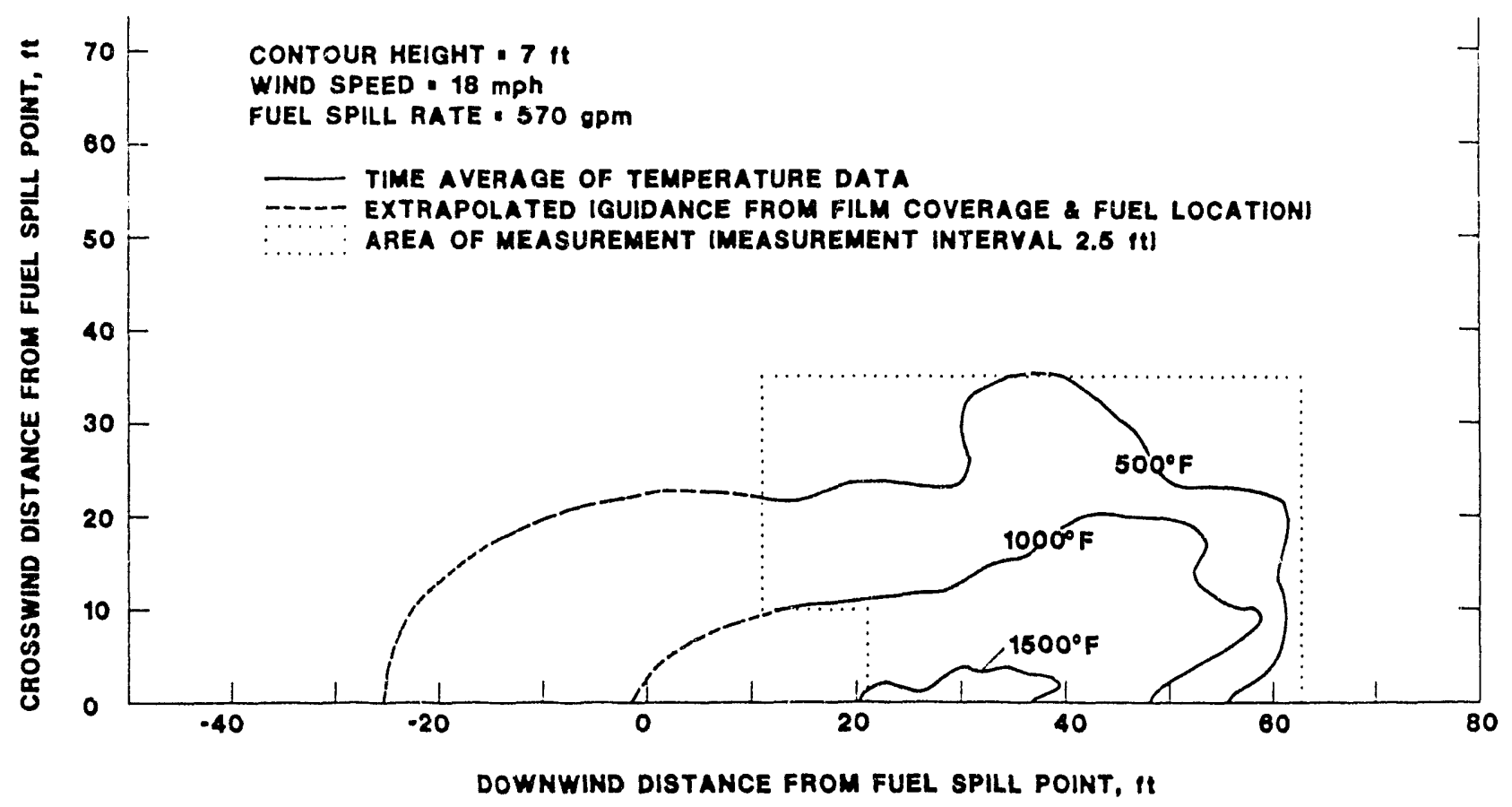

Figure A-5.3 Time-Average Temperature Contours at 7 foot height for $15 \mathrm{mph}$ Wind Speed and $600 \mathrm{gpm}$ Fuel Spill Rate (Test \#6)

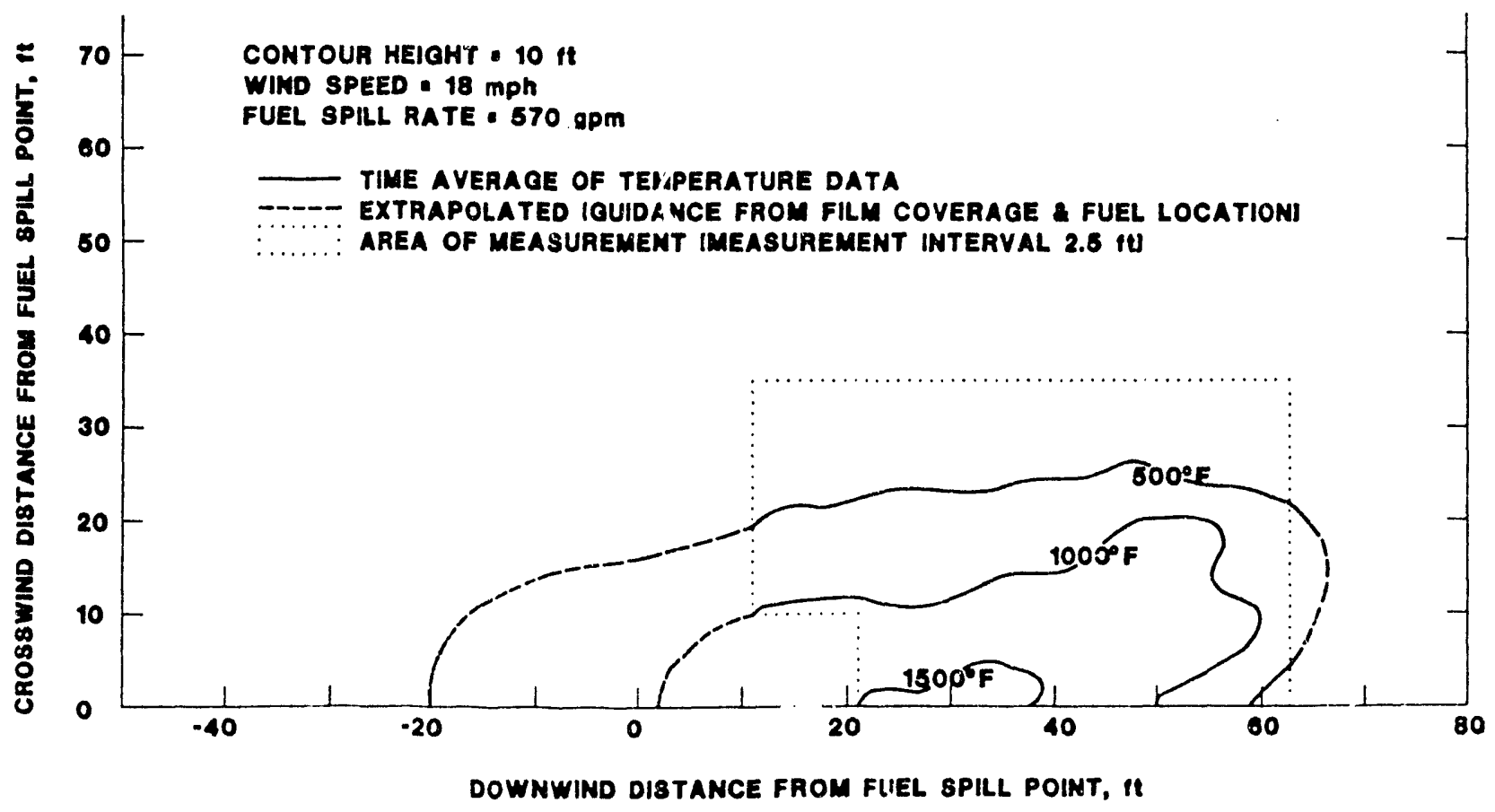

Figure A-5.4 Time-Average Temperature Contours at 10 foot height for $15 \mathrm{mph}$ Wind Speed and $600 \mathrm{gpm}$ Fuel Spill Rate (Test \#6) 


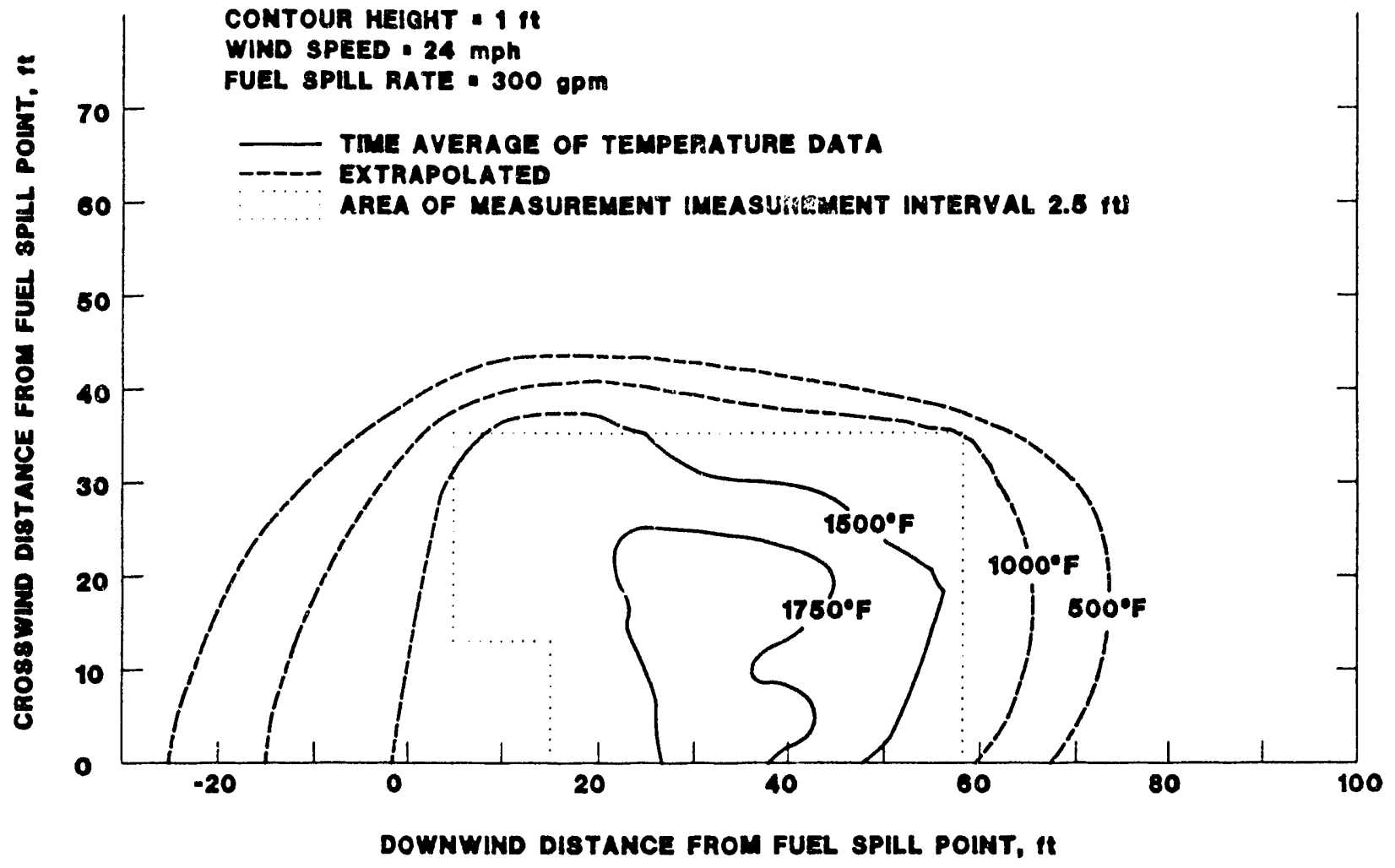

Figure A-6.1 Time-Average Temperature Contours at 1 foot height for $30 \mathrm{mph}$ Wind Speed and $300 \mathrm{gpm}$ Fuel Spill Rate (Test \#3)

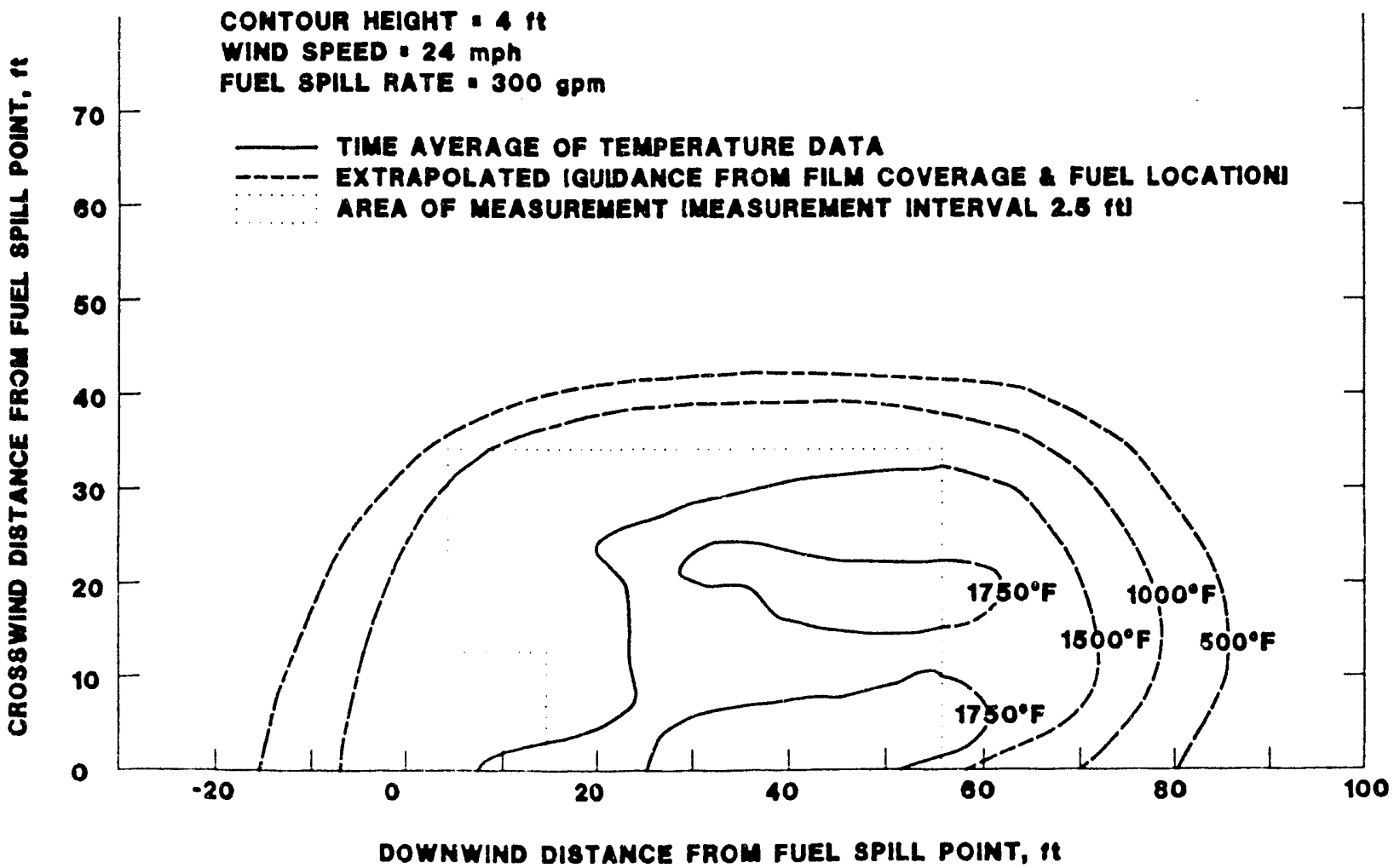

Figure A-6.2 Time-Average Temperature Contours at 4 foot height for 30 inph Wind Speed and 300 gpm Fuel Spill Rate (Test \#3) 


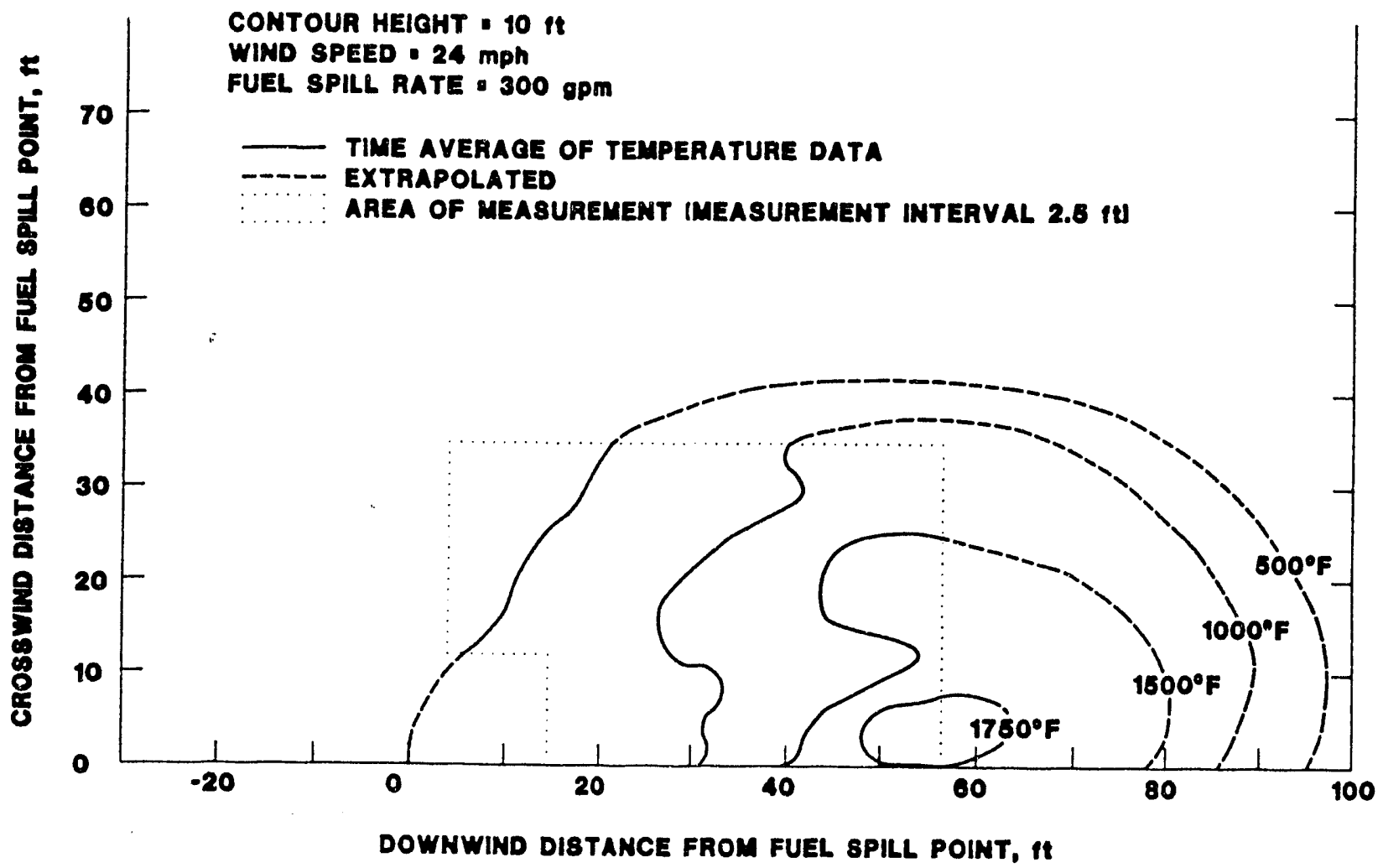

Figure A-6.3 Time-Average Temperature Contours at 10 foot height for $30 \mathrm{mph}$ Wind Speed and $\mathbf{3 0 0} \mathrm{gpm}$ Fuel Spill Rate (Test \#3) 


\section{Appendix B \\ AIRCRAFT/AIR BASE ORIENTATION}

There are twelve air force bases included in the evaluation of temperature exposure. The evaluation requires knowledge for each base of the direction of the runway, the directions that the alert aircraft are pointed, and which end of the runway the alert paid is located. Ground accident evaluation requires in addition the number of alert aircraft pointed in each direction. Appendix B provides a summary of this required data.

The directions that the alert aircraft are pointed, and the number of aircraft in each direction, are given in Table B-1. Sketches indicating the runway and alert aircraft orientations are given for each base on Figures B-1 through B-6.

TABLE B-1. Number of Aircraft at Each Parking Angle

\begin{tabular}{|c|c|c|c|c|c|}
\hline \multirow[b]{2}{*}{$\begin{array}{l}\text { TYPE OF ALERT } \\
\text { AIRCRAFT }\end{array}$} & \multirow[b]{2}{*}{ BASE } & \multicolumn{2}{|c|}{ 1st DIRECTION } & \multicolumn{2}{|c|}{ 2nd DIRECTION } \\
\hline & & $\begin{array}{c}\text { PARKING } \\
\text { ANGLE, deg CW } \\
\text { from } N\end{array}$ & $\begin{array}{l}\text { NUMBER OF } \\
\text { AIRCRAFT }\end{array}$ & $\begin{array}{c}\text { PARKING } \\
\text { ANGLE, deg CW } \\
\text { from N }\end{array}$ & $\begin{array}{l}\text { NUMBER OF } \\
\text { AIRCRAFT }\end{array}$ \\
\hline \multirow{8}{*}{ B-52 } & BARKSDALE & 170 & 4 & 80 & 4 \\
\hline & CARSWELL & 180 & 4 & 270 & 4 \\
\hline & CASTLE & 320 & 2 & 50 & 2 \\
\hline & EAKER & 0 & 4 & 270 & 4 \\
\hline & FAIRCHILD & 220 & 2 & 130 & 2 \\
\hline & MINOT & 330 & 4 & 240 & 4 \\
\hline & SAWYER & 10 & 4 & 100 & 5 \\
\hline & WURTSMITH & 230 & 4 & 140 & 5 \\
\hline \multirow{4}{*}{$B-1 B$} & DYESS & 120 & 5 & $\cdots$ & -.- \\
\hline & ELLSWORTH & 30 & 4 & 120 & 4 \\
\hline & GRAND FORKS & 80 & 4 & 30 & 4 \\
\hline & MCCONNELL & 190 & 5 & 10 & 5 \\
\hline
\end{tabular}




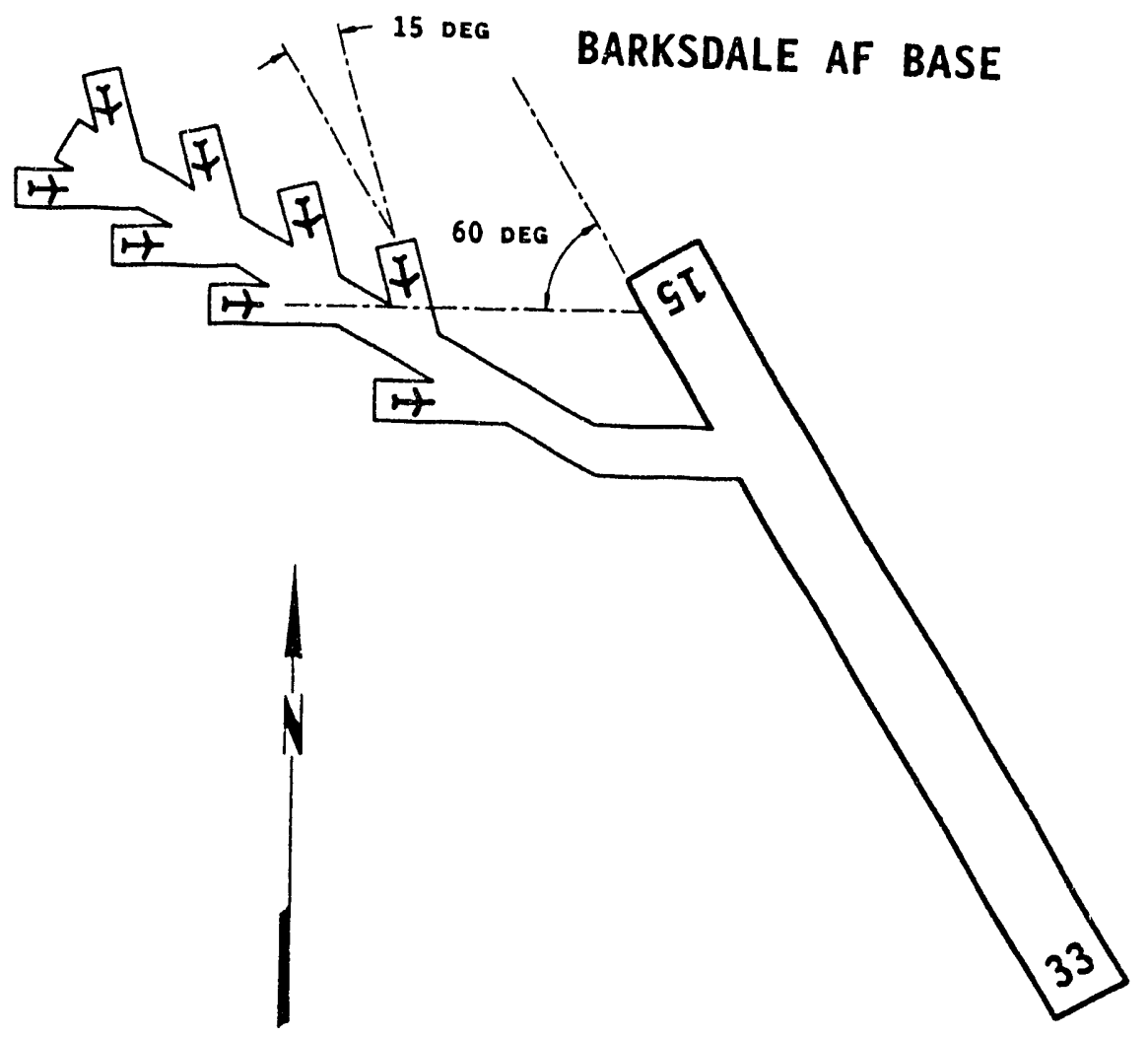

CARSWELL AF BASE, TX

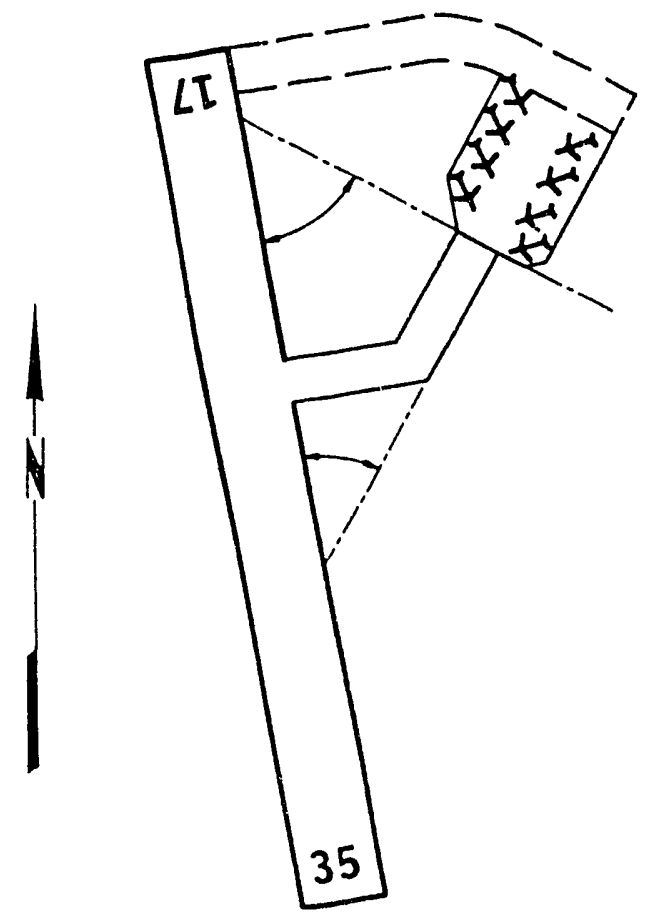

Figure B-1 Aircraft/Air Base Orientation at Barksdale AFB and Carswell AFB 


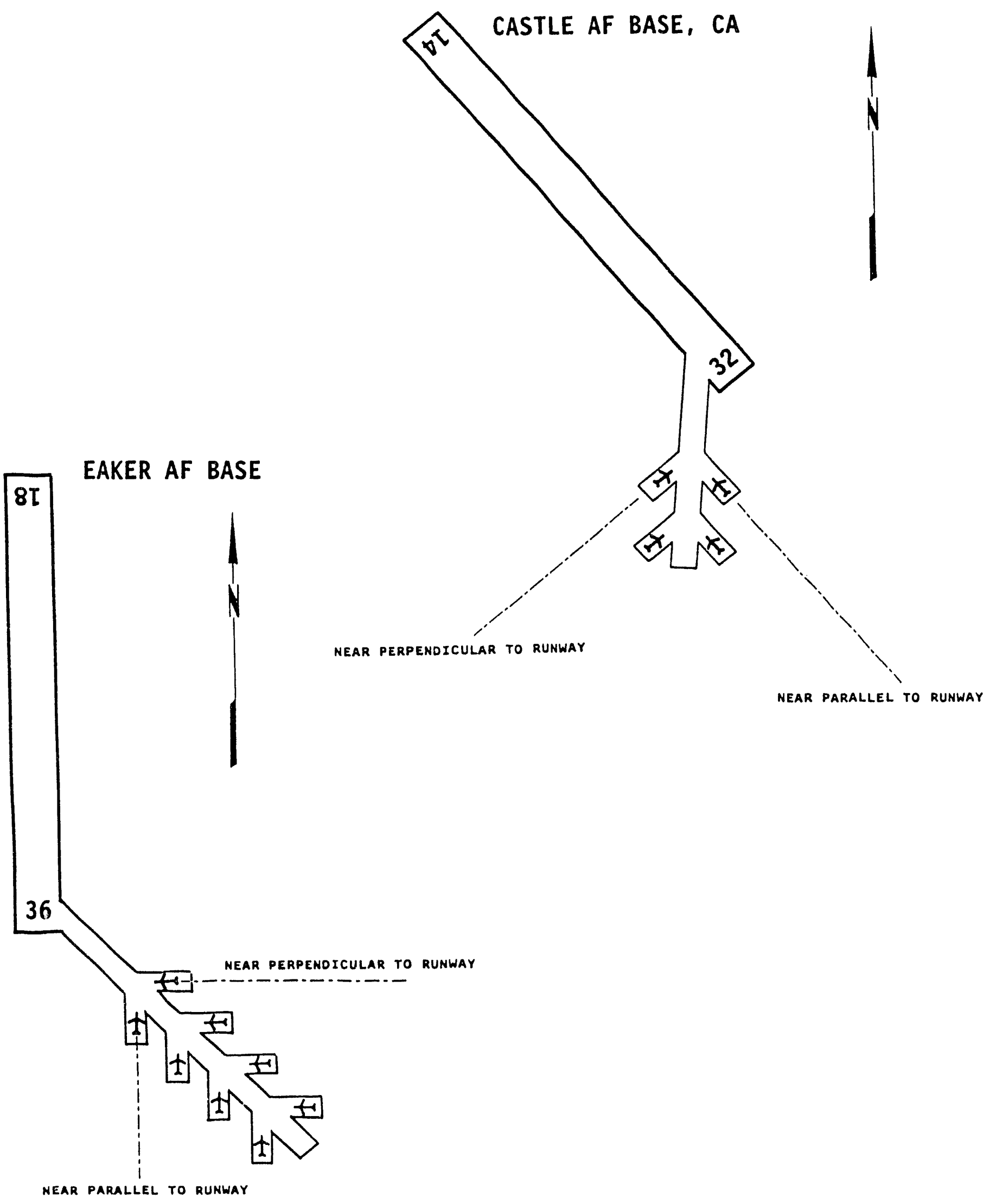

Figure B-2 Aircraft/Air Base Orientation at Castle AFB and Eaker AFB 
FAIRCHILD AF BASE
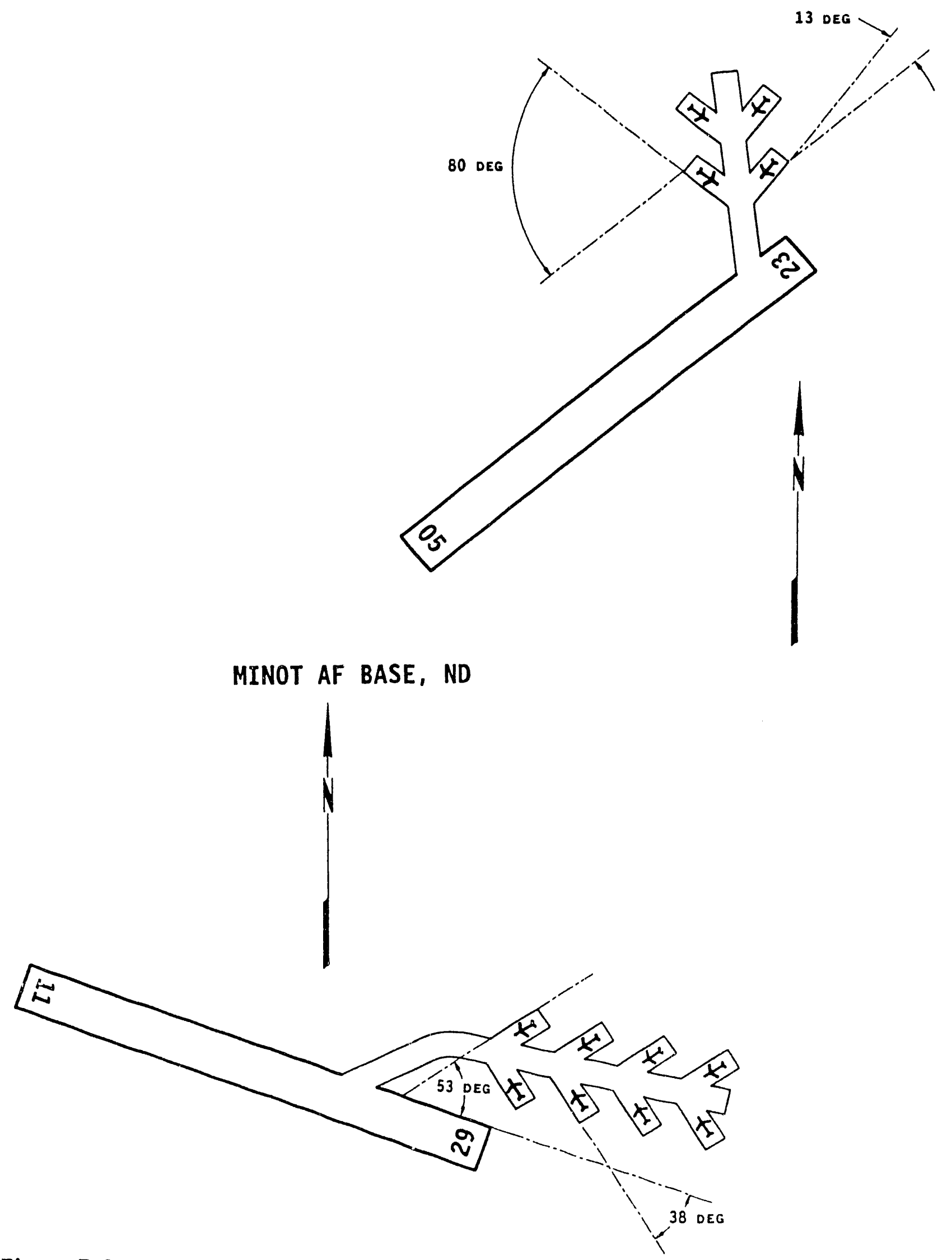

Figure B-3 Aircraft/Air Base Orientation at Fairchild AFB and Minot AFB 


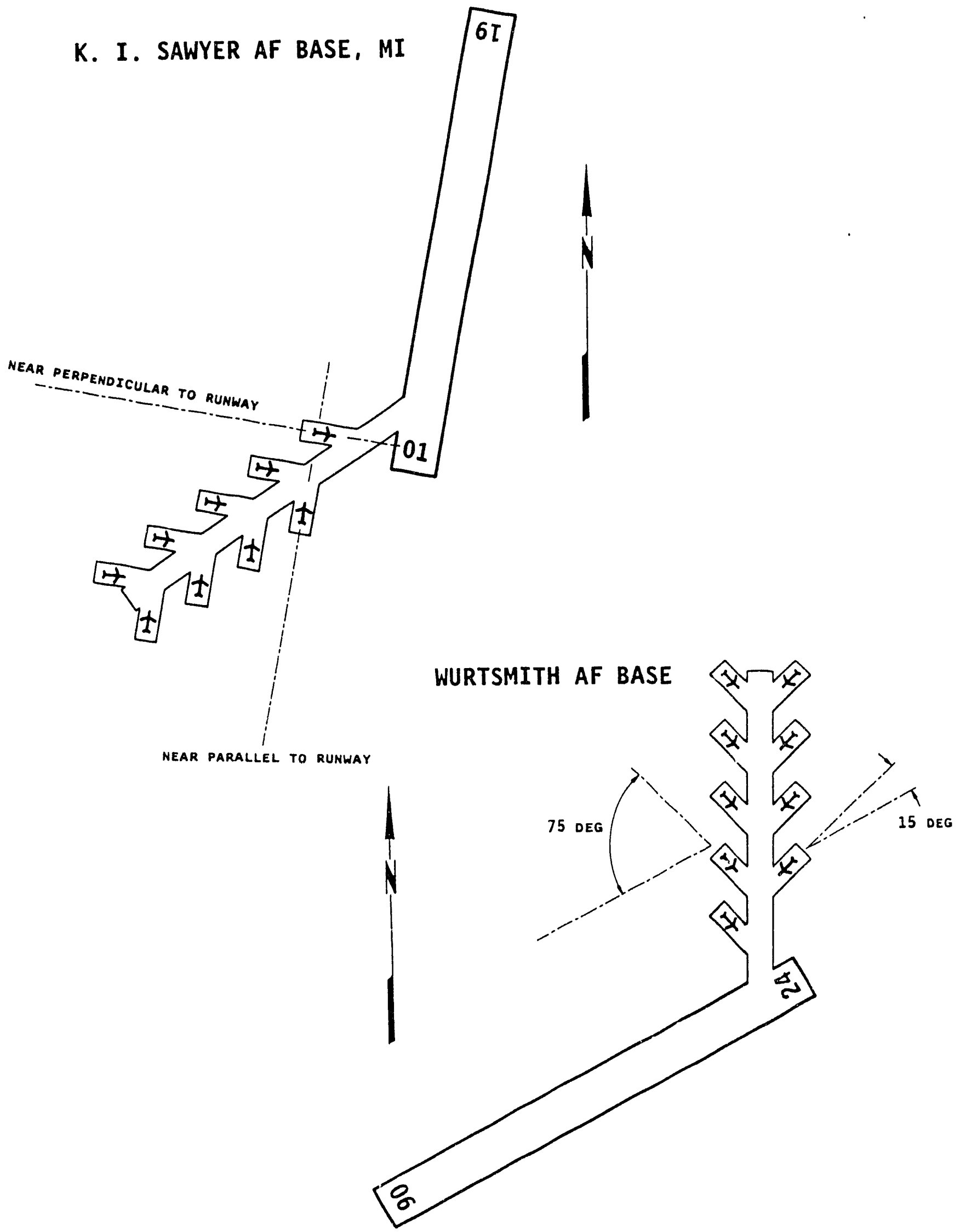

Figure B-4 Aircraft/Air Base Orientation at Sawyer AFB and Wurtsmith AFB 


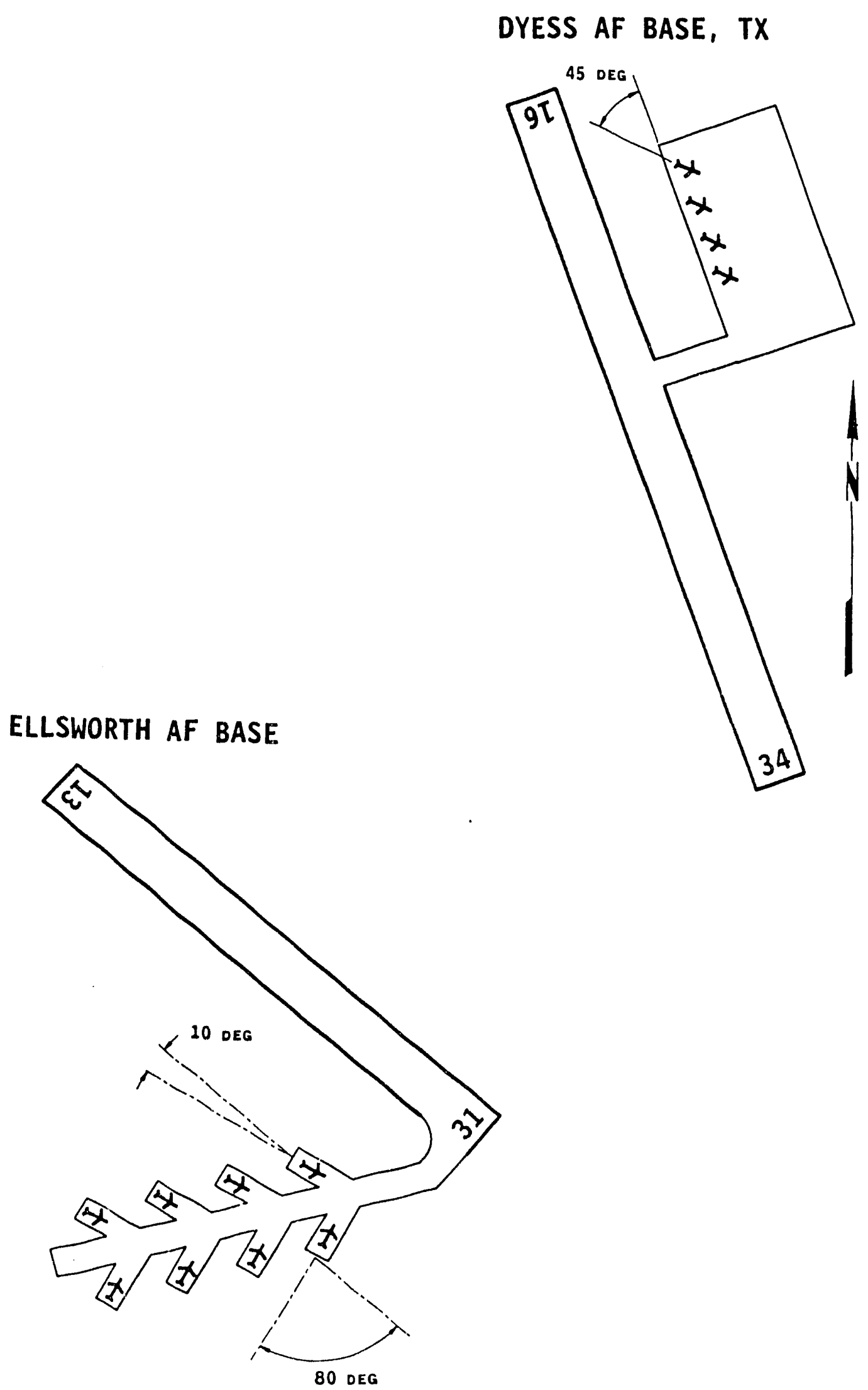

Figure B-5 Aircraft/Air Base Orientaiion at Dyess AFB and Ellsworth AFB 


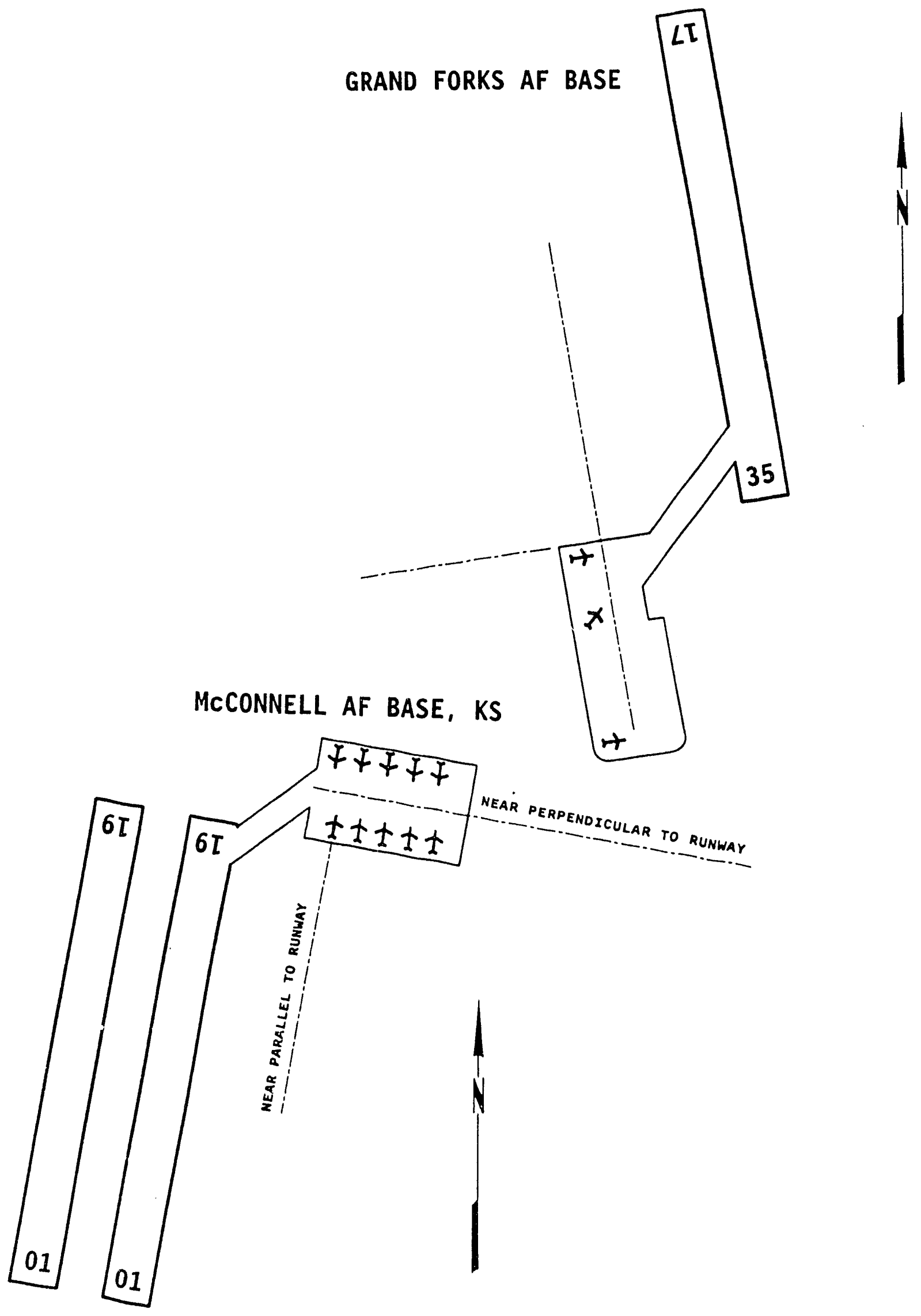

Figure B-6 Aircraft/Air Base Orientation at Grand Forks AFB and McConnell AFB 


\section{Appendix C WEATHER DATA}

Weather data used in the exposure evaluation comprised wind direction and wind speed readings at one-half hour intervals over a period of one year (January 1989 to January 1990) for each of the twelve bases (this data was provided by the Air Force*). Since the above data was, as a practical matter, limited to one year, it was verified that the weather for this year was typical by comparison with weather data compiled over a seven year period for the same locations (the 7 year data was provided by Lawrence Livermore National Laboratory). Appendix $C$ provides summary plots of the wind directions and wind speed and shows various example comparisons between the 1-year and 7-year data. Data discs of the raw weather data or the processed weather data (data required as input to the computer program) are available from Lawrence Livermore Laboratory (Douglas Stephens) or JM Technical (Joe Mansfield).

It is noted that the effects of rain and snow were ignored in the evaluation. Since rain and perhaps snow would most likely reduce the severity of the fire, including the effect of rain or snow, although probably a negligible effect, would tend to reduce the overall probability of exposure to given temperatures.

Summary plots of the relative probability of wind directions and wind speeds are given in Figures C-1 and C-2, respectively (1-year Air Force data).

Regarding the comparison between 1-year and 7-year data, the data was separated into the wind speed categories of 0 to $10 \mathrm{mph}, 10$ to $20 \mathrm{mph}$ and greater than $20 \mathrm{mph}$. Then the probability vs wind direction for each wind speed category was compared. Example comparative plots for Carswell, Castle, Sawyer, McConnell and Eaker AF Bases are given on Figures C-3 through C-7.

* Department of the Air Force, OL-A, USAF Environmental Technical Applications Center (MAC), Federal Building, Asheville, NC 28801 (Contact Mr. Henry Fountain). 
It is noted that the 1-year data was provided in terms of 10 degree angular intervals in wind direction, whereas the 7-year data was in 22.5 degree intervals. Similarly, the wind speed categories were not exactly equivalent. It is thereby not possible to make a point by point comparison, but the trends and their magnitudes are relevant. In this context, the 1-year data appears to be from a typical year.

The wind speeds averaged over a year for each of the twelve bases, and for all bases, is listed in Table C-1.

\section{TABLE C- 1. AVERAGE WIND SPEED AT VARIOUS SAC BASES}

\begin{tabular}{lc} 
BASE & SPEED (moh) \\
\cline { 2 - 2 } DYESS & 8.31 \\
ELLSWORTH & 8.96 \\
GRAND FORKS & 10.41 \\
MCCONNELL & 8.59 \\
& \\
BARKSDALE & 5.31 \\
CARSWELL & 8.92 \\
CASTLE & 5.02 \\
EAKER & 8.01 \\
FAIRCHILD & 6.52 \\
MINOT & 9.62 \\
SAWYER & 8.31 \\
WURTSMITH & 7.06 \\
& \\
WIND SPEED AVERAGED OVER ALL BASES $7.92 \mathrm{mph}$
\end{tabular}

The rate of airport operations (landings and take-offs) varies with the time of day, and the weather conditions also tend to vary with time of day. Using the assumption that the rate of accidents is proportional to the rate of airport operatior s, it was explained in Section 2 that data on the operation rate vs time of day was combined with wind speed and direction data to establish the relative probability that an accident will occur for given wind speeds and directions. Relative airport operation rates vs time of day were obtained from Reference 13. The operation rate data used is summarized in Table C-2. It can be seen from Table C-2 that the base ti base variation in rate at given times of day is not particularly significant, and that for four bases the average for the other eight bases was used. 


\section{TABLE C-2. FRACTIONS OF LANDINGS OR TAKE-OFFS AT VARIOUS TIMES OF DAY ${ }^{1}$}

\begin{tabular}{|l|c|c|c|c|}
\hline \multicolumn{1}{|c|}{ BASE } & \multicolumn{5}{|c|}{ TIME OF DAY (GMT) } \\
\hline \multirow{2}{*}{ CARSWELL } & $0000-0600$ & $0600-1200$ & $1200-1800$ & $1800-2400$ \\
\cline { 2 - 5 } DYESS & .069 & .279 & .450 & .202 \\
ELLSWORTH & .059 & .216 & .510 & .215 \\
FAIRCHILD & .059 & .315 & .463 & .163 \\
GRAND FORKS & .058 & .284 & .501 & .157 \\
McCONNELL & .060 & .279 & .519 & .142 \\
MINOT & .022 & .354 & .531 & .093 \\
SAWYER & .076 & .286 & .530 & .108 \\
\hline $\begin{array}{l}\text { AVERAGE FRACTION: } \\
\text { USED FOR }\end{array}$ & .061 & .244 & .527 & .168 \\
$\begin{array}{l}\text { BARKSDALE, CASTLE, } \\
\text { EAKER \& WURTSMITH }\end{array}$ & .058 & .282 & .504 & .156 \\
\hline
\end{tabular}

'Based on data from Jan 89 to Jan 90 . Includes military and general aviation.

The probabilities listed in Table $\mathrm{C}-3$ indicate the relative probability that accidents will occur for various wind speed/wind direction combinations at various times of day for $\mathrm{MCC}$ Connell $\mathrm{AF}$ Base. Probability numiver files that correspond to those on Table C-3 have been compiled for all twelve bases. These files are required input to the computer program for evaluating the probability of weapon exposure to given temperatures, and are available from Lawrence Livermore National Laboratory (Douglas Stephens) or JM Technical (Joe Mansfield). 


\section{TABLE C-3. RELATIVE PROBABILITY OF ACCIDENT FOR GIVEN WIND SPEEDS/WIND DIRECTIONS AT GIVEN TIMES OF DAY FOR MCCONNELL AF BASE.}

\begin{tabular}{|c|c|c|c|}
\hline $\begin{array}{l}\text { TIME OF } \\
\text { DAY, hours }\end{array}$ & $\begin{array}{l}\text { WIND SPEED } \\
\text { RANGE, mph }\end{array}$ & $\begin{array}{l}\text { WIND DIRECTION } \\
\text { RANGE, degrees }\end{array}$ & $\begin{array}{l}\text { RELATIVE } \\
\text { PROBABILITY of } \\
\text { ACCIDENT }\end{array}$ \\
\hline \multirow{46}{*}{$0000-0600$} & \multirow{29}{*}{$0-10$} & $355 \cdot 5$ & 0.000397 \\
\hline & & $\frac{5 \cdot 15}{15 \cdot 25}$ & 0.000618 \\
\hline & & $\frac{15 \cdot 25}{25 \cdot 35}$ & $\frac{0.000453}{0.000629}$ \\
\hline & & 35.45 & 0.000596 \\
\hline & & 45.55 & 0.000408 \\
\hline & & 55.65 & 0.000353 \\
\hline & & $65 \cdot 75$ & 0.000453 \\
\hline & & $\frac{75 \cdot 85}{85.95}$ & 0.000287 \\
\hline & & $\frac{85.95}{95.105}$ & 0.000519 \\
\hline & & 105.115 & $\begin{array}{l}0.000740 \\
0.000419\end{array}$ \\
\hline & & $115 \cdot 125$ & 0.000353 \\
\hline & & $\frac{125 \cdot 135}{125.115}$ & 0.000773 \\
\hline & & 135.145 & 0.001236 \\
\hline & & $\frac{145 \cdot 155}{155 \cdot 165}$ & 0.001093 \\
\hline & & $165 \cdot 175$ & 0.000883 \\
\hline & & $175-185$ & 0.000430 \\
\hline & & $185 \cdot 195$ & 0.000563 \\
\hline & & $\frac{205-215}{215-225}$ & 0.000232 \\
\hline & & $\frac{215 \cdot-225}{225 \cdot 235}$ & 0.000254 \\
\hline & & 235.245 & $\frac{0.000166}{0.000077}$ \\
\hline & & $245 \cdot 255$ & 0.000144 \\
\hline & & 255.265 & 0.000144 \\
\hline & & $265 \cdot 275$ & 0.000088 \\
\hline & & $275 \cdot 285$ & 0.000166 \\
\hline & & $285 \cdot 295$ & 0.000144 \\
\hline & & 295.305 & 0.000110 \\
\hline & & $305-315$ & 0.000121 \\
\hline & & $\begin{array}{l}325-335 \\
335-345 \\
\end{array}$ & $\frac{0.000088}{0.000166}$ \\
\hline & & 345.355 & $\begin{array}{l}0.000166 \\
0.000254\end{array}$ \\
\hline & \multirow{17}{*}{$10-20$} & 355.5 & 0.000442 \\
\hline & & $\frac{5 \cdot 15}{15 \cdot 25}$ & 0.000552 \\
\hline & & $\frac{15 \cdot 25}{25 \cdot 35}$ & 0.000442 \\
\hline & & 35.45 & $\frac{0.000309}{0.000166}$ \\
\hline & & 45.55 & 0.000088 \\
\hline & & $55-65$ & 0.000110 \\
\hline & & 65.75 & 0.000011 \\
\hline & & $-75 \cdot 85$ & 0.000000 \\
\hline & & $85 \cdot 95$ & 0.000055 \\
\hline & & $\frac{105 \cdot 115}{115 \cdot 125}$ & 0.000055 \\
\hline & & $125-135$ & 0.000055 \\
\hline & & $135 \cdot 145$ & 0.000210 \\
\hline & & $145 \cdot 155$ & 0.000342 \\
\hline & & $155 \cdot 1165$ & 0.000729 \\
\hline & & 165.175 & 0.000960 \\
\hline & & $175 \cdot 185$ & 0.000718 \\
\hline & & $185 \cdot 1 \mathrm{s5}$ & 0.000486 \\
\hline
\end{tabular}




\begin{tabular}{|c|c|c|c|}
\hline \multirow{49}{*}{$0000-0600$} & \multirow{15}{*}{$10-20$} & $195 \cdot 205$ & 0.000221 \\
\hline & & $205 \cdot 215$ & 0.000033 \\
\hline & & $215 \cdot 225$ & 0.000055 \\
\hline & & $225 \cdot 235$ & 0.000011 \\
\hline & & 235.245 & 0.000000 \\
\hline & & $245 \cdot 255$ & 0.000000 \\
\hline & & $255 \cdot 265$ & 0.000000 \\
\hline & & $265 \cdot 275$ & 0.000000 \\
\hline & & $275 \cdot 285$ & 0.000000 \\
\hline & & $285 \cdot 295$ & 0.000011 \\
\hline & & $\frac{295 \cdot 305}{305 \cdot 315}$ & $\frac{0.000033}{0.000055}$ \\
\hline & & $315 \cdot 325$ & 0.000033 \\
\hline & & $325 \cdot 335$ & 0.000022 \\
\hline & & $335 \cdot 345$ & 0.000188 \\
\hline & & $345 \cdot 355$ & 0.000166 \\
\hline & & $355 \cdot 5$ & 0.000044 \\
\hline & & $5 \cdot 15$ & 0.000088 \\
\hline & & $15 \cdot 25$ & 0.000066 \\
\hline & & $25 \cdot 35$ & 0.000000 \\
\hline & & $35-45$ & 0.000000 \\
\hline & & $\frac{45 \cdot 55}{55 \cdot 65}$ & 0.000000 \\
\hline & & $\frac{55 \cdot 65}{65 \cdot 75}$ & 0.000000 \\
\hline & & $\frac{65}{75} \cdot \frac{75}{85}$ & 0.000000 \\
\hline & & $\frac{15 \cdot 85}{85 \cdot 95}$ & 0.000000 \\
\hline & & $95 \cdot 105$ & 0.000000 \\
\hline & & $105 \cdot 115$ & 0.000000 \\
\hline & & $115 \cdot 125$ & $\frac{0.000011}{0.000011}$ \\
\hline & & $125 \cdot 135$ & $\frac{0.000011}{0.000000}$ \\
\hline & & $135-145$ & 0.000011 \\
\hline & & $145 \cdot 155$ & 0.000011 \\
\hline & & $155 \cdot 165$ & 0.000055 \\
\hline & $>20$ & $165 \cdot 175$ & 0.000055 \\
\hline & & $175 \cdot 185$ & 0.000099 \\
\hline & & $185-195$ & 0.000066 \\
\hline & & 195.205 & 0.000077 \\
\hline & & $205 \cdot 215$ & 0.000000 \\
\hline & & $215 \cdot 225$ & 0.000019 \\
\hline & & $225 \cdot 235$ & 0.000000 \\
\hline & & $235-245$ & 0.000000 \\
\hline & & $\frac{245 \cdot 255}{255 \cdot 265}$ & $\frac{0.000000}{0.000000}$ \\
\hline & & $\frac{255 \cdot 265}{265 \cdot 275}$ & $\frac{0.000000}{0.000000}$ \\
\hline & & 275.285 & $\frac{0.000000}{0.000000}$ \\
\hline & & $285 \cdot 295$ & 0.000000 \\
\hline & & $295 \cdot 305$ & 0.000000 \\
\hline & & $305 \cdot 315$ & 0.000000 \\
\hline & & $315 \cdot 325$ & 0.000022 \\
\hline & & $325 \cdot 335$ & 0.000022 \\
\hline & & $335 \cdot 345$ & 0.000055 \\
\hline & & $345 \cdot 355$ & 0.000033 \\
\hline & & $355 \cdot 5$ & 0.009320 \\
\hline & & $5-15$ & 0.010052 \\
\hline & & $15 \cdot 25$ & 0.011879 \\
\hline & & $\frac{25 \cdot 35}{25 \cdot 15}$ & 0.007311 \\
\hline & & $\frac{35.45}{45 \cdot 55}$ & 0.007311 \\
\hline & & $55 \cdot 65$ & 0.006031 \\
\hline & & $65 \cdot 75$ & 0.006213 \\
\hline n6nn-1200 & $0-10$ & $75 \cdot 85$ & 0.006579 \\
\hline $0000-1<00$ & & $85 \cdot 95$ & $\frac{0.006761}{0.006761}$ \\
\hline & & $95 \cdot 105$ & $\frac{0.006761}{0.007494}$ \\
\hline & & $105 \cdot 115$ & $\frac{0.007494}{0.007311}$ \\
\hline & & $115 \cdot 125$ & $\frac{0.00 / 311}{0.005848}$ \\
\hline & & $125 \cdot 135$ & 0.008407 \\
\hline$\cdot$ & & $135 \cdot 145$ & 0.012427 \\
\hline & & $145 \cdot 155$ & 0.014072 \\
\hline & & $155 \cdot 165$ & 0.019007 \\
\hline
\end{tabular}

\section{C-5}




\begin{tabular}{|c|c|c|c|}
\hline \multirow{69}{*}{$0600-1200$} & \multirow{18}{*}{$0 \cdot 10$} & 165.175 & 0.014072 \\
\hline & & $175 \cdot 185$ & 0.010052 \\
\hline & & $185 \cdot 195$ & 0.013342 \\
\hline & & $195 \cdot 205$ & 0.007676 \\
\hline & & $205 \cdot 215$ & 0.003472 \\
\hline & & $215 \cdot 225$ & 0.002376 \\
\hline & & $225 \cdot 235$ & 0.002011 \\
\hline & & $235 \cdot 245$ & 0.001096 \\
\hline & & $245 \cdot 255$ & 0.002193 \\
\hline & & $255 \cdot 265$ & 0.001463 \\
\hline & & $265 \cdot 275$ & 0.001463 \\
\hline & & $275 \cdot 285$ & 0.003107 \\
\hline & & $285 \cdot 295$ & 0.001645 \\
\hline & & 295.305 & 0.002011 \\
\hline & & $305-315$ & 0.002741 \\
\hline & & $\frac{375 \cdot 325}{325 \cdot 335}$ & 0.002924 \\
\hline & & $335 \cdot 345$ & 0003107 \\
\hline & & $345 \cdot 355$ & 0.004020 \\
\hline & & $355 \cdot 5$ & 0.004935 \\
\hline & & 5.15 & 0.008955 \\
\hline & & $15 \cdot 25$ & 0.005848 \\
\hline & & $25 \cdot 35$ & 0.004204 \\
\hline & & 35.45 & 0.003107 \\
\hline & & $45 \cdot 55$ & 0.000548 \\
\hline & & $55 \cdot 65$ & 0.001645 \\
\hline & & $65 \cdot 75$ & 0.000548 \\
\hline & & $75 \cdot 85$ & 0.000548 \\
\hline & & 85.95 & 0.000731 \\
\hline & & 95.105 & 0.001279 \\
\hline & & $105 \cdot 115$ & 0.000183 \\
\hline & $1 n-9$ & $115 \cdot 125$ & 0.001463 \\
\hline & $10-20$ & $125 \cdot 135$ & 0.000731 \\
\hline & & 135.145 & 0.000365 \\
\hline & & $145 \cdot 155$ & 0.002924 \\
\hline & & $155-165$ & 0.006944 \\
\hline & & $165 \cdot 175$ & 0.011514 \\
\hline & & $175 \cdot 185$ & 0.015718 \\
\hline & & $185 \cdot 195$ & 0.010235 \\
\hline & & 195.205 & 0.008590 \\
\hline & & $205 \cdot 215$ & 0.003837 \\
\hline & & $215 \cdot 225$ & 0.000913 \\
\hline & & $225 \cdot 235$ & 0.000548 \\
\hline & & 235.245 & 0000103 \\
\hline & & $245 \cdot 255$ & 0.0000103 \\
\hline & & $\frac{255 \cdot 265}{255 \cdot 275}$ & 0.000103 \\
\hline & & $\frac{265 \cdot 275}{275.285}$ & 0.000000 \\
\hline & & $\frac{275 \cdot 285}{285-295}$ & $\frac{0.000548}{0.000365}$ \\
\hline & & $295 \cdot 305$ & 0.000731 \\
\hline & & $305 \cdot 315$ & 0.000365 \\
\hline & & 315.325 & 0.000365 \\
\hline & & $325 \cdot 335$ & 0.001645 \\
\hline & & $335 \cdot 345$ & 0.003655 \\
\hline & & $345 \cdot 355$ & 0.003472 \\
\hline & & 355.5 & 0.000731 \\
\hline & & $5 \cdot 15$ & 0.001645 \\
\hline & & $\frac{15 \cdot 25}{25}$ & 0.001828 \\
\hline & & $\frac{25 \cdot 35}{25}$ & 0.000548 \\
\hline & & 35.45 & 0.000000 \\
\hline & & $45 \cdot 55$ & 0.000000 \\
\hline & & $55 \cdot 65$ & 0.000000 \\
\hline & $>20$ & $65 \cdot 75$ & 0.000000 \\
\hline & & $75 \cdot 85$ & 0.000000 \\
\hline & & $85 \cdot 95$ & 0.000000 \\
\hline & & $95-105$ & 0.000000 \\
\hline & & $105-115$ & 0.000000 \\
\hline & & 115.125 & 0.000000 \\
\hline & & $125 \cdot 135$ & 0.000000 \\
\hline & & $135 \cdot 145$ & 0.000183 \\
\hline & & $145 \cdot 155$ & 0.000000 \\
\hline
\end{tabular}




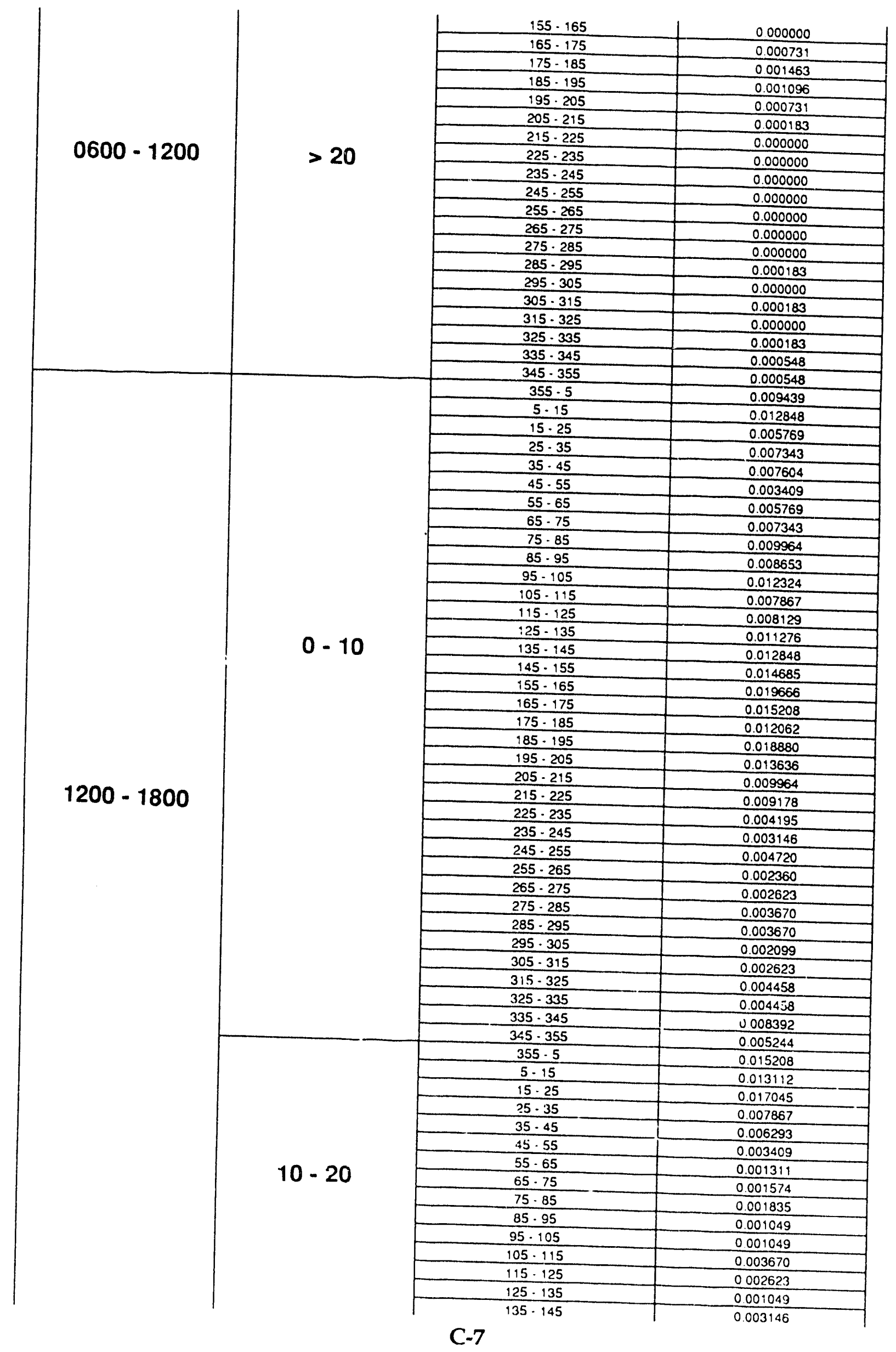




\begin{tabular}{|c|c|c|c|}
\hline \multirow{55}{*}{$1200-1800$} & \multirow{20}{*}{$10-20$} & $145 \cdot 155$ & 0.004195 \\
\hline & & $155 \cdot 165$ & 0.008129 \\
\hline & & $165 \cdot 175$ & 0.014161 \\
\hline & & $175 \cdot 185$ & 0.017306 \\
\hline & & $185 \cdot 195$ & 0020979 \\
\hline & & $195 \cdot 205$ & 0.017045 \\
\hline & & $205 \cdot 215$ & 0.008653 \\
\hline & & $215 \cdot 225$ & 0.004720 \\
\hline & & $225 \cdot 235$ & 0.000525 \\
\hline & & 235.245 & 0.000786 \\
\hline & & $245 \cdot 255$ & 0.001574 \\
\hline & & $255 \cdot 265$ & 0.000786 \\
\hline & & $265 \cdot 275$ & 0.000000 \\
\hline & & 275.285 & 0.000525 \\
\hline & & $285 \cdot 295$ & 0.001574 \\
\hline & & $295 \cdot 305$ & 0.002623 \\
\hline & & $305 \cdot 315$ & 0.002099 \\
\hline & & $315 \cdot 325$ & 0.002099 \\
\hline & & $325 \cdot 335$ & 0.002360 \\
\hline & & $335 \cdot 345$ & 0.008392 \\
\hline & & $345 \cdot 355$ & 0.012848 \\
\hline & & $355 \cdot 5$ & 0.003934 \\
\hline & & $5 \cdot 15$ & 0.002884 \\
\hline & & $15 \cdot 25$ & 0.002360 \\
\hline & & 25.35 & 0.000525 \\
\hline & & $\frac{35 \cdot 45}{45 \cdot 55}$ & 0.000000 \\
\hline & & $\frac{45 \cdot 55}{55 \cdot 65}$ & 0.000000 \\
\hline & & $\frac{55 \cdot 65}{65 \cdot 75}$ & 0.000000 \\
\hline & & $\frac{65 \cdot 75}{75 \cdot 85}$ & 0.000000 \\
\hline & & $\frac{75 \cdot 85}{85 \cdot 95}$ & 0.000000 \\
\hline & & $\frac{85 \cdot 95}{95 \cdot 105}$ & 0.000000 \\
\hline & & $\frac{95 \cdot 105}{105 \cdot 115}$ & 0.000000 \\
\hline & & $\frac{105 \cdot 115}{115 \cdot 12.5}$ & $\frac{0.000000}{0.000000}$ \\
\hline & & $125 \cdot 135$ & 0.000000 \\
\hline & & $135 \cdot 145$ & 0.000000 \\
\hline & & $145 \cdot 155$ & 0.000000 \\
\hline & $>20$ & $155 \cdot 165$ & 0.000261 \\
\hline & & $165 \cdot 175$ & 0.001049 \\
\hline & & $175 \cdot 185$ & 0.001835 \\
\hline & & $185-195$ & 0.002884 \\
\hline & & 195.205 & 0.002099 \\
\hline & & $205 \cdot 215$ & 0.001311 \\
\hline & & $215 \cdot 225$ & 0.000261 \\
\hline & & $225 \cdot 235$ & 0.000000 \\
\hline & & $235 \cdot 245$ & 0.000000 \\
\hline & & $245 \cdot 255$ & 0.000000 \\
\hline & & $255 \cdot 265$ & 0.000000 \\
\hline & & $265 \cdot 275$ & 0.000000 \\
\hline & & $275 \cdot 285$ & 0.000000 \\
\hline & & $285 \cdot 295$ & 0.000261 \\
\hline & & $295 \cdot 305$ & 0.000000 \\
\hline & & $305 \cdot 3 \cdot 5$ & 0.000525 \\
\hline & & $315 \cdot 325$ & 0.040525 \\
\hline & & $325 \cdot 335$ & 0.001049 \\
\hline & & $325 \cdot 345$ & 0.000525 \\
\hline & & $345 \cdot 355$ & 0.001574 \\
\hline & & $355 \cdot 5$ & 0001431 \\
\hline & & $\frac{5 \cdot 15}{15 \cdot 25}$ & $\frac{0.001562}{0.001431}$ \\
\hline & & 25.35 & 0,001258 \\
\hline & & $35 \cdot 45$ & 0.001431 \\
\hline & & $45 \cdot 55$ & 0.000954 \\
\hline $1800-2400$ & $0-10$ & $55 \cdot 65$ & 0.001041 \\
\hline & & $65 \cdot 75$ & 0.000954 \\
\hline & & $75 \cdot 85$ & 0001084 \\
\hline & & $85-95$ & 0000998 \\
\hline & & $95 \cdot 105$ & 0.001648 \\
\hline & & $105 \cdot 115$ & 0.001431 \\
\hline & & $115 \cdot 125$ & 0001345 \\
\hline & & $125 \cdot 135$ & 0001605 \\
\hline
\end{tabular}

\section{C-8}




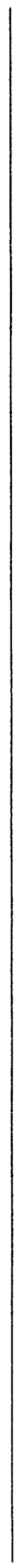

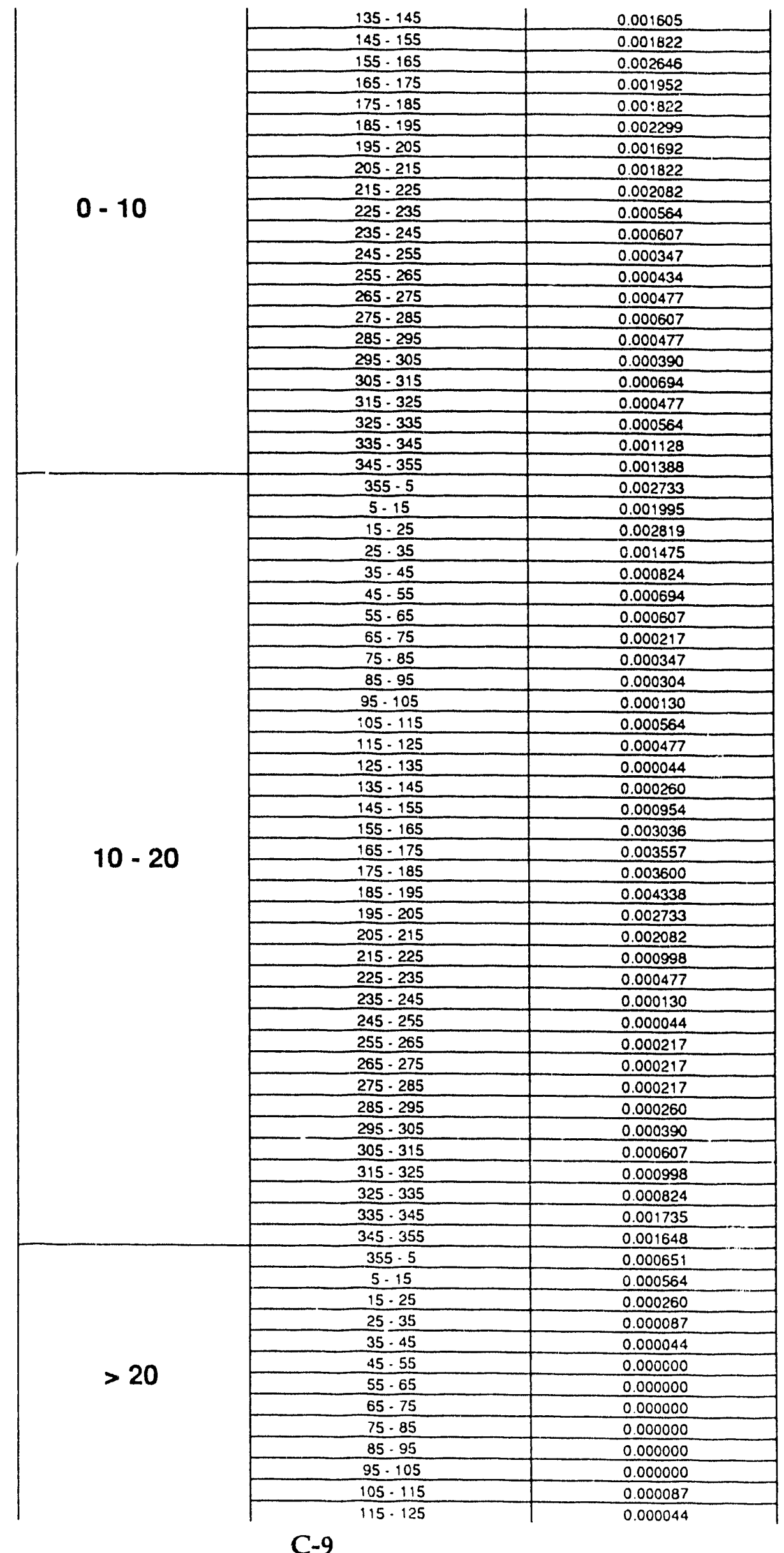




\begin{tabular}{|c|c|c|c|}
\hline \multirow{23}{*}{$1800-2400$} & \multirow{23}{*}{$>20$} & $125 \cdot 135$ & 0.000000 \\
\hline & & $135 \cdot 145$ & 0.000000 \\
\hline & & $145 \cdot 155$ & 0.000044 \\
\hline & & $155 \cdot 165$ & 0.000304 \\
\hline & & $165 \cdot 175$ & 0.000477 \\
\hline & & $175 \cdot 185$ & 0.000651 \\
\hline & & $185 \cdot 195$ & 0.000824 \\
\hline & & $195-205$ & 0.000694 \\
\hline & & $205 \cdot 215$ & 0.000260 \\
\hline & & $215-225$ & 0.000347 \\
\hline & & $225 \cdot 235$ & 0.000087 \\
\hline & & $235-245$ & 0.000044 \\
\hline & & $245 \cdot 255$ & 0.000087 \\
\hline & & $255 \cdot 265$ & 0.000000 \\
\hline & & 265.275 & 0.000000 \\
\hline & & $275 \cdot 285$ & 0.000000 \\
\hline & & $285 \cdot 295$ & 0.000000 \\
\hline & & $295-305$ & 0.000044 \\
\hline & & $305-315$ & 0.000000 \\
\hline & & $315 \cdot 325$ & 0.000087 \\
\hline & & $325 \cdot 335$ & 0.000087 \\
\hline & & $335 \cdot 345$ & 0.000173 \\
\hline & & $345 \cdot 355$ & 0.000434 \\
\hline
\end{tabular}

\section{C-10}



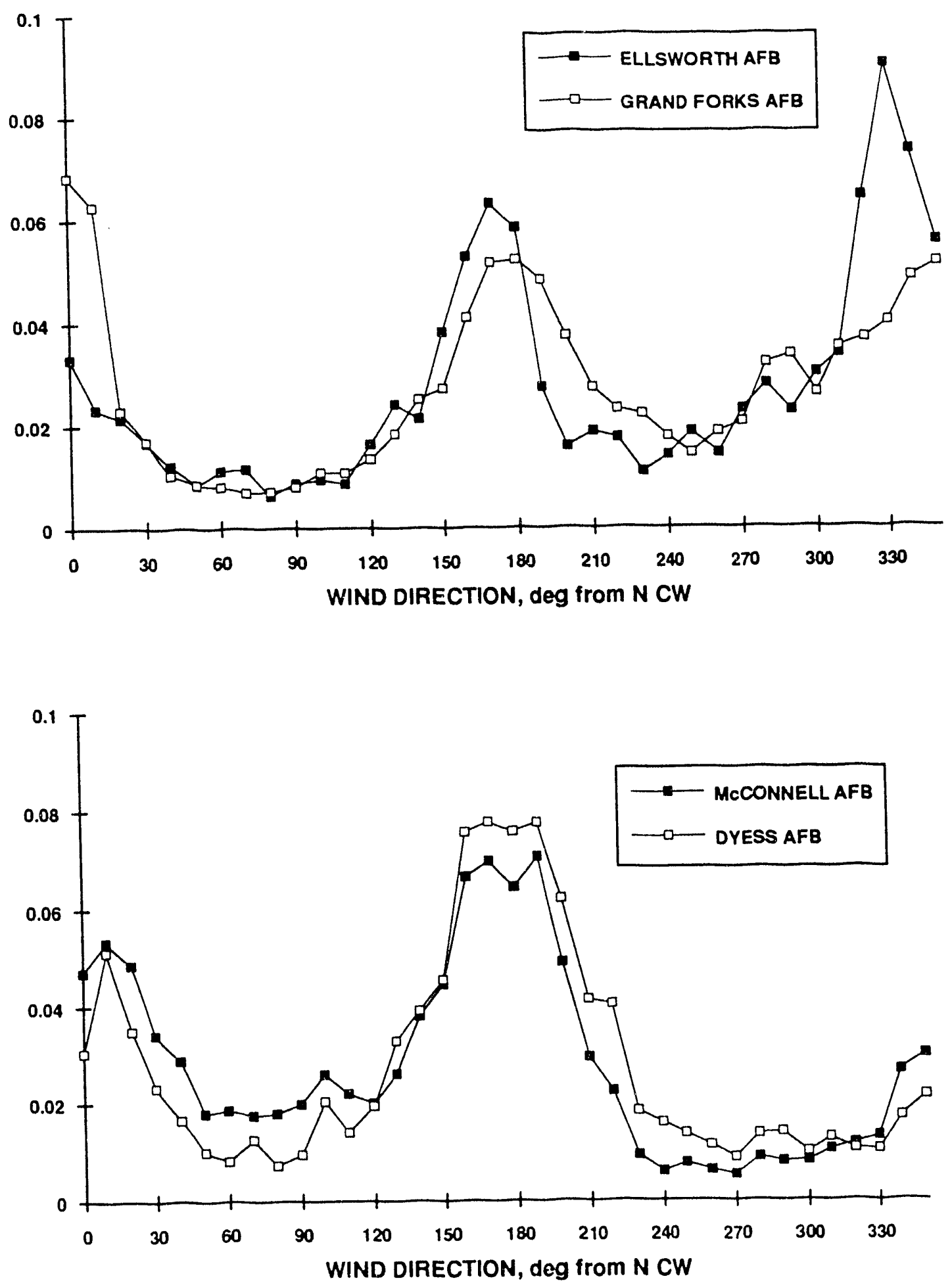

Figure C-1 Probability that the Wind will be in a Given Direction for Twelve Air Bases (continued on next two pages) 

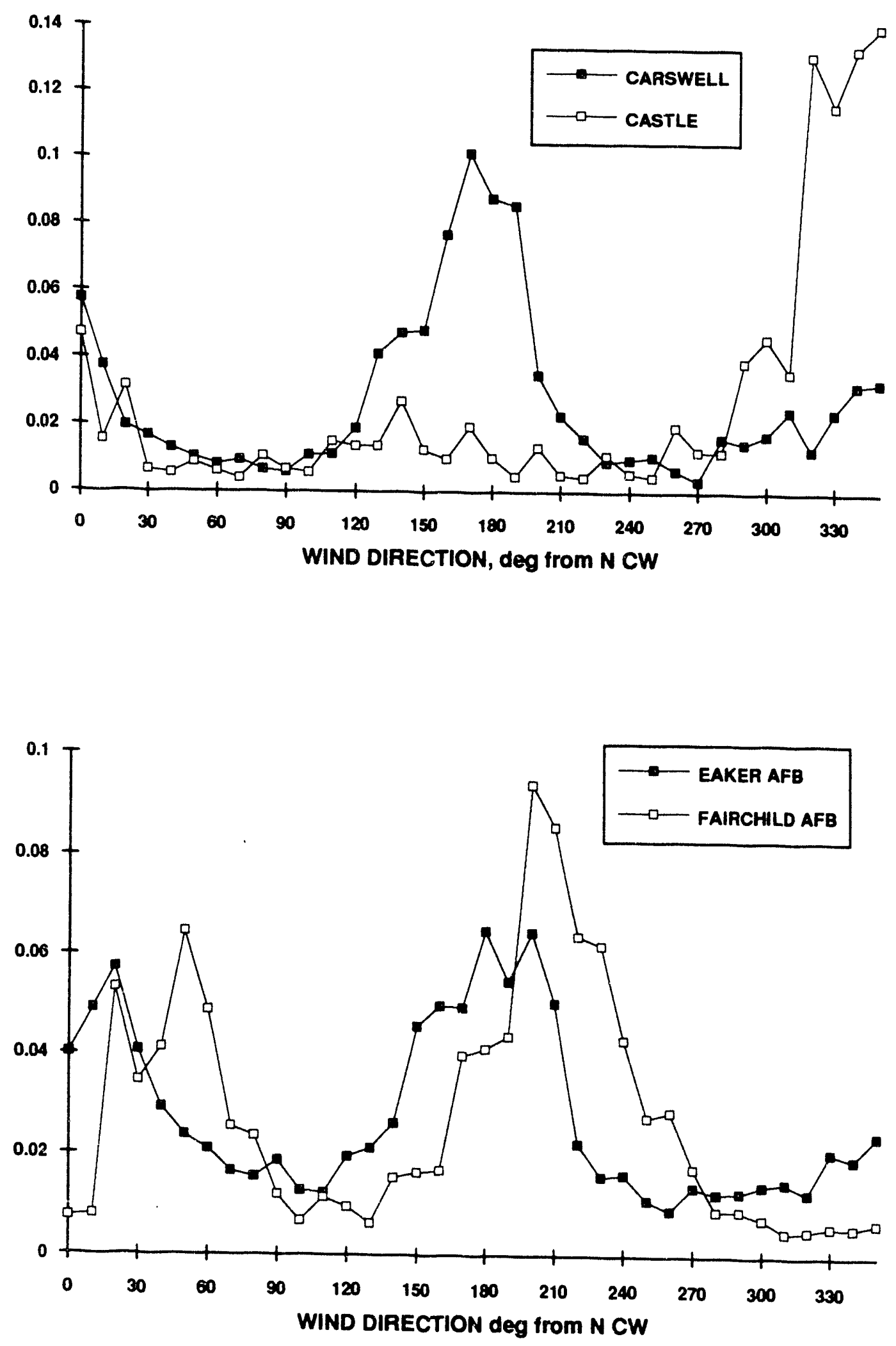

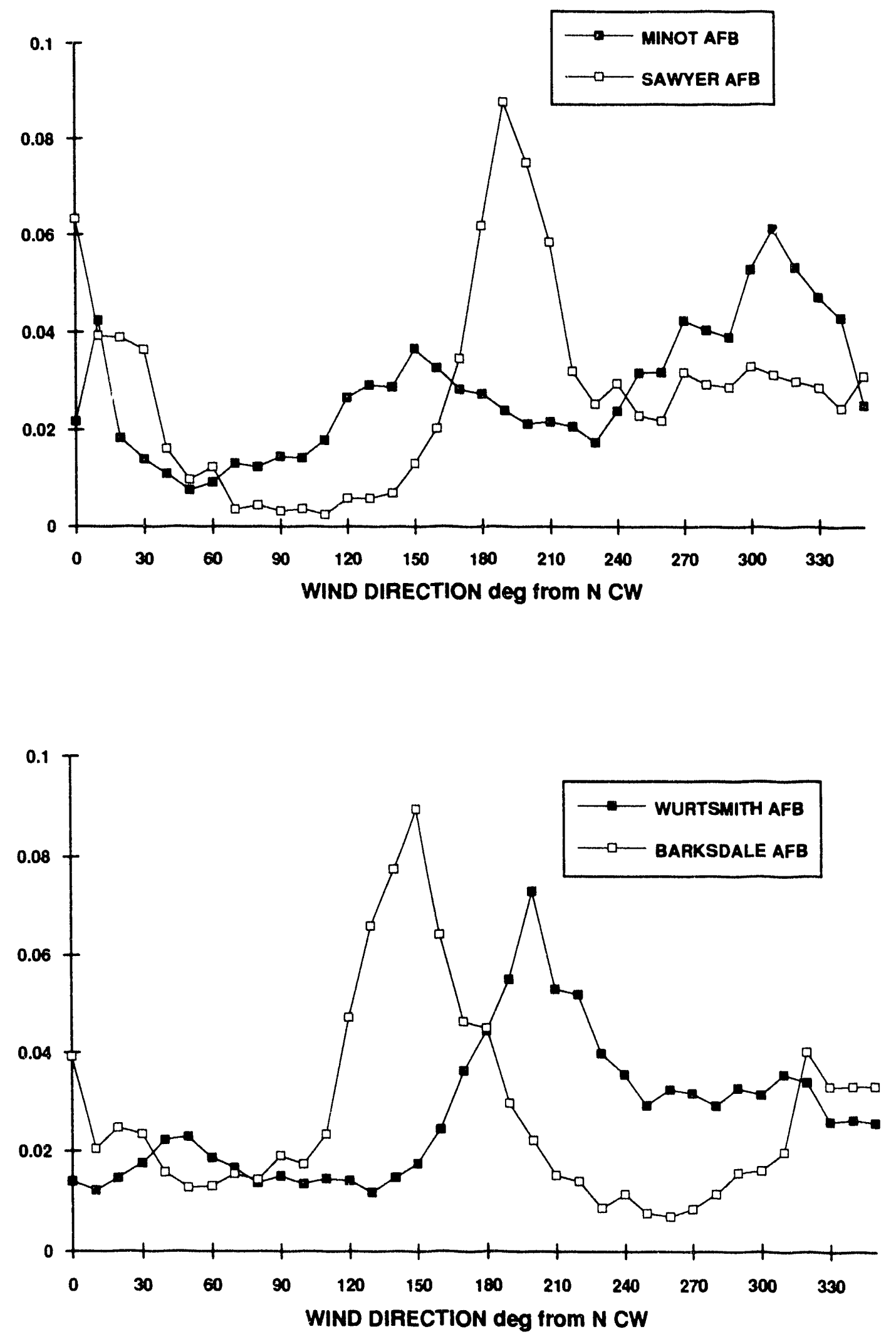

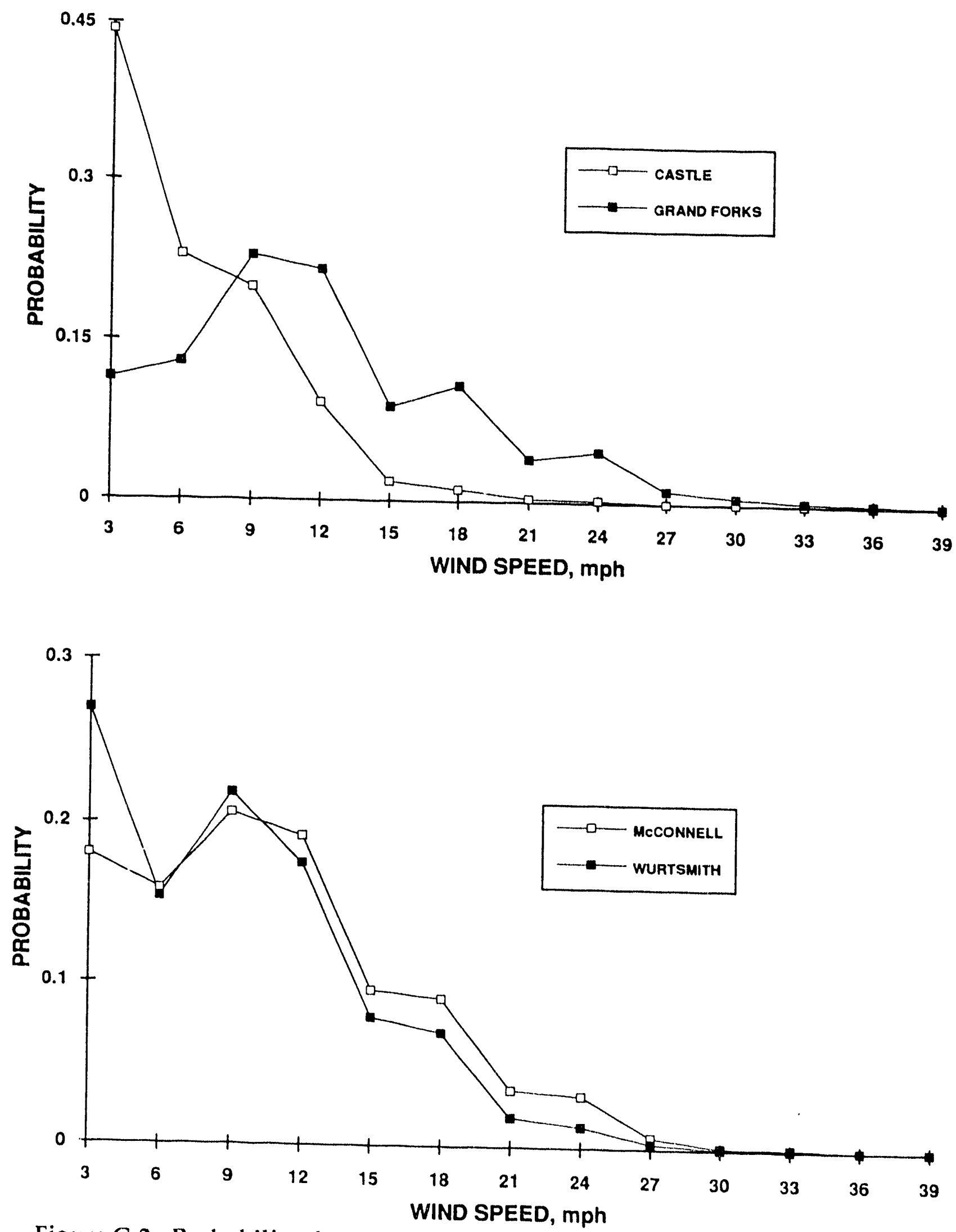

Figure C-2 Probability that the Wind Speed will be a Given Value for Various Air Bases 


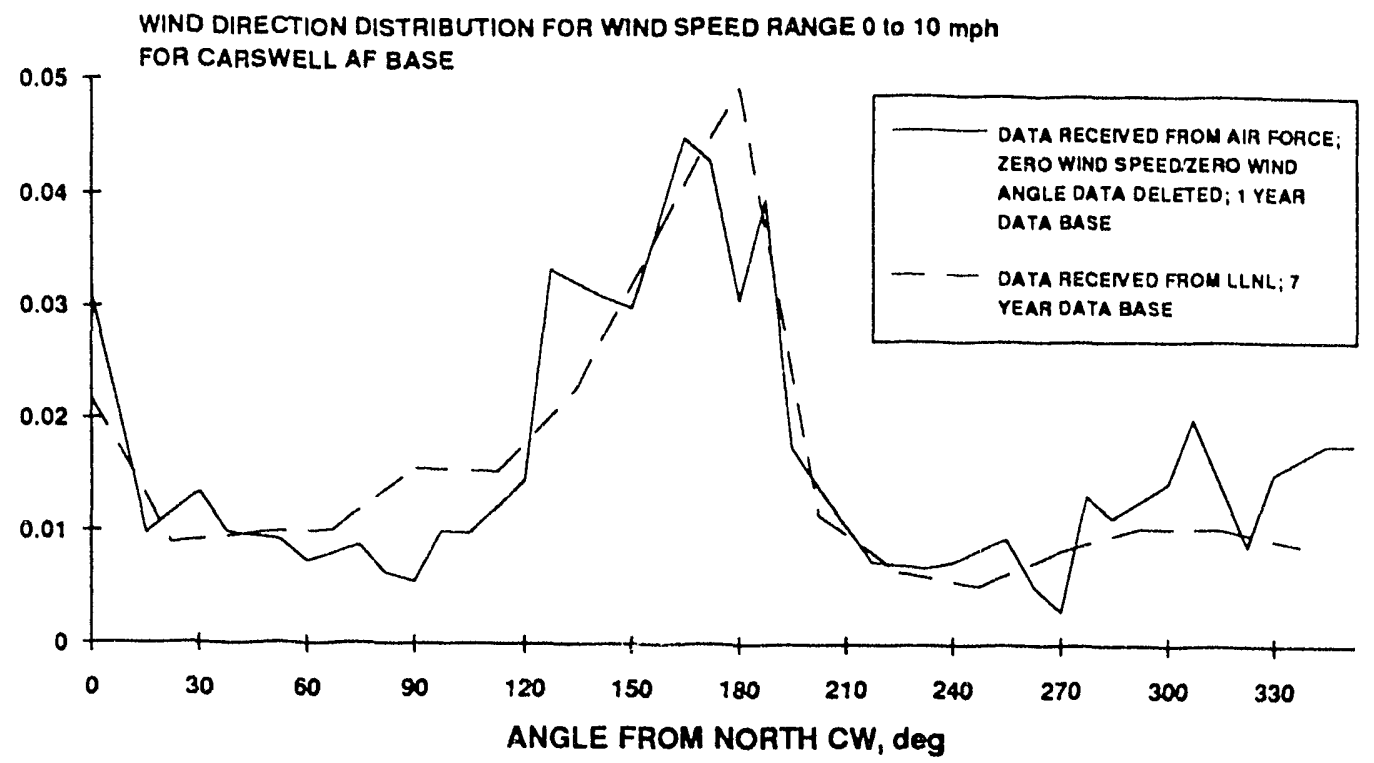

WIND DIRECTION DISTRIBUTION FOR WIND SPEED RANGE 10 to $20 \mathrm{mph}$ 0.06 T FOR CARSWELL AF BASE
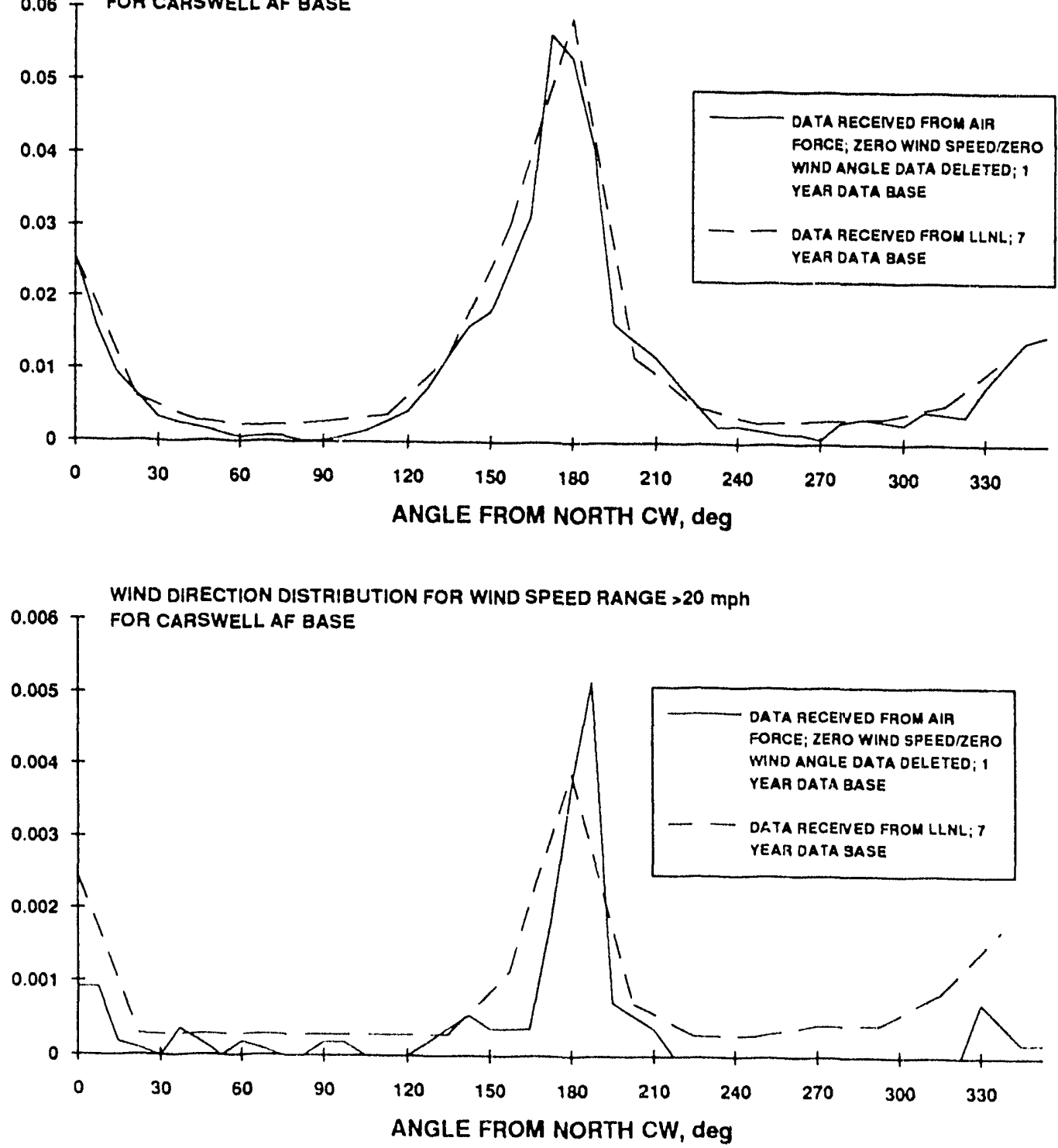

Figure C-3 Comparison Between Wind Data Obtained Throughout One-year and Seven-year Durations for Carswell AF Base 

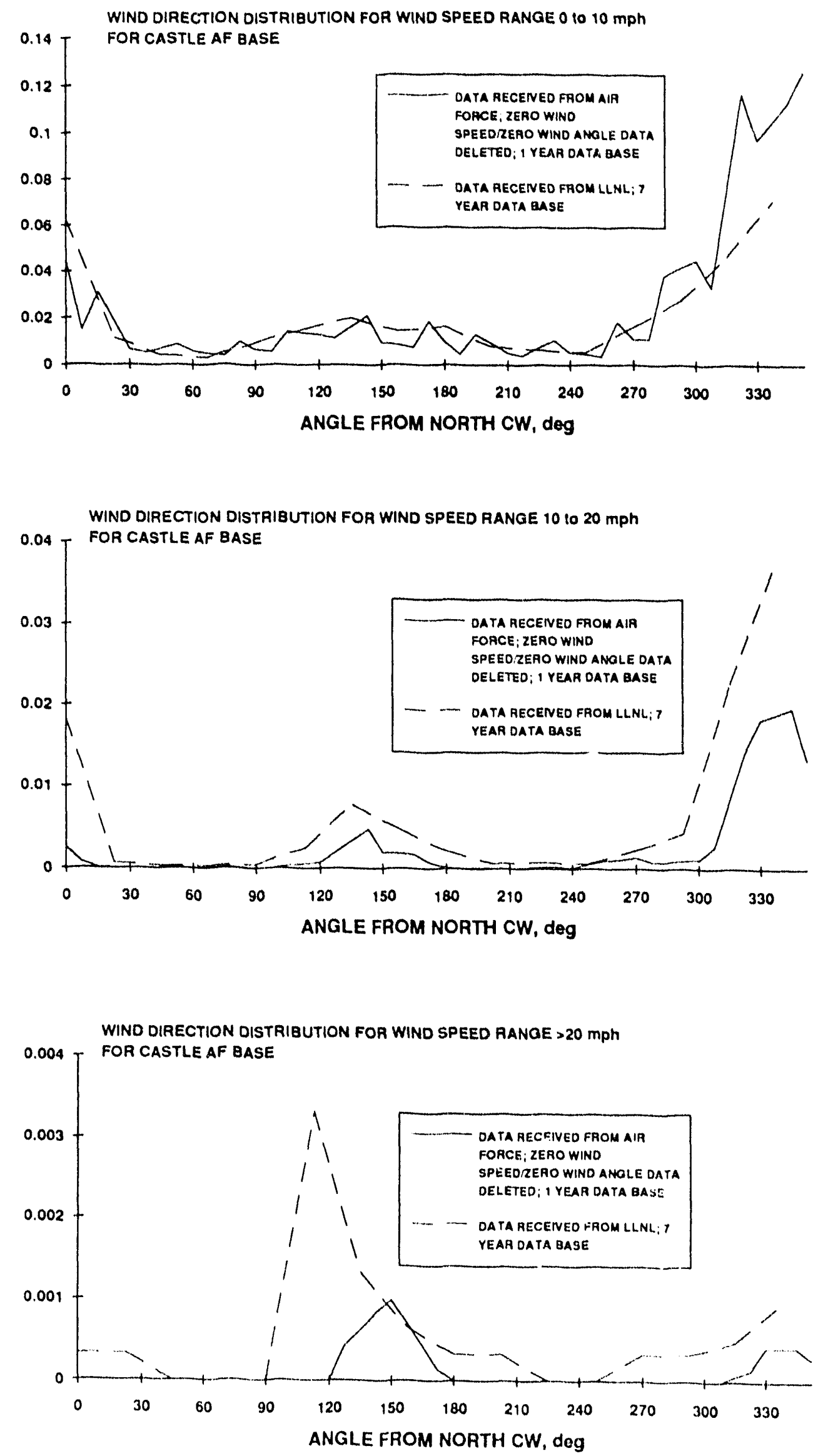

Figure C-4 Comparison Between Wind Data Obtained Throughout One-year and Seven-year Durations for Castle AF Base 

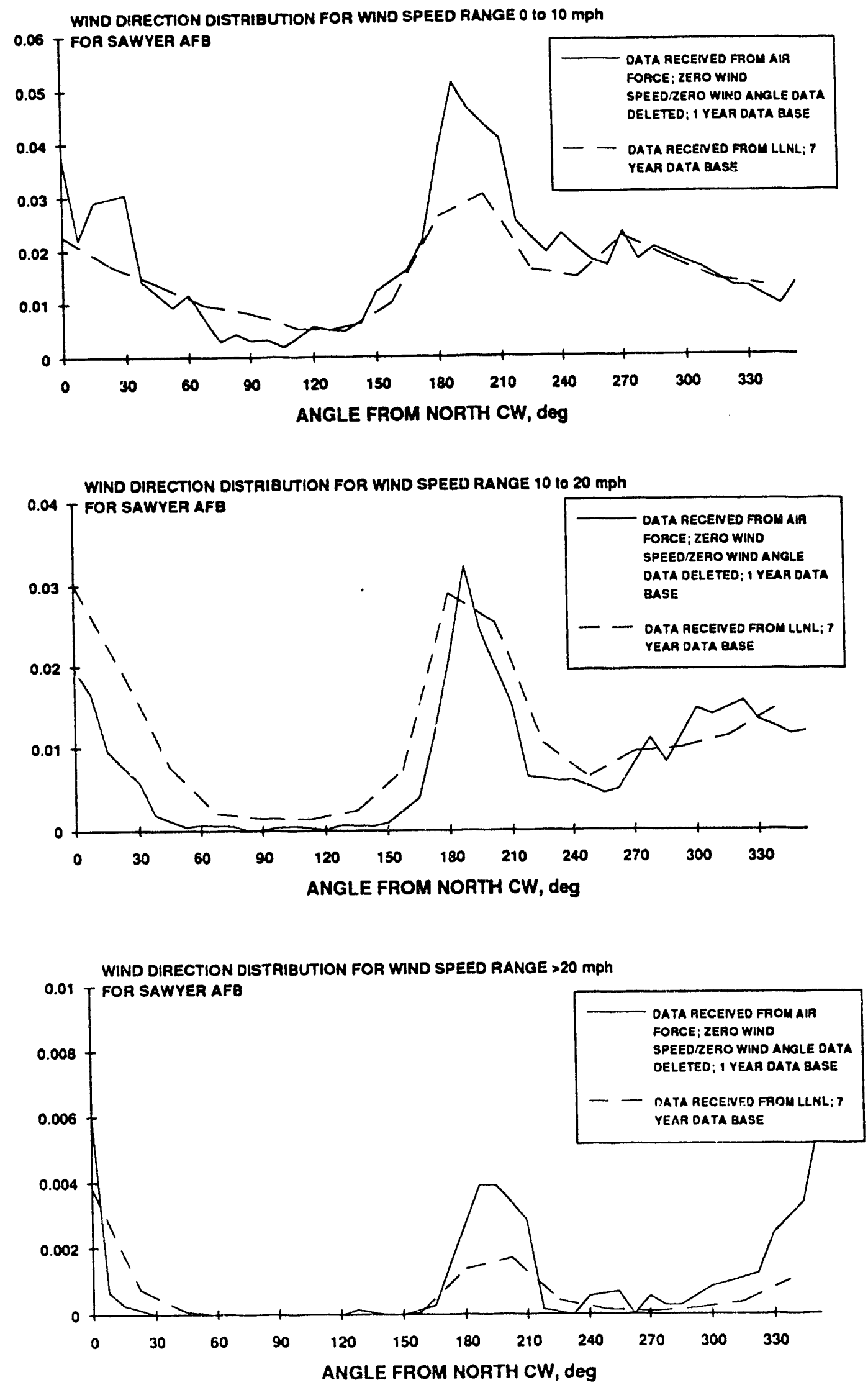

Figure C-5 Comparison Between Wind Data Obtained Throughout One-year and Seven-year Durations for Sawyer AF Base 
WIND DIRECTION DISTRIBUTION FOR WIND SPEED RANGE $01010 \mathrm{mph}$

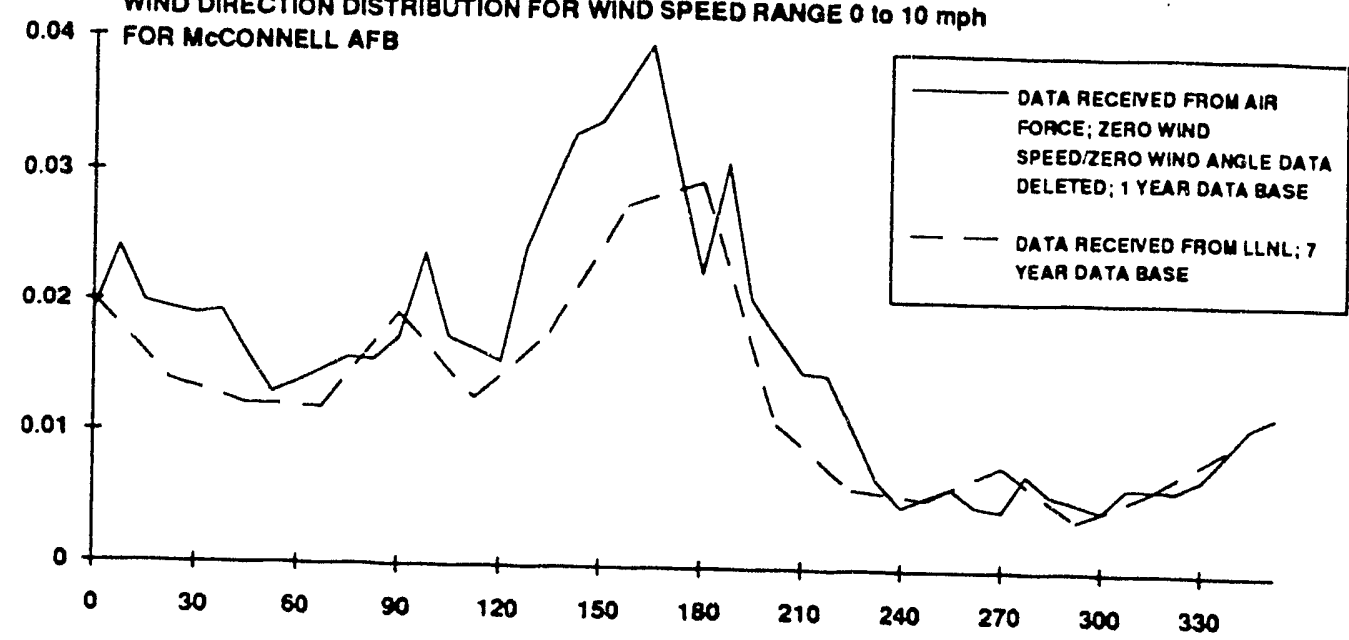

ANGLE FROM NORTH CW, dog
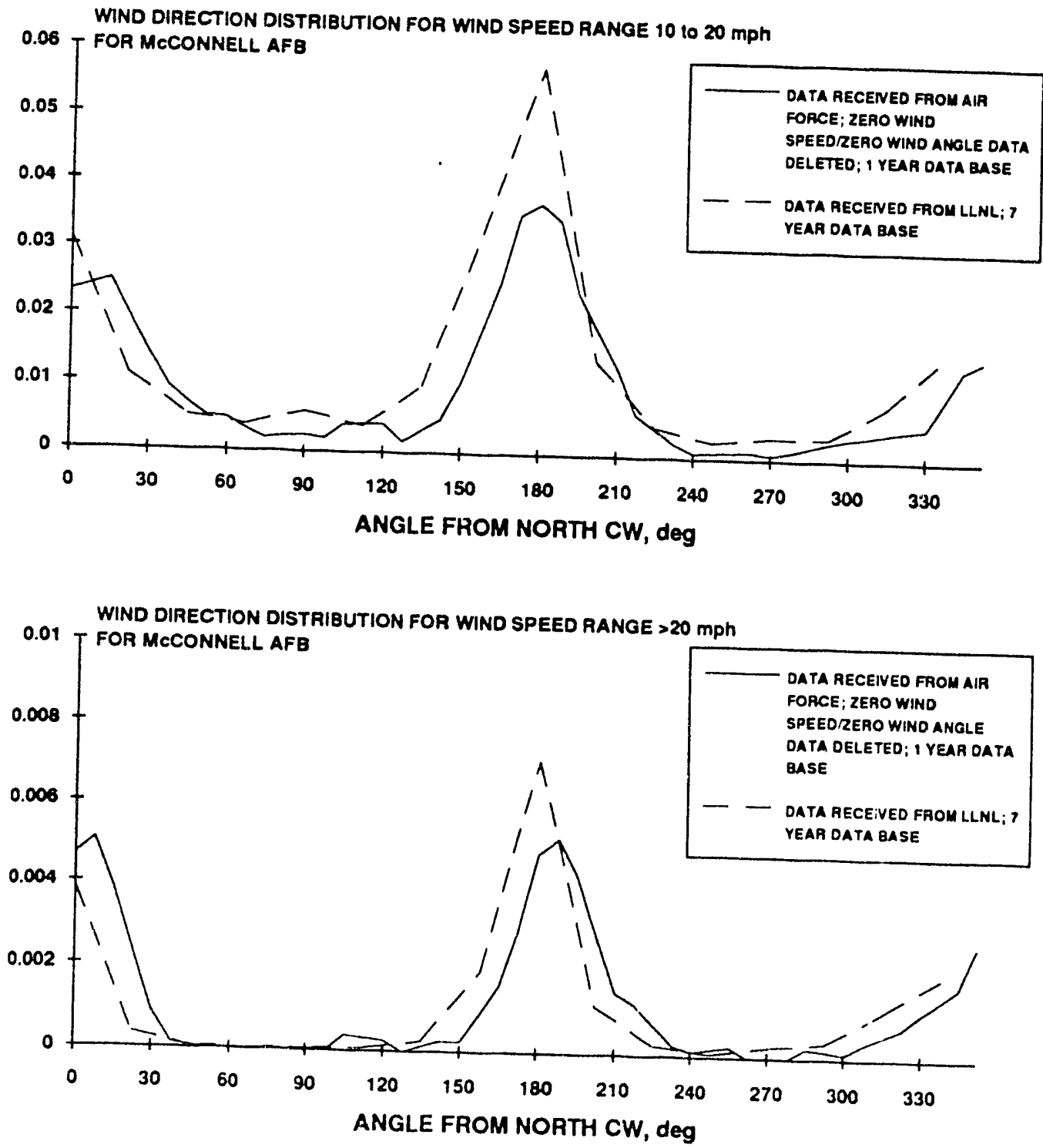

Figure C-6 Comparison Between Wind Data Obtained Throughout One-year and Seven-year Durations for McConnell AF Base 

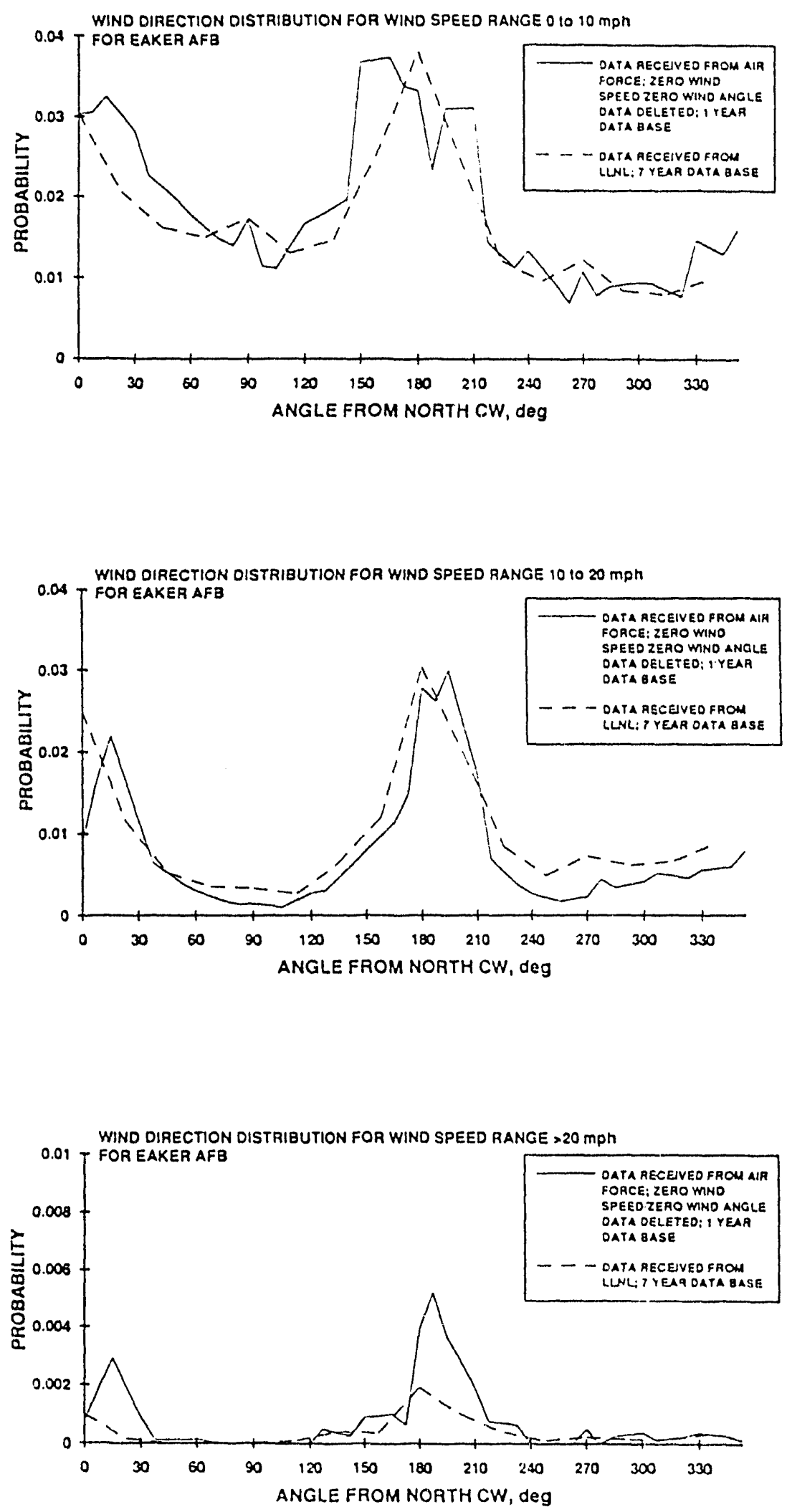

Figure C-7 Comparison Between Wind Data Obtained Throughout One-year and Seven-year Durations for Eaker AF Base 


\section{Appendix D FUEL SPILL LOCATION/RATE DATA}

A procedure to estimate the locations and rates of fuel spills that are likely to occur during landing and take-off collisions into the alert aircraft is described in Section 2. All data associated with these fuel spills are given in this appendix.

Figure D-1 shows the location of fuel tanks for the B-52 and the B-1B. For purposes of analysis, a distribution of potential fuel spill locations on the alert aircraft were designated on the basis of fuel tank locations; these discrete fuel spill locations, and a symbol associated with each location (R2, F4, etc.) are indicated in Figure D-2. The exact location of these discrete spill locations is given in terms of coordinate positions in what is called the aircraft coordinate system (a coordinate system attached to the alert aircraft) on Tables D-1 and D-2 for the B-52 and B-1B, respectively. In the aircraft coordinate system, the $Y$ axis is along the fuselage axis, with the coordinate origin 85 feet behind the forward tip of the fuselage (for both the B-52 and B-1B); the $X$ coordinates are positive to the right of the fuselage axis (right wing spill points have positive $X$ coordinate values), and $X$ coordinates are negative to the left of the fuselage axis.

Damage to and fuel spills from the aircraft that collides with the alert aircraft will also occur, and the colliding aircraft fuel spill locations must also be identified. These locations are identified in the coordinate system attached to the alert aircraft, the so-called aircraft coordinate system. A grid of potential spill coordinates in the aircraft coordinate system is designated, as illustrated in Figure D-3a. The spacing between grid points is 25 feet in the $X$ or $Y$ direction. This figure shows the $(x, y)$ coordinates for the grid of locations, and also a "C\#" designation. Figure D-3b shows, for example, the location of the B-52 in the aircraft coordinate system. 

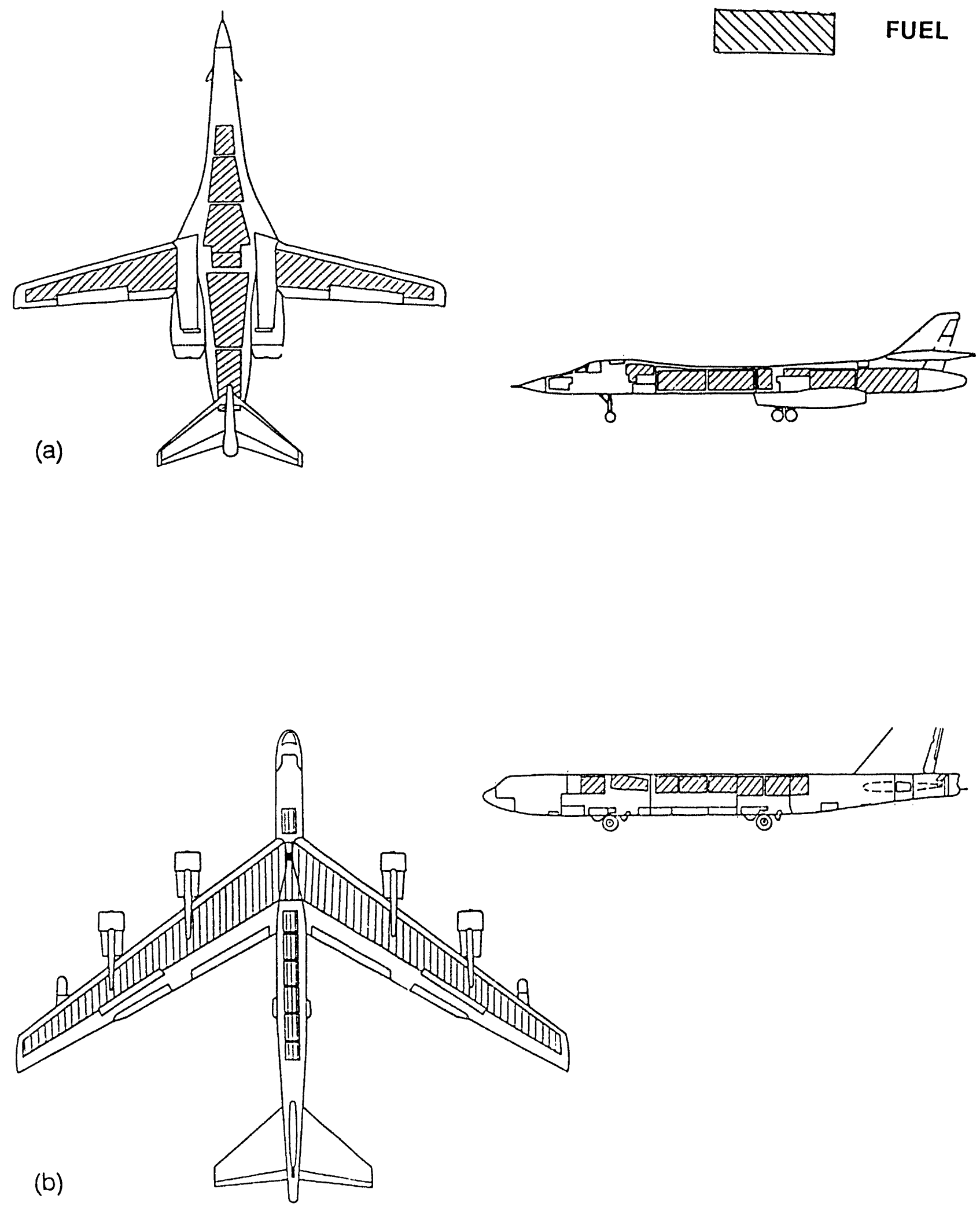

Figure D-1 Fuel Tank Locations in the Alert Aircraft 


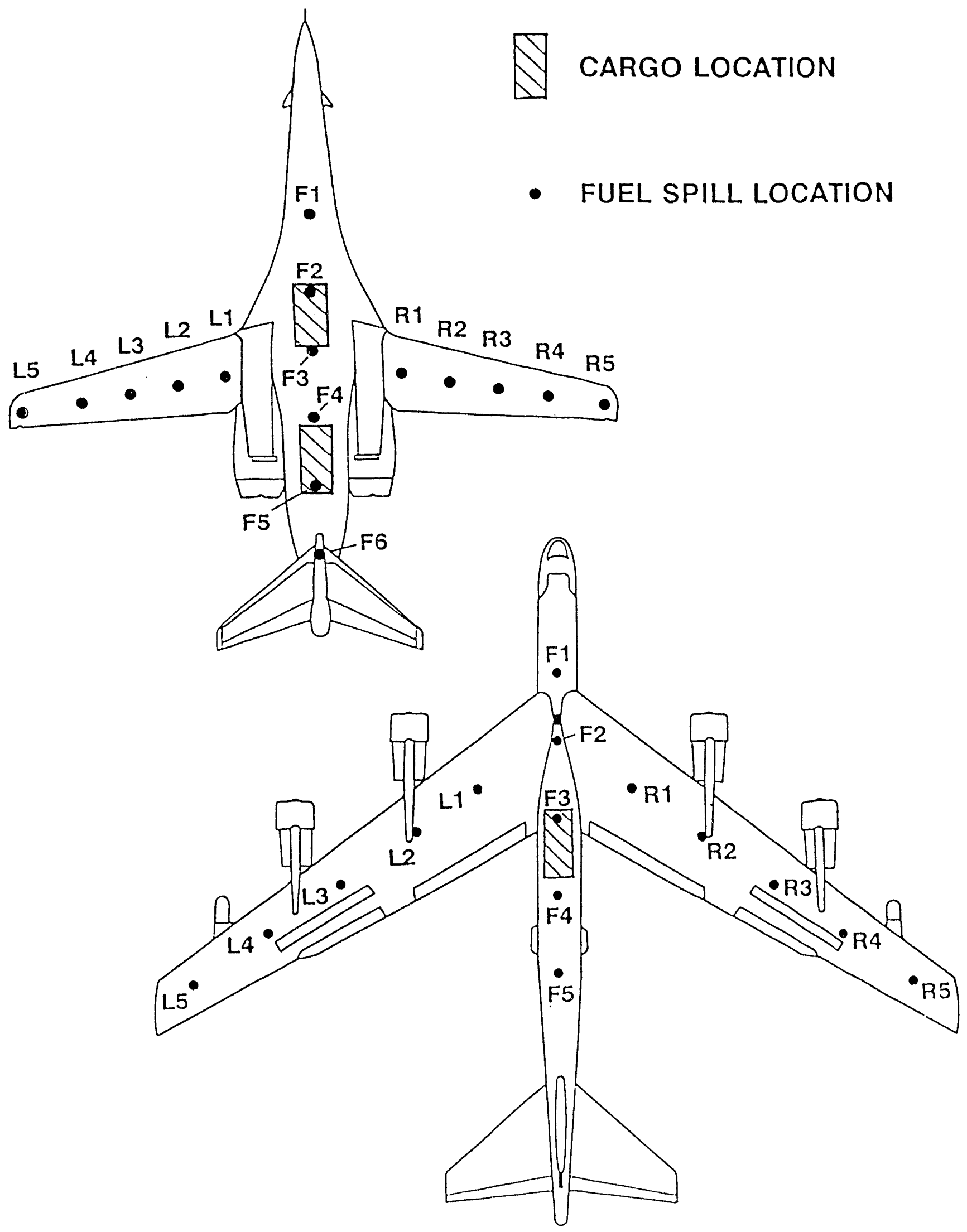

Figure D-2 Designated Fuel Spill Locations in the Alert Aircraft 
FUEL SPILL LOCATION DESIGNATIONS FOR AIRCRAFT

COLLIDING WITH ALERT AIRCRAFT

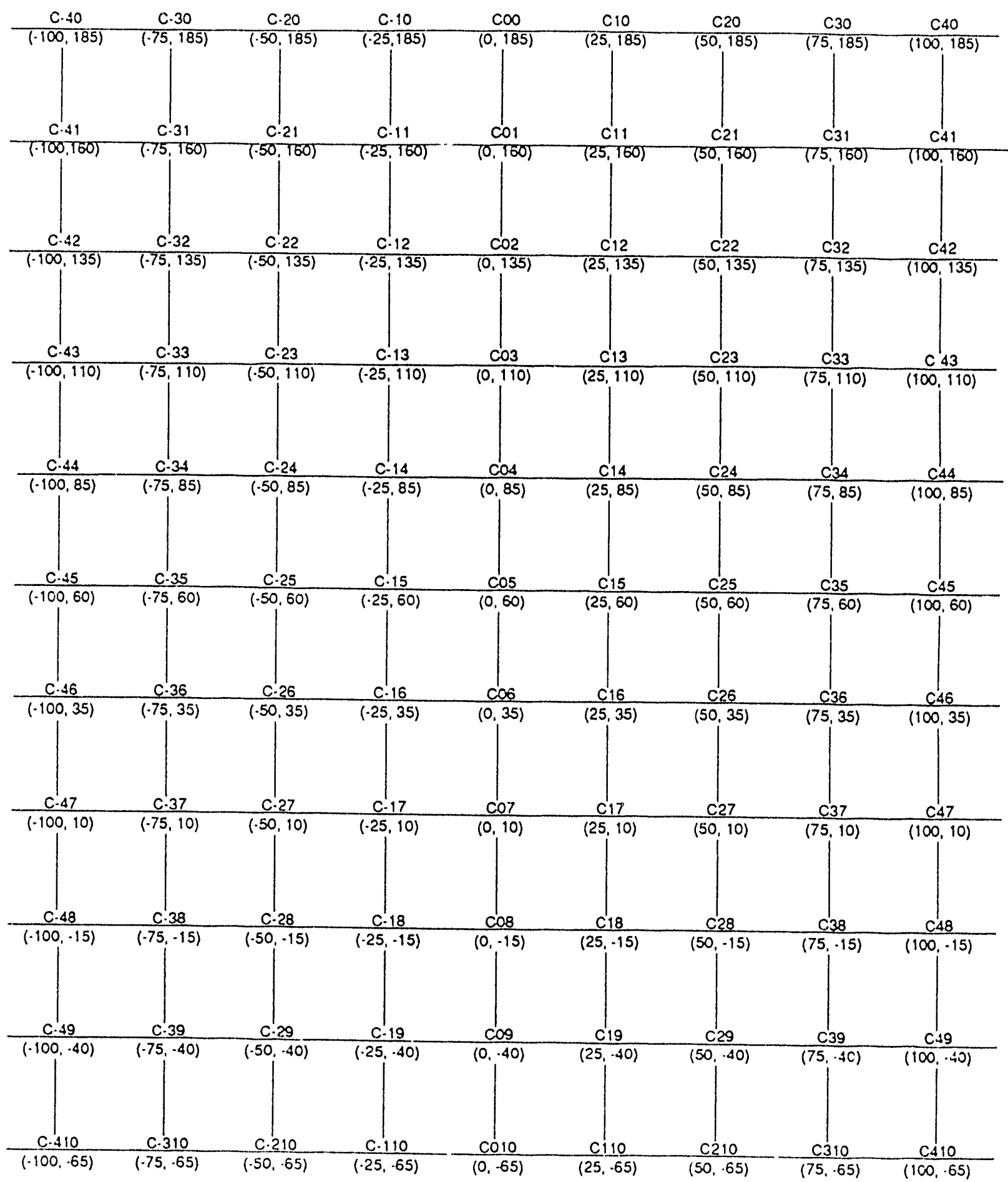

$(x, y)$ in Aircraft coordinate system

$\mathrm{C} \#$ \# location designation

Figure D-3a Fuel Spill Location Designation for the Aircraft that Collides with the Alert Aircraft 


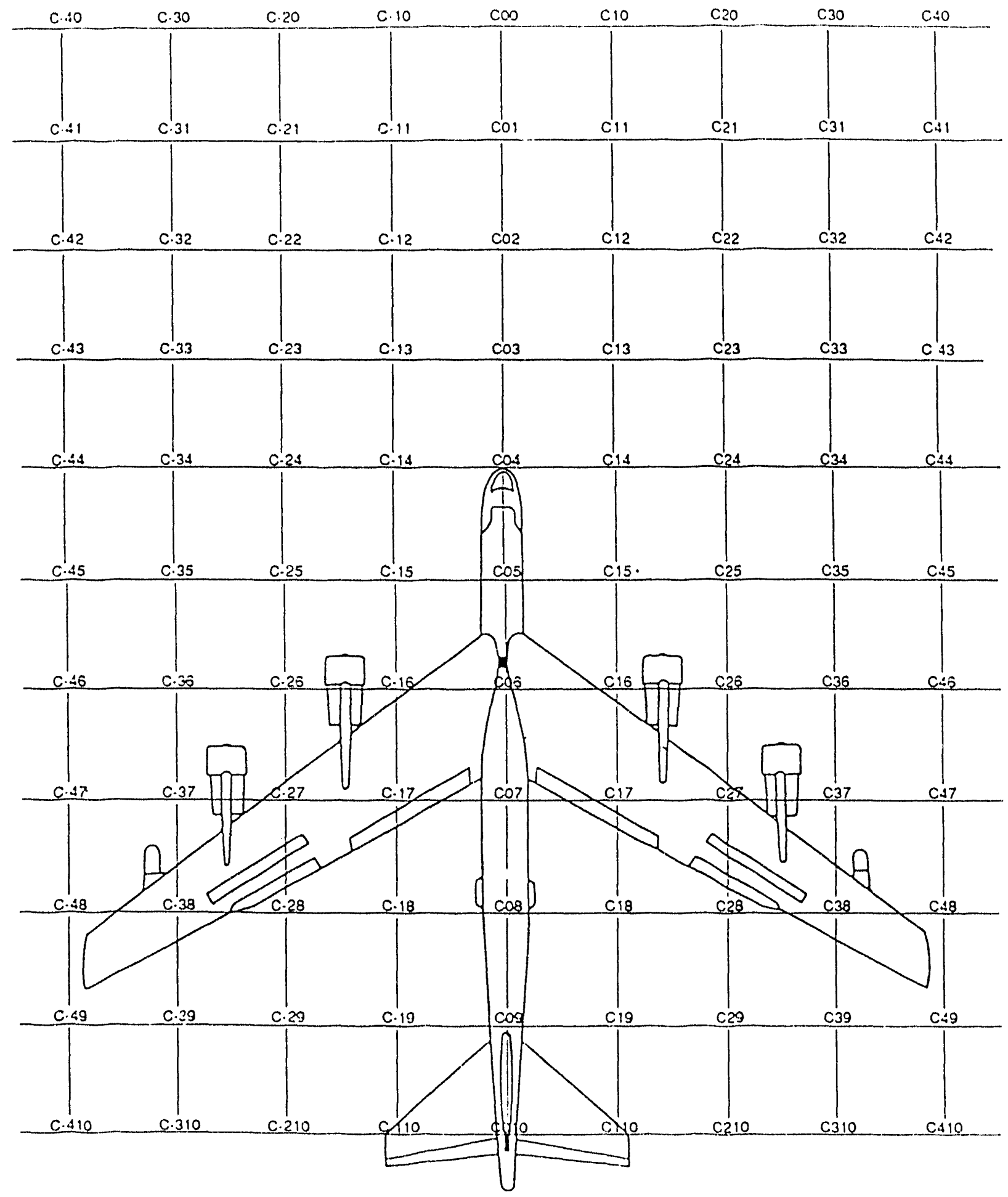

Figure D-3b Fuel Spill Location Designation for the Aircraft that Collides with the Alert Aircraft 
TABLE D-1. Fuel Spill Coordinate Locations for the B-52

FUEL SPILL LOCATION ON B-52

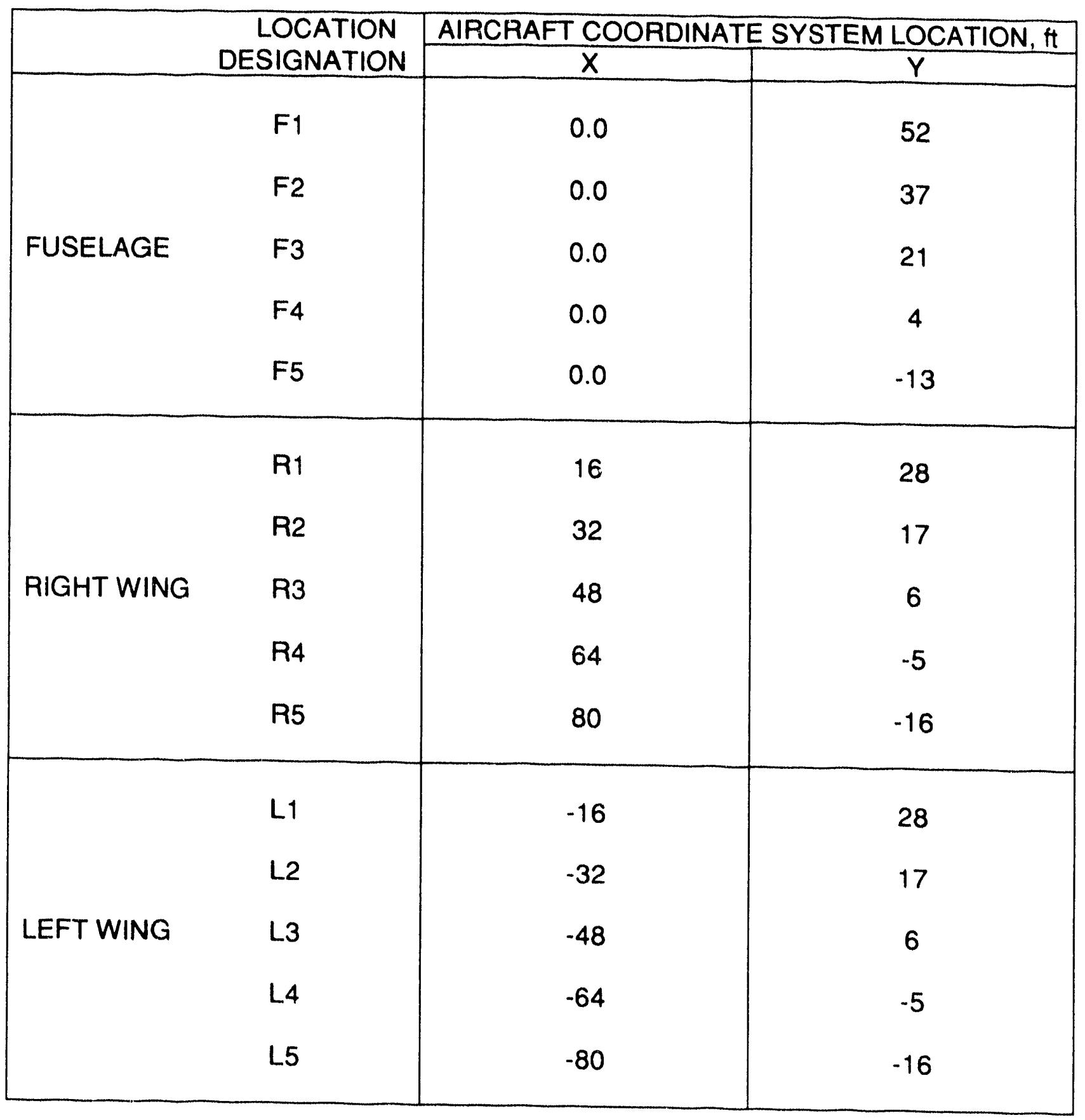


TABLE D-2. Fuel Spill Coordinate Locations for the B-1B

FUEL SPILL LOCATION ON B-1B

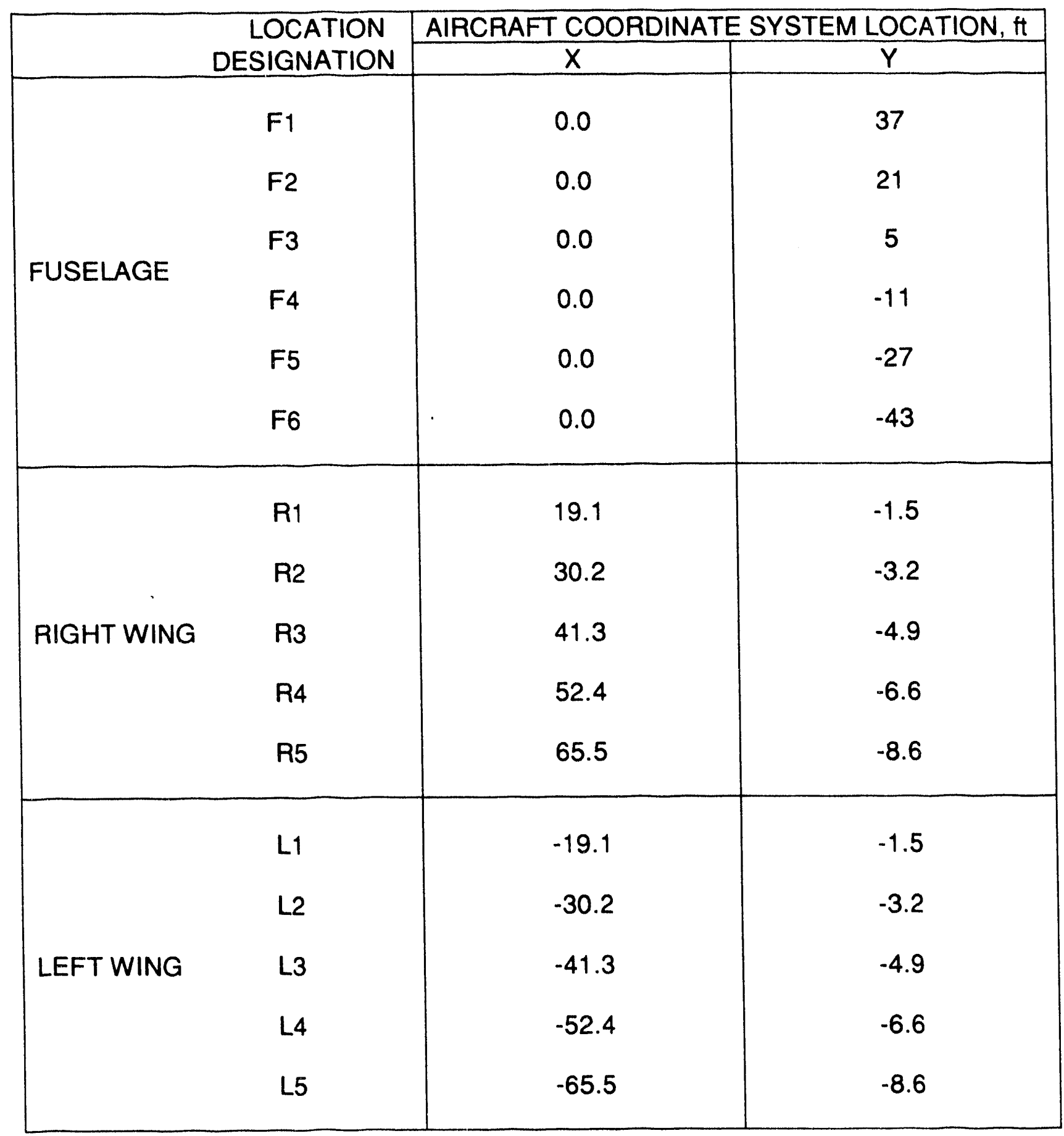


The procedure for evaluating the relative probability that fuel spills will occur at given locations and at given volumetric flow rates due to a collision of a moving aircraft (landing or taking-off) into an alert (parked) aircraft is described in Section 2. This probability distribution was evaluated for seven collision angles as illustrated in Figure D-4; specifically, distributions for collisions individually for the B-52 and B-1B were developed where the direction of motion of the colliding aircraft is in the direction

(a) that the alert aircraft is pointed (rear end collision),

(b) 45 degrees $\mathrm{CW}$ and $\mathrm{CCW}$ from the direction the alert aircraft is pointed (referred to as $45^{\circ}$ from rear),

(c) 90 degrees $\mathrm{CW}$ and $\mathrm{CCW}$ from the direction the alert aircraft is pointed (broadside collision), and

(d) 135 degrees $\mathrm{CW}$ and CCW from the direction the alert aircraft is pointed (referred to as $45^{\circ}$ from front).

For each of these collision angles, the location or locations and extent of fuel tank damage will depend in the relative lateral position of the colliding and alert aircraft; three distinct lateral positions are illustrated in Figure D-5, and the resulting fuel spill locations for both aircraft would be entirely different for the three positions. In evaluating the fuel spill location probability distribution, the lateral position at collision was separated into several "collision regions;" the broadside collision into the B-52, for example, has been separated into twelve collision regions as illustrated in Figure D-6. Figure D-6a shows specifically a broadside collision into Region 1, meaning that the left wing tip guide line of the colliding aircraft is within the dashed lines defining Region 1. Figure D-6b illustrates the broadside collision into Region 12. These collision regions were determined on the basis that the general aircraft response to collision and thereby the resulting fuel tank damage and spill locations for collision in a given region are different from that for other regions.

Figure D-7 shows the collision regions for all collision angles for the B-52, and similarly, Figure D-8 shows the collision regions for the same collision angles for collisions into the B-1B.

A concise listing of the fuel spill locations and rates and the corresponding relative probabilities of spills at these locations and rates that were determined by the procedure described in Section 2 is given in Tables D-3 and D-4 for the $\mathrm{B}-52$ and the B-1B, respectively. The first column in these tables indicates the collision region. The second column indicates the location of fuel spills in terms 


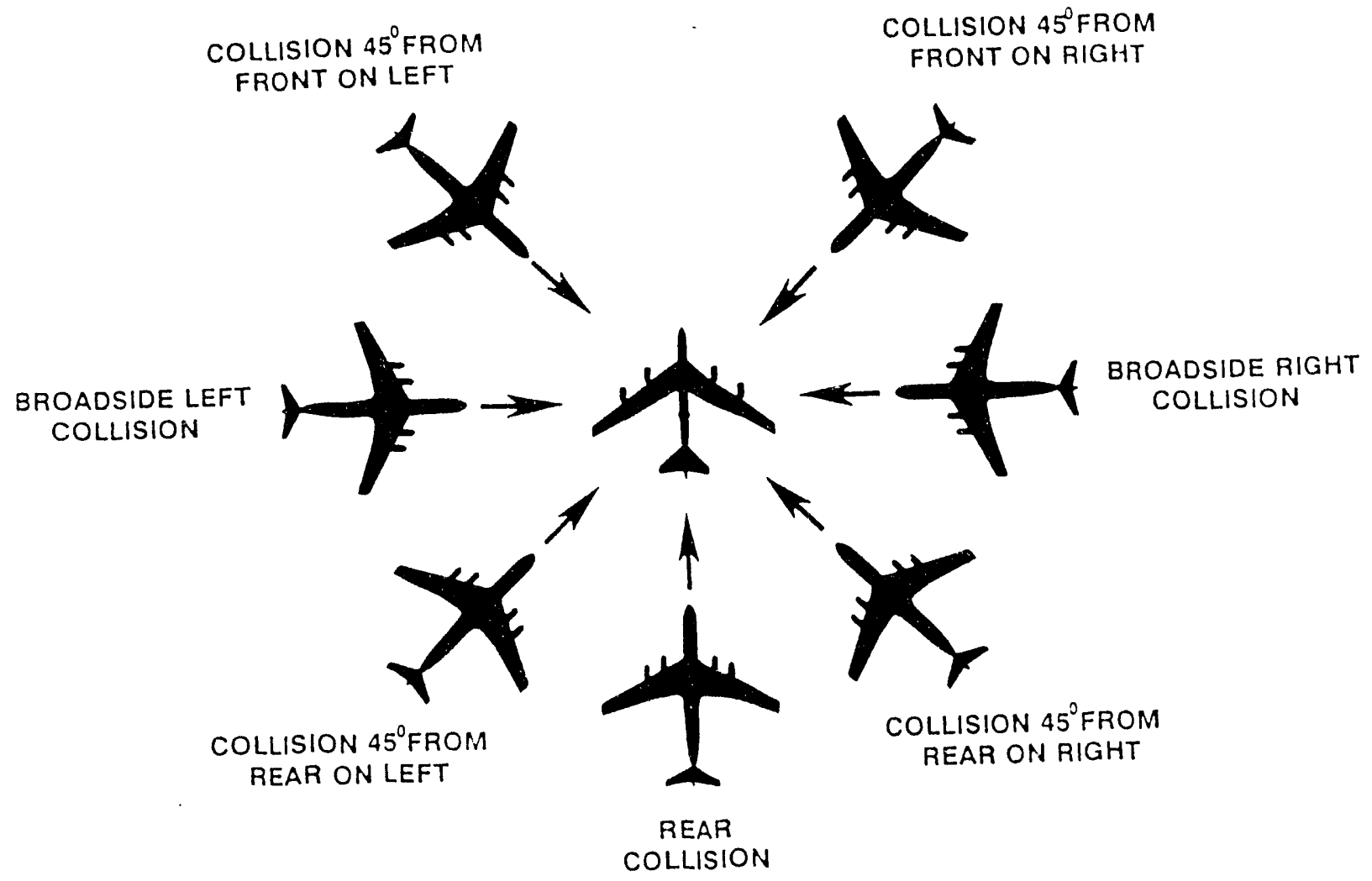

Figure D-4 Collision Angles Evaluated
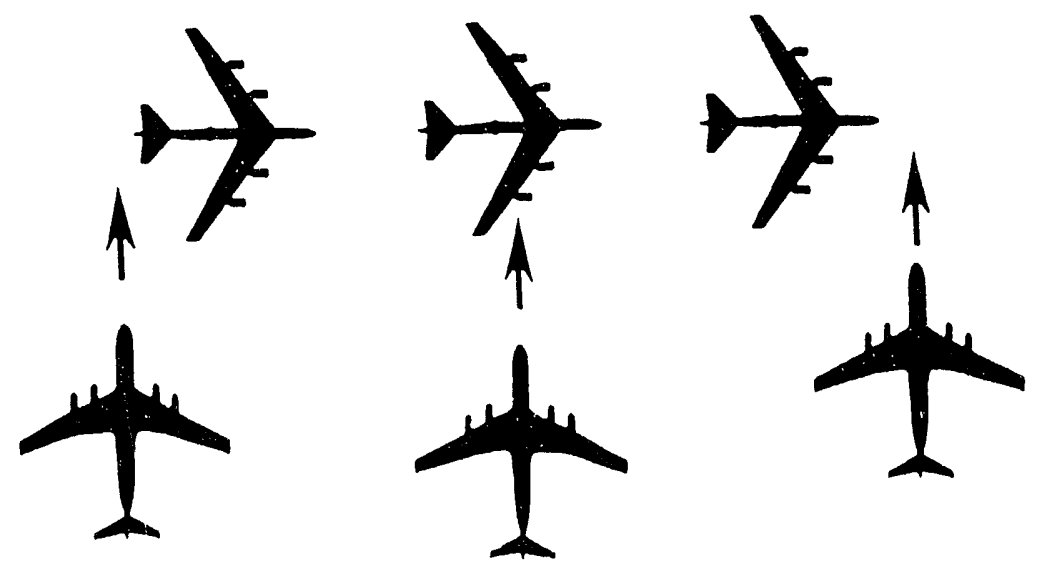

Figure D-5 Various Lateral Positions for Broadside Collision 


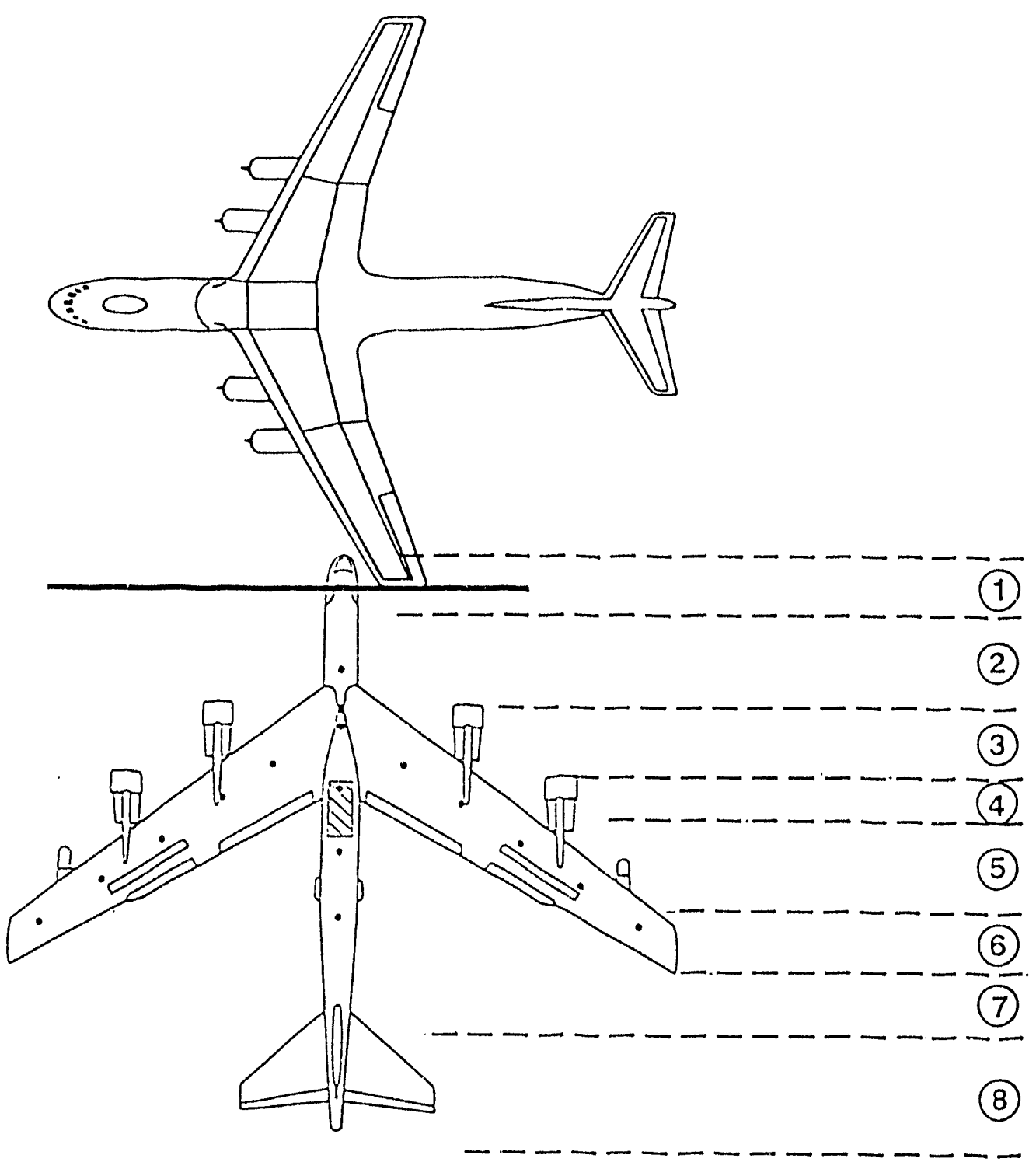

(9)

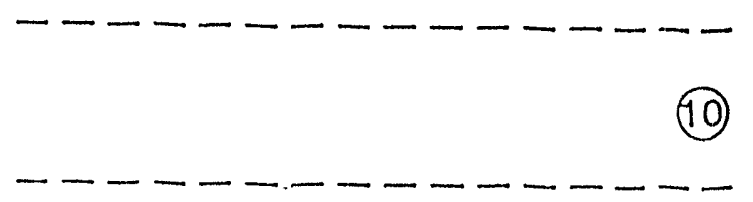

(11)

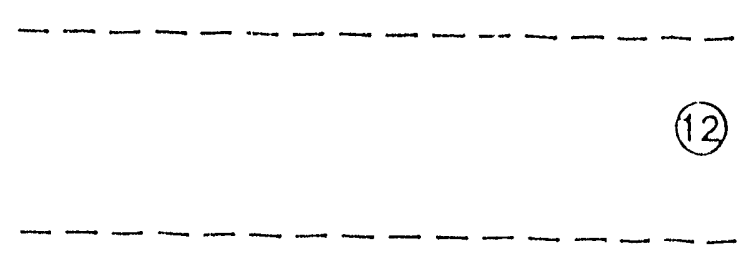

Figure D-6a Broadside Collision into B-52 at Region 1 


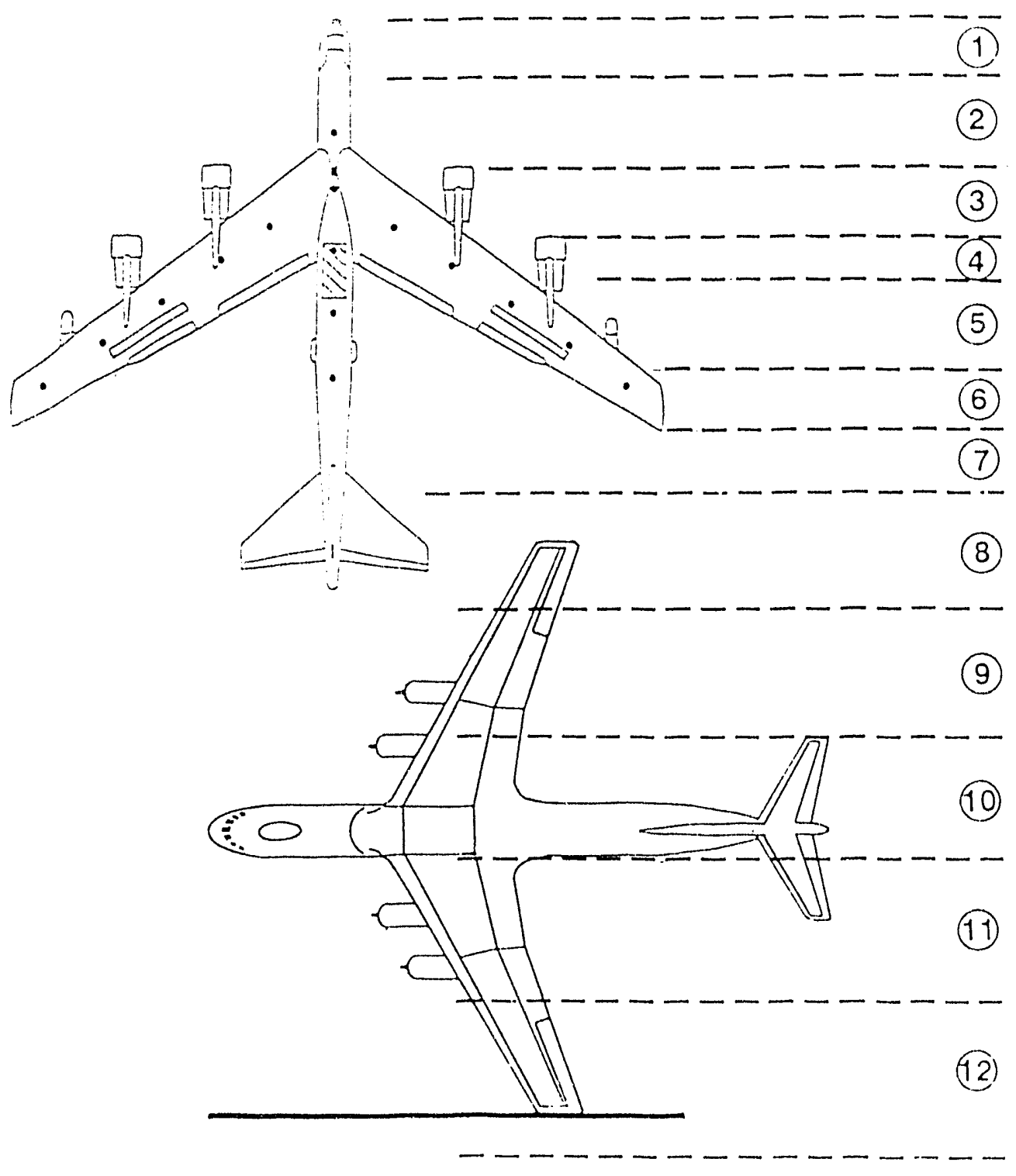

Figure D-6b Broadside Collision into B-52 at Region 12 


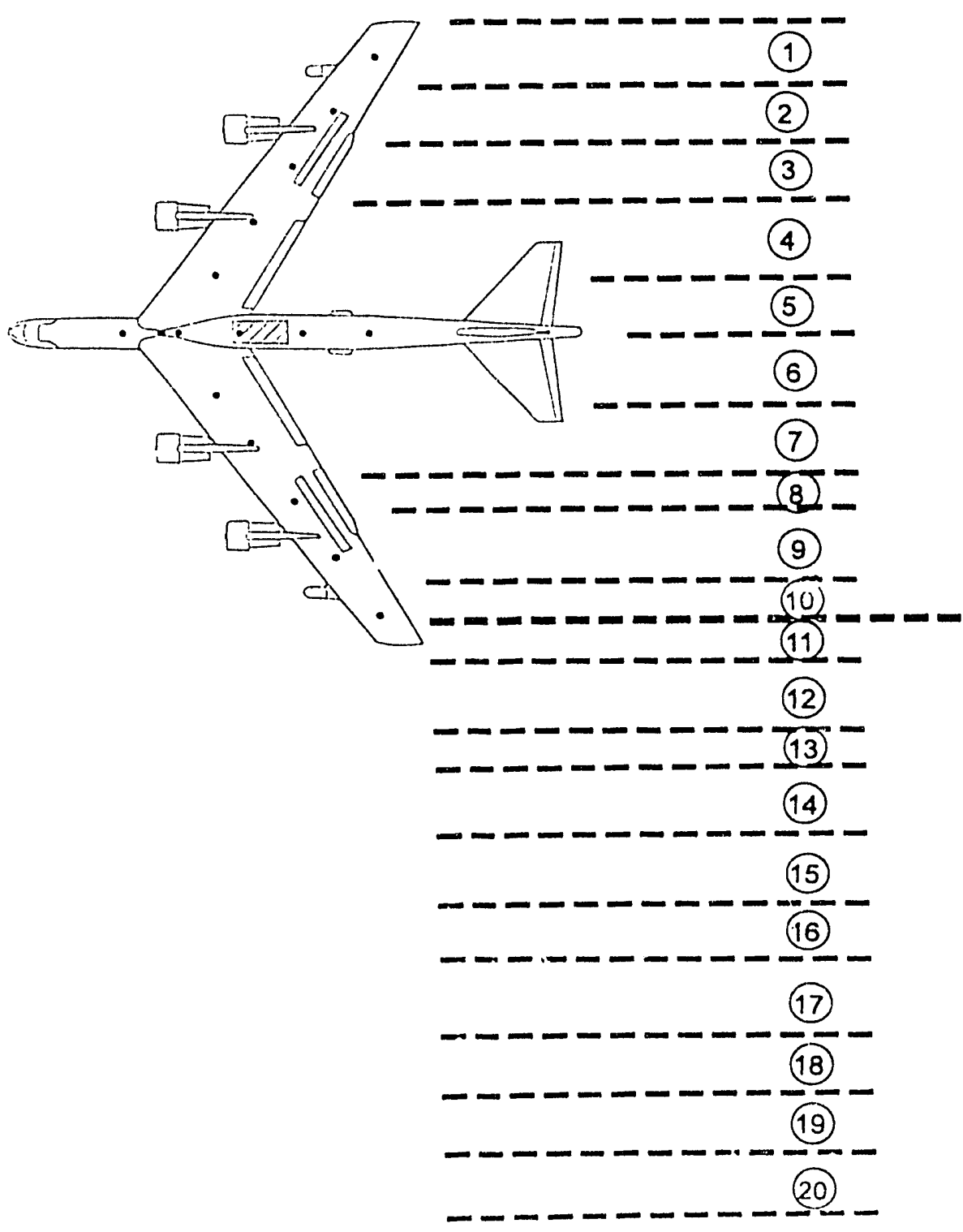

Figure D-7a Collision Regions for all Collision Angles into the B-52 


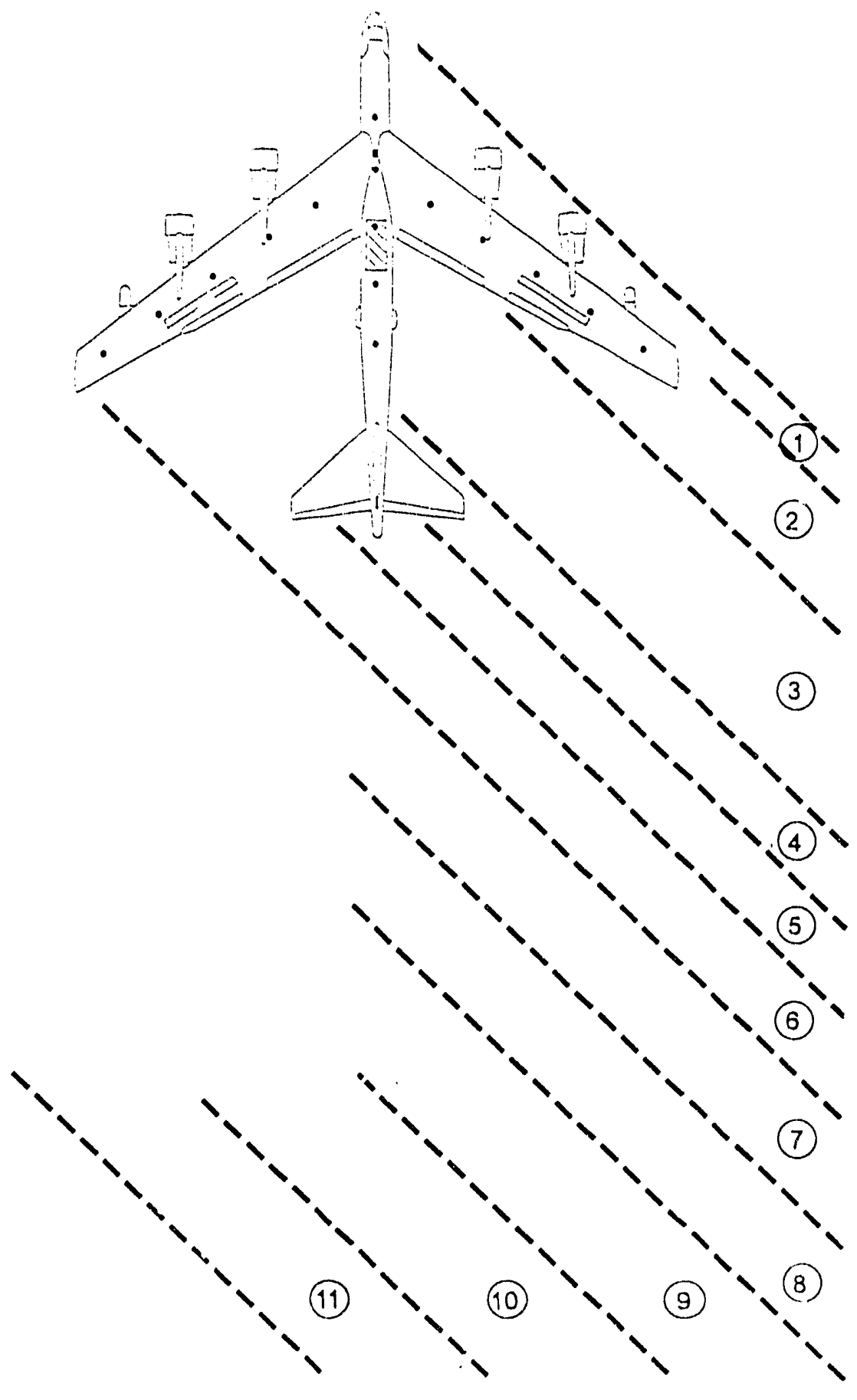

Figure D-7b Collision Regions for all Collision Angles into the B-52 


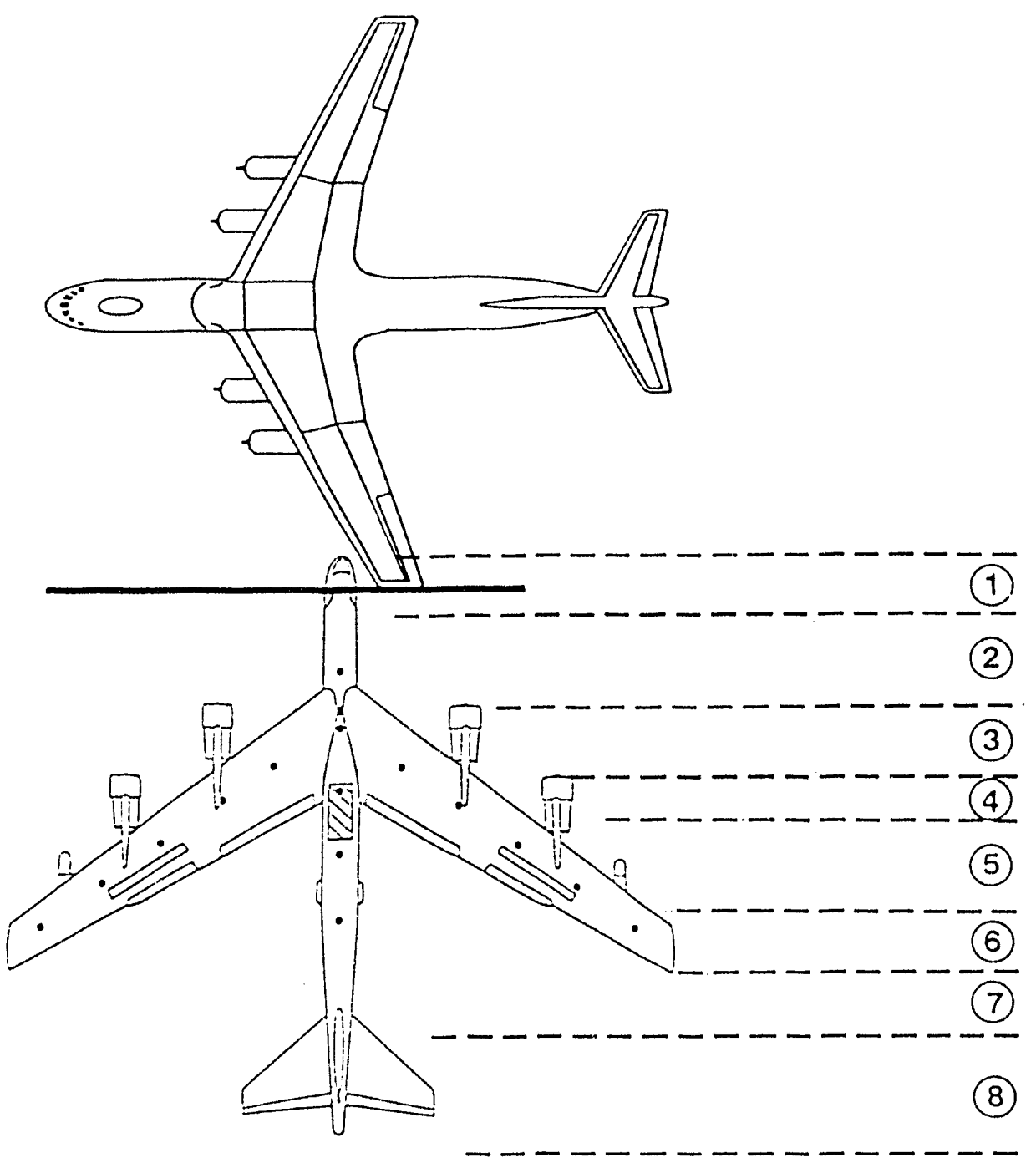

(9)

$-\cdots-\cdots-\cdots-\cdots-\cdots$

(10)

$---\ldots-\cdots-\cdots-$

$-\cdots-\cdots-\cdots-\cdots$

$-\cdots-\cdots-\cdots-\cdots$

Figure D-7c Collision Regions for all Collision Angles into the B-52 


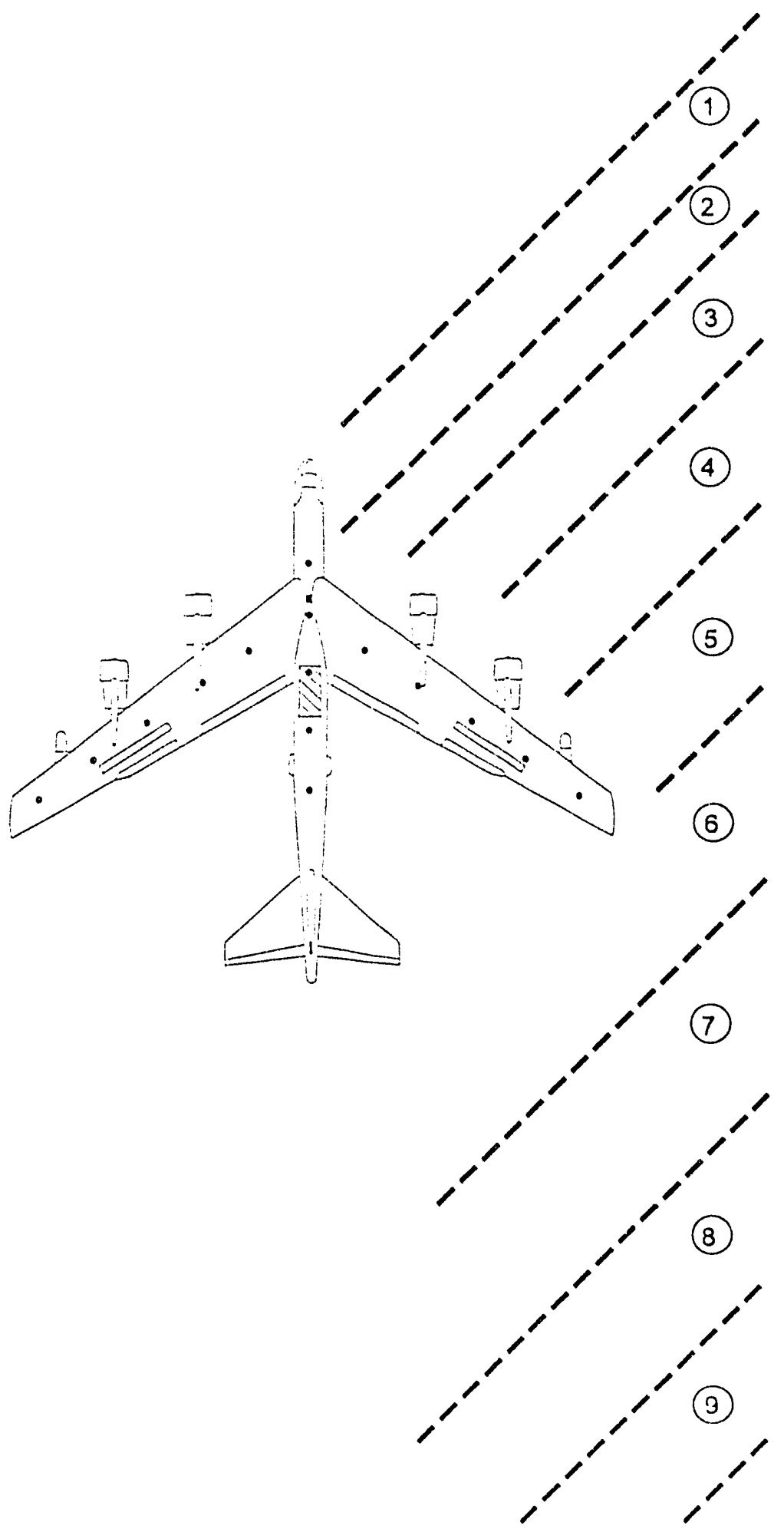

Figure D-7d Collision Regions for all Collision Angles into the B-52 


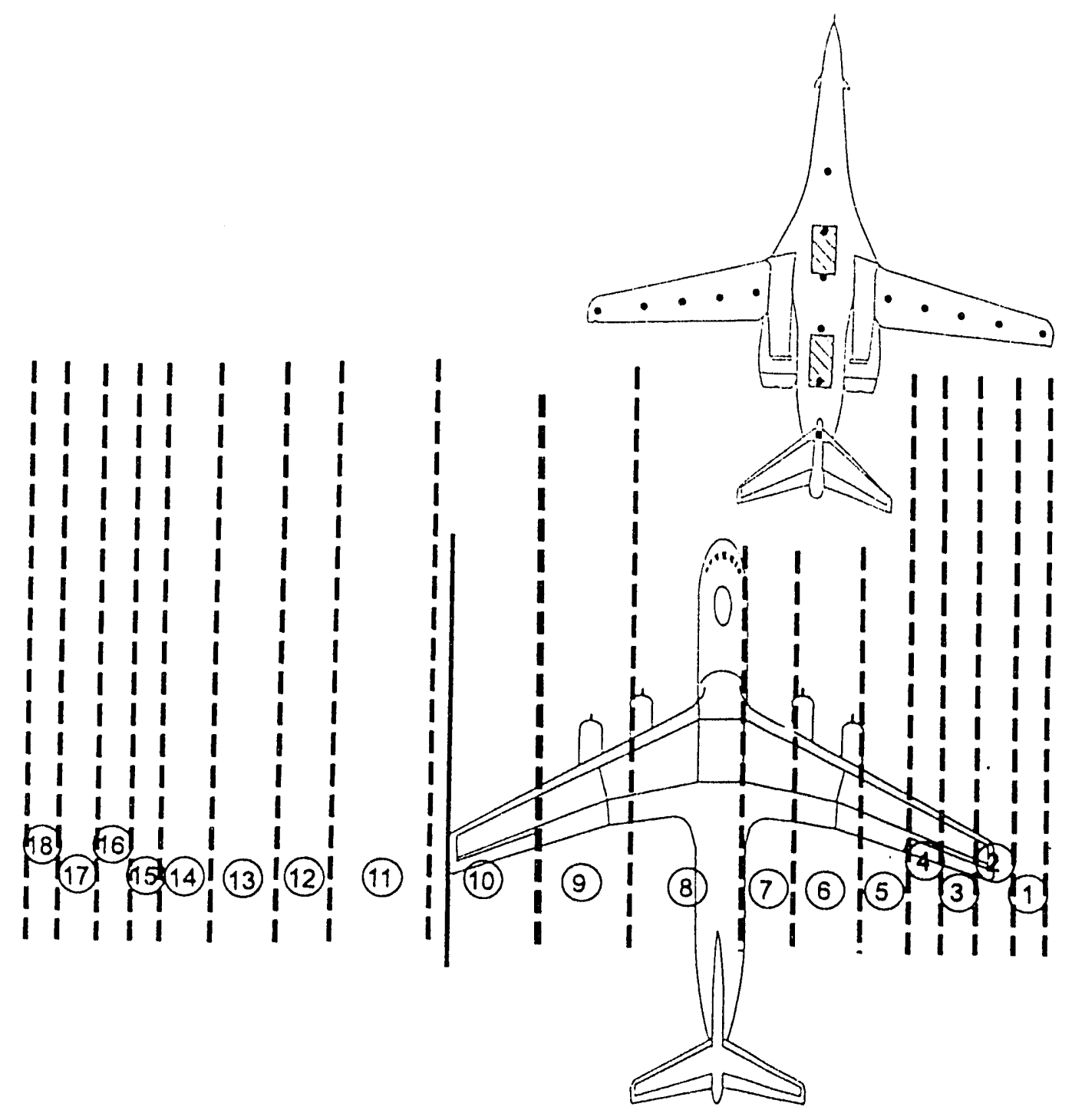

Figure D-8a Collision Regions for all Collision Angles into the B-1B 


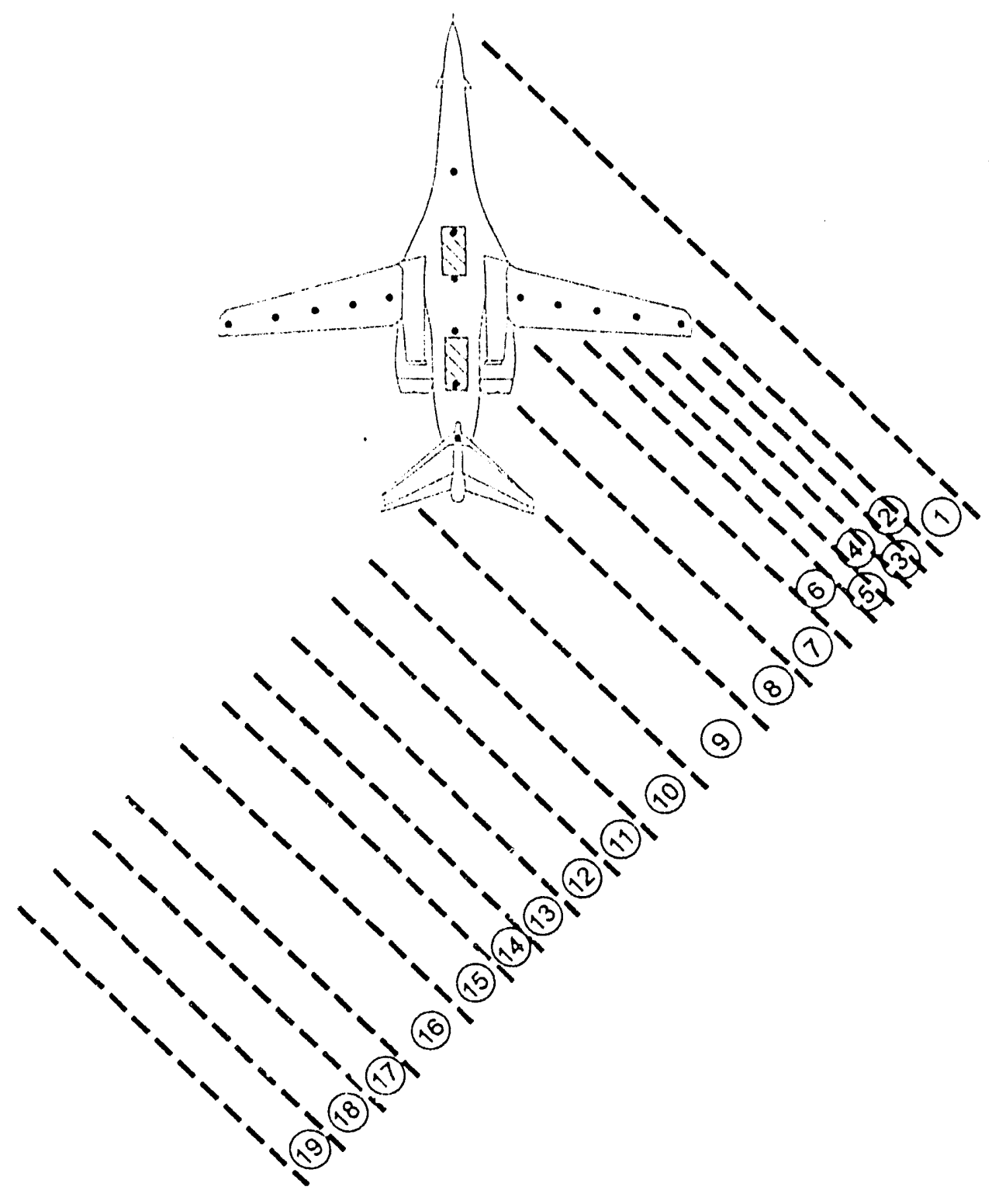

Figure D-8b Collision Regions for all Collision Angles into the B-1B 


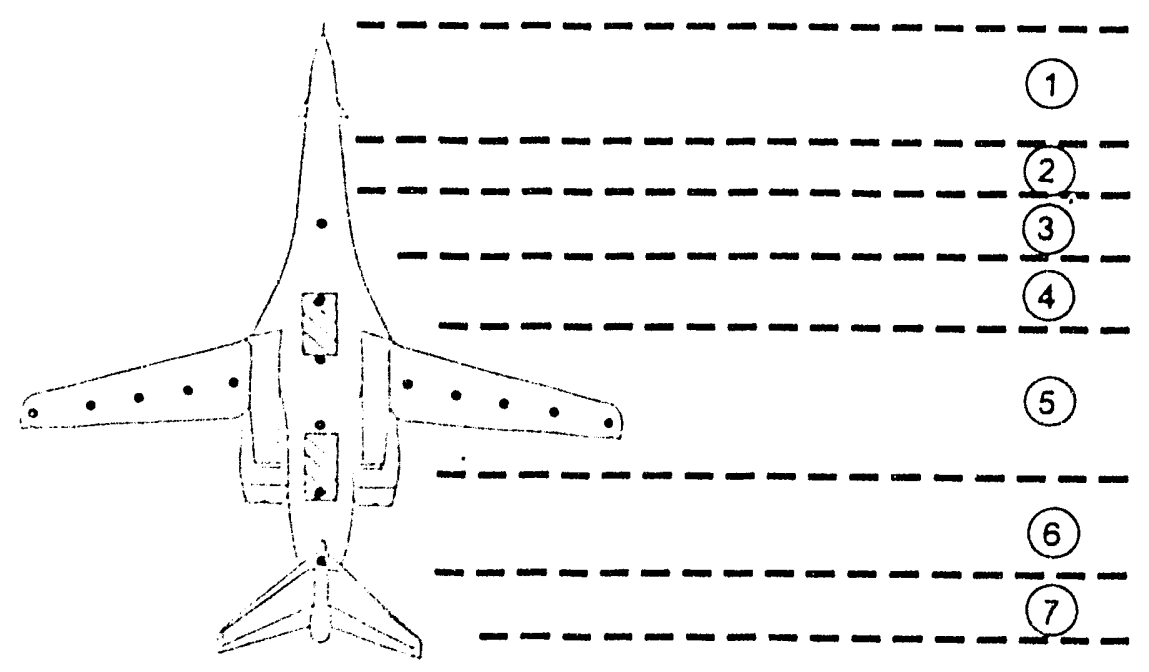

(8)
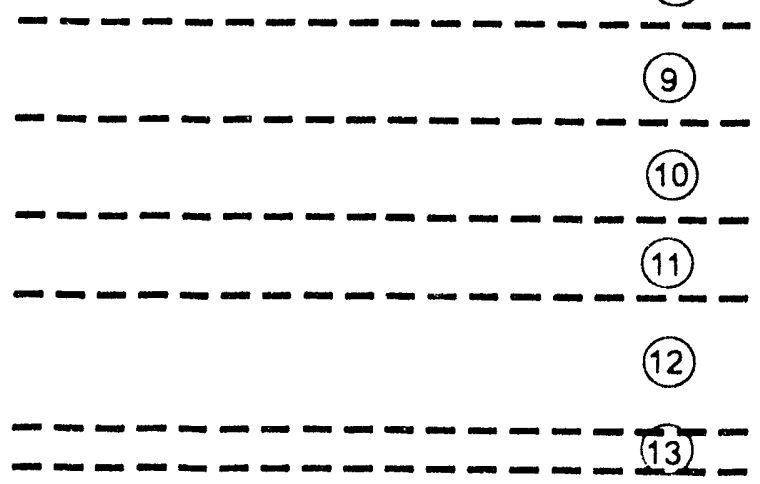

(14)

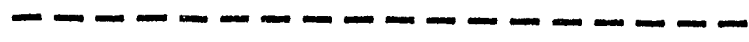

Figure D-8c Collision Regions for all Collision Angles into the B-1B 


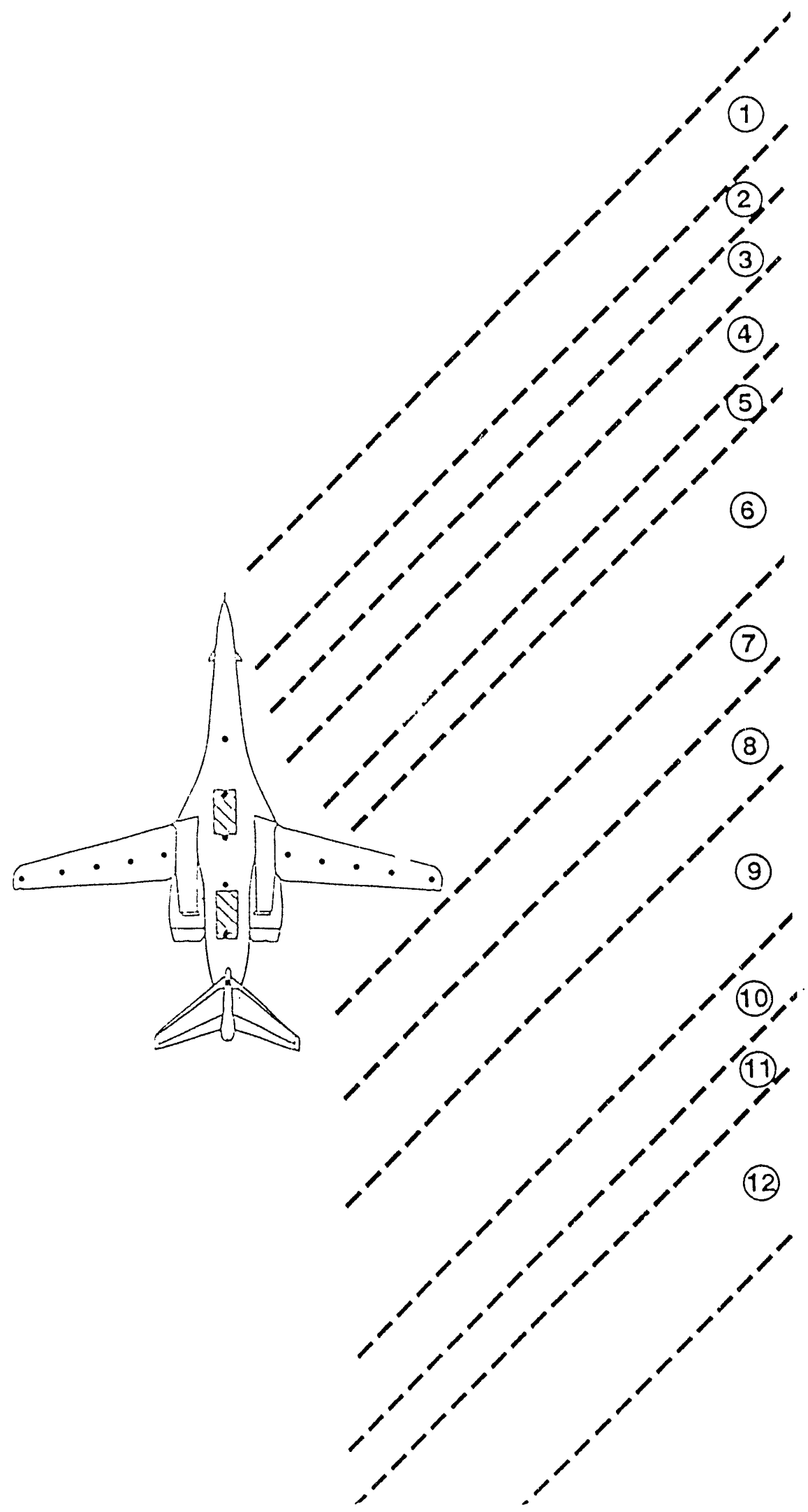

Figure D-8d Collision Regions for all Collision Angles into the B-15 
of the symbols designated on Figures D-2 and D-3. In column 2, the symbol "/" means "or," and the symbol " $X$ " means "and". The third column indicates the corresponding fuel spill rate category, where the categories 1,2 and 3 correspond, respectively, to the fuel spill rate ranges of zero to $225 \mathrm{gpm}, 225$ to $450 \mathrm{gpm}$, and greater than $450 \mathrm{gpm}$. The fourth column lists the relative probability for the spill location/rate combination.

Except for special cases, all spill probability distributions developed are given in Tables D-3 and D-4. Special cases not given include modifications of the distributions shown that were used in the uncertainty analysis and preliminary distributions that were generated during the procedure development process. 


\section{TABLE D-3a. FUEL SPILL LOCATIONS, RATES AND PROBABILITIES FOR DIRECT REAR COLLISION INTO B-52}

\section{A. COLLISION IN HIGH SPEED IMPACT RANGE}

\begin{tabular}{cl}
$\begin{array}{c}\text { COLLISION } \\
\text { REGION }\end{array}$ & \multicolumn{1}{c}{ FUEL SPILL LOCATIONS } \\
& \\
1 & $\mathrm{R} 5$ \\
2 & $\mathrm{R} 4 / \mathrm{R} 5$ \\
3 & $\mathrm{R} 3 / \mathrm{R} 4 / \mathrm{R} 5$ \\
4 & $\mathrm{R} 2 / \mathrm{R} 3 \times \mathrm{R} 4 / \mathrm{R} 5 \times \mathrm{C} 30 / \mathrm{C} 40 / \mathrm{C} 31 / \mathrm{C} 41$ \\
5 & $\mathrm{R} 1 / \mathrm{R} 2 / \mathrm{R} 3 / \mathrm{R} 4 \times \mathrm{R} 5 \times \mathrm{C} 31 / \mathrm{C} 21 / \mathrm{C} 12 / \mathrm{C} 22$ \\
6 & $\mathrm{R} 4 / \mathrm{R} 5 \times \mathrm{R} 1 / \mathrm{R} 2 / \mathrm{R} 3 \times$ \\
& $\mathrm{C} 25 / \mathrm{C} 26 / \mathrm{C} 27 / \mathrm{C} 24 / \mathrm{C} 16 / \mathrm{C} 14 / \mathrm{C} 15 / \mathrm{C} 23$ \\
7 & $\mathrm{R} 2 / \mathrm{R} 3 / \mathrm{R} 4 \times \mathrm{F} 4 / \mathrm{F} 5 \times \mathrm{C} 16 / \mathrm{C} 26 / \mathrm{C} 15$ \\
8 & $\mathrm{~F} 3 / \mathrm{F} 4 \times \mathrm{R} 1 / \mathrm{R} 2 \times \mathrm{R} 3 / \mathrm{R} 4 / \mathrm{R} 5 \times \mathrm{C} 19 / \mathrm{C} 110 / \mathrm{C} 111$ \\
9 & $\mathrm{~F} 2 / \mathrm{F} 3 / \mathrm{F} 4 / \mathrm{F} 5 \times \mathrm{R} 1 / \mathrm{R} 2 \times \mathrm{R} 3 / \mathrm{R} 4 \times \mathrm{L} 1 / \mathrm{L} 2$ \\
10 & $\mathrm{~F} 2 / \mathrm{F} 3 / \mathrm{F} 4 / \mathrm{F} 5 \times \mathrm{R} 1 / \mathrm{R} 2 / \mathrm{R} 3 \times \mathrm{L} 1 / \mathrm{L} 2 \times \mathrm{C}-19$ \\
11 & $\mathrm{~F} 2 / \mathrm{F} 3 / \mathrm{F} 4 / \mathrm{F} 5 \times \mathrm{L} 1 / \mathrm{L} 2 / \mathrm{L} 3 \times \mathrm{L} 1 / \mathrm{L} 2 \times \mathrm{C} 19$ \\
12 & $\mathrm{~F} 2 / \mathrm{F} 3 / \mathrm{F} 4 / \mathrm{F} 5 \times \mathrm{L} 1 / \mathrm{L} 2 \times \mathrm{L} 3 / \mathrm{L} 4 \times \mathrm{L} 1 / \mathrm{L} 2$ \\
13 & $\mathrm{~F} 3 / \mathrm{F} 4 \times \mathrm{L} 1 / \mathrm{L} 2 \times \mathrm{L} 3 / \mathrm{L} 4 / \mathrm{L} 5 \times \mathrm{C} 19 / \mathrm{C}-110 / \mathrm{C}-111$ \\
14 & $\mathrm{~L} 2 / \mathrm{L} 3 / \mathrm{L} 4 \times \mathrm{F} 4 / \mathrm{F} 5 \times \mathrm{C}-16 / \mathrm{C}-26 / \mathrm{C}-15$ \\
15 & $\mathrm{~L} 4 / \mathrm{L} 5 \times \mathrm{L} 1 / \mathrm{L} 2 / \mathrm{L} 3 \times \mathrm{C}$ \\
& $\mathrm{C}-25 / \mathrm{C}-26 / \mathrm{C}-27 / \mathrm{C}-24 / \mathrm{C}-16 / \mathrm{C}-14 / \mathrm{C}-15 / \mathrm{C}-23$ \\
16 & $\mathrm{~L} 1 / \mathrm{L} 2 / \mathrm{L} 3 / \mathrm{L} 4 \times \mathrm{L} 5 \times \mathrm{C}-31 / \mathrm{C}-21 / \mathrm{C}-12 / \mathrm{C}-22$ \\
17 & $\mathrm{~L} 2 / \mathrm{L} 3 \times \mathrm{L} 4 / \mathrm{L} 5 \times \mathrm{C}-30 / \mathrm{C}-40 / \mathrm{C}-31 / \mathrm{C}-41$ \\
18 & $\mathrm{~L} 3 / \mathrm{L} 4 / \mathrm{L} 5$ \\
19 & $\mathrm{~L} 4 / \mathrm{L} 5$ \\
20 & $\mathrm{~L} 5$
\end{tabular}
FUEL SPILL RATE CATEGORIES

\author{
RELATIVE \\ PROBABILITY
}

$\begin{array}{cc}1 & .026882 \\ 1 & .024194 \\ 1 & .024194 \\ 1 \times 2 \times 2 & .032258 \\ 1 \times 3 \times 1 & .024194 \\ 3 \times 2 \times 2 & .028226 \\ 3 \times 1 \times 3 & .028226 \\ 2 \times 3 \times 1 \times 2 & .014785 \\ 3 \times 3 \times 1 \times 3 & .029570 \\ 2 \times 3 \times 1 \times 3 & .017473 \\ 2 \times 3 \times 1 \times 3 & .017473 \\ 3 \times 3 \times 1 \times 3 & .029570 \\ 2 \times 3 \times 1 \times 2 & .014785 \\ 3 \times 1 \times 3 & .028226 \\ 3 \times 2 \times 2 & .028226 \\ 1 \times 3 \times 1 & .024194 \\ 1 \times 2 \times 2 & .032258 \\ 1 & .024194 \\ 1 & .024194 \\ 1 & .026882\end{array}$

\section{B. COLLISION IN MEDIUM SPEED IMPACT RANGE}

$\begin{array}{cl}1 & \mathrm{R} 5 \times \mathrm{C} 45 / \mathrm{C} 44 / \mathrm{C} 43 / \mathrm{C} 42 \\ 2 & \mathrm{R} 4 / \mathrm{R} 5 \times \mathrm{C} 35 / \mathrm{C} 34 / \mathrm{C} 33 / \mathrm{C} 45 / \mathrm{C} 44 / \mathrm{C} 43 \\ 3 & \mathrm{R} 3 / \mathrm{R} 4 / \mathrm{R} 5 \times \mathrm{C} 35 / \mathrm{C} 34 / \mathrm{C} 44 / \mathrm{C} 43 / \mathrm{C} 33 / \mathrm{C} 42 \\ 4 & \mathrm{R} 4 / \mathrm{R} 5 \times \mathrm{R} 2 / \mathrm{R} 3 \times \mathrm{C} 37 / \mathrm{C} 36 / \mathrm{C} 35 / \mathrm{C} 34 / \mathrm{C} 27 \\ 5 & \mathrm{R} 2 / \mathrm{R} 3 / \mathrm{R} 4 / \mathrm{R} 5 \times \mathrm{C} 26 / \mathrm{C} 27 / \mathrm{C} 35 / \mathrm{C} 36 / \mathrm{C} 37 \\ 6 & \mathrm{R} 2 / \mathrm{R} 3 \times \mathrm{R} 4 / \mathrm{R} 5 \times \mathrm{C} 26 / \mathrm{C} 27 \\ 7 & \mathrm{R} 2 / \mathrm{R} 3 / \mathrm{R} 4 \times \mathrm{C} 19 / \mathrm{C} 110 / \mathrm{C} 26 / \mathrm{C} 27 \\ 8 & \mathrm{~F} 2 / \mathrm{F} 3 \times \mathrm{R} 1 / \mathrm{R} 2 \times \mathrm{R} 3 / \mathrm{R} 4 \times \mathrm{C} 011 / \mathrm{C} 110 / \mathrm{C} 11 \\ 9 & \mathrm{~F} 3 / \mathrm{F} 4 / \mathrm{F} 5 \times \mathrm{R} 1 / \mathrm{R} 2 \times \mathrm{R} 3 / \mathrm{R} 4 \times \mathrm{C}-19 \\ 10 & \mathrm{~F} 3 \times \mathrm{F} 4 / \mathrm{F} 5 \times \mathrm{R} 1 / \mathrm{R} 2 \times \mathrm{R} 3 / \mathrm{R} 4 \\ 11 & \mathrm{~F} 3 \times \mathrm{F} 4 / \mathrm{F} 5 \times \mathrm{L} 1 / \mathrm{L} 2 \times \mathrm{L} 3 / \mathrm{L} 4\end{array}$

$\begin{array}{cc}1 \times 2 & .013441 \\ 1 \times 1 & .012097 \\ 1 \times 1 & .012097 \\ 1 \times 1 & .016129 \\ 2 \times 1 & .012097 \\ 3 \times 2 \times 2 & .014133 \\ 2 \times 2 & .014133 \\ 1 \times 2 \times 1 \times 2 & .007392 \\ 3 \times 3 \times 1 \times 3 & .014785 \\ 2 \times 3 \times 3 \times 1 & .008737 \\ 2 \times 3 \times 3 \times 1 & .008737\end{array}$




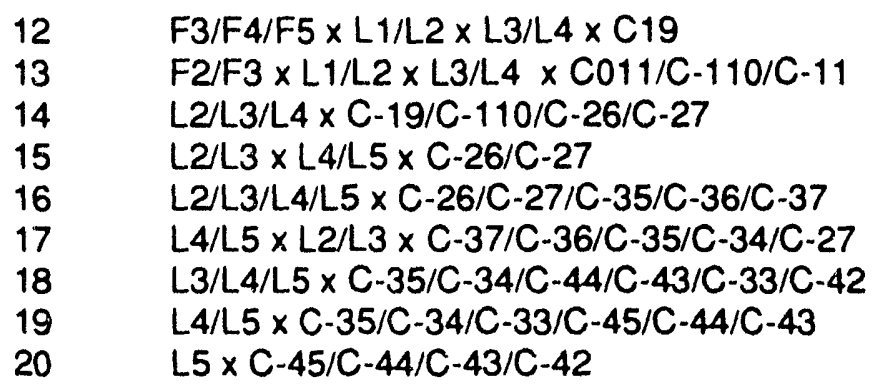

\section{COLLISION IN LOW SPEED IMPACT RANGE}

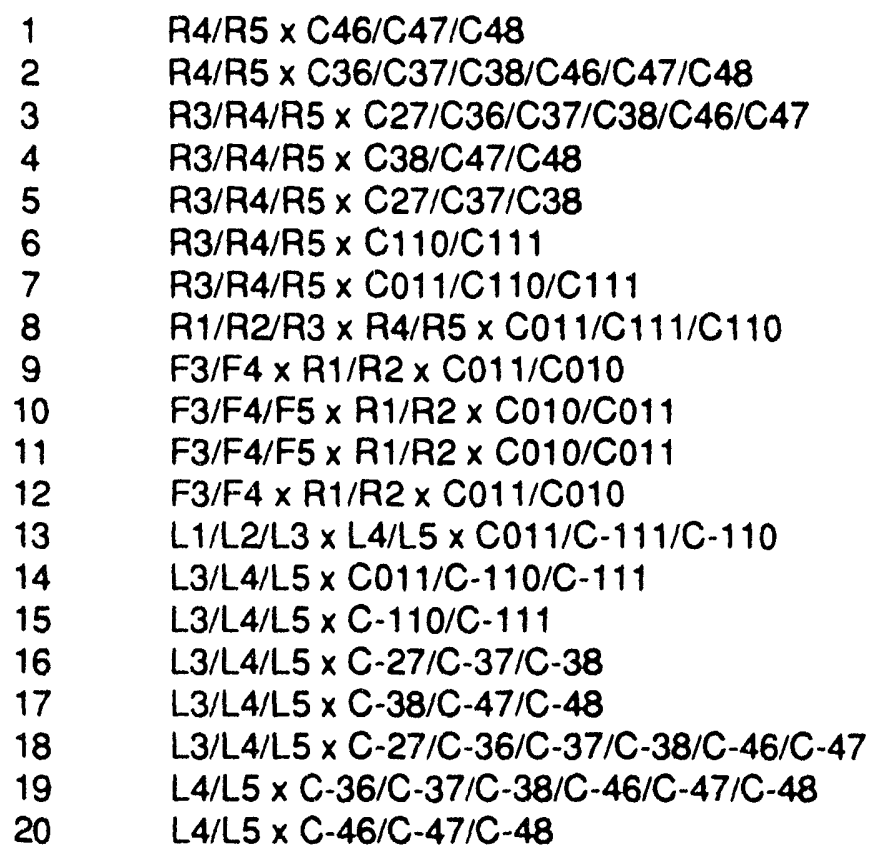

$\begin{array}{cc}3 \times 3 \times 1 \times 3 & .014785 \\ 1 \times 2 \times 1 \times 2 & .007392 \\ 2 \times 2 & .014133 \\ 3 \times 2 \times 2 & .014133 \\ 2 \times 1 & .012097 \\ 1 \times 1 & .016129 \\ 1 \times 1 & .012097 \\ 1 \times 1 & .012097 \\ 1 \times 2 & .013441\end{array}$

$\begin{array}{ll}1 \times 1 & .013441 \\ 1 \times 1 & .012097 \\ 1 \times 1 & .012097 \\ 1 \times 1 & .016129 \\ 3 \times 2 & .012097 \\ 2 \times 1 & .014133 \\ 2 \times 2 & .014133 \\ 2 \times 1 \times 2 & .007392 \\ 1 \times 2 \times 3 & .014785 \\ 1 \times 2 \times 3 & .008737 \\ 1 \times 2 \times 3 & .008737 \\ 1 \times 2 \times 3 & .014785 \\ 2 \times 1 \times 2 & .007392 \\ 2 \times 2 & .014133 \\ 2 \times 1 & .014133 \\ 3 \times 2 & .012097 \\ 1 \times 1 & .016129 \\ 1 \times 1 & .012097 \\ 1 \times 1 & .012097 \\ 1 \times 1 & .013441\end{array}$




\section{TABLE D-3b. FUEL SPILL LOCATIONS, RATES AND PROBABILITIES FOR $45^{\circ}$ FROM REAR COLLISION INTO B-52}

\section{A. COLLISION IN HIGH SPEED IMPACT RANGE}

\begin{tabular}{cl}
$\begin{array}{c}\text { COLLISION } \\
\text { REGION }\end{array}$ & \multicolumn{1}{c}{ FUEL SPILL LOCATIONS } \\
& \\
1 & $\mathrm{R} 4 \times \mathrm{C}-21 / \mathrm{C} 20$ \\
2 & $\mathrm{R} 2 / \mathrm{R} 3 / \mathrm{R} 4 / \mathrm{R} 5 \times \mathrm{C}-13 / \mathrm{C}-22 / \mathrm{C}-31$ \\
3 & $\mathrm{~F} 1 / \mathrm{F} 2 \times \mathrm{R} 1 / \mathrm{R} 2 / \mathrm{R} 3 / \mathrm{R} 4 / \mathrm{R} 5 \times \mathrm{C} 03 / \mathrm{C} 15$ \\
4 & $\mathrm{~L} 5 \times \mathrm{R} 1 / \mathrm{R} 2 / \mathrm{R} 3 / \mathrm{R} 4 / \mathrm{R} 5 \times \mathrm{F} 1 / \mathrm{F} 2 / \mathrm{F} 3 / \mathrm{F} 4$ \\
5 & $\mathrm{R} 1 / \mathrm{R} 2 / \mathrm{R} 3 / \mathrm{R} 4 / \mathrm{R} 5 \times \mathrm{F} 2 / \mathrm{F} 3 / \mathrm{F} 4 / \mathrm{F} 5 \times \mathrm{L} 5$ \\
6 & $\mathrm{R} 1 / \mathrm{R} 2 / \mathrm{R} 3 / \mathrm{R} 4 / \mathrm{R} 5 \times \mathrm{F} 2 / \mathrm{F} 3 / \mathrm{F} 4 / \mathrm{F} 5$ \\
& $\times \mathrm{C}-18 / \mathrm{C}-29 / \mathrm{C} 010$ \\
7 & $\mathrm{~F} 2 / \mathrm{F} 3 / \mathrm{F} 4 / \mathrm{F} 5 \times \mathrm{R} 2 / \mathrm{R} 3 \times \mathrm{C}-18 / \mathrm{C}-19 \times \mathrm{R} 5$ \\
8 & $\mathrm{~F} 4 / \mathrm{F} 5 \times \mathrm{L} 2 / \mathrm{L} 3 \times \mathrm{C}-19 / \mathrm{C}-29 / \mathrm{C}-310$ \\
9 & $\mathrm{~L} 3 / \mathrm{L} 4 / \mathrm{L} 5 \times \mathrm{C}-36 / \mathrm{C}-46 / \mathrm{C}-47$ \\
10 & $\mathrm{~L} 4 / \mathrm{L} 5 \times \mathrm{C}-47 / \mathrm{C}-57 / \mathrm{C}-56 / \mathrm{C}-66$ \\
11 & $\mathrm{~L} 5 \times \mathrm{C}-49$
\end{tabular}

\author{
FUEL SPILL \\ RATE \\ CATEGORIES \\ RELATIVE \\ PROBABILITY
}

$\begin{array}{cc}2 \times 2 & .017164 \\ 2 \times 2 & .047015 \\ 2 \times 3 \times 2 & .071642 \\ 1 \times 3 \times 3 & .029104 \\ 3 \times 2 \times 1 & .031343 \\ 3 \times 3 \times 2 & .038806 \\ & \\ 3 \times 2 \times 2 \times 1 & .044776 \\ 3 \times 2 \times 2 & .046269 \\ 3 \times 3 & .055970 \\ 2 \times 2 & .061194 \\ 1 \times 1 & .056716\end{array}$

B. COLLISION IN MEDIUM SPEED IMPACT RANGE

$\begin{array}{cl}1 & \mathrm{R} 4 \times \mathrm{C}-13 / \mathrm{C}-12 / \mathrm{C}-22 \\ 2 & \mathrm{R} 2 / \mathrm{R} 3 / \mathrm{R} 4 / \mathrm{R} 5 \times \mathrm{C} 15 / \mathrm{C} 04 \\ 3 & \mathrm{R} 2 / \mathrm{R} 3 / \mathrm{R} 4 / \mathrm{R} 5 \times \mathrm{C} 15 / \mathrm{C} 26 \\ 4 & \mathrm{R} 2 / \mathrm{R} 3 / \mathrm{R} 4 / \mathrm{R} 5 \times \mathrm{F} 2 / \mathrm{F} 3 / \mathrm{F} 4 \\ 5 & \mathrm{R} 2 / \mathrm{R} 3 / \mathrm{R} 4 / \mathrm{R} 5 \times \mathrm{F} 2 / \mathrm{F} 3 / \mathrm{F} 4 \\ 6 & \mathrm{R} 1 / \mathrm{R} 2 / \mathrm{R} 3 / \mathrm{R} 4 / \mathrm{R} 5 \times \mathrm{F} 2 / \mathrm{F} 3 / \mathrm{F} 4 / \mathrm{F} 5 \times \mathrm{C} 19 \\ 7 & \mathrm{~F} 2 / \mathrm{F} 3 / \mathrm{F} 4 / \mathrm{F} 5 \times \mathrm{R} 2 / \mathrm{R} 3 \times \mathrm{C}-19 \\ 8 & \mathrm{~F} 4 / \mathrm{F} 5 \times \mathrm{L} 2 / \mathrm{L} 3 \times \mathrm{C}-110 / \mathrm{C}-19 \\ 9 & \mathrm{~L} 3 / \mathrm{L} 4 / \mathrm{L} 5 \times \mathrm{C}-18 \\ 10 & \text { L4/L5 } \times \mathrm{C}-48 / \mathrm{C}-47 / \mathrm{C}-57 \\ 11 & \mathrm{~L} 5 \times \mathrm{C}-57 / \mathrm{C}-66 / \mathrm{C}-75\end{array}$

\section{COLLISION IN LOW SPEED IMPACT RANGE}

$\begin{array}{cl}1 & \mathrm{R} 4 \times \mathrm{C} 26 / \mathrm{C} 15 / \mathrm{C} 04 \\ 2 & \mathrm{R} 4 / \mathrm{R} 5 \times \mathrm{C} 37 / \mathrm{C} 26 \\ 3 & \mathrm{R} 3 / \mathrm{R} 4 / \mathrm{R} 5 \times \mathrm{C} 26 / \mathrm{C} 37 \\ 4 & \mathrm{R} 3 / \mathrm{R} 4 / \mathrm{R} 5 \\ 5 & \mathrm{R} 2 / \mathrm{R} 3 / \mathrm{R} 4 / \mathrm{R} 5 \times \mathrm{F} 3 \\ 6 & \mathrm{R} 2 / \mathrm{R} 3 / \mathrm{R} 4 / \mathrm{R} 5 \times \mathrm{F} 2 / \mathrm{F} 3 / \mathrm{F} 4 \times \mathrm{C} 010 \\ 7 & \mathrm{~F} 3 / \mathrm{F} 4 / \mathrm{F} 5 \times \mathrm{R} 3 \times \mathrm{C} 09 / \mathrm{C} 010 \\ 8 & \mathrm{~F} 4 / \mathrm{F} 5 \times \mathrm{C} 010 \\ 9 & \text { L4/L5 } \times \mathrm{C} 010 / \mathrm{C}-19 / \mathrm{C}-29 \\ 10 & \text { L5 } \times \mathrm{C}-29 / \mathrm{C}-39 \\ 11 & \text { L5 } \times \mathrm{C}-48 / \mathrm{C}-57 / \mathrm{C}-66\end{array}$

$\begin{array}{cc}1 \times 1 & .008582 \\ 2 \times 1 & .023507 \\ 2 \times 2 & .035821 \\ 3 & .014552 \\ 3 \times 1 & .015672 \\ 3 \times 2 \times 2 & .019403 \\ 2 \times 1 \times 2 & .022388 \\ 2 \times 1 & .023134 \\ 1 \times 2 & .027985 \\ 1 \times 2 & .030597 \\ 1 \times 1 & .028358\end{array}$




\section{TABLE D-3C. FUEL SPILL LOCATIONS, RATES AND PROBABILITIES FOR BROADSIDE COLLISION INTO B-52}

\section{A. COLLISION IN HIGH SPEED IMPACT RANGE}

\begin{tabular}{cl}
$\begin{array}{c}\text { COLLISION } \\
\text { REGION }\end{array}$ & \multicolumn{1}{c}{ FUEL SPILL LOCATIONS } \\
& \\
1 & None \\
2 & $\mathrm{~F} 1 \times \mathrm{C}-73 / \mathrm{C}-63 / \mathrm{C}-53 / \mathrm{C}-84 / \mathrm{C}-74 / \mathrm{C}-75 / \mathrm{C}-64 / \mathrm{C}-55$ \\
3 & $\mathrm{~F} 1 / \mathrm{F} 2 \times \mathrm{R} 1 / \mathrm{R} 2 \times \mathrm{C}-54 / \mathrm{C}-44 / \mathrm{C}-34 / \mathrm{C}-24 / \mathrm{C}-43 / \mathrm{C}-33$ \\
4 & $\mathrm{~F} 1 / \mathrm{F} 2 \times \mathrm{R} 2 / \mathrm{R} 3 / \mathrm{R} 4$ \\
& $\times \mathrm{C} 15 / \mathrm{C} 04 / \mathrm{C}-13 / \mathrm{C}-14 / \mathrm{C}-23 / \mathrm{C}-33 / \mathrm{C}-24 / \mathrm{C}-34$ \\
5 & $\mathrm{~F} 1 \times \mathrm{R} 1 / \mathrm{R} 2 \times \mathrm{R} 4 / \mathrm{R} 5 \times \mathrm{C} 14 / \mathrm{C} 15 / \mathrm{CO} / \mathrm{C}-13$ \\
6 & $\mathrm{~F} 1 \times \mathrm{R} 1 / \mathrm{R} 2 / \mathrm{R} 3 / \mathrm{R} 4 \times \mathrm{C} 18 / \mathrm{C} 16 / \mathrm{C} 15 / \mathrm{C} 14$ \\
7 & $\mathrm{~L} 5 \times \mathrm{R} 1 / \mathrm{R} 2 / \mathrm{R} 3 / \mathrm{R} 4 \times \mathrm{F} 1 / \mathrm{F} 2 / \mathrm{F} 3 / \mathrm{F} 4$ \\
8 & $\mathrm{~L} 5 \times \mathrm{F} 2 / \mathrm{F} 3 / \mathrm{F} 4 \times \mathrm{R} 3 / \mathrm{R} 4 \times \mathrm{C} 29 / \mathrm{C} 18$ \\
9 & $\mathrm{R} 2 / \mathrm{R} 3 / \mathrm{R} 4 / \mathrm{R} 5 \times \mathrm{F} 4 / \mathrm{F} 5 \times \mathrm{C} 19 / \mathrm{C} 29$ \\
10 & $\mathrm{R} 3 / \mathrm{R} 4 / \mathrm{R} 5 \times \mathrm{F} 5 \times \mathrm{C} 010 / \mathrm{C} 110 / \mathrm{C}-110$ \\
11 & $\mathrm{~F} 5 \times \mathrm{R} 4 / \mathrm{R} 5 \times \mathrm{C}-510 / \mathrm{C}-410 / \mathrm{C}-310$ \\
12 & $\mathrm{C}-811 / \mathrm{C}-711 / \mathrm{C}-611 / \mathrm{C}-511$
\end{tabular}

B. COLLISION IN MEDIUM SPEED IMPACT RANGE

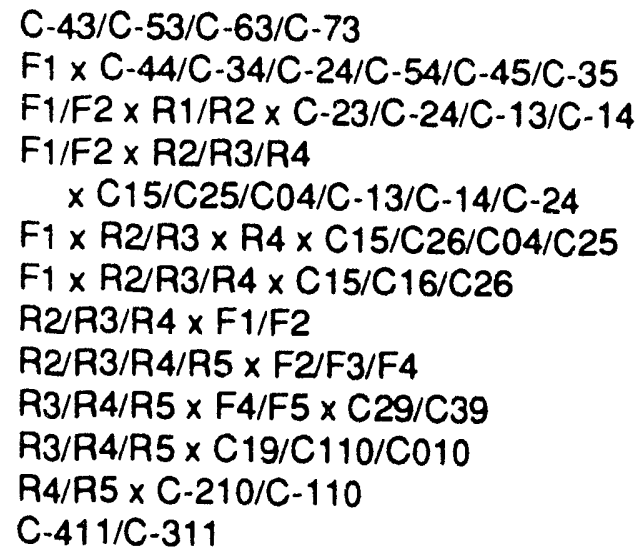

FUEL SPILL RATE CATEGORIES

3
$2 \times 3$
$2 \times 3 \times 3$
$3 \times 2 \times 3$
$3 \times 2 \times 1 \times 3$
$3 \times 2 \times 2$
$1 \times 3 \times 2$
$1 \times 3 \times 3 \times 1$
$2 \times 3 \times 1$
$2 \times 2 \times 2$
$1 \times 1 \times 3$
2

RELATIVE PROBABILITY

.025788
.038682
.030802

.020057
.038682
.027221
.030086
.051576
.055874
.054441
.059456
.067335

.025788

.038682

(067335

$\begin{array}{cc}2 & .012894 \\ 1 \times 2 & .019341 \\ 1 \times 2 \times 3 & .015401 \\ 3 \times 2 \times 3 & .010029 \\ 2 \times 2 \times 1 \times 3 & .019341 \\ 3 \times 2 \times 2 & .013610 \\ 3 \times 2 & .015043 \\ 3 \times 2 & .025788 \\ 3 \times 2 \times 2 & .027937 \\ 2 \times 2 & .027221 \\ 1 \times 3 & .029728 \\ 2 & .033668\end{array}$


C. COLLISION IN LOW SPEED IMPACT RANGE

C04/C-14/C-24/C-34

$\begin{array}{cc}2 & .012894 \\ 1 \times 1 & .019341 \\ 1 \times 2 \times 2 & .015401 \\ 2 \times 1 \times 2 & .010029 \\ 1 \times 2 \times 1 \times 2 & .019341 \\ 2 \times 2 \times 2 & .013610 \\ 2 \times 1 & .015043 \\ 2 & .025788 \\ 2 & .027937 \\ 2 \times 2 & .027221 \\ 1 \times 2 & .029728 \\ 1 & .033668\end{array}$


TABLE D-3d. FUEL SPILL LOCATIONS, RATES AND PROBABILITIES FOR $45^{\circ}$ FROM FRONT COLLISION INTO B-52

A. COLLISION IN HIGH SPEED IMPACT RANGE

\begin{tabular}{|c|c|}
\hline $\begin{array}{l}\text { COI.LISION } \\
\text { REGION }\end{array}$ & FUEL SPILL LOCATIONS \\
\hline $\begin{array}{l}1 \\
2 \\
3 \\
4 \\
5 \\
6 \\
7 \\
8 \\
9\end{array}$ & 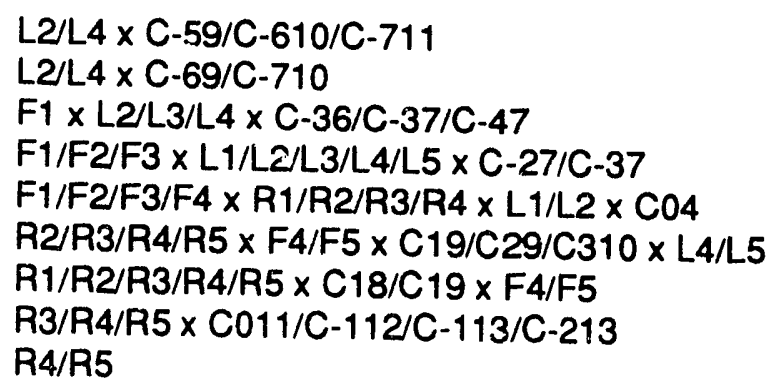 \\
\hline
\end{tabular}

B. COLLISION IN MEDIUM SPEED IMPACT RANGE

$\mathrm{L} 2 \times \mathrm{C}-26 / \mathrm{C}-47 / \mathrm{C}-48$

$\mathrm{L} 2 / \mathrm{L} 4 \times \mathrm{C}-26 / \mathrm{C}-37 / \mathrm{C}-47$

F1 x L.ZLZ/L4 x C,-15/C-26

F1/F2 2 L2/I $3 \times C-16 / C-26$

$\mathrm{F} 1 / \mathrm{F} 2 / \mathrm{F3} \times \mathrm{L} 1 / \mathrm{L}: \mathrm{IL} 3 / \mathrm{L} 4 \times \mathrm{CO} 4$

$\mathrm{R} 2 / \mathrm{R3} / \mathrm{R} 4 / \mathrm{R} 5 \times \mathrm{F} 4 / \mathrm{F} 5 \times \mathrm{C} 29 / \mathrm{C} 39$

$\mathrm{R} 1 / \mathrm{R} 2 / \mathrm{R} 3 \times \mathrm{C} 18 / \mathrm{C} 29 / \mathrm{C} 39 \times \mathrm{F} 5$

$\mathrm{R} 4 / \mathrm{R} 5 \times \mathrm{C} 110 / \mathrm{C} 111 / \mathrm{C} 010 / \mathrm{C} 011$

$\mathrm{R} 4 / \mathrm{R} 5 \times \mathrm{C} 011 / \mathrm{C} 012 / \mathrm{C}-113 / \mathrm{C}-114$

$\begin{array}{cc}\text { FUEL SPILL } & \text { RELATIVE } \\ \text { RATE } & \text { PROBABILITY } \\ \text { CATEGORIES } & \end{array}$

$2 \times 1$
$2 \times 1$
$1 \times 3 \times 3$
$3 \times 3 \times 2$
$3 \times 3 \times 1 \times 2$
$3 \times 3 \times 2 \times 1$
$3 \times 2 \times 1$
$2 \times 2$
2

.037879

.031818

.046970

.057576

.065152

.068182

.075758

.054545

.062121

\section{COLLISION IN LOW SPEED IMPACT RANGE}

$\begin{array}{ll}1 & \mathrm{~L} 2 \times \mathrm{CO} 4 / \mathrm{C}-15 \\ 2 & \mathrm{~L} 2 \times \mathrm{CO} 4 / \mathrm{C}-25 \\ 3 & \mathrm{~L} 2 / \mathrm{L} 3 \times \mathrm{C} 04 / \mathrm{C}-15 \\ 4 & \mathrm{~F} 1 / \mathrm{F} 2 \times \mathrm{CO} 4 \\ 5 & \mathrm{~F} 1 / \mathrm{F} 2 / \mathrm{F} 3 \times \mathrm{R} 2 / \mathrm{R} 3 \\ 6 & \mathrm{R} 2 / \mathrm{R} 3 \times \mathrm{R} 4 / \mathrm{R} 5 \\ 7 & \mathrm{R} 3 / \mathrm{R} 4 / \mathrm{R} 5 \\ 8 & \mathrm{R} 3 / \mathrm{R} 4 / \mathrm{R} 5 \times \mathrm{C} 39 / \mathrm{C} 310 / \mathrm{C} 29 / \mathrm{C} 2 ! \mathrm{O} \\ 9 & \mathrm{R} 4 / \mathrm{R} 5 \times \mathrm{C} 39 / \mathrm{C} 310 / \mathrm{C} 210 / \mathrm{C} 211\end{array}$

$\begin{array}{cc}1 \times 1 & .018939 \\ 2 \times 1 & .015909 \\ 1 \times 2 \times 3 & .023485 \\ 3 \times 2 \times 2 & .028788 \\ 2 \times 2 \times 1 & .03<576 \\ 3 \times 2 \times 1 & .034091 \\ 2 \times 2 \times 1 & .037879 \\ 2 \times 2 & .027273 \\ 2 \times 2 & .031061\end{array}$

$\begin{array}{cl}1 \times 1 & .018939 \\ 1 \times 1 & .015909 \\ 1 \times 2 & .023485 \\ 2 \times 1 & .028788 \\ 2 \times 1 & .032576 \\ 3 \times 2 & .034091 \\ 3 & .037879 \\ 2 \times 2 & .027273 \\ 1 \times 1 & .031061\end{array}$




\section{TABLE D-4a. FUEL SPILL LOCATIONS, RATES AND PROBABILITIES}

FOR CIIRECT REAR COLLISION INTO B-1B

\section{A. COLLISION IN HIGH SPEED IMPACT RANGE}

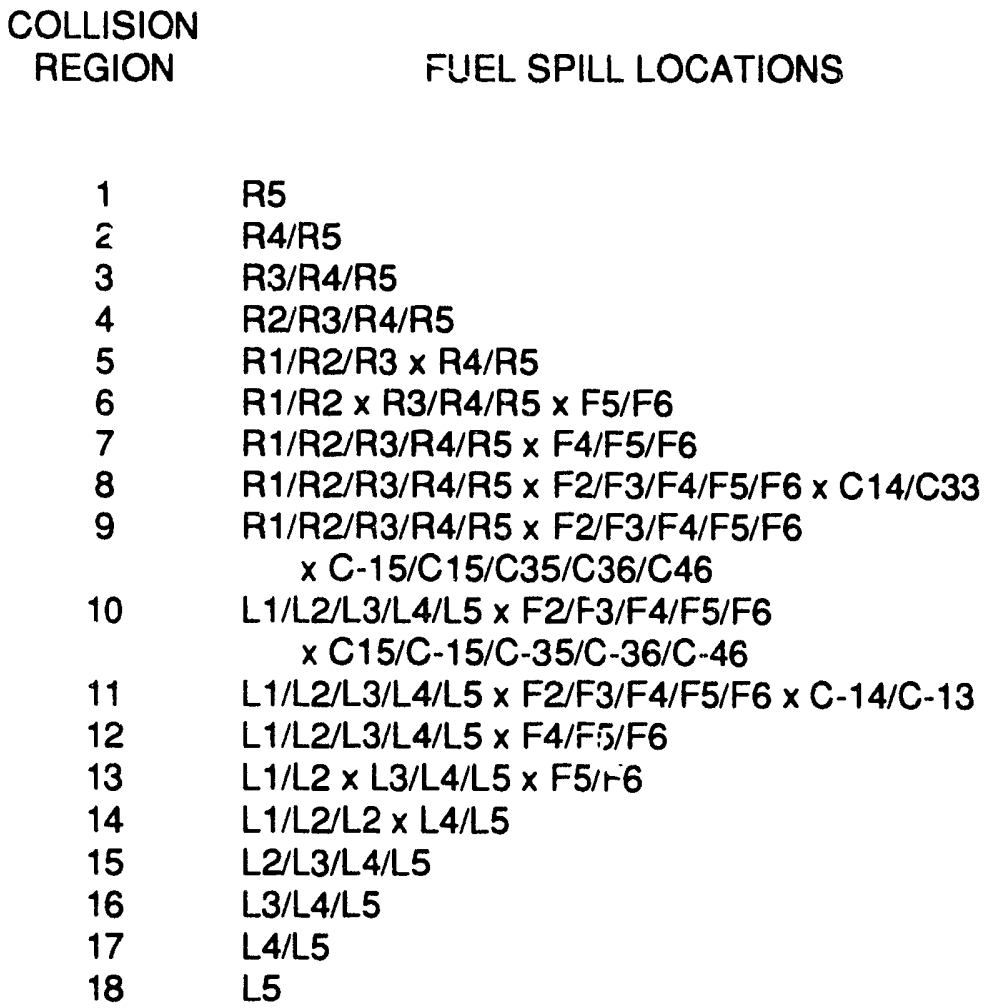

\author{
FUEL SPILL \\ RATE \\ CATEGORIES \\ RELATIVE \\ PROBABILITY
}

$\begin{array}{cc}1 & .015576 \\ 1 & .017912 \\ 1 & .017912 \\ 1 & .017912 \\ 1 \times 3 & .024922 \\ 1 \times 3 \times 1 & .031153 \\ 2 \times 1 & .023364 \\ 2 \times 2 \times 3 & .054517 \\ 3 \times 3 \times 3 & .046729 \\ 3 \times 3 \times 3 & .046729 \\ 2 \times 2 \times 3 & .054517 \\ 2 \times 1 & .023364 \\ 1 \times 2 \times 1 & .031153 \\ 1 \times 3 & .024926 \\ 1 & .017912 \\ 1 & .017912 \\ 1 & .017912 \\ 1 & .015576\end{array}$

B. COLLISION IN MEDIUM SPEED IMPACT RANGE

R5

R4/R5

R3/R4/R5

$\mathrm{R} 2 / \mathrm{R} 3 / \mathrm{R} 4 / \mathrm{R} 5$

$\mathrm{R} 1 / \mathrm{R} 2 \times \mathrm{R} 3 / \mathrm{R} 4 / \mathrm{R} 5 \times \mathrm{C} 23 / \mathrm{C} 24 \times \mathrm{C} 32 / \mathrm{C} 33 / \mathrm{C} 43$

$\mathrm{R} 2 / \mathrm{R} 3 / \mathrm{R} 4 / \mathrm{R} 5 \times \mathrm{F} 5 / \mathrm{F} 6$ $\times C 14 / C 15 / C 16 / C 23 / C 24 / C 25$

$\mathrm{R} 2 / \mathrm{R} 3 / \mathrm{R} 4 / \mathrm{R} 5 \times \mathrm{F} 5 / \mathrm{F} 6$ $\times C 14 / C 15 / C 16 / C 23 / C 24 / C 25$

$\mathrm{R} 2 / \mathrm{R} 3 / \mathrm{R} 4 \times \mathrm{F} 3 / \mathrm{F} 4 / \mathrm{F} 5$

$\times$ - $19 / \mathrm{COS} / \mathrm{C} 18 / \mathrm{C} 27 / \mathrm{C} 08 / \mathrm{C} 17 / \mathrm{C} 26$

$\mathrm{R} 1 / \mathrm{R} 2 \times \mathrm{F} 3 / \mathrm{F} 4 /, \mathrm{C} 5 / \mathrm{F} 6 \times \mathrm{C}-19 / \mathrm{C} 09 / \mathrm{C} 19 / \mathrm{CO} / \mathrm{C} 18$

$\mathrm{L} 1 / \mathrm{L} 2 \times \mathrm{F} 3 / \mathrm{F} 4 / \mathrm{F} 5 / \mathrm{F} 6 \times \mathrm{C} 19 / \mathrm{C09} / \mathrm{C}-19 / \mathrm{C} 08 / \mathrm{C}-18$

$\mathrm{L} 2 / \mathrm{L} 3 / \mathrm{L} 4 \times \mathrm{F} 3 / \mathrm{F} 4 / \mathrm{F5}$

$\times$ C19/C09/C-18/C-27/C08/C-19/C-26
.007788

.008956

.008956

.008956

$1 \times 2 \times 1 \times 2 \quad .012461$

$2 \times 1 \times 2 \quad .015576$

$2 \times 1 \times 2 \quad .011682$

$2 \times 2 \times 2 \quad .027259$

$2 \times 2 \times 2 \quad .023364$

$2 \times 2 \times 2 \quad .023364$

$2 \times 2 \times 2 \quad .027259$ 
$12 \quad \mathrm{~L} 2 / \mathrm{L} 3 / \mathrm{L} 4 / \mathrm{L} 5 \times \mathrm{F} 5 / \mathrm{F} 6$

$\times C-14 / C-15 / C-16 / C-23 / C-24 / C-25 \quad 2 \times 1 \times 2 \quad .011682$

$13 \quad \mathrm{~L} 2 / \mathrm{L} 3 / \mathrm{L} 4 / \mathrm{L} 5 \times \mathrm{F} 5 / \mathrm{F} 6$

x C-14/C-15/C-16/C-23/C-24/C-25 $2 \times 1 \times 2 \quad .015576$

$14 \quad L 1 / L 2 \times L 3 / L 4 / L 5 \times C-23 / C-24 \times C-32 / C-33 / C-43$

$15 \quad$ L2/L3/L4/L5

$16 \quad$ L3/L4/L5

$17 \quad$ L $4 /$ L5

18 L5

$\begin{array}{cc}1 \times 2 \times 1 \times 2 & .012461 \\ 1 & .008956 \\ 1 & .008956 \\ 1 & .008956 \\ 1 & .007788\end{array}$

C. COLLISION IN LOW SPEED IMPACT RANGE

$\begin{array}{cl}1 & \mathrm{R} 5 \times \mathrm{C} 36 / \mathrm{C} 37 \\ 2 & \mathrm{R} 4 / \mathrm{R} 5 \times \mathrm{C} 36 / \mathrm{C} 37 \\ 3 & \mathrm{R} 3 / \mathrm{R} 4 / \mathrm{R} 5 \times \mathrm{C} 26 / \mathrm{C} 27 / \mathrm{C} 36 / \mathrm{C} 37 \\ 4 & \mathrm{R} 2 / \mathrm{R} 3 / \mathrm{R} 4 / \mathrm{R} 5 \times \mathrm{C} 27 / \mathrm{C} 28 / \mathrm{C} 37 / \mathrm{C} 38 \\ 5 & \mathrm{R} 1 / \mathrm{R} 2 / \mathrm{R} 3 \times \mathrm{R} 4 / \mathrm{R} 5 \times \mathrm{C} 19 / \mathrm{C} 26 / \mathrm{C} 27 / \mathrm{C} 28 \\ 6 & \mathrm{R} 1 / \mathrm{R} 2 \times \mathrm{R} 3 / \mathrm{R} 4 / \mathrm{R} 5 \times \mathrm{F} 5 / \mathrm{F} 6 \\ & \times \mathrm{C} 17 / \mathrm{C} 18 / \mathrm{C} 27 / \mathrm{C} 28 / \mathrm{C} 36 \\ 7 & \mathrm{R} 1 / \mathrm{R} 2 \times \mathrm{R} 3 / \mathrm{R} 4 / \mathrm{R} 5 \times \mathrm{F} 5 / \mathrm{F} 6 \\ & \times \mathrm{C} 17 / \mathrm{C} 18 / \mathrm{C} 27 / \mathrm{C} 28 / \mathrm{C} 36 \\ 8 & \mathrm{R} 2 / \mathrm{R} 3 / \mathrm{R} 4 \times \mathrm{F} 4 / \mathrm{F} 5 \times \mathrm{C}-19 / \mathrm{C} 09 / \mathrm{C} 19 \\ 9 & \mathrm{R} 1 / \mathrm{R} 2 \times \mathrm{F} 2 / \mathrm{F} 3 / \mathrm{F} 4 \times \mathrm{C}-110 / \mathrm{C} 010 / \mathrm{C} 110 \\ 10 & \mathrm{~L} 1 / \mathrm{L} 2 \times \mathrm{F} 2 / \mathrm{F} 3 / \mathrm{F} 4 \times \mathrm{C} 110 / \mathrm{C} 010 / \mathrm{C}-110 \\ 11 & \mathrm{~L} 2 / \mathrm{L} 3 / \mathrm{L} 4 \times \mathrm{F} 4 / \mathrm{F} 5 \times \mathrm{C} 19 / \mathrm{C} 09 / \mathrm{C}-19 \\ 12 & \mathrm{~L} 1 / \mathrm{L} 2 / \times \mathrm{L} 3 / \mathrm{L} 4 / \mathrm{L} 5 \times \mathrm{F} 5 / \mathrm{F} 6 \\ & \times \mathrm{C}-17 / \mathrm{C}-18 / \mathrm{C}-27 / \mathrm{C}-28 / \mathrm{C}-36 \\ 13 & \mathrm{~L} 1 / \mathrm{L} 2 \times \mathrm{L} 3 / \mathrm{L} 4 / \mathrm{L} 5 \times \mathrm{F} 5 / \mathrm{F} 6 \\ & \times \mathrm{C}-17 / \mathrm{C}-18 / \mathrm{C}-27 / \mathrm{C}-28 / \mathrm{C}-36 \\ 14 & \mathrm{~L} 1 / \mathrm{L} 2 / \mathrm{L} 3 \times \mathrm{L} 4 / \mathrm{L} 5 \times \mathrm{C}-19 / \mathrm{C}-26 / \mathrm{C}-27 / \mathrm{C}-28 \\ 15 & \mathrm{~L} 2 / \mathrm{L} 3 / \mathrm{L} 4 / \mathrm{L} 5 \times \mathrm{C}-27 / \mathrm{C}-28 / \mathrm{C}-37 / \mathrm{C}-38 \\ 16 & \mathrm{~L} 3 / \mathrm{L} 4 / \mathrm{L} 5 \times \mathrm{C}-26 / \mathrm{C}-27 / \mathrm{C}-36 / \mathrm{C}-37 \\ 17 & \mathrm{~L} 4 / \mathrm{L} 5 \times \mathrm{C}-36 / \mathrm{C}-37 \\ 18 & \mathrm{~L} 5 \times \mathrm{C}-36 / \mathrm{C}-37\end{array}$

$\begin{array}{cc}1 \times 1 & .007788 \\ 1 \times 1 & .008956 \\ 1 \times 1 & .008956 \\ 1 \times 1 & .008956 \\ 1 \times 2 \times 1 & .012461 \\ & \\ 1 \times 2 \times 1 \times 2 & .015576 \\ & \\ 1 \times 2 \times 1 \times 2 & .011682 \\ 2 \times 1 \times 2 & .027259 \\ 2 \times 2 \times 1 & .023364 \\ 2 \times 2 \times 1 & .023364 \\ 2 \times 1 \times 2 & .027259 \\ 1 \times 2 \times 1 \times 2 & .011682 \\ & \\ 1 \times 2 \times 1 \times 2 & .015576 \\ 1 \times 2 \times 1 & .012461 \\ 1 \times 1 & .008956 \\ 1 \times 1 & .008956 \\ 1 \times 1 & .008956 \\ 1 \times 1 & .007788\end{array}$




\section{TABLE D-4b. FUEL SPILL LOCATIONS, RATES AND PROBABILITIES FOR $45^{\circ}$ FROM REAR COLLISION INTO B-1B}

\section{A. COLLISION IN HIGH SPEED IMPACT RANGE}

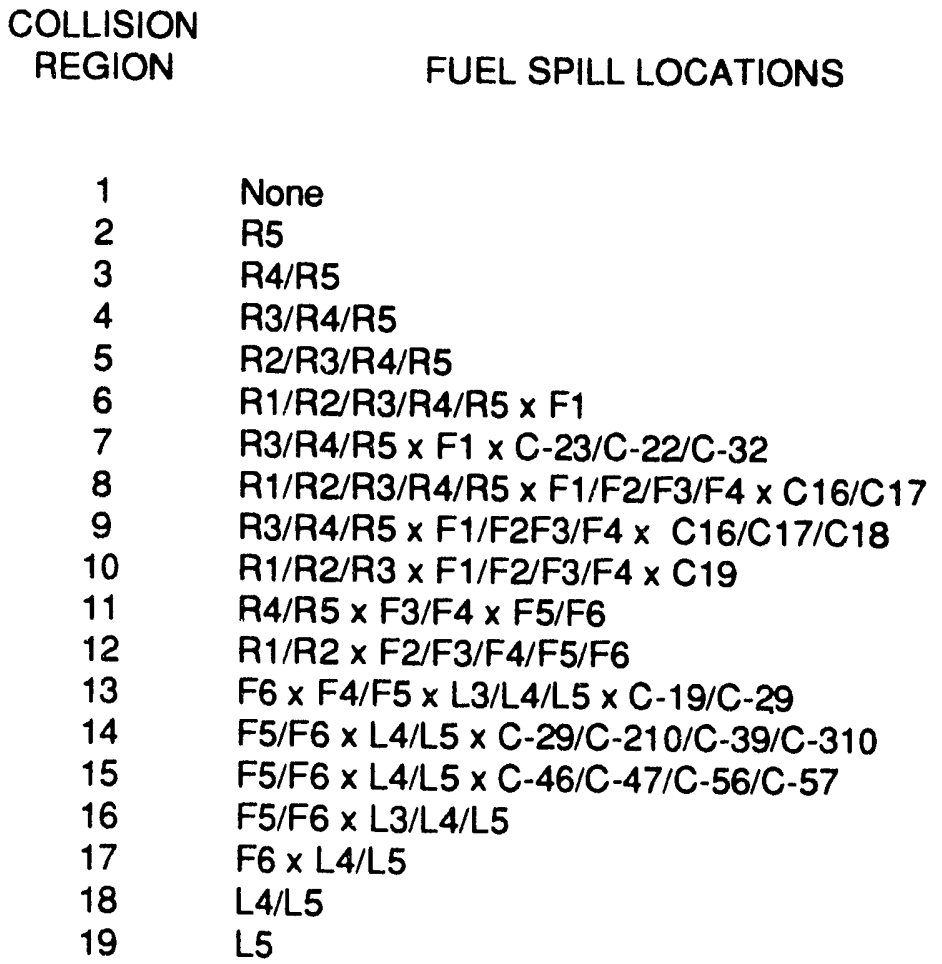

\author{
FUEL SPILL \\ RATE \\ CATEGORIES \\ RELATIVE \\ PROBABILITY
}

$\begin{array}{cc}1 & .023810 \\ 1 & .012755 \\ 1 & .012755 \\ 1 & .012755 \\ 1 & .012755 \\ 1 \times 1 & .022959 \\ 2 \times 1 \times 2 & .033163 \\ 3 \times 2 \times 3 & .033163 \\ 2 \times 2 \times 2 & .036565 \\ 3 \times 3 \times 2 & .036565 \\ 3 \times 3 \times 3 & .029762 \\ 3 \times 3 & .029762 \\ 2 \times 2 \times 1 \times 3 & .027211 \\ 2 \times 2 \times 3 & .030612 \\ 2 \times 2 \times 2 & .030612 \\ 1 \times 2 & .038265 \\ 1 \times 1 & .025510 \\ 1 & .025510 \\ 1 & .025510\end{array}$

B. COLLISION IN MEDIUM SPEED IMPACT RANGE

$\begin{array}{cl}1 & \mathrm{C}-14 / \mathrm{C}-23 / \mathrm{C}-32 \\ 2 & \mathrm{R} 5 \times \mathrm{C} 04 / \mathrm{C}-13 / \mathrm{C}-22 \\ 3 & \mathrm{R} 4 / \mathrm{R} 5 \times \mathrm{C} 04 / \mathrm{C}-13 / \mathrm{C}-22 \\ 4 & \mathrm{R} 3 / \mathrm{R} 4 / \mathrm{R} 5 \times \mathrm{C} 04 / \mathrm{C}-13 / \mathrm{C}-22 \\ 5 & \mathrm{R} 2 / \mathrm{R} 3 / \mathrm{R} 4 / \mathrm{R} 5 \times \mathrm{C}-23 / \mathrm{C}-13 / \mathrm{C}-14 / \mathrm{C} 05 \\ 6 & \mathrm{R} 1 / \mathrm{R} 2 / \mathrm{R} 3 / \mathrm{R} 4 / \mathrm{R} 5 \times \mathrm{F} 1 \times \mathrm{C} 04 / \mathrm{C} 05 / \mathrm{C}-13 / \mathrm{C}-14 \\ 7 & \mathrm{R} 3 / \mathrm{R} 4 / \mathrm{R} 5 \times \mathrm{F} 1 \times \mathrm{C} 04 / \mathrm{C} 05 / \mathrm{C}-14 \\ 8 & \mathrm{R} 1 / \mathrm{R} 2 / \mathrm{R} 3 / \mathrm{R} 4 / \mathrm{R} 5 \times \mathrm{F} 1 \times \mathrm{C} 16 / \mathrm{C} 17 / \mathrm{C} 15 / \mathrm{C} 05 \\ 9 & \mathrm{R} 3 / \mathrm{R} 4 / \mathrm{R} 5 \times \mathrm{F} 1 / \mathrm{F} 2 / \mathrm{F} 3 / \mathrm{F} 4 \times \mathrm{C} 16 \\ 10 & \mathrm{R} 1 / \mathrm{R} 2 / \mathrm{R} 3 \times \mathrm{F} 1 / \mathrm{F} 2 / \mathrm{F} 3 / \mathrm{F} 4 \\ 11 & \mathrm{R} 4 / \mathrm{R} 5 \times \mathrm{F} 3 / \mathrm{F} 4 \times \mathrm{F} 5 / \mathrm{F} 6 \\ 12 & \mathrm{R} 4 / \mathrm{R} 5 \times \mathrm{F} 3 / \mathrm{F} 4 / \mathrm{F} 5 / \mathrm{F} 6 \\ 13 & \mathrm{~F} 6 \times \mathrm{F} 4 / \mathrm{F} 5 \times \mathrm{L} 3 / \mathrm{L} 4 / \mathrm{L} 5 \\ 14 & \mathrm{~F} 5 / \mathrm{F} 6 \times \mathrm{L} 4 / \mathrm{L} 5 \times \mathrm{C}-19 / \mathrm{C}-110 \\ 15 & \mathrm{~F} 5 / \mathrm{F} 6 \times \mathrm{L} 4 / \mathrm{L} 5 \times \mathrm{C}-29 / \mathrm{C}-39 \\ 16 & \mathrm{~F} 5 / \mathrm{F} 6 \times \mathrm{L} 3 / \mathrm{L} 4 / \mathrm{L} 5 \times \mathrm{C}-19 / \mathrm{C}-29 / \mathrm{C}-39 \\ 17 & \mathrm{~L} 4 / \mathrm{L} 5 \times \mathrm{C}-47 / \mathrm{C}-57 \\ 18 & \mathrm{~L} 4 / \mathrm{L} 5 \times \mathrm{C}-47 / \mathrm{C}-57 \\ 19 & \mathrm{~L} 5 \times \mathrm{C}-55\end{array}$

$\begin{array}{cc}1 & .011905 \\ 1 \times 1 & .006378 \\ 1 \times 1 & .006378 \\ 1 \times 1 & .006378 \\ 1 \times 1 & .006378 \\ 1 \times 1 \times 1 & .011480 \\ 2 \times 1 \times 2 & .016582 \\ 3 \times 1 \times 2 & .016582 \\ 2 \times 2 \times 2 & .018282 \\ 2 \times 3 & .018282 \\ 3 \times 3 \times 2 & .014881 \\ 3 \times 3 & .014881 \\ 1 \times 3 \times 1 & .013605 \\ 2 \times 1 \times 2 & .015306 \\ 2 \times 2 \times 2 & .015306 \\ 1 \times 2 \times 1 & .019133 \\ 1 \times 1 & .012755 \\ 1 \times 1 & .012755 \\ 1 \times 1 & .012755\end{array}$


C. COLLISION IN LOW SPEED IMPACT RANGE

$\begin{array}{ll}1 & \mathrm{C} 04 \\ 2 & \mathrm{R} 5 \times \mathrm{C} 27 / \mathrm{C} 16 / \mathrm{C} 05 \\ 3 & \mathrm{R} 4 / \mathrm{R} 5 \times \mathrm{C} 27 / \mathrm{C} 16 / \mathrm{C} 05 \\ 4 & \mathrm{R} 3 / \mathrm{R} 4 / \mathrm{R} 5 \times \mathrm{C} 17 / \mathrm{C} 27 / \mathrm{C} 16 / \mathrm{C} 05 \\ 5 & \mathrm{R} 2 / \mathrm{R} 3 / \mathrm{R} 4 / \mathrm{R} 5 \times \mathrm{C} 17 / \mathrm{C} 27 / \mathrm{C} 16 / \mathrm{C} 05 \\ 6 & \mathrm{R} 1 / \mathrm{R} 2 / \mathrm{R} 3 / \mathrm{R} 5 / \mathrm{R} 5 \times \mathrm{F} 1 \times \mathrm{CO} 6 / \mathrm{C} 16 / \mathrm{C} 17 / \mathrm{C} 27 \\ 7 & \mathrm{R} 3 / \mathrm{R} 4 / \mathrm{R} 5 \times \mathrm{F} 1 \times \mathrm{C} 16 / \mathrm{C} 17 / \mathrm{C} 27 \\ 8 & \mathrm{R} 1 / \mathrm{R} 2 / \mathrm{R} 3 / \mathrm{R} 4 / \mathrm{R} 5 \times \mathrm{F} 1 \times \mathrm{C} 18 / \mathrm{C} 28 / \mathrm{C} 29 \\ 9 & \mathrm{R} 3 / \mathrm{R} 4 / \mathrm{R} 5 \times \mathrm{F} 1 \\ 10 & \mathrm{R} 1 / \mathrm{R} 2 / \mathrm{R} 3 \times \mathrm{F} 1 / \mathrm{F} 2 \\ 11 & \mathrm{~F} 3 / \mathrm{F} 4 \times \mathrm{F} 5 \\ 12 & \mathrm{R} 4 / \mathrm{R} 5 \times \mathrm{F} 5 / \mathrm{F} 6 \\ 13 & \mathrm{~F} 6 \times \mathrm{F} 4 / \mathrm{F} 5 \times \mathrm{L} 3 / \mathrm{L} 4 / \mathrm{L} 5 \\ 14 & \mathrm{~F} 5 / \mathrm{F} 6 \times \mathrm{L} 4 / \mathrm{L} 5 \\ 15 & \mathrm{~F} 5 / \mathrm{F} 6 \times \mathrm{L} 4 / \mathrm{L} 5 \\ 16 & \mathrm{~F} 5 / \mathrm{F} 6 \times \mathrm{L} 4 / \mathrm{L} 5 \times \mathrm{C}-19 \\ 17 & \mathrm{~L} 5 \times \mathrm{C}-19 \\ 18 & \mathrm{~L} 4 / \mathrm{L} 5 \times \mathrm{C}-19 \\ 19 & \mathrm{~L} 5 \times \mathrm{C}-47 / \mathrm{C}-56\end{array}$

$\begin{array}{cl}1 & .001905 \\ 1 \times 1 & .006378 \\ 1 \times 1 & .006378 \\ 1 \times 1 & .006378 \\ 1 \times 1 & .006378 \\ 1 \times 1 \times 1 & .011480 \\ 2 \times 1 \times 1 & .016582 \\ 3 \times 1 \times 2 & .016582 \\ 1 \times 1 & .018282 \\ 2 \times 1 & .018282 \\ 1 \times 2 & .014881 \\ 2 \times 1 & .014881 \\ 1 \times 2 \times 1 & .013605 \\ 2 \times 1 & .015306 \\ 2 \times 1 & .015306 \\ 2 \times 1 \times 1 & .019133 \\ 1 \times 1 & .012755 \\ 1 \times 1 & .012755 \\ 1 \times 1 & .012755\end{array}$


TABLE D-4C. FUEL SPILL LOCATIONS, RATES AND PROBABILITIES FOR BROADSIDE COLLISION INTO B-1B

A. COLLISION IN HIGH SPEED IMPACT RANGE

COLLISION

FUEL SPILL LOCATIONS

C's only (Out of Exposure Range)

$\mathrm{F} 1 \times \mathrm{C}$ 's (Out of Exposure Range)

$\mathrm{F} 1 \times \mathrm{C}-55 / \mathrm{C}-56$

$\mathrm{F} 1 \times \mathrm{C}-45 / \mathrm{C}-55 / \mathrm{C}-46$

F1/F2 $\times$ R2/R3/R4/R5 $\times$ C-24/C-25/C-16/C-34/C-15

F1/F2/F3 $\times$ R1/R2/R3/R4/R5 $\times$ C-16/C-17/C05

F1/F2/F3/F4/F5 $\times$ R1/R2/R3/R4/R5 $\times$ C05

$\mathrm{F} 2 / \mathrm{F} 3 / \mathrm{F} 4 / \mathrm{F} 5 / \mathrm{F} 6 \times \mathrm{R} 1 / \mathrm{R} 2 / \mathrm{R} 3 / \mathrm{R} 4 / \mathrm{R} 5 \times \mathrm{L} 5$

F2/F3/F4/F5/F6 $\times$ R1/R2/R3/R4/R5 x L5

F4/F5/F6 x R1/R2/R3/R4/R5 x C-19

$\mathrm{F} 4 / \mathrm{F} 5 / \mathrm{F} 6 \times \mathrm{R} 3 / \mathrm{R} 4 / \mathrm{R} 5 \times \mathrm{C}-19 / \mathrm{C}-110 / \mathrm{C}-29 / \mathrm{C}-210$

F5/F5 $\times$ R3/R4/R5 $\times$ C-29/C-39/C-310/C-410

F5/F6 $\times$ C-610/C-611

F6

B. COLLISION IN MEDIUM SPEED IMPACT RANGE
C-45/C-55

$\mathrm{F} 1 \times \mathrm{C}-45 / \mathrm{C}-55$

$\mathrm{F} 1 \times \mathrm{C}-36 / \mathrm{C}-35 / \mathrm{C}-45 / \mathrm{C}-55$

$\mathrm{F} 1 \times \mathrm{C}-25 / \mathrm{C}-26 / \mathrm{C}-35 / \mathrm{C}-36 / \mathrm{C}-45$

F1/F2 x R1/R2/R3/R4/R5

F1/F2/F3 $\times$ R1/R2/R3/R4/R5 $\times$ C05

F1/F2/F3 $\times$ R1/R2/R3/R4/R5 $\times$ C05

F2/F3/F4/F5 x R1/R2/R3/R4/R5 x L5

F2/F3/F4/F5 x R1/R2/R3/R4/R5 x L5

F4/F5/F6 $\times$ R2/R3/R4/R5

F4/F5/F6 x R2/R3/R4/R5 x C-19/C-110

F5/F6 $\times$ R3/R4/R5 $\times$ C-19/C-29/C-210

F5/F6 $\times$ C-39/C-310/C-49/C-410

$\mathrm{F} 6 \times \mathrm{C}-410 / \mathrm{C}-510$

\author{
FUEL SPILL \\ RATE \\ RELATIVE \\ CATEGORIES \\ PROBABILITY
}

$\begin{array}{cc}1 & .023463 \\ 1 \times 2 & .012136 \\ 1 \times 3 & .012136 \\ 1 \times 3 & .012136 \\ 3 \times 1 & .025890 \\ 3 \times 2 \times 1 & .024272 \\ 3 \times 2 \times 1 & .014159 \\ 2 \times 2 \times 1 & .020227 \\ 2 \times 2 \times 1 & .020227 \\ 2 \times 2 & .020227 \\ 3 \times 1 \times 2 & .014968 \\ 2 \times 1 \times 2 & .025890 \\ 1 \times 1 & .009709 \\ 1 \times 1 & .014563\end{array}$


C. COLLISION IN LOW SPEED IMPACT RANGE

$\begin{array}{cl}1 & \text { C-15/C-25/C-35/C-45 } \\ 2 & \text { C-15/C-25/C-35/C-45 } \\ 3 & \text { F1 } \times \text { C-14/C-15/C-24/C-25 } \\ 4 & \text { F1 } \times \text { C-15/C-25 } \\ 5 & \text { F1/F2 } \times \text { R2/R3/R4/R5 } \\ 6 & \text { F1/F2 } \times \text { R1/R2/R3/R4/R5 } \\ 7 & \text { F1/F2/F3 } \times \text { R1/R2/R3/R4/R5 } \\ 8 & \text { F2/F3 } \times \text { R2/R3/R4/R5 } \\ 9 & \text { F3/F4/F5 } \times \text { R2/R3/R4/R5 } \\ 10 & \text { F5/F6 } \times \text { R3/R4/R5 } \\ 11 & \text { F5/F6 } \times \text { R2/R3/R4/R5 } \\ 12 & \text { F5/F6 } \times \text { R4/R5 } \times \text { C-110/C-111 } \times \text { C19 } \\ 13 & \text { F5/F6 } \times \text { C-19/C-110/C-29/C-210/C-39/C-310 } \\ 14 & \text { F6 } \times \text { C-110/C-210/C-310 }\end{array}$

$\begin{array}{cc}1 & .023463 \\ 2 & .012136 \\ 1 \times 2 & .012136 \\ 1 \times 2 & .012136 \\ 3 \times 1 & .025890 \\ 3 \times 2 & .024272 \\ 2 \times 1 & .014159 \\ 2 \times 1 & .020227 \\ 2 \times 1 & .020227 \\ 2 \times 1 & .020227 \\ 3 \times 1 & .014968 \\ 2 \times 1 \times 1 \times 1 & .025890 \\ 1 \times 1 & .009709 \\ 1 \times 1 & .014563\end{array}$


TABLE D-4d. FUEL SPILL LOCATIONS, RATES AND PROBABILITIES FOR $45^{\circ}$ FROM FRONT COLLISION INTO B-1B

A. COLLISION IN HIGH SPEED IMPACT RANGE

COLLISION
REGION

1

2

3

4

5

6

7

8

9

10

11

12

\section{B. COLLISION IN MEDIUM SPEED IMPACT RANGE}

\section{COLLISION IN LOW SPEED IMPACT RANGE}

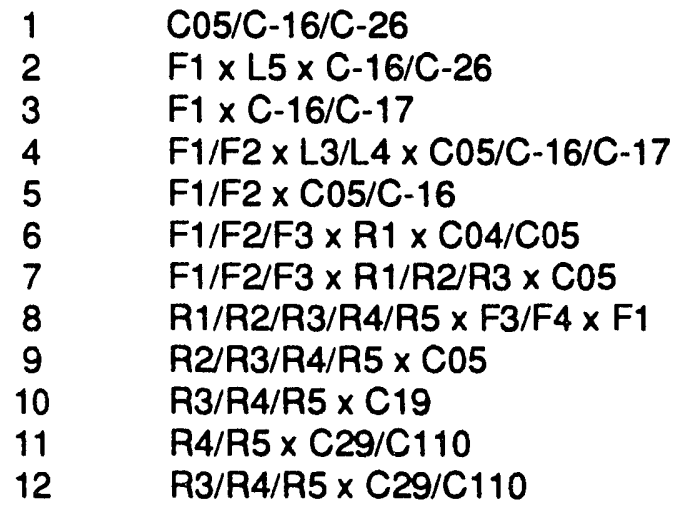

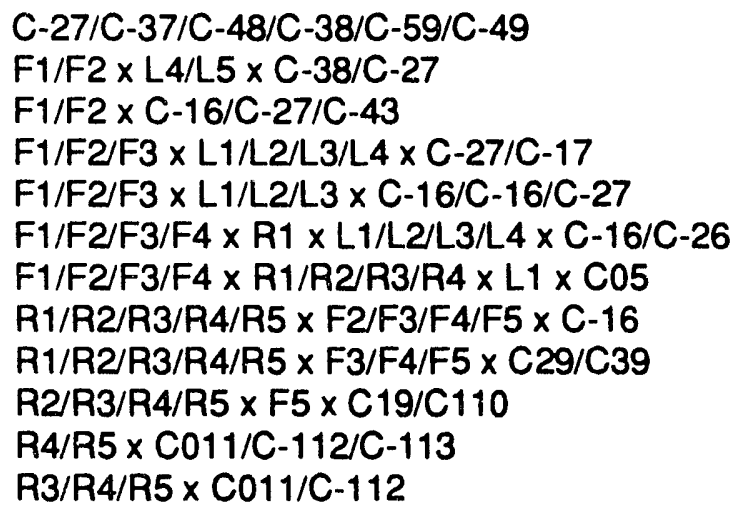

\author{
FUEL SPILL \\ RATE \\ CATEGORIES \\ RELATIVE \\ PROBABILITY
}

1
$1 \times 1 \times 2$
$2 \times 1 \times 2$
$2 \times 2 \times 3$
$2 \times 3 \times 3$
$3 \times 2 \times 2 \times 2$
$3 \times 3 \times 1 \times 3$
$3 \times 2 \times 3$
$3 \times 3 \times 1 \times 1$
$2 \times 1 \times 2$
$2 \times 3$
$2 \times 2$

.044369

.025597

.029010

.032423

.013652

.068259

.039249

.046075

.058020

.044369

.023891

.075085

$\begin{array}{cc}1 & .022184 \\ 1 \times 1 \times 1 & .012799 \\ 2 \times 2 & .014505 \\ 2 \times 3 \times 3 & .016212 \\ 2 \times 3 \times 3 & .006826 \\ 2 \times 2 \times 2 \times 2 & .034130 \\ 3 \times 2 \times 1 \times 2 & .019625 \\ 3 \times 2 \times 2 & .023038 \\ 2 \times 2 \times 2 & .029010 \\ 2 \times 1 \times 2 & .022184 \\ 2 \times 2 & .011945 \\ 1 \times 2 & .037543\end{array}$

$\begin{array}{cc}1 & .022184 \\ 1 \times 1 \times 1 & .012799 \\ 1 \times 2 & .014505 \\ 3 \times 1 \times 1 & .016212 \\ 1 \times 2 & .006826 \\ 2 \times 1 \times 2 & .034130 \\ 2 \times 1 \times 2 & .019625 \\ 2 \times 1 \times 2 & .023038 \\ 2 \times 1 & .029010 \\ 2 \times 2 & .022184 \\ 2 \times 2 & .011945 \\ 1 \times 1 & .037543\end{array}$




\section{Appendix E \\ COMPUTER PROGRAM: LISTING, EXPLANATION, USER INSTRUCTION AND EXAMPLE OUTPUT}

The computer program called "TPROB6" was developed to evaluate the probability of cargo (weapon) exposure to given flame temperatures (program language FORTRAN 77L). The details and use of TPROB6 are described in Appendix E. A listing of TPROB6 which includes program line numbers is given below, and the discussion throughout Appendix $\mathrm{E}$ makes frequent reference to the program in terms of line numbers and statement number; a readily accessible copy of the program listing will facilitate comprehensive reading of Appendix E.

Four output files created by the program and a summary of the required input to the program are described in the first 71 lines of the program listing. The first output file, named "PROBOUT," provides a self-explanatory summary of results; two examples of the PROBOUT file are given on Figure E-6. The remaining three output files are number files of results, and are generated for convenience of plotting; the details of these files are described on lines 8 through 25 of the program listing.

Data is input into the program in two ways, by program calling of number data files, and as a number in various statements of the program. The input number files that must be available for call by the program are:

(1), the flame temperature files, which give the time-average temperature vs location in the fire for various wind speeds and fuel spill rates, (2), the weather files for each of the twelve air bases, which give the relative probability that an accident will occur at a given time of day with given wind speeds and given wind directions, and

(3), the fuel spill location/rate/probability distribution files for various collision angles into the B-1B and the B-52, and for engine start accidents.

These files are described in detail below and in lines 27 through 71 of the program.

All other data required is input as a number in various statements of the program and specifically within a program section identified in the program as "INPUT DATA." This input data establishes the conditions of the accident to be evaluated, including the air base of interest, ground vs collision accident, parking directions of alert aircraft, location of cargo (weapon), and so on. The INPUT DATA section of the program is on lines 83 through 187 of the program, and the comments in this program section explain the meaning of each input. 
The program always evaluates the probability that the exposure will exceed given temperatures for two accident cases, where the two cases may differ in any one of or any combination of the following three ways: (1), in the pointing direction of the alert aircraft, (2), for collision accidents, in the angle of collision in to the alert aircraft (broadside, rear-end, etc.), and (3), for collision accidents, in that one case may be a collision into an alert aircraft by a landing aircraft, the other by an aircraft on take-off. (The two cases are always for the same air base and same type of alert aircraft, and both are for a ground accident or a collision accident.) Thus, input data in the INPUT DATA section will often require data input pairs corresponding to the conditions of the first and second cases to be evaluated, and the program (and summary output file) will often refer to the first and second accident cases.

Once the probability that exposure will exceed given temperatures is evaluated for each of the two accident cases, these probabilities are statistically weighted to provide an overall probability that the exposure will exceed given temperatures. The information. required for statistical weighting (also input in the INPUT DATA section) is the ratio of landing to take-off collisions that result in fires for the collision accidents (input as RLTO in the INPUT DATA section), and is the number of alert aircraft pointed in each of the two directions for ground accidents (input as values for NLA and NTA in the INPUT DATA section).

As described on lines 112 through 141, the values assigned for NLANDT and NLAND2 establish whether the evaluation is for the ground accident or collision accident, and if it is a collision accident, whether the two cases are both for landing collisions, both for take-off collisions, or a mixture (the mixture is usually the pair of interest). It should be recognized that there are wind directions that are excluded in a landing collision accident case or a take-off collision case; the reason for these excluded directions is explained early in Section 3.4. The program automatically makes these exclusions once the air base and landing or take-off collision has been designated. Input related to fuel spill distribution is described and values are assigned on lines 155 through 183. The spill related values to be assigned for the first accident case are NDISTR, NSPTOT, NSINGL, NDOUBL and NTRIPL, and correspondingly for the second case accident are NDIST2, NSPTO2, NSING2, NDOUB2 and NTRIP2. The meaning of these input parameters is also given on lines 155 through 183. These input parameters depend on the type of accident (ground vs collision), type of alert aircraft, and for collision accidents on the angle of collision; values for these parameters are given in Table E-1. By way of example, suppose that the first 
accident case of interest is a broadside collision into a B-1B; then from Table E-1, the assigned values would be: NDISTR $=5, \mathrm{NSINGL}=11, \mathrm{NDOUBL}=291$, NTRIPL $=1152$, and NSPTOT $=1184 .^{*}$

\section{TABLE E-1. Fuel Spill Distribution Data Required for Operation of TPROB6 Program}

\section{(a) B-1B Collision Accident}

\begin{tabular}{|c|c|c|c|c|}
\hline $\begin{array}{l}\text { DIRECTION } \\
\text { ALERT AIRCRAFT } \\
\text { IMPACTED } \\
\text { INPUT } \\
\text { DESIGNATION }\end{array}$ & $\begin{array}{l}\text { DIRECT } \\
\text { REAR } \\
\end{array}$ & $\begin{array}{l}45 \text { deg } \\
\text { FROM } \\
\text { REAR }\end{array}$ & BROADSIDE & $\begin{array}{l}45 \text { deg } \\
\text { FROM } \\
\text { FRONT }\end{array}$ \\
\hline $\begin{array}{l}\text { DISTRIBUTION \#; } \\
\text { NDISTR or NDIST2 = }\end{array}$ & 3 & 4 & 5 & 6 \\
\hline $\begin{array}{l}\text { NUMBER OF SINGLE } \\
\text { SPILL LOCATION CASES; } \\
\text { NSINGL or NSING2 = }\end{array}$ & 40 & 10 & 11 & 12 \\
\hline $\begin{array}{l}\text { SUM OF NUMBER OF SINGLE } \\
\text { AND DOUBLE SPILL } \\
\text { LOCATION CASES; } \\
\text { NDOUBL Or NDOUB2 = }\end{array}$ & 196 & 292 & 291 & 118 \\
\hline $\begin{array}{c}\text { SUM OF NUMBER OF } \\
\text { SINGLE, DOUBLE AND } \\
\text { TRIPLE SPILL LOCATION } \\
\text { CASES; } \\
\text { NTRIPL or NTRIP2 = }\end{array}$ & 2206 & 1168 & 1152 & 1126 \\
\hline $\begin{array}{l}\text { TOTAL NUMBER OF CASES; } \\
\text { NSPTOT or NSPTO2 = }\end{array}$ & 2998 & 1216 & 1184 & 1766 \\
\hline
\end{tabular}

* For a given selection for the value of the distribution number NDISTR, the four associated values of NSINGL, NDOUBL, NTRIPL and NSPTOT are inherently established, as specified in Table E-1. The program could thereby have been written to reduce the input burden by automatic assignment of the four associated values. However, circumstances frequently occurred throughout the project where the flexibility of manipulating distributions was desirable, and this program flexibility has been retained. 
TABLE E-1 (b). B-52 Collision Accident

\begin{tabular}{|c|c|c|c|c|}
\hline $\begin{array}{l}\text { DIRECTION ALERT } \\
\text { AIRCRAFT } \\
\text { IMPACTED } \\
\text { FROM } \\
\text { INPUT } \\
\text { DESIGNATION }\end{array}$ & $\begin{array}{l}\text { DIRECT } \\
\text { REAR }\end{array}$ & $\begin{array}{l}45 \mathrm{deg} \\
\text { FROM } \\
\text { REAR }\end{array}$ & BROADSIDE & $\begin{array}{l}45 \text { deg } \\
\text { FROM } \\
\text { FRONT }\end{array}$ \\
\hline $\begin{array}{l}\text { DISTRIBUTION \#; } \\
\text { NDISTR or NDIST2 = }\end{array}$ & 8 & 9 & 10 & 11 \\
\hline $\begin{array}{c}\text { NUMBER OF SINGLE SPILL } \\
\text { LOCATION CASES; } \\
\text { NSINGL or NSING2 = }\end{array}$ & 6 & 3 & 21 & 5 \\
\hline $\begin{array}{c}\text { SUM OF NUMBER OF } \\
\text { SINGLE AND DOUBLE SPILL } \\
\text { LOCATION CASES; } \\
\text { NDOUBL or NDOUB2 = }\end{array}$ & 550 & 237 & 159 & 179 \\
\hline $\begin{array}{l}\text { SUM OF NUMBER OF } \\
\text { SINGLE, DOUBLE AND } \\
\text { TRIPLE SPILL } \\
\text { LOCATION CASES; } \\
\text { NTRIPL Or NTRIP2 = }\end{array}$ & 1534 & 795 & 867 & 509 \\
\hline $\begin{array}{l}\text { TOTAL NUMBER OF } \\
\text { CASES; } \\
\text { NSPTOT or NSPTO2 = }\end{array}$ & 2718 & 859 & 1019 & 829 \\
\hline
\end{tabular}


TABLE E-1 (c). Ground Accident

\begin{tabular}{|c|c|c|}
\hline $\begin{array}{c}\text { TYPE OF } \\
\text { ALERT AIRCRAFT } \\
\text { DESIGNATION }\end{array}$ & B-1B & B-52 \\
\hline DISTRIBUTION \#; NDISTR Or NDIST2 $=$ & 12 & 13 \\
\hline $\begin{array}{c}\text { NUMBER OF SINGLE SPILL LOCATION } \\
\text { CASES; } \\
\text { NSINGL Or NSING2 }\end{array}$ & 4 & 4 \\
\hline $\begin{array}{c}\text { SUM OF NUMBER OF SINGLE AND } \\
\text { DOUBLE SPILL } \\
\text { LOCATION CASES; NDOUBL Or NDOUB2 }\end{array}$ & $\cdots$ & $\cdots$ \\
\hline $\begin{array}{c}\text { SUM OF NUMBER OF SINGLE,DOUBLE } \\
\text { AND TRIPLE SPILL LOCATION CASES; } \\
\text { NTRIPL OR NTRIP2 }=\end{array}$ & $\cdots$ & 4 \\
\hline $\begin{array}{c}\text { TOTAL NUMBER OF CASES; } \\
\text { NSPTOT Or NSPTO2 }\end{array}$ & $-\cdots$ & \\
\hline
\end{tabular}

For collision accidents, it must also be specified in the input (see lines 176 through 183) whether the collision is on the right or left (or rear) of the alert aircraft (LEFTRI and LEFTR2). The fuel location/rate/probability distributions were developed on the basis of collisions on the right side (and rear) of the alert aircraft. For an equivalent angle from the rear, the distributions for a collision from the left are the same as those for the right except that the $X$ coordinate value of the spill location differs in sign. The program automatically converts the sign of the $X$ coordinate value when a left side collision is specified (conversion at lines 858 through 863 ).

In the next stage of the program, appropriate temperature and weather number files are opened and read into the program (lines 305 through 464). Temperatures are read into the program as subscripted (integer) variables, 
NT\#(I), where the \# corresponds to the test numbers $(3,4,6,7,8$ or 9$)$ and each I corresponds to a different location in the fire.

Regarding weather files, the relative probabilities of an accident at a given time of day, at a given wind speed and at a given wind direction are read in (lines 365 through 464) as the subscripted variable $\mathrm{PW}(\mathrm{I})$, where I corresponds to a given time/speed/direction combination. The weather probability distribution file is opened and read in only for the air base of interest (the air base assigned at line 111). The organization of the weather files is described in lines 58 through 71 of the program (and in Appendix C).

It was mentioned above that for collision accidents, only wind directions which have a component of air flow opposing the motion of the aircraft landing or taking-off are included in the calculation. It is then necessary to identify angles that are perpendicular to the runway, and this is done on lines 466 through 480. This is evaluated from the value of "ANGLRW," which is defined as the direction of motion of an aircraft landing on the alert pad end of the runway; ANGLRW depends solely on the selection of airbase, and it's value is automatically assigned at lines 365 through 464 when the weather data file for the selected base is opened and read in. It can be seen in line 466 through 480 that the angles perpendicular to the runway are determined in terms of the angle clockwise of ANGLRW divided by 10, "NCW," and the angle counter clockwise of ANGLRW divided by 10, "NCC." NCW and NCC are then applied later in the program when wind direction restrictions are imposed for collision accidents.

The program next evaluates (lines 490 through 518) what is called the "cargo (or weapon) volume of analysis," which simply identifies the location of the weapon "volume" in the aircraft coordinate system (the coordinate system attached to the alert aircraft). This volume location then depends on the selected height and horizontal position of the weapon, weapon length, and the selected weapon bay in the case of the B-1B (see weapon input data on lines 87 through 95). From lines 515 through 518 it can be seen that the width of the volume of analysis ( $X$ coordinate dimension) is always 2.5 feet, extending 1.25 feet in either $X$ direction from the selected position of the axis of the weapon (axis position selected on lines 92/93). The diameter of all weapons considered is less than 2.5 feet, and a 2.5 foot minimum width dimension was chosen to insure that the volume of analysis encompasses one or more temperature data locations (recall that temperature data is arranged over horizontal girds with 2.5 foot spacing). For similar reasons, the volume of analysis is 2.5 feet longer than the actual input length of the weapon (1.25 feet is added to each end at lines 503/504). With regard to the vertical dimensions, the temperature data is given at the heights of $1,4,7$ and 10 feet, and the temperature data used is for the height that most nearly approaches the input weapon height (see lines 505 through 514). Later in Appendix E, it will be seen that the flame exposure analysis will involve a 
transformation of the location of the volume in the aircraft coordinate system to the so called fire coordinate system for each set of conditions considered (each winc speed, wind direction, etc.).

It was explained above that the program always evaluates two accident cases. After the first acidident case has been evaluated, input conditions for evaluating the second case are automatically assigned, and program control is returned to statement 860 (line 522) for evaluation of the second case. The value of NDEC, first assigned the value of 1 at line 520, controls whether the program is evaluating the first or second case accident. After the first case accident has been evaluated, NDEC is assigned the value of 2 at statement 873 (line 1588).

It is noted that the integer variables "LL" and "KK" are assigned an initial value of 1 at lines 551 and 552, respectively. It will be seen below that these two variables are used as counters to control the number of cycles in the two major calculational loops. LL is the counter for the weather variation loop, and KK for the fuel spill location/rate variation loop.

T. e program next opens and reads in the appropriate set of fuel spill ristribution files (lines 555 through 856), where a set comprises the four files $\therefore C R \#$, YCR\#, NRATE\# and PRF\#. The symbol "\#" takes on the assigned value of NDISTR, where the value of NDISTR specifies the type of accident; for example, a B-52 ground accident (NDISTR = 13), a broadside collision into a B-1B (NDISTR = 5), and so on. The correspondence between the type of accident and the NDISTR value is given on lines 555 through 630 . The values in the XCR\# and $Y C R \#$ files represent, respectively, the $X$ and $Y$ coordinate values of spill locati ns in the aircraft coordinate system (in feet). The values in the NRATE\# file are 1,2 or 3 corresponding to the fuel spill rate of 0 to $225 \mathrm{gpm}, 225$ to $45 \mathrm{C}$ gpri, or greater than $450 \mathrm{gpm}$, respectively. Values in the PRF\# files represent the relative probability that a fuel spill will occur at a given $(X, Y)$ coordinate location within a given spill rate range. Each of the four files is a onedimensional file, that is, there is one value per file line. The fi." "s are arranged so that the probability valixe on a given line of the PRF\# file corresponds to the probability that a spill svill occur at the $(X, Y)$ location given on the same line of the XCR\# and YCR\# files and at the rate given on the same line of the NRATE\# tile. Thus, the four files for a given "\#" value (given type of accident) must be equal in length (the same number of file lines), but the file lengths will vary with the value of "\#". The values in the four files XCR\#, YCR\#, NRATE\# and PRF\# are read into the program (lines 652 through 856 ) as the subscripted variables XCOORD (I), YCOORD (I), NSR (I) and PFS (I), respectively, where I ranges from 1 to the total number of spills involved for the particular type of accident, NSPTOT.

The main calculational loop commences at statement 206 (line 875), and an internal l- Jp at statement 250 (line 955); a highly abbreviated block diagram of these loops is shown in Figure E-1. Starting at statement 206, the mathematics is 


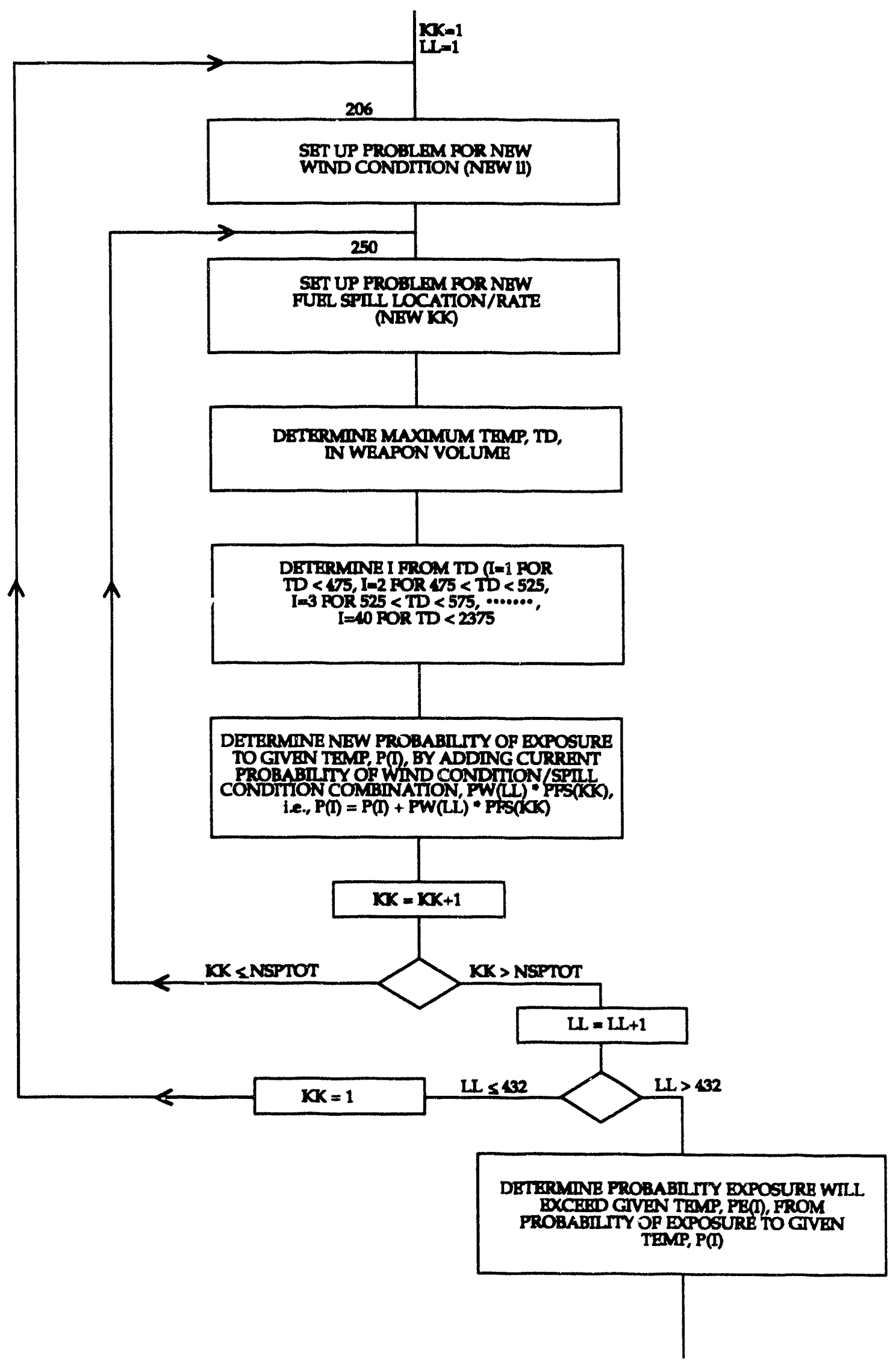

Figure E-1 
set-up for the current wind speed and wind directions according to the current value of LL. Then the mathematics is set-up for the current fuel spill location/rate according to the current value of KK. On the basis of the current wind condition/fuel spill condition combination, the temperatures at various locations within the weapon volume are determined. The maximum from among these temperatures, TD, is then determined. The particular temperature range that the current TD falls within is now identified; specifically, a value for "I" is determined, where $\mathrm{I}=1$ for $\mathrm{TD}<475^{\circ} \mathrm{F}, \mathrm{I}=2$ for $475^{\circ} \mathrm{F}<\mathrm{TD}<525^{\circ} \mathrm{F}$, $\mathrm{I}=3$ for $525^{\circ} \mathrm{F}<\mathrm{TD}<575^{\circ} \mathrm{F}, \ldots \ldots, \mathrm{I}=40$ for $\mathrm{TD}>2375^{\circ} \mathrm{F}$. Subsequently, the contribution for the current wind/spill condition combination to the probability of weapon exposure, $\mathrm{P}(\mathrm{I})$, to the temperature range associated with $\mathrm{I}$, is evaluated. For example, if TD was within the temperature range 525 to $575^{\circ} \mathrm{F}$, then the probability of exposure to this temperature range, $\mathrm{P}(3)$, would be increased by the probability of the current wind/spill combination, $\mathrm{PW}(\mathrm{LL}) * \mathrm{PFS}(\mathrm{KK})$. The value of KK is now increased by 1 . If not all of the spill location/rate combinations have been considered (if KK $\leq$ NSPTOT, the total number of spill combinations), program control will be returned to statement 250, and the evaluation will be repeated for the next spill combination (but with the same wind conditions). When KK eventually exceeds NSPTOT, the value of $L L$ is increased by 1 . If not all wind condition combinations have been considered (if LL $\leq 432$ ), $\mathrm{KK}$ will be set to 1 , and program control returned to statement 206 where the mathematics of the next wind condition combination will be set up. When the value of LL eventually exceeds 432 , the probability that the exposure will exceed a given temperature, $\mathrm{PE}(\mathrm{I})$, is evaluated from the probability of exposure to a given temperature, $\mathrm{P}(\mathrm{I})$. The above program organization is given in greater detail later.

The discussion returns now to the start of the calculational loops for a more detailed description of the program. At statement 206 (line 875), the wind speed category, NWS, is first determined on the basis of the current value of LL (NWS is 1,2 or 3 corresponding to the speed ranges $0-10 \mathrm{mph}, 10-20 \mathrm{mph}$, or $>20 \mathrm{mph}$, respectively). Next the wind direction category, NWD, is determined on the basis of the current value of KK (NWD is $1,2, \ldots \ldots, 36$ corresponding to the ranges 355 to 5 degrees, 5 to 15 degrees, ...., 345 to 355 degrees, respectively). At line 923 through 942 , wind directions are eliminated which do not have a component of wind flow opposing the motion of the aircraft landing or taking off. If the accident case is a ground accident, wind directions restrictions are not imposed; for ground accidents, at statement 220 (line 921), the direction restrictions are by-passed (to statement 248, line 943). At lines 943 through 953, the sine and cosine are determined for the angle between the wind flow direction and the direction the alert aircraft is parked (the positive $Y$ direction in the aircraft coordinate system). 
At the start of the internal loop, there is a coordinate transformation of the corner points of the weapon volume from the aircraft coordinate system to the so called fire coordinate system, where the $Y$ and $X$ axes of the fire coordinate system are parallel and perpendicular to the wind flow direction, respectively, and where the origin is at the fuel spill point. This transformation defines a rectangle in the fire coordinate system that encompasses the weapon volume, where the rectangle is defined by the four coordinate values, XMA, XMI, YMA and YMI. Subsequently, the temperature data grid points (2.5 foot spacing) within the above rectangle are identified (see lines 965 through 968); an illustration of the grid points (as dots) within the rectangle is given on Figure E-2 for three spill points and a wind direction of 20 degrees. Finally, the grid points solely within the weapon volume in the fire coordinate system are identified (lines 1009 through 1035) by demanding that the perpendicular distance from the center line of the volume to a given grid point in the rectangle is under 1.3 feet. The grid points within the volume are shown as circled points in Figure $\mathrm{E}-2$. The coordinates of these points are given as $\mathrm{XF}(\mathrm{I})$ and $\mathrm{YF}(\mathrm{I})$ on lines 1023 and 1024, and the number of these points is given as NPOINT on line 1035.

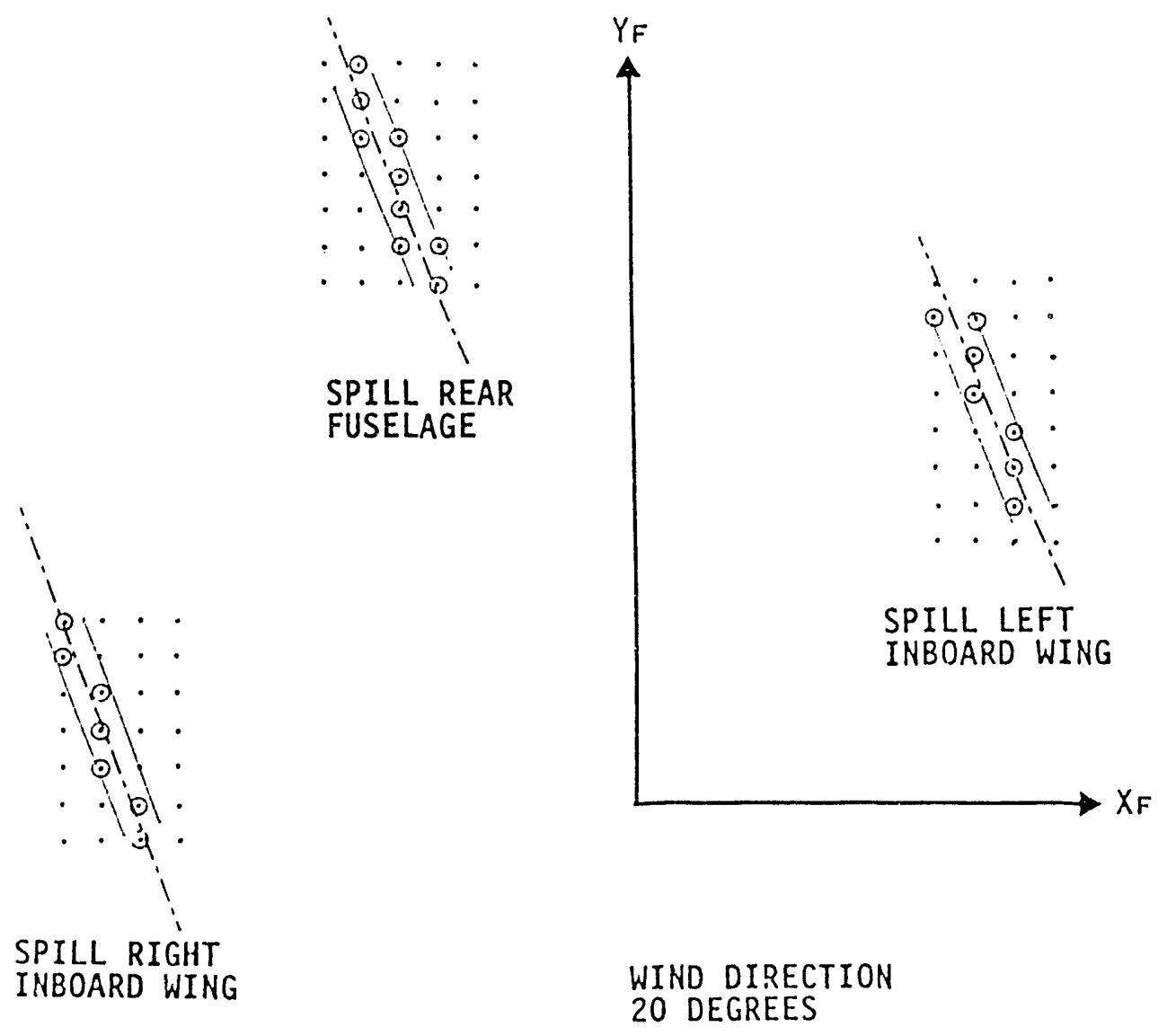

Figure E-2. Cargo Volume in Fire Coordinate System 
Once the grid points within the weapon volume have been identified, the flame temperature at these points is determined. This is done for each of the NPOINT grid points on lines 1039 through 1242. The general control organization of this program section is shown in the block diagram given on Figure E-3. Control enters this section of the program with current values for: NWS, the wind speed category; NSR(KK), the spill rate category; NPOINT, the number of temperature grid points within the weapon volume; and $\mathrm{XF}(\mathrm{I}), \mathrm{YF}(\mathrm{I})$ and $\mathrm{ZF}$, the coordinate values of the grid points from $\mathrm{I}=1$ to $\mathrm{I}=\mathrm{NPOINT}$. The output temperatures in the volume are NTB(I) from I=1 to I=NPOINT. Control is subsequently shifted to statement 500 (line 1245). At lines 1245 through 1248, the values of NTB(I) for I larger than NPOINT and less than 19 are arbitrarily set to the value 2. Then at statement 504 (lines 1249 through 1251), the highest temperature, $\mathrm{TB}$, from the temperatures $\mathrm{NTB}(\mathrm{I})$, is selected; the highest exposure temperature along the surface of the weapon is the temperature of interest.

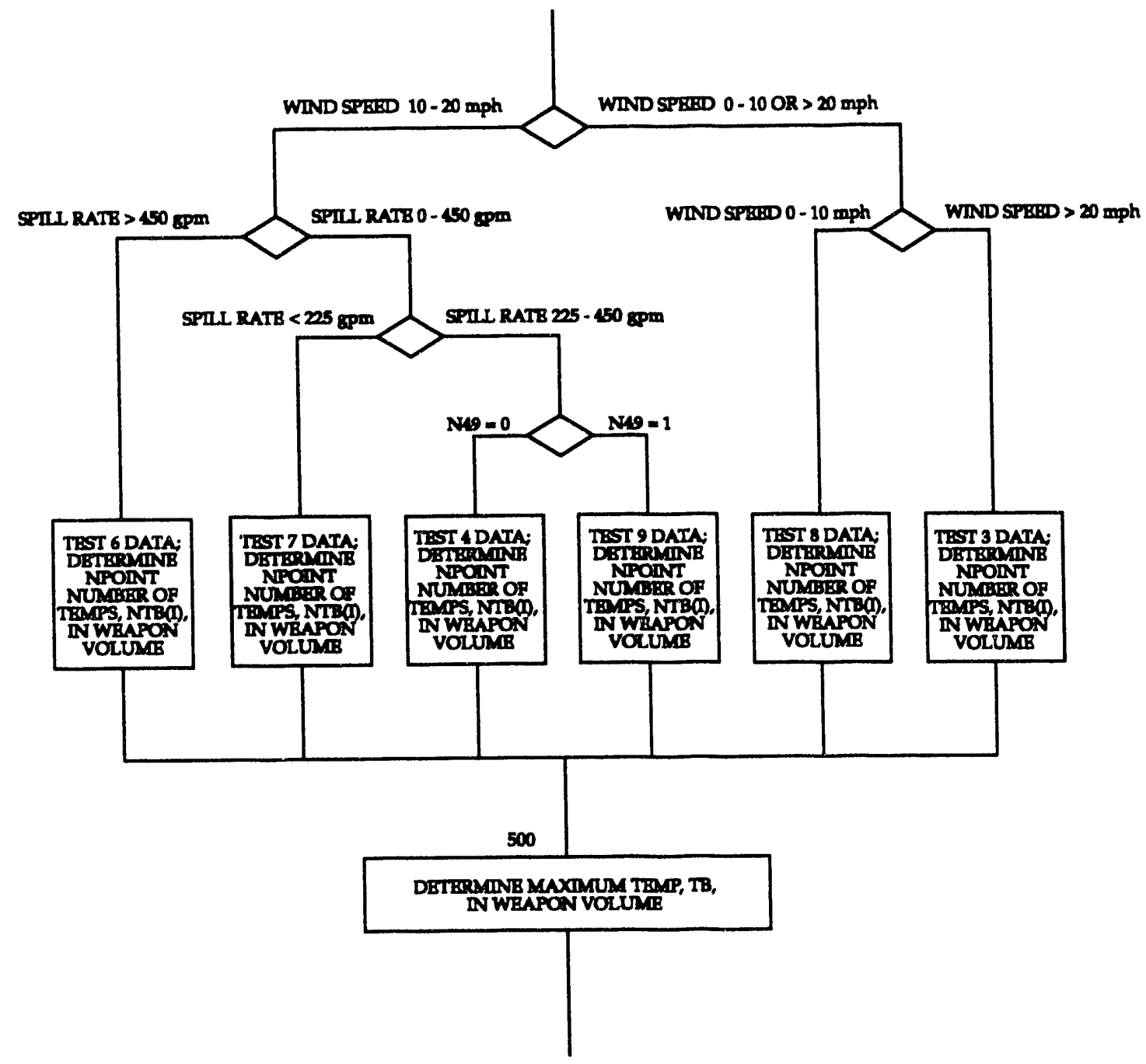

Figure E-3 
It is appropriate here to examine how the program deals with the issue of multiple concurrent fuel spills. As described in Sections 2, 3, and Appendix D, a collision at a given collision angle, lateral position and collision impact speed can result in fuel tank breaches at one or more than one location, so that there can be concurrent fires. Usually, the flame from only one of the fires will expose the weapon for a given set of conditions (wind direction, etc.), and the idea with multiple concurrent spills is to determine which fire is causing the exposure (or the most severe exposure) of the weapon. The program individually determines the maximum temperature in the weapon volume for each of the concurrent fires, and then retains and uses only the maximum temperature from the fire that caused the most severe exposure. In examining how the program accomplishes this, it is first noted that the fuel spill location/rate/probability files are arranged so that:

- The data for all single fires are listed first; this corresponds to values of KK $\leq$ NSINGL, that is, the data on the first NSINGL file lines is associated with single spills.

- The data for all double concurrent fires are listed second; this corresponds to values of KK such that NSINGL $<$ KK $\leq$ NDOUBL, that is, data on file lines above the value of NSINGL and less than or equal to the value of NDOUBL are associated with double concurrent spills; there are two file lines associated with each accident scenario, one of which indicates the location, spill rate and probability for one of the two fires, the other indicating the location, rate and probability for the second fire (the two probabilities are of course equal).

- The data for all triple fires are listed third; this corresponds to values of KK such that NDOUBL , KK $\leq$ NTRIPL, that is, data on file lines above the value of NDOUBL and less than or equal to the value of NTRIPL are associated with triple concurrent spills; there are three file lines associated with each accident case, where a given file line of the three indicates the location, rate and probability of one of the three concurrent fires.

- The data for all quadruple fires are listed last; this corresponds to values of KK such that NTRIPL < KK $\leq$ NSPTOT; there are four file lines associated with each accident case. 
With the above file arrangement in mind, the upper section of the diagram on Figure E-4 indicates the procedure with regard to single or multiple concurrent spills (also see lines 1260 through 1297 of the program). After the maximum temperature in the weapon volume is determined, program operation is directed to different program sections according to whether the value of $\mathrm{KK}$ is in the single, double, triple or quadruple section of the fuel spill files. If $\mathrm{KK} \leq$ NSINGL, TD is simply assigned the value of TB, and TD is applied to the evaluation of $\mathrm{P}(\mathrm{I})$, the probability of exposure to a given temperature. $\mathrm{KK}$ is then increased by 1 and a new maximum temperature, TB, is evaluated for the next fuel spill location/rate. When the value of KK becomes NSINGL + 1, program operation is directed to the double spill section (statement 510), JCOUNT is equal to 1 , and the value of TB1 is assigned the current value of TB. JCOUNT is then increased by 1 (to 2), the section for evaluating $P(1)$ is by-passed, $K K$ is increased by 1 (to NSINGL + 2), and TB for the second fire of the concurrent pair of fires is evaluated. In this instance, JCOUNT is equal to 2 , and the value of TB2 is assigned the new current value of TB. TD is then given the maximum value of the two values TB1 and TB2. JCOUNT is then reset to the value 1 (so that the next fire pair can be evaluated), and TD is applied to the evaluation of $\mathrm{P}(\mathrm{I})$. In a similar fashion, the results for triple and quadruple concurrent spills are evaluated.

Referring again to Figure E-4, the mid-section of this diagram shows (in greater detail than was shown earlier) the manner in which the probability of weapon exposure to a given temperature, $\mathrm{P}(\mathrm{I})$, is evaluated; programmatically, this evaluation occurs on lines 1304 through 1461 of the program. It can be seen that when a new value of TD is obtained, the program is directed to one of forty sub-sections according to the current value of TD. Then the probability, $\mathrm{P}(\mathrm{I})$, for the appropriate value of $I$ is increased by the product, PW(LL) * PFS(KK). 


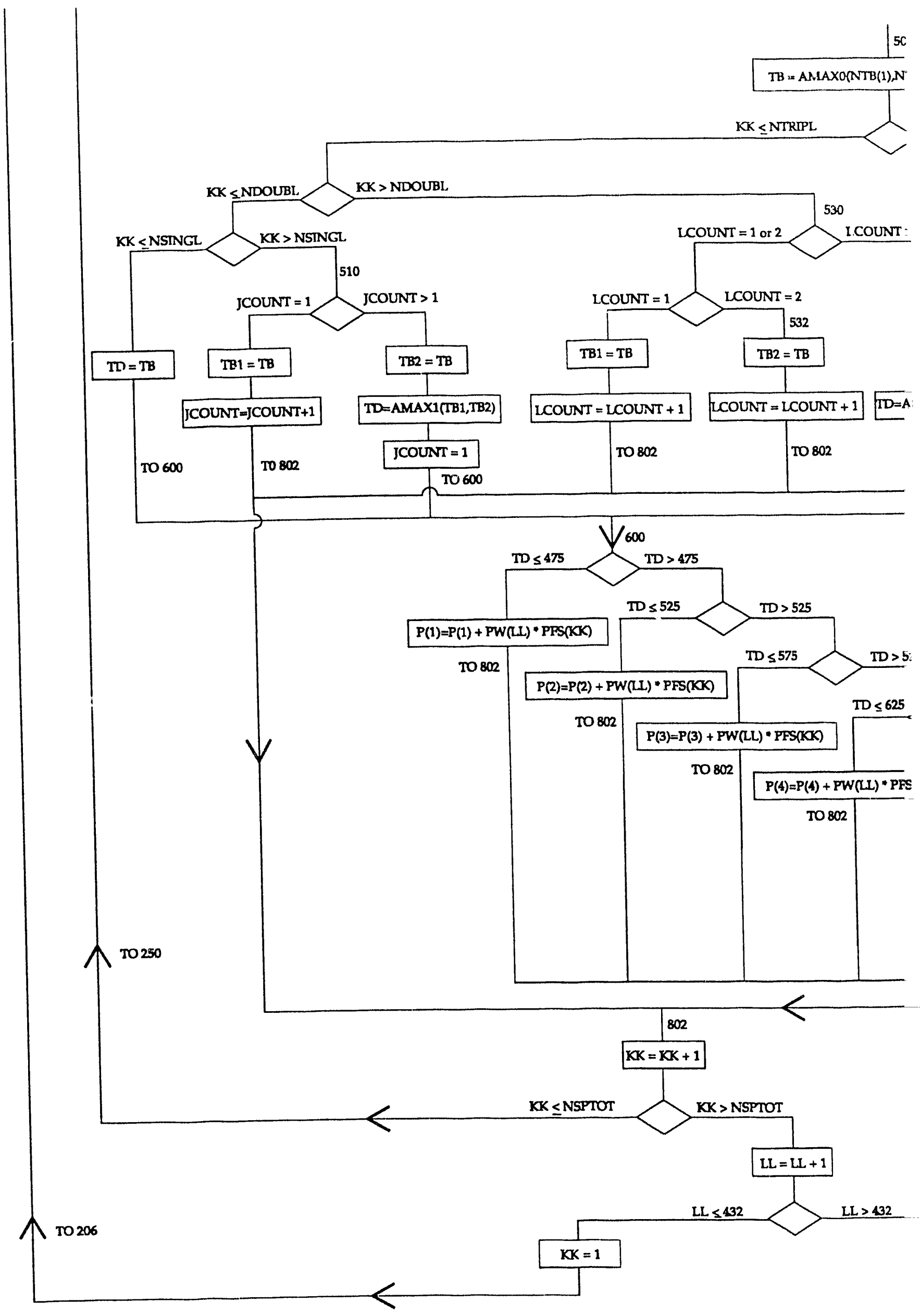

Figure E-4 


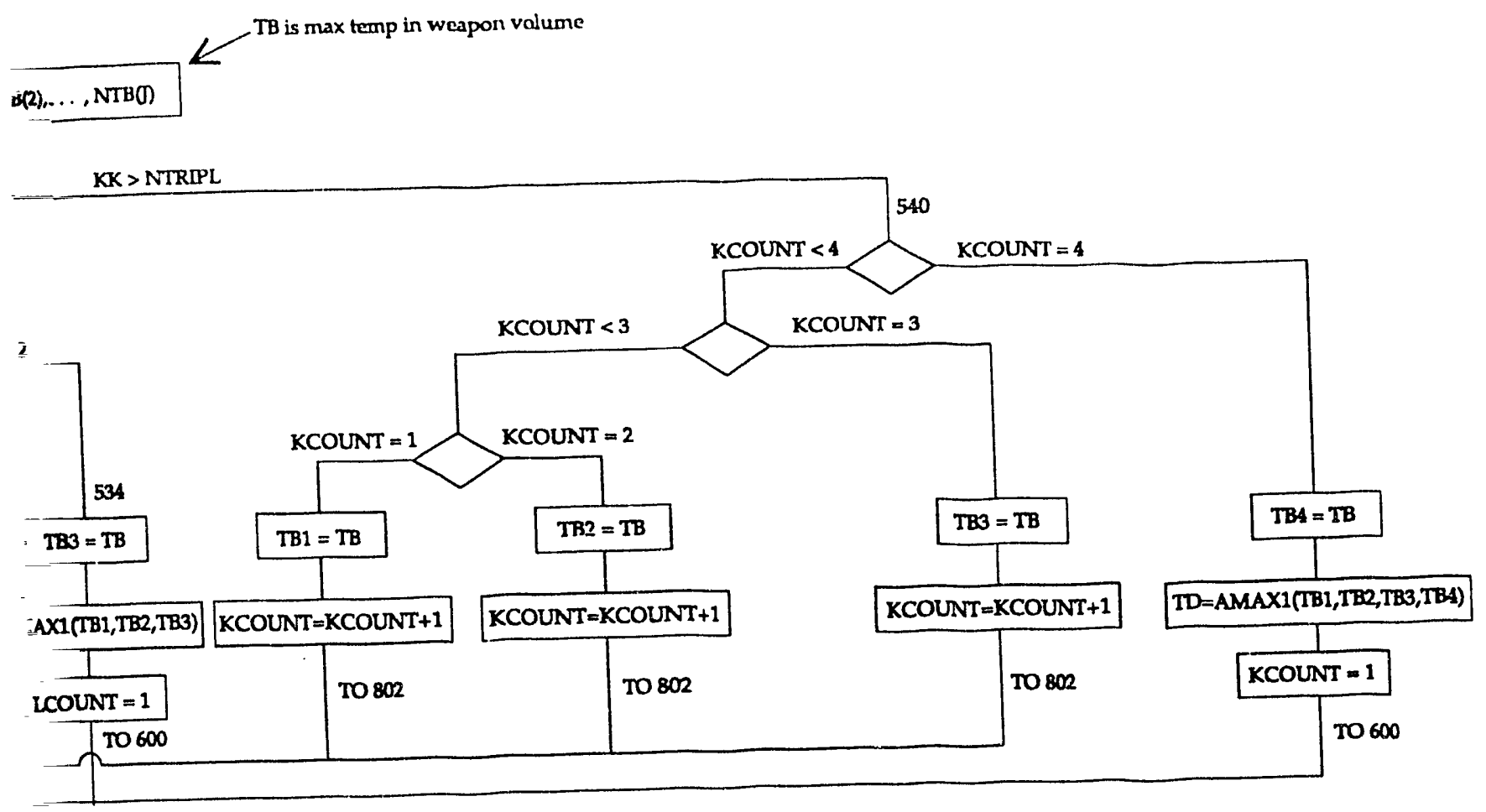

$P(1)$ is probability temp is in the Ith temp interval

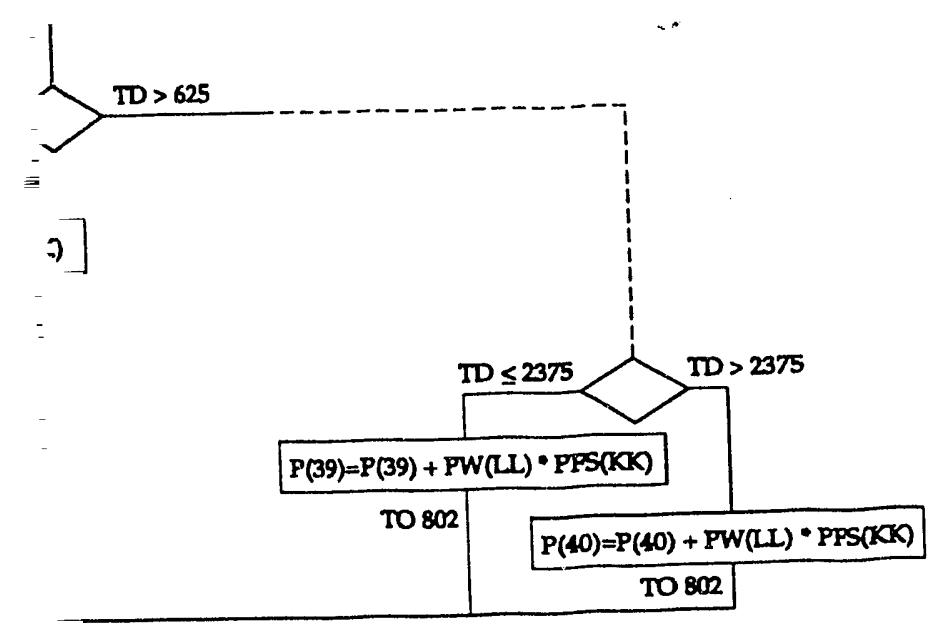




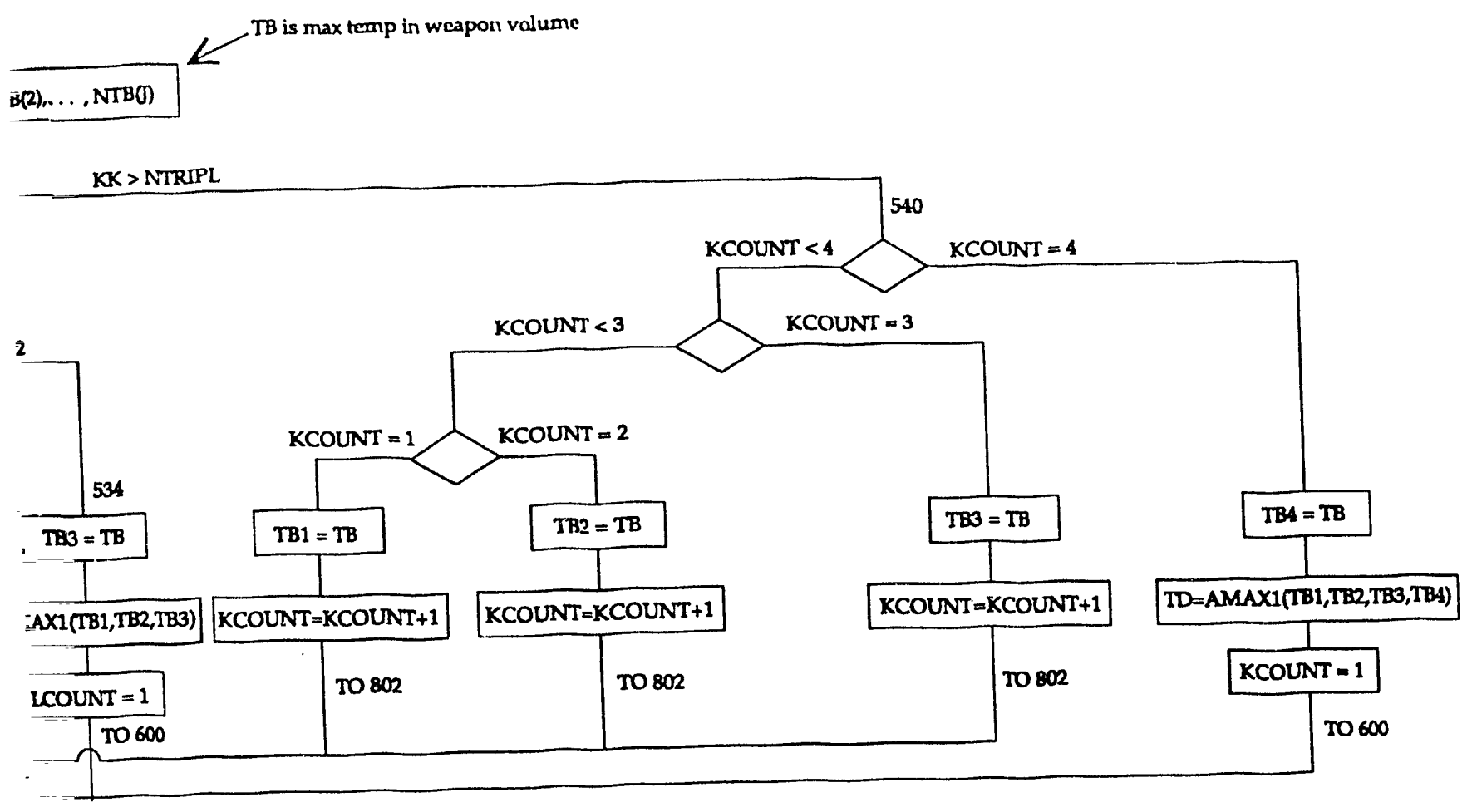

$P(M)$ is probability temp is in the Ith temp interval

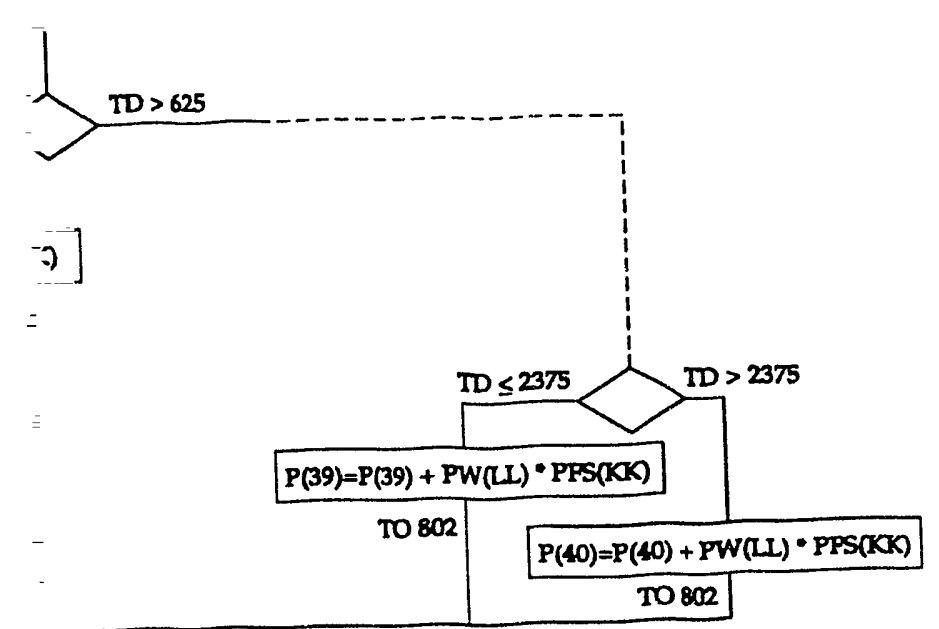

TO 830 
After $\mathrm{P}(\mathrm{I})$ is increased, it can be seen in the lower section of Figure E-4 that the value of $\mathrm{KK}$ is increased by 1 . If the new value of $\mathrm{KK}$ has not exceeded the total number of fuel spill location/rate combinations, program control is returned to statement 250 (line 957) for evaluation of exposure temperatures for the new fuel spill location/rate. If $\mathrm{KK}$ has exceeded all fuel spill conditions, the value of LL is increased by 1 . If the value of LL has not exceeded the total number of wind conditions (432), the value of $\mathrm{KK}$ is reset to 1, and program control is returned to statement 206 (line 875), and the exposure temperatures will be evaluated for all fuel spill conditions for the new set of wind conditions. When the value of LL eventually exceeds the total number of wind conditions, program control will be directed to statement 830 (line 1478).

The program from statement 830 onward pertains to: (1), generation of the output files (PROBOUT, PROBX, PROBY and PROBZ), (2), evaluation of the probability that the exposure will exceed given temperatures (PE(I) and PE1(I) for the first and second accident cases, respectively) from the probability of exposure to given temperatures $(P(I))$, and (3), changing various input parameters from first case accident values to second case accident values. A diagram showing the general organization of this final program section is given on Figure E-5. 


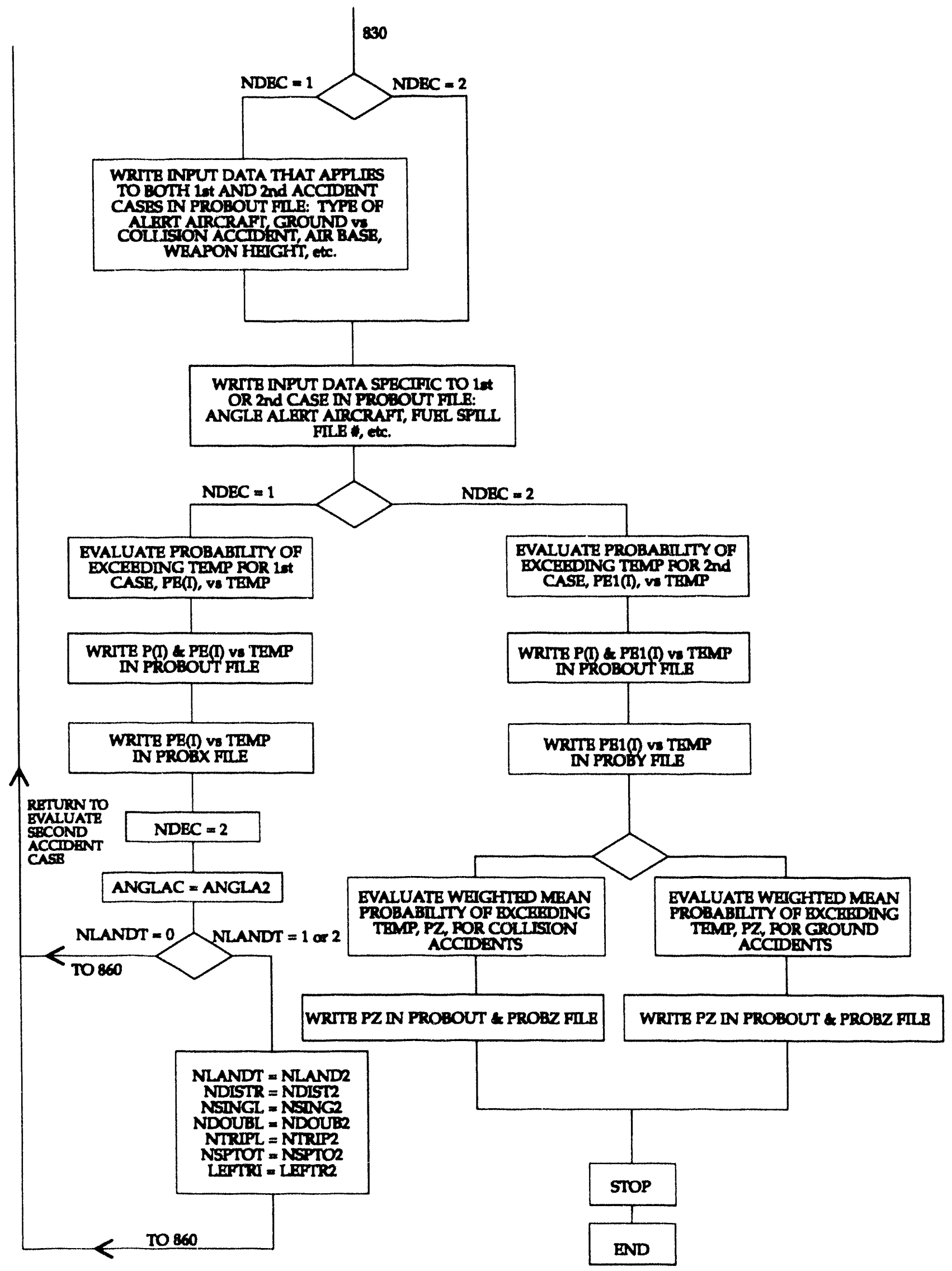

Figure E-5 


\section{PROGRAM LISTING}

PROGRAM TPROB6

(IN FORTRAN 77L)

$12 / 6 / 92$ REV

PROGRAM FOR EVALUATING THE PROBABILITY THAT THE TEMPERATURE OF AIRCRAFT CARGO FLAME EXPOSURE WILL EXCEED A GIVEN VALUE FOR AIRCRAFT ACCIDENTS INVOLVING AIRCRAFT FUEL TANK SPILLAGE.

AN OUTPUT SUMMARY OF RESULTS IS GIVEN IN A FILE CALLED 'PROBOUT'.

THERE ARE ALSO THREE OUTPUT NUMBER FILES (FOR PLOTTING PURPOSES, ETC.) CALLED 'PROBX', 'PROBY', \& 'PROBZ'. ALL THREE FILES GIVE THE PROBABILITY OF EXCEEDING A TEMPERATURE AT 50 DEG F INTERVALS FROM 500 THRU 2250 DEG F. FOR GROUND ACCIDENTS, PROBX \& PROBY ARE THE INDIVIDUAL RESULTS FOR THE TWO ALERT AIRCRAFT ORIENTATIONS (ANGLAC \& ANGLA2 BELOW), AND PROBZ IS THE MEAN OF PROBX \& PROBY RESULTS WEIGHTED ACCORDING TO THE RELATIVE NUMBER OF ALERT AIRCRAFT AT EACH ORIENTATION. FOR AIRCRAFT CRASH ACCIDENTS, PROBX \& PROBY ARE THE INDIVIDUAL RESULTS FOR TWO TYPES OF COLIISION (WHICH MAY DIFFER IN FUEL SPILL LOCATION/RATE DISTRIBUTION, ALERT AIRCRAFT ORIENTATION, LANDING VS TAKE-OFF COLLISION, OR SOME OR ALI OF THE ABOVE). WHEN BOTH LANDING AND TAKE-OFF COLLISIONS ARE INCLUUDED IN THE CALCULATION, PROBZ IS THE MEAN RESULT WEIGHTED BY THE RELATIVE FREQUENCY OF LANDING TO TAKEOFF CRASHES. WHEN LANDING ONLY OR TAKE-OFF ONLY COLLISIONS ARE INVOLVED (e.g., WHEN THE CALCULATION CONCERNS TWO TYPES OF LANDING COLLISION), PROBZ IS THE MEAN RESULT WEIGHTED AS APPROPRIATE (USUALLY WEIGHTED EQUALLY) .

CERTAIN INPUT PARAMETERS (SUCH AS AIR BASE OF INTEREST, TYPE OF AIRCRAFT ON ALERT, LOCATION OF CARGO, ETC.) ARE INSERTED BELOW IN A PROGRAM SECTION CALLED 'INPUT DATA.' OTHER INPUT INFORMTION INCLUDING FLAME TEMPERATURE DISTRIBUTIONS, WEATHER PROBABILITY DISTRIBUTIONS AT VARIOUS BASES, AND FUEL SPILL LOCATION AND RATE PROBABILITIES ARE INPUT AS DATA FILES.

FLAME TEMPERATURE DATA FOR VARIOUS WIND SPEEDS AND FUEL SPILL RATES ARE CONTAINED IN FILES CALLED 'TAX\#' FILES, WHERE \# IS $3,4,6,7,8$ OR 9 CORRESPONDING TO A FIRE TEST NUMBER DESIGNATION. TAX4 AND TAX9 FILES ARE FROM REPLICATE TESTS CORRESPONDING TO SPILI RATES OF ABOUT 300 GPM AND WIND SPEEDS OF ABOUT $16 \mathrm{MPH}$. THE OPTION OF USING TEST 4 OR TEST 9 DATA IS GIVEN BELOW (N49 VALUE).

PROBABILITY DATA FOR VARIOUS FUEL SPILL LOCATION/SPILL RATE COMBINATIONS ARE GIVEN IN FILE SETS, WITH EACH SET COMPRISING THE FILES XCR\#, YCR\#, NRATE\# \& PRF\#, WHERE '\#' IS A NUMBER ASSOCIATED WITH A GIVEN SET, AND EACH SET CORRESPONDS TO A PARTICULAR TYPE OF ACCIDENT (e.g., THE ANGLE OF COLLISION BETWEEN TWO AIRCRAFT). THE VALUES IN THE FILES XCR\# \& YCR\# CORRESPOND TO THE (X, Y) COORDINATES OF FUEL SPILL LOCATIONS FROM THE ALERT AIRCRAFT \& FOR AIRCRAFT/AIRCRAFT COLLISIONS ALSO FROM THE MOVING/COLLIDING AIRCRAFT. NRATE\# DESIGNATES THE SPILL RATE CLASS FOR EACH OF THE ABOVE LOCATIONS. THE VALUES IN PRF\# ARE THE PROBABILITIES OF EACH SPILL LOCATION/SPILI RATE COMBINATION. THE FOUR FILES OF A GIVEN SET ARE EQUAL IN LENGTH, BUT THE FILE LENGTH VARIES EROM SET TO SET (TO 2998). \#=3,4,5 \& 6 ARE COLIISION INTO REAR, 45 DEG FROM REAR, BROADSIDE \& 45 DEG EROM FRONT, RESPECTIVELY, OF B-1B. \#=8, 9,10 , \& 11 ARE COLLISIONS AS ABOVE INTO B-52. \#=12\&13 ARE GROUND ACCIDENTS OF B-1B \& B-52, RESPECTIVELY.

PROBABILITY DATA FOR THE COMBINATIONS OF WIND DIRECTION/WIND SPEED/TRAFFIC (TIME OF DAY) FOR EACH AIR BASE IS GIVEN IN FILES 


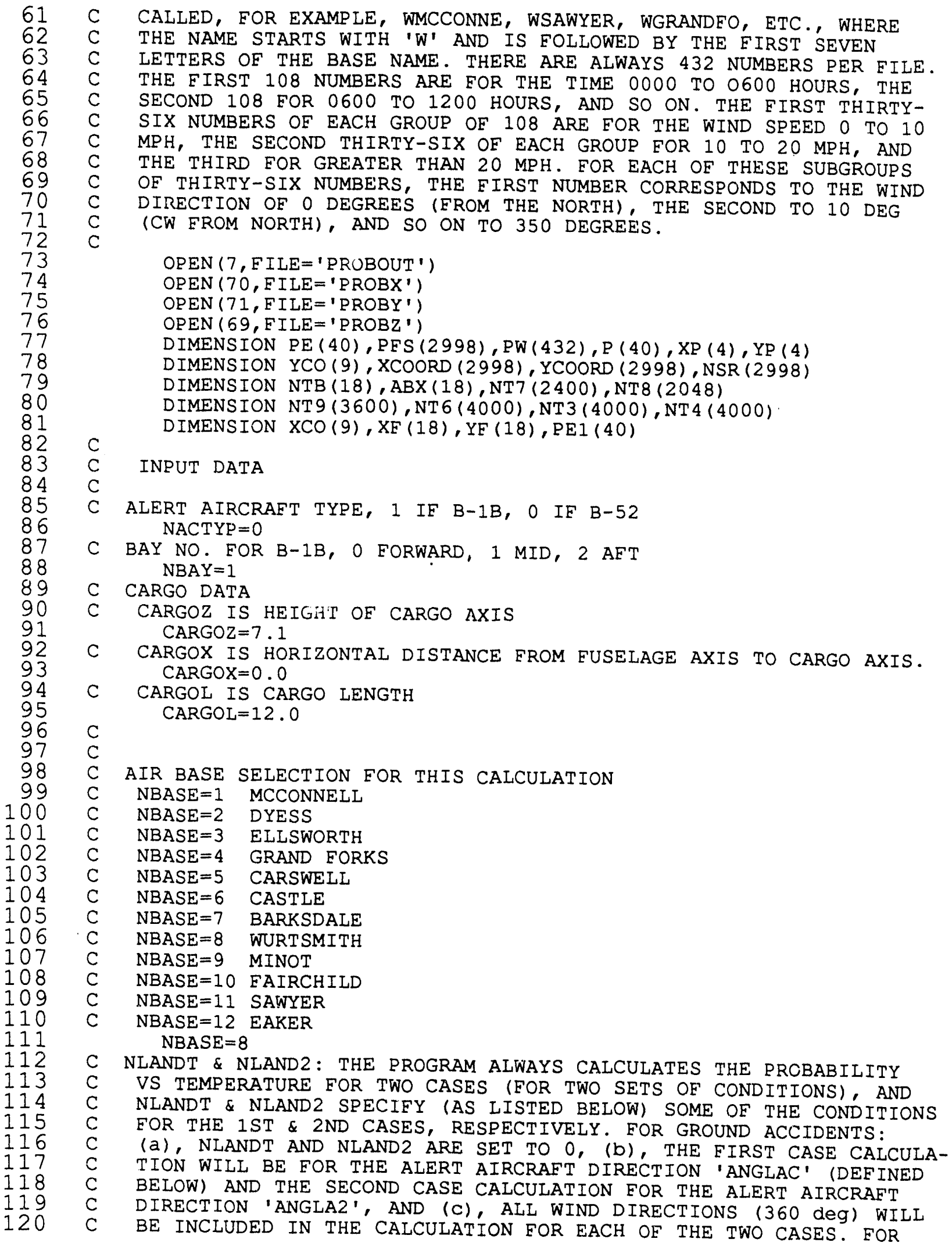




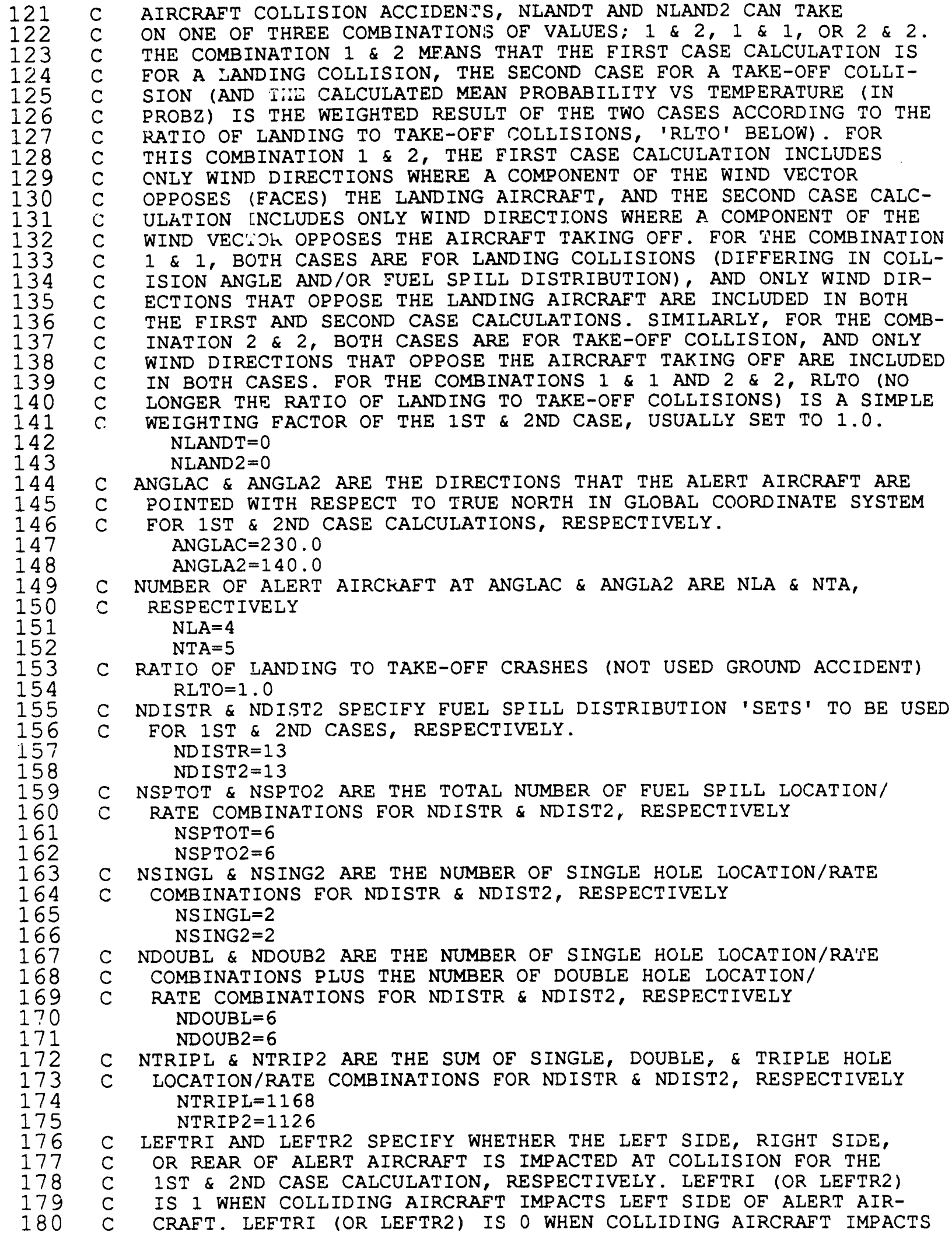




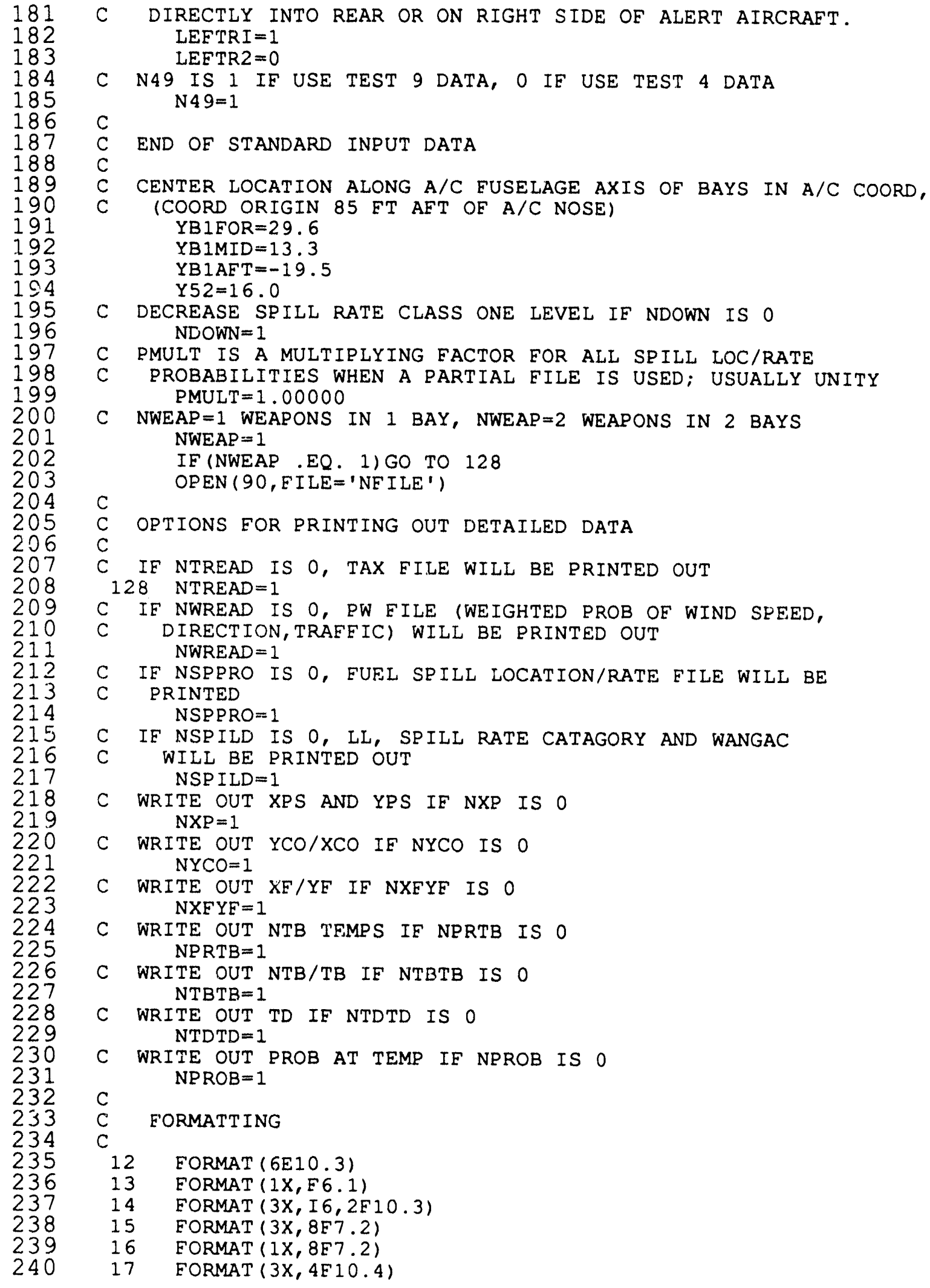




\begin{tabular}{|c|c|c|}
\hline 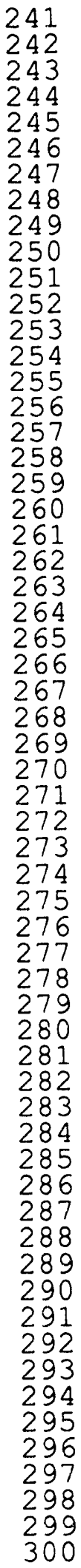 & $\begin{array}{l}19 \\
20 \\
21 \\
22 \\
23 \\
24 \\
25 \\
26 \\
27 \\
28 \\
29 \\
30 \\
31 \\
32 \\
33 \\
34 \\
35 \\
36 \\
37 \\
38 \\
39 \\
40 \\
41 \\
42 \\
43 \\
44 \\
45 \\
46 \\
47 \\
48 \\
49 \\
50 \\
51 \\
52 \\
53 \\
54 \\
55 \\
56 \\
57 \\
58 \\
59 \\
60 \\
61 \\
62 \\
63 \\
64 \\
65 \\
66 \\
67 \\
68 \\
69 \\
70 \\
71 \\
72 \\
73 \\
74 \\
75 \\
76\end{array}$ & 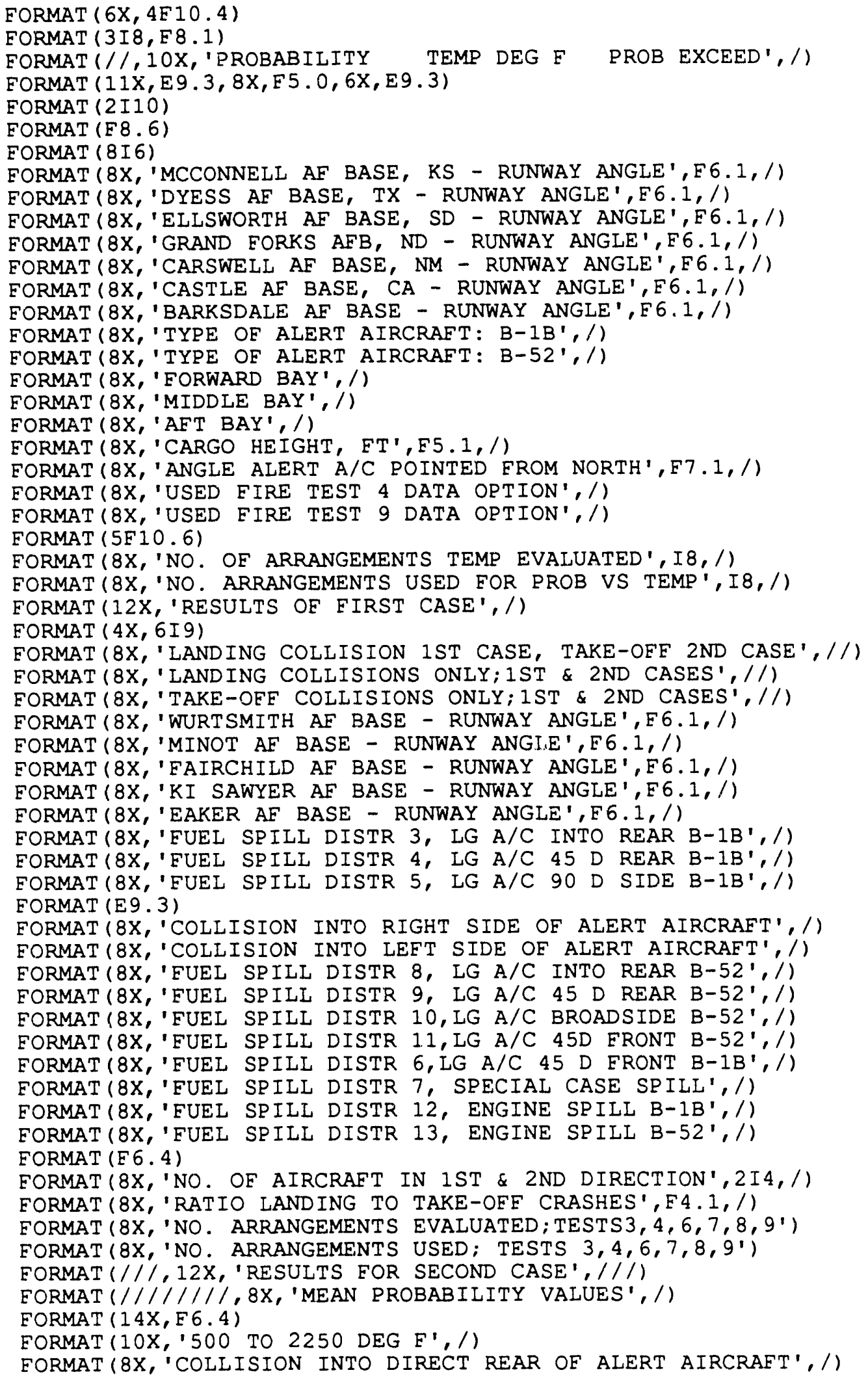 \\
\hline
\end{tabular}




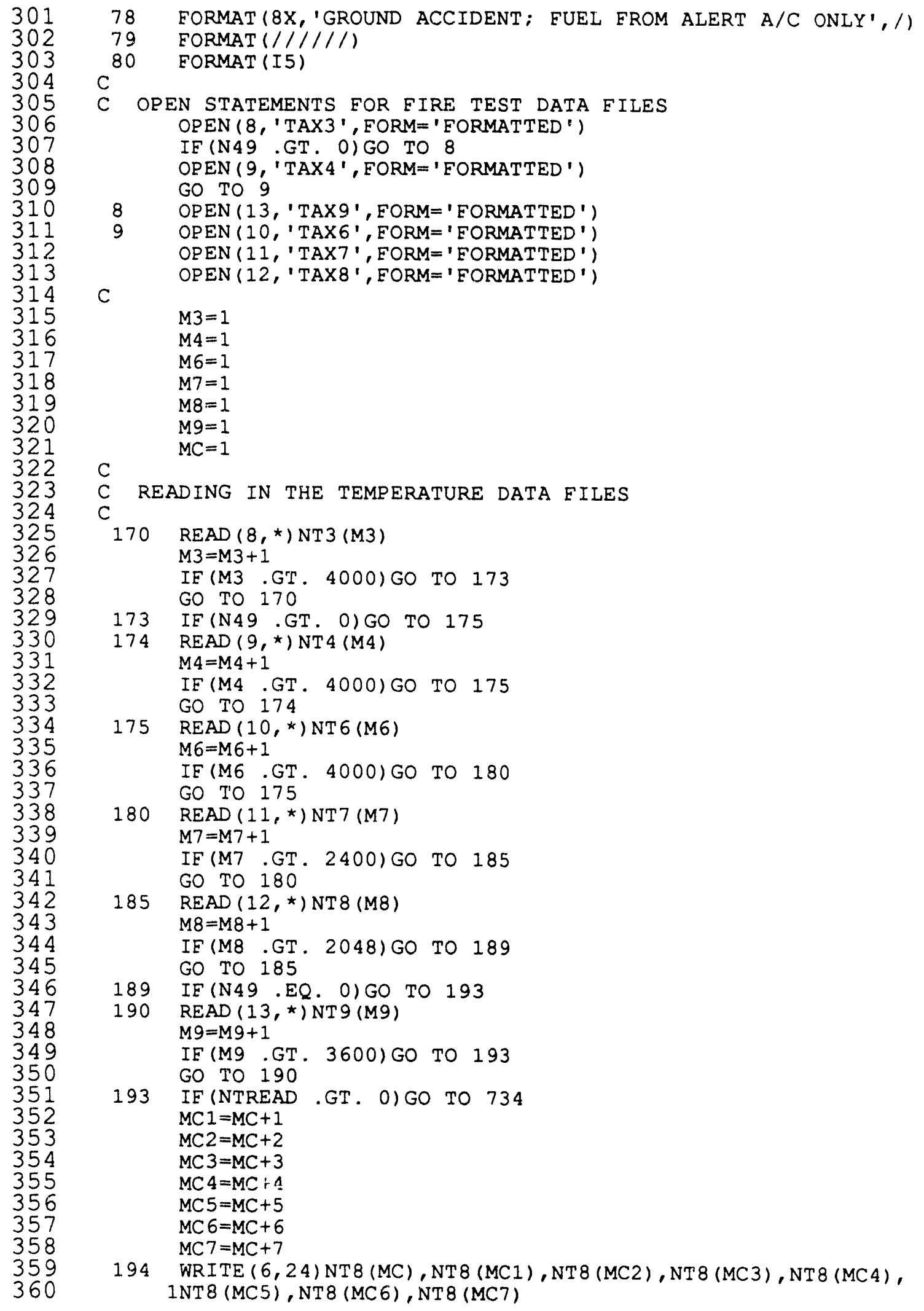




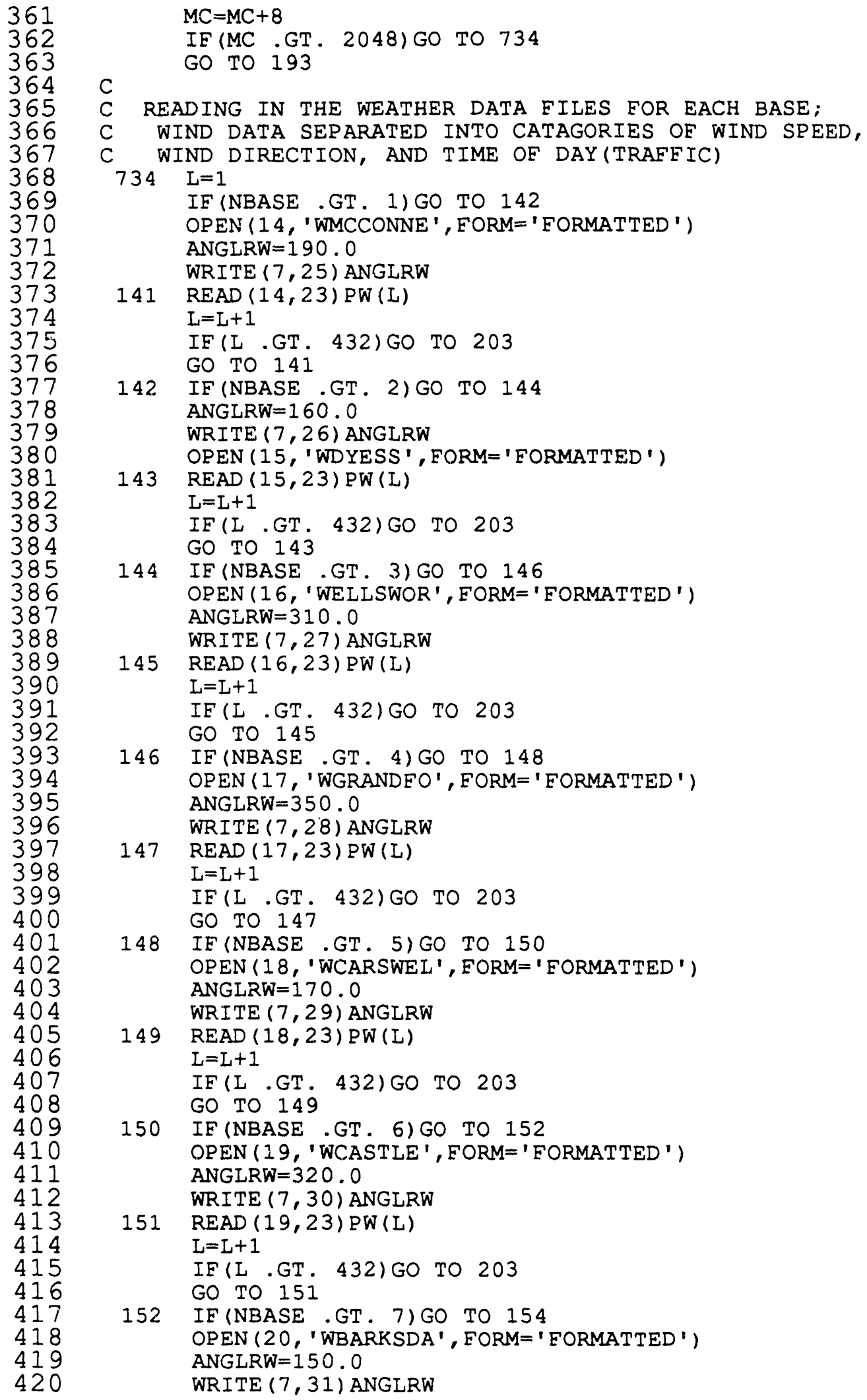




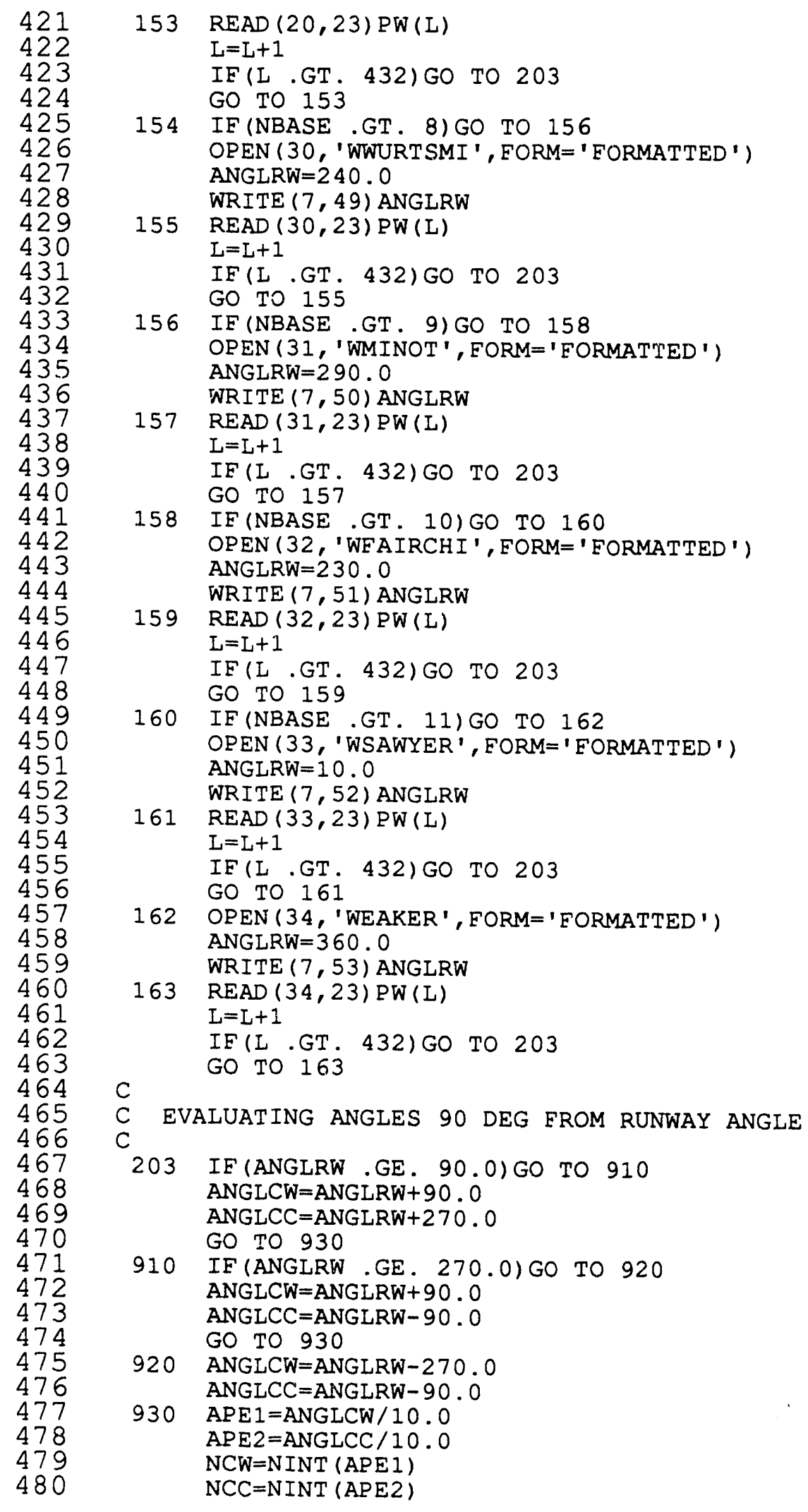




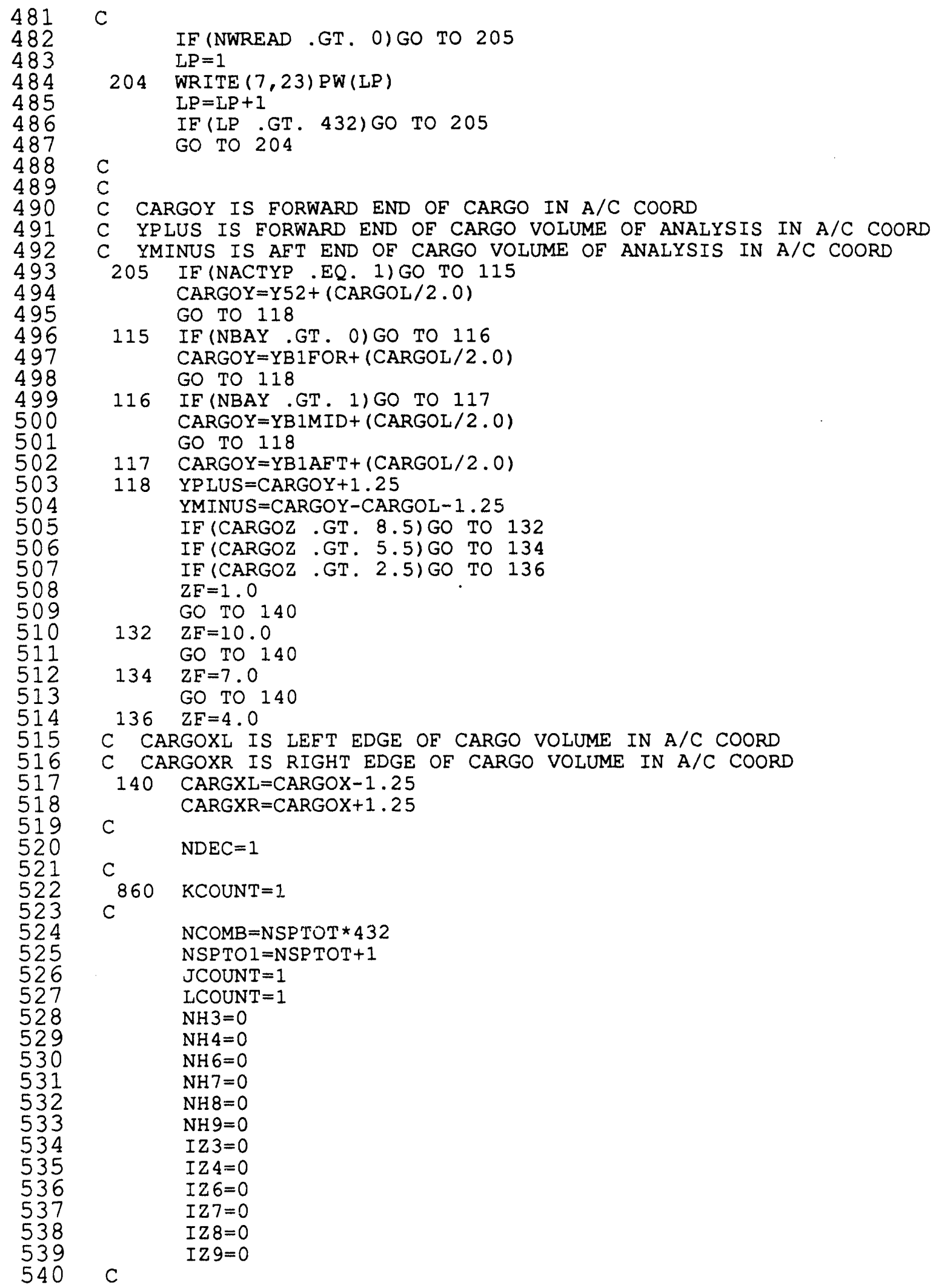




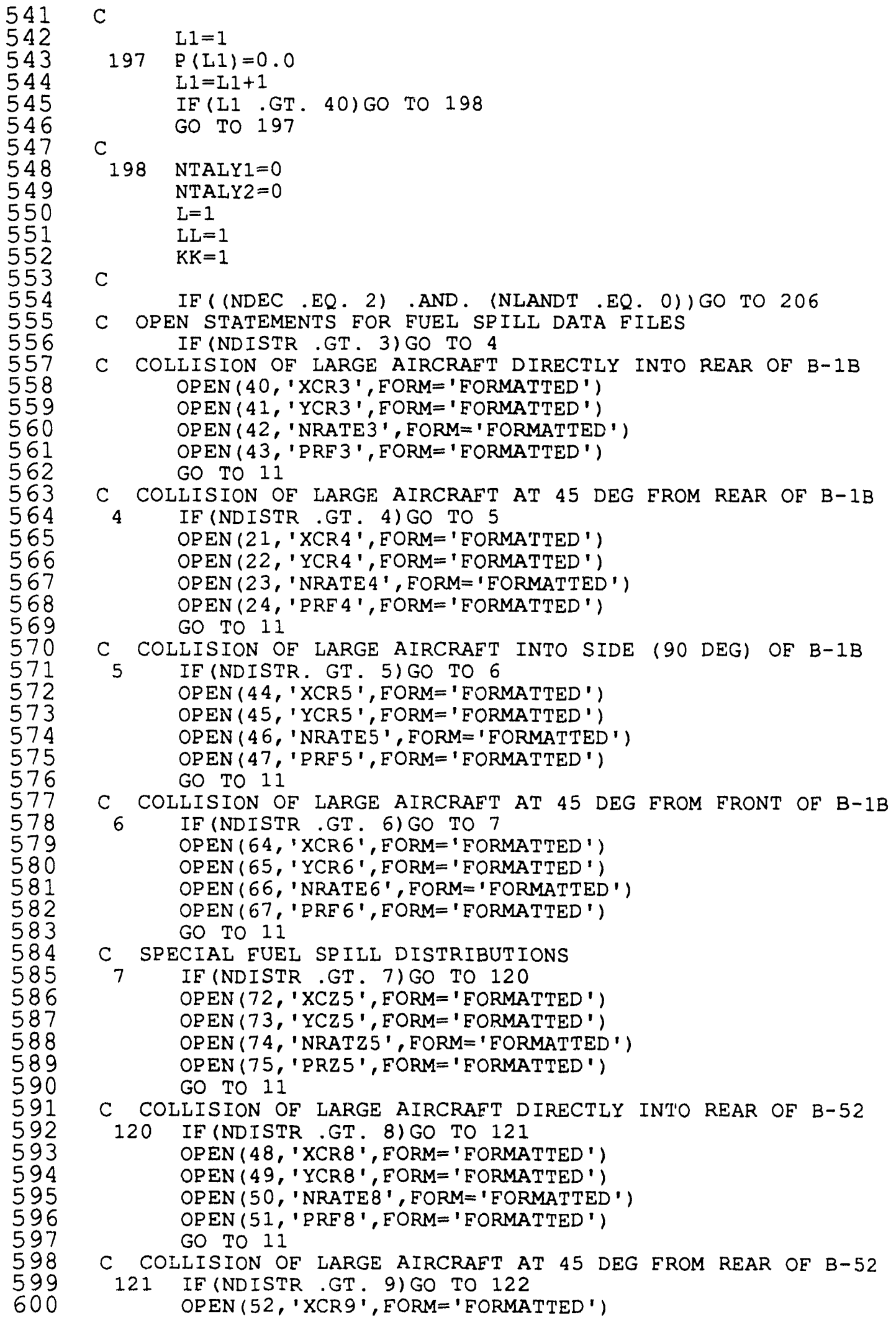




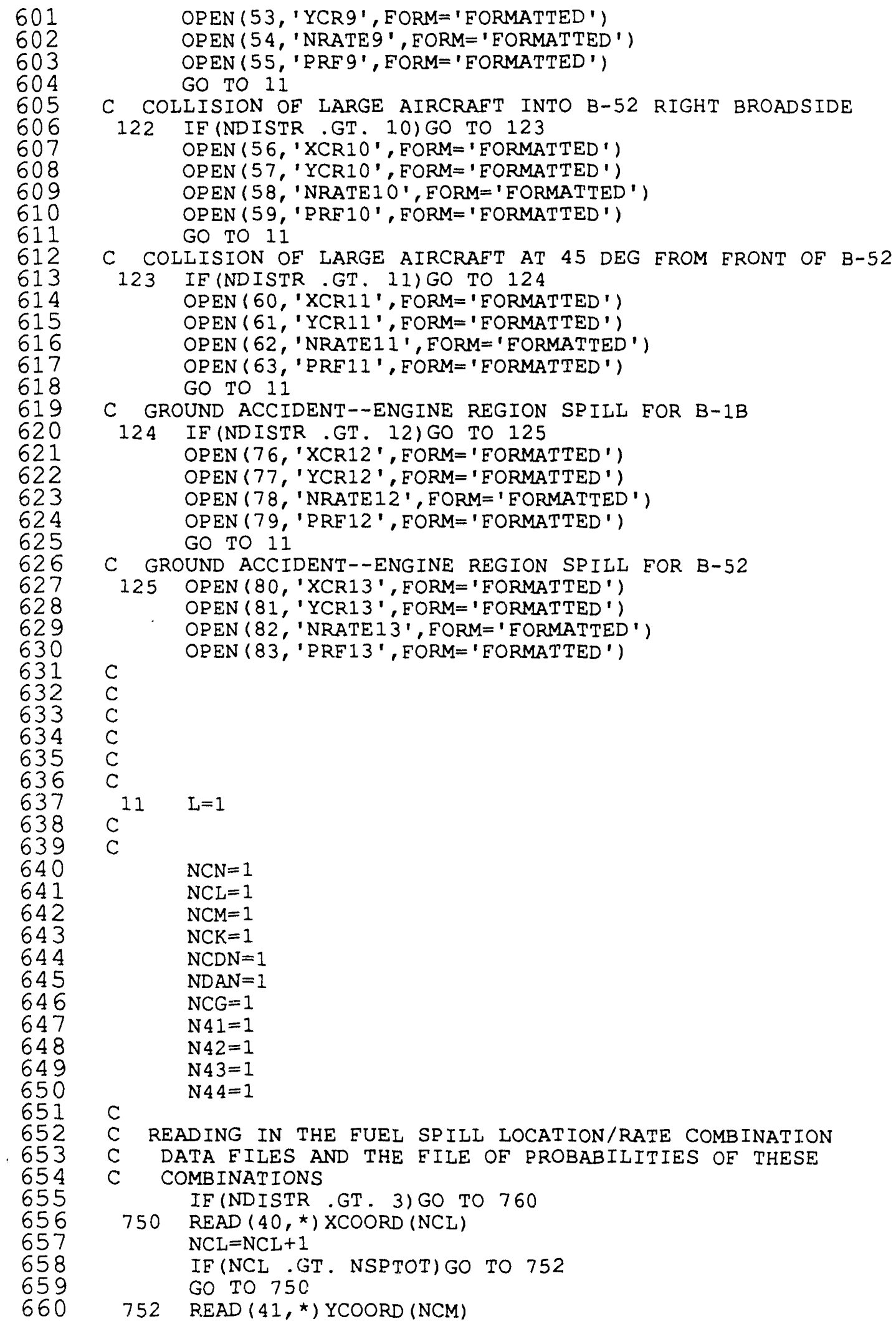




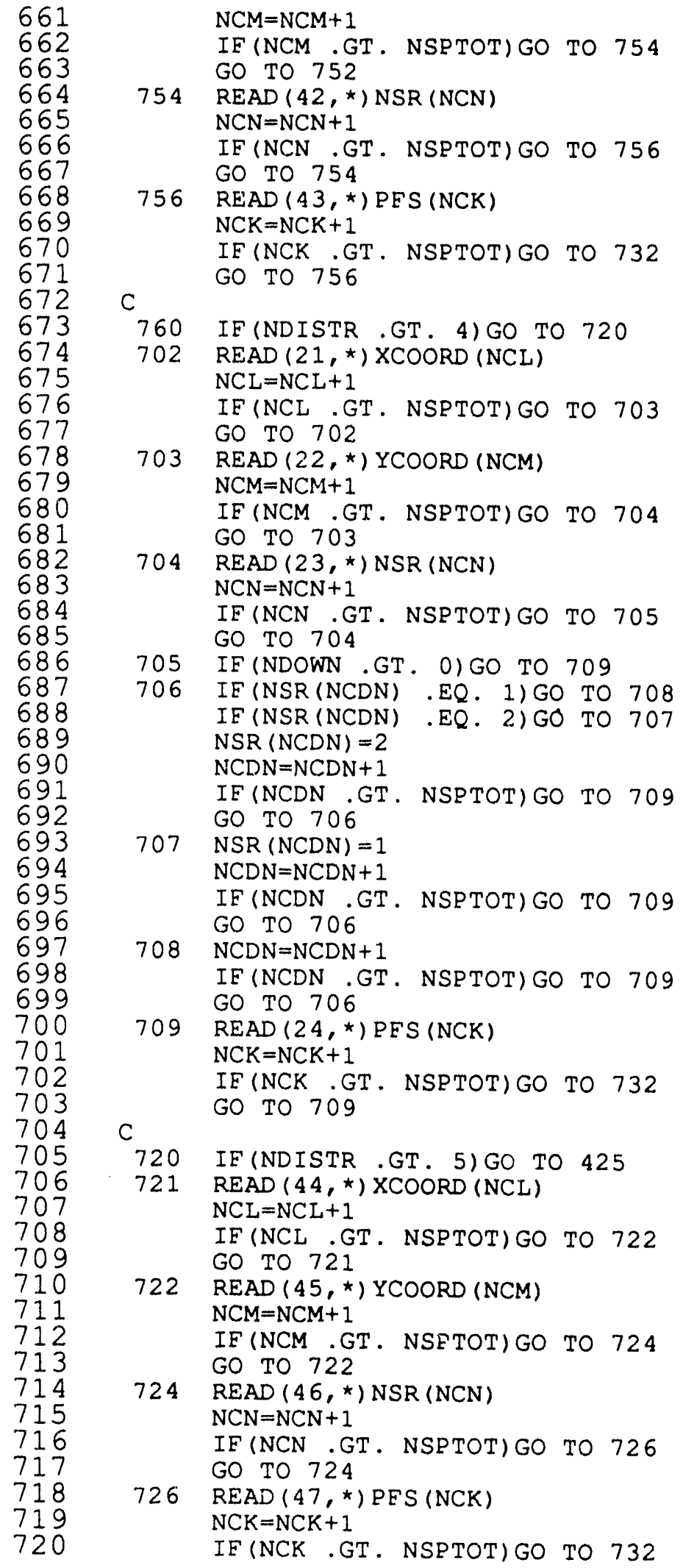




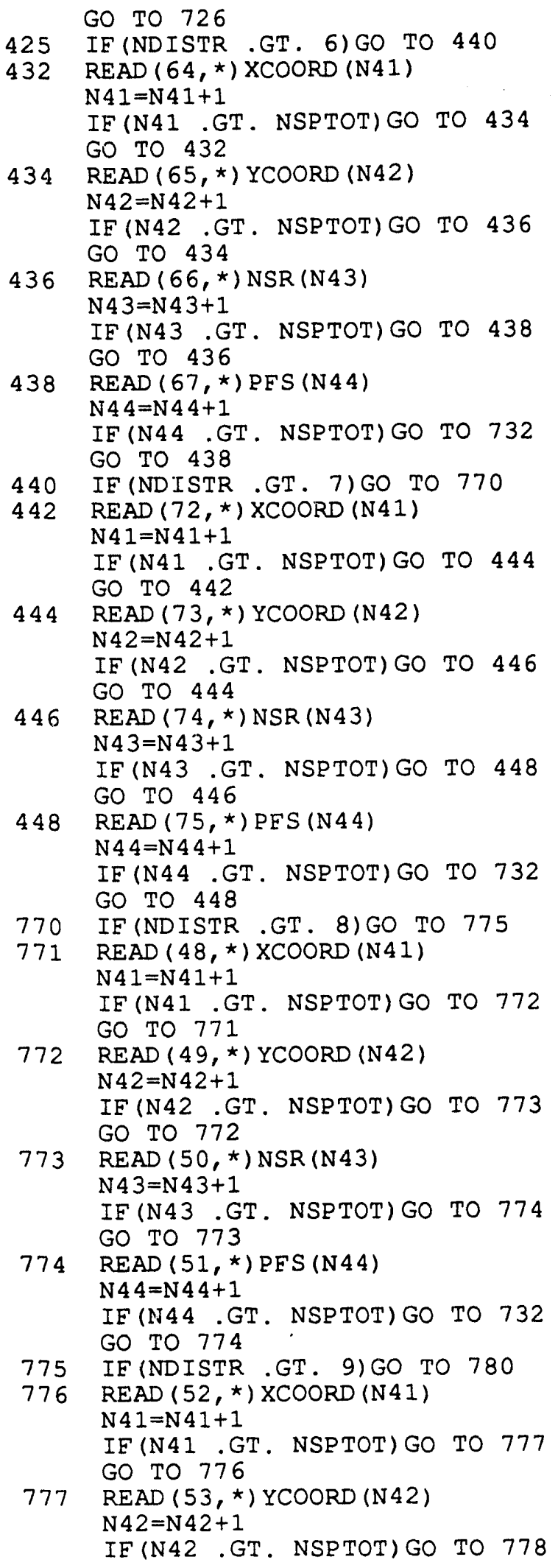




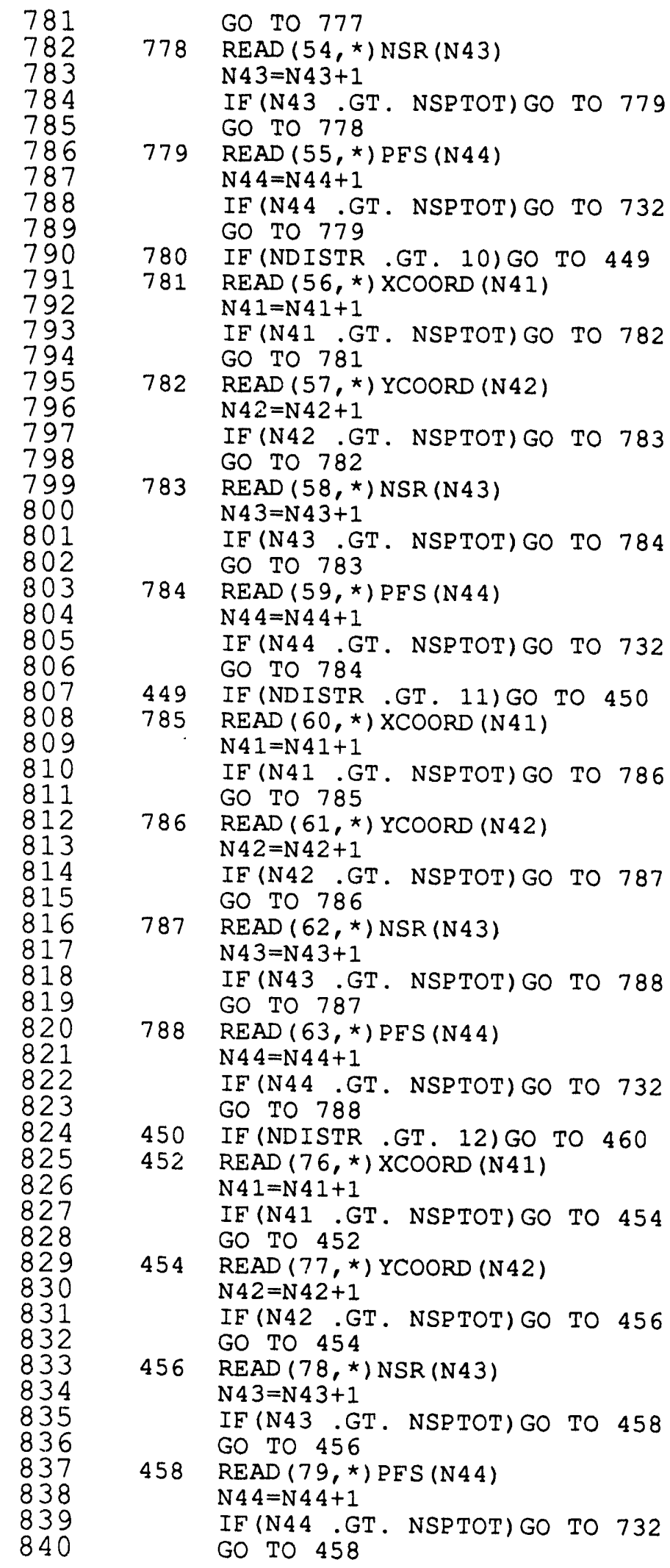




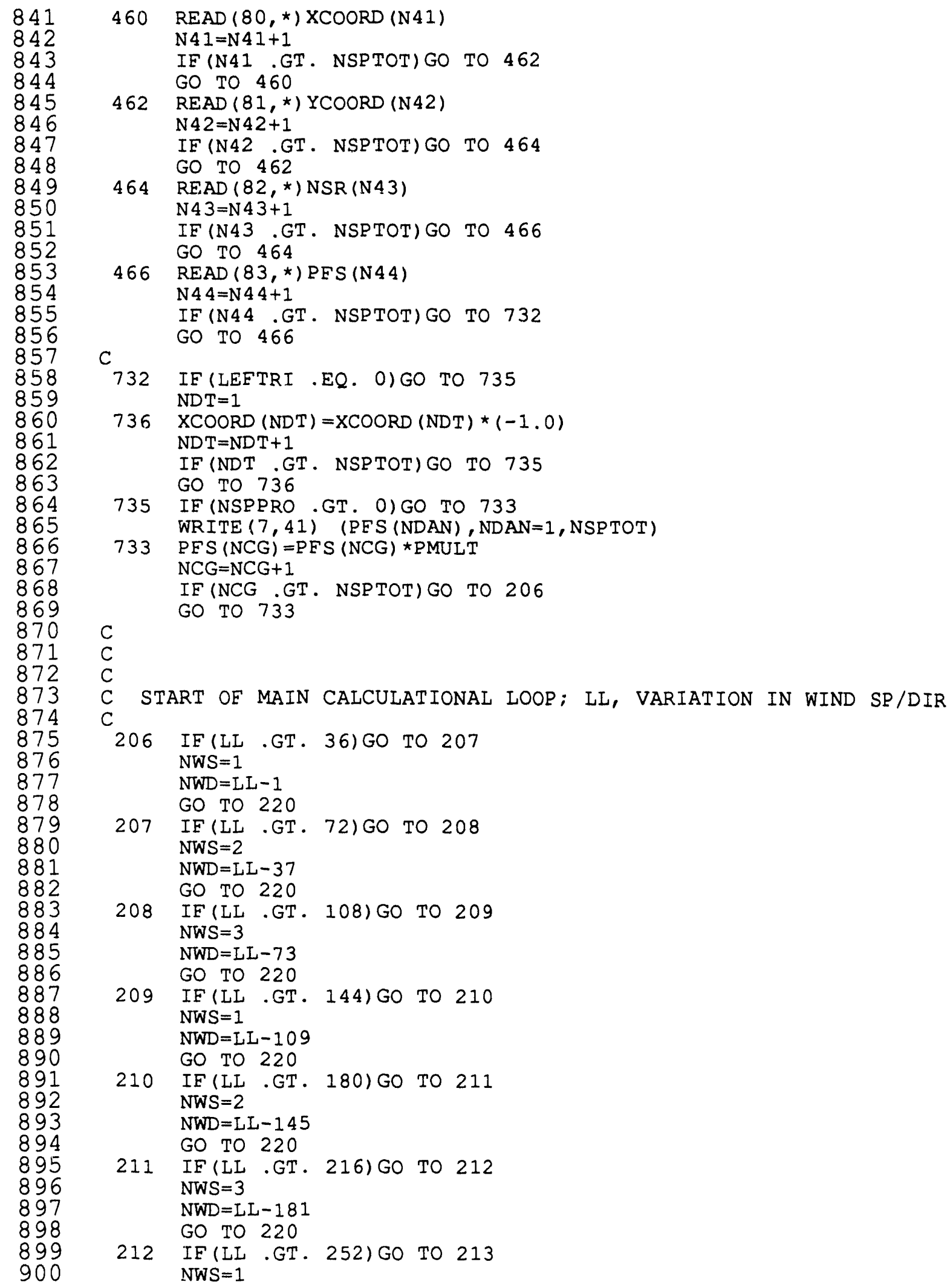




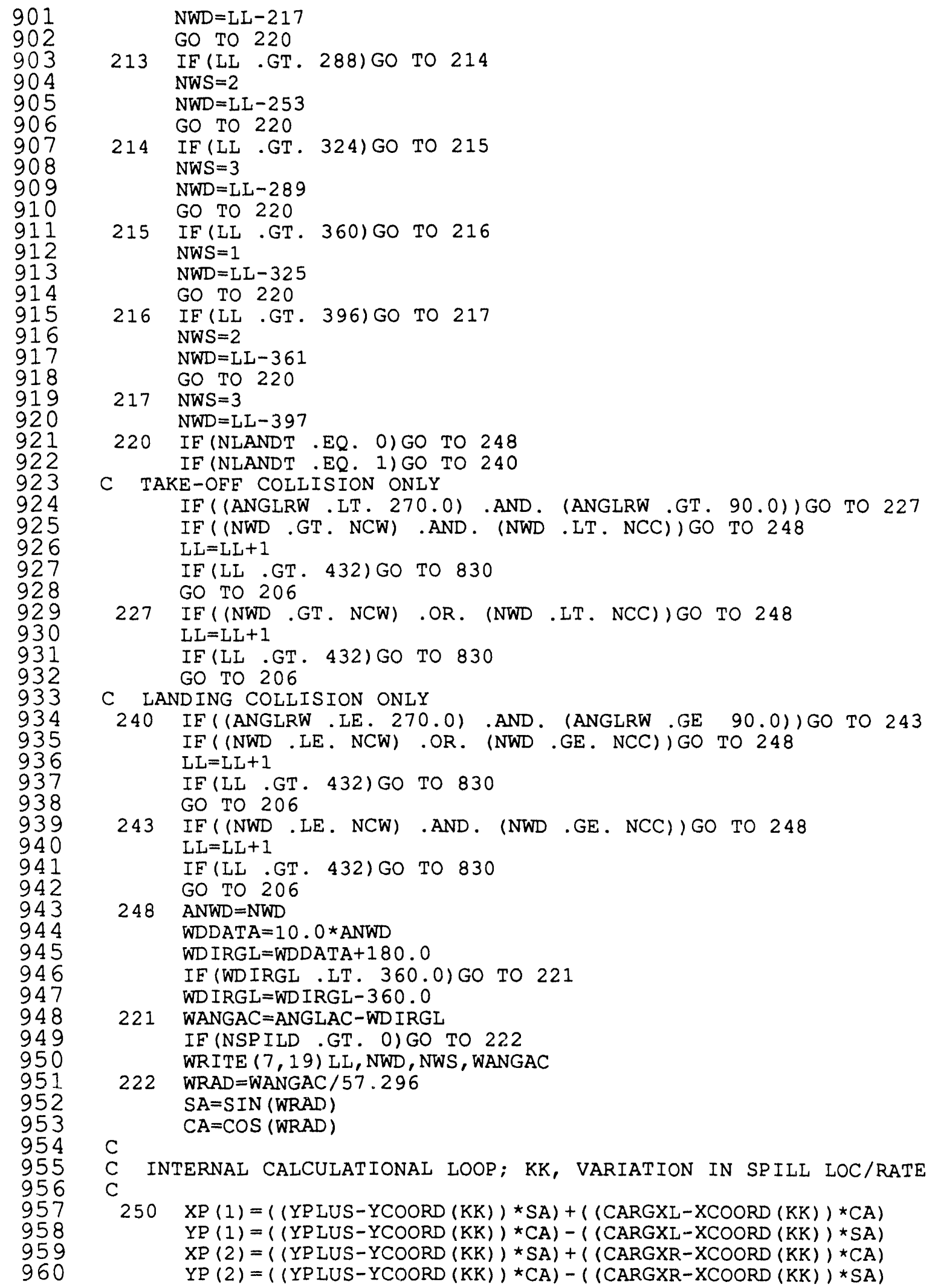




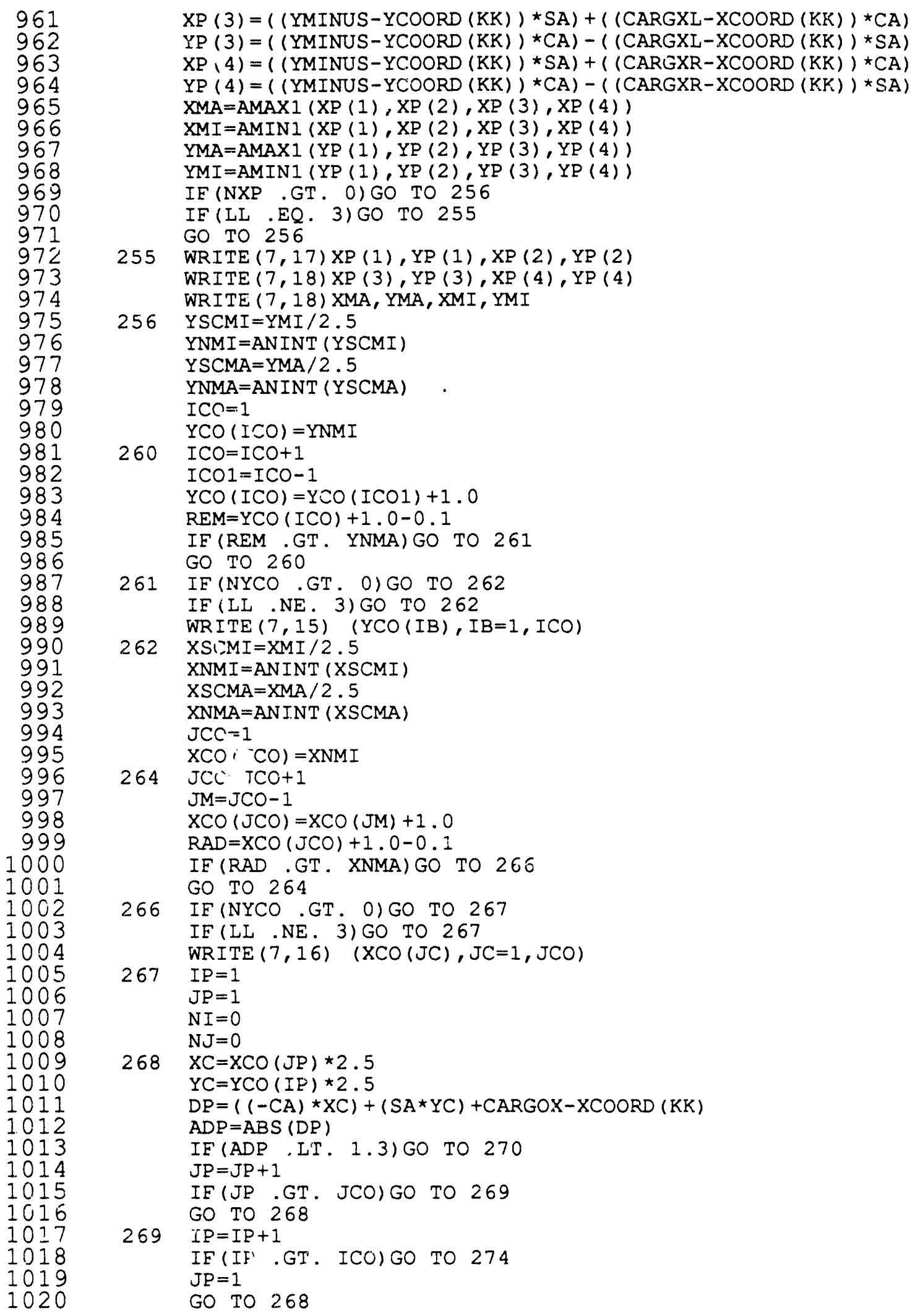




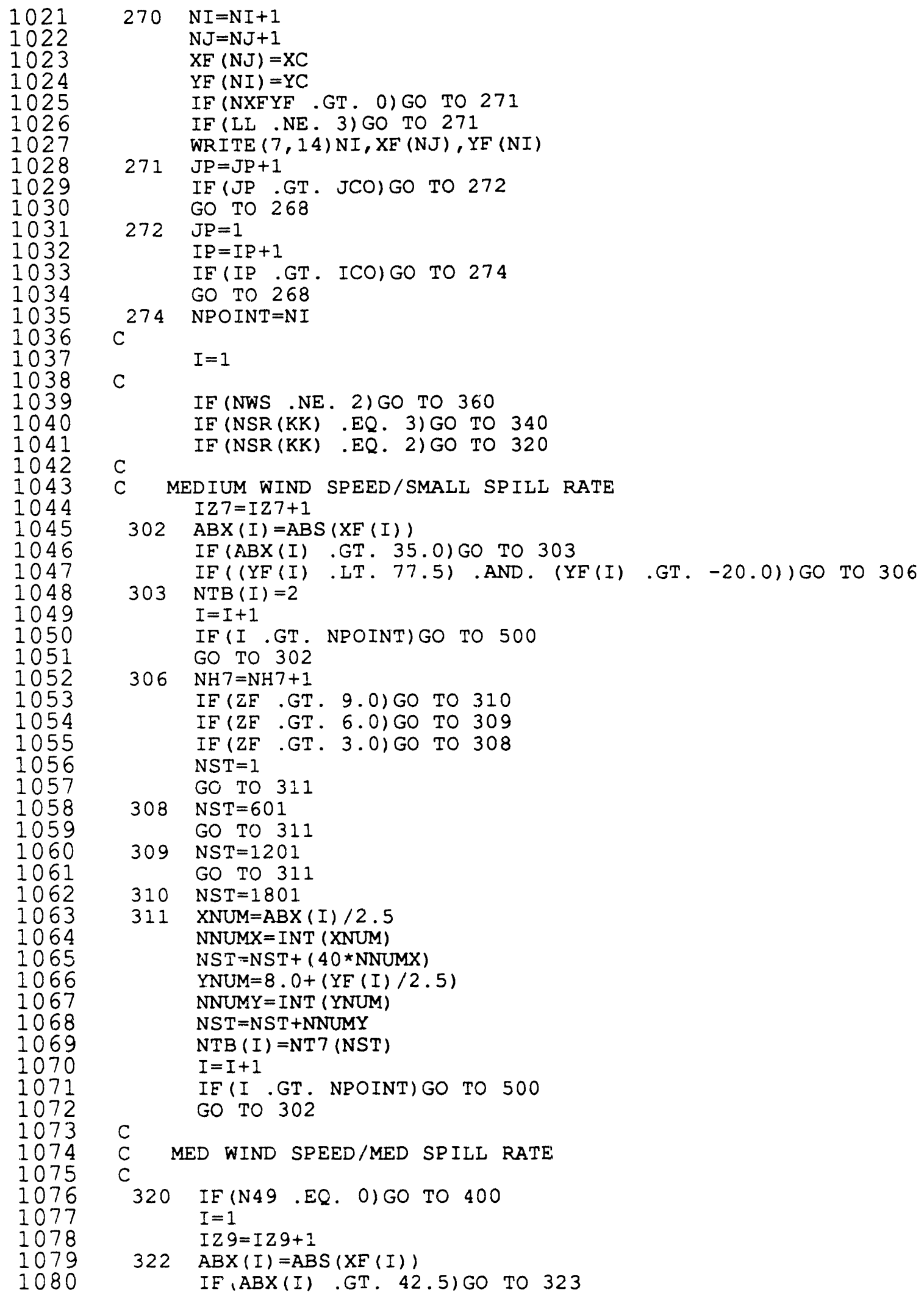




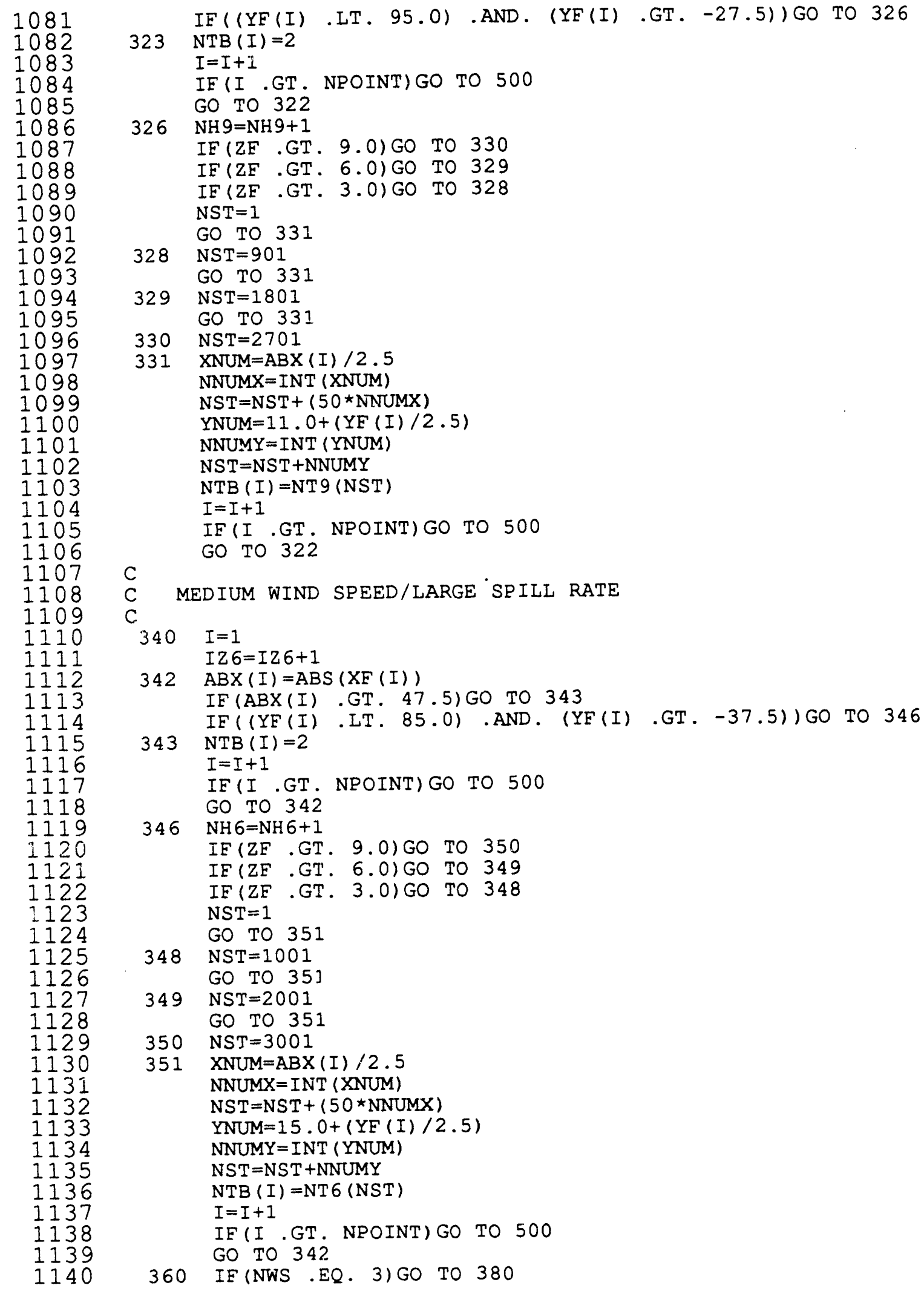




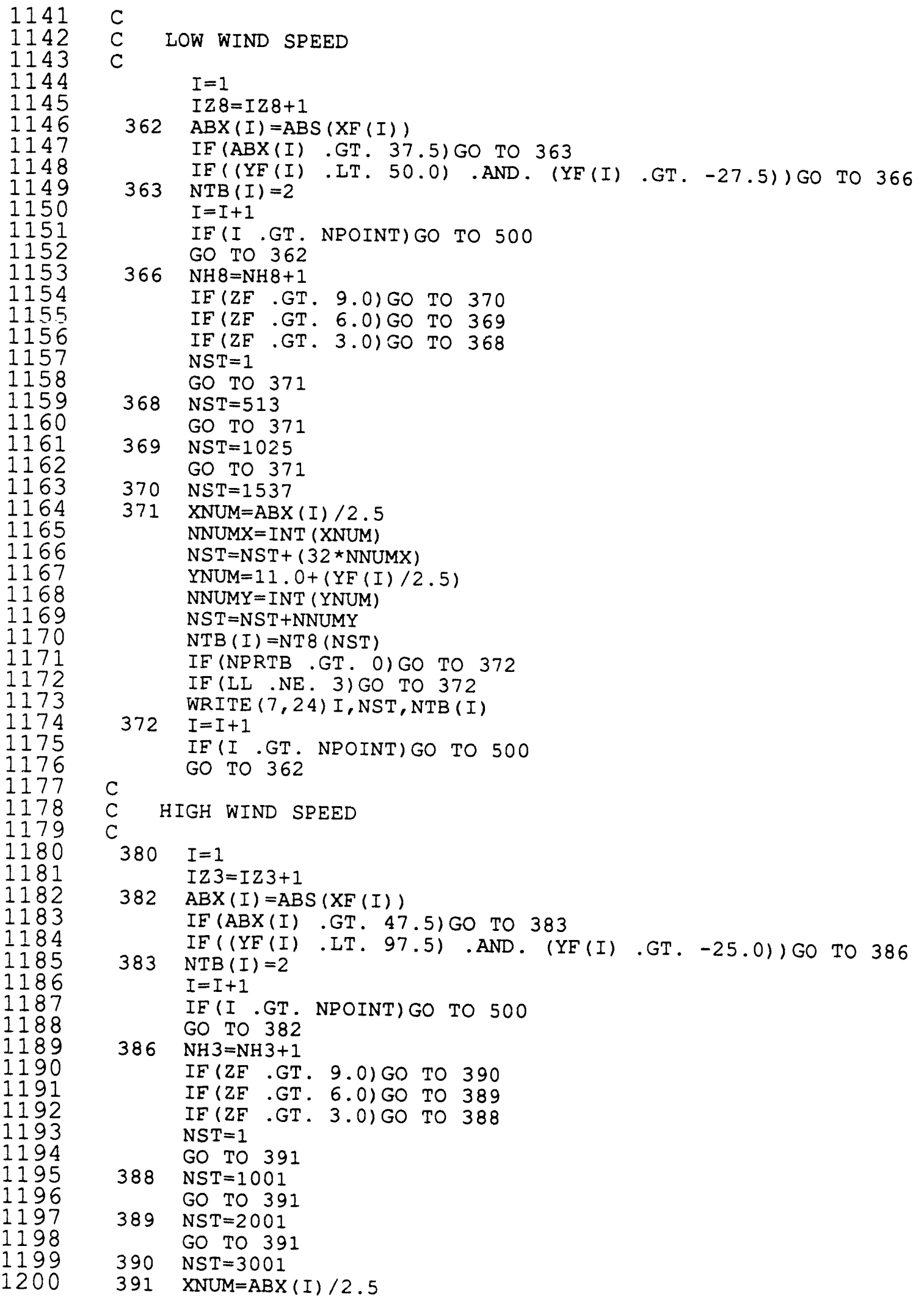




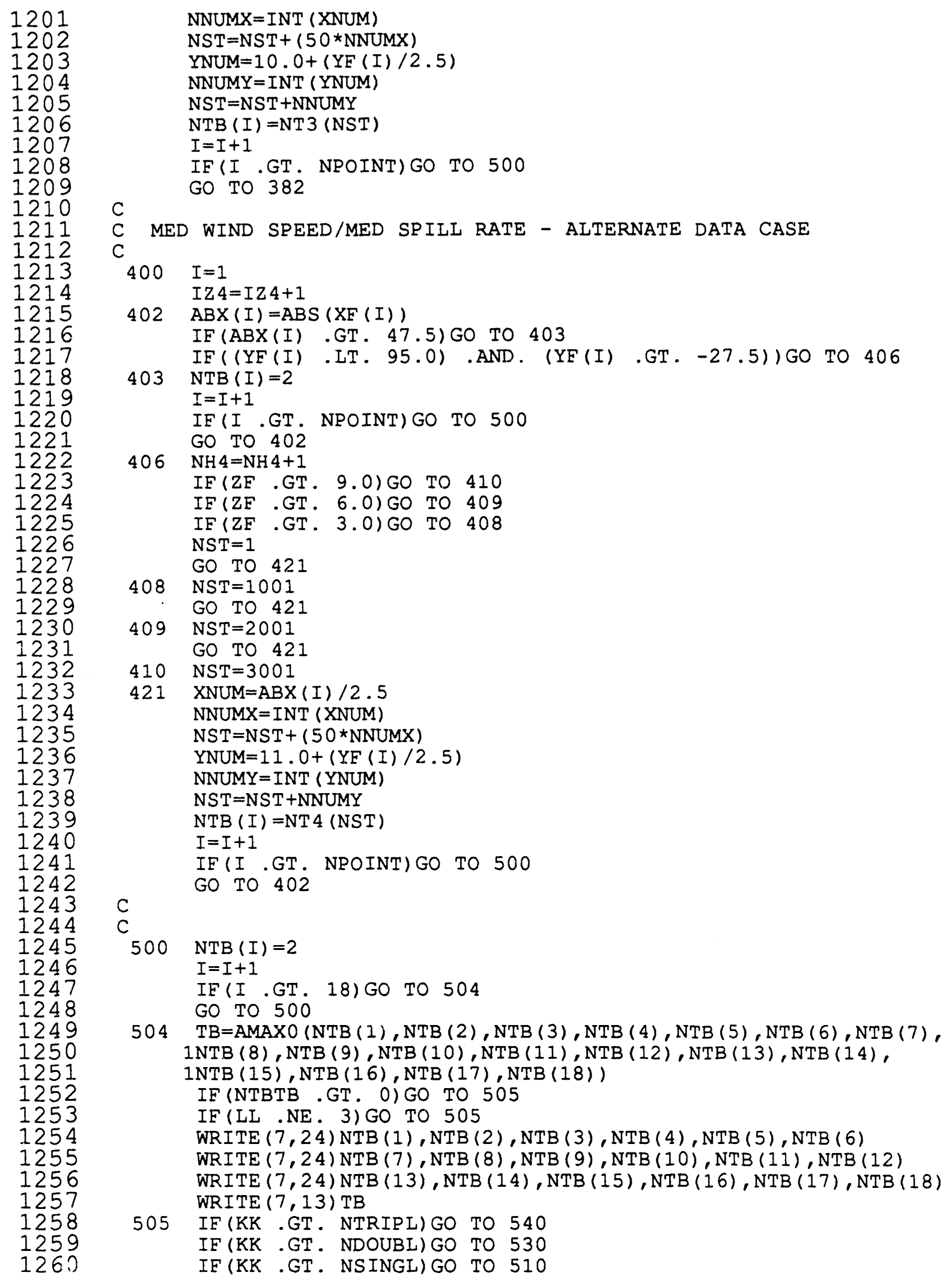




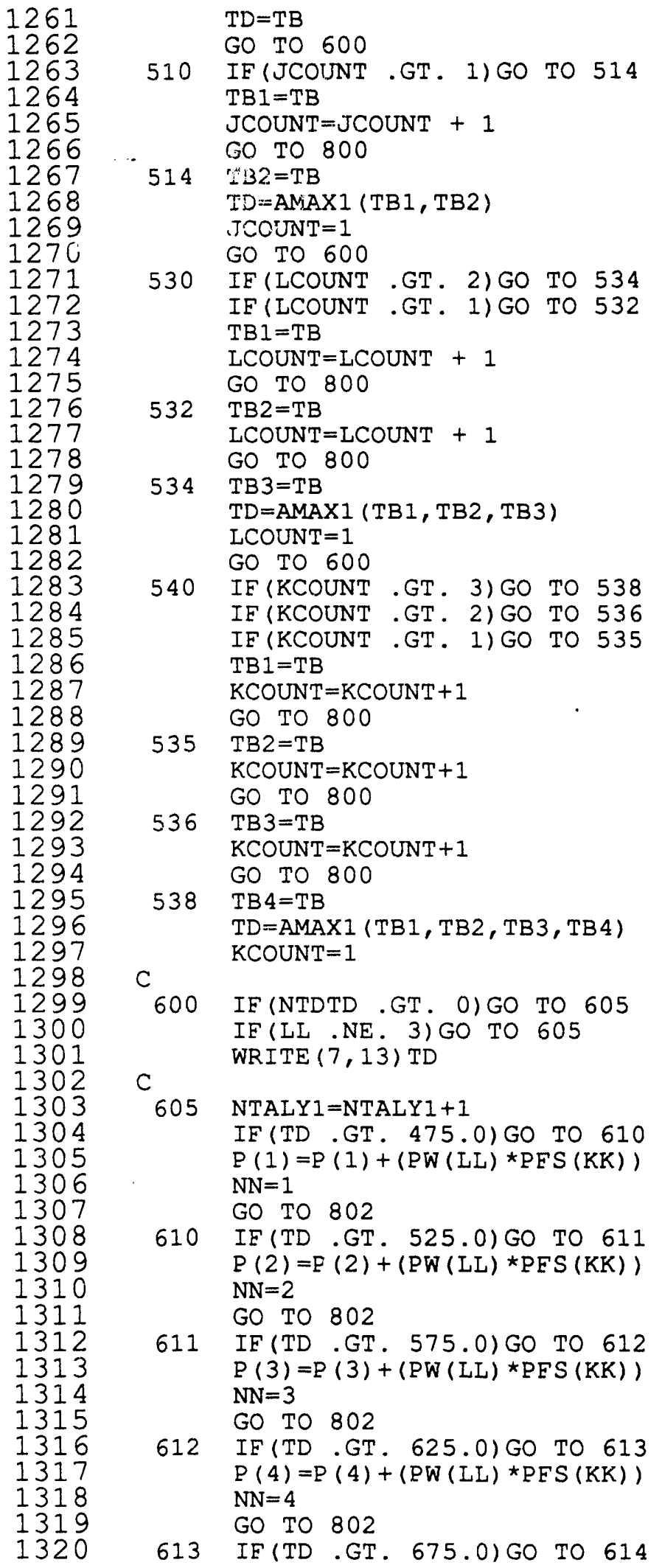




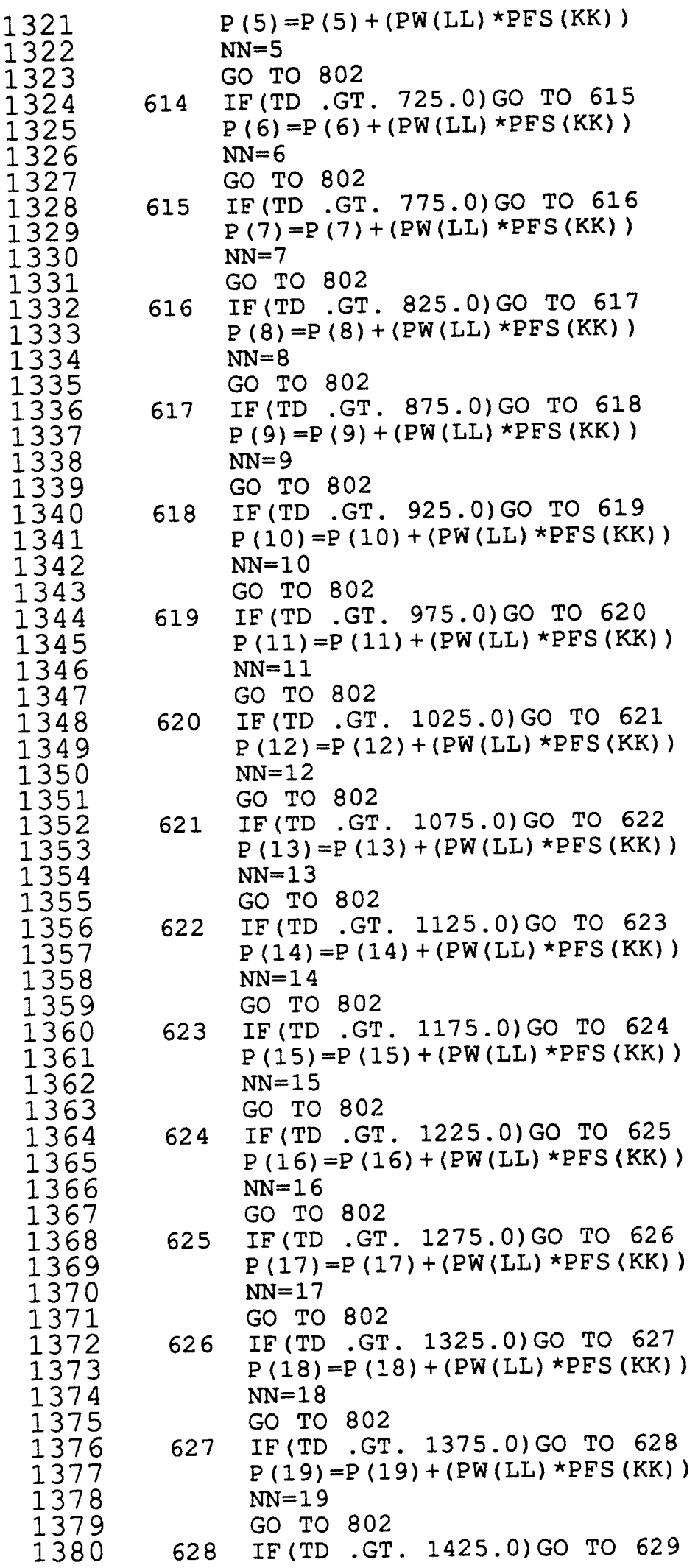




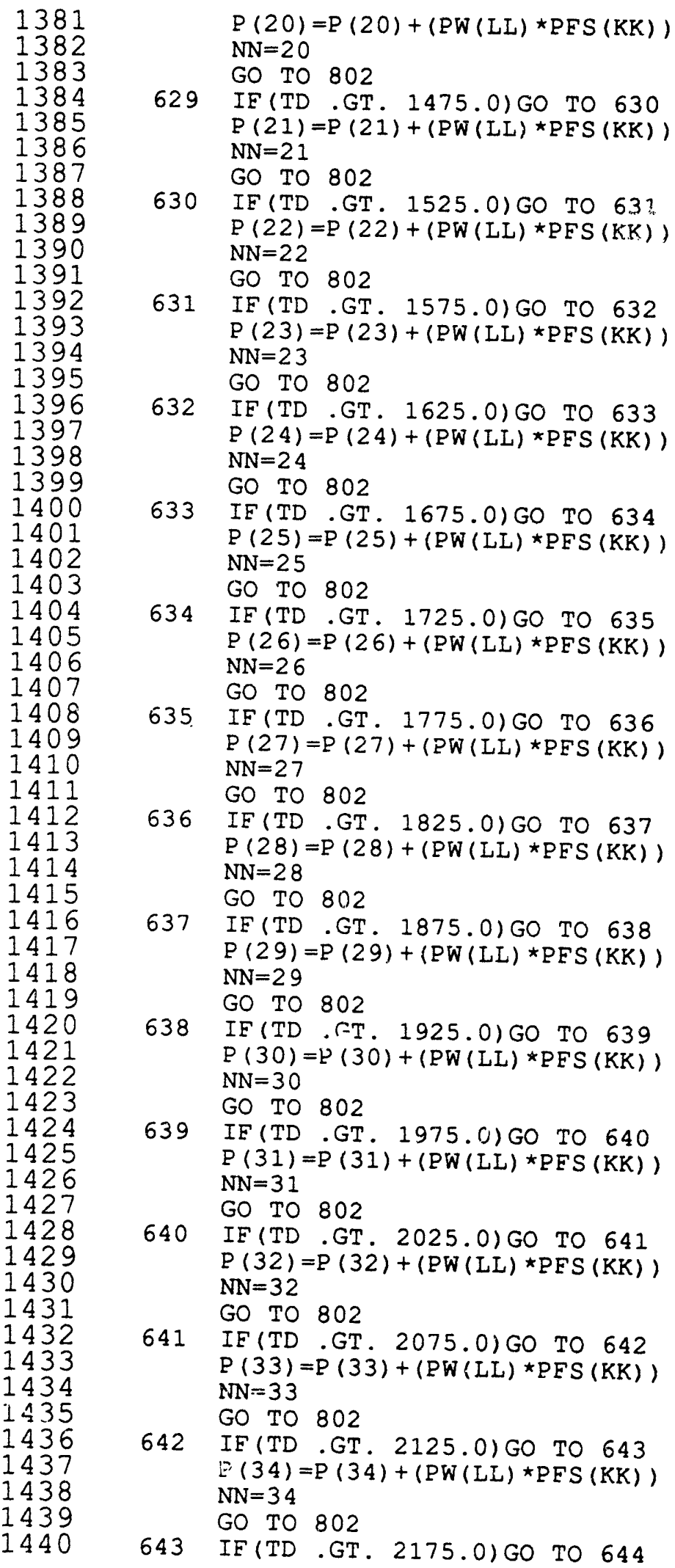




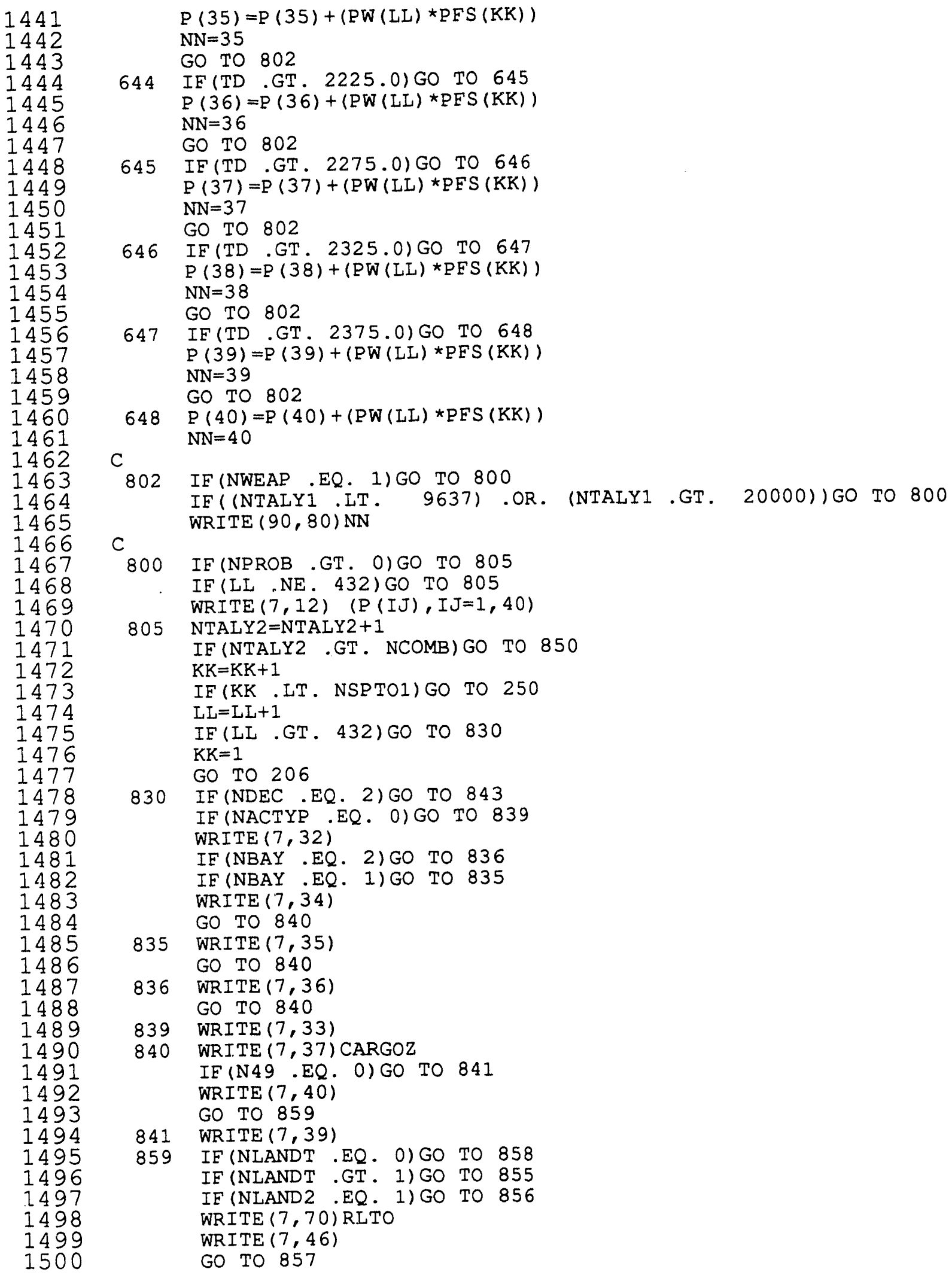




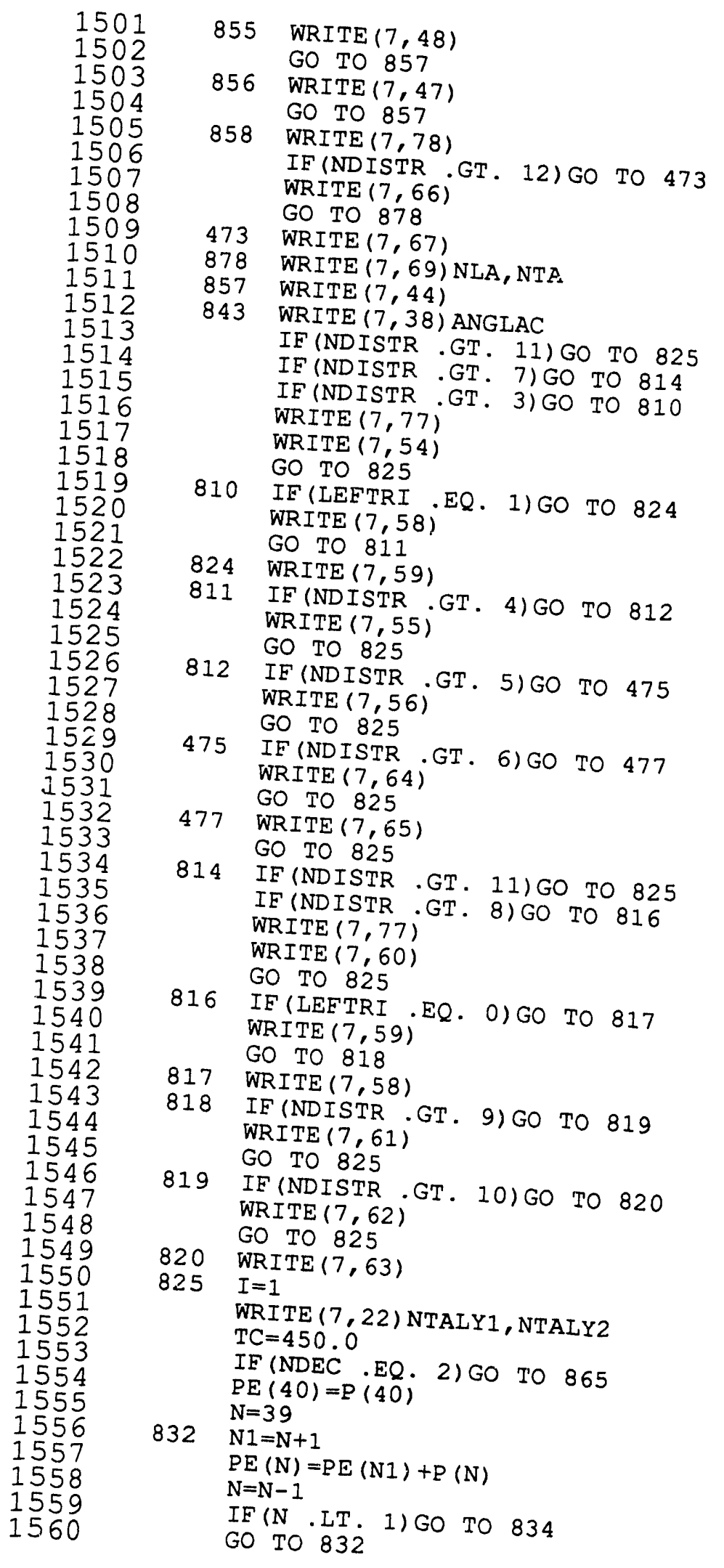




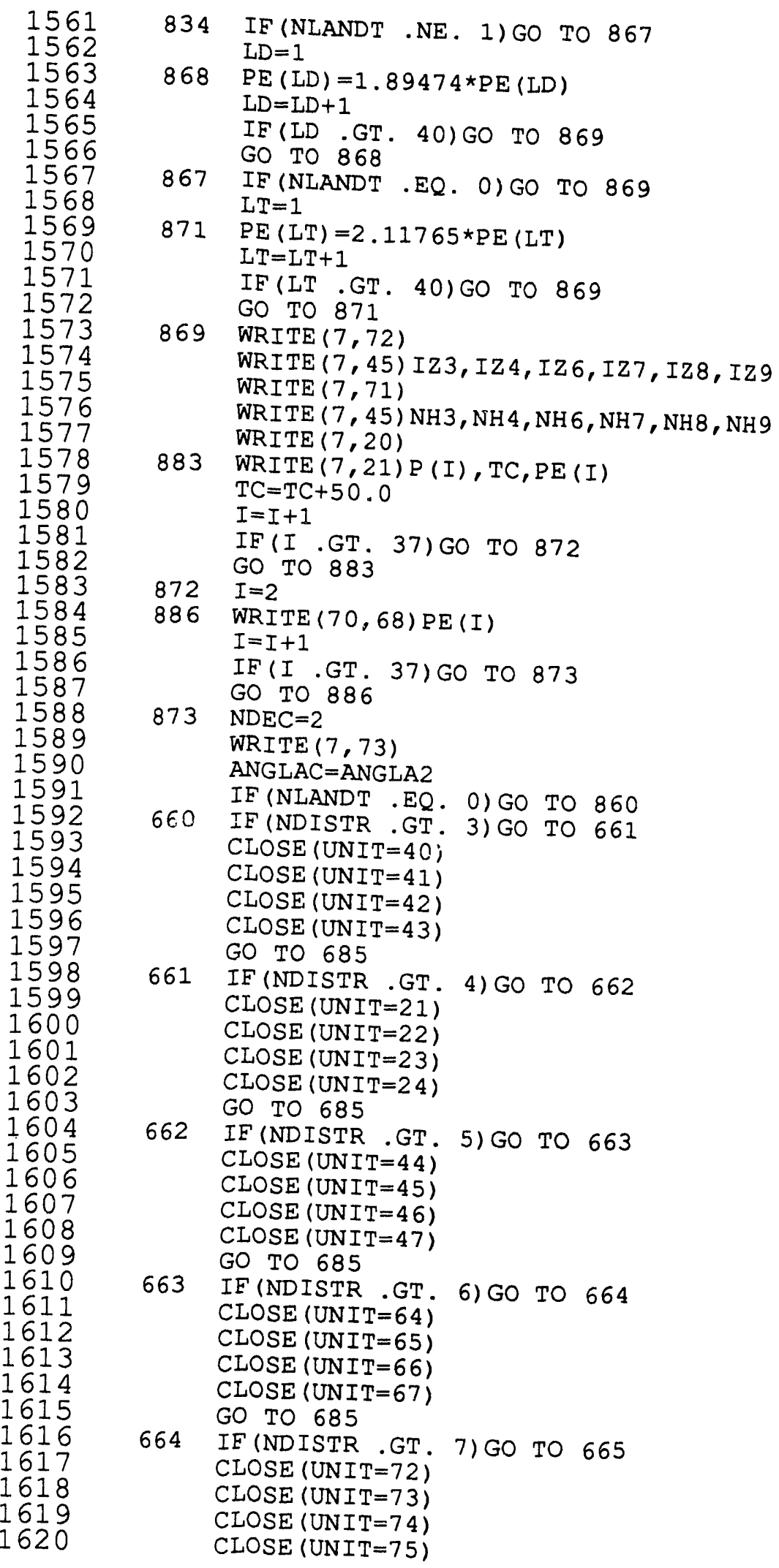




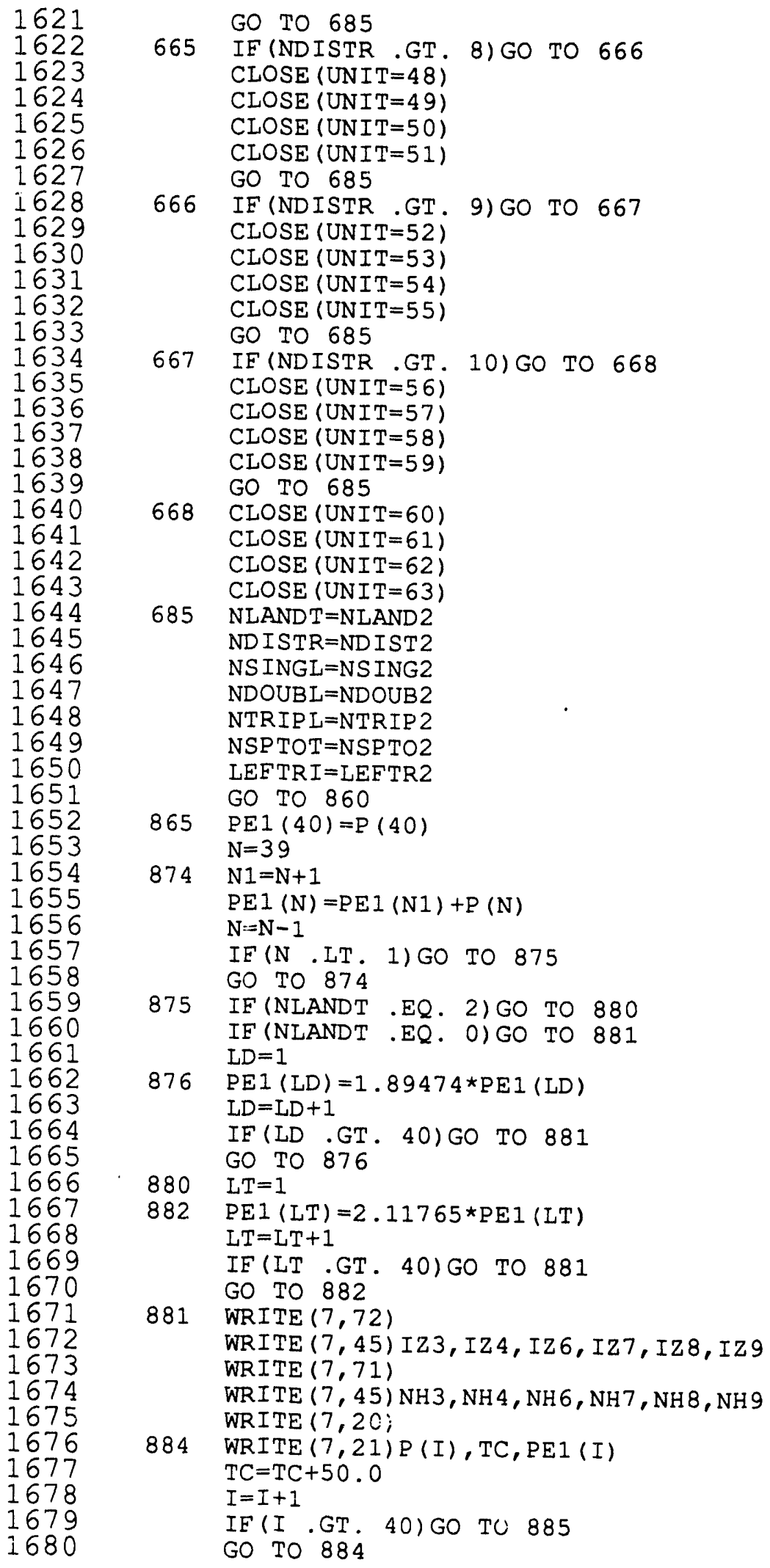




\begin{tabular}{|c|c|c|}
\hline $\begin{array}{l}686 \\
687 \\
688 \\
689 \\
690 \\
691 \\
692 \\
693 \\
694 \\
695 \\
696 \\
697 \\
698 \\
699 \\
700 \\
701 \\
702\end{array}$ & 889 & 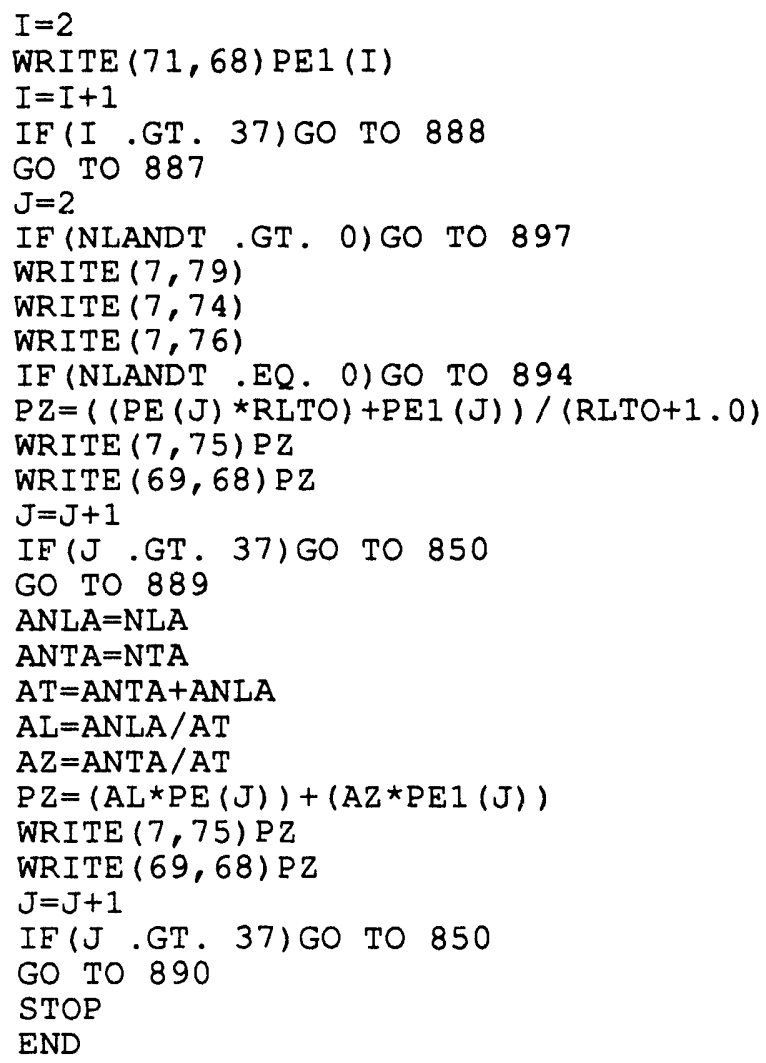 \\
\hline
\end{tabular}




\title{
EXAMPLE OUTPUT \#1
}

\author{
ELLSWORTH AF BASE, SD - RUNWAY ANGLE 310.0 \\ TYPE OF ALERT AIRCRAFT: B-1B \\ MIDDLE BAY \\ CARGO HEIGHT, FT 10.1 \\ USED FIRE TEST 9 DATA OPTION \\ RATIO LANDING TO TAKE-OFE CRASHES 3.0 \\ IAANDING COLLISION 1ST CASE, TAKE-OFF 2ND CASE \\ RESULTS OF FIRST CASE
}

ANGLE ALERT A/C POINTED FROM NORTH 30.0

COLLISION INTO RIGHT SIDE OF ALERT AIRCRAFT

FUEL SPILL DISTR 5, IG A/C 90 D SIDE B-1B

$\begin{array}{ccllll}101688 & 269952 & & & \\ \text { NO. ARRANGEMENTS } & \text { USED; TESTS } 3,4,6,7,8,9 & \\ 89984 & 0 & 23636 & 31388 & 89984 & 34960 \\ \text { NO. ARRANGEMENTS } & \text { EVALUATED: TESTS3, } 4,6,7,8,9 & \\ 345564 & 0 & 128548 & 63180 & 258840 & 122732\end{array}$

$\begin{array}{lcc}\text { PROBABILITY } & \text { TEMP DEG } & \text { PROB EXCEED } \\ 0.283 E+00 & 450 . & 0.113 \mathrm{E}+01 \\ 0.166 \mathrm{E}-01 & 500 . & 0.595 \mathrm{E}+00 \\ 0.213 \mathrm{E}-01 & 550 . & 0.564 \mathrm{E}+00 \\ 0.172 \mathrm{E}-01 & 600 . & 0.523 \mathrm{E}+00 \\ 0.973 \mathrm{E}-02 & 650 . & 0.491 \mathrm{E}+00 \\ 0.124 \mathrm{E}-01 & 700 . & 0.472 \mathrm{E}+00 \\ 0.121 \mathrm{E}-01 & 750 . & 0.449 \mathrm{E}+00 \\ 0.159 \mathrm{E}-01 & 800 . & 0.426 \mathrm{E}+00 \\ 0.775 \mathrm{E}-02 & 850 . & 0.396 \mathrm{E}+00 \\ 0.828 \mathrm{E}-02 & 900 . & 0.381 \mathrm{E}+00 \\ 0.822 \mathrm{E}-02 & 950 . & 0.365 \mathrm{E}+00 \\ 0.288 \mathrm{E}-01 & 1000 . & 0.350 \mathrm{E}+00 \\ 0.391 \mathrm{E}-01 & 1050 . & 0.295 \mathrm{E}+00 \\ 0.918 \mathrm{E}-03 & 1100 . & 0.221 \mathrm{E}+00 \\ 0.425 \mathrm{E}-02 & 1150 . & 0.219 \mathrm{E}+00 \\ 0.653 \mathrm{E}-02 & 1200 . & 0.211 \mathrm{E}+00 \\ 0.140 \mathrm{E}-01 & 1250 . & 0.199 \mathrm{E}+00 \\ 0.511 \mathrm{E}-02 & 1300 . & 0.173 \mathrm{E}+00 \\ 0.171 \mathrm{E}-02 & 1350 . & 0.163 \mathrm{E}+00 \\ 0.408 \mathrm{E}-02 & 1400 . & 0.160 \mathrm{E}+00 \\ 0.811 \mathrm{E}-02 & 1450 . & 0.152 \mathrm{E}+00 \\ 0.310 \mathrm{E}-02 & 1500 . & 0.137 \mathrm{E}+00 \\ 0.149 \mathrm{E}-01 & 1550 . & 0.131 \mathrm{E}+00 \\ 0.308 \mathrm{E}-02 & 1600 . & 0.102 \mathrm{E}+00 \\ 0.523 \mathrm{E}-02 & 1650 . & 0.966 \mathrm{E}-01 \\ 0.118 \mathrm{E}-02 & 1700 . & 0.867 \mathrm{E}-01 \\ 0.409 \mathrm{E}-02 & 1750 . & 0.845 \mathrm{E}-01 \\ 0.686 \mathrm{E}-02 & 1800 . & 0.767 \mathrm{E}-01 \\ 0.202 \mathrm{E}-02 & 1850 . & 0.637 \mathrm{E}-01 \\ 0.285 \mathrm{E}-02 & 1900 . & 0.599 \mathrm{E}-01 \\ 0.433 \mathrm{E}-02 & 1950 . & 0.545 \mathrm{E}-01 \\ 0.106 \mathrm{E}-02 & 2000 . & 0.463 \mathrm{E}-01 \\ 0.128 \mathrm{E}-01 & 2050 . & 0.443 \mathrm{E}-01 \\ 0.000 \mathrm{E}+00 & 2100 . & 0.200 \mathrm{E}-01 \\ 0.105 \mathrm{E}-01 & 2150 . & 0.200 \mathrm{E}-01 \\ 0.000 \mathrm{E}+00 & 2200 . & 0.000 \mathrm{E}+00 \\ 0.000 \mathrm{E}+00 & 2250 . & 0.000 \mathrm{E}+00 \\ & & \\ 0.02 & \end{array}$

Figure E-6 


\section{EXAMPLE OUTPUT \#1 (continued)}

RESULTS FOR SECOND CASE

ANGLE ALERT A/C POINTED FROM NORTH 120.0

COLLISION INTO DIRECT REAR OF ALERT AIRCRAFT

FUEL SPILL DISTR 3, LG A/C INTO REAR B-1B

$\begin{array}{cccccc}201144 & 611592 & & & \\ \text { NO. ARRANGEMENTS } & \text { USED; TESTS } 3,4,6,7,8,9 & \\ 203864 & 0 & 60384 & 48756 & 203864 & 94724 \\ \text { NO. ARRANGEMENTS } & \text { EVALUATED;TESTS3, } 4,6,7,8,9 & \\ 671668 & 0 & 254452 & 62872 & 508888 & 325440\end{array}$

\begin{tabular}{|c|c|c|}
\hline PROBABILITY & TEMP DEG $F$ & PROB EXCEED \\
\hline $\begin{array}{l}0.214 \mathrm{E}+00 \\
0.610 \mathrm{E}-02 \\
0.189 \mathrm{E}-02 \\
0.475 \mathrm{E}-02 \\
0.559 \mathrm{E}-02 \\
0.663 \mathrm{E}-02 \\
0.455 \mathrm{E}-02 \\
0.379 \mathrm{E}-02 \\
0.550 \mathrm{E}-02 \\
0.464 \mathrm{E}-02 \\
0.310 \mathrm{E}-02 \\
0.135 \mathrm{E}-01 \\
0.734 \mathrm{E}-02 \\
0.489 \mathrm{E}-02 \\
0.153 \mathrm{E}-02 \\
0.224 \mathrm{E}-02 \\
0.168 \mathrm{E}-02 \\
0.278 \mathrm{E}-02 \\
0.592 \mathrm{E}-04 \\
0.498 \mathrm{E}-02 \\
0.200 \mathrm{E}-02 \\
0.248 \mathrm{E}-03 \\
0.548 \mathrm{E}-02 \\
0.254 \mathrm{E}-03 \\
0.146 \mathrm{E}-02 \\
0.335 \mathrm{E}-03 \\
0.276 \mathrm{E}-02 \\
0.149 \mathrm{E}-03 \\
0.386 \mathrm{E}-03 \\
0.200 \mathrm{E}-02 \\
0.711 \mathrm{E}-03 \\
0.271 \mathrm{E}-03 \\
0.191 \mathrm{E}-02 \\
0.000 \mathrm{E}+00 \\
0.492 \mathrm{E}-03 \\
0.000 \mathrm{E}+00 \\
0.000 \mathrm{E}+00 \\
0.000 \mathrm{E}+00 \\
0.000 \mathrm{E}+00 \\
0.000 \mathrm{E}+00\end{array}$ & $\begin{array}{l}450 . \\
500 . \\
550 . \\
600 . \\
650 . \\
700 . \\
750 . \\
800 . \\
850 . \\
900 . \\
950 . \\
1000 . \\
1050 . \\
1100 . \\
1150 . \\
1200 . \\
1250 . \\
1300 . \\
1350 . \\
1400 . \\
1450 . \\
1500 . \\
1550 . \\
1600 . \\
1650 . \\
1700 . \\
1750 . \\
1800 . \\
1850 . \\
1900 . \\
1950 . \\
2000 . \\
2050 . \\
2100 . \\
2150 . \\
2200 . \\
2250 . \\
2300 . \\
2350 . \\
2400 .\end{array}$ & $\begin{array}{l}0.673 E+00 \\
0.220 \mathrm{E}+00 \\
0.207 \mathrm{E}+00 \\
0.203 \mathrm{E}+00 \\
0.193 \mathrm{E}+00 \\
0.181 \mathrm{E}+00 \\
0.167 \mathrm{E}+00 \\
0.158 \mathrm{E}+00 \\
0.150 \mathrm{E}+00 \\
0.138 \mathrm{E}+00 \\
0.128 \mathrm{E}+00 \\
0.122 \mathrm{E}+00 \\
0.931 \mathrm{E}-01 \\
0.775 \mathrm{E}-01 \\
0.672 \mathrm{E}-01 \\
0.640 \mathrm{E}-01 \\
0.592 \mathrm{E}-01 \\
0.557 \mathrm{E}-01 \\
0.498 \mathrm{E}-01 \\
0.496 \mathrm{E}-01 \\
0.391 \mathrm{E}-01 \\
0.348 \mathrm{E}-01 \\
0.343 \mathrm{E}-01 \\
0.227 \mathrm{E}-01 \\
0.222 \mathrm{E}-01 \\
0.191 \mathrm{E}-01 \\
0.184 \mathrm{E}-01 \\
0.125 \mathrm{E}-01 \\
0.122 \mathrm{E}-01 \\
0.114 \mathrm{E}-01 \\
0.716 \mathrm{E}-02 \\
0.566 \mathrm{E}-02 \\
0.508 \mathrm{E}-02 \\
0.104 \mathrm{E}-02 \\
0.104 \mathrm{E}-02 \\
0.000 \mathrm{E}+00 \\
0.000 \mathrm{E}+00 \\
0.000 \mathrm{E}+00 \\
0.000 \mathrm{E}+00 \\
0.000 \mathrm{E}+00\end{array}$ \\
\hline
\end{tabular}

Figure E-6 (continued) 
MEAN PROBABILITY VALUES

500 TO 2250 DEG E
0.5013
0.4745
0.4432
0.4163
0.3996
0.3785
0.3588
0.3342
0.3203
0.3060
0.2927
0.2447
0.1853
0.1814
0.1745
0.1641
0.1433
0.1346
0.1322
0.1237
0.1111
0.1066
0.0825
0.0780
0.0698
0.0679
0.0607
0.0508
0.0478
0.0427
0.0361
0.0345
0.0153
0.0153
0.0000
0.0000

Figure E-6 (continued) 


\section{EXAMPLE OUTPUT \#2}

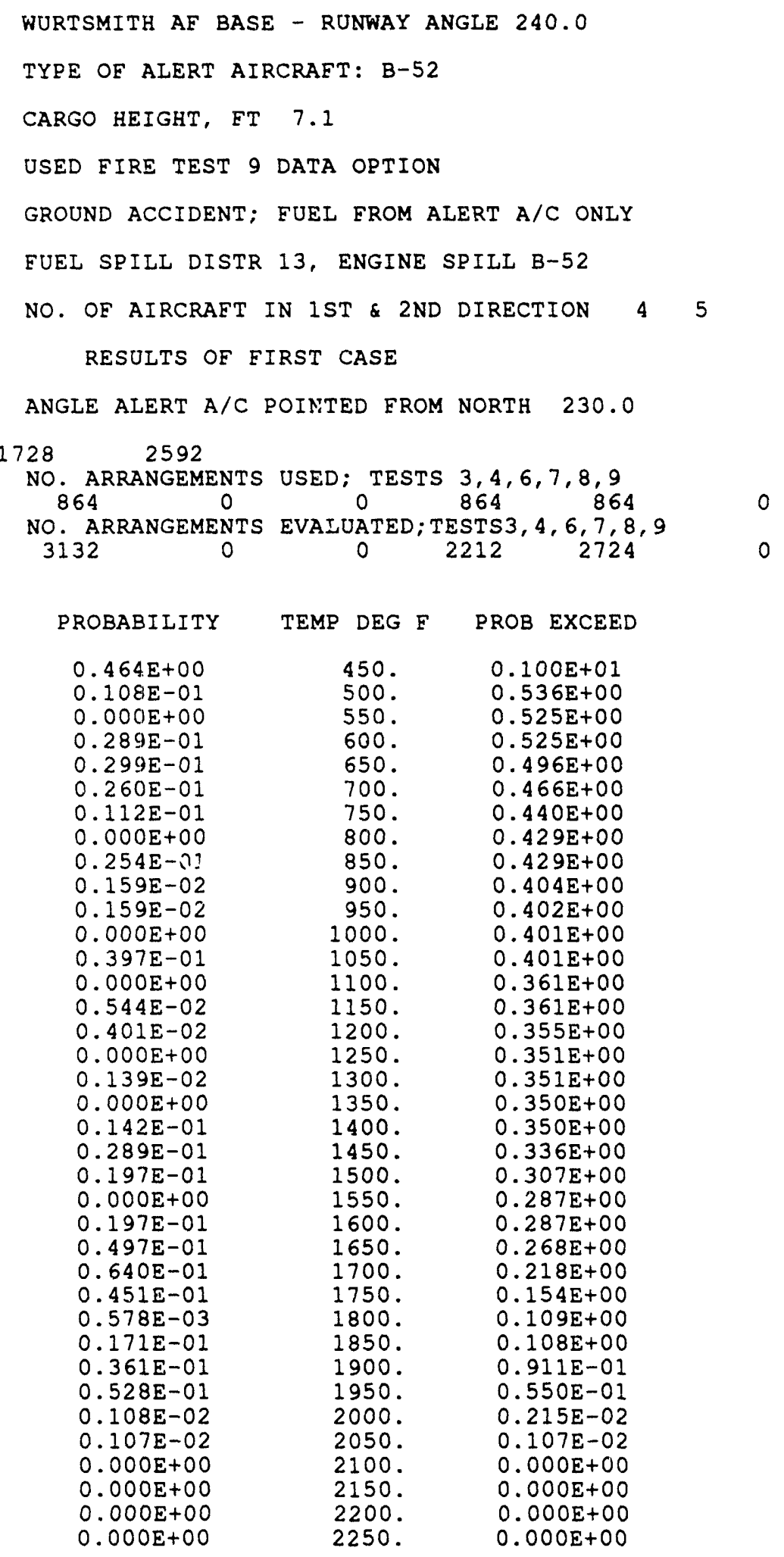


EXAMPLE OUTPUT \#2 (continued)

RESULTS FOR SECOND CASE

ANGLE ALERT A/C POINTED FROM NORTH 140.0

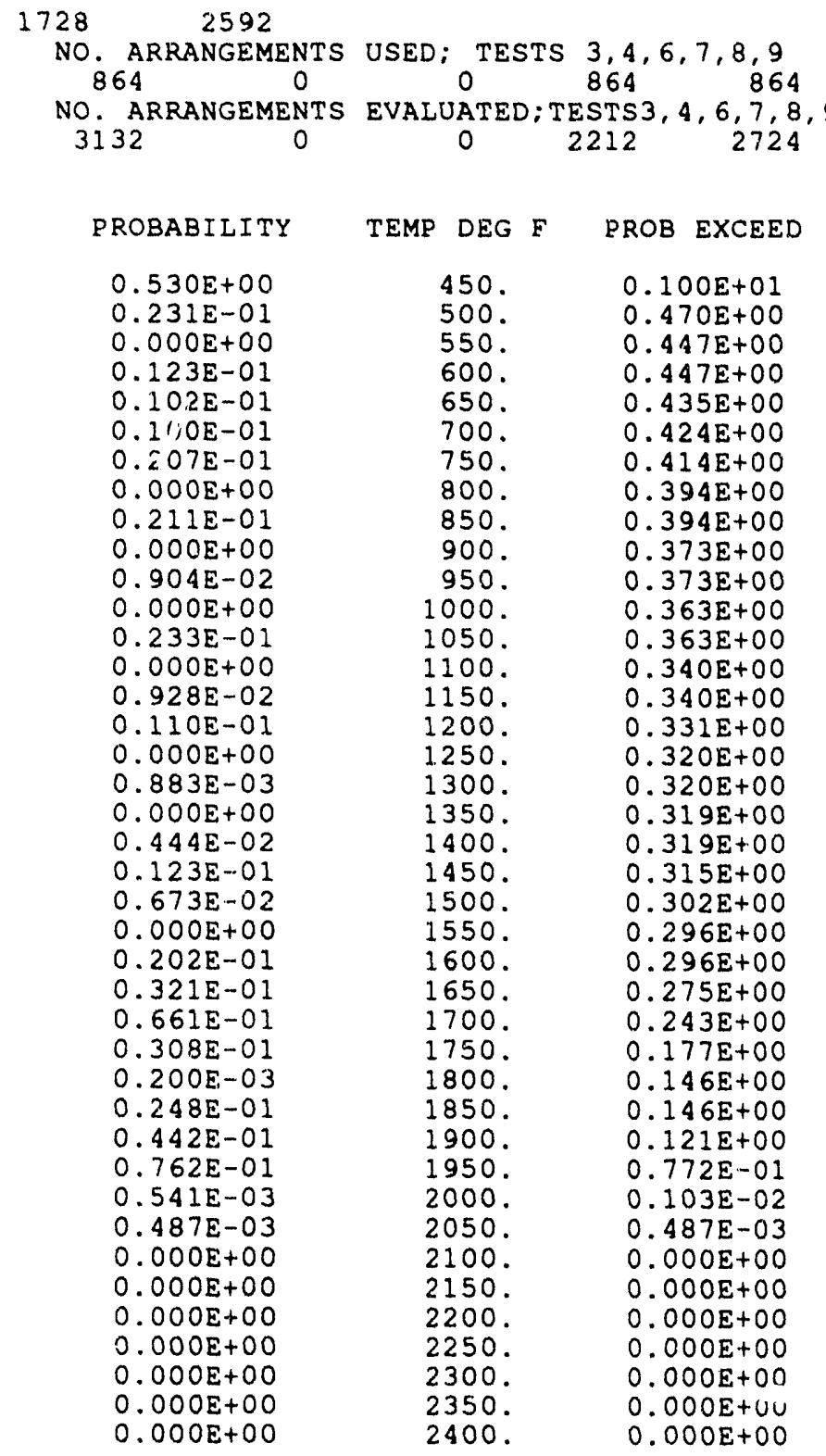


EXAMPLE OUTPUT \#2 (continued)

MEAN PROBABILITY VALUES
500 TO 2250 DEG F
0.4993
0.4816
0.4816
0.4620
0.4430
0.4259
0.4095
0.4095
0.3864
0.3857
0.3800
0.3800
0.3494
0.3494
0.3418
0.3339
0.3339
0.3328
0.3328
0.3241
0.3044
0.2919
0.2919
0.2719
0.2320
0.1668
0.1297
0.1293
0.1080
0.0673
0.0015
0.0007
0.0000
0.0000
0.0000
0.0000

Figure E-6 (continued) 


\section{Appendix F SUPPLEMENTARY RESULTS}

This appendix contains various results that were calculated and plotted but not included in the main body of the report. 

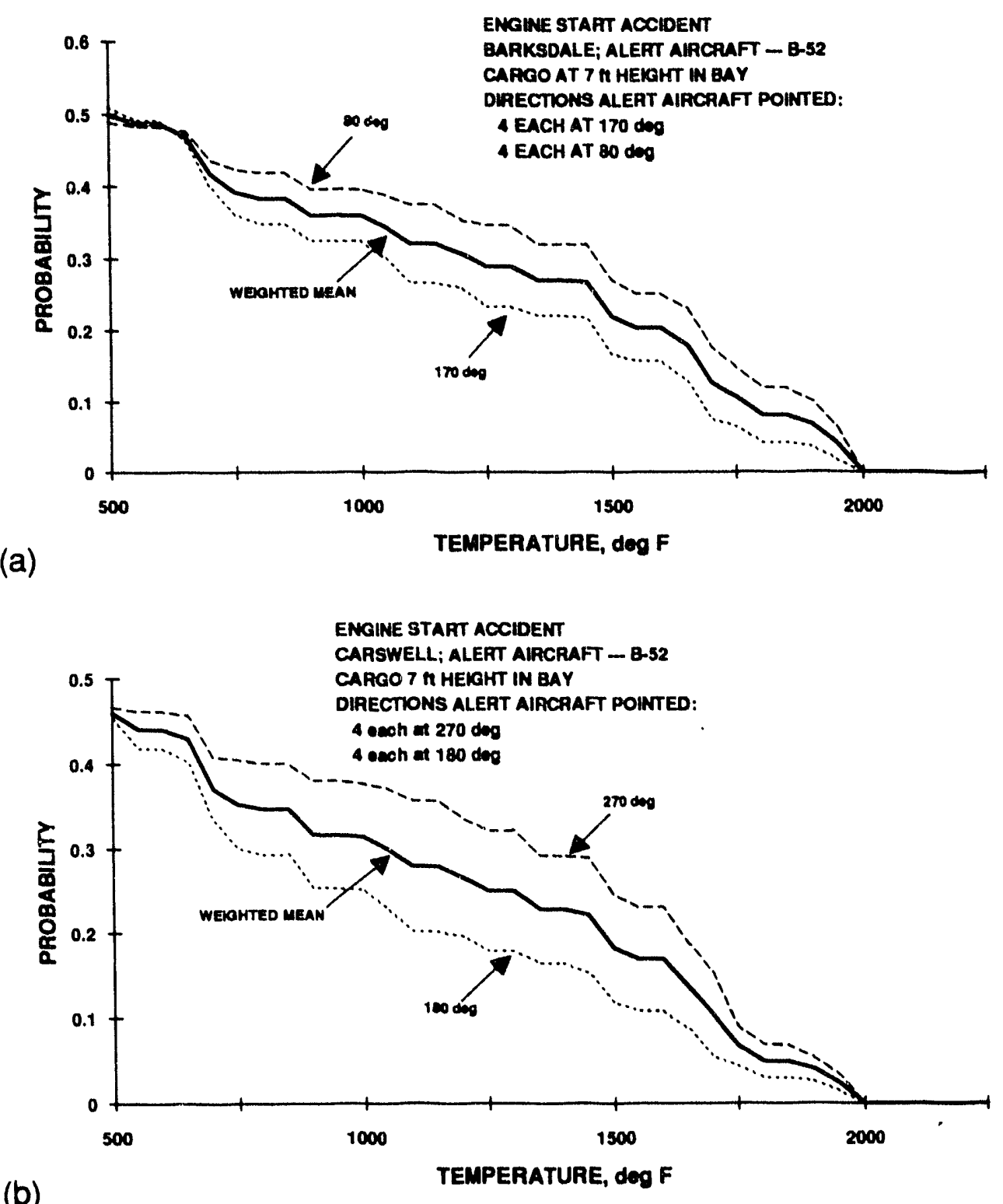

(b)

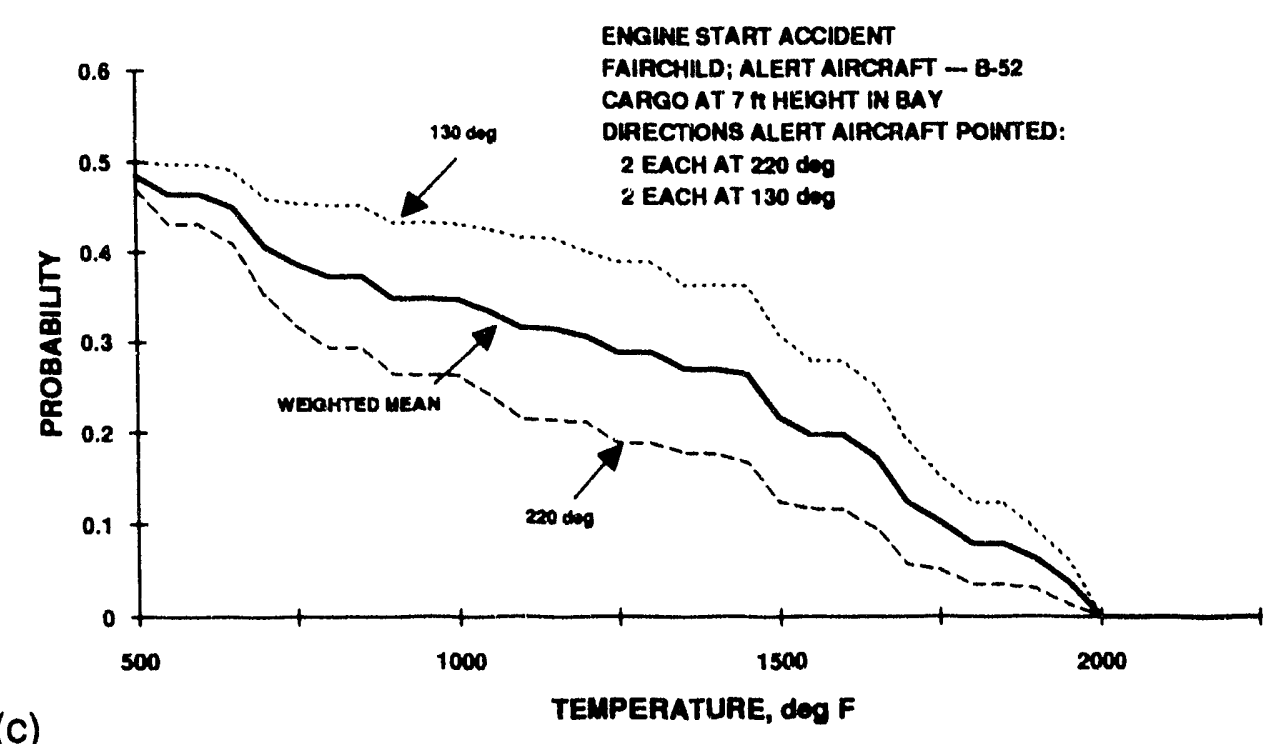

Figure F-1 Probability of Exceeding Given Temperatures for Various B-52 Bases 


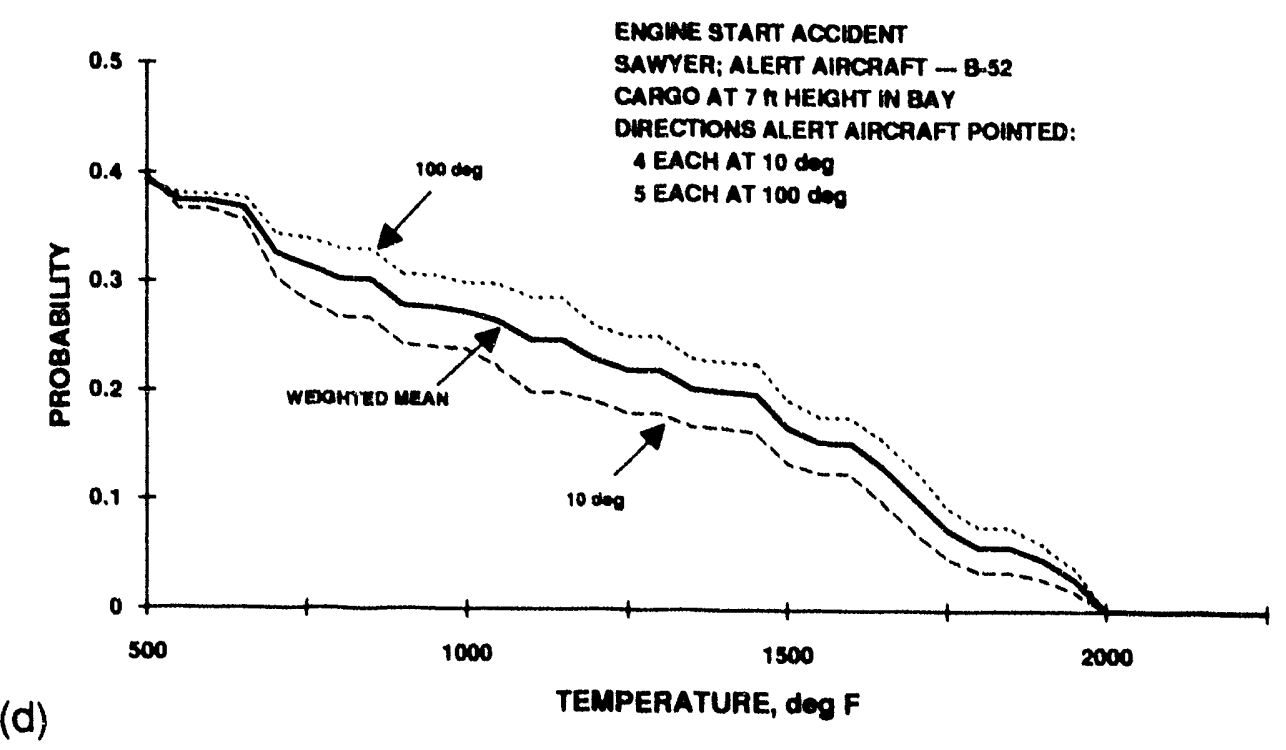

(d)
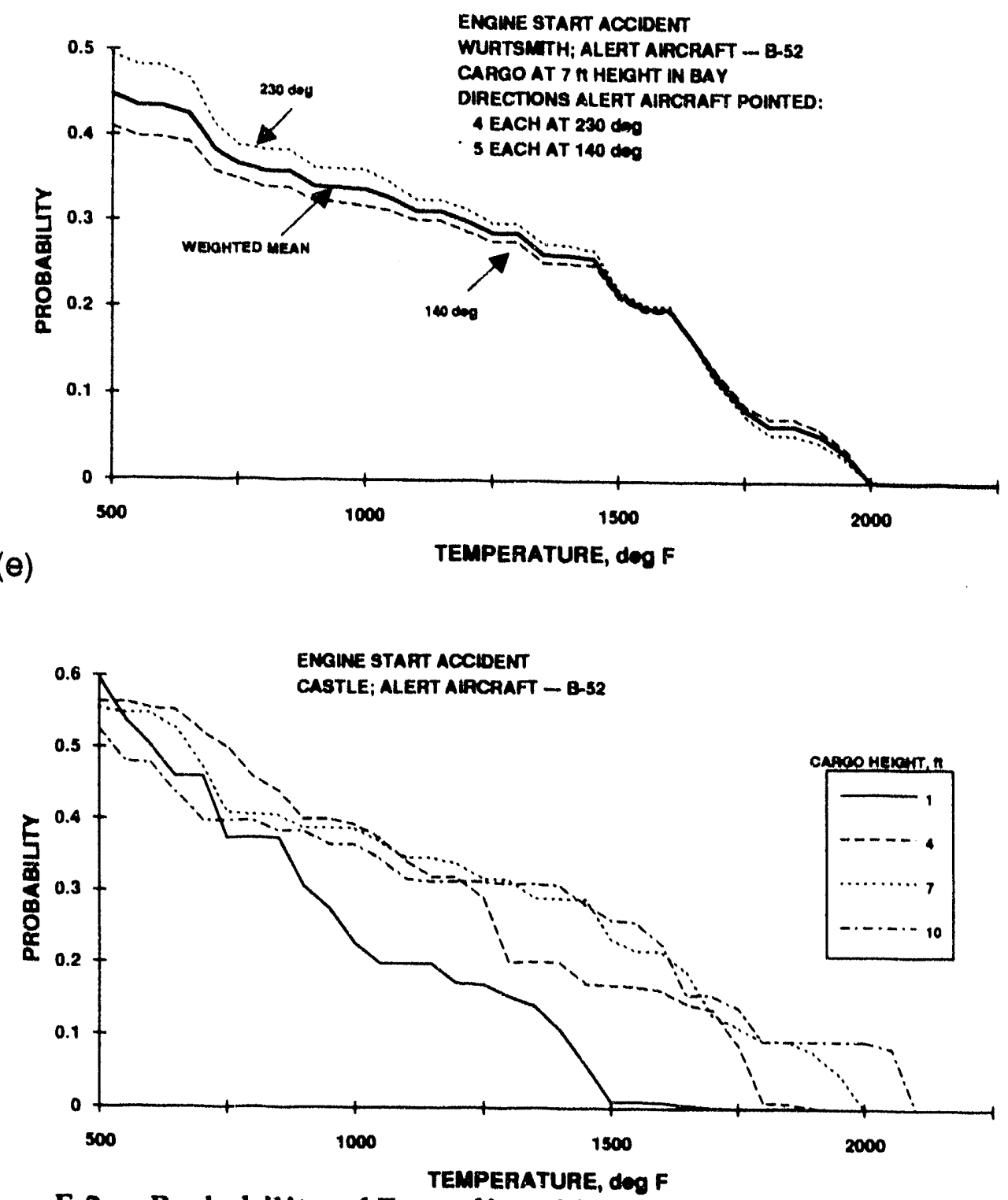

Figure F-2 Probability of Exceeding Given Temperatures for Various Heights at Castle AF Base 


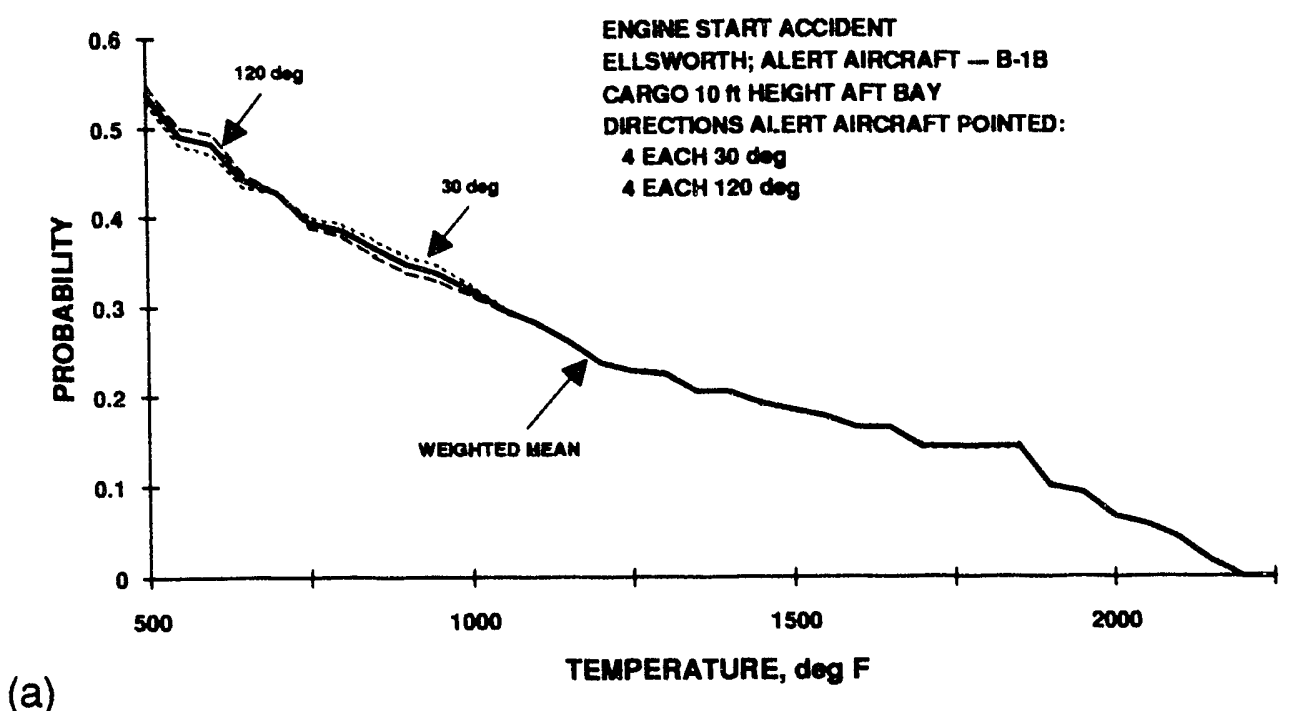

(a)

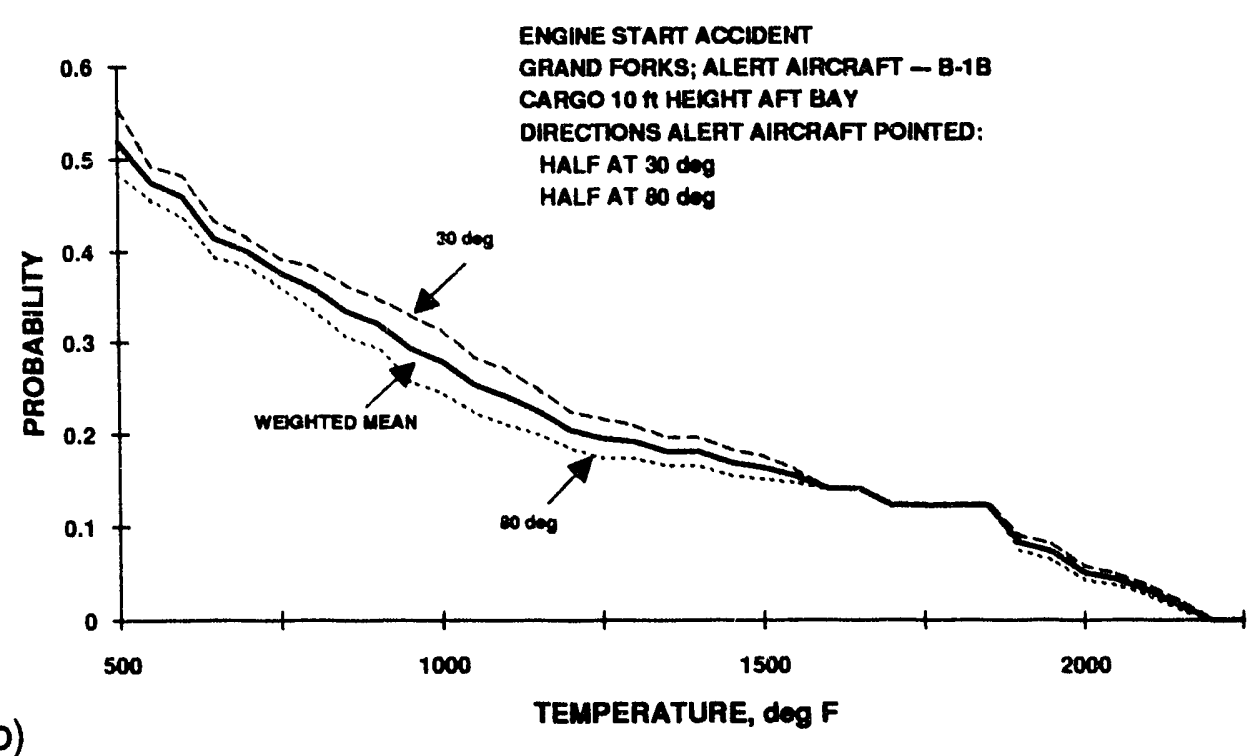

(b)

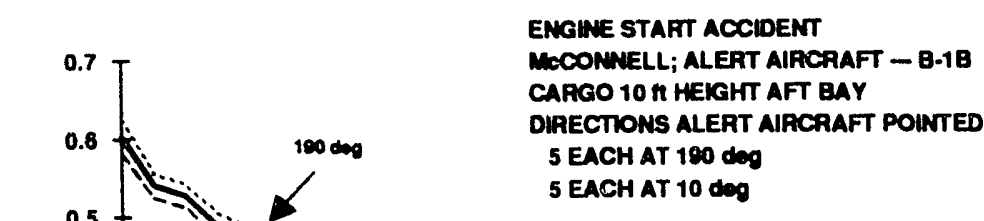

(c)

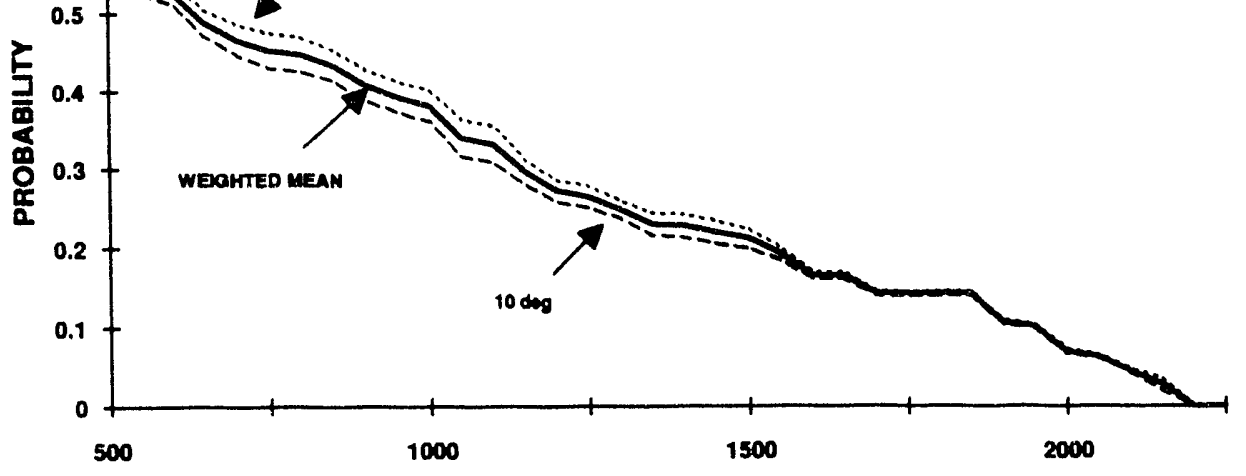

TEMPERATURE, dog F

Figure F-3 Probability of Exceeding Given Temperature for Weapons in Aft Bay of B-1B 


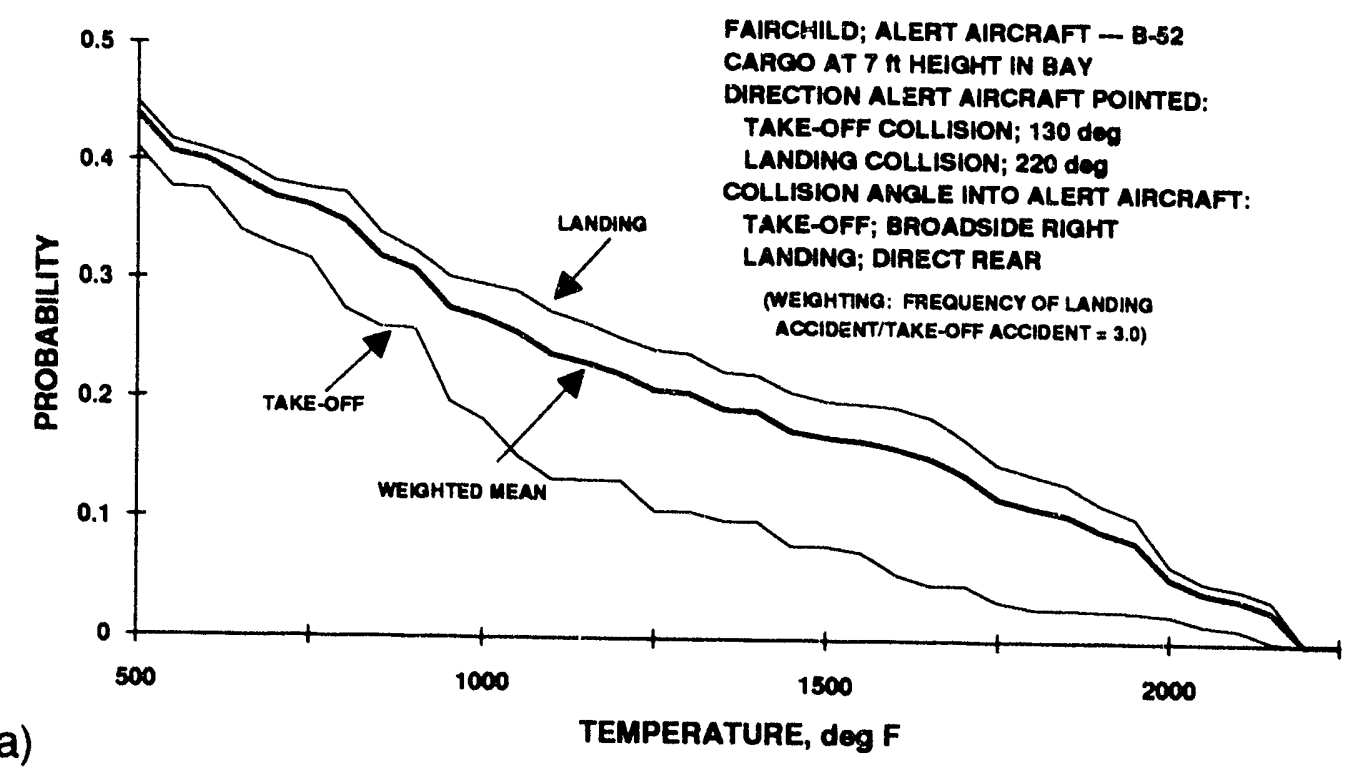

(a)

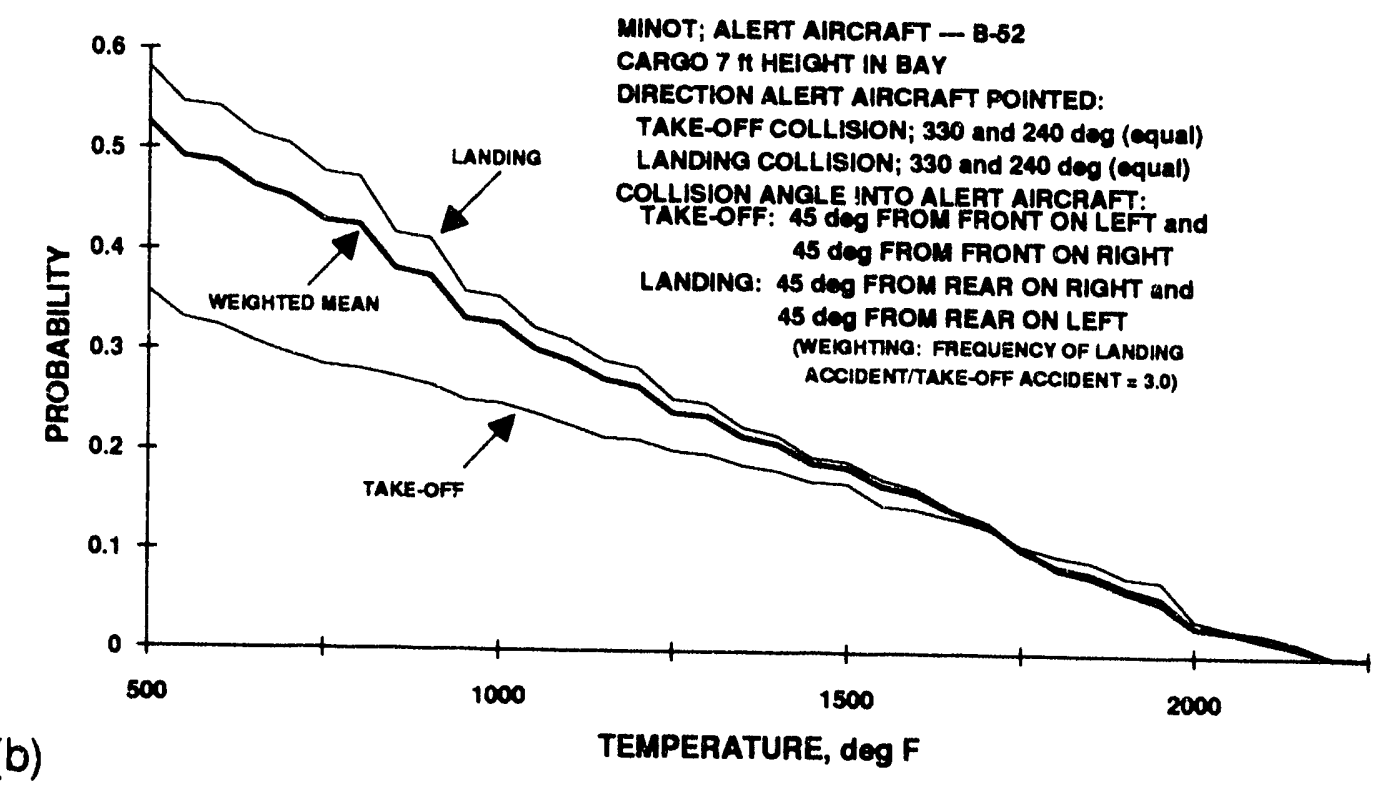

Figure F-4 Probability of Exceeding Given Temperatures for Various B-52 Bases 

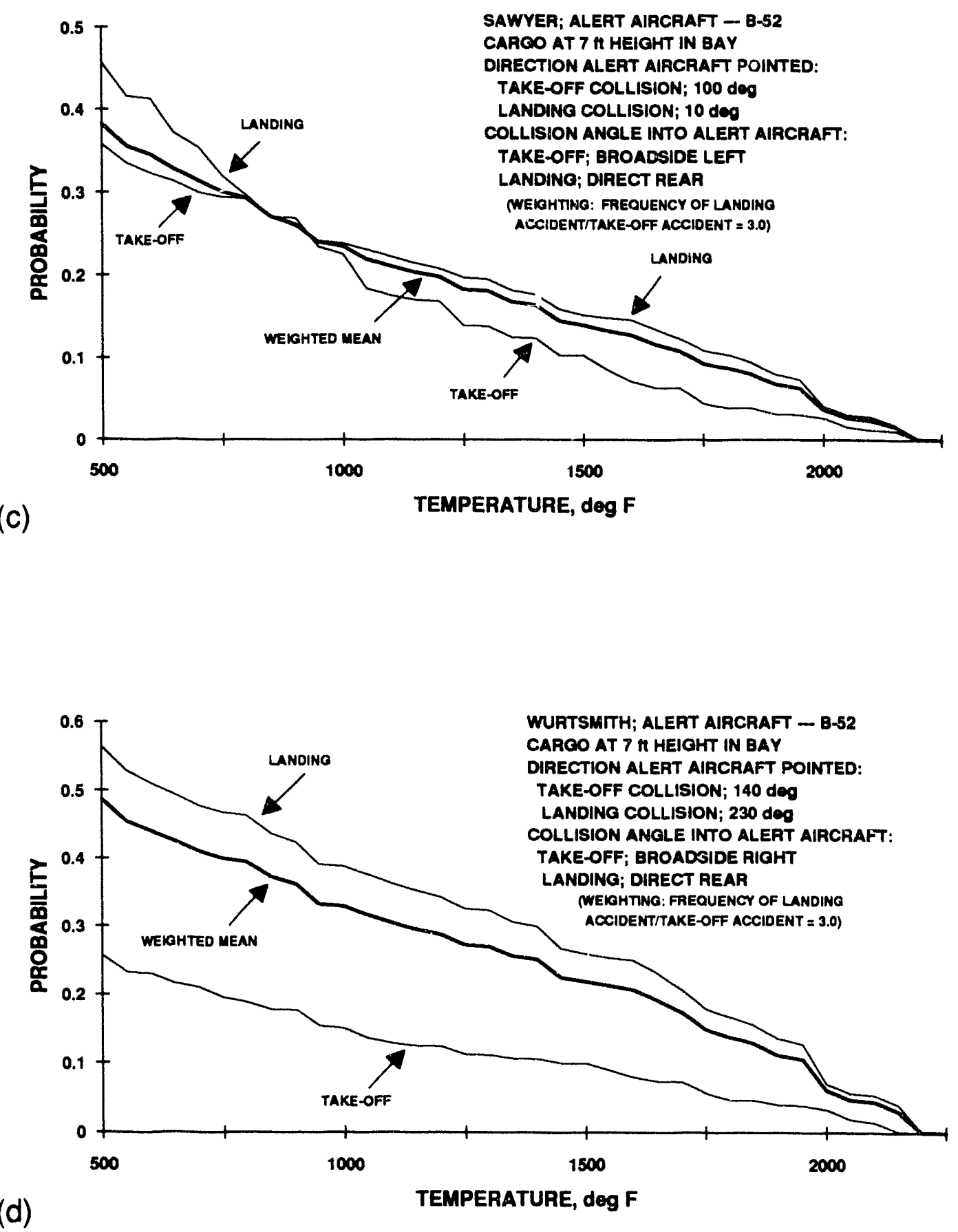

Figure F-4 Probability of Exceeding Given Temperatures for Various B-52 Bases (continued) 

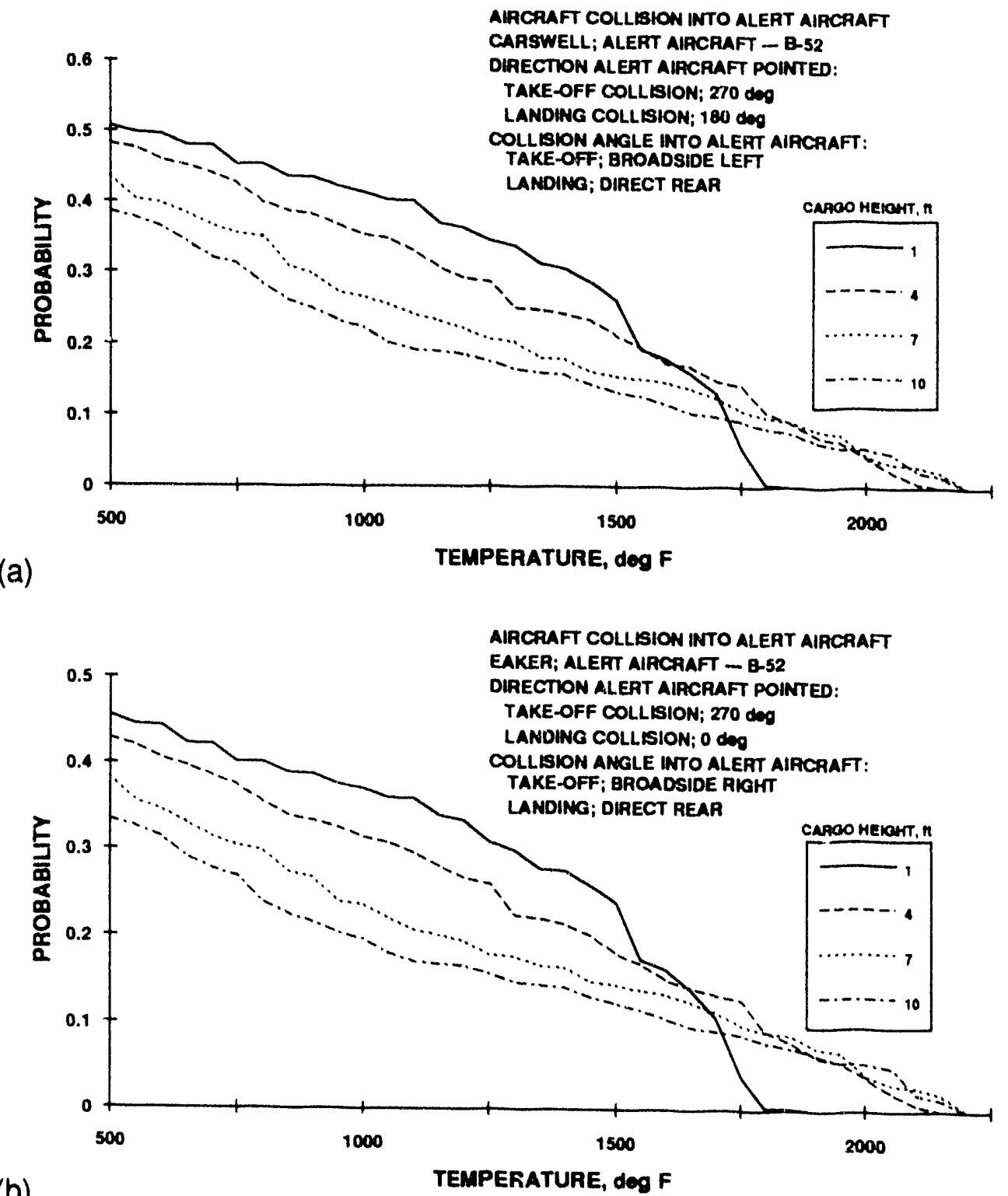

(b)

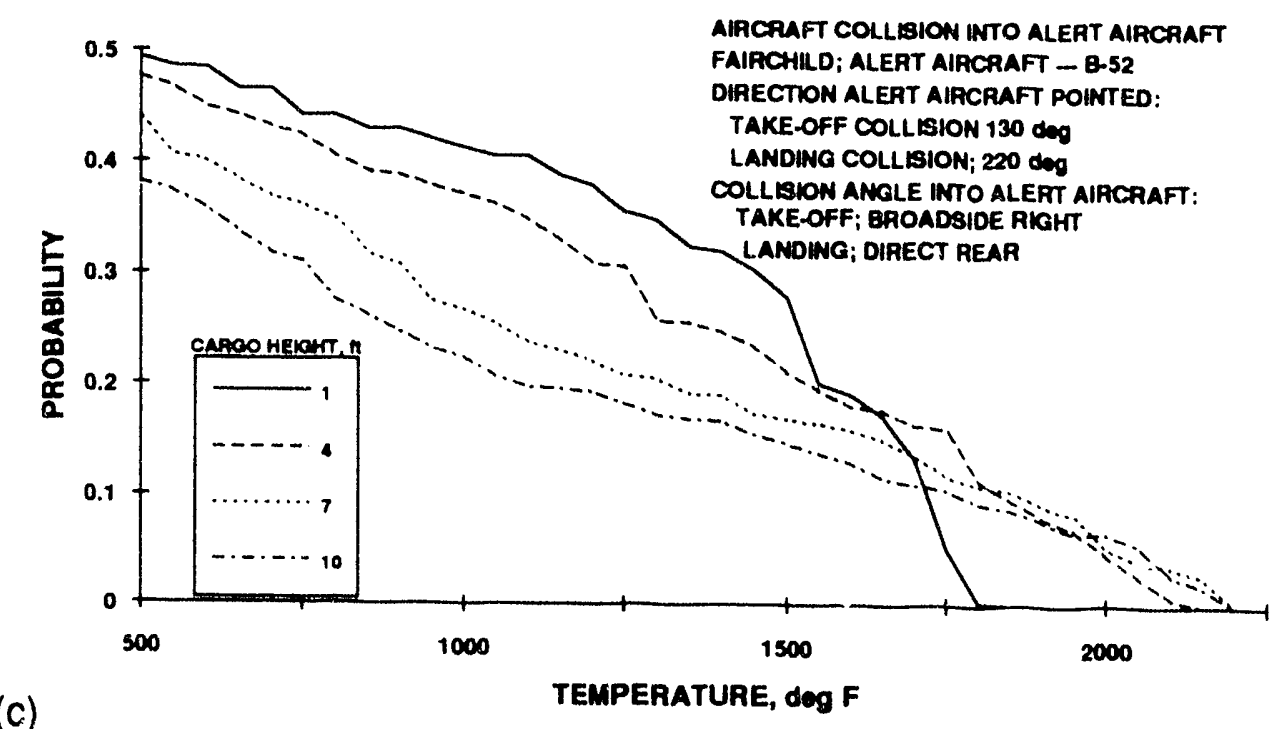

Figure F-5 Probability of Exceeding Given Temperatures vs Height for Various B-52 Bases 


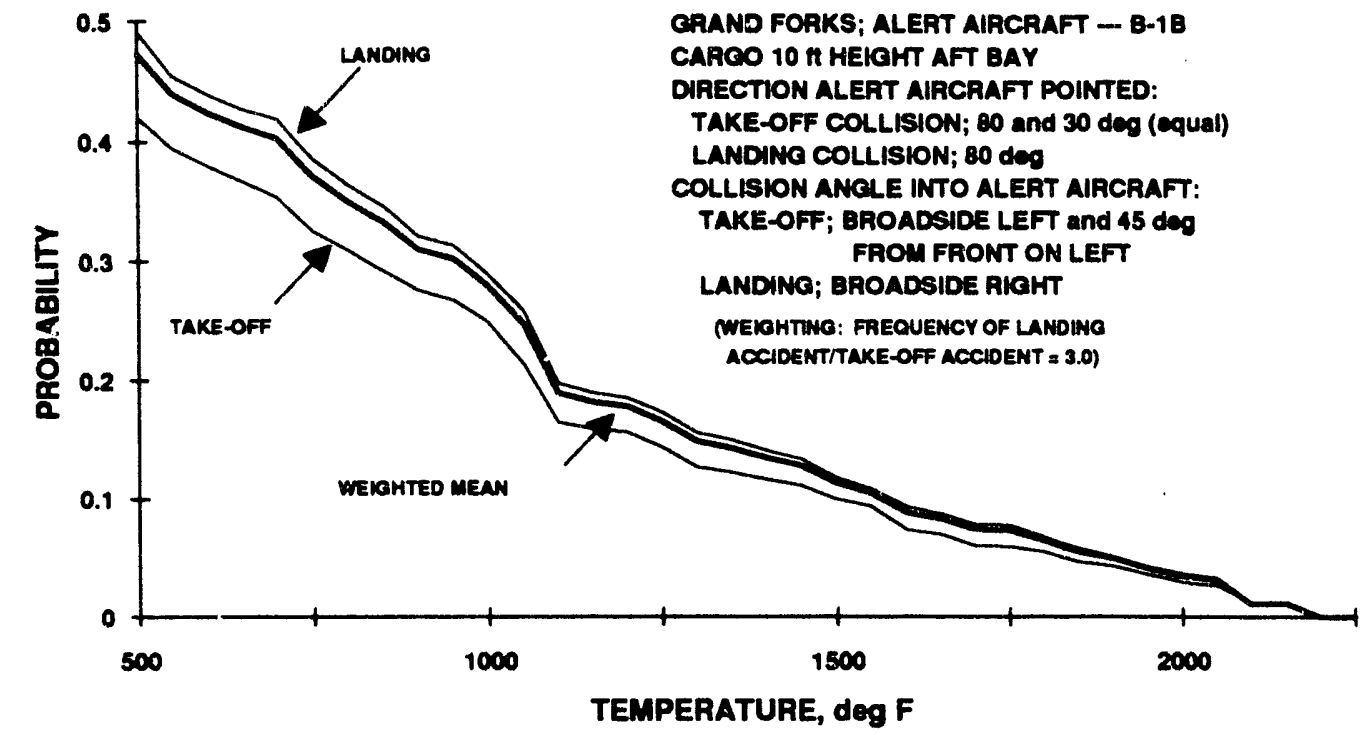

Figure F-6 Probability of Exceeding Given Temperature for Aft Bay at Grand Forks AF Base

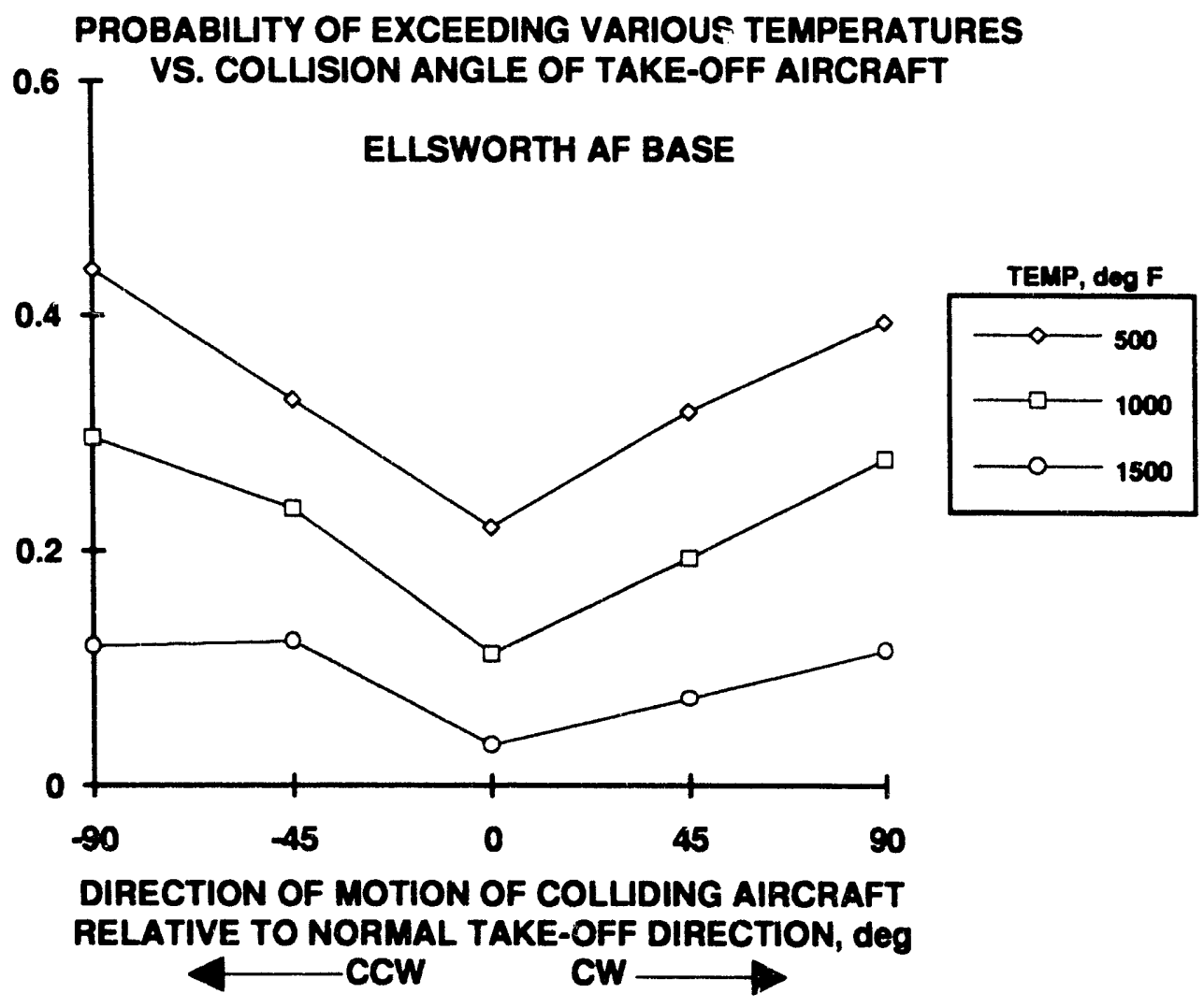

Figure F-7 Probability vs Collision Angle for Take-off Collision at Ellsworth AFB 


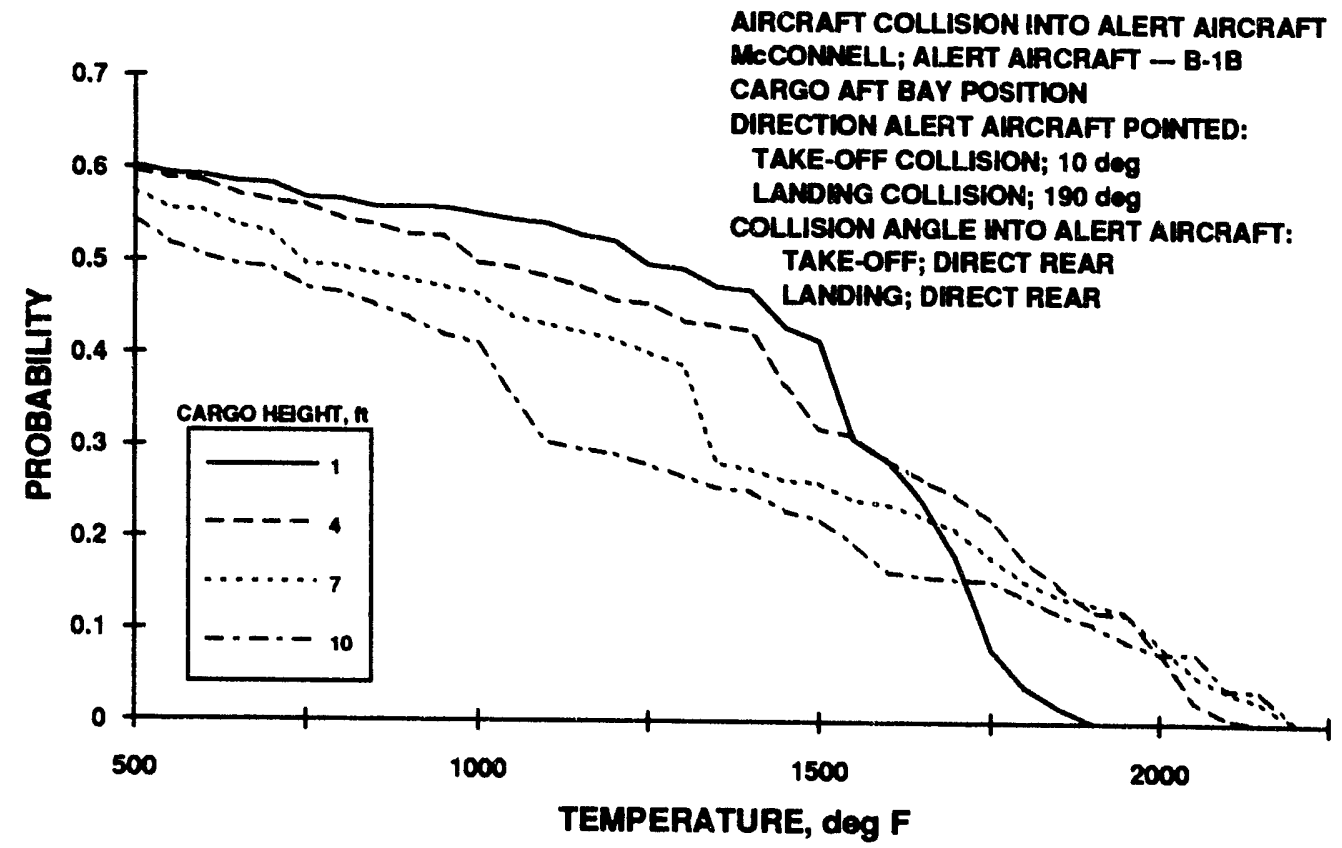

Figure F-8 Probability of Exceeding Given Temperature for Aft Bay for Various Heights at McConnell AF Base 

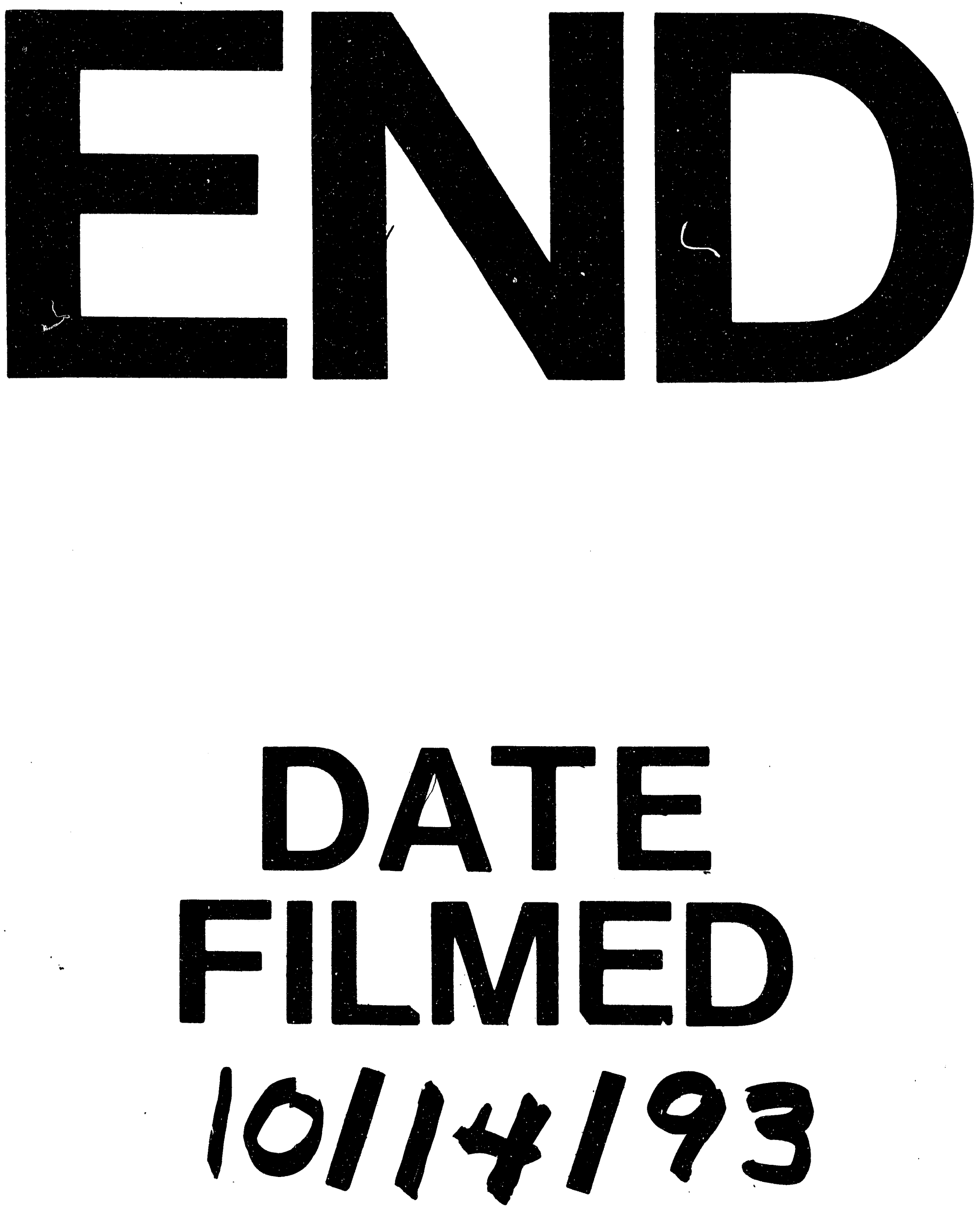
